\title{
Regulation of the long non-coding RNA FAM83H-AS1 by human papillomavirus in cervical cancer
}

\author{
Jamie Ann Barr Ph.D. \\ West Virginia University, jbarr3@mix.wvu.edu
}

Follow this and additional works at: https://researchrepository.wvu.edu/etd

Part of the Cancer Biology Commons, Cell Biology Commons, Molecular Biology Commons, Oncology Commons, and the Virus Diseases Commons

\section{Recommended Citation}

Barr, Jamie Ann Ph.D., "Regulation of the long non-coding RNA FAM83H-AS1 by human papillomavirus in cervical cancer" (2019). Graduate Theses, Dissertations, and Problem Reports. 3926.

https://researchrepository.wvu.edu/etd/3926

This Dissertation is protected by copyright and/or related rights. It has been brought to you by the The Research Repository @ WVU with permission from the rights-holder(s). You are free to use this Dissertation in any way that is permitted by the copyright and related rights legislation that applies to your use. For other uses you must obtain permission from the rights-holder(s) directly, unless additional rights are indicated by a Creative Commons license in the record and/ or on the work itself. This Dissertation has been accepted for inclusion in WVU Graduate Theses, Dissertations, and Problem Reports collection by an authorized administrator of The Research Repository @ WVU.

For more information, please contact researchrepository@mail.wvu.edu. 


\title{
Regulation of the long non-coding RNA
}

\section{FAM83H-AS1 by human papillomavirus in cervical cancer}

\author{
Jamie A. Barr \\ Dissertation submitted \\ to the West Virginia University School of Medicine \\ at West Virginia University \\ in partial fulfillment for the degree of \\ Doctor of Philosophy \\ in \\ Cancer Cell Biology \\ J. Michael Ruppert, MD, PhD, Chair \\ Laura F. Gibson, PhD \\ John B. Barnett, $\mathrm{PhD}$ \\ Peter Stoilov, $\mathrm{PhD}$ \\ William P. Petros, PharmD \\ Ivan Martinez, $\mathrm{PhD}$, Mentor \\ Cancer Cell Biology Program \\ Morgantown, WV \\ 2019
}

Key Words: FAM83H-AS1, long non-coding RNA, lncRNA, human papillomavirus, HPV, cervical cancer

Copyright 2019 Jamie A. Barr 


\section{Abstract \\ Regulation of the long non-coding RNA FAM83H-AS1 by human papillomavirus in cervical cancer}

Jamie A. Barr

Non-coding RNAs (NcRNAs), such as long non-coding RNAs (lncRNAs) and microRNAs (miRNAs), have been found to be involved in a variety of critical biological processes, and dysregulation of ncRNAs have been involved with several human diseases including cancer.

High-risk human papillomavirus (HPV) infection is one of the first events in the process of carcinogenesis in cervical and a subset of head and neck cancers. The expression of the viral oncoproteins $\mathrm{E} 6$ and E7 is essential in this process by inactivating the tumor suppressor proteins p53 and Rb, respectively, in addition to their interactions with other host proteins and regulation of ncRNAs. Our group identified novel regulation of host lncRNAs by HPV oncoprotein E6. More specifically, we discovered that a lncRNA known as FAM83H-AS1 is involved with proliferation, migration, and apoptosis in cervical cells, and high expression of this lncRNA correlates with poor overall cervical cancer patient survival. FAM83H-AS1 is a nuclear RNA, and mechanistically it is regulated through the E6-p300 pathway in a p53-independent manner. These findings provide knowledge of a specific lncRNA that could be studied further as a biomarker and/or therapeutic target not only in HPV-related cancers but also in other types of cancers where FAM83H-AS1 expression is dysregulated.

In parallel with these studies, our group identified a specific subgroup of miRNAs that are induced during quiescence and processed by a non-canonical biogenesis pathway by using primary human cells. miRNA expression is dysregulated when cells undergo a reversible state of growth arrest known as quiescence. These primary (pri-)miRNAs are modified with a 2,2,7-trimethylguanosine (TMG)cap such that they are processed downstream in an Exportin-1 (XPO1)-dependent manner, independent of the canonical Exportin-5 (XPO5) protein used for exportation to the cytoplasm. The discovery of a new alternative miRNA pathway in quiescent primary human cells opens the door to future studies in other types of cells, such as stem cells and cancer stem cells, where the state of quiescence is important in their biological functions. 


\section{Dedication}

This dissertation is dedicated to my parents, Ron and Regina Barr, who raised me to be hardworking and taught me persistence to strive to accomplish my goals while still enjoying the journey along the way. I am truly blessed to have such loving parents who have supported me through every aspect of my life. This dissertation work would not have been possible without them. I also want to thank my loving fiancé, Chris Juric, who provided constant optimism, laughter, and happiness when life was challenging, and motivation to continue working hard to complete this work. This dissertation is also dedicated to my Godparents, Larry Barr and Carol Pacofsky, who provided multiple care packages to brighten my day and reassurance that everything would work out.

I would also like to dedicate this dissertation to my supportive friends and family who were personally affected by cancer, especially my grandmothers, Irene Barr and Filomena Pacofsky, and grandfather, Larry Barr. Their experiences instigated my desire to enter into oncology research, and they kept me motivated when experiments failed. I am blessed to have the love and support of all of my friends and family, and I will forever be grateful for their contributions. 


\section{Acknowledgements}

I would like to take this opportunity to thank my mentor, Dr. Ivan Martinez, for his guidance, constant support, and optimism throughout my graduate career. Being his first graduate student has provided us the opportunity to learn and grow together, therefore making us closer than the typical graduate student-principal investigator relationship. Looking back on how I progressed from a naïve to experienced scientist, I gained a multitude of knowledge from him, which made that transition and this dissertation possible.

I would also like to thank the Post-Doctoral Fellow, Karen Hayes, who was in the Martinez Lab for the bulk of my PhD years. She provided an unending amount of support both in and out of the laboratory, not only while she was a member of the lab, but even after she moved on to a new position. Her optimism, love for research, technical advice, and desire to help me succeed aided significantly in the completion of my dissertation. Additionally, my lab mates Tayvia Brownmiller and Abby Harold (e.g. the CRISPR whisperer) collaborated with me on multiple research projects and provided friendship in and out of the lab. We helped each other out along the way by sharing lab responsibilities, which allowed me to progress my work easier.

Lastly, I would like to acknowledge my committee members, Dr. J. Michael Ruppert, Dr. Laura F. Gibson, Dr. John B. Barnett, Peter Stoilov, and William P. Petros for their guidance and expertise throughout my $\mathrm{PhD}$ dissertation work. 


\section{Table of Contents}

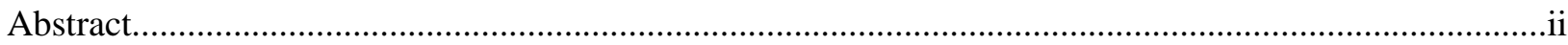

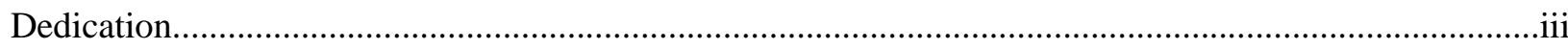

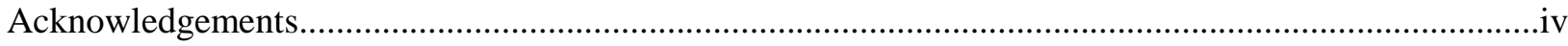

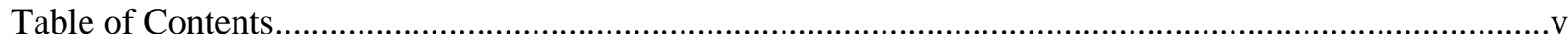

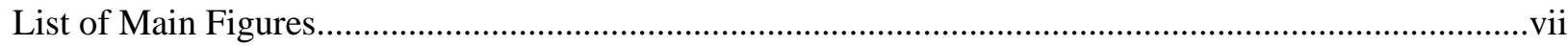

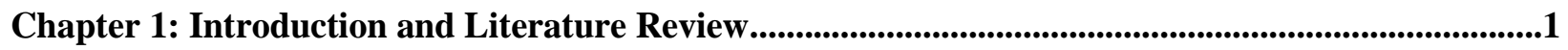

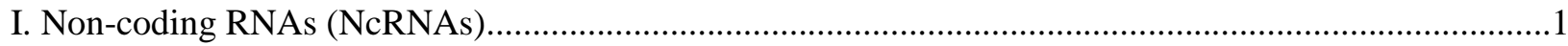

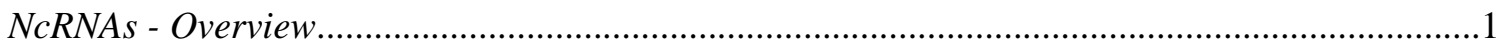

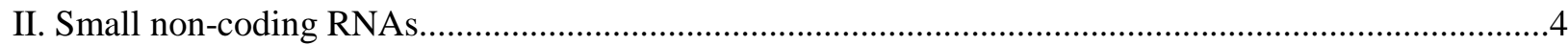

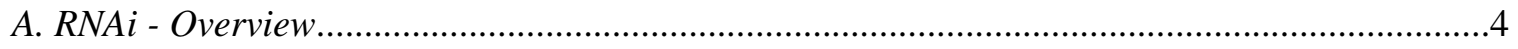

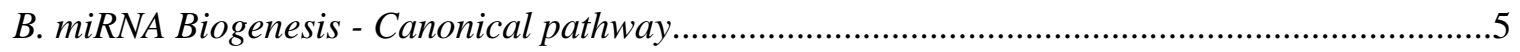

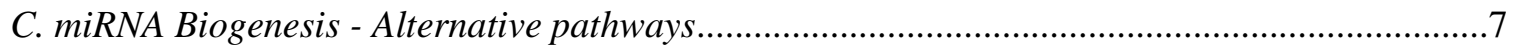

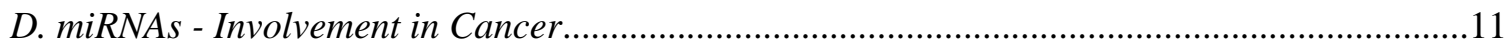

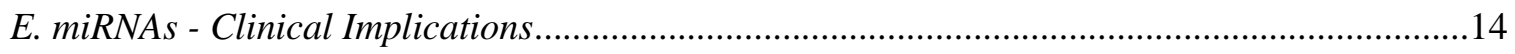

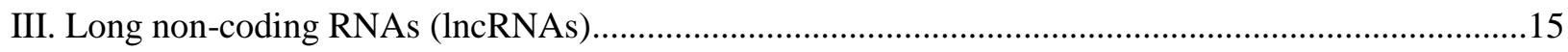

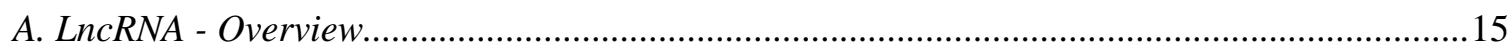

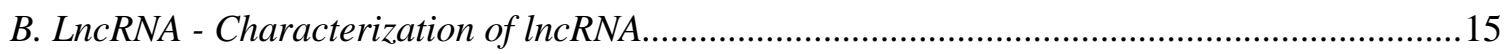

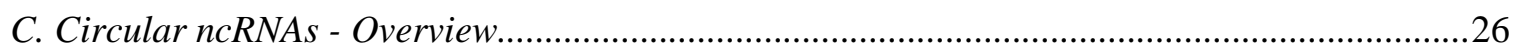

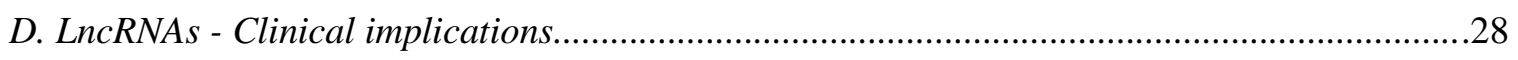

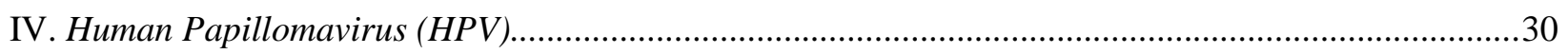

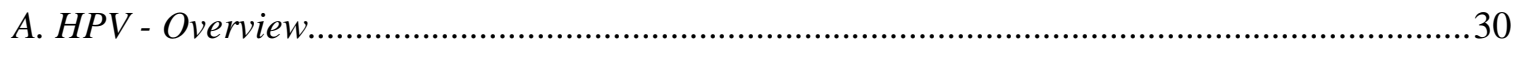

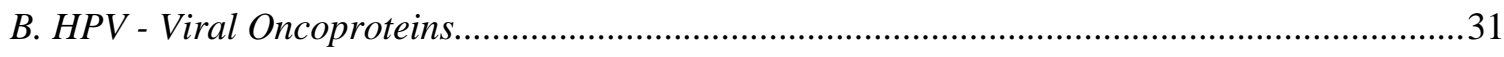

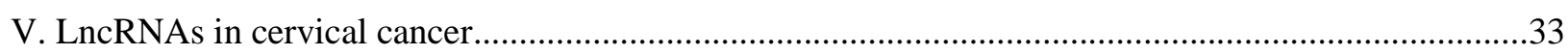

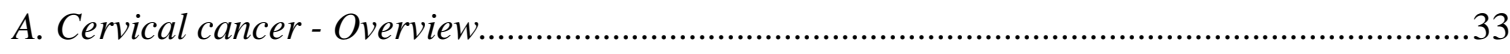

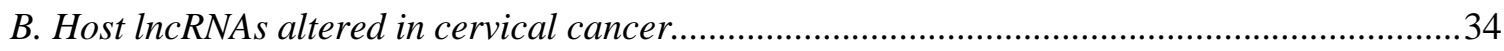

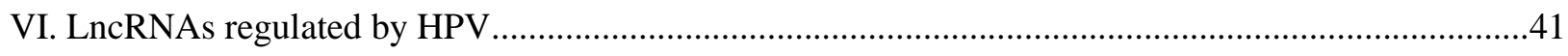

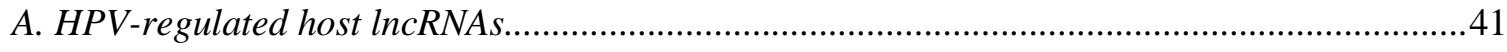

B. HPV regulation of lncRNAs in cancer - Clinical implications.................................................43

VII. Oncogenic lncRNA FAM83H-AS1 (also known as 'onco-lncRNA-3')...........................................43 
Chapter 2: Long non-coding RNA FAM83H-AS1 is regulated by human papillomavirus 16 E6 independently of $\mathbf{p 5 3}$ in cervical cancer cells.

Chapter 3: An Exportin-1 dependent microRNA biogenesis pathway during human cell quiescence

Chapter 4: Overall Discussion and Future Directions.................................................................186

Appendix: Immunoprecipitation of Tri-methylated Capped RNA........................................................204

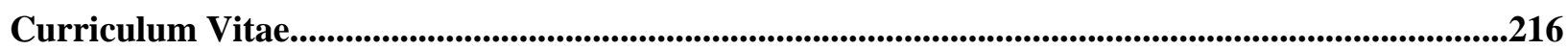




\section{List of Main Figures:}

\section{Chapter 1: Introduction and Literature Review}

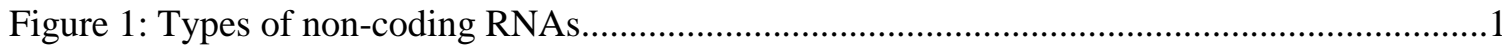

Figure 2: Non-coding gene abundance with evolutionary organisms...........................................

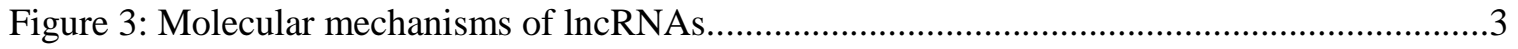

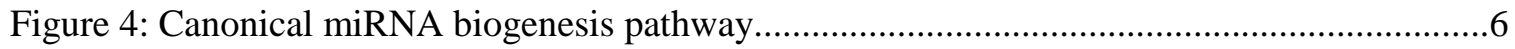

Figure 5: Alternative miRNA biogenesis pathway in quiescent cells.........................................10

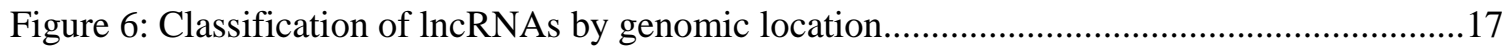

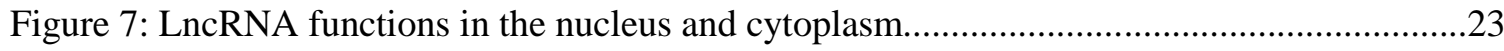

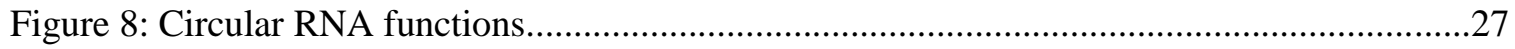

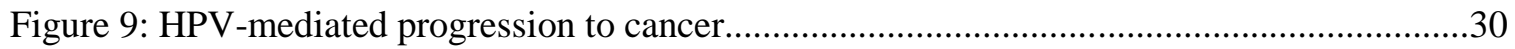

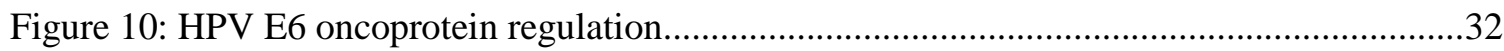

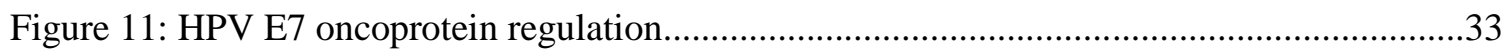

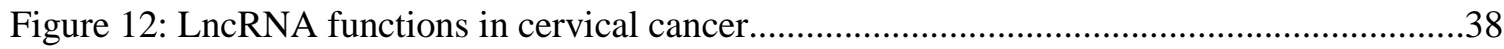

Figure 13: Heat map of FAM83H-AS1 expression alteration in multiple cancer types.................47

\section{Chapter 2: Long non-coding RNA FAM83H-AS1 is regulated by human papillomavirus 16 E6} independently of $\mathbf{p 5 3}$ in cervical cancer cells

Figure 1: Differential expression of host lncRNAs after expression of HPV-16 E6 in primary foreskin keratinocytes.

Figure 2: Increased FAM83H-AS1 expression in primary cervical keratinocytes containing the HPV-16 genome as well as in HPV-16 positive cervical cancer and HNSCC lines

Figure 3: Regulation of FAM83H-AS1 expression by HPV-16 E6 in a p53-independent, p300dependent manner.

Figure 4: FAM83H-AS1 is localized in the nucleus in cervical pre-malignant and cancerous cell lines

Figure 5: FAM83H-AS1 knockdown altered cell proliferation, migration, and apoptosis in CaSki cells.

Figure 6: FAM83H-AS1 expression is increased in human cervical cancer tissues and correlates with poor overall survival.

Chapter 3: Exportin-1 dependent microRNA biogenesis pathway during human cell quiescence 
Figure 1: Differential expression of mature miRNAs and their corresponding pri-miRNAs during

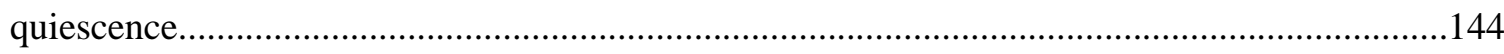

Figure 2: Regulation of Exportin-5 during quiescence by autophagy and miR-34a....................145

Figure 3: Biogenesis of quiescence-induced miRNAs required for Exportin-1 but not

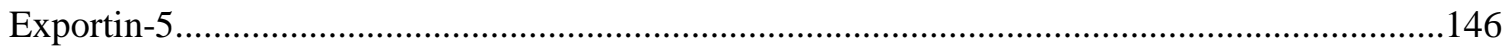

Figure 4: Quiescence-induced primary miRNAs are (TMG)-capped by TGS1

Figure 5: Detection of cytoplasmic pri-miR-34a is dependent on Exportin-1 expression............148

Figure 6: Detection of a small cytoplasmic isoform of Drosha during quiescence......................149

\section{Chapter 4: Overall Discussion and Future Directions}

Figure 1: Heat map of FAM83H-AS1 expression alterations in multiple cancer types...............189

Figure 2: HPV-16 was the only high-risk strain analyzed that demonstrated E6 oncoprotein

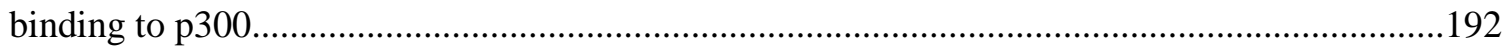

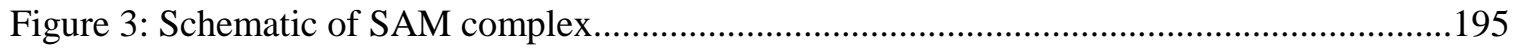

Figure 4: CRISPR Cas9 mutant technology to over-express FAM83H-AS1 in C-33A cell line.

\section{Appendix: Immunoprecipitation of Tri-methylated Capped RNA}

Figure 1: Main steps diagram of this protocol.

Figure 2: RNA immunoprecipitation of (TMG)-capped primary miRNAs (pri-miRNAs) in quiescent human foreskin fibroblasts (HFFs) 


\section{Chapter 1}

\section{Introduction and Literature Review}

\section{$\underline{\text { I. Non-coding RNAs (NcRNAs) }}$}

\section{NcRNAs - Overview}

In 1961, the concept of messenger RNAs (mRNAs) was determined as the intermediate messenger between DNA and protein synthesis(1). This led to the central dogma of biology that DNA containing the genetic information is transcribed to mRNA, which in turn is translated to a protein; the protein is considered the critical functional product. Non-coding RNAs contribute to the translational process, including ribosomal RNA (rRNA) and transfer RNA (tRNA) processing. rRNA associates with proteins to form ribosomes, which catalyze the assembly of amino acids into protein chains. Each amino acid is covalently bound to a specific tRNA, which guides it to the polypeptide chain and helps decipher the next desired amino acid to add to the chain(2).

In 1982, the first "enzymatically active" RNA was identified (ribozyme) with a specific biological

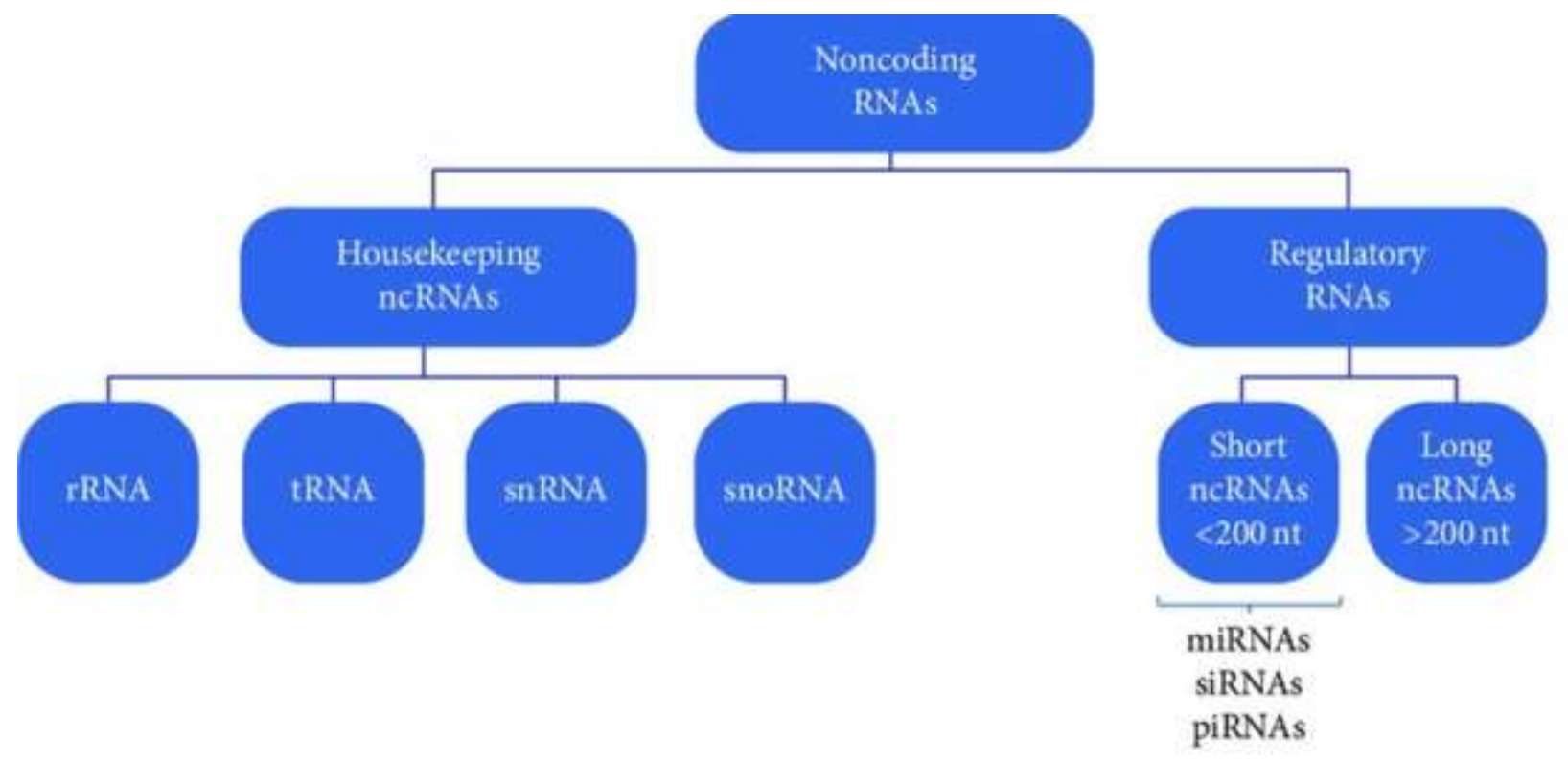

Figure 1: Types of non-coding RNAs.

Modified from: Losko et al., Mediators of Inflammation, 2016.

function(3). These RNA enzymes catalyze their own intronic excision during RNA splicing (self-splicing 
introns)(4). These early findings opened the door to research additional RNAs exhibiting non-coding functions and, to date, there are various other functional housekeeping and regulatory ncRNAs summarized in Figure 1.

Advancement of sequencing technologies, the Encyclopedia of DNA Elements (ENCODE) work(5), and completion of the Human Genome Project revealed that $\sim 80 \%$ of the human genome is transcribed and the majority of transcribed genes do not code for proteins $(<3 \%$ of the human genome codes for proteins)(6). It was initially thought that the majority of the non-coding genomic regions were "transcriptional noise", but it is now appreciated that many of these have more biologically relevant functions.

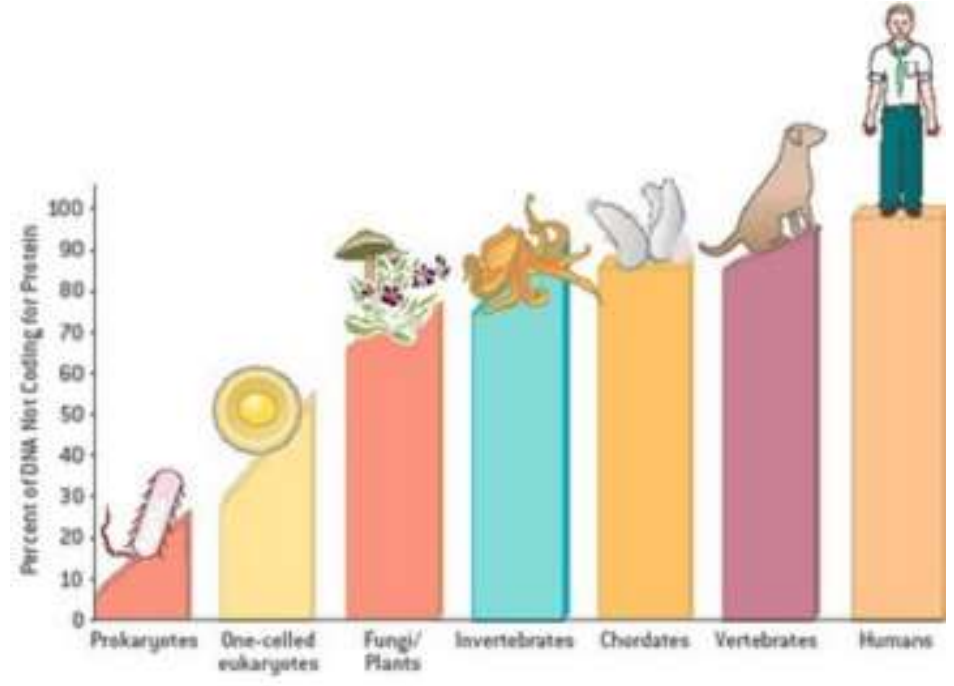

Figure 2: Non-coding gene abundance with evolutionary organisms. Adapted from: Mattick JS, Scientific American, 2004.
These various ncRNAs once considered to be "junk" have been identified to rebel against the status quo and have other biological functions besides coding for proteins. NcRNAs typically lack an open reading frame (ORF) such that they do not code for proteins. Some ncRNAs contain very small ORFs that produce small peptides $(7,8)$, but this does not prove they are functional. More evolutionary complex organisms have a greater abundance of ncRNAs than less complex organisms

(Figure 2) leading towards the theory that ncRNAs contribute to the development and control of more complex cellular processes. Most ncRNAs form RNA-protein complexes called ribonucleoproteins (RNPs), however, they can have a multitude of alternative interactions. NcRNAs can also act as competing endogenous RNAs (ceRNAs) such that the ncRNA can mimic an RNA binding partner and inhibit the function of the alternative binding partner interaction. NcRNAs are typically classified based on their size, into small and long non-coding RNAs (lncRNAs). Common small ncRNAs include small nuclear RNAs (snRNAs), small nucleolar RNAs (snoRNAs), microRNAs (miRNAs), small interfering RNAs (siRNAs), and PIWI-interacting RNAs (piRNAs) (Figure 1).

In mRNA processing, pre-mRNA is the initial product transcribed, which contains both exons and introns. In 1993, a Nobel Prize in Physiology and Medicine was earned for identifying that introns can be 
spliced out by spliceosomes (Philip Sharp and Richard Roberts). The spliceosomes are composed of several small nuclear (sn) RNP-specific proteins interacting with non-coding snRNAs. The snRNA can form an RNA-RNA pair with pre-mRNA in the required alignment for downstream splicing steps(9).

RNA interference (RNAi) is the process of RNAs directing enzymes to mRNAs to induce posttranscriptional gene silencing and therefore regulate vital cellular processes. The first RNAi type of phenomenon was reported in 1990 in plants(10). In 1998, RNAi was identified in C. Elegans and they further identified that it was double-stranded RNAs triggering gene silencing(11). In 2006, Andrew Z. Fire and Craig C. Mello were awarded the Nobel prize for discovering RNAi. Two central types of ubiquitously expressed RNAi are miRNAs and siRNAs, while piRNAs are another form of RNAi that are expressed mainly in germline cells(12). Pseudogenes resemble functional genes, but have coding-sequence deficiencies. RNAi genes, such as siRNA(13), can derive from pseudogenes. Additionally, pseudogene ncRNAs can act as RNAi, such as miRNA sponges, to regulate gene expression(14).

Whereas RNAi mechanisms of

action in gene regulation are well understood, lncRNAs are not yet well characterized. LncRNAs are defined as RNAs greater than 200 nucleotides in length that are not translated to proteins. In 2002, IncRNAs were officially identified as a significant new class of non-protein coding RNA molecules after the sequencing of a full-length cDNA library in mouse(15). Since then, they have been found to function as signal, guide, decoy, and scaffold RNAs (Figure 3).

All of these types of ncRNAs have been found to play roles in a variety of pathological conditions, including cancer. Specific ncRNAs expression has been correlated with survival and therefore are being studied as biomarkers for disease diagnosis and prognosis. Additionally,
I. Signal

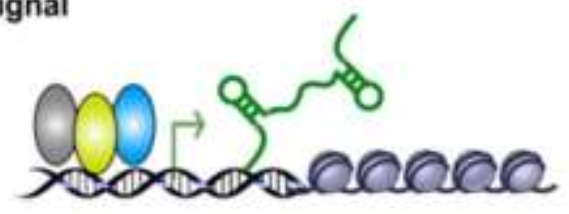

II. Decoy

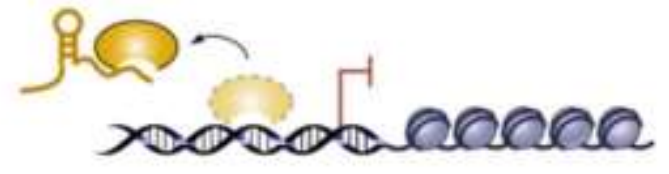

III. Guide

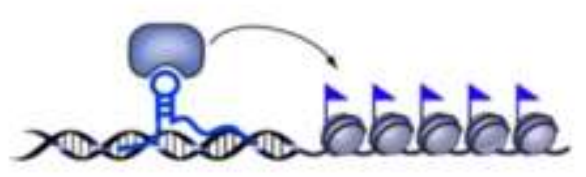

IV. Scaffold

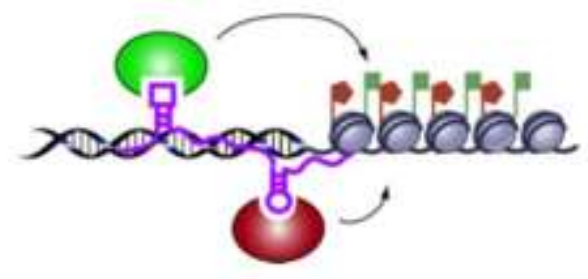

Figure 3: Molecular mechanisms of lncRNAs. Adapted from: Wang and Chang. Molecular Cell, 2011. 
specific ncRNAs have been identified to be functionally related to hallmarks of cancer and are being pursued as therapeutic targets.

\section{Small non-coding RNAs}

\section{A. RNAi - Overview}

In 1993, the first microRNA, lin-4, was identified in C. Elegans, by the Ambros and Ruvkun labs(16). However, miRNAs were not respected as functionally critical until 2002 when Carlos Croce at the Kimmel Cancer Institute (now at The Ohio State University) identified, for the first time, two miRNAs involved in human cancer(17). Around that time, hundreds of other miRNAs were reported and discovered to be conserved amongst species(18). These small ncRNAs were initially referred to as "small temporal RNAs (stRNAs)" prior to 3 publications in Science in 2001 that transitioned the field towards the term "microRNAs"(19-21).

MicroRNAs are short, single stranded ncRNAs that are $\sim 22$ nucleotides long. They function by binding to the miRNA response elements (MREs) in the 3' untranslated region (3'-UTR) of mRNAs and targeting them for degradation and/or preventing their translation. MiRNAs contain an eight-base seed region that is well conserved and binds with perfect complementarity to the mRNA target. miRNAs with similar seed sequences with likely target a similar set of genes, however miRNAs with different seed sequences can still target and regulate similar gene sets.

miRNAs have been found to be well conserved in many species, including humans, and are master regulators of almost every biological process. In humans, it is predicted that miRNAs can regulate more than $90 \%$ of genes, and a single miRNA can target multiple genes(22). They are involved with immunity, development, and metabolism, and dysregulation of these miRNAs is implicated in most human diseases, including cancer, cardiovascular diseases, and neurological diseases. Currently, there are 2,654 annotated human miRNAs(23).

In 1999, a similar ncRNA known as small interfering RNA (siRNA) was identified in plants(24). A long double-stranded RNA is cleaved by the endonuclease Dicer to form a small interfering RNA (siRNA) that is $\sim 20-25$ nucleotides in length. SiRNA can enter into a cell, get incorporated into other proteins to form the RNA-Induced Silencing Complex (RISC). At this point, the siRNA is unwound and separated to form a single stranded siRNA. The single-stranded siRNA that remains part of the RISC 
complex then binds to complementary target mRNA to induce mRNA cleavage, and leads to mRNA degradation.

miRNAs and siRNAs show many similarities, but there are some distinctive traits between the two RNAs. siRNAs are derived from longer regions of double-stranded RNA. Both siRNA and miRNA are similar in length and mediate downregulation of their specific mRNA target. miRNAs are regulators of endogenous genes, while siRNAs regulate in response to foreign entities (e.g. viruses). It is suggested that siRNA destabilizes mRNA, while miRNA primarily inhibits mRNA translation without affecting the mRNA level. miRNA can sequester and degrade mRNA by P-bodies, which are sites for RNA decay(25). The main difference between siRNA and miRNA is that siRNA has perfect complementarity to its target, while most miRNA only has complementarity in the seed region with variable complementarity of the target to the rest of the miRNA. This feature allows miRNAs to have greater plasticity to target several mRNAs, while siRNAs only can target mRNA that have perfect complementarity. Additionally, miRNAs and siRNAs can be synthetically developed and utilized as a research tool as well as clinical therapeutic applications.

More recently, another form of RNAi known as piRNAs were identified to prevent genome instability in germ line cells. piRNAs interact with piwi proteins to form RNA-protein complexes that are involved in post-transcriptional silencing of transposons and other cellular genes. piRNAs are transcribed from single-stranded precursor transcripts that are processed in a Dicer-independent mechanism and are slightly longer ( 24-31 nucleotides) than miRNAs and siRNAs (12).

\section{B. MiRNA Biogenesis - Canonical Pathway}

The canonical biogenesis of miRNAs has been elucidated as a stepwise process in normal and aberrant cells (Figure 4). The initial step of miRNA biogenesis is transcription of a long primary (pri)miRNA, typically by RNA Polymerase II (Pol II)(26). Some miRNAs are transcribed by RNA Polymerase I (Pol I). The majority of pri-miRNA are transcribed from intergenic regions or antisense to host genes while others are transcribed from intronic regions; thus, miRNA may be formed independently or as part of host genes. The size of a pri-miRNA ranges from several hundred nucleotides to several kilobases, and contains a stable 7-methylguanosine (m7G) cap at its 5' end and polyadenylated cap at its 3' end. These primiRNAs contain one or more bulged structures. The canonical biogenesis pathway then consists of two cleavage events, one nuclear and one cytoplasmic, to produce a mature miRNA. 
In the nucleus, the microprocessor consisting of the RNase III nuclease Drosha(27) and double stranded RNA binding protein DiGeorge Syndrome Critical Region 8 (DGCR8)(28) recognizes the bulged hairpin structure of the pri-miRNA and cleaves the pri-miRNA to precursor (pre-) miRNA. This leaves the pre-miRNA with a 70 nucleotide stem-loop structure. More specifically, Drosha cleavage generates 3' protruding ends that are later recognized by Exportin-5 (XPO5) and Dicer(27, 29, 30).

Notably, RNase III protein homologs conservation and interactions can vary between species. Initially Pasha was identified as Drosha's partner required for miRNA maturation and miRNA-mediated gene regulation in Drosophila and Caenorhabditis elegans (C. Elegans)(31) and it was later identified as DGCR8 in other organisms(28).

Pre-miRNA intermediates are bound by XPO5 and exported from the nucleus to the cytoplasm(30, 32, 33) in a RanGTP dependent manner. Subsequently, pre-miRNAs are cleaved by the cytoplasmic RNase III protein Dicer1 and Dicertransactivating response RNAbinding protein (TRBP) complex into $\sim 22$ nucleotide miRNA duplexes(34-38). This $\mathrm{miR} / \mathrm{miR}^{*}$ (miR-3p/miR-5p) duplex is then unwound by a helicase. One strand of the cleaved duplex, sometimes referred to as the "passenger strand" (miRNA*), can be nonfunctional and degraded by a nuclease (39). The other "guide strand" preferentially remains a functional mature miRNA $(40,41)$. The miRNA* terminology is now used less, however, because some
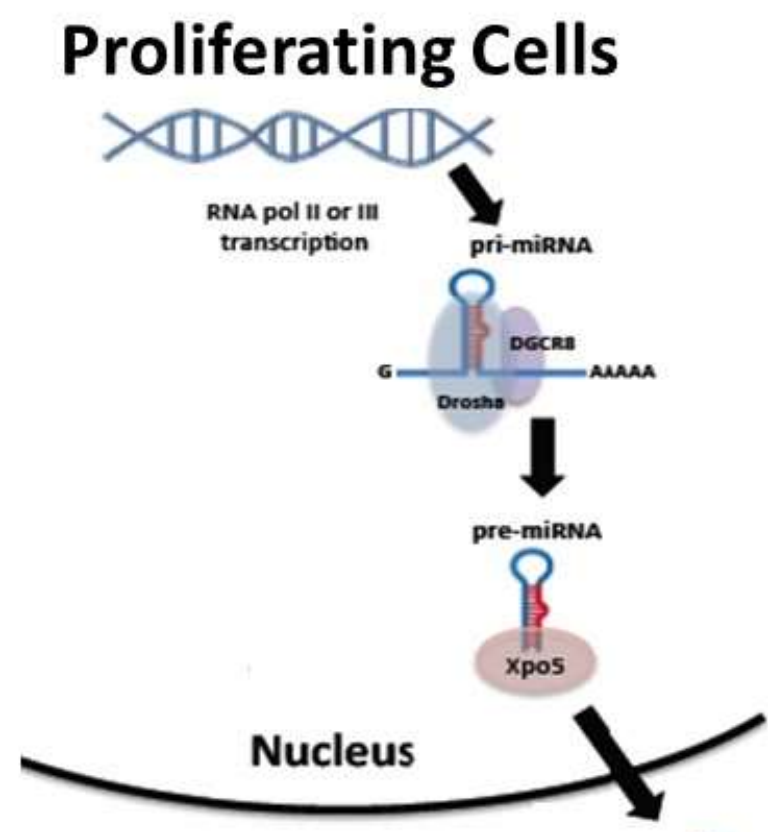

\section{Cytoplasm}

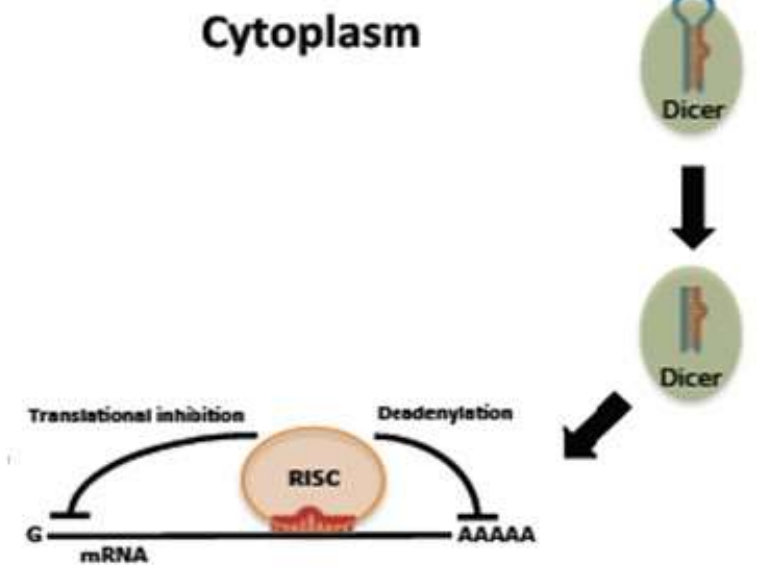

Figure 4: Canonical miRNA biogenesis pathway. Adapted from: Martinez et al., PNAS, 2017. 
miRNA* sequences were found to be functional. Currently, the two miRNA strands are named miR-3p and miR-5p based on the direction of the strand it derived from, and both strands have the potential to be functional(42). The single-stranded mature miRNA guide strand is loaded into the Argonaute-2 (Ago2) containing RNA-induced silencing complex (RISC). Each RISC can only contain one of the two strands. The stability of the base pairs at the 5' end of each predetermines which strand is loaded into the RISC (41); the strand with the less stable 5' end usually survives. The mature miRNA contained in the RISC will then base-pair incompletely with the target mRNA. Depending on the degree of complementarity between the miRNA and mRNA target will determine the fate of the mRNA. Most miRNAs exhibit imperfect complementarity with target mRNA and will inhibit the mRNA's translation(43) by inhibiting the ribosome progression. Perfect complementarity will induce degradation of the mRNA(44). For example, miR-196 is perfectly complementary to its mRNA target HOXB8 (except for a wobble base pairing) and the miRNA induces degradation of the mRNA(44). miRNA binding destabilizes the mRNA by speeding up the deadenylation of the mRNA and contributing to mRNA degradation(45).

\section{C. miRNA Biogenesis- Alternative Pathways}

Even though the canonical pathway is well studied, deep sequencing of systems with deletions of miRNA biogenesis pathway components have identified non-canonical biogenesis pathways in different organisms and cells that can still produce functional miRNAs. These alternative pathways can differ in the number of cleavage events and enzymes responsible for miRNA processing. It appears that the biogenesis pathway that a miRNA takes to be processed is determined by its genomic site of origin, sequence, and thermodynamic stability.

In the same human cell line, Drosha, XPO5, and Dicer were deleted to analyze for effects on miRNA biogenesis. Drosha-depleted cells exhibited completely abolished canonical miRNA biogenesis. From this analysis, they detected three miRNAs processed independent of Drosha (miR-7706, miR-3615, and miR-1254), while canonical miRNA production was completely abolished in cells with Drosha depletion. Dicer depletion led to reduced levels canonical miRNAs, but many were still detected. In cells with Dicer knockdown, pre-miRNAs were loaded directly onto Argonaute protein and trimmed at the 3' end to produce miRNAs from the 5 ' strand (5p miRNAs). Unexpectedly, XPO5 depletion only partially affected miRNAs expression(46), suggesting there are alternative miRNA exportation pathways. This study contributes to the idea that certain miRNAs can be processed independent of the main proteins involved with canonical biogenesis. 


\section{Mirtrons}

In 2007, the Bartel and Lai laboratories described a group of RNAs in Drosophila melanogaster(47, 48) and Caenorhabditis elegans (C. Elegans)(48) termed "mirtrons" (pre-miRNA/intron) that follow a noncanonical miRNA biogenesis pathway as they are Drosha- and DGCR8-independent. Similar to canonical miRNAs, mirtrons are encoded within short stem-loop structures. However, while miRNAs utilize the microprocessor complex for processing, mirtrons are instead generated from the excised intron of proteincoding genes that are released during mRNA splicing. More specifically, a small intron lariat is linearized by the lariat debranching enzyme to produce a pre-miRNA-like short hairpin. Thus, mirtrons are processed independently of the microprocessor complex, but follow the canonical miRNA pathway during nuclear export and thereafter $(47,48)$. Subsequently, mirtrons were found to be well-conserved and expressed in diverse vertebrates(49-52).

With conventional mirtrons, both of their ends are defined by splicing; however, some mirtrons contain 5'-(52) or 3'-(52,53) single-stranded RNA tails that need to be trimmed by nucleases before Dicer processing. For example, certain 3'-tailed mirtrons (e.g. hsa-miR-4745 and hsa-miR-6869) are trimmed by the RNA exosome $(52,53)$.

\section{Simtrons}

While mirtrons are splicing-dependent, a subset termed "simtrons" (splicing-independent mirtronlike miRNAs) do not require DGCR8, Dicer, Exportin-5, or Argonaute 2 (AGO2). They can however interact with Argonaute proteins. They are exported from the nucleus in an unknown mechanism. They are bound by, and dependent on, Drosha. Simtrons function by silencing target transcripts and are found in the RISC complex. Some examples are miR-1225 and miR-1228(54).

SnoRNA-miRNAs

In 2008, Ender C. et al. discovered small RNAs derived from snoRNA ACA45 that are processed independent of the microprocessor Drosha/DGCR8 complex and function like miRNAs. The processing of this RNA is still dependent on Dicer and interacted with Ago proteins to silence mRNA (CDC2L6) expression post-transcriptionally in a miRNA-like manner(55). 
Since then, other snoRNAs with dual roles as miRNAs (e.g. miR-140, miR-605) have been identified. Based on sequence and structure, there are two classes of snoRNAs (box H/ACA and box C/D), both of which produce snoRNA-derived RNAs(55-59).

\section{miRNAs from tRNAs}

In 2008, Babiarz et al. identified a pre-tRNA (miR-1983) that was Dicer-dependent and DGCR8independent(50). Later studies demonstrated other tRNA-derived small RNAs (tsRNAs) involved in gene silencing that were Dicer-dependent (type I tsRNAs) $(60,61)$, as well as some tsRNAs processed by RNase $\mathrm{Z}$ cleavage downstream from a tRNA gene in a Dicer-independent manner (type II tsRNAs)(61). These tsRNAs localize to the cytoplasm and associate with Argonaute proteins(61).

\section{Virus-derived miRNAs}

Other tRNA-derived small RNAs that were miRNA-like were identified to be generated from both host and viral genomes(62-64). Murine gamma-herpesvirus 68 (MHV68) miRNAs are co-transcribed fused to a tRNA from RNA polymerase III promoters(63). The resulting pri-miRNA is then processed by tRNase $\mathrm{Z}$, independent of Drosha, to form a pre-miRNA that then exhibits canonical processing to yield mature viral miRNAs (e.g. MHV68 miR-M1-7)(64).

In certain instances, potentially to combat viral infection (e.g. Sinbis RNA virus), pri-miRNAs can be incorporated into a cytoplasmic virus. The pri-miRNA is downstream processed in a Dicer-dependent, DGCR8-independent manner to produce a viral mirtron-like product (virtron)(65). Following viral infection, Drosha is relocalized to the cytoplasm to aid in this processing of virus-derived miRNAs(66).

Expression of viral miRNAs from herpesvirus saimiri (HVS) relies on elements required for the synthesis and processing of Sm class U RNAs known as HVS U RNAs (HSURs). More specifically, these viral miRNAs are processed independent of the Microprocessor complex. Instead the Integrator complex (mediates processing of snRNA) cleaves to generate the 3' end of the HSUR and the pre-miRNA hairpin. To generate these mature viral miRNAs, the pre-miRNA is then processed downstream by the canonical pathway involving XPO5 and Dicer(67). 
Recently, viral miRNAs produced from certain low risk strains of HPV (17, 37, and 41) were identified. Further studies found that these viral miRNAs are processed by canonical miRNA biogenesis and can regulate viral transcripts (e.g. E1) and consequently control their own viral life cycle(68).

\section{Dicer-independent miRNA biogenesis}

miR-451 is a conserved vertebrate miRNA that is cleaved by Drosha/DGCR8 to generate a premiRNA that is too short to be a substrate for Dicer. Instead, the pre-miRNA is loaded directly into Argonaute proteins. If the pre-miRNA is loaded into an Ago2 protein, it is cleaved(69-72).

\section{XPO1-dependent miRNA biogenesis}

Previously, capped RNAs shorter than 200 nucleotides were found to be bound by CBC (capbinding complex)-PHAX and exported via XPO1 rather than XPO5.(73) In a similar manner, certain premiRNAs (e.g. pre-miR-320 and pre-miR-688) were found to be processed differently with regards to pre-miRNA synthesis, nuclear-cytoplasmic transport, and guide strand selection in mice. They have a 7-methylguanosine $\left(\mathrm{m}^{7} \mathrm{G}\right)$-cap employed by eIF4E that is recognized by XPO1 via the adapter molecule PHAX. These certain capped pre-miRNAs were processed independently of the Microprocessor. Once exported in an XPO5independent manner, they are cleaved by Dicer and only the 3p-miRNA is efficiently loaded onto Argonaute to form mature, functional miRNA(74).

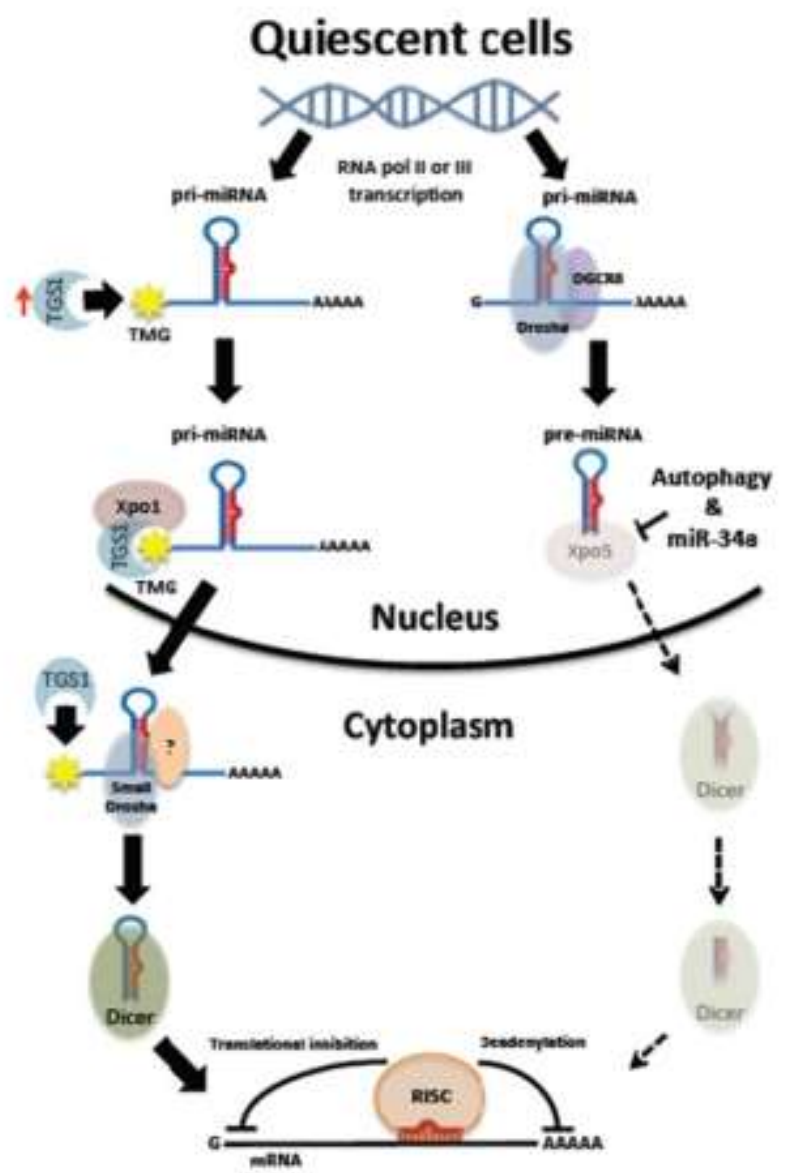

Figure 5: Alternative miRNA biogenesis pathway in quiescent cells. Adapted from: Martinez et al., PNAS, 2017. 
The Martinez lab recently discovered another XPO1-independent, non-canonical pathway of miRNA biogenesis in normal, human foreskin fibroblast (HFF) quiescent cells (Figure 5). XPO5 expression is lost in quiescent cells by autophagy and the regulation of miR-34a. A group of miRNAs are induced during quiescence in HFFs and their biogenesis is XPO1-dependent (XPO5-independent). These pri-miRNAs (e.g. pri-miR-34a and pri-miR-3188) are modified with a 2,2,7-trimethylguanosine (TMG)cap by trimethylguanosine synthase 1 (TGS1), which allows them to bind to and be exported by XPO1. Additionally, pri-miR-34a was present in the cytoplasm along with a small isoform of Drosha, implying further potential alternative processing of this miRNA in quiescent cells.

In summary, the importance of miRNAs is essential in so many processes that evolution gives the plasticity to process these miRNAs in non-canonical pathways as needed.

\section{D. miRNAs - Involvement in Cancer}

MiRNAs involvement in cancer has been comprehensively reviewed(75-78). Global differential expression analyses of miRNAs in human cancer cells and tissues have revealed dysregulation of many miRNAs in human cancer and that most miRNAs are repressed in cancer tissues compared to normal(79, 80). Global depletion of miRNAs via impaired miRNA processing, led to cell transformation and tumorigenesis, which implies miRNA alteration contributes to cancer development(81). Functionally, miRNAs can be both tumor suppressive or oncogenic depending on the context, and are involved in many aspects of cancer biology, including proliferation and apoptosis. In many cases, the trend of miRNA expression correlates with many clinical variables (e.g. tumor stage and overall survival) such that miRNA profiling of a patient can lead to improved diagnosis and prognosis in certain cancers(77). Additionally, some have proposed that miRNA profiling is even better at predicting clinical variables than mRNA profiling in certain cases explored thus far(79).

\section{Mechanisms of miRNA alterations in cancer}

There are multiple mechanisms of miRNA deregulation in cancers, including genomic abnormality, epigenetic factors, transcriptional regulation, and miRNA biogenesis regulation as described below. Notably, stoichiometry analysis of miRNA to target gene abundance can allow for the speculation of how likely the miRNA is to regulate the target and contribute to a biological effect. Higher levels of mRNA expression results in varied downregulation of targets by miRNA(82). 
The first example of a miRNA involved in cancer was miR-15a and -16-1, which are clustered at a region of the genome that is frequently deleted in B cell chronic lymphocytic leukemias (B-CLL). Thus, in the majority of CLL cases, both genes are deleted or down-regulated(17). Other groups have additionally showed deregulation of miRNAs in cancer due to chromosomal abnormalities (e.g. fragile sites)(83).

miRNA expression can be regulated by epigenetic factors, as many $\mathrm{CpG}$ islands are located near miRNAs(84). CpG hypermethylation repressed miRNAs (e.g. miR-9-1 and miR-124a) in cancer tissues compared to normal, and this miRNA hypermethylation affected known tumor suppressors and oncogenic factors $(84,85)$. Alternatively, miRNAs (e.g. miR-29) can counteract CpG methylation by directly targeting DNA methyltransferases and therefore de-repress downstream targets regulated by promoter methylation in cancer cells(86).

Well-known transcription factors involved in cancer can up-regulate the expression of certain miRNAs to contribute to carcinogenesis. For example, Myc(87) and p53(88) can alter expression of the miR-17-92 cluster and miR-34, respectively, in cancer cells.

Alterations in expression of mature miRNAs can occur due to decreased miRNA biogenesis processing efficiency or miRNA stability. In cancer, key regulators of the miRNA biogenesis pathway (Drosha or Dicer) have altered expression, which affected steady-state miRNA levels and led to tumor development $(81)$.

In some cases, miRNA expression levels are not altered, but rather there is sequence variation in the miRNA binding sites in the target gene that affect miRNA-mRNA interactions and contribute to cancer(89).

\section{Oncogenic or tumor suppressive miRNAs}

Certain miRNAs are up-regulated in a cancer lineage-specific manner, while others are altered in many different types of cancers and thus critical tumor suppressors or oncogenes. Some of the most wellknown miRNAs that are altered in many cancers from different lineages are miR-17-92 cluster, miR-21, miR-155, let-7, and miR34s(77). Since miRNAs target and regulate expression of mRNAs, a miRNA is determined to be oncogenic or tumor suppressive depending on its target gene. 
The oncogenic miR-17-92 cluster (composed of miR-17, -18a, -19a, -20a, -19b-1, and -92a-1) is up-regulated in many human cancers(76). It is up-regulated by MYCN and binds the 3'UTR of the cyclindependent kinase inhibitor CDKN1A (p21) and pro-apoptotic regulator BCL2L11 (Bim) mRNAs in neuroblastoma(90).

miR-21 regulates multiple target genes, including p53 and NF-kB, involved in hallmarks of cancer(91). miR-21 expression is up-regulated in many different types of cancers(76) and is considered to be a ubiquitous oncogene as it is expressed in all epithelial-cell derived solid tumors and many other cells(91). In the IL-6 signaling pathway, Stat3 transcriptionally activates miR-21 such that downstream it regulates invasion and metastasis(92).

miR-155 has been identified as a bona fide oncogene in breast cancer because it binds key cancer targets (e.g. FOXO3A and RhoA) for degradation or inhibition of translation to accelerate tumor development(93). miR-155 has also been found to drive therapy resistance in various tumor types and is being studied as a target for miRNA-based therapeutics(94).

Let-7 is a tumor suppressor that targets well-known oncogenes RAS, c-Myc(81), and HMGA2(76). The expression of let-7 has been reported to be down-regulated in many human cancers, including lung and $\operatorname{colon}(95)$.

miR-34a acts as a tumor suppressor by regulating of at least 700 transcripts involved in cellular processes such as proliferation (cyclin-dependent kinases), apoptosis (BCL2), and motility (SNAI1). miR34a is transcriptionally regulated by the tumor suppressor p53 in normal conditions, however miR-34a expression is decreased in many cancers likely due to alterations in p53(96).

\section{Virus-regulation of miRNAs}

The involvement of miRNAs in the pathogenesis of oncogenic viruses was comprehensively reviewed by Lin et al. in 2011(97). Certain oncogenic viruses can alter host miRNAs(98, 99) while others can encode their own viral miRNAs $(100,101)$ to control their viral life cycle, but consequently this can contribute to carcinogenesis(97). 
The high-risk human papillomavirus (HPV) E6 oncoprotein inhibits expression of the tumor suppressor miR-34a(99). Additionally, Martinez et al. showed that high-risk HPV E6 inhibits miR-218, which up-regulates LAMB3 to contribute to HPV's tumor promoting function(98).

Kaposi sarcoma-associated herpesvirus (KSHV)(100) and Epstein-Barr virus (EBV)(101), can produce viral miRNAs to regulate carcinogenesis. For example, a cluster of KSHV miRNA decreases an anti-angiogenic, tumor suppressor thrombospondin 1 (THBS1)(100, 102).

\section{E. miRNA - Clinical Implications}

Regulating miRNAs for application in a clinical setting has evolved by using antagomir and miRNA mimics. To counteract the up-regulation of oncogenic miR-17p-92 cluster in therapy-resistant neuroblastoma, in vitro and in vivo antagomir-17-5p treatment was used to abolish the growth of MYCNamplified cancer cells by up-regulating p21 and BIM to blockade the cell cycle and activate apoptosis(90). A Phase 1 clinical trial was completed that tested intravenous injection of TargomiRs (targeted minicells containing a miRNA mimic) for the first time in humans. It was tested as a second- or third-line treatment for patients with recurrent malignant pleural mesothelioma (MPM) and non-small cell lung cancer (NSCLC). The TagomiR consisted of a synthetic miR-16 mimic, non-living bacterial minicells (nanoparticles), and an anti-EGFR specific antibody. At this time, no results have been posted for this specific study (NCT02369198).

Additionally, there is the potential of analyzing for expression of miRNA(s) for use as diagnostic and/or prognostic marker. There are various clinical trials underway to analyze circulating miRNAs in blood and plasma as biomarkers of different types of cancers to diagnose cancer and stratify patients by stage (to determine treatment). Certain Phase I clinical trials (e.g. NCT02509052). are combining their primary objectives with secondary and tertiary objectives to preliminarily analyze miRNA signatures associated with responses of cancer cells to therapeutic treatments. The Spanish Breast Cancer Research Group is sponsoring a clinical trial to analyze whole blood of metastatic breast cancer patients for a miRNA signature to predict response to bevacizumab therapy (NCT01598285). Analysis of ncRNA expression in exosomes is currently an actively pursued direction of clinical research. One clinical study is currently recruiting patients to analyze ncRNA expression via genetic sequencing of miRNA/lncRNA in the exosome of epithelia ovarian cancer patients. (NCT03738319). There are clinical trials researching miR-155 as a diagnostic marker in non-muscle invasive bladder cancer (NCT03591367). Notably, many clinical trials are looking for a miRNA signature of multiple miRNAs rather than relying on a single miRNA biomarker. 
One specific colon cancer study is currently analyzing for expression of 6 miRNAs (miR-21, -20a-5p, 103a-3p, -106b-5p, -143-5p, -215) in clinical surgical specimens to identify if a patient should additionally receive adjuvant chemotherapy (NCT02466113). A Phase 4 clinical trial detecting fifteen circulating miRNAs (identified from preclinical studies) as biomarkers of hormone sensitivity in breast cancer patients before and after first-line therapy was completed. In parallel, they analyzed a larger scale analysis of circulating miRNAs in these patients (NCT01612871). Prospective studies are being conducted, such as profiling plasma miRNA expression in heavy smokers to assess its efficacy as a first-line screening for lung cancer screening of high-risk population (NCT02247453). There is a completed clinical trial analyzing for expression of miRNA biogenesis proteins (Dicer and Drosha) in epithelial skin cancer (NCT00849914). A clinical trial sponsored by MiraKind is validating the role of miRNA binding site mutations in cancer risk, prevention, and treatment (NCT02253251). Unfortunately, at this time, no results have been posted on clinicaltrials.gov for any of the studies mentioned above.

As miRNA mechanisms of action to function in gene regulation are well understood, lncRNAs are not yet well characterized.

\section{Long non-coding RNAs (LncRNAs)}

\section{A. LncRNA - Overview}

In 2009, Dr. John Rinn and Dr. Eric Lander's labs at the Broad Institute identified over a thousand lncRNAs that are highly conserved in mammals(103). This contributed to the great increase in research publications on lncRNAs in the past $\sim 10$ years(104). To date, there are currently over 127,000 annotated human IncRNA transcripts in the LNCipedia public database (lncipedia.org), however, few of these have been fully characterized. Admittedly, lncRNA transcription does not necessarily mean that it has a biological function, however many lncRNAs have been characterized as functionally important and it is critical to continue to dissect functions. LncRNAs make up the majority of the non-coding portion of the genome (https://www.gencodegenes.org/human/stats.html), so it is critical to further research and characterize this class of RNAs.

\section{B. LncRNA - Characterization of IncRNA}


LncRNAs were initially identified as mRNA-like transcripts that do not code for proteins; however, further characterization of IncRNAs has distinguished them as their own class. It is still challenging to distinguish between coding and lncRNA transcripts.

Since lncRNAs as a class are not well characterized yet, there are only trends, not standard "rules", to classify lncRNA based on biogenesis, processing, localization, functions, etc. As expected, there are also lncRNAs that have been identified as exceptions to the trends of the majority of IncRNAs. A review in 2016 summarized certain research techniques that could be used to identify and characterize a newly identified lncRNA(105). Once a specific lncRNA is identified, researchers can determine: biogenesis/processing, genomic location, non-protein coding potential; conservation; expression level, size, and primary sequence; secondary structure; localization; and biological function.

\section{LncRNA - Biogenesis and Processing}

In terms of biogenesis, IncRNAs and mRNAs share similar characteristics. LncRNAs are typically transcribed by RNA Polymerase II (Pol II) from genomic loci with similar chromatin states to mRNAs(103). In many cases, lncRNAs differ from mRNAs solely in that lncRNAs are lacking a translated ORF. Similar to mRNAs, lncRNAs are modified with a 5' cap and 3' polyadenylated tail(103) and undergo pre-RNA splicing. Some lncRNAs undergo non-canonical processing at their 5' and 3' ends(106-108).

The promoter regions of the majority of lncRNAs display similar epigenetic marks [e.g. increased trimethylation of lysine 4 of histone 3 (H3K4me3)] to mRNAs. LncRNAs have higher enrichment of acetylation of lysine of histone $3(\mathrm{H} 3 \mathrm{~K} 27 \mathrm{ac})$ and are more strongly repressed by certain chromatin remodeling complexes (e.g. Swr1, Isw2) than mRNAs(106). Some eukaryotic promoters are bi-directional such that a sense protein-coding gene could be transcribed while an antisense is transcribed in the opposite direction(109). Polyadenylation signals differ between sense (protein coding) and antisense strands (lncRNA) produced from divergent promoters; polyadenylation usually occurs during splicing of mRNAs, while it typically occurs during cleavage in lncRNAs.

Similar to mature tRNAs, some lncRNAs (e.g. MALAT1) use an alternative processing method consisting of cleavage by the RNase $\mathrm{P}$ ribonucleoprotein complex. These specific lncRNAs contain a tRNA-like structure at their 3' ends that RNase P cleaves to produce mature lncRNA transcripts. After RNase P cleavage, the mature lncRNA has an RNA triple helix at the 3' end (similar to a polyA tail) that 
increases its stability. Additionally, both mRNAs and lncRNAs can be spliced, and some lncRNAs can be back-spliced and circularized (circular RNAs) (110).

\section{LncRNA - Genomic location}

One method of classifying lncRNAs is based on their location in the genome relative to proteincoding genes. Based on this genomic location of a lncRNA, the functional role of the lncRNA can be better predicted. As shown in Figure 6, lncRNAs can be defined as sense, antisense, bidirectional or divergent, intronic, intergenic or intervening $(111,112)$. Importantly, lncRNAs can overlap protein-coding genes and be expressed in a similar pattern as the coding gene(113) (Figure 6, IIa and IIb). The V3.0 lncRNA classifications by Arraystar Incorporated shows their analysis of the number of each subgroup. The majority of lncRNAs are classified as lincRNAs $(19,590)$ followed by intronic $(4,409)$, antisense $(3,691)$, sense overlapping (1,597), and bidirectional $(1,299)$ lncRNAs (www.arraystar.com).

Sense lncRNAs are transcribed in the same direction as exons of protein-coding genes (Figure 6, Ia) and have been found to be non-coding variants of genes that can regulate gene expression. A lncRNA can also partially overlap a protein-coding gene on the same genomic strand, and both are transcribed in the same direction (Figure 6, IIa). These sense-overlapping
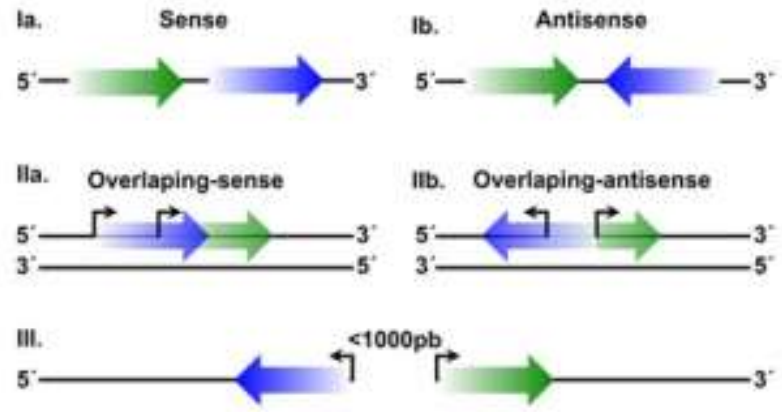

Bidlirectional or Divergent
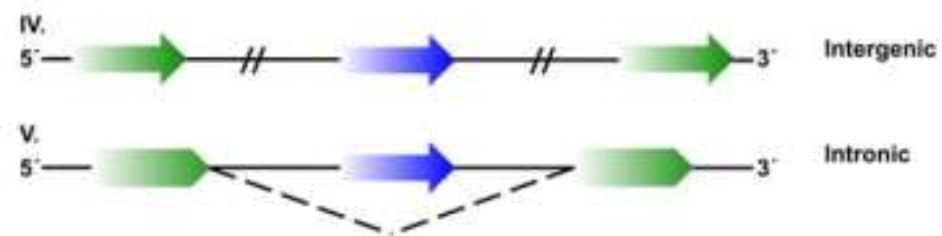

Figure 6: Classification of IncRNAs by genomic location. Adapted from: Herrera-Solorio et al., Clin Epigenetics, 2017. lncRNAs can be transcript variants of protein-coding genes, and the majority of these lncRNAs lack a substantial ORF for protein translation.

Bidirectional, or divergent, lncRNAs are transcribed in the opposite direction as a protein-coding gene within the vicinity (within 1kb) (Figure 6, III). There is similarity in expression patterns between some 
lncRNA and its protein-coding counterpart such they may be regulated by similar mechanisms, but this is does not hold true for all bidirectional lncRNAs. Bidirectional expression of lncRNA-protein-coding gene pairs are prevalent in cancer.

Antisense lncRNAs are transcribed from the antisense strand, in the opposite direction as a proteincoding gene (Figure 6, Ib). A lncRNA can also partially overlap a protein-coding gene, and both are transcribed in the opposite direction (Figure 6, IIb). Antisense lncRNAs can share the same promoter as coding gene and their expressions can highly correlate(114). Antisense lncRNA may pair with a proteincoding gene on the sense strand to regulate epigenetic silencing, transcription, and mRNA stability(115). As an example of how genomic location can aid in characterization of lncRNAs, mouse sense-antisense transcripts tend to be poly(A) negative and localized in the nucleus(116). Antisense lncRNA transcription of genes affects both the expression and splicing of sense genes(117). Natural antisense transcripts' (NATs) expression regulate tumor suppressors and oncogenes in cancer. OncoNAT is useful tool that catalogs cancer-related genes with significant antisense transcription.

Intergenic lncRNAs (lincRNAs) are transcribed between two protein-coding genes and are at least $1 \mathrm{~kb}$ away from the nearest protein-coding genes (Figure 6, IV). Very long intergenic ncRNAs (vlincRNAs) can range in size from 50 kilobases to 1 megabase. As of 2009, more than 1,000 lincRNAs were identified to be encoded by the mammalian genome and are clearly conserved across mammals. Some lincRNAs (e.g. HOTAIR) have been found to associate with chromatin modifying proteins and regulate gene expression(118). They have also been found to be important in a variety of cellular processes including apoptosis, cell-cycle progression, and cellular senescence. Intergenic lncRNAs can be subcategorized as being enhancer-associated (elncRNA) (present close to the promoter, in an enhancer region) or promoterassociated (plncRNA). ElncRNAs are more tissue-specific, less highly expressed, and less well conserved during evolution(119) and have been found to be important in certain diseases(120).

Intronic lncRNAs are transcribed from an intronic region of a protein-coding gene (Figure 6, V). Intronic lncRNAs can be transcribed in the sense or antisense direction. Intronic lncRNAs are expressed in similar tissues as the corresponding protein-coding gene, and the lncRNA may regulate alternative splicing or stability(121).

Additionally, there are certain regions of the genome that are associated with diseases, and these regions contain lncRNAs. Thus, these lncRNAs could be critical regulators in these diseases. 


\section{LncRNA - Excluding protein-coding potential}

Classifying RNAs as non-coding and coding transcripts is not a straightforward process as mRNA and lncRNA exhibit similar structure, and a single RNA can function as an mRNA and also have a noncoding function. This discernment is also difficult, as lncRNAs overlap protein-coding genes (Figure 6, IIa and IIb). Certain IncRNAs have already been identified to encode functional peptides(8) (7), while some RNAs currently classified as lncRNAs may indeed encode proteins if researched further. These aforementioned difficulties of differentiating between coding and non-coding RNA are comprehensively reviewed by Dinger et al.(122).

Protein-coding transcripts contain a long open reading frame (ORF) capable of translating a protein, which can separate mRNAs from lncRNAs in some instances. Solely using ORF size is not acceptable as some RNAs classified functionally as lncRNAs also contain long ORFs (e.g. H19 and Xist). On the other hand, some mRNAs contain shorter ORFs. If an RNA contains an ORF, the conservation of that ORF can be analyzed to indirectly guide towards discerning between mRNA and lncRNA; certain codons for amino acids show preservation of protein-coding potential(111).

Certain RNAs classified as lncRNAs can contain ORFs by chance, and they might instead function solely as a ncRNA. On the other hand, if a lncRNA is identified to code for a protein it does not eliminate the possibility that it also has a critical non-coding function. Interestingly, there are specific bifunctional RNA that can have both protein-coding and coding-independent functions $(123,124)$. There are certain mRNAs that have regulatory non-coding RNA elements; for example, oskar RNA can act as a scaffold, independent of Oska protein(125).

Research techniques (such as mass spectroscopy proteomics and in vitro translation assays) can be used to identify lncRNAs that encode functional peptides. Ribosome footprint profiling can be used to visually show the direct binding of transcripts to ribosomes, and this technique specifically can be used to analyze lncRNAs in mammalian cells $(126,127)$. Software tools, such as the Coding-Potential Assessment Tool (CPAT)(128), can be used to predict the likelihood that a transcript can encode for a protein by analyzing an ORF for the presence of known protein motifs.

\section{LncRNA - Tissue specificity and evolutionary conservation}


LncRNAs are typically tissue- and cell-type specific, even more than protein-coding genes(129132). Since many lncRNAs are expressed in certain tissues, this could be such that they have critical functions in the development and maintenance of that specific tissue. Alternatively, in 2015, a group conducted a pan-cancer analysis of IncRNAs from multiple tumor tissues to their matched normal counterparts and found a class of lncRNAs that are altered in multiple cancers. They classified these as 'onco-IncRNAs' that are predicted to have conserved oncogenic and/or tumor suppressive across multiple cancer types(133).

Many lncRNAs have poor evolutionary conservation across species(134), but it is intriguing to characterize lncRNAs that are conserved and thus speculated to be critical for biological functions. Additionally, there are specific human lncRNAs that lack evolutionary conservation in non-human species, but are still functionally relevant in humans and should be studied further(134). LncRNAs have been identified in a variety of species, including yeast, viruses, and humans, but to further determine conservation of the lncRNA, samples from multiple species could be further analyzed for lncRNA expression. Lack of conservation does pose certain challenges in determining the research model for studying lncRNAs as many lncRNAs identified in non-human models have not progressed towards applicability in humans. LncRNAs can be spliced to produce multiple isoforms, which creates challenges in that a certain lncRNA could be spliced differently in a certain species, tissues, and under certain cellular stresses, thus making it difficult to determine the biologically functionally isoform.

LncRNAs tend to exhibit poorer primary sequence conservation than mRNAs(134). Certain lncRNAs consist of highly repetitive sequences. The repetition of these elements can contribute to identification of function. For example, certain lncRNAs with Alu repeats can bind to hnRNPK and localize in the nucleus(135).

Secondary and tertiary structure are highly conserved and analyzing RNA structure could also help identify functionally important lncRNAs and their binding partners. LncRNAs are complex, high molecular weight molecules, which therefore makes crystallization difficult. The structure of an RNA can be determined by various in vitro and in vivo technologies, including dimethyl sulfate-sequencing (DMS-seq) and selective 2'-hydroxyl acylation analyzed by primer extension-sequencing (SHAPE-seq) (136). The presence of certain structural motifs can lead to the interaction with certain binding partners. For example, a G-rich motif in the lncRNA Braveheart interacts with certain nucleic acid binding partners such as a zincfinger transcription factor (137). 


\section{LncRNA - Size, expression level, and stability}

The size cut off of lncRNAs (>200 nucleotides) is an arbitrary cutoff that is used to separate from distinctly small ncRNAs. When comparing lncRNAs to mRNAs, lncRNAs tend to be shorter than mRNAs and have fewer but longer exons. Most lncRNAs range in size from less than $1 \mathrm{~kb}(73 \%)$, between $1 \mathrm{~kb}$ and $2 \mathrm{~kb}(14 \%)$, and more than $2 \mathrm{~kb}(13 \%)(138)$. Few lncRNAs are exceptions in that they can span 100 kilobases. For example, the promoter for the an antisense lnRNA Air (antisense Igf2r RNA) lies 107,796 base pairs away from the $3^{\prime}$ end, in an intron flanking the Mas1 gene(139). Tol determine RNA size, Northern Blot analysis can be conducted.

There are a variety of cellular stresses that regulate lncRNA expression, including, but not limited to, DNA damage(140), viral infection(141), and serum starvation (quiescence)(142). LncRNA expression levels are dysregulated in many diseases and their expression can correlate with clinical parameters (e.g. overall survival). Many lncRNAs are expressed at low expression levels compared to protein-coding mRNAs(108). This poses research challenges and the debate about if lncRNAs are expressed at high enough levels to be functionally important. The stoichiometry of a lncRNA to its target gene can predict the likelihood of this interaction having a critical importance. To determine relative expression level of a lncRNA, quantitative reverse polymerase chain reaction (qRT-PCR) can be used, and if absolute expression is desired, in vitro transcription followed by qRT-PCR can be used.

In 2012, Clark et al. compared the stability of mouse lncRNAs and mRNAs. Based on half-lives, lncRNA stability is comparable to, but on average less than, mRNAs. Intergenic and antisense lncRNAs that act in cis are more stable than those derived from introns. Additionally, spliced lncRNAs are more stable than unspliced (single exon) lncRNAs. LncRNAs localized in the nucleus are more likely to be unstable(143). Comparing lncRNAs to mRNAs, mRNAs are primarily degraded by 5'-to-3' exonuclease digestion in the nucleus or decapping at their 5' end in the cytoplasm, while many unstable lncRNAs are degraded by the nuclear exosome or nonsense-mediated decay (NMD) in the cytoplasm(144).

\section{LncRNA - Cellular localization}

Comparing lncRNAs to mRNAs, mRNAs usually localize to ribosomes in the cytoplasm while lncRNAs can vary in their localization. LncRNAs are primarily localized in the nucleus but can also localize in the cytoplasm(131) (Figure 7). The localization of lncRNAs primarily in the nucleus is consistent with lncRNAs main function of contributing to epigenetic regulation of gene expression in the nucleus(129). 
Many lncRNAs are exported from the nucleus to the cytoplasm through the canonical mRNA pathway, via the major export complex TREX and NXF1/NXT1 nuclear transport receptor(145).

The sequence of an RNA determines if it is a substrate for retention in the nucleus or exportation to the cytoplasm $(145,146)$. There are specialized cis-elements in RNAs that can promote retention in the nucleus. During splicing of an RNA, the spliceosome recruits export factors to the RNA that can promote exportation. There are splicing-independent factors that instigate nuclear export via association with the same exportation factors(145).

Certain lncRNAs are restricted to localization in the nucleus. For example, the most abundant and conserved lncRNA is MALAT1 (metastasis-associated lung adenocarcinoma transcript 1), also known as nuclear-enriched abundant transcripts 2 (NEAT2). NEAT1 is also restricted to the nucleus and both NEAT lncRNAs interact with nuclear speckles in human cells (147).

Common technique to determine lncRNA cellular localization are cellular fractionation and RNA fluorescence in situ hybridization (FISH).

Alterations in mRNA localization are associated with various diseases. Under cellular stresses, nucleocytoplasmic transport is disrupted by sequestering critical transport factors into stress granules to exacerbate neurodegeneration(148). Thus, it is considered that lncRNA mis-localization could also be related to diseases.

Knowledge of lncRNA localization provides insight into interactions and functions. Nuclear lncRNA can regulate transcription and chromatin remodeling in various ways, as shown in Figure 6. They can regulate transcription by acting as enhancer RNA (eRNA) (Figure 7A), or interacting with and recruiting chromatin modifying complexes (Figure 7B) or transcription factors (Figure 7C). LncRNA can also be the mediators to spatially bring chromosomes in close proximity (Figure 7D) and pre-mRNA splicing (Figure 7E). Cytoplasmic lncRNAs can regulate mRNA stability (Figure 7F) or mRNA translation (Figure 7G). Linear and circular RNAs can bind to and sponge miRNAs (Figure 7H). Some lncRNAs contain small ORFs that can be translated to small peptides (Figure 7I).

\section{LncRNA -Biological function}


Studying lncRNA biological function is critical, as lncRNAs already have been found to be associated with developmental processes (149) and dysregulation of lncRNAs is associated with diseases(150). Additionally, lncRNAs have been found to be involved with many biological processes, including proliferation, apoptosis, and differentiation by regulating gene expression, interacting with epigenetic modifiers, mRNA splicing and many others. LncRNA location can give a hint towards the function of a lncRNA, and some known nuclear and cytoplasmic functions are summarized in Figure 7. Broadly, lncRNAs molecular functions are subdivided into four categories: signals (HOTAIR), guides (Evf2)(151), decoys (GAS5), and scaffolds (ANRIL) (Figure 3). LncRNA commonly form ribonuceloprotein (RNP) complexes to control the location of protein regulators (Figures 3 and 7).

LncRNAs can act in cis in the nucleus, where they modify chromatin and/or regulate nearby gene expression, or in trans, where they leave the site of their transcription to perform biological functions. It

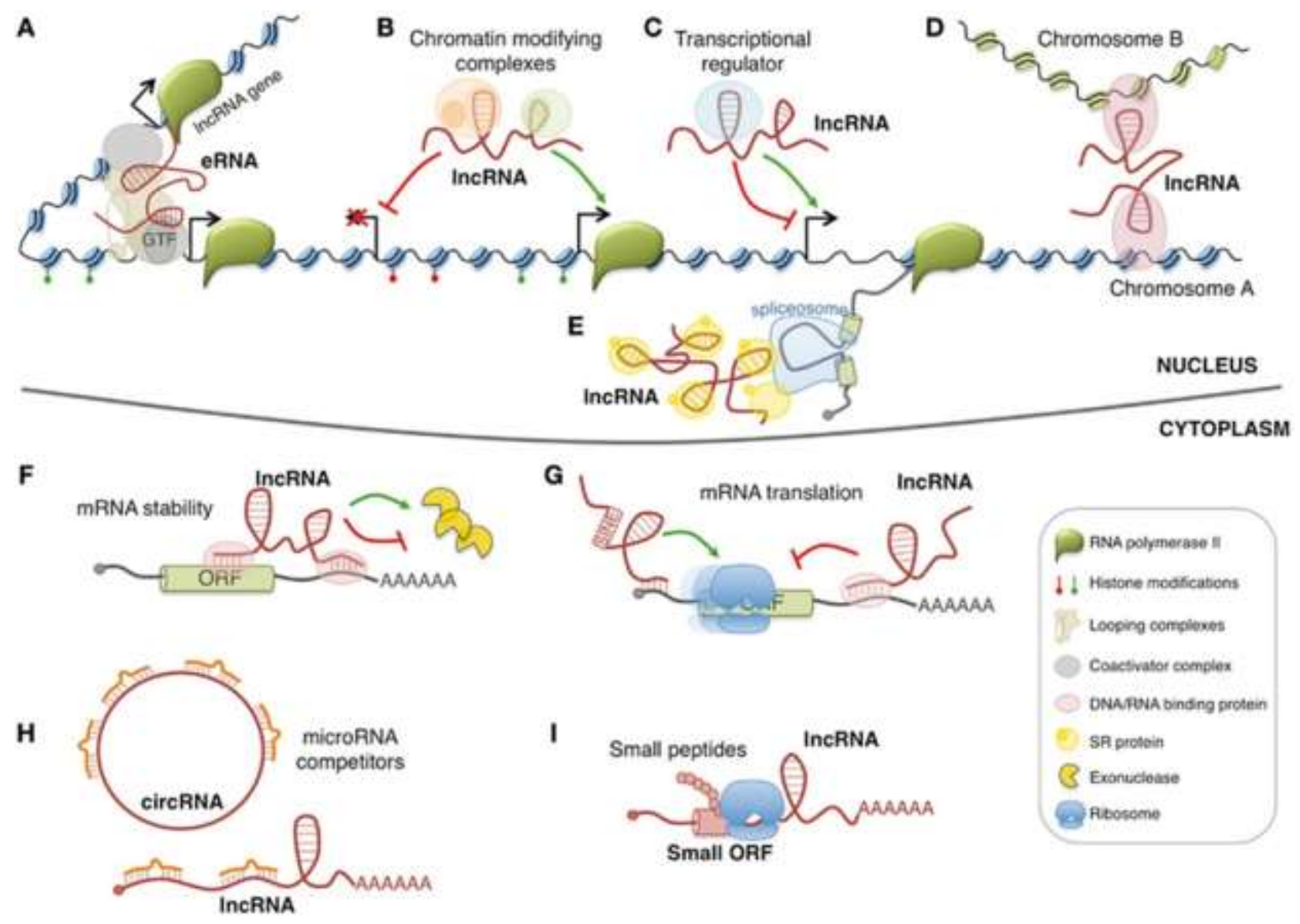

Figure 7: LncRNA functions in the nucleus and cytoplasm.

Adapted from: Morlando et al., Mini Reviews in Medicine. 2015.

can be difficult to determine the function of cis-acting lncRNAs as their expression can be directly, indirectly, or not important for downstream biological effects. The capability to act in cis makes them 
unique from mRNAs, and allows lncRNAs to be involved in transcriptional regulation and chromosome looping.

\section{Enhancer $\operatorname{lncRNA}$}

Figure 7A and 7D visually represent enhancer activity by a lncRNA. Recently, Miao et al. identified an enhancer lncRNA-mediated transcriptional regulation. The LEENE (lncRNA that enhances eNOS expression) lncRNA transcripts (located on chromosome 14) associates with the eNOS (endothelial nitric oxide synthase) locus (located in chromosome 7). Both chromosomes are brought into close proximity to permit the association and modulate eNOS mRNA synthesis(152).

\section{Chromatin Remodeling}

LncRNA can act as guides to recruit chromatin modifying enzymes to DNA to increase gene expression (Figures 7B and 3III). A recent study found that out of 3,300 human lincRNAs analyzed, 20\% were bound by chromatin-modifying complexes including Polycomb Repressive Complex 2 (PRC2)(118). Certain lncRNAs can mediate chromatin remodeling in cis, while others can regulate in trans. In cis, transcribed lncRNAs such as Air can recruit the transcriptional repressive histone methyltransferase G9a to interact with the promoter of one of its imprinted genes Slc22a3 and silence Slc22a3 expression(153). Intrans, lncRNA such as HOTAIR can act as a scaffold and bind to key PRC2 components (Suz12 and Ezh2) and the LSD1-coREST complex to methylate gene histones and promote gene silencing(154).

\section{Interaction with Transcription Factors}

Nuclear lncRNA can bind to transcription factors (Figure 7C) and guide them to DNA (Figure 3III) or away from DNA (Figure 3II) to regulate transcription. LncRNAs have been found to activate transcription, while others suppress transcription. One example of a lncRNA contributing to transcriptional activation is $E v f-2$ that is transcribed from an ultra-conserved enhancer, forms a stable complex with Dlx2, which consequently activates Dlx-2 to enhance transcription of Dlx-5/6(151). LncRNAs can repress transcription by binding to and sequestering transcription factors. An example of a lncRNA contributing to transcriptional repression is PANDA, which associates with the transcription factor NF-YA to act as decoy to keep NF-YA away from target gene chromatin. Due to this, pro-apoptotic genes are not expressed by NF-YA(155). 


\section{Splicing Regulation}

As shown in Figure 7E, lncRNA can regulate splicing. More specifically, lncRNAs can regulate alternative splicing and interact with splicing factor as recently summarized (156). MALAT1 controls the activity of the SR protein family of splicing factors to regulate alternative splicing(157).

mRNA Stability

Figure $7 F$ visually shows that lncRNA can regulate mRNA stability. For example, the lncRNA LAST (IncRNA-assisted stabilization of transcripts) stabilizes CCND1 mRNA. c-Myc regulates the lncRNA LAST, which in collaboration with CNBP binds to the 5' UTR of CCND1 mRNA to protect against possible targeting by nuclease(158).

\section{mRNA Translation}

As shown in Figure 7G, lncRNA can regulate mRNA translation. For example, lincRNA-p21 associates with JunB and $\beta$-catenin mRNAs and selectively decreases their translation by diminishing their polysomes (159). A lncRNA antisense to, and overlapping, the Uchl1 protein-coding gene was found to be required for Uchl1 translation. Furthermore, this was shown to be due to an embedded repetitive sequence (short interspersed nuclear element/SINE) in the lncRNA that regulates recruitment of polysomes to the mRNA for translation(115).

\section{miRNA Regulation}

As shown in Figure 7H, lncRNAs can interact with and sequester miRNAs and act as competing endogenous RNAs (ceRNAs). Multiple studies have analyzed for lncRNAs acting as miRNA sponges(160, 161). Targetscan (targetscan.org)(162) can be used to predict miRNA targets, while spongeScan (http://spongescan.rc.ufl.edu) (163) can be used to specifically identify miRNA binding elements in lncRNA sequences.

p53-regulated lncRNAs

Expression of the tumor suppressor p53 correlates with many host lncRNAs and these lncRNAs were induced in a p53-dependent manner(164). Alternatively, lncRNAs regulate p53 expression levels. For 
example, the nuclear lncRNA PURPL (p53 upregulated regulator of p53 levels) associates with MYBBP1A (a protein that binds to and stabilizes p53) to decrease p53 stabilization, decrease p53 basal expression levels, and promote tumorigenicity in colorectal cancer(165).

Overall, IncRNAs lack a standard classification framework, which makes categorizing and analyzing lncRNAs difficult. Currently, in an attempt to standardize this process, IncRNAs are primarily classified by genomic location and function.

\section{Circular ncRNAs}

\section{Circular ncRNAs - Overview}

Circular RNAs (CircRNAs) are produced when precursor mRNA back-splice and the head to tail region bind each other. Due to their circular structure, they are thought to be stable as they would be resistant to degradation by exonucleases that typically degrade linear RNA. CircRNAs are typically cell- and tissuespecific, fairly conserved, and expressed at low levels(166). They are, however, expressed in high levels in brain, indicating they have neuronal functions. Additionally, circRNAs play a role in pathological conditions such as development of cancer and other diseases. For example, circRNAs are associated with EMT-related functions(167).

\section{Circular ncRNAs - Functions}

The functions of circRNAs are currently being researched. Specific techniques are required to identify circRNAs, such that large scale high-throughput sequencing of ncRNAs looking for linear, polyA+ RNAs would not detect circRNA expression. When desired, circRNA detection efficiency of RNA-seq with a specific microarray platform could be used to efficiently profile circRNAs by circRNA microarray. Using a specific microarray platform, thousands of circRNAs could be detected in cell-free plasma samples(168). Some known functions are sequestering miRNAs and proteins, modulating transcription, interfering with splicing, and translating proteins (Figure 8).

CircRNAs can function as miRNA sponges, thus preventing the miRNA from targeting mRNA and permitting translation (Figure 8A). One of the most well-known circRNAs is ciRS-7 (also known as CDR1as), which contains 70 binding sites for miR-7 and has been identified as a miRNA inhibitor. Consequently, this regulation is involved with many different cancers since miR-7 directly targets several 
oncogenes(169). Additionally, many other circRNAs acts as miRNA sponges. A testis-specific circRNA known as sex-determining region $\mathrm{Y}$ (circSry) serves as a miR-138 sponge to regulate testes development (170).

Exon-intron circRNAs (EIcircRNAs) can enhance transcription of their parental genes. For example, after EIcircRNA formation, it can interact with RNA Pol II and U1 snRNP to enhance transcription (Figure 8B).

CircRNAs can also act as a protein scaffold (Figure 8C) or bind and sequester proteins (Figure 8D) to particular cellular locations to modulate protein interactions(171). For example, circMbl can bind to the muscleblind (MBL) protein to regulate MBL function(172).

a
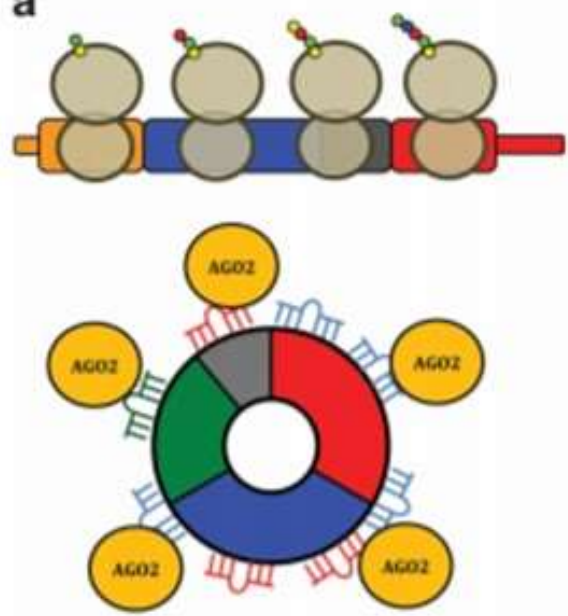

miRNA sponges or decoys

c

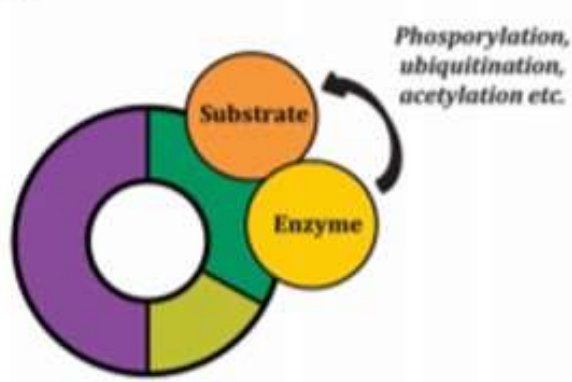

Protein scaffolding

b

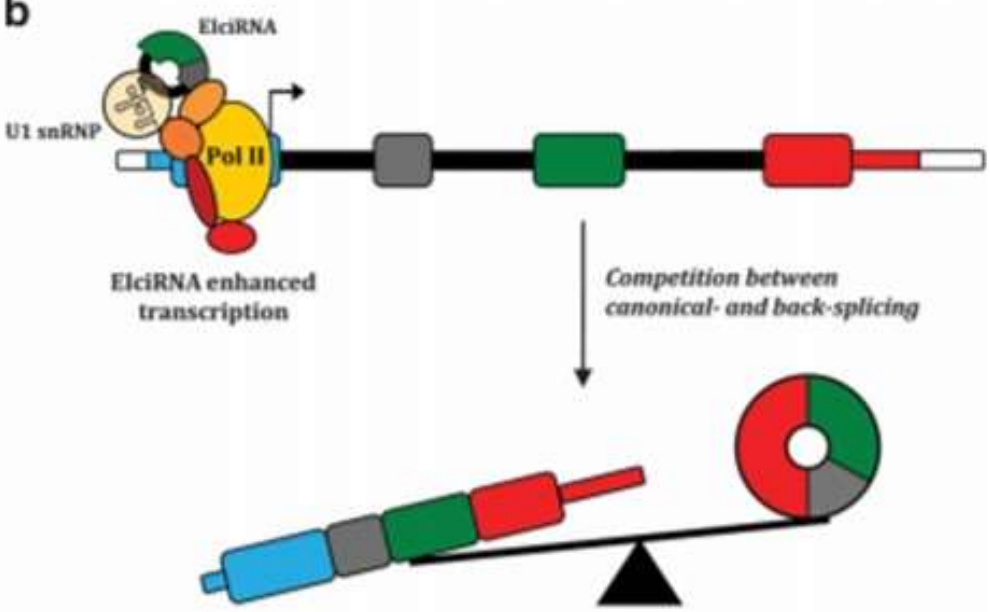

Splicing and transcription

d

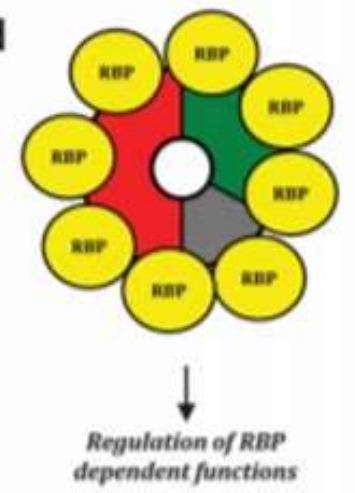

Protein sponges or decoys

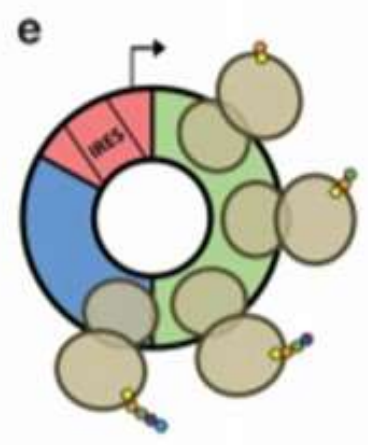

Translation

Figure 8: Circular RNA functions.

Adapted from: Kristensen et al., Oncogene, 2018. 
Hundreds of endogenous circRNAs containing an internal ribosome entry site (IRES) and AUG start codon can interact with ribosomes and have translation abilities(173) (Figure 8E). Using human embryonic kidney 293 cells and HPV-18 positive cervical cancer cells (HeLa), a group identified dependence of N6-methyladenosine $\left(\mathrm{m}^{6} \mathrm{~A}\right)$ motifs is the sequence of these circRNA molecules capable of translating proteins(174).

As host cells produced circRNAs, certain viruses also produce circRNAs. For example, Epstein Barr virus (EBV) encodes latent and lytic viral circRNAs, some of which are dysregulated in stomach cancer samples(175). Kaposi's sarcoma herpesvirus also can encode viral circRNAs that are up-regulated during the lytic phase of infection and alter cell growth(176).

\section{LncRNAs - Clinical Implications}

Currently, cervical cancer screening is the combination of high-risk HPV DNA testing with cytological morphological assessment during Pap smear. In the case of cervical cancer, identification of biomarkers associated with oncogenic progression would be useful. It is proposed by some that panels of epigenetic biomarkers (e.g. high CpG methylation rates of DAPK(177), CDH1(178), CCNA1(179), and CADM1(180)) analyzed during cervical cancer screening could be more beneficial predicters of oncogenic progression than standard methods. Certain epigenetic markers suggested are expressed in plasma or serum and can detect stages less invasively (DAPK(177) and CDH1(178)), while others are expressed in the tissue specimen to distinguish between stage and duration of disease (CCNA1(179) and CADM1(180)). Epigenetic alterations related to ncRNAs (including lncRNAs) contribute to cervical cancer and are being evaluated to determine disease progression and potentially target them for therapeutic benefit(181).

LncRNAs are strikingly more tissue specific than protein coding genes(129-132). Clinically, this allows for the advantage that these specific lncRNAs can be targeted for therapy to alter solely the tissue it is expressed in to reduce off-target effects. LncRNAs could be potentially targeted by multiple methods, including RNAi (siRNA), antisense oligonucleotides (ASOs), and small molecules(182, 183). Additionally, lncRNAs are being researched as clinical biomarkers for cancer detection and prognosis.

There are various basic science and clinical research studies being conducted to analyze for clinical potential of lncRNAs. For example, inhibiting the oncogenic lncRNA HOTAIR in vitro can inhibit cancer invasiveness, especially in cells with high PRC2 activity(184). Certain groups are working to identify a 
gene signature to predict prognosis of cancer patients. One group specifically identified a prognostic 15lncRNA signature for cervical squamous cell carcinoma (CSCC) that are associated with patient survival(185). There is a Phase $2 / 3$ clinical trial designed to validate a previously identified and independently validated prognostic and predictive mRNA-lncRNA signature for triple-negative breast cancer (TNBC), which could be used to classify TNBC patients into high- or low-risk of recurrence. This signature was used to identify high-risk TNBC patients, such that this decision could influence the decision of alternative therapeutic treatments on this set of high-risk patients compared to low-risk. This study is actively recruiting patients (NCT02641847). A recently completed clinical trial is analyzing expression of various ncRNAs (lncRNAs, snRNAs, snoRNAs, miRNAs) in radiation-induced fibrosis in breast cancer patients. In addition to ncRNA expression, they are also analyzing for alterations pre- and post-radiation in cellular localization. This study was completed in 2018 (NCT03000764).

The idea of collecting a patient's body fluid (e.g. urine and blood) to analyze for expression of circulating lncRNA(s) has become intriguing in the field. It could eliminate the current invasive diagnostic and prognostic method to biopsy patient tissue. As examples, lncRNA PVT1 is a potential serum biomarker for detection of cervical cancer(186), and GAS5 is proposed as a diagnostic biomarker in non-small cell lung cancer(187). Excitingly, detection of lncRNAs in body fluids of patients has translated to being valuable to detect cancer in human patients. The lncRNA prostate cancer antigen 3 (PCA3) is leading the field of lncRNAs commercially available for cancer detection. The urine test PROGENSA PCA3 was FDAapproved in 2012 for use in men who are considering repeat biopsy after an initially negative result for prostate cancer. It is considered to be more specific and effective in identifying prostate cancer than the prostate-specific antigen (PSA) urine test. Certain clinical trials being conducted, in addition to their primary objective, are collecting blood/serum samples at diagnosis and later stages (e.g. at time of surgery, pre- and post-surgery) to detect alterations in lncRNA biomarkers. For example, a Phase 2/3 clinical trial to treat hormone receptor positive and triple-negative breast cancer (TNBC) is additionally collecting samples to test patients for angiogenic serum markers (miRNA, IncRNA, and circRNA) measured at diagnosis and time of surgery. This study is active, but currently not recruiting patients (NCT02221999). HOTAIR, as a single oncogenic lncRNA, expression is monitored in the peripheral blood of thyroid cancer patients. This study is not yet recruiting patients (NCT03469544). Analysis of ncRNA expression in exosomes is currently an actively pursued direction of clinical research. One newly posted clinical trial is currently recruiting patients to analyze ncRNA expression via genetic sequencing of miRNA/lncRNA in the exosome of epithelia ovarian cancer patients to determine biomarkers for detection and prognosis of patients. This study is currently recruiting patients(NCT03738319). Unfortunately, there are no results posted on clinicaltrials.gov for any lncRNA studies at this time. 


\section{$\underline{\text { IV. Human Papillomavirus (HPV) }}$}

\section{A. HPV-Overview}

Human papillomavirus (HPV) is now recognized as one of the most prevalent sexually transmitted infection in the world. It is a member of the Papillomaviridae family, which is a group of small, nonenveloped viruses with double stranded DNA circular genomes that are $\sim 8$ kilobase pairs. More specifically, HPV is classified as a mucosal alpha-virus. In the 1980s, Harald zur Hausen's group discovered that cervical carcinoma samples contained DNA from certain strains of HPV, which suggested HPV as a etiological factor in cervical cancer(188). A schematic of the HPV-mediated progression to carcinogenesis is presented in Figure 9. Upon micro-abrasions in cutaneous and mucosal sites, the basal layer of stratified squamous epithelia is exposed and the virus can infect keratinocytes. Infected cells in this region have a highly organized process of tissue renewal that viruses utilize to replicate and produce virions to infect other cells. In early stages of HPV infection, the viral genome is maintained in an episomal state; as the infected cells differentiate, the viral genome replicates in the nucleus of the infected cells using the host cells replication machinery. As the virus-infected cells differentiate and progress towards the surface of the epithelium, the virus replicates alongside the host cell in a differentiationdependent manner. Once the surface of an infected cell reaches the surface of the epithelium, virions are assembled and released to infect other target cells.

There are more than 100 HPV strains, which are divided into the two subtypes high-risk and low-risk, depending on the clinical prognosis of lesions after HPV infection. Low-risk HPVs (e.g. HPV-6 and -11) can cause

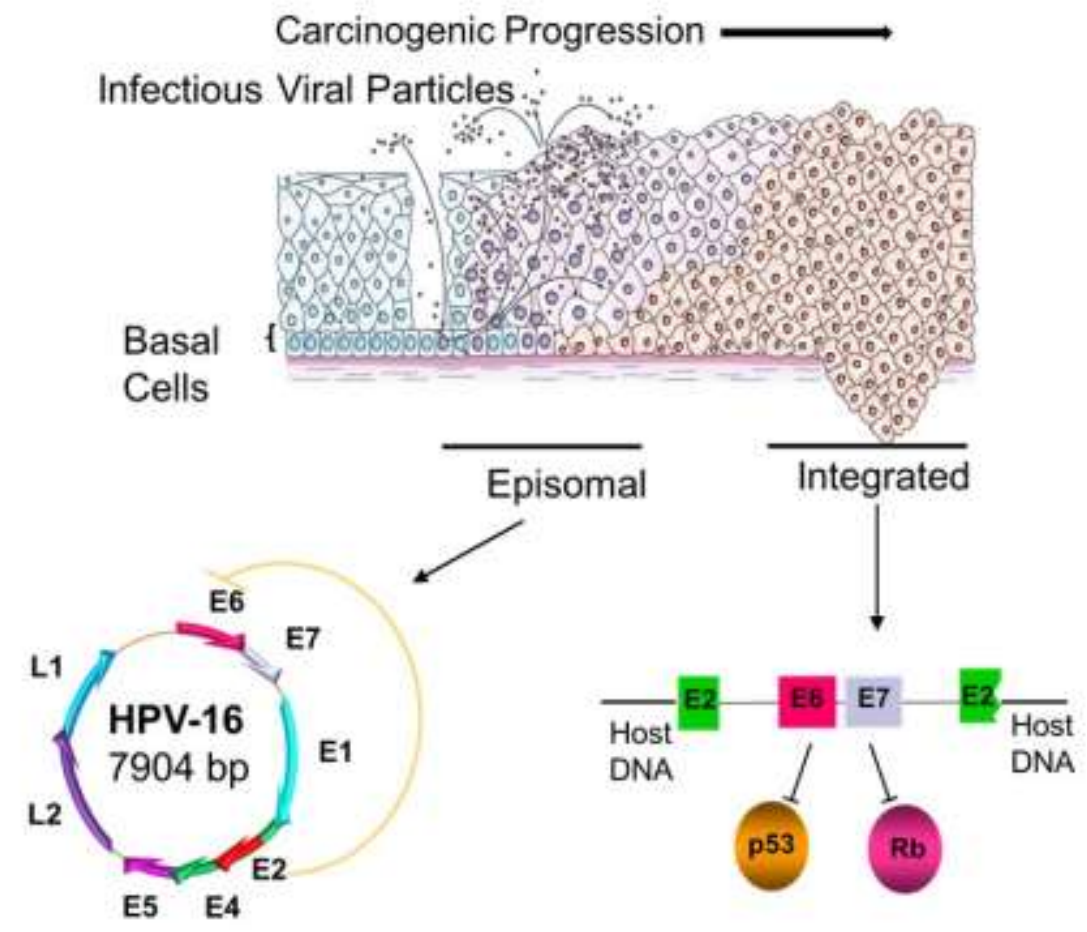

Figure 9: HPV-mediated progression to cancer. benign epithelial lesions and 
are associated with $90 \%$ of genital warts. There are fifteen strains associated with cancer, and these are classified as high-risk HPVs (e.g. HPV-16 and -18). Each of these high-risk strains encode for 6 genes in the early region (E1, E2, E4, E5, E6, E7) and two genes in the late region (L1 and L2). The genes encoded by the early region are responsible for modulating the cellular environment for viral replication and immune evasion, while the genes from the late region produce capsid, structural proteins(189).

As stated above, the viral genome is maintained in an episomal state in early HPV infection. While the genome is episomal, the E2 gene can repress the expression of the E6 and E7 oncogenes. However, upon persistent infection, over many years, the virus can integrate into the host genome. When integration occurs, the E2 gene is disrupted such that it cannot repress the promoter of E6 and E7, and there is a robust increase in the expression of these oncogenes. Up-regulation of E6 and E7 adds to carcinogenic progression because they contribute to the degradation of two important tumor suppressors p53 and $\mathrm{Rb}$, respectively(189) (Figure 9). The co-expression of the oncogenes immortalizes the cells(190). After many years of persistent HPV infection, other mutations in host genes occur that transform the cells and contribute to formation of a tumor(191). In addition to viral infection, genetic mutations as well as epigenetic alterations contribute to carcinogenesis(181).

HPV infections are asymptomatic and usually cure spontaneously by the immune system; however, persistent high-risk infection is an etiological agent in a variety of different cancers. In the United States, there are more than 42,000 HPV-associated cancers each year related to cervical (91\%), vaginal (75\%), vulvar (69\%), penile (63\%), anal (91\%) and oropharynx (70\%) cancers in the United States. In 2012, worldwide, $4.5 \%$ of all cancers worldwide (1.2 million) are related to HPV infection, with $73 \%$ of these cases related to the two strains HPV-16 and -18 combined(192).

There are currently three FDA-approved prophylactic vaccinations against HPV infection. Gardasil 9 protects against HPV-16 and -18, low-risk HPV-6 and -11, as well as five additional high-risk strains HPV-31, -33, -45, -52, and -58. Gardasil 9 has now replaced Gardasil (protects against HPV-16 and -18, as well as low-risk strains HPV-6 and -11). Cervarix protects against the two most carcinogenic strains (HPV16 and -18). A review published in 2017 summarizes these prophylactic vaccines in more detail, describing that Gardasil and Cervarix approach 90\% effectiveness in preventing HPV-16/-18 infections in women older than 25 years(193).

\section{B. HPV - Viral Oncoproteins}


Both E6 and E7 are small (18 and $13 \mathrm{kDa}$, respectively) and primarily localize in the nucleus(194). Both proteins lack enzymatic activities and function instead by binding to other cellular factors. High-risk E7 alone can immortalize human keratinocytes, while E6 alone is not sufficient alone to immortalize primary cells; immortalization efficiency was increased when E6 and E7 were co-expressed(190). The combination of E6 and E7 is not sufficient to transform cells; additional oncogenes are required to induce tumorigenesis(191). These oncoproteins contribute to carcinogenesis both individually, but also by synergizing with each other(195).

Notably, the E6 and E7 proteins are not conserved between strains. For example, there is variability in p53 degradation activities between different HPV strains(196).

Both of the Infected cell

oncoproteins are involved with epigenetic regulation. E6 upregulates DNMT1 by suppression of p53(197). E7 binds to DMNT1 to induce its DNA methyltransferase activity(198). One effect of this interaction is suppression of E-cadherin, which consequently decreases adhesion between epithelial cells(199). E6 can also regulate E-cadherin expression(200). E7 may be

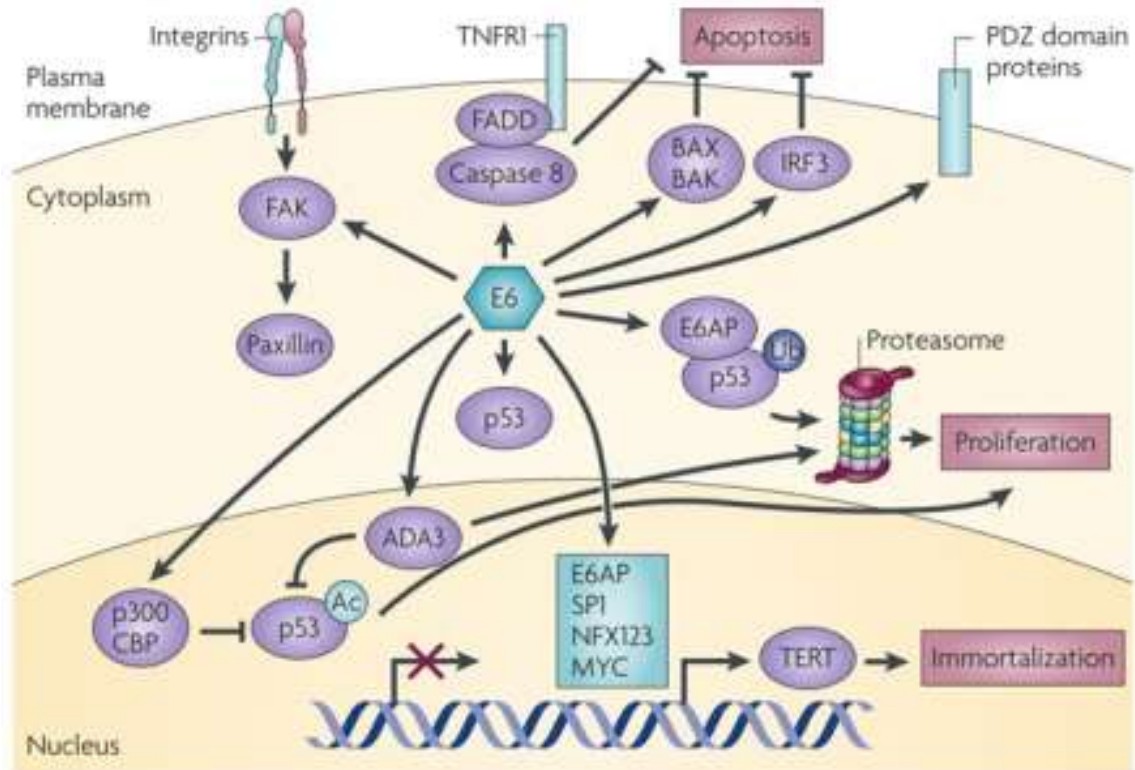

Figure 10: HPV E6 oncoprotein regulation.

Extracted from: Moody et al., Nature Reviews Cancer. 2010. able to activate transcription of DNMT1 through the pRB/E2F pathway(201).

The HPV E6 oncoprotein downstream regulators are presented in Figure 10. The most well-known E6 regulation is that it interacts with E6 associated protein (E6AP) ubiquitin ligase that in turn binds to and degrades p53(202). This results in inhibition of p53 tumor suppressive functions in regulating growth arrest and apoptosis in response to aberrant proliferation. E6 can also abrogate p53 function by targeting the p53 co-activator CBP-p300(203, 204). Additionally, E6 regulates a plethora of other proteins both directly and indirectly to contribute to regulate apoptosis, proliferation and immortalization to contribute to 
carcinogenesis. E6 interacts with many proteins to activate transcription of telomerase reverse transcription (TERT); combined with Rb inactivation by E7, this is an essential step to immortalization. E6 also mediates degradation of PDZ proteins, which leads to loss of cell polarity and induces hyperplasia.

Some known downstream effectors of HPV E7 are shown in Figure 11. E7 is most well-known for being involved in the regulation of retinoblastoma $(\mathrm{Rb})$ family of proteins, contributing to cell cycle activation and proliferation downstream. E7 mediates pRB and pRB-related proteins (p107 and p130) destabilization by a proteasome-dependent mechanism. When E7 binds Rb, this dissociates the transcription factor E2F-1 that is bound to $\mathrm{Rb}$ such that unbound $\mathrm{E} 2 \mathrm{~F}-1$ is free to drive expression of $\mathrm{S}$ phase genes and induce cell cycle activation and proliferation. E7 abrogation of Rb function leads to increased expression of the tumor suppressor p53. Consequently, E6 has evolved to target p53. E7 can also regulate many other proteins to influence genomic instability, apoptosis, and proliferation and contribute to carcinogenesis.

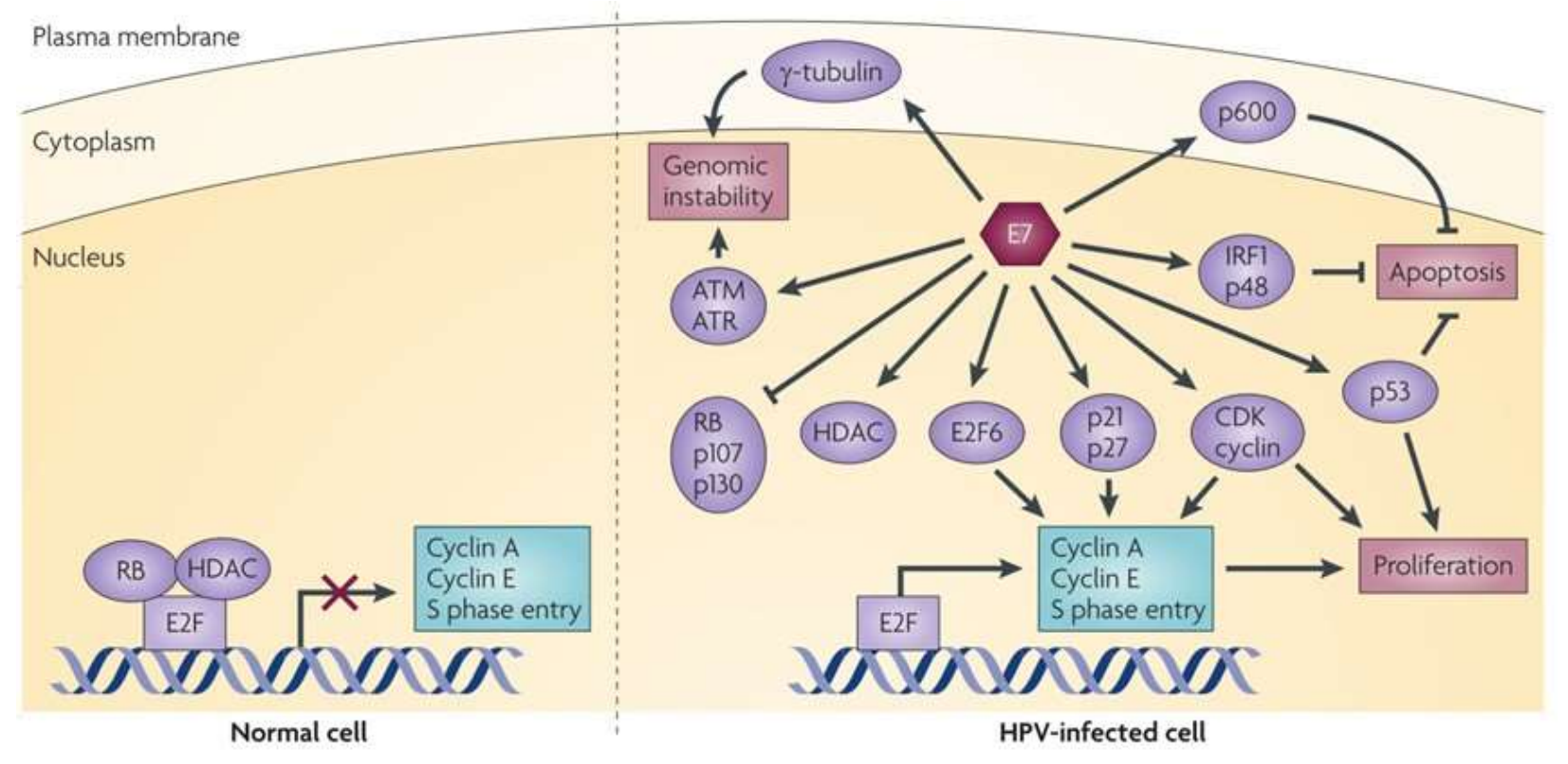

Figure 11: HPV E7 oncoprotein regulation

Extracted from: Moody et al., Nature Reviews Cancer. 2010.

\section{$\underline{\text { V. LncRNAs in cervical cancer }}$}

\section{A. Cervical Cancer - Overview}

Cervical cancer is one of the most common gynecological cancers. Worldwide, it is responsible for the second highest number of deaths in female cancers (exceeded only by breast cancer), as 10-15\% of all 
female cancer-related deaths were due to cervical cancer(205). $80 \%$ of patients have already developed invasive cancer at the time of diagnosis.

A combination of radiation therapy and low-dose chemotherapy is often used to treat early stages of cervical cancer. Alternatively, radiation therapy alone or surgery could be used for a small, early-stage tumor. Patients at high risk for recurrence could be administered radiation therapy and chemotherapy after surgery. Recurrent cervical cancer or patients with metastasis will be administered a combination therapy of platinum-based chemotherapy with the targeted therapy bevacizumab (Avastin) (https://www.cancer.net/cancer-types/cervical-cancer/treatment-options).

Identifying biomarkers for early detection as well as for prognosis are desirable. Somatic mutations in PIK3CA, PTEN, TP53, and KRAS are involved with the pathogenesis of cervical cancer(205). In the future, to predict, diagnose, and treat cervical cancer, it is proposed that utilizing lncRNAs could be beneficial.

\section{B. Host IncRNAs are altered in cervical cancer}

Unfortunately, The Cancer Genome Atlas (TCGA) does not directly include all of the human lncRNAs. However, due to the identification of IncRNAs role in cancer, many databases have been developed to summarize specific lncRNAs expression correlated with survival. MD Anderson developed TANRIC (The Atlas of non-coding RNA in Cancer), which extracted RNA sequencing data from TCGA and provided lncRNA expression correlative to survival in cancer patients. Currently, there are 307 cases of cervical squamous cell carcinoma and endocervical adenocarcinoma (CESC) available (https://portal.gdc.cancer.gov/projects/TCGA-CESC).

There have been multiple analyses of global differential expression of host lncRNAs in cervical cancer that have assisted in identifying specific lncRNAs playing a role in cervical cancer. Interestingly, a single lncRNA could be oncogenic in one tissue type, but tumor suppressive in another tissue lineage. Expression changes of lncRNAs in cancer versus normal samples as well as how altering lncRNA expression affects cancer phenotype (e.g. proliferation, migration, invasion, etc.) are useful; however, further elucidation of specific mechanisms of their regulation is critical for applicability and clinical potential from these lncRNA studies. 
Host lncRNAs are altered at the three pre-malignant stages of cervical dysplasia as well as in established cervical cancer samples compared to normal samples. One group conducted differential lncRNA expression profile analysis between mild (CIN1), moderate (CIN2), and severe (CIN3) cervical dysplasia compared to non-neoplastic cervical tissue. Out of the 1,056 lncRNAs analyzed, 13 were aberrantly expressed in all three CIN stages(206).RNA-seq was performed on HPV-16 positive cervical cancer samples and matched adjacent non-tumor samples to identify differential expression of lncRNAs, circRNAs, miRNAs, and mRNAs in human patients, including 3 novel lncRNAs and 44 novel circRNAs. Additionally, this study analyzed the ceRNA network to show that each miRNA targeted multiple lncRNAs and circRNAs(207). In HPV-18 positive cervical cancer samples, the differential expression of circular RNAs with and without radiation was analyzed by RNA-seq. Further, the mechanistic role of these circular RNAs was evaluated in radioresistance. Circular RNA functions and pathways were analyzed, and more specifically circRNA-miRNA target gene interaction network studies were conducted(208).A recent study provided 41 microarray datasets and 10 RNA-seq data sets that can be analyzed for circRNA expression in cervical cancer(168). Overall, this provides resources that show host lncRNA alterations, as well as provides resources to extract host lncRNAs potentially involved in cervical cancer initiation and progression.

Many specific lncRNAs have been found to be involved in cervical cancer, some of which are HOTAIR, MALAT1, CCHE1, and $\operatorname{lncRNA-EBIC}(209,210)$ and a summary of cervical cancer-related lncRNAs can be seen in Figure 12 below.

\section{HOTAIR (Hox transcript antisense RNA)}

HOTAIR is one of the most studied lncRNAs in cervical cancer. It is an oncogenic lincRNA that, in comparison to normal tissue, is up-regulated in many human cancers, including breast, gastric, colorectal, and cervical cancer(211-213). It interacts with the PRC2 complex leads to epigenetic regulation of many different cellular pathways(213).

In the context of cervical cancer, increased HOTAIR expression correlates with lymph node metastasis(211) and tumor size(211), tumor stage(212), and depth of cervical invasion(212). HOTAIR expression has been shown as a prognostic factor as high HOTAIR expression significantly correlated with poor overall survival(212) and disease-free survival/recurrence $(211,212)$ in cervical cancer patients. Knockdown of HOTAIR expression reduced cell proliferation, migration, and invasion in cervical cancer cell lines. HOTAIR could be exerting these oncogenic effects by regulating vascular endothelial growth 
factor (VEGF), matrix metalloproteinase-9 (MMP-9), and epithelial-to-mesenchymal transition (EMT)related genes(211) (Figure 12a). HOTAIR also enhances radio-resistance by inhibiting p21 in cervical cancer(214). Thus, HOTAIR could be used as a prognostic marker or therapeutic target in cervical cancer.

\section{MALAT1 (Metastasis-associated lung adenocarcinoma transcript 1)}

LncRNA MALAT1 [also known as nuclear-enriched transcript 2 (NEAT2)] is dysregulated in many cancers, including lung(215) and cervical cancer(216). Depending on the cancer type, it can be oncogenic or tumor suppressive.

In the context of cervical cancer, MALAT1 is up-regulated in tumor tissues compared to adjacent normal tissue, and this high expression correlates with tumor size, stage, invasion, lymph node metastasis, and poor overall survival(217). MALAT1 is involved with cervical cancer cell proliferation, cell cycle, and invasion and can regulate gene expression of apoptotic regulations (e.g. Bcl-2 and caspase-3)(216). Furthermore, MALAT1 regulates cancer cell growth and invasion at least in part by regulating miR-124 and its downstream target RBG2(218) (Figure 12f). MALAT1 could be a diagnostic or prognostic marker in cervical cancer.

\section{LncRNA-EBIC (EZH2-binding lncRNA in cervical cancer)}

Sun et al. conducted a microarray analysis of lncRNA and mRNA differentially expressed in cervical cancer and paired peritumoral tissues. They identified 708 up- and 836 down-regulated lncRNAs, including the up-regulated lncRNA EBIC (also known as lncRNA-TI17313). This lncRNA plays a role in migration and invasion in cervical cancer cells. Additionally, lncRNA-EBIC binds to EZH2 to regulate transcription in cervical cancer, and this interaction is required for the repression of E-cadherin (tumor suppressor that inhibits epithelial to mesenchymal transition (EMT) and prevent malignant progression)(219) (Figure 12e).

ANRIL (Antisense non-coding RNA in the INK4 locus)

ANRIL lncRNA plays an oncogenic role in many cancers, including melanoma, osteosarcoma, and cervical cancer(220).High ANRIL expression in cervical cancer patients correlated with tumor, lymph node metastasis, and poor overall survival(220). ANRIL plays a role in cell proliferation, migration, and invasion 
in cervical cancer(220) (Figure 12g). ANRIL can also promote cervical cancer developing by sponging miR-186(221).

\section{HOXA11-AS (Homeobox A11 antisense)}

Microarray analysis has been used to identify differential expression of lncRNAs in human cervical cancer tissue samples(222, 223). A specific microarray analysis of mRNAs and lncRNAs expression identified differential lncRNA expression, number of differentially up- or down-regulated lncRNAs and mRNAs in each chromosome, and types (sense, antisense, intronic, intergenic, or bidirectional) of lncRNAs up- and down-regulated in cervical cancer(222). A specific microarray analysis identified HOXA11-AS lncRNA alteration in cervical cancer compared to normal cervix. Further analysis of this lncRNAs showed that HOXA11-AS is a regulator of HOXA11 that contributes to cervical cancer(223).

\section{SNHG1 (Small nucleolar host gene 1)}

Another group sent three pairs of cervical cancer tissue samples and corresponding adjacent normal samples for lncRNA microarray identify differential expression of lncRNAs. From this analysis, they identified that lncRNA SNHG1 is up-regulated in cervical cancer, and knockdown of this lncRNA resulted in a decrease in cell proliferation, migration, and invasion in both HPV-positive and HPV-negative cervical cancer cell lines(224). A recent review summarized that SNHG1 expression is up-regulated in at least 11 types of cancers and contributes to proliferation, migration, and invasion of cancer cells; therefore, it is proposed to consider SNHG1 as a prognostic marker and/or therapeutic target(225).

\section{LncRNA CCHE1(Cervical carcinoma high-expressed 1)}

In cervical cancer, increased CCHE1 expression is associated with tumor size, stage, and poor survival(226, 227). CCHE1 is involved with proliferation and physically associates with proliferating cell nuclear antigen (PCNA) mRNA to enhance the PCNA expression (Figure 12h). Thus, CCHE1 could potentially serve as a diagnostic or prognostic marker in cervical cancer(227).

LncRNA CRNDE (Colorectal neoplasia differentially expressed RNA)

LncRNA CRNDE (colorectal neoplasia differentially expressed RNA) is altered many cancers, including colorectal cancer(228), glioma(229) and expression correlates with patient prognosis(230). Up- 
regulation of CRNDE expression in cervical cancer was determined from a lncRNA microarray analysis of cervical cancer tissues compared to normal adjacent tissues. Knockdown of CRNDE is HPV-positive and -negative cervical cancer cell lines decreased cell proliferation, migration, and invasion(231). Recently, CRNDE was found to be associated with poor prognosis in cervical cancer patients, and it targets $\mathrm{PI} 3 \mathrm{~K} / \mathrm{AKT}$ to promote cervical cancer cell proliferation and inhibition of apoptosis(232).

\section{MEG 3 (Maternally expressed gene 3)}

MEG3 is a tumor suppressor that mediates p53 signaling and inhibits cell proliferation. It has been found to be down-regulated in a variety of human cancers, including cervical. The MEG3 is a tumor suppressor in cervical cancer, as it inhibits cervical cancer cell proliferation through induction of cell cycle arrest and apoptosis(233) by regulating miR-21-5p(234) (Figure 12i).

\section{BCAR4 (Breast cancer anti-estrogen resistance 4)}

The lncRNA BCAR4 has been shown to enhance cell proliferation and promote metastasis in breast cancer by activating the HER2/3 pathway(235). Due to this, in cervical cancer, lapatinib (EGFR/HER2

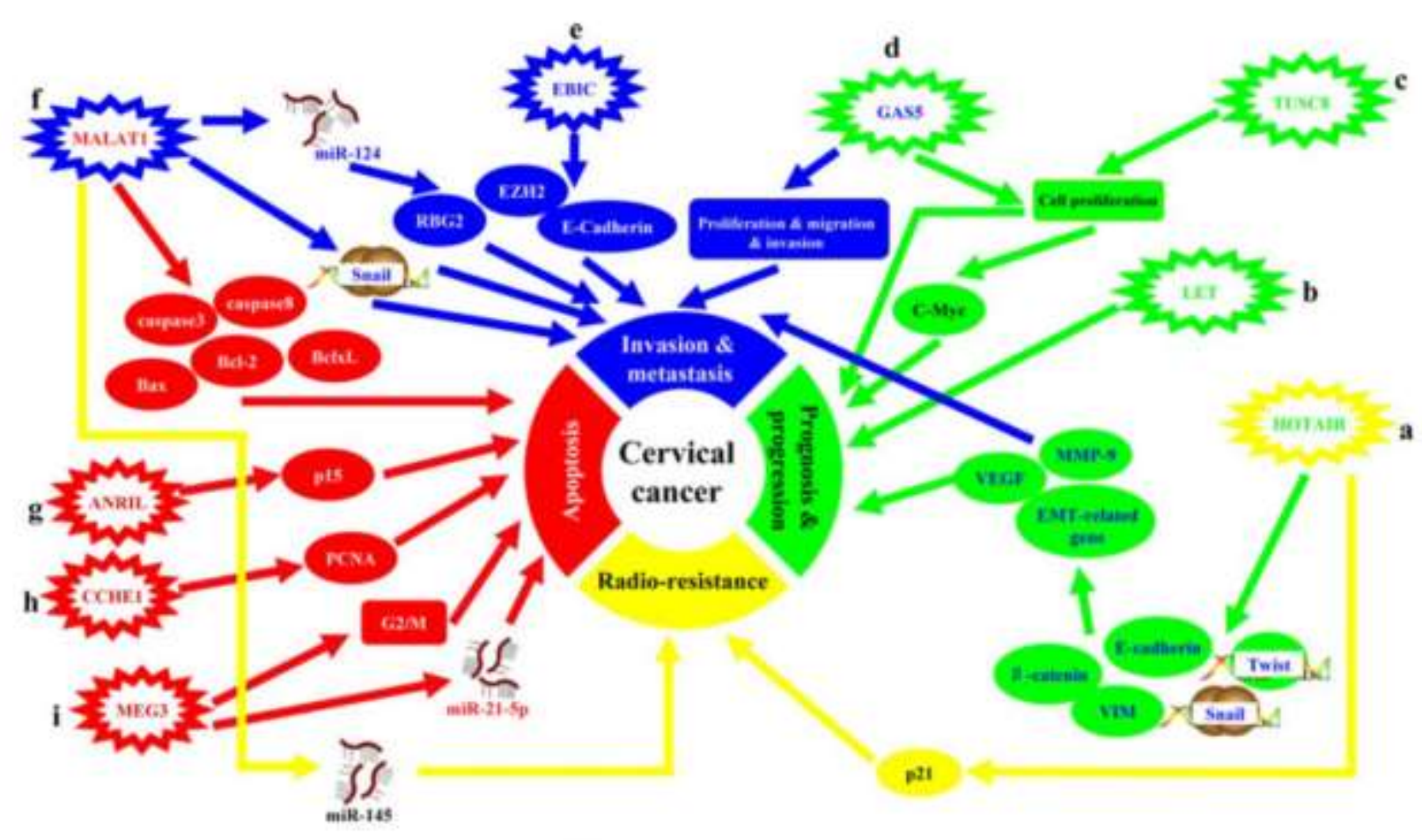

Figure 12: LncRNA functions in cervical cancer.

Extracted from: Peng et al., Tumor Biology, 2016. 
inhibitor) BCAR4 lncRNA has been found to be associated with response to lapatinib in human patients(236).

\section{LncRNA Loc554202}

LncRNA Loc554202 is the host gene of miR-31(237). It has been shown to be involved in the development of breast(238), colorectal(239), and cervical cancer(240). Loc554202 exhibits higher expression in cervical cancer tissues than adjacent non-cancer tissues. High expression of the lncRNA correlates with poor overall survival, tumor size, stage, and lymph node metastasis. Knockdown of Loc554202 inhibits cell proliferation and induces apoptosis in cervical cancer cell lines(240).

\section{$H 19$}

H19 can be oncogenic(241) or tumor suppressive (242), depending on the tissue lineage. In cervical cancer, it has been shown to be oncogenic and regulate cell proliferation(243). Additionally, in cervical cancer, H19 can sponge miR-138-5p to regulate proliferation and apoptosis(244).

\section{GAS5 (growth arrest-specific transcript 5)}

GAS5 is down-regulated in cervical cancer tissues as well as many other cancer types (e.g. breast(245) and lung(246))(247). GAS5 is a tumor suppressor lncRNA that when knocked down in vitro led to increased cell proliferation, migration, and invasion in cervical cancer (Figure 12d). The decrease in GAS5 expression significantly correlated with advanced cancer progression(248), thus GAS5 is considered a potentially useful biomarker for cervical cancer patients.

\section{TUSC8 (tumor suppressor candidate 8)}

Another tumor suppressor lincRNA in cervical cancer is TUSC8 (also known as XLOC_010588). In cervical cancer, TUSC8 expression is dramatically reduced, and this lower expression correlates with tumor stage and tumor size. With low TUSC8 expression in cervical cancer, c-myc is up-regulated and cervical cancer cells exhibit increased proliferation. Mechanistically, TUSC8 interacts with c-myc to consequently down-regulate c-Myc expression, and exogenous expression of TUSC8 in cervical cancer cells revealed decreased c-myc expression and decreased proliferation(249) (Figure 12c). Therefore, TUSC8 could be a potential therapeutic target in cervical cancer. 


\section{LncRNA LET}

LncRNA LET is down-regulated in cervical cancer, as well as many other cancers (colorectal, hepatocellular, and lung), and low expression correlates with poor overall survival. Decreased expression of IncRNA LET correlates with tumor stage and lymph node metastasis(250) such that it might be a useful prognostic marker and therapeutic target for cervical cancer (Figure 12b).

AC017078.1 and XLOC_011152

Recently, a group analyzed systemic lncRNA expression in serum from cervical cancer patients via microarray analysis. Further validation discovered two potential candidate lncRNA biomarkers whose expression was down-regulated in cervical cancer (AC017078.1 and XLOC_011152)(251). Further elucidation of circulating lncRNAs that could be used as blood-based biomarkers in cervical cancer diagnosis could be beneficial.

hsa_circ_0018289

Microarray analysis was used to identify differentially expressed circRNAs in cervical cancer tissues and reveal up-regulation of hsa_circ_0018289. Further analysis of this specific lncRNA showed its role in proliferation, migration, and invasion of cervical cancer cells as well as binding and sponging miR497 to contribute to cervical cancer(252).

hsa_circ_0023404

Hsa_circ_0023404 was shown to be up-regulated in cervical cancer tissue compared to normal tissue, and high expression of this circRNA correlated with poor overall survival. Knockdown of hsa_circ_0023404 reduced proliferation, migration, and invasion in cervical cancer cells. This circRNA binds to and sponges miR-136 such that the miRNA cannot regulate its target TFCP2, which consequently leads to activation of YAP signaling and cervical cancer progression(253).

circRNA-000284 
Another group conducted microarray analysis of cervical cancer cells (HPV-16 positive, HPV-18 positive, and HPV-negative) compared to normal cervical cell line to identify differential circRNA expressions and more specifically identify circRNA-000284 as significantly up-regulated in all cervical cancer cell lines analyzed. This specific circRNA binds to and sponges miR-506 to promote proliferation and invasion in cervical cancer(254).

\section{CircRNA8924}

Human circRNA microarray V2.0 was used to identify up-regulation of circRNA8924 expression in cervical cancer tissues. Further studies showed the involvement of the circRNA in proliferation, invasion, and migration by competitively binding to miR-518d-5p/miR-519a-5p family to modulate CBX8 expression in cervical cancer(255).

\section{CircPAIP2}

An intron containing circRNA (EIciRNA) circPAIP2 was shown to regulates memory-related genes through PABP reactivation in human cervical cancer cells(256).

\section{LncRNAs regulated by HPV}

\section{A. HPV-regulated host lncRNAs}

Many host lncRNAs are dysregulated upon HPV infection with the entire HPV genome. A 2016 review summarized lncRNAs that were altered upon HPV status, including many well-studied lncRNAs (e.g. XIST, H19, and MEG3) (DOI: 10.4137/ATV.S29816). CDKN2B-AS, EGOT (Eosinophil granule ontogeny transcript), NCRNA00185, PRINS (Psoriasis Susceptibility-related RNA Gene Induced by Stress), TTTY14, TTTY15, XIST, and LINC00152 were all identified to have altered expression in HPV active versus HPV negative oral tumor samples(257). LncRNA CCHE1 expression is correlated with HPV status in cervical cancer(226). Microarray analysis of HPV-16 positive cervical cancer cells compared to HPV-negative cervical cancer cells was conducted and 4750 differentially expressed lncRNAs were identified(258). RNA-seq analysis of HPV-16 positive tumor tissues compared to normal adjacent tissues identified lncRNAs and circRNAs that are differentially expressed(207). Nohata et al. conducted RNA-seq analysis of HPV-positive versus HPV-negative HNSCC tumors and identified 140 lncRNAs significantly differentially expressed between the groups. Multiple other molecular changes occur between HPV-positive 
and -negative HNSCC samples, however, it can be speculated that HPV is contributing to alterations in lncRNA expression profiles(259).

Additionally, many host lncRNAs are regulated specifically by the sole presence of both the HPV E6 and E7 oncoproteins. MEG3, PCNA-AS, and H19 were all differentially expressed in human foreskin keratinocytes expressing HPV-16E6/E7(260). HPV proteins can alter mitochondrial lncRNA expression to potentially regulate the cell cycle. More specifically, HPV proteins induces the expression of a sense mitochondrial ncRNA (SncmtRNA-2)(261).

Certain lncRNAs can be altered solely by the expression of the HPV E6 oncoprotein. For example, CCEPR (Cervical carcinoma expressed PCNA regulatory) lncRNA expression is regulated by HPV E6 oncoprotein to alter cell proliferation(262). Differential expression of lncRNAs was determined in human foreskin keratinocytes with and without HPV-16 E6 expression. FAM83H-AS1 expression is regulated by HPV-16 E6 oncoprotein in a p53-independent, p300-dependent manner. FAM83H-AS1 up-regulation altered cell proliferation and migration in HPV-16 positive cervical cancer cells (Chapter 2).

Alternatively, certain lncRNAs can be altered by the sole expression of the HPV E7 oncoprotein. A group conducted a study in the HPV-18 positive HeLa cervical cancer cell line where they knockdown HPV-18 E7 with siRNA and conducted lncRNA microarray analysis. They identified LINC01101 and LINC00277 whose expression increased with HPV-18 E7 knockdown. Both of these lncRNAs exhibited decreased expression in pre-cancerous lesion and cervical cancer compared to control tissues in a patient cohort of HPV-16 and -18 positive status. Additionally, lncRNA LINC01101 and LINC00277 expressions were significantly decreased in high-risk HPV-positive samples compared to HPV-negative samples; these two lncRNAs are more drastically decreased in HPV-18 samples than HPV-16, but both follow the same trend(263). CircRNA expression changes altered by the high-risk HPV-16 E7 oncoprotein were analyzed in a cervical cancer cell line (CaSki). More specifically, CaSki cells with knockdown of endogenous HPV16 E7 underwent microarray analysis to identify 352 up-regulated and 174 down-regulated circRNAs at least 2-fold change compared to control(264). HOTAIR expression in down-regulated in HPV-16 positive cervical cancer samples compared to HPV-negative normal samples(265). In cervical cancer, HOTAIR directly interacts with HPV-16 E7 and is inactivated by the HPV protein.

MALAT1 has been the most extensively studied HPV-regulated lncRNA. MALAT1 expression is increased in HPV-16 and HPV-18 positive human cervical cancer samples compared to normal human cervical samples(141, 218). Additionally, separating HPV-positive and HPV-negative samples, increased 
MALAT1 expression correlated with HPV-positive status. In cervical cancer cells, MALAT1 has been shown to be involved in the regulation of miRNAs to contribute to carcinogenesis $(218,266)$. One group specifically showed that in high-risk HPV cervical cancer, MALAT1 expression is higher in radioresistant than radiosensitive cancer cases and that MALAT1 sponges miR-145 to mediate this radioresistance(267). Decreasing MALAT1 expression in cervical cancer cells altered proliferation and invasion(216). Knockdown of HPV-16 E6/E7 led to decreased MALAT1 expression, showing a potential E6/E7 regulation of MALAT1(141). Additionally, MALAT1 is up-regulated in oral keratinocytes expressing HPV-16 $\mathrm{E} 6 / \mathrm{E} 7(268)$.

Overall, host lncRNAs are altered by individual expression of each of the HPV oncoproteins, coexpression of both oncoproteins, as well as expression of the entire HPV genome. As miRNAs are altered by HPV to regulate the viral life cycle and additionally cause alterations that contribute to cancer, it is speculated that many lncRNAs are being altered by HPV for similar functions.

\section{B. HPV regulation of lncRNAs in cancer - Clinical implications}

A clinical trial sponsored in China is currently recruiting uterine cervical adenocarcinoma patients to analyze multi-omics results of patients with and without HPV infection, with the goal of revealing the role of HPV integration in molecular mechanism(s) of tumorigenesis and prognosis of patients. They will analyze for changes in transcriptomics (IncRNA, miRNA, mRNA), whole-exome (DNA), and metabolomics (NCT03742869).

\section{Oncogenic IncRNA FAM83H-AS1 (also known as 'onco-IncRNA-3')}

In 2014, LCAL7 (lung cancer associated long non-coding RNA 7), which contains at least part of FAM83H-AS1, ENST00000533004, ENST00000534398, and ENST00000435097 in the genome, was first identified as up-regulated in lung adenocarcinoma (LUAD) and lung squamous cell carcinoma (LUSC) human tissues compared to controls. LCAL7 is located in chr8: 144,823,553-144,828,510 and a partial sequence of LCAL7 was identified by 5' and 3' RACE in a lung cancer cell line and can be found in GenBank (accession number: KF773847)(269). Overall, LCAL7 (containing a portion of FAM83H-AS1) is up-regulated in lung cancer.

In 2015, 'onco-lncRNA-3' expression was further analyzed using publicly available TCGA RNAseq data from tumors and matched normal tissues. Onco-lncRNA-3 is up-regulated in breast invasive 
carcinoma (BRCA), colorectal and rectal cancer (CRC), lung adenocarcinoma (LUAD), and lung squamous cell carcinoma (LUSC). Additionally, analysis showed 'outlier' expression in head and neck squamous cell carcinoma (HNSC); 'outlier' referred to subset of patients from the certain cancer type exhibiting consistent gene expression changes. Onco-lncRNA-3 was not found to be altered in bladder urothelial carcinoma (BLCA), kidney renal cell carcinoma (KIRC), or uterine corpus endometrial carcinoma (UCEC). Functionally, onco-lncRNA-3 exhibited a 'guilt by association' effect as it was co-expressed with many protein coding genes involved in cell cycle regulation, such that it was speculated that onco-lncRNA-3 plays a role in cell cycle progression. Onco-lncRNA-3 was shown to play a role in S-phase cell cycle in cancer as knockdown of the lncRNA in color cancer and lung cancer cells resulted in decreased EdU incorporation compared to control(133). In this study, onco-lncRNA-3 was found to be up-regulated in breast cancer, colorectal cancer, and lung cancer and plays a role in cell cycle progression.

Yang et al. identified FAM83H-AS1 (family with sequence similarity 83 member H-antisense 1) as the most up-regulated lncRNA in their RNA-seq analysis of breast cancer tissues compared to normal tissues. Additionally, analysis of 626 breast cancer samples compared to 105 non-cancerous breast tissues showed a significantly higher FAM83H-AS1 RPKM value in the cancerous tissues. Analyzing TCGA data from luminal subtype breast cancer patients revealed higher expression of FAM83H-AS1 correlates with poorer overall survival. Analyzing a TCGA cohort of breast cancer patients (containing all subtypes) revealed high expression of FAM83H-AS1 correlated with poorer overall survival(270). Taken together, FAM83H-AS1 is an independent prognostic marker in breast cancer.

Analysis of multiple colorectal cancer microarrays for differential expression of lncRNAs showed FAM83H-AS1 was the top up-regulated lncRNA analyzed in colorectal cancer (CRC) tissues compared to adjacent normal tissues. Further analysis of human colorectal cancer cell lines confirmed expression of FAM83H-AS1 in all but one cell line analyzed. High FAM83H-AS1 expression in CRC patients correlated with poorer overall survival than those with low FAM83H-AS1 expression(271). FAM83H-AS1 could be a potential prognosis biomarker in colorectal cancer patients.

RNA-seq analysis of LUAD tissues compared to controls in TCGA showed increased FAM83HAS1. Further studies analyzing FAM83H-AS1 expression in tumor versus adjacent normal tissue of the same lineage revealed significant up-regulation of FAM83H-AS1 in LUAD, lung large cell carcinoma (LULC), LUSC, bladder cancer, breast cancer, gastric cancer, head and neck cancer, and prostate cancer while no significant differences in kidney cancer, liver cancer, or thyroid cancer was observed in their analysis(272). Notably, data from a different study showed gastric cancer patients in TCGA revealed no 
consistent differential expression of FAM83H-AS1(273). In lung cancer clinical patients, high expression of FAM83H-AS1 correlated with poorer patient survival. Functionally, knockdown of FAM83H-AS1 expression in lung cancer cell lines decreased proliferation, migration, and invasion as well as decreased percentage of cells in S-phase of the cell cycle. Notably, proliferation was affected in most EGFR-mutated lung cancer cells. FAM83H-AS1 is primarily localized in the nucleus of lung cancer cells. Mechanistically, knockdown of FAM83H-AS1 decreased protein expression of MET, EGFR and their downstream proteins AKT and ERK1/2 in lung cancer cell lines. Notably, knockdown of FAM83H-AS1 regulated MET at the transcriptional and translational level(272). In lung cancer, FAM83H-AS1 could potentially be used as a diagnostic and prognostic marker or a therapeutic target to impact MET/EGFR signaling involved with cell proliferation and invasion.

RNA-seq data in TCGA of pancreas-adenocarcinoma ductal type (PDA) was analyzed for differential lncRNA expression. Analyzing relative enrichment in the stroma or epithelium identified FAM83H-AS1 as one of the top epithelial lncRNAs altered in their study. FAM83H-AS1 and the nearby protein coding gene FAM83H likely share the same promoter region, but are transcribed in opposite directions. FAM83H-AS1 is located in a genomic region frequently amplified in PDA (8q23.3-8q24.3), and its expression does in fact correlate significantly with amplification. Analysis indicates that is has four exons, and is located in an actively transcribed region in a pancreatic cancer cell line. FAM83H-AS1 expression is shown to be up-regulated in PDA lines as well as a breast cancer cell line. Clinically, high expression of FAM83H-AS1 correlates with poorer overall survival in PDA. Knockdown of FAM83H-AS1 in a pancreatic cancer cell line by two different siRNAs revealed 719 genes differentially expressed in cells with siRNA1 and siRNA2. Regulatory network analysis predicted 146 target genes of FAM83H-AS1 (78 positive/activated and 68 negative/inhibited). Of these target genes, the negatively regulated targets were more associated with benign processes while positively regulated targets are associated with more malignant processes(274). Thus, FAM83H-AS1 is involved with malignant progression in pancreatic cancer.

Bi et al. identified FAM83H-AS1 is up-regulated in glioma tissues and cell lines compared to controls. FAM83H-AS1 expression significantly correlates with tumor grade. Patients with high FAM83HAS1 expression were associated with worse overall survival in glioma. High-grade tumors FAM83H-AS1 expression correlates with patients' prognosis. FAM83H-AS1 expression could be an independent prognostic factor in glioma. Functionally, for the first time, a group expressed FAM83H-AS1 to observe functional alterations in the cells. Over-expression of FAM83H-AS1 in glioma cells increased cell viability and colony formation. Knockdown of FAM83H-AS1 in glioma cells reduced cell viability, colony 
formation, percentage of cells in S-phase of the cell cycle, and cyclin-dependent kinases (CDK2, CDK4, CDK6), while it increased cellular apoptosis in glioma cells. Additionally, knockdown of FAM83H-AS1 in glioma cells resulted in increased CDK inhibitors CDKN1A (also known as p21) mRNA expression. FAM83H-AS1 is primarily localized in the nucleus and contributes to epigenetic transcriptional regulation of p21 expression by binding to EZH2 in glioma cells. Decrease in p21 expression potentially contributes to FAM83H-AS1 oncogenic function(275). Together, FAM83H-AS1 could be a prognostic marker in glioma patients, and/or therapeutically targeting FAM83H-AS1 and in turn modulating its downstream targets might improve glioma patient outcome.

FAM83H-AS1 expression is up-regulated in colorectal cancer (CRC) tissues and cells. In CRC clinical patients, FAM83H-AS1 expression correlates with tumor stage and size and high expression of FAM83H-AS1 is associated with decreased overall survival. Functionally, knockdown of FAM83H-AS1 in colorectal cancer cells with specific short-hairpin RNA (shRNA) led to decreased proliferation, colony formation, and migration as well as increased cell apoptosis. Additionally, knockdown of FAM83H-AS1 led to a decrease in Notch1 mRNA expression. Treatment of colorectal cancer cells with a widely used gamma-secretase inhibitor (DAPT) that represses receptors and ligands in the Notch signaling pathway (including Notch1) decreased FAM83H-AS1 expression. These results are indicative that FAM83H-AS1 regulates through the Notch pathway.(276) Overall, analysis of FAM83H-AS1 expression in colorectal cancer tissues can act as an independent prognostic factor in colorectal carcinoma. Therapeutically targeting FAM83H-AS1 to modulate downstream Notch signaling might be clinically useful as Notch signaling is involved with regulation of cell proliferation, differentiation, and death.

Our group identified FAM83H-AS1 expression is up-regulated in cervical cancer tissues and cells, and clinical patients with high FAM83H-AS1 expression exhibited a significantly worse overall survival than those with low FAM83H-AS1 expression. Notably, in both cervical and HNSCC cells, FAM83H-AS1 expression was significantly higher in HPV-positive compared to HPV-negative samples. Mechanistically, FAM83H-AS1 expression is regulated in a p53-independent and at least partially by HPV-16 E6 expression and p300 in cervical cancer. In cervical cancer cells, FAM83H-AS1 expression is primarily localized in the nucleus and does not appear to regulate its nearby protein coding gene FAM83H. Knockdown of FAM83HAS1 expression in cervical cancer cells decreased proliferation (and increased percentage of cells in the Sphase of cell cycle), migration as well as increased apoptosis (Chapter 2). Together, FAM83H-AS1 could be a potential prognostic marker in cervical cancer, further studies could reveal that FAM83H-AS1 could be a diagnostic marker for HPV infection progression to cervical cancer, and therapeutically targeting FAM83H-AS1 could improve patient outcome. 


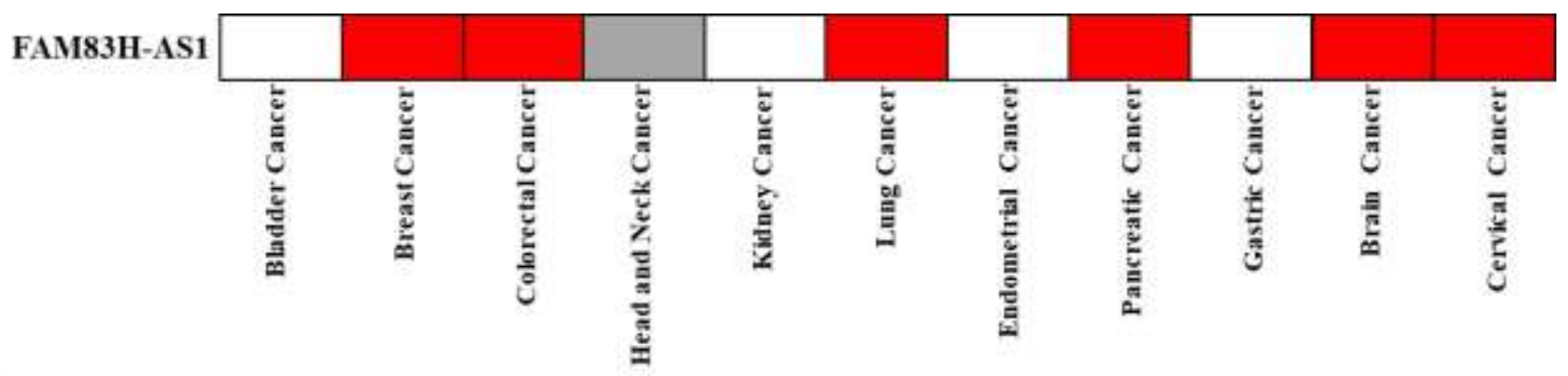

Up-regulated

Outlier

Not altered

Figure 13: Heat map of FAM83H-AS1 expression alterations in multiple cancer types. Adapted from: Cabanski et al., RNA Blology; 2015.

Overall, FAM83H-AS1 is up-regulated in many $\operatorname{lung}(133,269,272)$, breast(133, 270, 272), colorectal(133, 271, 276), pancreatic(274), glioma brain(275), bladder(272), prostate(272), and cervical cancers (Figure 13 and Chapter 2). FAM83H-AS1 expression is upregulated in certain gastric cancer(272) and HNSCC(272) studies, while others show variable alterations in gastric(273) and HNSCC(133) tissues. Thus, FAM83H-AS1 could potentially be a diagnostic marker in those cancers. High expression of FAM83H-AS1 correlates with worse overall survival in breast(270), colorectal(271, 276), lung(272), pancreatic(274), glioma brain(275), and cervical cancers (Chapter 2); therefore, FAM83H-AS1 could potentially be a prognostic marker in those cancer types. FAM83H-AS1 is mainly localized in the epithelium in pancreatic cancer cells(274) and specifically in the nucleus in lung cancer(272), cervical cancer (Chapter 2), and glioma brain cancer(275) cells. FAM83H-AS1 expression is up-regulated at least in part by HPV-16 E6 and p300 in cervical cancer cells (Chapter 2). Downstream, FAM83H-AS1 regulates Notch signaling in colorectal cancer(276), MET/EGFR signaling in lung cancer(272), and p21 expression in glioma(275). Further studies need to be conducted to determine if these upstream and downstream targets involved with FAM83H-AS1 expression are consistent between cancer types, and novel upstream and downstream regulators of FAM83H-AS1 expression need to be elucidated to increase therapeutic potential of targeting FAM83H-AS1. 


\section{References:}

1. Jacob F, Monod J. 1961. Genetic regulatory mechanisms in the synthesis of proteins. J Mol Biol 3:318-356.

2. Fox GE. 2010. Origin and evolution of the ribosome. Cold Spring Harb Perspect Biol 2:a003483.

3. Kruger K, Grabowski PJ, Zaug AJ, Sands J, Gottschling DE, Cech TR. 1982. Self-splicing RNA: autoexcision and autocyclization of the ribosomal RNA intervening sequence of Tetrahymena. Cell 31:147-157.

4. Garriga G, Lambowitz AM. 1984. RNA splicing in neurospora mitochondria: self-splicing of a mitochondrial intron in vitro. Cell 39:631-641.

5. Anonymous. 2012. An integrated encyclopedia of DNA elements in the human genome. Nature 489:57-74.

6. Birney E, Stamatoyannopoulos JA, Dutta A, Guigo R, Gingeras TR, Margulies EH, Weng Z, Snyder M, Dermitzakis ET, Thurman RE, Kuehn MS, Taylor CM, Neph S, Koch CM, Asthana S, Malhotra A, Adzhubei I, Greenbaum JA, Andrews RM, Flicek P, Boyle PJ, Cao H, Carter NP, Clelland GK, Davis S, Day N, Dhami P, Dillon SC, Dorschner MO, Fiegler H, Giresi PG, Goldy J, Hawrylycz M, Haydock A, Humbert R, James KD, Johnson BE, Johnson EM, Frum TT, Rosenzweig ER, Karnani N, Lee K, Lefebvre GC, Navas PA, Neri F, Parker SC, Sabo PJ, Sandstrom R, Shafer A, Vetrie D, et al. 2007. Identification and analysis of functional elements in 1\% of the human genome by the ENCODE pilot project. Nature 447:799816.

7. Bazzini AA, Johnstone TG, Christiano R, Mackowiak SD, Obermayer B, Fleming ES, Vejnar CE, Lee MT, Rajewsky N, Walther TC, Giraldez AJ. 2014. Identification of small ORFs in vertebrates using ribosome footprinting and evolutionary conservation. Embo j 33:981-993.

8. Galindo MI, Pueyo JI, Fouix S, Bishop SA, Couso JP. 2007. Peptides encoded by short ORFs control development and define a new eukaryotic gene family. PLoS Biol 5:e106.

9. Lee Y, Rio DC. 2015. Mechanisms and Regulation of Alternative Pre-mRNA Splicing. Annu Rev Biochem 84:291-323.

10. Napoli C, Lemieux C, Jorgensen R. 1990. Introduction of a Chimeric Chalcone Synthase Gene into Petunia Results in Reversible Co-Suppression of Homologous Genes in trans. Plant Cell 2:279289.

11. Fire A, Xu S, Montgomery MK, Kostas SA, Driver SE, Mello CC. 1998. Potent and specific genetic interference by double-stranded RNA in Caenorhabditis elegans. Nature 391:806-811.

12. Iwasaki YW, Siomi MC, Siomi H. 2015. PIWI-Interacting RNA: Its Biogenesis and Functions. Annu Rev Biochem 84:405-433.

13. Wen YZ, Zheng LL, Liao JY, Wang MH, Wei Y, Guo XM, Qu LH, Ayala FJ, Lun ZR. 2011. Pseudogene-derived small interference RNAs regulate gene expression in African Trypanosoma brucei. Proc Natl Acad Sci U S A 108:8345-8350.

14. Guo X, Lin M, Rockowitz S, Lachman HM, Zheng D. 2014. Characterization of human pseudogene-derived non-coding RNAs for functional potential. PLoS One 9:e93972.

15. Okazaki Y, Furuno M, Kasukawa T, Adachi J, Bono H, Kondo S, Nikaido I, Osato N, Saito R, Suzuki H, Yamanaka I, Kiyosawa H, Yagi K, Tomaru Y, Hasegawa Y, Nogami A, Schonbach C, Gojobori T, Baldarelli R, Hill DP, Bult C, Hume DA, Quackenbush J, Schriml LM, Kanapin A, Matsuda H, Batalov S, Beisel KW, Blake JA, Bradt D, Brusic V, Chothia C, Corbani LE, Cousins S, Dalla E, Dragani TA, Fletcher CF, Forrest A, Frazer KS, Gaasterland T, Gariboldi M, Gissi C, Godzik A, Gough J, Grimmond S, Gustincich S, Hirokawa N, Jackson IJ, Jarvis ED, Kanai A, et al. 2002. Analysis of the mouse transcriptome based on functional annotation of 60,770 full-length cDNAs. Nature 420:563-573.

16. Lee RC, Feinbaum RL, Ambros V. 1993. The C. elegans heterochronic gene lin-4 encodes small RNAs with antisense complementarity to lin-14. Cell 75:843-854. 
17. Calin GA, Dumitru CD, Shimizu M, Bichi R, Zupo S, Noch E, Aldler H, Rattan S, Keating M, Rai K, Rassenti L, Kipps T, Negrini M, Bullrich F, Croce CM. 2002. Frequent deletions and down-regulation of micro- RNA genes miR15 and miR16 at 13q14 in chronic lymphocytic leukemia. Proc Natl Acad Sci U S A 99:15524-15529.

18. Berezikov E, Guryev V, van de Belt J, Wienholds E, Plasterk RH, Cuppen E. 2005. Phylogenetic shadowing and computational identification of human microRNA genes. Cell 120:21-24.

19. Lau NC, Lim LP, Weinstein EG, Bartel DP. 2001. An abundant class of tiny RNAs with probable regulatory roles in Caenorhabditis elegans. Science 294:858-862.

20. Lagos-Quintana M, Rauhut R, Lendeckel W, Tuschl T. 2001. Identification of novel genes coding for small expressed RNAs. Science 294:853-858.

21. Lee RC, Ambros V. 2001. An extensive class of small RNAs in Caenorhabditis elegans. Science 294:862-864.

22. Lewis BP, Shih IH, Jones-Rhoades MW, Bartel DP, Burge CB. 2003. Prediction of mammalian microRNA targets. Cell 115:787-798.

23. Kozomara A, Griffiths-Jones S. 2014. miRBase: annotating high confidence microRNAs using deep sequencing data. Nucleic Acids Res 42:D68-73.

24. Hamilton AJ, Baulcombe DC. 1999. A species of small antisense RNA in posttranscriptional gene silencing in plants. Science 286:950-952.

25. Liu J, Valencia-Sanchez MA, Hannon GJ, Parker R. 2005. MicroRNA-dependent localization of targeted mRNAs to mammalian P-bodies. Nat Cell Biol 7:719-723.

26. Lee Y, Kim M, Han J, Yeom KH, Lee S, Baek SH, Kim VN. 2004. MicroRNA genes are transcribed by RNA polymerase II. Embo j 23:4051-4060.

27. Lee Y, Ahn C, Han J, Choi H, Kim J, Yim J, Lee J, Provost P, Radmark O, Kim S, Kim VN. 2003. The nuclear RNase III Drosha initiates microRNA processing. Nature 425:415-419.

28. Han J, Lee Y, Yeom KH, Kim YK, Jin H, Kim VN. 2004. The Drosha-DGCR8 complex in primary microRNA processing. Genes Dev 18:3016-3027.

29. Basyuk E, Suavet F, Doglio A, Bordonne R, Bertrand E. 2003. Human let-7 stem-loop precursors harbor features of RNase III cleavage products. Nucleic Acids Res 31:6593-6597.

30. Lund E, Guttinger S, Calado A, Dahlberg JE, Kutay U. 2004. Nuclear export of microRNA precursors. Science 303:95-98.

31. Denli AM, Tops BB, Plasterk RH, Ketting RF, Hannon GJ. 2004. Processing of primary microRNAs by the Microprocessor complex. Nature 432:231-235.

32. Yi R, Qin Y, Macara IG, Cullen BR. 2003. Exportin-5 mediates the nuclear export of premicroRNAs and short hairpin RNAs. Genes Dev 17:3011-3016.

33. Bohnsack MT, Czaplinski K, Gorlich D. 2004. Exportin 5 is a RanGTP-dependent dsRNAbinding protein that mediates nuclear export of pre-miRNAs. Rna 10:185-191.

34. Bernstein E, Caudy AA, Hammond SM, Hannon GJ. 2001. Role for a bidentate ribonuclease in the initiation step of RNA interference. Nature 409:363-366.

35. Grishok A, Pasquinelli AE, Conte D, Li N, Parrish S, Ha I, Baillie DL, Fire A, Ruvkun G, Mello CC. 2001. Genes and mechanisms related to RNA interference regulate expression of the small temporal RNAs that control C. elegans developmental timing. Cell 106:23-34.

36. Hutvagner G, McLachlan J, Pasquinelli AE, Balint E, Tuschl T, Zamore PD. 2001. A cellular function for the RNA-interference enzyme Dicer in the maturation of the let-7 small temporal RNA. Science 293:834-838.

37. Ketting RF, Fischer SE, Bernstein E, Sijen T, Hannon GJ, Plasterk RH. 2001. Dicer functions in RNA interference and in synthesis of small RNA involved in developmental timing in C. elegans. Genes Dev 15:2654-2659.

38. Knight SW, Bass BL. 2001. A role for the RNase III enzyme DCR-1 in RNA interference and germ line development in Caenorhabditis elegans. Science 293:2269-2271. 
39. O'Toole AS, Miller S, Haines N, Zink MC, Serra MJ. 2006. Comprehensive thermodynamic analysis of 3' double-nucleotide overhangs neighboring Watson-Crick terminal base pairs. Nucleic Acids Res 34:3338-3344.

40. Khvorova A, Reynolds A, Jayasena SD. 2003. Functional siRNAs and miRNAs exhibit strand bias. Cell 115:209-216.

41. Schwarz DS, Hutvagner G, Du T, Xu Z, Aronin N, Zamore PD. 2003. Asymmetry in the assembly of the RNAi enzyme complex. Cell 115:199-208.

42. Huang CJ, Nguyen PN, Choo KB, Sugii S, Wee K, Cheong SK, Kamarul T. 2014. Frequent co-expression of miRNA-5p and $-3 p$ species and cross-targeting in induced pluripotent stem cells. Int J Med Sci 11:824-833.

43. Bartel DP. 2004. MicroRNAs: genomics, biogenesis, mechanism, and function. Cell 116:281-297.

44. Yekta S, Shih IH, Bartel DP. 2004. MicroRNA-directed cleavage of HOXB8 mRNA. Science 304:594-596.

45. Eulalio A, Huntzinger E, Nishihara T, Rehwinkel J, Fauser M, Izaurralde E. 2009. Deadenylation is a widespread effect of miRNA regulation. Rna 15:21-32.

46. Kim YK, Kim B, Kim VN. 2016. Re-evaluation of the roles of DROSHA, Export in 5, and DICER in microRNA biogenesis. Proc Natl Acad Sci U S A 113:E1881-1889.

47. Okamura K, Hagen JW, Duan H, Tyler DM, Lai EC. 2007. The mirtron pathway generates microRNA-class regulatory RNAs in Drosophila. Cell 130:89-100.

48. Ruby JG, Jan CH, Bartel DP. 2007. Intronic microRNA precursors that bypass Drosha processing. Nature 448:83-86.

49. Berezikov E, Chung WJ, Willis J, Cuppen E, Lai EC. 2007. Mammalian mirtron genes. Mol Cell 28:328-336.

50. Babiarz JE, Ruby JG, Wang Y, Bartel DP, Blelloch R. 2008. Mouse ES cells express endogenous shRNAs, siRNAs, and other Microprocessor-independent, Dicer-dependent small RNAs. Genes Dev 22:2773-2785.

51. Glazov EA, Cottee PA, Barris WC, Moore RJ, Dalrymple BP, Tizard ML. 2008. A microRNA catalog of the developing chicken embryo identified by a deep sequencing approach. Genome Res 18:957-964.

52. Ladewig E, Okamura K, Flynt AS, Westholm JO, Lai EC. 2012. Discovery of hundreds of mirtrons in mouse and human small RNA data. Genome Res 22:1634-1645.

53. Flynt AS, Greimann JC, Chung WJ, Lima CD, Lai EC. 2010. MicroRNA biogenesis via splicing and exosome-mediated trimming in Drosophila. Mol Cell 38:900-907.

54. Havens MA, Reich AA, Duelli DM, Hastings ML. 2012. Biogenesis of mammalian microRNAs by a non-canonical processing pathway. Nucleic Acids Res 40:4626-4640.

55. Ender C, Krek A, Friedlander MR, Beitzinger M, Weinmann L, Chen W, Pfeffer S, Rajewsky N, Meister G. 2008. A human snoRNA with microRNA-like functions. Mol Cell 32:519-528.

56. Brameier M, Herwig A, Reinhardt R, Walter L, Gruber J. 2011. Human box C/D snoRNAs with miRNA like functions: expanding the range of regulatory RNAs. Nucleic Acids Res 39:675686.

57. Scott MS, Avolio F, Ono M, Lamond AI, Barton GJ. 2009. Human miRNA precursors with box H/ACA snoRNA features. PLoS Comput Biol 5:e1000507.

58. Scott MS, Ono M. 2011. From snoRNA to miRNA: Dual function regulatory non-coding RNAs. Biochimie 93:1987-1992.

59. Ono M, Scott MS, Yamada K, Avolio F, Barton GJ, Lamond AI. 2011. Identification of human miRNA precursors that resemble box C/D snoRNAs. Nucleic Acids Res 39:3879-3891.

60. Cole C, Sobala A, Lu C, Thatcher SR, Bowman A, Brown JW, Green PJ, Barton GJ, Hutvagner G. 2009. Filtering of deep sequencing data reveals the existence of abundant Dicerdependent small RNAs derived from tRNAs. Rna 15:2147-2160.

61. Haussecker D, Huang Y, Lau A, Parameswaran P, Fire AZ, Kay MA. 2010. Human tRNAderived small RNAs in the global regulation of RNA silencing. Rna 16:673-695. 
62. Bowden RJ, Simas JP, Davis AJ, Efstathiou S. 1997. Murine gammaherpesvirus 68 encodes tRNA-like sequences which are expressed during latency. J Gen Virol 78 ( Pt 7):1675-1687.

63. Reese TA, Xia J, Johnson LS, Zhou X, Zhang W, Virgin HW. 2010. Identification of novel microRNA-like molecules generated from herpesvirus and host tRNA transcripts. J Virol 84:10344-10353.

64. Bogerd HP, Karnowski HW, Cai X, Shin J, Pohlers M, Cullen BR. 2010. A mammalian herpesvirus uses noncanonical expression and processing mechanisms to generate viral MicroRNAs. Mol Cell 37:135-142.

65. Shapiro JS, Varble A, Pham AM, Tenoever BR. 2010. Noncanonical cytoplasmic processing of viral microRNAs. Rna 16:2068-2074.

66. Shapiro JS, Langlois RA, Pham AM, Tenoever BR. 2012. Evidence for a cytoplasmic microprocessor of pri-miRNAs. Rna 18:1338-1346.

67. Cazalla D, Xie M, Steitz JA. 2011. A primate herpesvirus uses the integrator complex to generate viral microRNAs. Mol Cell 43:982-992.

68. Chirayil R, Kincaid RP, Dahlke C, Kuny CV, Dalken N, Spohn M, Lawson B, Grundhoff A, Sullivan CS. 2018. Identification of virus-encoded microRNAs in divergent Papillomaviruses. PLoS Pathog 14:e1007156.

69. Cheloufi S, Dos Santos CO, Chong MM, Hannon GJ. 2010. A dicer-independent miRNA biogenesis pathway that requires Ago catalysis. Nature 465:584-589.

70. Cifuentes D, Xue H, Taylor DW, Patnode H, Mishima Y, Cheloufi S, Ma E, Mane S, Hannon GJ, Lawson ND, Wolfe SA, Giraldez AJ. 2010. A novel miRNA processing pathway independent of Dicer requires Argonaute2 catalytic activity. Science 328:1694-1698.

71. Yang JS, Lai EC. 2010. Dicer-independent, Ago2-mediated microRNA biogenesis in vertebrates. Cell Cycle 9:4455-4460.

72. Yang JS, Maurin T, Robine N, Rasmussen KD, Jeffrey KL, Chandwani R, Papapetrou EP, Sadelain M, O'Carroll D, Lai EC. 2010. Conserved vertebrate mir-451 provides a platform for Dicer-independent, Ago2-mediated microRNA biogenesis. Proc Natl Acad Sci U S A 107:1516315168.

73. McCloskey A, Taniguchi I, Shinmyozu K, Ohno M. 2012. hnRNP C tetramer measures RNA length to classify RNA polymerase II transcripts for export. Science 335:1643-1646.

74. Xie M, Li M, Vilborg A, Lee N, Shu MD, Yartseva V, Sestan N, Steitz JA. 2013. Mammalian 5'-capped microRNA precursors that generate a single microRNA. Cell 155:1568-1580.

75. Garzon R, Calin GA, Croce CM. 2009. MicroRNAs in Cancer. Annu Rev Med 60:167-179.

76. Lee YS, Dutta A. 2009. MicroRNAs in cancer. Annu Rev Pathol 4:199-227.

77. Di Leva G, Garofalo M, Croce CM. 2014. MicroRNAs in cancer. Annu Rev Pathol 9:287-314.

78. Peng Y, Croce CM. 2016. The role of MicroRNAs in human cancer. Signal Transduct Target Ther 1:15004.

79. Lu J, Getz G, Miska EA, Alvarez-Saavedra E, Lamb J, Peck D, Sweet-Cordero A, Ebert BL, Mak RH, Ferrando AA, Downing JR, Jacks T, Horvitz HR, Golub TR. 2005. MicroRNA expression profiles classify human cancers. Nature 435:834-838.

80. Gaur A, Jewell DA, Liang Y, Ridzon D, Moore JH, Chen C, Ambros VR, Israel MA. 2007. Characterization of microRNA expression levels and their biological correlates in human cancer cell lines. Cancer Res 67:2456-2468.

81. Kumar MS, Lu J, Mercer KL, Golub TR, Jacks T. 2007. Impaired microRNA processing enhances cellular transformation and tumorigenesis. Nat Genet 39:673-677.

82. Arvey A, Larsson E, Sander C, Leslie CS, Marks DS. 2010. Target mRNA abundance dilutes microRNA and siRNA activity. Mol Syst Biol 6:363.

83. Calin GA, Sevignani C, Dumitru CD, Hyslop T, Noch E, Yendamuri S, Shimizu M, Rattan S, Bullrich F, Negrini M, Croce CM. 2004. Human microRNA genes are frequently located at fragile sites and genomic regions involved in cancers. Proc Natl Acad Sci U S A 101:2999-3004. 
84. Lehmann U, Hasemeier B, Christgen M, Muller M, Romermann D, Langer F, Kreipe H. 2008. Epigenetic inactivation of microRNA gene hsa-mir-9-1 in human breast cancer. J Pathol 214:17-24.

85. Lujambio A, Ropero S, Ballestar E, Fraga MF, Cerrato C, Setien F, Casado S, SuarezGauthier A, Sanchez-Cespedes M, Git A, Spiteri I, Das PP, Caldas C, Miska E, Esteller M. 2007. Genetic unmasking of an epigenetically silenced microRNA in human cancer cells. Cancer Res 67:1424-1429.

86. Fabbri M, Garzon R, Cimmino A, Liu Z, Zanesi N, Callegari E, Liu S, Alder H, Costinean S, Fernandez-Cymering C, Volinia S, Guler G, Morrison CD, Chan KK, Marcucci G, Calin GA, Huebner K, Croce CM. 2007. MicroRNA-29 family reverts aberrant methylation in lung cancer by targeting DNA methyltransferases 3A and 3B. Proc Natl Acad Sci U S A 104:15805-15810.

87. Dews M, Homayouni A, Yu D, Murphy D, Sevignani C, Wentzel E, Furth EE, Lee WM, Enders GH, Mendell JT, Thomas-Tikhonenko A. 2006. Augmentation of tumor angiogenesis by a Myc-activated microRNA cluster. Nat Genet 38:1060-1065.

88. He L, He X, Lim LP, de Stanchina E, Xuan Z, Liang Y, Xue W, Zender L, Magnus J, Ridzon D, Jackson AL, Linsley PS, Chen C, Lowe SW, Cleary MA, Hannon GJ. 2007. A microRNA component of the p53 tumour suppressor network. Nature 447:1130-1134.

89. He H, Jazdzewski K, Li W, Liyanarachchi S, Nagy R, Volinia S, Calin GA, Liu CG, Franssila K, Suster S, Kloos RT, Croce CM, de la Chapelle A. 2005. The role of microRNA genes in papillary thyroid carcinoma. Proc Natl Acad Sci U S A 102:19075-19080.

90. Fontana L, Fiori ME, Albini S, Cifaldi L, Giovinazzi S, Forloni M, Boldrini R, Donfrancesco A, Federici V, Giacomini P, Peschle C, Fruci D. 2008. Antagomir-17-5p abolishes the growth of therapy-resistant neuroblastoma through p21 and BIM. PLoS One 3:e2236.

91. Buscaglia LE, Li Y. 2011. Apoptosis and the target genes of microRNA-21. Chin J Cancer 30:371380.

92. Loffler D, Brocke-Heidrich K, Pfeifer G, Stocsits C, Hackermuller J, Kretzschmar AK, Burger R, Gramatzki M, Blumert C, Bauer K, Cvijic H, Ullmann AK, Stadler PF, Horn F. 2007. Interleukin-6 dependent survival of multiple myeloma cells involves the Stat3-mediated induction of microRNA-21 through a highly conserved enhancer. Blood 110:1330-1333.

93. Mattiske S, Suetani RJ, Neilsen PM, Callen DF. 2012. The oncogenic role of miR-155 in breast cancer. Cancer Epidemiol Biomarkers Prev 21:1236-1243.

94. Bayraktar R, Van Roosbroeck K. 2018. miR-155 in cancer drug resistance and as target for miRNA-based therapeutics. Cancer Metastasis Rev 37:33-44.

95. Wang X, Cao L, Wang Y, Wang X, Liu N, You Y. 2012. Regulation of let-7 and its target oncogenes (Review). Oncol Lett 3:955-960.

96. Slabakova E, Culig Z, Remsik J, Soucek K. 2017. Alternative mechanisms of miR-34a regulation in cancer. Cell Death Dis 8:e3100.

97. Lin Z, Flemington EK. 2011. miRNAs in the pathogenesis of oncogenic human viruses. Cancer Lett 305: 186-199.

98. Martinez I, Gardiner AS, Board KF, Monzon FA, Edwards RP, Khan SA. 2008. Human papillomavirus type 16 reduces the expression of microRNA-218 in cervical carcinoma cells. Oncogene 27:2575-2582.

99. Wang X, Wang HK, McCoy JP, Banerjee NS, Rader JS, Broker TR, Meyers C, Chow LT, Zheng ZM. 2009. Oncogenic HPV infection interrupts the expression of tumor-suppressive miR34a through viral oncoprotein E6. Rna 15:637-647.

100. Samols MA, Skalsky RL, Maldonado AM, Riva A, Lopez MC, Baker HV, Renne R. 2007. Identification of cellular genes targeted by KSHV-encoded microRNAs. PLoS Pathog 3:e65.

101. Pfeffer S, Zavolan M, Grasser FA, Chien M, Russo JJ, Ju J, John B, Enright AJ, Marks D, Sander C, Tuschl T. 2004. Identification of virus-encoded microRNAs. Science 304:734-736. 
102. Taraboletti G, Benelli R, Borsotti P, Rusnati M, Presta M, Giavazzi R, Ruco L, Albini A. 1999. Thrombospondin-1 inhibits Kaposi's sarcoma (KS) cell and HIV-1 Tat-induced angiogenesis and is poorly expressed in KS lesions. J Pathol 188:76-81.

103. Guttman M, Amit I, Garber M, French C, Lin MF, Feldser D, Huarte M, Zuk O, Carey BW, Cassady JP, Cabili MN, Jaenisch R, Mikkelsen TS, Jacks T, Hacohen N, Bernstein BE, Kellis M, Regev A, Rinn JL, Lander ES. 2009. Chromatin signature reveals over a thousand highly conserved large non-coding RNAs in mammals. Nature 458:223-227.

104. Miao Y, Xu SY, Chen LS, Liang GY, Pu YP, Yin LH. 2017. Trends of long noncoding RNA research from 2007 to 2016: a bibliometric analysis. Oncotarget 8:83114-83127.

105. Kashi K, Henderson L, Bonetti A, Carninci P. 2016. Discovery and functional analysis of IncRNAs: Methodologies to investigate an uncharacterized transcriptome. Biochim Biophys Acta 1859:3-15.

106. Quinn JJ, Chang HY. 2016. Unique features of long non-coding RNA biogenesis and function. Nat Rev Genet 17:47-62.

107. Wu H, Yang L, Chen LL. 2017. The Diversity of Long Noncoding RNAs and Their Generation. Trends Genet 33:540-552.

108. Sun Q, Hao Q, Prasanth KV. 2018. Nuclear Long Noncoding RNAs: Key Regulators of Gene Expression. Trends Genet 34:142-157.

109. Grzechnik P, Tan-Wong SM, Proudfoot NJ. 2014. Terminate and make a loop: regulation of transcriptional directionality. Trends Biochem Sci 39:319-327.

110. Salzman J, Gawad C, Wang PL, Lacayo N, Brown PO. 2012. Circular RNAs are the predominant transcript isoform from hundreds of human genes in diverse cell types. PLoS One 7:e30733.

111. Rinn JL, Chang HY. 2012. Genome regulation by long noncoding RNAs. Annu Rev Biochem 81:145-166.

112. Herrera-Solorio AM, Armas-Lopez L, Arrieta O, Zuniga J, Pina-Sanchez P, Avila-Moreno F. 2017. Histone code and long non-coding RNAs (lncRNAs) aberrations in lung cancer: implications in the therapy response. Clin Epigenetics 9:98.

113. Ning Q, Li Y, Wang Z, Zhou S, Sun H, Yu G. 2017. The Evolution and Expression Pattern of Human Overlapping lncRNA and Protein-coding Gene Pairs. Sci Rep 7:42775.

114. Balbin OA, Malik R, Dhanasekaran SM, Prensner JR, Cao X, Wu YM, Robinson D, Wang R, Chen G, Beer DG, Nesvizhskii AI, Chinnaiyan AM. 2015. The landscape of antisense gene expression in human cancers. Genome Res 25:1068-1079.

115. Carrieri C, Cimatti L, Biagioli M, Beugnet A, Zucchelli S, Fedele S, Pesce E, Ferrer I, Collavin L, Santoro C, Forrest AR, Carninci P, Biffo S, Stupka E, Gustincich S. 2012. Long non-coding antisense RNA controls Uchl1 translation through an embedded SINEB2 repeat. Nature 491:454-457.

116. Kiyosawa H, Mise N, Iwase S, Hayashizaki Y, Abe K. 2005. Disclosing hidden transcripts: mouse natural sense-antisense transcripts tend to be poly(A) negative and nuclear localized. Genome Res 15:463-474.

117. Morrissy AS, Griffith M, Marra MA. 2011. Extensive relationship between antisense transcription and alternative splicing in the human genome. Genome Res 21:1203-1212.

118. Khalil AM, Guttman M, Huarte M, Garber M, Raj A, Rivea Morales D, Thomas K, Presser A, Bernstein BE, van Oudenaarden A, Regev A, Lander ES, Rinn JL. 2009. Many human large intergenic noncoding RNAs associate with chromatin-modifying complexes and affect gene expression. Proc Natl Acad Sci U S A 106:11667-11672.

119. Marques AC, Hughes J, Graham B, Kowalczyk MS, Higgs DR, Ponting CP. 2013. Chromatin signatures at transcriptional start sites separate two equally populated yet distinct classes of intergenic long noncoding RNAs. Genome Biol 14:R131.

120. Ounzain S, Pezzuto I, Micheletti R, Burdet F, Sheta R, Nemir M, Gonzales C, Sarre A, Alexanian M, Blow MJ, May D, Johnson R, Dauvillier J, Pennacchio LA, Pedrazzini T. 2014. 
Functional importance of cardiac enhancer-associated noncoding RNAs in heart development and disease. J Mol Cell Cardiol 76:55-70.

121. Nakaya HI, Amaral PP, Louro R, Lopes A, Fachel AA, Moreira YB, El-Jundi TA, da Silva AM, Reis EM, Verjovski-Almeida S. 2007. Genome mapping and expression analyses of human intronic noncoding RNAs reveal tissue-specific patterns and enrichment in genes related to regulation of transcription. Genome Biol 8:R43.

122. Dinger ME, Pang KC, Mercer TR, Mattick JS. 2008. Differentiating protein-coding and noncoding RNA: challenges and ambiguities. PLoS Comput Biol 4:e1000176.

123. Chooniedass-Kothari S, Emberley E, Hamedani MK, Troup S, Wang X, Czosnek A, Hube F, Mutawe M, Watson PH, Leygue E. 2004. The steroid receptor RNA activator is the first functional RNA encoding a protein. FEBS Lett 566:43-47.

124. Warden CD, Kim SH, Yi SV. 2008. Predicted functional RNAs within coding regions constrain evolutionary rates of yeast proteins. PLoS One 3:e1559.

125. Jenny A, Hachet O, Zavorszky P, Cyrklaff A, Weston MD, Johnston DS, Erdelyi M, Ephrussi A. 2006. A translation-independent role of oskar RNA in early Drosophila oogenesis. Development 133:2827-2833.

126. Eastman G, Smircich P, Sotelo-Silveira JR. 2018. Following Ribosome Footprints to Understand Translation at a Genome Wide Level. Comput Struct Biotechnol J 16:167-176.

127. Guttman M, Russell P, Ingolia NT, Weissman JS, Lander ES. 2013. Ribosome profiling provides evidence that large noncoding RNAs do not encode proteins. Cell 154:240-251.

128. Wang L, Park HJ, Dasari S, Wang S, Kocher JP, Li W. 2013. CPAT: Coding-Potential Assessment Tool using an alignment-free logistic regression model. Nucleic Acids Res 41:e74.

129. Derrien T, Johnson R, Bussotti G, Tanzer A, Djebali S, Tilgner H, Guernec G, Martin D, Merkel A, Knowles DG, Lagarde J, Veeravalli L, Ruan X, Ruan Y, Lassmann T, Carninci P, Brown JB, Lipovich L, Gonzalez JM, Thomas M, Davis CA, Shiekhattar R, Gingeras TR, Hubbard TJ, Notredame C, Harrow J, Guigo R. 2012. The GENCODE v7 catalog of human long noncoding RNAs: analysis of their gene structure, evolution, and expression. Genome Res 22:1775-1789.

130. Cabili MN, Trapnell C, Goff L, Koziol M, Tazon-Vega B, Regev A, Rinn JL. 2011. Integrative annotation of human large intergenic noncoding RNAs reveals global properties and specific subclasses. Genes Dev 25:1915-1927.

131. Djebali S, Davis CA, Merkel A, Dobin A, Lassmann T, Mortazavi A, Tanzer A, Lagarde J, Lin W, Schlesinger F, Xue C, Marinov GK, Khatun J, Williams BA, Zaleski C, Rozowsky J, Roder M, Kokocinski F, Abdelhamid RF, Alioto T, Antoshechkin I, Baer MT, Bar NS, Batut P, Bell K, Bell I, Chakrabortty S, Chen X, Chrast J, Curado J, Derrien T, Drenkow J, Dumais E, Dumais J, Duttagupta R, Falconnet E, Fastuca M, Fejes-Toth K, Ferreira P, Foissac S, Fullwood MJ, Gao H, Gonzalez D, Gordon A, Gunawardena H, Howald C, Jha S, Johnson R, Kapranov P, King B, et al. 2012. Landscape of transcription in human cells. Nature 489:101108.

132. Ravasi T, Suzuki H, Pang KC, Katayama S, Furuno M, Okunishi R, Fukuda S, Ru K, Frith MC, Gongora MM, Grimmond SM, Hume DA, Hayashizaki Y, Mattick JS. 2006. Experimental validation of the regulated expression of large numbers of non-coding RNAs from the mouse genome. Genome Res 16:11-19.

133. Cabanski CR, White NM, Dang HX, Silva-Fisher JM, Rauck CE, Cicka D, Maher CA. 2015. Pan-cancer transcriptome analysis reveals long noncoding RNAs with conserved function. RNA Biol 12:628-642.

134. Johnsson P, Lipovich L, Grander D, Morris KV. 2014. Evolutionary conservation of long noncoding RNAs; sequence, structure, function. Biochim Biophys Acta 1840:1063-1071.

135. Lubelsky Y, Ulitsky I. 2018. Sequences enriched in Alu repeats drive nuclear localization of long RNAs in human cells. Nature 555:107-111. 
136. Li R, Zhu H, Luo Y. 2016. Understanding the Functions of Long Non-Coding RNAs through Their Higher-Order Structures. Int J Mol Sci 17.

137. Xue Z, Hennelly S, Doyle B, Gulati AA, Novikova IV, Sanbonmatsu KY, Boyer LA. 2016. A G-Rich Motif in the IncRNA Braveheart Interacts with a Zinc-Finger Transcription Factor to Specify the Cardiovascular Lineage. Mol Cell 64:37-50.

138. Li J, Ma W, Zeng P, Wang J, Geng B, Yang J, Cui Q. 2015. LncTar: a tool for predicting the RNA targets of long noncoding RNAs. Brief Bioinform 16:806-812.

139. Lyle R, Watanabe D, te Vruchte D, Lerchner W, Smrzka OW, Wutz A, Schageman J, Hahner L, Davies C, Barlow DP. 2000. The imprinted antisense RNA at the Igf2r locus overlaps but does not imprint Mas1. Nat Genet 25:19-21.

140. Su M, Wang H, Wang W, Wang Y, Ouyang L, Pan C, Xia L, Cao D, Liao Q. 2018. LncRNAs in DNA damage response and repair in cancer cells. Acta Biochim Biophys Sin (Shanghai) 50:433439.

141. Jiang Y, Li Y, Fang S, Jiang B, Qin C, Xie P, Zhou G, Li G. 2014. The role of MALAT1 correlates with HPV in cervical cancer. Oncol Lett 7:2135-2141.

142. Bierhoff H, Dammert MA, Brocks D, Dambacher S, Schotta G, Grummt I. 2014. Quiescenceinduced LncRNAs trigger H4K20 trimethylation and transcriptional silencing. Mol Cell 54:675682.

143. Clark MB, Johnston RL, Inostroza-Ponta M, Fox AH, Fortini E, Moscato P, Dinger ME, Mattick JS. 2012. Genome-wide analysis of long noncoding RNA stability. Genome Res 22:885898.

144. Atkinson SR, Marguerat S, Bitton DA, Rodriguez-Lopez M, Rallis C, Lemay JF, Cotobal C, Malecki M, Smialowski P, Mata J, Korber P, Bachand F, Bahler J. 2018. Long noncoding RNA repertoire and targeting by nuclear exosome, cytoplasmic exonuclease, and RNAi in fission yeast. Rna 24:1195-1213.

145. Palazzo AF, Lee ES. 2018. Sequence Determinants for Nuclear Retention and Cytoplasmic Export of mRNAs and lncRNAs. Front Genet 9:440.

146. Prasanth KV, Prasanth SG, Xuan Z, Hearn S, Freier SM, Bennett CF, Zhang MQ, Spector DL. 2005. Regulating gene expression through RNA nuclear retention. Cell 123:249-263.

147. Hutchinson JN, Ensminger AW, Clemson CM, Lynch CR, Lawrence JB, Chess A. 2007. A screen for nuclear transcripts identifies two linked noncoding RNAs associated with SC35 splicing domains. BMC Genomics 8:39.

148. Zhang K, Daigle JG, Cunningham KM, Coyne AN, Ruan K, Grima JC, Bowen KE, Wadhwa H, Yang P, Rigo F, Taylor JP, Gitler AD, Rothstein JD, Lloyd TE. 2018. Stress Granule Assembly Disrupts Nucleocytoplasmic Transport. Cell 173:958-971.e917.

149. Amaral PP, Mattick JS. 2008. Noncoding RNA in development. Mamm Genome 19:454-492.

150. Esteller M. 2011. Non-coding RNAs in human disease. Nat Rev Genet 12:861-874.

151. Feng J, Bi C, Clark BS, Mady R, Shah P, Kohtz JD. 2006. The Evf-2 noncoding RNA is transcribed from the Dlx-5/6 ultraconserved region and functions as a Dlx-2 transcriptional coactivator. Genes Dev 20:1470-1484.

152. Miao Y, Ajami NE, Huang TS, Lin FM, Lou CH, Wang YT, Li S, Kang J, Munkacsi H, Maurya MR, Gupta S, Chien S, Subramaniam S, Chen Z. 2018. Enhancer-associated long noncoding RNA LEENE regulates endothelial nitric oxide synthase and endothelial function. Nat Commun 9:292.

153. Nagano T, Mitchell JA, Sanz LA, Pauler FM, Ferguson-Smith AC, Feil R, Fraser P. 2008. The Air noncoding RNA epigenetically silences transcription by targeting G9a to chromatin. Science 322: 1717-1720.

154. Tsai MC, Manor O, Wan Y, Mosammaparast N, Wang JK, Lan F, Shi Y, Segal E, Chang HY. 2010. Long noncoding RNA as modular scaffold of histone modification complexes. Science 329:689-693. 
155. Hung T, Wang Y, Lin MF, Koegel AK, Kotake Y, Grant GD, Horlings HM, Shah N, Umbricht C, Wang P, Wang Y, Kong B, Langerod A, Borresen-Dale AL, Kim SK, van de Vijver M, Sukumar S, Whitfield ML, Kellis M, Xiong Y, Wong DJ, Chang HY. 2011. Extensive and coordinated transcription of noncoding RNAs within cell-cycle promoters. Nat Genet 43:621-629.

156. Romero-Barrios N, Legascue MF, Benhamed M, Ariel F, Crespi M. 2018. Splicing regulation by long noncoding RNAs. Nucleic Acids Res 46:2169-2184.

157. Tripathi V, Ellis JD, Shen Z, Song DY, Pan Q, Watt AT, Freier SM, Bennett CF, Sharma A, Bubulya PA, Blencowe BJ, Prasanth SG, Prasanth KV. 2010. The nuclear-retained noncoding RNA MALAT1 regulates alternative splicing by modulating SR splicing factor phosphorylation. Mol Cell 39:925-938.

158. Cao L, Zhang P, Li J, Wu M. 2017. LAST, a c-Myc-inducible long noncoding RNA, cooperates with CNBP to promote CCND1 mRNA stability in human cells. Elife 6.

159. Yoon JH, Abdelmohsen K, Srikantan S, Yang X, Martindale JL, De S, Huarte M, Zhan M, Becker KG, Gorospe M. 2012. LincRNA-p21 suppresses target mRNA translation. Mol Cell 47:648-655.

160. Militello G, Weirick T, John D, Doring C, Dimmeler S, Uchida S. 2017. Screening and validation of lncRNAs and circRNAs as miRNA sponges. Brief Bioinform 18:780-788.

161. Olgun G, Sahin O, Tastan O. 2018. Discovering lncRNA mediated sponge interactions in breast cancer molecular subtypes. BMC Genomics 19:650.

162. Agarwal V, Bell GW, Nam JW, Bartel DP. 2015. Predicting effective microRNA target sites in mammalian mRNAs. Elife 4.

163. Furio-Tari P, Tarazona S, Gabaldon T, Enright AJ, Conesa A. 2016. spongeScan: A web for detecting microRNA binding elements in lncRNA sequences. Nucleic Acids Res 44:W176-180.

164. Zhang A, Xu M, Mo YY. 2014. Role of the lncRNA-p53 regulatory network in cancer. J Mol Cell Biol 6:181-191.

165. Li XL, Subramanian M, Jones MF, Chaudhary R, Singh DK, Zong X, Gryder B, Sindri S, Mo M, Schetter A, Wen X, Parvathaneni S, Kazandjian D, Jenkins LM, Tang W, Elloumi F, Martindale JL, Huarte M, Zhu Y, Robles AI, Frier SM, Rigo F, Cam M, Ambs S, Sharma S, Harris CC, Dasso M, Prasanth KV, Lal A. 2017. Long Noncoding RNA PURPL Suppresses Basal p53 Levels and Promotes Tumorigenicity in Colorectal Cancer. Cell Rep 20:2408-2423.

166. Guo JU, Agarwal V, Guo H, Bartel DP. 2014. Expanded identification and characterization of mammalian circular RNAs. Genome Biol 15:409.

167. Conn SJ, Pillman KA, Toubia J, Conn VM, Salmanidis M, Phillips CA, Roslan S, Schreiber AW, Gregory PA, Goodall GJ. 2015. The RNA binding protein quaking regulates formation of circRNAs. Cell 160:1125-1134.

168. Li S, Teng S, Xu J, Su G, Zhang Y, Zhao J, Zhang S, Wang H, Qin W, Lu ZJ, Guo Y, Zhu Q, Wang D. 2018. Microarray is an efficient tool for circRNA profiling. Brief Bioinform doi:10.1093/bib/bby006.

169. Hansen TB, Kjems J, Damgaard CK. 2013. Circular RNA and miR-7 in cancer. Cancer Res 73:5609-5612.

170. Hansen TB, Jensen TI, Clausen BH, Bramsen JB, Finsen B, Damgaard CK, Kjems J. 2013. Natural RNA circles function as efficient microRNA sponges. Nature 495:384-388.

171. Du WW, Zhang C, Yang W, Yong T, Awan FM, Yang BB. 2017. Identifying and Characterizing circRNA-Protein Interaction. Theranostics 7:4183-4191.

172. Ashwal-Fluss R, Meyer M, Pamudurti NR, Ivanov A, Bartok O, Hanan M, Evantal N, Memczak S, Rajewsky N, Kadener S. 2014. circRNA biogenesis competes with pre-mRNA splicing. Mol Cell 56:55-66.

173. Pamudurti NR, Bartok O, Jens M, Ashwal-Fluss R, Stottmeister C, Ruhe L, Hanan M, Wyler E, Perez-Hernandez D, Ramberger E, Shenzis S, Samson M, Dittmar G, Landthaler M, Chekulaeva M, Rajewsky N, Kadener S. 2017. Translation of CircRNAs. Mol Cell 66:9-21.e27. 
174. Yang Y, Fan X, Mao M, Song X, Wu P, Zhang Y, Jin Y, Yang Y, Chen LL, Wang Y, Wong CC, Xiao X, Wang Z. 2017. Extensive translation of circular RNAs driven by N(6)methyladenosine. Cell Res 27:626-641.

175. Ungerleider N, Concha M, Lin Z, Roberts C, Wang X, Cao S, Baddoo M, Moss WN, Yu Y, Seddon M, Lehman T, Tibbetts S, Renne R, Dong Y, Flemington EK. 2018. The Epstein Barr virus circRNAome. PLoS Pathog 14:e1007206.

176. Tagawa T, Gao S, Koparde VN, Gonzalez M, Spouge JL, Serquina AP, Lurain K, Ramaswami R, Uldrick TS, Yarchoan R, Ziegelbauer JM. 2018. Discovery of Kaposi's sarcoma herpesvirus-encoded circular RNAs and a human antiviral circular RNA. Proc Natl Acad Sci U S A doi:10.1073/pnas.1816183115.

177. Zhong ZJ, Yang JX, Cao DY, Sun Y, Sun LL, Cheng XM, Chen J, Lang JH, Shen K. 2012. [Promoter methylation of DAPK1, RAR-beta and MGMT in exfoliated cervical cytology and its clinical application]. Zhonghua Fu Chan Ke Za Zhi 47:196-200.

178. Shivapurkar N, Sherman ME, Stastny V, Echebiri C, Rader JS, Nayar R, Bonfiglio TA, Gazdar AF, Wang SS. 2007. Evaluation of candidate methylation markers to detect cervical neoplasia. Gynecol Oncol 107:549-553.

179. Lim EH, Ng SL, Li JL, Chang AR, Ng J, Ilancheran A, Low J, Quek SC, Tay EH. 2010. Cervical dysplasia: assessing methylation status (Methylight) of CCNA1, DAPK1, HS3ST2, PAX1 and TFPI2 to improve diagnostic accuracy. Gynecol Oncol 119:225-231.

180. Bierkens M, Hesselink AT, Meijer CJ, Heideman DA, Wisman GB, van der Zee AG, Snijders PJ, Steenbergen RD. 2013. CADM1 and MAL promoter methylation levels in hrHPV-positive cervical scrapes increase proportional to degree and duration of underlying cervical disease. Int $\mathbf{J}$ Cancer 133:1293-1299.

181. Fang J, Zhang H, Jin S. 2014. Epigenetics and cervical cancer: from pathogenesis to therapy. Tumour Biol 35:5083-5093.

182. Parasramka MA, Maji S, Matsuda A, Yan IK, Patel T. 2016. Long non-coding RNAs as novel targets for therapy in hepatocellular carcinoma. Pharmacol Ther 161:67-78.

183. Arun G, Diermeier SD, Spector DL. 2018. Therapeutic Targeting of Long Non-Coding RNAs in Cancer. Trends Mol Med 24:257-277.

184. Gupta RA, Shah N, Wang KC, Kim J, Horlings HM, Wong DJ, Tsai MC, Hung T, Argani P, Rinn JL, Wang Y, Brzoska P, Kong B, Li R, West RB, van de Vijver MJ, Sukumar S, Chang HY. 2010. Long non-coding RNA HOTAIR reprograms chromatin state to promote cancer metastasis. Nature 464:1071-1076.

185. Mao X, Qin X, Li L, Zhou J, Zhou M, Li X, Xu Y, Yuan L, Liu QN, Xing H. 2018. A 15-long non-coding RNA signature to improve prognosis prediction of cervical squamous cell carcinoma. Gynecol Oncol doi:10.1016/j.ygyno.2017.12.011.

186. Yang JP, Yang XJ, Xiao L, Wang Y. 2016. Long noncoding RNA PVT1 as a novel serum biomarker for detection of cervical cancer. Eur Rev Med Pharmacol Sci 20:3980-3986.

187. Liang W, Lv T, Shi X, Liu H, Zhu Q, Zeng J, Yang W, Yin J, Song Y. 2016. Circulating long noncoding RNA GAS5 is a novel biomarker for the diagnosis of nonsmall cell lung cancer. Medicine (Baltimore) 95:e4608.

188. Durst M, Gissmann L, Ikenberg H, zur Hausen H. 1983. A papillomavirus DNA from a cervical carcinoma and its prevalence in cancer biopsy samples from different geographic regions. Proc Natl Acad Sci U S A 80:3812-3815.

189. Moody C. 2017. Mechanisms by which HPV Induces a Replication Competent Environment in Differentiating Keratinocytes. Viruses 9.

190. Halbert CL, Demers GW, Galloway DA. 1991. The E7 gene of human papillomavirus type 16 is sufficient for immortalization of human epithelial cells. J Virol 65:473-478.

191. Moody CA, Laimins LA. 2010. Human papillomavirus oncoproteins: pathways to transformation. Nat Rev Cancer 10:550-560. 
192. de Martel C, Plummer M, Vignat J, Franceschi S. 2017. Worldwide burden of cancer attributable to HPV by site, country and HPV type. Int J Cancer 141:664-670.

193. Harper DM, DeMars LR. 2017. HPV vaccines - A review of the first decade. Gynecol Oncol 146:196-204.

194. Tao M, Kruhlak M, Xia S, Androphy E, Zheng ZM. 2003. Signals that dictate nuclear localization of human papillomavirus type 16 oncoprotein E6 in living cells. J Virol 77:1323213247.

195. Jabbar S, Strati K, Shin MK, Pitot HC, Lambert PF. 2010. Human papillomavirus type 16 E6 and E7 oncoproteins act synergistically to cause head and neck cancer in mice. Virology 407:6067.

196. Mesplede T, Gagnon D, Bergeron-Labrecque F, Azar I, Senechal H, Coutlee F, Archambault J. 2012. p53 degradation activity, expression, and subcellular localization of E6 proteins from 29 human papillomavirus genotypes. J Virol 86:94-107.

197. Yeung CL, Tsang TY, Yau PL, Kwok TT. 2017. Human papillomavirus type 16 E6 suppresses microRNA-23b expression in human cervical cancer cells through DNA methylation of the host gene C9orf3. Oncotarget 8:12158-12173.

198. Burgers WA, Blanchon L, Pradhan S, de Launoit Y, Kouzarides T, Fuks F. 2007. Viral oncoproteins target the DNA methyltransferases. Oncogene 26:1650-1655.

199. Laurson J, Khan S, Chung R, Cross K, Raj K. 2010. Epigenetic repression of E-cadherin by human papillomavirus 16 E7 protein. Carcinogenesis 31:918-926.

200. D'Costa ZJ, Jolly C, Androphy EJ, Mercer A, Matthews CM, Hibma MH. 2012. Transcriptional repression of E-cadherin by human papillomavirus type 16 E6. PLoS One 7:e48954.

201. McCabe MT, Davis JN, Day ML. 2005. Regulation of DNA methyltransferase 1 by the pRb/E2F1 pathway. Cancer Res 65:3624-3632.

202. Scheffner M, Huibregtse JM, Vierstra RD, Howley PM. 1993. The HPV-16 E6 and E6-AP complex functions as a ubiquitin-protein ligase in the ubiquitination of p53. Cell 75:495-505.

203. Zimmermann H, Degenkolbe R, Bernard HU, O'Connor MJ. 1999. The human papillomavirus type 16 E6 oncoprotein can down-regulate p53 activity by targeting the transcriptional coactivator CBP/p300. J Virol 73:6209-6219.

204. Patel D, Huang SM, Baglia LA, McCance DJ. 1999. The E6 protein of human papillomavirus type 16 binds to and inhibits co-activation by CBP and p300. Embo j 18:5061-5072.

205. Ojesina AI, Lichtenstein L, Freeman SS, Pedamallu CS, Imaz-Rosshandler I, Pugh TJ, Cherniack AD, Ambrogio L, Cibulskis K, Bertelsen B, Romero-Cordoba S, Trevino V, Vazquez-Santillan K, Guadarrama AS, Wright AA, Rosenberg MW, Duke F, Kaplan B, Wang R, Nickerson E, Walline HM, Lawrence MS, Stewart C, Carter SL, McKenna A, Rodriguez-Sanchez IP, Espinosa-Castilla M, Woie K, Bjorge L, Wik E, Halle MK, Hoivik EA, Krakstad C, Gabino NB, Gomez-Macias GS, Valdez-Chapa LD, Garza-Rodriguez ML, Maytorena G, Vazquez J, Rodea C, Cravioto A, Cortes ML, Greulich H, Crum CP, Neuberg DS, Hidalgo-Miranda A, Escareno CR, Akslen LA, Carey TE, Vintermyr OK, et al. 2014. Landscape of genomic alterations in cervical carcinomas. Nature 506:371-375.

206. Gibb EA, Becker-Santos DD, Enfield KS, Guillaud M, Niekerk D, Matisic JP, Macaulay CE, Lam WL. 2012. Aberrant expression of long noncoding RNAs in cervical intraepithelial neoplasia. Int J Gynecol Cancer 22:1557-1563.

207. Wang H, Zhao Y, Chen M, Cui J. 2017. Identification of Novel Long Non-coding and Circular RNAs in Human Papillomavirus-Mediated Cervical Cancer. Front Microbiol 8:1720.

208. Yu D, Li Y, Ming Z, Wang H, Dong Z, Qiu L, Wang T. 2018. Comprehensive circular RNA expression profile in radiation-treated HeLa cells and analysis of radioresistance-related circRNAs. PeerJ 6:e5011.

209. Dong J, Su M, Chang W, Zhang K, Wu S, Xu T. 2017. Long non-coding RNAs on the stage of cervical cancer (Review). Oncol Rep 38:1923-1931. 
210. Aalijahan H, Ghorbian S. 2018. Long non-coding RNAs and cervical cancer. Exp Mol Pathol doi:10.1016/j.yexmp.2018.11.010.

211. Kim HJ, Lee DW, Yim GW, Nam EJ, Kim S, Kim SW, Kim YT. 2015. Long non-coding RNA HOTAIR is associated with human cervical cancer progression. Int J Oncol 46:521-530.

212. Huang L, Liao LM, Liu AW, Wu JB, Cheng XL, Lin JX, Zheng M. 2014. Overexpression of long noncoding RNA HOTAIR predicts a poor prognosis in patients with cervical cancer. Arch Gynecol Obstet 290:717-723.

213. Hajjari M, Salavaty A. 2015. HOTAIR: an oncogenic long non-coding RNA in different cancers. Cancer Biol Med 12:1-9.

214. Jing L, Yuan W, Ruofan D, Jinjin Y, Haifeng Q. 2015. HOTAIR enhanced aggressive biological behaviors and induced radio-resistance via inhibiting p21 in cervical cancer. Tumour Biol 36:36113619.

215. Gutschner T, Hammerle M, Diederichs S. 2013. MALAT1 -- a paradigm for long noncoding RNA function in cancer. J Mol Med (Berl) 91:791-801.

216. Guo F, Li Y, Liu Y, Wang J, Li Y, Li G. 2010. Inhibition of metastasis-associated lung adenocarcinoma transcript 1 in CaSki human cervical cancer cells suppresses cell proliferation and invasion. Acta Biochim Biophys Sin (Shanghai) 42:224-229.

217. Yang L, Bai HS, Deng Y, Fan L. 2015. High MALAT1 expression predicts a poor prognosis of cervical cancer and promotes cancer cell growth and invasion. Eur Rev Med Pharmacol Sci 19:3187-3193.

218. Liu S, Song L, Zeng S, Zhang L. 2016. MALAT1-miR-124-RBG2 axis is involved in growth and invasion of HR-HPV-positive cervical cancer cells. Tumour Biol 37:633-640.

219. Sun NX, Ye C, Zhao Q, Zhang Q, Xu C, Wang SB, Jin ZJ, Sun SH, Wang F, Li W. 2014. Long noncoding RNA-EBIC promotes tumor cell invasion by binding to EZH2 and repressing Ecadherin in cervical cancer. PLoS One 9:e100340.

220. Zhang D, Sun G, Zhang H, Tian J, Li Y. 2017. Long non-coding RNA ANRIL indicates a poor prognosis of cervical cancer and promotes carcinogenesis via PI3K/Akt pathways. Biomed Pharmacother 85:511-516.

221. Zhang JJ, Wang DD, Du CX, Wang Y. 2018. Long Noncoding RNA ANRIL Promotes Cervical Cancer Development by Acting as a Sponge of miR-186. Oncol Res 26:345-352.

222. Zhu H, Chen X, Hu Y, Shi Z, Zhou Q, Zheng J, Wang Y. 2017. Long non-coding RNA expression profile in cervical cancer tissues. Oncol Lett 14:1379-1386.

223. Chen J, Fu Z, Ji C, Gu P, Xu P, Yu N, Kan Y, Wu X, Shen R, Shen Y. 2015. Systematic gene microarray analysis of the lncRNA expression profiles in human uterine cervix carcinoma. Biomed Pharmacother 72:83-90.

224. Liu Y, Yang Y, Li L, Liu Y, Geng P, Li G, Song H. 2018. LncRNA SNHG1 enhances cell proliferation, migration, and invasion in cervical cancer. Biochem Cell Biol 96:38-43.

225. Huang L, Jiang X, Wang Z, Zhong X, Tai S, Cui Y. 2018. Small nucleolar RNA host gene 1: A new biomarker and therapeutic target for cancers. Pathol Res Pract 214:1247-1252.

226. Chen Y, Wang CX, Sun XX, Wang C, Liu TF, Wang DJ. 2017. Long non-coding RNA CCHE1 overexpression predicts a poor prognosis for cervical cancer. Eur Rev Med Pharmacol Sci 21:479483.

227. Yang M, Zhai X, Xia B, Wang Y, Lou G. 2015. Long noncoding RNA CCHE1 promotes cervical cancer cell proliferation via upregulating PCNA. Tumour Biol 36:7615-7622.

228. Graham LD, Pedersen SK, Brown GS, Ho T, Kassir Z, Moynihan AT, Vizgoft EK, Dunne R, Pimlott L, Young GP, Lapointe LC, Molloy PL. 2011. Colorectal Neoplasia Differentially Expressed (CRNDE), a Novel Gene with Elevated Expression in Colorectal Adenomas and Adenocarcinomas. Genes Cancer 2:829-840.

229. Wang Y, Wang Y, Li J, Zhang Y, Yin H, Han B. 2015. CRNDE, a long-noncoding RNA, promotes glioma cell growth and invasion through mTOR signaling. Cancer Lett 367:122-128. 
230. Xie H, Ma B, Gao Q, Zhan H, Liu Y, Chen Z, Ye S, Li J, Yao L, Huang W. 2018. Long noncoding RNA CRNDE in cancer prognosis: Review and meta-analysis. Clin Chim Acta 485:262271.

231. Meng Y, Li Q, Li L, Ma R. 2017. The long non-coding RNA CRNDE promotes cervical cancer cell growth and metastasis. Biol Chem 399:93-100.

232. Yang HY, Huang CP, Cao MM, Wang YF, Liu Y. 2018. Long non-coding RNA CRNDE may be associated with poor prognosis by promoting proliferation and inhibiting apoptosis of cervical cancer cells through targeting PI3K/AKT. Neoplasma doi:10.4149/neo_2018_171225N841.

233. Qin R, Chen Z, Ding Y, Hao J, Hu J, Guo F. 2013. Long non-coding RNA MEG3 inhibits the proliferation of cervical carcinoma cells through the induction of cell cycle arrest and apoptosis. Neoplasma 60:486-492.

234. Zhang J, Yao T, Wang Y, Yu J, Liu Y, Lin Z. 2016. Long noncoding RNA MEG3 is downregulated in cervical cancer and affects cell proliferation and apoptosis by regulating miR-21. Cancer Biol Ther 17:104-113.

235. Godinho MF, Wulfkuhle JD, Look MP, Sieuwerts AM, Sleijfer S, Foekens JA, Petricoin EF, 3rd, Dorssers LC, van Agthoven T. 2012. BCAR4 induces antioestrogen resistance but sensitises breast cancer to lapatinib. Br J Cancer 107:947-955.

236. Anonymous. 2017. Integrated genomic and molecular characterization of cervical cancer. Nature 543:378-384.

237. Corcoran DL, Pandit KV, Gordon B, Bhattacharjee A, Kaminski N, Benos PV. 2009. Features of mammalian microRNA promoters emerge from polymerase II chromatin immunoprecipitation data. PLoS One 4:e5279.

238. Shi Y, Lu J, Zhou J, Tan X, He Y, Ding J, Tian Y, Wang L, Wang K. 2014. Long non-coding RNA Loc554202 regulates proliferation and migration in breast cancer cells. Biochem Biophys Res Commun 446:448-453.

239. Yang L, Wei H, Xiao HJ. 2016. Long non-coding RNA Loc554202 expression as a prognostic factor in patients with colorectal cancer. Eur Rev Med Pharmacol Sci 20:4243-4247.

240. Chen J, Zhu J. 2017. Elevated Expression Levels of Long Non-Coding RNA, Loc554202, Are Predictive of Poor Prognosis in Cervical Cancer. Tohoku J Exp Med 243:165-172.

241. Shima H, Kida K, Adachi S, Yamada A, Sugae S, Narui K, Miyagi Y, Nishi M, Ryo A, Murata S, Taniguchi H, Ichikawa Y, Ishikawa T, Endo I. 2018. Lnc RNA H19 is associated with poor prognosis in breast cancer patients and promotes cancer stemness. Breast Cancer Res Treat doi:10.1007/s10549-018-4793-Z.

242. Zhu M, Chen Q, Liu X, Sun Q, Zhao X, Deng R, Wang Y, Huang J, Xu M, Yan J, Yu J. 2014. lncRNA H19/miR-675 axis represses prostate cancer metastasis by targeting TGFBI. Febs j 281:3766-3775.

243. Iempridee T. 2017. Long non-coding RNA H19 enhances cell proliferation and anchorageindependent growth of cervical cancer cell lines. Exp Biol Med (Maywood) 242:184-193.

244. Ou L, Wang D, Zhang H, Yu Q, Hua F. 2018. Decreased Expression of miR-138-5p by lncRNA H19 in Cervical Cancer Promotes Tumor Proliferation. Oncol Res 26:401-410.

245. Mourtada-Maarabouni M, Pickard MR, Hedge VL, Farzaneh F, Williams GT. 2009. GAS5, a non-protein-coding RNA, controls apoptosis and is downregulated in breast cancer. Oncogene 28:195-208.

246. Shi X, Sun M, Liu H, Yao Y, Kong R, Chen F, Song Y. 2015. A critical role for the long noncoding RNA GAS5 in proliferation and apoptosis in non-small-cell lung cancer. Mol Carcinog $\mathbf{5 4}$ Suppl 1:E1-e12.

247. Ma C, Shi X, Zhu Q, Li Q, Liu Y, Yao Y, Song Y. 2016. The growth arrest-specific transcript 5 (GAS5): a pivotal tumor suppressor long noncoding RNA in human cancers. Tumour Biol 37:14371444.

248. Cao S, Liu W, Li F, Zhao W, Qin C. 2014. Decreased expression of lncRNA GAS5 predicts a poor prognosis in cervical cancer. Int J Clin Exp Pathol 7:6776-6783. 
249. Liao LM, Sun XY, Liu AW, Wu JB, Cheng XL, Lin JX, Zheng M, Huang L. 2014. Low expression of long noncoding XLOC_010588 indicates a poor prognosis and promotes proliferation through upregulation of c-Myc in cervical cancer. Gynecol Oncol 133:616-623.

250. Jiang S, Wang HL, Yang J. 2015. Low expression of long non-coding RNA LET inhibits carcinogenesis of cervical cancer. Int J Clin Exp Pathol 8:806-811.

251. Iempridee T, Wiwithaphon S, Piboonprai K, Pratedrat P, Khumkhrong P, Japrung D, Temisak S, Laiwejpithaya S, Chaopotong P, Dharakul T. 2018. Identification of reference genes for circulating long noncoding RNA analysis in serum of cervical cancer patients. FEBS Open Bio 8:1844-1854.

252. Gao YL, Zhang MY, Xu B, Han LJ, Lan SF, Chen J, Dong YJ, Cao LL. 2017. Circular RNA expression profiles reveal that hsa_circ_0018289 is up-regulated in cervical cancer and promotes the tumorigenesis. Oncotarget 8:86625-86633.

253. Zhang J, Zhao X, Zhang J, Zheng X, Li F. 2018. Circular RNA hsa_circ_0023404 exerts an oncogenic role in cervical cancer through regulating miR-136/TFCP2/YAP pathway. Biochem Biophys Res Commun 501:428-433.

254. Ma HB, Yao YN, Yu JJ, Chen XX, Li HF. 2018. Extensive profiling of circular RNAs and the potential regulatory role of circRNA-000284 in cell proliferation and invasion of cervical cancer via sponging miR-506. Am J Transl Res 10:592-604.

255. Liu J, Wang D, Long Z, Liu J, Li W. 2018. CircRNA8924 Promotes Cervical Cancer Cell Proliferation, Migration and Invasion by Competitively Binding to MiR-518d-5p /519-5p Family and Modulating the Expression of CBX8. Cell Physiol Biochem 48:173-184.

256. Li Z, Huang C, Bao C, Chen L, Lin M, Wang X, Zhong G, Yu B, Hu W, Dai L, Zhu P, Chang Z, Wu Q, Zhao Y, Jia Y, Xu P, Liu H, Shan G. 2015. Exon-intron circular RNAs regulate transcription in the nucleus. Nat Struct Mol Biol 22:256-264.

257. Tomar S, Graves CA, Altomare D, Kowli S, Kassler S, Sutkowski N, Gillespie MB, Creek KE, Pirisi L. 2016. Human papillomavirus status and gene expression profiles of oropharyngeal and oral cancers from European American and African American patients. Head Neck 38 Suppl 1:E694-704.

258. Yang L, Yi K, Wang H, Zhao Y, Xi M. 2016. Comprehensive analysis of IncRNAs microarray profile and mRNA-lncRNA co-expression in oncogenic HPV-positive cervical cancer cell lines. Oncotarget 7:49917-49929.

259. Nohata N, Abba MC, Gutkind JS. 2016. Unraveling the oral cancer lncRNAome: Identification of novel lncRNAs associated with malignant progression and HPV infection. Oral Oncol 59:5866.

260. Gyongyosi E, Szalmas A, Ferenczi A, Poliska S, Konya J, Veress G. 2015. Transcriptional regulation of genes involved in keratinocyte differentiation by human papillomavirus 16 oncoproteins. Arch Virol 160:389-398.

261. Villota C, Campos A, Vidaurre S, Oliveira-Cruz L, Boccardo E, Burzio VA, Varas M, Villegas J, Villa LL, Valenzuela PD, Socias M, Roberts S, Burzio LO. 2012. Expression of mitochondrial non-coding RNAs (ncRNAs) is modulated by high risk human papillomavirus (HPV) oncogenes. J Biol Chem 287:21303-21315.

262. Sharma S, Munger K. 2018. Expression of the cervical carcinoma expressed PCNA regulatory (CCEPR) long noncoding RNA is driven by the human papillomavirus E6 protein and modulates cell proliferation independent of PCNA. Virology 518:8-13.

263. Iancu IV, Anton G, Botezatu A, Huica I, Nastase A, Socolov DG, Stanescu AD, Dima SO, Bacalbasa N, Plesa A. 2017. LINC01101 and LINC00277 expression levels as novel factors in HPV-induced cervical neoplasia. J Cell Mol Med 21:3787-3794.

264. Zheng SR, Zhang HR, Zhang ZF, Lai SY, Huang LJ, Liu J, Bai X, Ding K, Zhou JY. 2018. Human papillomavirus 16 E7 oncoprotein alters the expression profiles of circular RNAs in Caski cells. J Cancer 9:3755-3764. 
265. Sharma S, Mandal P, Sadhukhan T, Roy Chowdhury R, Ranjan Mondal N, Chakravarty B, Chatterjee T, Roy S, Sengupta S. 2015. Bridging Links between Long Noncoding RNA HOTAIR and HPV Oncoprotein E7 in Cervical Cancer Pathogenesis. Sci Rep 5:11724.

266. Liu S, Song L, Yao H, Zhang L, Xu D, Gao F, Li Q. 2016. MiR-375 Is Epigenetically Downregulated by HPV-16 E6 Mediated DNMT1 Upregulation and Modulates EMT of Cervical Cancer Cells by Suppressing lncRNA MALAT1. PLoS One 11:e0163460.

267. Lu H, He Y, Lin L, Qi Z, Ma L, Li L, Su Y. 2016. Long non-coding RNA MALAT1 modulates radiosensitivity of HR-HPV+ cervical cancer via sponging miR-145. Tumour Biol 37:1683-1691.

268. Jeffers LK, Duan K, Ellies LG, Seaman WT, Burger-Calderon RA, Diatchenko LB, WebsterCyriaque J. 2013. Correlation of transcription of MALAT-1, a novel noncoding RNA, with deregulated expression of tumor suppressor p53 in small DNA tumor virus models. J Cancer Ther 4.

269. White NM, Cabanski CR, Silva-Fisher JM, Dang HX, Govindan R, Maher CA. 2014. Transcriptome sequencing reveals altered long intergenic non-coding RNAs in lung cancer. Genome Biol 15:429.

270. Yang F, Lv SX, Lv L, Liu YH, Dong SY, Yao ZH, Dai XX, Zhang XH, Wang OC. 2016. Identification of IncRNA FAM83H-AS1 as a novel prognostic marker in luminal subtype breast cancer. Onco Targets Ther 9:7039-7045.

271. Yang L, Xu L, Wang Q, Wang M, An G. 2016. Dysregulation of long non-coding RNA profiles in human colorectal cancer and its association with overall survival. Oncol Lett 12:4068-4074.

272. Zhang J, Feng S, Su W, Bai S, Xiao L, Wang L, Thomas DG, Lin J, Reddy RM, Carrott PW, Lynch WR, Chang AC, Beer DG, Guo YM, Chen G. 2017. Overexpression of FAM83H-AS1 indicates poor patient survival and knockdown impairs cell proliferation and invasion via MET/EGFR signaling in lung cancer. Sci Rep 7:42819.

273. Baratieh Z, Khalaj Z, Honardoost MA, Emadi-Baygi M, Khanahmad H, Salehi M, Nikpour P. 2017. Aberrant expression of PlncRNA-1 and TUG1: potential biomarkers for gastric cancer diagnosis and clinically monitoring cancer progression. Biomark Med 11:1077-1090.

274. Arnes L, Liu Z, Wang J, Carlo Maurer H, Sagalovskiy I, Sanchez-Martin M, Bommakanti N, Garofalo DC, Balderes DA, Sussel L, Olive KP, Rabadan R. 2018. Comprehensive characterisation of compartment-specific long non-coding RNAs associated with pancreatic ductal adenocarcinoma. Gut doi:10.1136/gutjnl-2017-314353.

275. Bi YY, Shen G, Quan Y, Jiang W, Xu F. 2018. Long noncoding RNA FAM83H-AS1 exerts an oncogenic role in glioma through epigenetically silencing CDKN1A (p21). J Cell Physiol doi:10.1002/jcp.26813.

276. Lu S, Dong W, Zhao P, Liu Z. 2018. IncRNA FAM83H-AS1 is associated with the prognosis of colorectal carcinoma and promotes cell proliferation by targeting the Notch signaling pathway. Oncol Lett 15:1861-1868. 


\title{
Chapter 2
}

\section{Long non-coding RNA FAM83H-AS1 is regulated by human papillomavirus 16 E6 independently of p53 in cervical cancer cells}

\author{
Jamie A. Barr ${ }^{1}$, Karen E. Hayes ${ }^{1}$, Tayvia Brownmiller ${ }^{1}$, Abby D. Harold ${ }^{1}$, Rajaganapathi Jagannathan ${ }^{2}$, \\ Paul R. Lockman ${ }^{2}$, Saleem Khan ${ }^{3}$, and Ivan Martinez ${ }^{1, *}$ \\ ${ }^{1}$ Department of Microbiology, West Virginia University Cancer Institute, School of Medicine, West \\ Virginia University, Morgantown, West Virginia 26506; ${ }^{2}$ Department of Basic Pharmaceutical Sciences, \\ Health Sciences Center, School of Pharmacy, West Virginia University, Morgantown, West Virginia \\ 26506; ${ }^{3}$ Department of Microbiology and Molecular Genetics, School of Medicine, University of \\ Pittsburgh, Pittsburgh, Pennsylvania 15219
}

Key Words: Long non-coding RNA, Human papillomavirus, FAM83H-AS1, Cervical cancer *Correspondence: Ivan Martinez, (ivmartinez@hsc.wvu.edu)

*The authors disclose no potential conflicts of interest.

Published in: Scientific Reports

Sci Rep. 2019 Mar 6;9(1):3662. PMID: 30842470 


\section{$\underline{\text { I. Abstract }}$}

High-risk human papillomavirus (HPV) infection is one of the first events in the process of carcinogenesis in cervical and head and neck cancers. The expression of the viral oncoproteins E6 and E7 are essential in this process by inactivating the tumor suppressor proteins $\mathrm{p} 53$ and $\mathrm{Rb}$, respectively, in addition to their interactions with other host proteins. Non-coding RNAs, such as long non-coding RNAs (lncRNAs) have been found to be dysregulated in several cancers, suggesting an important role in tumorigenesis. In order to identify host lncRNAs affected by HPV infection, we expressed the high-risk HPV-16 E6 oncoprotein in primary human keratinocytes and measured the global lncRNA expression profile by high-throughput sequencing (RNA-seq). We found several host lncRNAs differentially expressed by E6 including GAS5, H19, and FAM83H-AS1. Interestingly, FAM83H-AS1 was found overexpressed in HPV-16 positive cervical cancer cell lines in an HPV-16 E6-dependent manner but independently of p53 regulation. Furthermore, FAM83H-AS1 was found to be regulated through E6-p300 pathway. Knockdown of FAM83H-AS1 by siRNAs decreased cellular proliferation, migration and increased apoptosis. FAM83H-AS1 was also found to be altered in human cervical cancer tissues and high expression of this lncRNA was associated with worse overall survival, suggesting an important role in cervical carcinogenesis.

\section{Introduction}

High-risk HPV infection (e.g. HPV-16) is one of the most common causes of cervical cancer(13), as well as a subset of head and neck squamous cell carcinoma (HNSCC)(1). The HPV oncoproteins E6 and E7 have been shown to contribute to carcinogenesis by modulating the degradation of human proteins, such as the tumor suppressors p53(4) and $\mathrm{Rb}(5)$ as well as a plethora of other cellular proteins(2, 3, 6-8). The HPV-16 E6 protein can abrogate 553 function by proteasomal degradation as it forms a complex with E6-associated protein (E6AP)(9), or by targeting the p53 coactivator CBP-p300(8, 10). Upon transmission, HPV infects the undifferentiated keratinocytes at the basal layer of the stratified epithelia and its genome remains episomal maintaining low copy numbers. During the course of cancer development, the viral genome frequently becomes integrated into the host cell DNA(11).

The recent discovery of different classes of non-coding RNAs (ncRNAs) expressed in human cells has opened a new chapter in the understanding of cellular processes, such as chromatin remodeling, transcriptional control, and post-transcriptional regulation. One of these classes of ncRNAs called long 
non-coding RNAs (lncRNAs) are defined as RNAs larger than 200 nucleotides that are not translated into proteins. Recent findings indicate that lncRNAs are involved in gene regulation at the transcriptional level by functioning as signal, guide, decoy, or scaffold RNAs(12-14). Dysregulation of lncRNAs occurs in a variety of cancers, suggesting a potential use of these ncRNAs as biomarkers for diagnosis, prognosis, stage of cancer, and response to therapy(15-18). LncRNAs have been shown to be altered in cervical cancer(19-21), however, only a few publications have studied lncRNAs that are specifically regulated by the HPV E6 oncoprotein, such as MALAT1 and $\operatorname{CCEPR}(22,23)$. These lncRNAs were found altered in cervical cancer but it is unknown if these alterations are part of the early events in cervical carcinogenesis. A few studies have looked at aberrant expression of lncRNAs in progression from pre-malignant cervical intraepithelial neoplasia (CIN) to cervical cancer $(24,25)$. For these reasons, it is important to understand if certain lncRNAs are important in the first stages of immortalization and transformation caused by HPV infections.

In this study, we demonstrated that the IncRNA FAM83H-AS1 (also known as onco-lncRNA-3) is up-regulated in primary keratinocytes expressing HPV-16 oncogene E6 as well as HPV-16 positive human cervical cancer cell lines and cervical tumor samples. We show that FAM83H-AS1 is regulated by HPV-16 E6 through the presence of p300 instead of the tumor suppressor p53. Finally, we show that FAM83H-AS1 is involved with cellular proliferation, migration, and apoptosis and is associated with worse overall survival in cervical cancer patients.

\section{Results}

\section{A. High-risk HPV-16 E6 oncoprotein alters host long non-coding RNAs in primary keratinocytes.}

As an initial screen to identify host lncRNAs that are regulated specifically by HPV-16 E6, we developed a system to look specifically at the effect of E6 expression alone in primary human foreskin keratinocytes (HEKa). HEKa were infected with a retroviral vector expressing HPV-16 E6 oncogene or GFP as a control. After puromycin selection and stable expression of HPV-16 E6 was confirmed by RTPCR (Fig. S1), RNA was extracted, and samples were analyzed by RNA high-throughput sequencing (RNA-seq) to determine gene expression alterations in long non-coding RNAs (lncRNAs). Following bioinformatics analysis, we found 151 up- and 100 down-regulated host lncRNAs altered greater than 1.5fold change when HPV-16 E6 was expressed in HEKa cells compared to GFP control (Fig. 1A, Table S1). From these host lncRNAs, we randomly chose 8 up- and 8 down-regulated host lncRNAs to validate our RNA-seq data by qRT-PCR. The expression of all the lncRNAs selected for validation followed the 
same trend of up- or down-regulation found by RNA-seq (Fig. 1B). In order to determine the importance of these 251 lncRNAs in cervical carcinogenesis, a variety of filtering methods were utilized to reduce our scope (see Methods section). After filtering, we performed preliminary experiments with many lncRNAs, however, some of our top altered lncRNAs from our RNAseq analysis of foreskin keratinocytes (HEKa) were not altered in cervical cells (HCK) with HPV-16 E6 expression (e.g. SNHG15). This is not surprising, as lncRNAs are known to typically be tissue specific(26). We also found differences in the expression of some lncRNAs between pre-malignant and cancerous cervical cells CaSki and W12/201402 to HCK (e.g. miR205HG). One of the up-regulated lncRNAs in our RNA-seq dataset, FAM83H-AS1 was intriguing. Its expression recently was found to correlate with poor overall survival in a variety of human cancers(27-32) but had not previously been shown in the context of cervical cancer. In addition, it was recently shown to be involved in regulating cellular processes associated with the hallmarks of cancer (e.g. proliferation and migration) $(28,31,32)$. To ensure that expression of FAM83H-AS1 is comparable to lncRNAs with well-known functions, we utilized our RNAseq dataset to compare their RPKM values (Table S2).

\section{B. FAM83H-AS1 expression is higher in HPV-16+ pre-malignant and cancerous samples.}

Because many lncRNAs are known to be tissue specific(26) and our RNA-seq screening was performed with foreskin keratinocytes expressing HPV-16 E6 oncogene, it was critical to confirm the expression changes of FAM83H-AS1 in epithelial keratinocytes of the cervix where HPV naturally infects(11). Additionally, we considered that the E6 and E7 oncogenes can be synergistic with each other(33), so we needed to develop a model that closely mimics HPV infection by using the entire HPV16 genome. Therefore, primary human cervical keratinocytes (HCK) were transfected with the entire HPV-16 genome (by releasing the viral genome from a plasmid construct and circularizing it by ligation before transfection), then passaged several times (around 10-15 divisions) for growth selection of HPV positive immortalized cells. HPV-16 E6 and E7 expression was confirmed by RT-PCR (Fig. S2A), as well as p53 degradation through the HPV-16 E6 pathway by Western Blot (Fig. S2B). After confirmation of HPV-16 oncogene expression in these cells, we named them JAMM-16. It was confirmed by qRT-PCR that FAM83H-AS1 is also up-regulated in JAMM-16 cervical keratinocytes expressing the entire HPV-16 genome in comparison to the parental cervical keratinocytes (Fig. 2A). We then used HPV-16 positive low-grade cervical (W12/20863 [episomal HPV-16], W12/201402 [integrated HPV-16]) and carcinoma (CaSki [integrated HPV-16]) cell lines to investigate the expression of FAM83H-AS1. As shown in Figure 2B, we found higher expression of FAM83H-AS1 in all the HPV-16 positive cell lines in comparison to HCK cells. Interestingly, FAM83H-AS1 was expressed at lower levels in HPV-negative 
cervical cancer C33A cells compared to HCK as well as HPV-16 positive cervical cells (Fig. 2B). Furthermore, HPV-16 positive and HPV-negative head and neck squamous cell carcinoma (HNSCC) cell lines were compared, and higher expression of FAM83H-AS1 was observed in the HPV-16 positive versus the HPV-negative HNSCC (Fig. 2C). Overall, we conclude that presence of HPV-16 correlates with elevated levels of FAM83H-AS1 expression in early stages of cervical carcinogenesis (newly immortalized JAMM-16 cells and cervical low-grade pre-malignant cell lines) as well as cervical cancer and HNSCC cell lines.

\section{FAM83H-AS1 expression is regulated by HPV-16 E6 in a p53-independent, p300-dependent manner.}

As shown in Figure 1, foreskin keratinocytes (HEKa) expressing HPV-16 E6 up-regulated FAM83H-AS1 expression. To further confirm HPV-16 E6 regulation of FAM83H-AS1 in cervical cells, cervical keratinocytes (HCK) stably expressing HPV-16 E6 were developed (Fig. S3). To measure if FAM83H-AS1 regulation could be affected by HPV-16 E7 oncogene in synergistic or antagonistic manner, we generated HCK stable cell lines expressing HPV-16 E7 or co-expressing HPV-16 E6 and E7 (Fig. S3). FAM83H-AS1 was up-regulated when cells expressed HPV-16 E6 or HPV-16 E6 and E7, but not when they expressed HPV-16E7 alone (Fig. 3A) suggesting a specific regulation by E6. To further confirm these findings, HPV-16 E6 was knocked down in CaSki (Fig. 3B, Fig. S4A) and W12/201402 (Fig. 3C, Fig. S4B) cell lines by two different siRNAs against HPV-16 E6-E7. FAM83H-AS1 expression was down-regulated after the reduction of HPV-16 E6 expression confirming the regulation of this lncRNA by HPV-16 E6. It is well known that one of the major HPV E6 targets is the tumor suppressor p53(34), which is involved in the regulation of cell proliferation, DNA repair, and apoptosis(35). Interestingly, when we knocked down p53 in HCK by using two different siRNAs, we observed that FAM83H-AS1 expression did not change (Fig. 3D, Fig. S4C), suggesting a regulation by HPV-16 E6 in a p53-independent manner. It is also known that HPV-16 E6 is able to regulate the expression of other important genes in carcinogenesis such as hTERT through the regulation of transcriptional coactivators such as p300(36). By using the UCSC Genome Browser (https://genome.ucsc.edu/) to investigate potential p300 binding site in the promoter region of FAM83H-AS1, we found three predicted p300 binding sites (Fig. 3E). In order to measure the potential regulation of FAM83H-AS1 by p300, we used two siRNAs against p300 and quantified FAM83H-AS1 expression. When p300 was knocked down in primary cervical keratinocytes, FAM83H-AS1 expression was also reduced (Fig. 3F, Fig. S4D) suggesting direct and/or indirect p300 regulation of FAM83H-AS1. Previous publications have shown a greater affinity of HPV-16 E6 to interact with p300 in comparison with other high-risk HPV E6 such as HPV-18 E6(37). Interestingly, FAM83H-AS1 expression was found to be up-regulated in HPV-16 
positive cell lines (Fig. 2), but down-regulated in HPV-18 positive HeLa cells and HPV-31b positive CIN-612 cells (Fig. S4E). Altogether, we found that FAM83H-AS1 is regulated by HPV-16 E6 independently of p53 but influenced by the presence of p300.

\section{FAM83H-AS1 is localized to the nucleus and does not regulate transcription of nearby FAM83H.}

The cellular localization of lncRNAs can provide information on their potential function. Nuclear lncRNAs can regulate at the transcriptional level by interacting with critical epigenetic regulators and enhancing chromatin looping, as well as interact with splicing factors to regulate splicing(38). Meanwhile cytoplasmic lncRNAs have been found to function at the post-transcriptional level as competing endogenous RNAs by acting as microRNA sponges and binding to mRNAs leading to the recruitment of RNA binding proteins that promote decay, suppress translation, or factors that initiate translation(39). For this reason, we investigated the cellular localization of FAM83H-AS1 in two HPV-16 positive cervical cell lines by cellular fractionation. We used U6 as a nuclear RNA control and $\beta$-actin mature mRNA as a cytoplasmic RNA control. We found significantly higher amplification of FAM83H-AS1 in the nuclear fractions in comparison to the cytoplasmic fractions by qRT-PCR in CaSki (Fig. 4A) and W12/201402 (Fig. 4B) cells, suggesting that FAM83H-AS1 is a nuclear lncRNA. Because many nuclear lncRNAs can act in cis(38), we hypothesized that FAM83H-AS1 could regulate its nearby protein coding gene FAM83H. The lncRNA FAM83H-AS1 and protein coding gene FAM83H share a promoter region but are transcribed in opposite directions(27) (Fig. 3E, Fig. S5). FAM83H is required for the organization of the keratin cytoskeleton in epithelial cells(40) and has been shown over-expressed in different tumor samples compared to their matching normal tissues(41). Interestingly, we found increased expression of FAM83H in HCK expressing HPV-16 E6 in comparison to parental HCK cells (Fig. S5B), but when we transfected an siRNA against FAM83H-AS1 in HPV-16 positive CaSki cells, we were unable to detect changes in FAM83H expression (Fig. S5C), suggesting that FAM83H-AS1 is not involved in regulation of FAM83H expression.

E. FAM83H-AS1 knockdown in cervical cancer cells causes reduced cellular proliferation and migration, as well as induction of apoptosis.

To understand the significance of FAM83H-AS1 in cervical cancer cells, we analyzed the effects on cellular proliferation when FAM83H-AS1 was knocked down by siRNA in CaSki and W12/201402 cervical cells. First, knockdown efficiency of a pool of 4 individual siRNAs (SMARTpool), as well as each of the individual siRNAs, was evaluated by qRT-PCR. Knockdown of FAM83H-AS1 in CaSki with 
the siRNA SMARTpool was maintained over a time-course from 24 to 120 hours, which was sufficient for all functional assays conducted (Fig. S6). All of the individual siRNAs, as well as the SMARTpool showed greater than 51\% knockdown of FAM83H-AS1 in CaSki (Fig. 5A) and greater than 49\% in W12/201402 cells (Fig. S7A). Therefore, we decided to randomly choose two of the individual siRNAs and SMARTpool to knockdown FAM83H-AS1 and monitor cellular functional changes. Two siRNAs against FAM83H-AS1 were transfected independently into CaSki and W12/201402 cells, cultured for 48 hours, and replated to measure cell proliferation by cell counting. In both CaSki and W12/201402 cells, we observed a decrease ( $\geq 48 \%$ ) in cell number with knockdown of FAM83H-AS1 compared to control (Fig. 5B, Fig. S7B). Cellular proliferation assay (CCK-8) showed that there was a decrease in cellular proliferation when FAM83H-AS1 is knocked down in CaSki cells and W12/201402 as monitored from 48 hours to 96 hours after replating. We found significantly less proliferation in CaSki (64\% decrease) and W12/201402 (73\% decrease), in the siRNA FAM83H-AS1 knockdown compared to siRNA control cells at the 96-hour time point (Fig. 5C, Fig. S7C). We observed similar functional changes between the two individual siRNAs and the SMARTpool so for future functional assays we only used the siRNA SMARTpool. In order to identify changes in cell cycle that could explain the differences found in cellular proliferation after FAM83H-AS1 knockdown, we performed cell cycle flow cytometry analysis. CaSki and W12/201402 cells had a significant reduction (43\% and 56\%, respectively) of cells in S-phase when FAM83H-AS1 was knocked down in comparison to control suggesting that FAM83H-AS1 is important in the G2/S-phase transition (Fig. 5D, Fig. S7D). Other important hallmarks of cancer such as migration and resistance to apoptosis were measured after FAM83H-AS1 knockdown. We found that cellular migration was significantly decreased in CaSki and W12/201402 after siRNA knockdown of FAM83HAS1 compared to siRNA control (Fig. 5E, Fig. S7E). Also, we measured a significant increase in early and late apoptosis in CaSki and W12/201402 cells with knockdown of FAM83H-AS1 compared to control cells by using Annexin V/PI staining and flow cytometry (Fig. 5F, Fig. S7F and S7G-H). W12/201402 cells with knockdown of FAM83H-AS1 showed a significant increase in necrosis (Fig. S7F), while only a slight increase in necrosis was observed in CaSki cells (Fig. 5F, Fig. S7H). Altogether, we observed significant alterations in cellular proliferation, cell cycle, migration, and apoptosis by the absence of FAM83H-AS1, suggesting an important role in cervical carcinogenesis.

\section{F. FAM83H-AS1 expression is increased in cervical cancer human tissues \& is associated with worse} overall survival.

In order to extrapolate our findings into a more clinically relevant setting, we extracted RNA from pre-malignant and cervical cancer patient samples and analyzed the expression of FAM83H-AS1 by 
qRT-PCR. We found high expression of FAM83H-AS1 in the pre-malignant sample (CIN3) as well as the cervical cancer $(\mathrm{CaCx})$ samples in comparison to normal cervix tissue (Fig. 6A). These findings corroborate our in vitro data suggesting an importance of FAM83H-AS1 in clinical tumor samples at different stages of carcinogenesis. We also took advantage of the cervical cancer samples deposit in the TCGA database to compare the expression of FAM83H-AS1 between normal cervix and cervical cancer samples obtained from different cancer stages (120 Stage I, 35 Stage II, 30 Stage III, 7 Stage IV, 4 Stage unavailable). The TCGA data showed elevated expression of FAM83H-AS1 (RPKM values) in cervical cancer patients compared to normal cervix control (Fig. 6B). This coincides with our previous observations of FAM83H-AS1 expression being higher in cervical cancer cells lines (Fig. 2B). Finally, we used the TCGA data set from the TANRIC database to divide cervical cancer patients into high versus low expression groups and measured overall survival based on FAM83H-AS1 expression. Interestingly, we found that patients with higher FAM83H-AS1 expression yielded a worse overall survival than patients with lower FAM83H-AS1 expression suggesting a biological importance of this lncRNA in cervical cancer reflected in patients' clinical outcomes (Fig. 6C). Overall, we conclude that FAM83HAS1 expression is elevated in cervical cancer patients and high expression correlates with overall poor survival.

\section{$\underline{\text { IV. Discussion }}$}

$4.5 \%$ of all cancers worldwide are attributable to HPV infection. Almost all cervical cancers and a substantial amount of other anogenital and oropharyngeal cancers have been found to be infected by high-risk HPVs. HPV-16 and -18 contribute to $73 \%$ of HPV-associated cancers(42), implying a higher ability to induce tumorigenesis in comparison to other types of HPVs. Although the prevalence of HPVassociated cancers has decreased due to development of the preventative vaccine and early detection screening methods(2), there is still a great need for prognostic and therapeutic options specially to people already infected with HPV as well as those affected in less developed countries where the access to the vaccine is limited.

Long noncoding RNAS (IncRNAs) have been shown to regulate a variety of critical cellular processes, including transcription and chromosome remodeling(12-14). Dysregulation of lncRNAs has been shown to be associated with the development and progression of many cancers(15-17), and interestingly they are commonly tissue specific(26) and only altered in one cancer type(32). Therefore, lncRNAs are currently being studied in the context of biomarkers for diagnosis and prognosis of cancer, as well as therapeutic targets(15-18). 
Previous studies have shown that the high-risk HPV E6 protein expressed is clearly involved in the progression of carcinogenesis. HPV E6-regulation of non-coding RNAs such as microRNAs has been well studied(43), however long non-coding RNA regulation by high-risk HPV E6 needs to be studied further; there are only a couple lncRNAs shown to be specifically HPV-16 E6 regulated, including MALAT1 and CCEPR(22, 23). To add to this field of study, we sought out to identify an HPV-16 E6 regulated gene that was altered from the early stages of HPV infection until carcinogenesis and therefore we considered to be important in both the development and progression of carcinogenesis. Thus, we developed a new HPV-16 positive cell line referred to as JAMM-16 to represent early infection, but also analyzed expression in established HPV-16 positive cervical cell lines such as CaSki and W12 cells as well as pre-malignant and malignant cervical tumor samples. We found FAM83H-AS1 overexpression in W12/20863 and W12/201402 (which came from a CIN2 tumor) similar to CaSki cells (cervical carcinoma). Additionally, in Figure 6A, the CIN3 (considered Stage 0 cervical carcinoma) patient sample shows similar expression to later stage cervical carcinoma $(\mathrm{CaCx})$ samples. For this reason, we believe that FAM83H-AS1 up-regulation in pre-malignant cervical samples could be linked to the expression of viral oncogenes in early HPV infection.

From the host lncRNAs altered by HPV-16 E6 in our RNA-seq analysis (Fig. 1), we found several of these lncRNAs previously described to be altered in cervical cancers, confirming that our data aligned with former studies. For example, it was previously found that decreased expression of GAS5 is associated with poor prognosis of cervical cancer patients $(44,45)$ as well as is tumor suppressive in other types of cancer such as breast cancer $(46,47)$ and prostate cancer(47). Furthermore, GAS5 expression was also found altered in vitro in HPV-16 positive CaSki cells(44) and HPV-18 positive HeLa cells(45). Another lncRNAs affected in our study was H19. It was shown previously that DNA methylation alterations at the IGF2/H19 imprinted domain may mediate the association between HPV and invasive cervical cancer(48) and high H19 expression has also been shown to be predictive of poor prognosis in cervical cancer(49) as well as in a variety of other human cancers, including HNSCC(50) and breast cancer(51).

A class of lncRNAs known as onco-lncRNAs were also interesting to us because, as the majority of lncRNAs are tissue specific(26), this group of lncRNAs exhibit differential expression across multiple cancers and are hypothesized to have conserved oncogenic or tumor suppressive functions. One such onco-lncRNA which was found in our RNA sequencing analysis of an E6-regulated lncRNA is oncolncRNA-3, referred to as FAM83H-AS1. This lncRNA is transcribed from chromosome 8 and its function 
in normal cells is unknown. It was first characterized in 2015(32), and as of now multiple publications have shown increased expression of FAM83H-AS1 in breast $(29,32), \operatorname{lung}(31,32)$, colorectal $(28,30,32)$, kidney(32), bladder(32), and pancreatic cancers(27) and increased expression correlates with worse overall survival in most of these cancers $(27,29-31)$. Our findings show for the first time that FAM83HAS1 is overexpressed in human cervical cancer (CESC) tissues and high expression in patients correlates with poor overall survival (Fig. 6).

According to previous studies, FAM83H-AS1 is an epithelial lncRNA(27) supporting our data obtained from foreskin and cervical keratinocytes. Determining the localization of a lncRNA can predict functionality of the lncRNA; our findings that FAM83H-AS1 is localized in the nucleus of cervical cancer cells (Fig. 4) is consistent with previous findings of its nuclear localization in lung cancer cells ${ }^{14}$. Functionally, it has been found to be co-expressed with protein coding genes that were enriched for cell cycle-related genes(32), and knockdown of FAM83H-AS1 altered cell cycle(31, 32), proliferation(28, $31)$, migration(28,31), invasion(31), and apoptosis(28) in certain cancers. Our group shows here that in the context of cervical cancer, FAM83H-AS1 is involved in cell cycle, proliferation, migration, and apoptosis (Fig. 5). It is unknown if FAM83H-AS1 elicits its functions in cis or trans, but our findings suggest that FAM83H-AS1 does not elicit cis regulation on the nearby protein coding gene FAM83H (Fig. S5), which is up-regulated in a variety of human cancers. For this reason, it will be interesting in future studies to identify the protein, RNA, and/or DNA interactions of FAM83H-AS1 in cervical cancers.

Previously, it was shown that FAM83H-AS1 regulates MET/EGFR signaling in lung cancer cells(31) and that when FAM83H-AS1 was downregulated it exhibited an anti-proliferative role by suppressing the Notch signaling pathway in colorectal cancer(28). To elucidate additional downstream targets of FAM83H-AS1, a group recently conducted RNA-seq on a pancreatic cancer cell line with siRNA knockdown of FAM83H-AS1 compared to control and identified gene alterations (78 activated and 68 inhibited targets)(27). Our group plans to determine if these downstream regulators are also involved in FAM83H-AS1 mediated functional changes observed in cervical cancer cells.

HPV-16 E6 and -18 E6 are well known to contribute to the degradation of p53, however, it is important to note that HPV-16 and HPV-18 vary in their interactions with other proteins to regulate carcinogenesis. For example, previous studies have shown that HPV-16 E6 directly interacts with CBP/p300(8, 10, 37), but HPV-18 E6 appears to be unable to interact with p300(37). This could be a possible explanation for variation in FAM83H-AS1 expression between HPV-16 and -18 positive cancers 
observed in our study (Fig. 2, Fig. S4E). Supporting our data, another publication previously showed low expression of FAM83H-AS1 in HeLa (HPV-18 positive) cells(27). This information led us to elucidate the mechanism of FAM83H-AS1 up-regulation by E6 in a p53-independent and p300-dependent manner (Fig. 3, Fig. S4). This regulation is interesting because the majority of E6 regulation of several coding and non-coding genes is primarily through the p53 pathway. Interestingly, a previous study showed that overexpression of cyclooxygenase (COX-2) gene was the result of the recruitment of p300 to its promoter region via the overexpression of HPV-16 E6 protein in CaSki cells as well as the exogenous expression of HPV-16 E6 in HPV-negative C-33A cells(52). Recently, p300 inhibitors such as C646 have been shown to be good candidates as anti-cervical cancer drugs, demonstrating the importance of p300 not only in the regulation of host genes but also of HPV viral genes(53).

In summary, the identification of FAM83H-AS1 up-regulation in the early stages of cervical carcinogenesis, correlation with overall survival in cervical cancer, and involvement in different hallmarks of cancer contributes further evidence of the importance of this lncRNA in cancer. Further studies on this lncRNA could enhance the use of FAM83H-AS1 as a potential biomarker or therapeutic target in multiple cancers.

\section{$\underline{\text { V. Methods }}$}

Detailed experimental protocols are described in the Supplementary Methods section. All experiments were performed in compliance with the Institutional Biosafety Committee at West Virginia University, number 15-03-03.

\section{A. Cells}

The following cell lines were used: human primary foreskin keratinocytes (HEKa) (Invitrogen, C005-5C); human primary cervical keratinocytes (HCK) and J2-3T3 murine fibroblast feeder cells (obtained from Dr. Alison McBride's laboratory, NIH, Bethesda, MD); 3T3M murine fibroblast feeder cells, as well as CaSki (HPV-16 positive), HeLa (HPV-18 positive), and C-33A (HPV negative) cervical carcinoma cells (obtained from Dr. Daniel DiMaio's laboratory, Yale University, New Haven, CT); W12/201402 (HPV-16 positive) and W12/20863 (HPV-16 positive) pre-malignant cervical cells (obtained from Paul F. Lambert, University of Wisconsin-Madison, Madison, WI); CIN-612 (HPV-31b positive) (obtained from Dr. Laimonis A. Laimins' laboratory, Northwestern University, Chicago, IL); UMSCC-1 (HPV negative), UMSCC-47 (HPV-16 positive), and UMSCC-104 (HPV-16 positive) head 
and neck squamous cell carcinoma (HNSCC) cell lines (obtained from Dr. Scott A. Weed's laboratory, West Virginia University, Morgantown, WV). Further details are in the Supplementary Methods section.

\section{B. High-throughput RNA sequencing}

Three replicates each of human foreskin keratinocytes (HEKa) stably expressing HPV-16 E6 or GFP were sent for RNA high-throughput sequencing (Illumina). FASTQ files were subsequently imported into Strand NGS suites for analysis. Reads were aligned to the human hg19 reference genomes using the Bowtie algorithm. These were then quantified against Ensemble transcript and including small and lncRNA annotations. Any lncRNA that were detected in human subjects were used for further analysis. Raw lncRNA counts were then normalized to the total number of lncRNA reads per sample and expression values calculated against the control samples. Further Mapping rate visualization done using Strand NGS software. In order to determine the importance of the lncRNAs obtained from the RNA-seq analysis, we used the following filtering strategy: First, only lncRNAs with reasonable expression (RPKM greater than 1) were analyzed further. Then, we used The Atlas of Noncoding RNAs in Cancer (TANRIC, MD Anderson Cancer Center)(54), which contains 297 sequenced human cervical squamous cell carcinoma and endocervical adenocarcinoma (CESC) patient data from The Cancer Genome Atlas (TCGA), to analyze the expression and clinical outcomes of these lncRNAs in patient samples. Finally, we searched previous publications to identify lncRNAs altered in other types of cancer and/or involved in hallmarks of cancer. To increase our novelty, we eliminated lncRNAs that were previously shown to be involved specifically in cervical cancer (e.g. H19).

\section{Functional Analysis}

For cell counting experiments, CaSki cells were transiently transfected with Lincode Human FAM83H-AS1 siRNA SMARTpool (Dharmacon, R-188909-00-000), Individual: Lincode FAM83H-AS1 siRNA (N-188909-02-0002 and N-188909-04-0002), or Lincode Non-targeting siRNA \#1 (Dharmacon, D-001320-01-05) using Lipofectamine ${ }^{\circledR}$ RNAiMAX according to manufacturer's instructions (Invitrogen).

CaSki cells were cultured with siRNA-containing media for 48 hours, re-plated in equal cell numbers $(200,000$ cells/well of 6-well), cultured for another 48 hours, and attached cells were re-counted with a hemacytometer. 
For all other functional assays (CCK-8 cell proliferation, FACS cell cycle, transwell migration, annexin V-FITC/PI apoptosis), CaSki cells were transiently transfected with Lincode Human FAM83HAS1 siRNA SMARTpool or Lincode Non-targeting siRNA \#1 (Dharmacon, D-001320-01-05) using Lipofectamine ${ }^{\circledR}$ RNAiMAX according to manufacturer's instructions (Invitrogen). Cells were incubated with siRNA-containing media for 24 hours then re-plated in equal cell numbers for to initiate experiments described below. To monitor cell proliferation, transfected cells were plated in 96 -well plates and after 24, 48, 72, and 96 hours analyzed with CCK-8 kit (Sigma-Aldrich) according to manufacturer's protocol. Alterations in cell cycle were determined by flow cytometry propidium iodide DNA staining. Transfected cells were plated in media containing $10 \%$ fetal bovine serum (FBS). Cells were allowed to attach and then were serum-starved for 24 hours. Samples were then fixed with ethanol, stained with propidium iodide, and analyzed by flow cytometry (Fortessa S10). For transwell migration assay, transfected cells were seeded onto upper chambers of transwell inserts ( $8 \mu \mathrm{m}$ pore size) with $20 \%$ FBS chemoattractant in the lower chamber of 24 -well plate. After 48 hours, migrated cells located on the underside of the transwell insert were stained with $0.5 \%$ crystal violet in methanol. Migrated cells were quantified using ImageJ software. To monitor apoptosis, transfected cells were plated and incubated 24, 48, and 72 hours. At desired time point, attached and floating cells were pelleted and co-stained with annexin V-FITC and propidium iodine and immediately analyzed by flow cytometry (Fortessa S10).

\section{Data Availability}

The RNA-seq raw data generated during and/or analyzed during the current study are available in the Gene Expression Omnibus (GEO-NCBI) repository, accession number: GSE115334.

\section{References}

1. Cubie HA. 2013. Diseases associated with human papillomavirus infection. Virology 445:21-34.

2. Moody CA, Laimins LA. 2010. Human papillomavirus oncoproteins: pathways to transformation. Nat Rev Cancer 10:550-560.

3. Yim EK, Park JS. 2005. The role of HPV E6 and E7 oncoproteins in HPV-associated cervical carcinogenesis. Cancer Res Treat 37:319-324.

4. Scheffner M, Werness BA, Huibregtse JM, Levine AJ, Howley PM. 1990. The E6 oncoprotein encoded by human papillomavirus types 16 and 18 promotes the degradation of p53. Cell 63:11291136.

5. Boyer SN, Wazer DE, Band V. 1996. E7 protein of human papilloma virus-16 induces degradation of retinoblastoma protein through the ubiquitin-proteasome pathway. Cancer Res 56:4620-4624.

6. Roman A, Munger K. 2013. The papillomavirus E7 proteins. Virology 445:138-168. 
7. Vande Pol SB, Klingelhutz AJ. 2013. Papillomavirus E6 oncoproteins. Virology 445:115-137.

8. Zimmermann H, Degenkolbe R, Bernard HU, O'Connor MJ. 1999. The human papillomavirus type 16 E6 oncoprotein can down-regulate p53 activity by targeting the transcriptional coactivator CBP/p300. J Virol 73:6209-6219.

9. Scheffner M, Huibregtse JM, Vierstra RD, Howley PM. 1993. The HPV-16 E6 and E6-AP complex functions as a ubiquitin-protein ligase in the ubiquitination of p53. Cell 75:495-505.

10. Patel D, Huang SM, Baglia LA, McCance DJ. 1999. The E6 protein of human papillomavirus type 16 binds to and inhibits co-activation by CBP and p300. Embo j 18:5061-5072.

11. Moody C. 2017. Mechanisms by which HPV Induces a Replication Competent Environment in Differentiating Keratinocytes. Viruses 9.

12. Long Y, Wang X, Youmans DT, Cech TR. 2017. How do lncRNAs regulate transcription? Sci Adv 3:eaao2110.

13. Marchese FP, Raimondi I, Huarte M. 2017. The multidimensional mechanisms of long noncoding RNA function. Genome Biol 18:206.

14. Wang KC, Chang HY. 2011. Molecular mechanisms of long noncoding RNAs. Mol Cell 43:904914.

15. Chen L, Dzakah EE, Shan G. 2018. Targetable long non-coding RNAs in cancer treatments. Cancer Lett 418:119-124.

16. Vitiello M, Tuccoli A, Poliseno L. 2015. Long non-coding RNAs in cancer: implications for personalized therapy. Cell Oncol (Dordr) 38:17-28.

17. Gutschner T, Diederichs S. 2012. The hallmarks of cancer: a long non-coding RNA point of view. RNA Biol 9:703-719.

18. Arun G, Diermeier SD, Spector DL. 2018. Therapeutic Targeting of Long Non-Coding RNAs in Cancer. Trends Mol Med 24:257-277.

19. Dong J, Su M, Chang W, Zhang K, Wu S, Xu T. 2017. Long non-coding RNAs on the stage of cervical cancer (Review). Oncol Rep 38:1923-1931.

20. Song J, Ye A, Jiang E, Yin X, Chen Z, Bai G, Zhou Y, Liu J. 2018. Reconstruction and analysis of the aberrant lncRNA-miRNA-mRNA network based on competitive endogenous RNA in CESC. J Cell Biochem doi:10.1002/jcb.26850.

21. Zhu H, Chen X, Hu Y, Shi Z, Zhou Q, Zheng J, Wang Y. 2017. Long non-coding RNA expression profile in cervical cancer tissues. Oncol Lett 14:1379-1386.

22. Sharma S, Munger K. 2018. Expression of the cervical carcinoma expressed PCNA regulatory (CCEPR) long noncoding RNA is driven by the human papillomavirus E6 protein and modulates cell proliferation independent of PCNA. Virology 518:8-13.

23. Liu S, Song L, Yao H, Zhang L, Xu D, Gao F, Li Q. 2016. MiR-375 Is Epigenetically Downregulated by HPV-16 E6 Mediated DNMT1 Upregulation and Modulates EMT of Cervical Cancer Cells by Suppressing lncRNA MALAT1. PLoS One 11:e0163460.

24. Gibb EA, Becker-Santos DD, Enfield KS, Guillaud M, Niekerk D, Matisic JP, Macaulay CE, Lam WL. 2012. Aberrant expression of long noncoding RNAs in cervical intraepithelial neoplasia. Int J Gynecol Cancer 22:1557-1563.

25. Iancu IV, Anton G, Botezatu A, Huica I, Nastase A, Socolov DG, Stanescu AD, Dima SO, Bacalbasa N, Plesa A. 2017. LINC01101 and LINC00277 expression levels as novel factors in HPV-induced cervical neoplasia. J Cell Mol Med 21:3787-3794.

26. Yan X, Hu Z, Feng Y, Hu X, Yuan J, Zhao SD, Zhang Y, Yang L, Shan W, He Q, Fan L, Kandalaft LE, Tanyi JL, Li C, Yuan CX, Zhang D, Yuan H, Hua K, Lu Y, Katsaros D, Huang Q, Montone K, Fan Y, Coukos G, Boyd J, Sood AK, Rebbeck T, Mills GB, Dang CV, Zhang L. 2015. Comprehensive Genomic Characterization of Long Non-coding RNAs across Human Cancers. Cancer Cell 28:529-540.

27. Arnes L, Liu Z, Wang J, Carlo Maurer H, Sagalovskiy I, Sanchez-Martin M, Bommakanti N, Garofalo DC, Balderes DA, Sussel L, Olive KP, Rabadan R. 2018. Comprehensive 
characterisation of compartment-specific long non-coding RNAs associated with pancreatic ductal adenocarcinoma. Gut doi:10.1136/gutjnl-2017-314353.

28. Lu S, Dong W, Zhao P, Liu Z. 2018. IncRNA FAM83H-AS1 is associated with the prognosis of colorectal carcinoma and promotes cell proliferation by targeting the Notch signaling pathway. Oncol Lett 15:1861-1868.

29. Yang F, Lv SX, Lv L, Liu YH, Dong SY, Yao ZH, Dai XX, Zhang XH, Wang OC. 2016. Identification of lncRNA FAM83H-AS1 as a novel prognostic marker in luminal subtype breast cancer. Onco Targets Ther 9:7039-7045.

30. Yang L, Xu L, Wang Q, Wang M, An G. 2016. Dysregulation of long non-coding RNA profiles in human colorectal cancer and its association with overall survival. Oncol Lett 12:4068-4074.

31. Zhang J, Feng S, Su W, Bai S, Xiao L, Wang L, Thomas DG, Lin J, Reddy RM, Carrott PW, Lynch WR, Chang AC, Beer DG, Guo YM, Chen G. 2017. Overexpression of FAM83H-AS1 indicates poor patient survival and knockdown impairs cell proliferation and invasion via MET/EGFR signaling in lung cancer. Sci Rep 7:42819.

32. Cabanski CR, White NM, Dang HX, Silva-Fisher JM, Rauck CE, Cicka D, Maher CA. 2015. Pan-cancer transcriptome analysis reveals long noncoding RNAs with conserved function. RNA Biol 12:628-642.

33. Jabbar S, Strati K, Shin MK, Pitot HC, Lambert PF. 2010. Human papillomavirus type 16 E6 and E7 oncoproteins act synergistically to cause head and neck cancer in mice. Virology 407:6067.

34. Munger K, Howley PM. 2002. Human papillomavirus immortalization and transformation functions. Virus Res 89:213-228.

35. Bieging KT, Mello SS, Attardi LD. 2014. Unravelling mechanisms of p53-mediated tumour suppression. Nat Rev Cancer 14:359-370.

36. James MA, Lee JH, Klingelhutz AJ. 2006. HPV16-E6 associated hTERT promoter acetylation is E6AP dependent, increased in later passage cells and enhanced by loss of p300. Int J Cancer 119:1878-1885.

37. White EA, Kramer RE, Tan MJ, Hayes SD, Harper JW, Howley PM. 2012. Comprehensive analysis of host cellular interactions with human papillomavirus E6 proteins identifies new E6 binding partners and reflects viral diversity. J Virol 86:13174-13186.

38. Yu B, Shan G. 2016. Functions of long noncoding RNAs in the nucleus. Nucleus 7:155-166.

39. Noh JH, Kim KM, McClusky WG, Abdelmohsen K, Gorospe M. 2018. Cytoplasmic functions of long noncoding RNAs. Wiley Interdiscip Rev RNA 9:e1471.

40. Kuga T, Sasaki M, Mikami T, Miake Y, Adachi J, Shimizu M, Saito Y, Koura M, Takeda Y, Matsuda J, Tomonaga T, Nakayama Y. 2016. FAM83H and casein kinase I regulate the organization of the keratin cytoskeleton and formation of desmosomes. Sci Rep 6:26557.

41. Snijders AM, Lee SY, Hang B, Hao W, Bissell MJ, Mao JH. 2017. FAM83 family oncogenes are broadly involved in human cancers: an integrative multi-omics approach. Mol Oncol 11:167179.

42. de Martel C, Plummer M, Vignat J, Franceschi S. 2017. Worldwide burden of cancer attributable to HPV by site, country and HPV type. Int J Cancer 141:664-670.

43. Martinez I, Gardiner AS, Board KF, Monzon FA, Edwards RP, Khan SA. 2008. Human papillomavirus type 16 reduces the expression of microRNA-218 in cervical carcinoma cells. Oncogene 27:2575-2582.

44. Cao S, Liu W, Li F, Zhao W, Qin C. 2014. Decreased expression of lncRNA GAS5 predicts a poor prognosis in cervical cancer. Int J Clin Exp Pathol 7:6776-6783.

45. Li Y, Wan YP, Bai Y. 2018. Correlation between long strand non-coding RNA GASS expression and prognosis of cervical cancer patients. Eur Rev Med Pharmacol Sci 22:943-949.

46. Mourtada-Maarabouni M, Pickard MR, Hedge VL, Farzaneh F, Williams GT. 2009. GAS5, a non-protein-coding RNA, controls apoptosis and is downregulated in breast cancer. Oncogene 28:195-208. 
47. Ma C, Shi X, Zhu Q, Li Q, Liu Y, Yao Y, Song Y. 2016. The growth arrest-specific transcript 5 (GAS5): a pivotal tumor suppressor long noncoding RNA in human cancers. Tumour Biol 37:14371444.

48. Vidal AC, Henry NM, Murphy SK, Oneko O, Nye M, Bartlett JA, Overcash F, Huang Z, Wang F, Mlay P, Obure J, Smith J, Vasquez B, Swai B, Hernandez B, Hoyo C. 2014. PEG1/MEST and IGF2 DNA methylation in CIN and in cervical cancer. Clin Transl Oncol 16:266272.

49. Peng L, Yuan XQ, Liu ZY, Li WL, Zhang CY, Zhang YQ, Pan X, Chen J, Li YH, Li GC. 2017. High lncRNA H19 expression as prognostic indicator: data mining in female cancers and polling analysis in non-female cancers. Oncotarget 8:1655-1667.

50. Guan GF, Zhang DJ, Wen LJ, Xin D, Liu Y, Yu DJ, Su K, Zhu L, Guo YY, Wang K. 2016. Overexpression of lncRNA H19/miR-675 promotes tumorigenesis in head and neck squamous cell carcinoma. Int J Med Sci 13:914-922.

51. Shima H, Kida K, Adachi S, Yamada A, Sugae S, Narui K, Miyagi Y, Nishi M, Ryo A, Murata S, Taniguchi H, Ichikawa Y, Ishikawa T, Endo I. 2018. Lnc RNA H19 is associated with poor prognosis in breast cancer patients and promotes cancer stemness. Breast Cancer Res Treat doi:10.1007/s10549-018-4793-z.

52. Subbaramaiah K, Dannenberg AJ. 2007. Cyclooxygenase-2 transcription is regulated by human papillomavirus 16 E6 and E7 oncoproteins: evidence of a corepressor/coactivator exchange. Cancer Res 67:3976-3985.

53. He H, Lai Y, Hao Y, Liu Y, Zhang Z, Liu X, Guo C, Zhang M, Zhou H, Wang N, Luo XG, Huo L, Ma W, Zhang TC. 2017. Selective p300 inhibitor C646 inhibited HPV E6-E7 genes, altered glucose metabolism and induced apoptosis in cervical cancer cells. Eur J Pharmacol 812:206-215.

54. Li J, Han L, Roebuck P, Diao L, Liu L, Yuan Y, Weinstein JN, Liang H. 2015. TANRIC: An Interactive Open Platform to Explore the Function of lncRNAs in Cancer. Cancer Res 75:37283737.

VIII. Acknowledgement

This work was supported in part by WVU Cancer Institute, National Cancer Institute (NCI) Plan (2V882), American Cancer Society IRG Internal Pilot Funding (IRG-09-061-04), WVCTSI Award (NIH/NIGMS Award Number U54GM104942), Tumor Microenvironment (TME) CoBRE Grant (NIH/NIGMS P20GM121322). Karen Hayes was supported in part by The Ladies Auxiliary to the VFW of the United States Cancer Research Fellowship (CK003229). Neil Infante (WVU Bioinformatics Core) assisted with the high-throughput RNA sequencing data analysis. We acknowledge Molly Lovern, a West Virginia IDeA Network of Biomedical Research Excellence (WV INBRE) undergraduate student, and WV-INBRE funding source [NIH (NIGMS) Grant \#P20GM103434] assisted in developing the JAMM-16 cell line. Sijin Wen (WVU Biostatistics Core) assisted with biostatistics on the TANRIC/TCGA data analysis. We thank Kathy Brundage in the WVU Flow Cytometry Core for assistance with FACS analysis, along with funding sources for the Fortessa S10 (OD016165) and overall core support (MBRCC CoBRE grant: GM103488). We thank Alison McBride at the NIH for kindly providing primary human cervical keratinocytes. 


\section{Author Contributions:}

J.A.B., K.E.H. and I.M. designed research; S.K. provided initial analysis and collected cervical tissue samples; J.A.B, K.E.H., T.B., A.D.H, and I.M. performed research; J.A.B, K.E.H., T.B., A.D.H, R.J. P.R.L., and I.M. analyzed data; J.A.B. and I.M. wrote the paper.

\section{$\underline{\text { X. Additional Information: }}$}

The authors declare no competing interests. 


\section{$\underline{\text { XI. Figure Legends: }}$}

Figure 1: Differential expression of host lncRNAs after expression of HPV-16 E6 in primary foreskin keratinocytes. (A) Waterfall plot of host lncRNAs altered 1.5-fold or greater in primary foreskin keratinocytes (HEKa) expressing HPV-16 E6 compared to uninfected HEKa by high-throughput RNA sequencing (RNA-seq) analysis. Triplicates for each sample were sent for RNA-seq. (B) qRT-PCR validation of representative differentially expressed host lncRNAs found by RNA-seq analysis. Red bars represent the lncRNAs up-regulated with HPV-16 E6 expression, and green bars represent the lncRNAs down-regulated with HPV-16 E6 expression. GAPDH mRNA was used to normalize the qRT-PCR analyses, which are shown relative to uninfected HEKa (grey bars).

Figure 2: Increased FAM83H-AS1 expression in primary cervical keratinocytes containing the HPV-16 genome as well as in HPV-16 positive cervical cancer and head and neck squamous cell carcinoma cell lines. (A) qRT-PCR analysis showing the increase of FAM83H-AS1 expression in newly immortalized cervical keratinocytes expressing entire HPV-16 genome (JAMM-16) compared to uninfected primary cervical keratinocytes (HCK). (B) qRT-PCR analysis showing the increase of FAM83H-AS1 expression in HPV-16 positive cervical cell lines (CaSki, W12/20863, W12/201402) and decrease of FAM83H-AS1 in HPV negative cervical cancer cell line (C-33A) compared to uninfected cervical keratinocytes (HCK). (C) qRT-PCR analysis showing the increase of FAM83H-AS1 expression in HPV-16 positive head and neck squamous cell carcinoma (HNSCC) cell lines (UMSCC-47 and UMSCC-104) compared to HPV negative HNSCC cell line (UMSCC-1). All graphs in the figure show the average of two individual experiments. Similar results were obtained in at least three independent experiments. GAPDH mRNA was used to normalize the qRT-PCR analyses. Two-tailed $t$ test results are indicted as ** $\mathrm{p} \leq 0.01$.

Figure 3: Regulation of FAM83H-AS1 expression by HPV-16 E6 in a p53-independent, p300-dependent manner. (A) FAM83H-AS1 expression by qRT-PCR analysis in cervical keratinocytes (HCK) stably individually expressing HPV-16 E6 or E7, or co-expressing E6/E7 compared to GFP control. (B-C) qRTPCR analysis of HPV-16 E6 and FAM83H-AS1 expression in HPV-16 positive CaSki (B) and W12/201402 (C) cervical cell lines transfected with an siRNA against HPV-16E6 compared to siRNA control. (D) qRT-PCR analysis of p53 and FAM83H-AS1 expression in HCK transfected with an siRNA against p53 compared to siRNA control. (E) Genome representative image showing location of FAM83H, FAM83H-AS1, and three predictive p300 binding sites in FAM83H-AS1 promoter region. (F) p300 and FAM83H-AS1 expression in HCK transfected with an siRNA against p300 compared to siRNA control. All graphs in the figure show the average of two individual experiments. Similar results were obtained in at least three independent experiments. GAPDH mRNA was used to normalize the qRT-PCR analyses. Two-tailed $t$ test results are indicted as $* \mathrm{p} \leq 0.05$ and $* * \mathrm{p} \leq 0.01$. CTRL, control.

Figure 4: FAM83H-AS1 is localized in the nucleus in cervical pre-malignant and cancerous cell lines. (A) qRT-PCR of FAM83H-AS1 expression in fractionated HPV-16 positive cervical cancer CaSki (A) and pre-malignant W12/201402 (B) cell lines. U6 small nuclear RNA (snRNA) was used as a nuclear control RNA and mature Beta Actin was used as a cytoplasmic control RNA. Representative images; similar results were obtained in at least three independent experiments. Normalization was done using $\mathrm{C}$. Elegans total RNA as an exogenous spike for the amplification of worm-specific ama-1 gene. Two-tailed $\mathrm{t}$ test results are indicted as $* * \mathrm{p} \leq 0.01$.

Figure 5: FAM83H-AS1 knockdown altered cell proliferation, migration, and apoptosis in CaSki cells. (A) Knockdown efficiency of individual and SMARTpool siRNA against FAM83H-AS1 in HPV-16 positive CaSki cell line, measured by qRT-PCR analysis. Because of variations in the expression of GAPDH after the knockdown of FAM83H-AS1, we used UBC mRNA to normalize the qRT-PCR analyses. The graph shows average of two individual experiments. (B) CaSki cells were transfected with individual siRNAs against FAM83H-AS1, siRNA SMARTpool against FAM83H-AS1, or siRNA control 
for 48 hours. Cells were then re-plated in equal numbers $(200,000$ cells/well, represented by dashed line in graph) and cultured another 48 hours prior to re-counting attached cells. Data were obtained in triplicate, and the graph shows the average of two individual experiments. (C) CaSki cells were transfected with individual siRNAs against FAM83H-AS1, siRNA SMARTpool against FAM83H-AS1, or siRNA control for 24 hours then plated in equal numbers. Transfected cells were analyzed for cellular proliferation assessment by CCK-8 assay at 48,72 , and 96 hours post-plating. The graph shows the average of two individual experiments; similar results were obtained in three independent experiments.

(D-F) CaSki cells were transfected with siRNA SMARTpool against FAM83H-AS1 or siRNA control for 24 hours then plated in equal numbers for experiments. (D) Transfected cells were analyzed for cell cycle alterations by FACS analysis. CaSki cells with knockdown of FAM83H-AS1 exhibit less cells in S-phase of cell cycle compared to control cells. The graph shows the average of two individual experiments; similar results were obtained in three independent experiments. (E) Transwell migration of transfected cells was analyzed 48 hours post-plating in upper chamber with chemoattractant in lower chamber. The graph shows the average of three individual experiments. (F) Transfected CaSki cells were collected at 1, 2, and 3 days post-plating, stained with Annexin V/PI, and analyzed by flow cytometry to show alterations in apoptosis compared to siRNA control. The graph shows the average of three individual experiments. Two-tailed t test results are indicted as $* \mathrm{p} \leq 0.05$ and $* * \mathrm{p} \leq 0.01$.

Figure 6: FAM83H-AS1 expression is increased in human cervical cancer tissues and correlates with poor overall survival. (A) Increased FAM83H-AS1 expression in human cervical cancer and cervical intraepithelial neoplasia (CIN) stage 3 patient samples compared to non-cancerous cervical tissue as measured by qRT-PCR analysis. (B) The Cancer Genome Atlas (TCGA) analysis of FAM83H-AS1 RPKM values in cervical cancer tissues $(n=196)$ compared to non-cancerous tissues $(n=3)$. (C) Survival plot of cervical squamous cell carcinoma and endocervical adenocarcinoma (CESC) patient data mined from TCGA with low $(n=22)$ vs. high $(n=174)$ expression of FAM83H-AS1. High expression of FAM83H-AS1 expression correlates with worse overall survival in CESC patients. 
Figure 1:

A

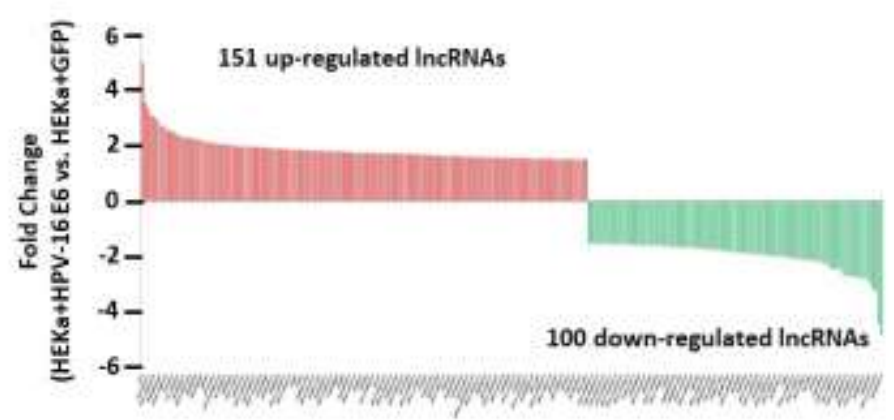

B

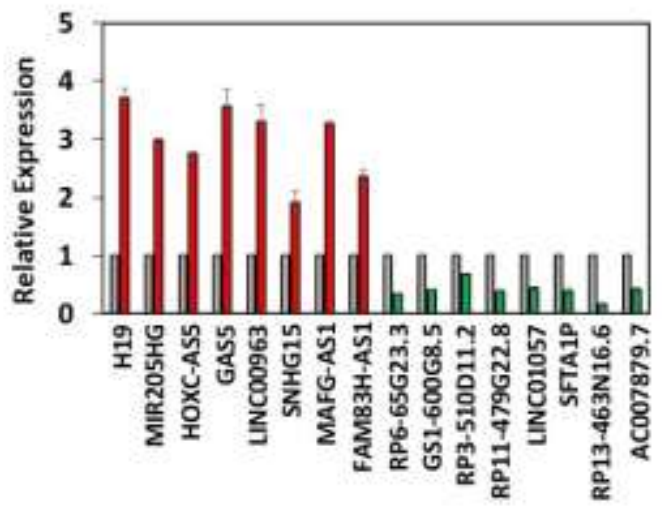

C

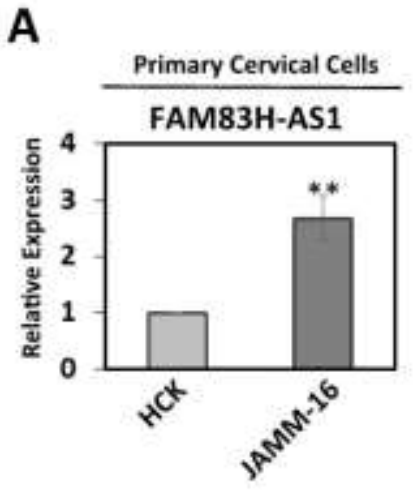

B

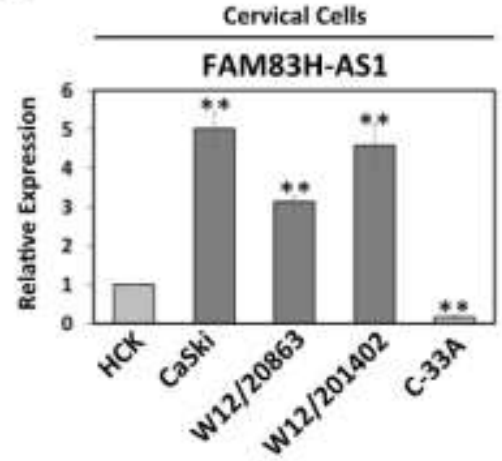

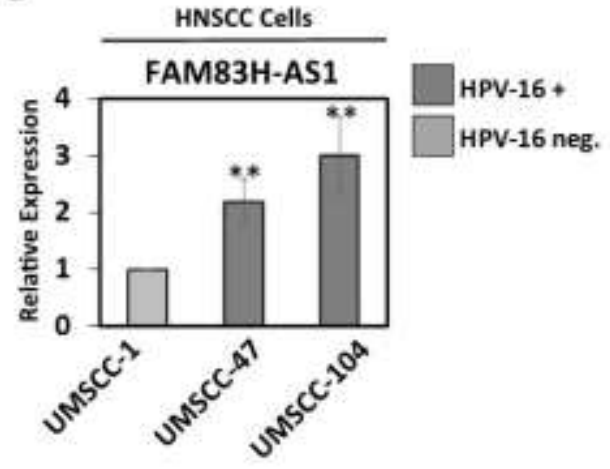


Figure 3:

A

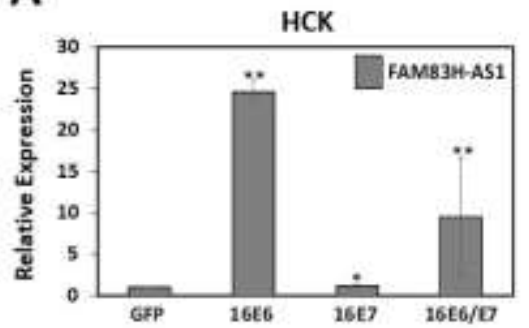

D

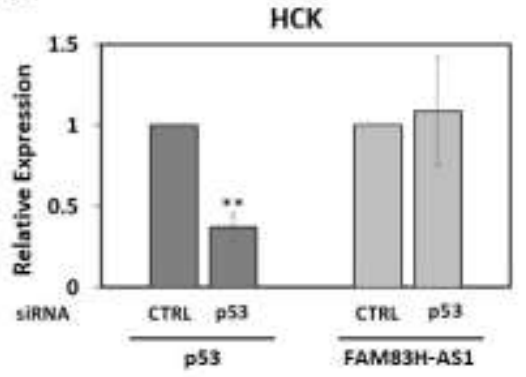

B

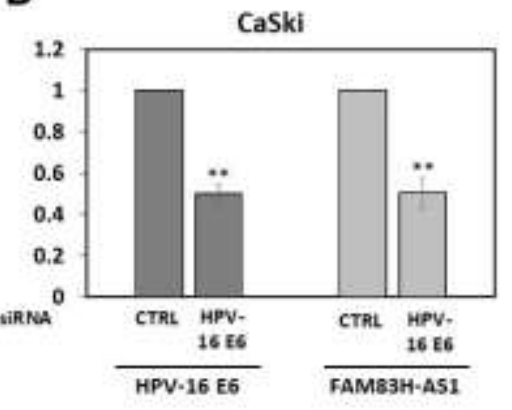

C

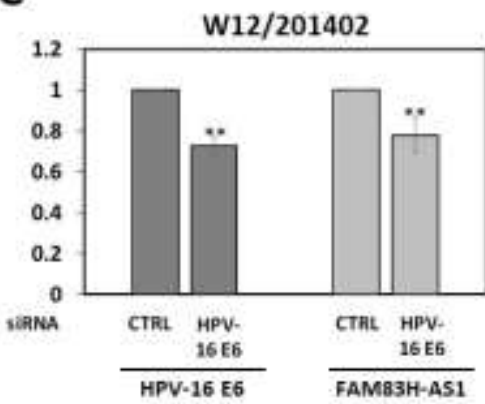

E

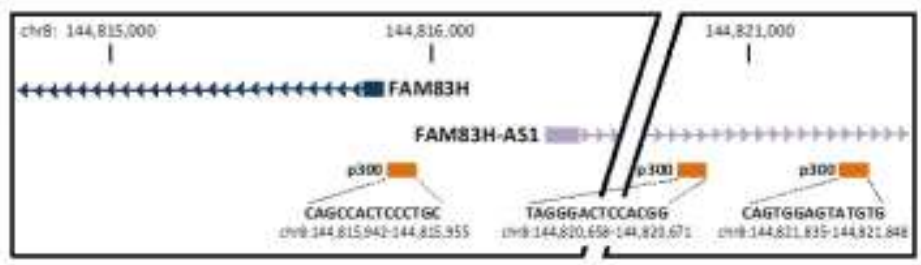

F

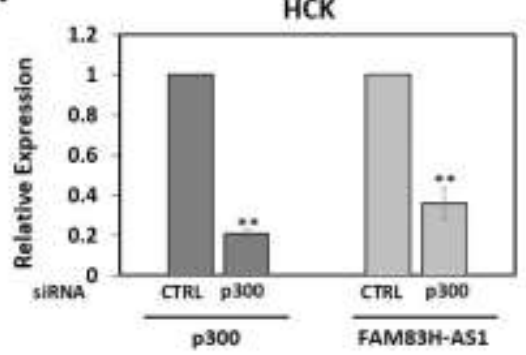


Figure 4:

A

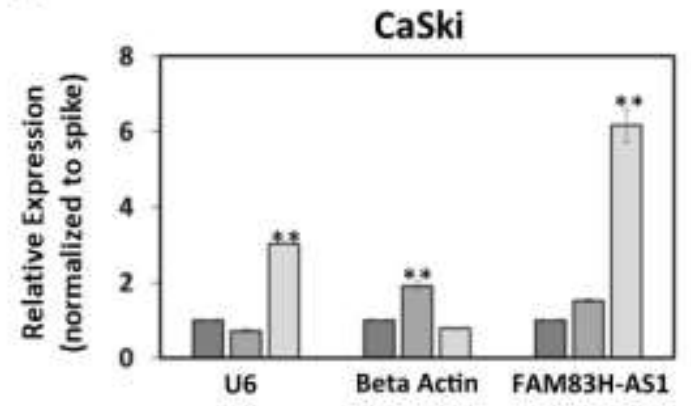

B

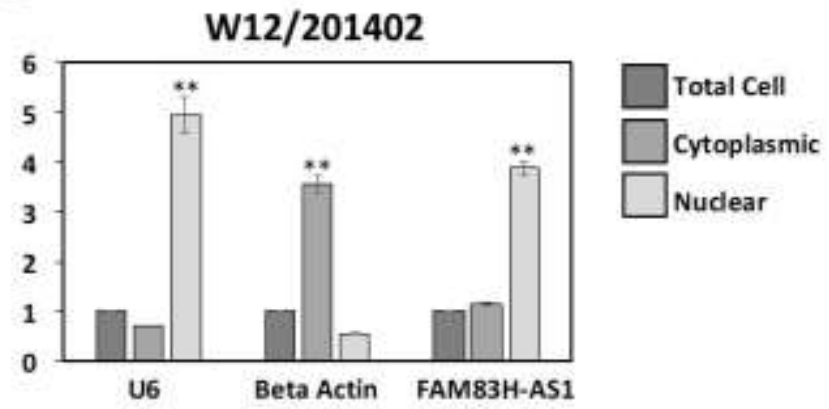

Figure 5:
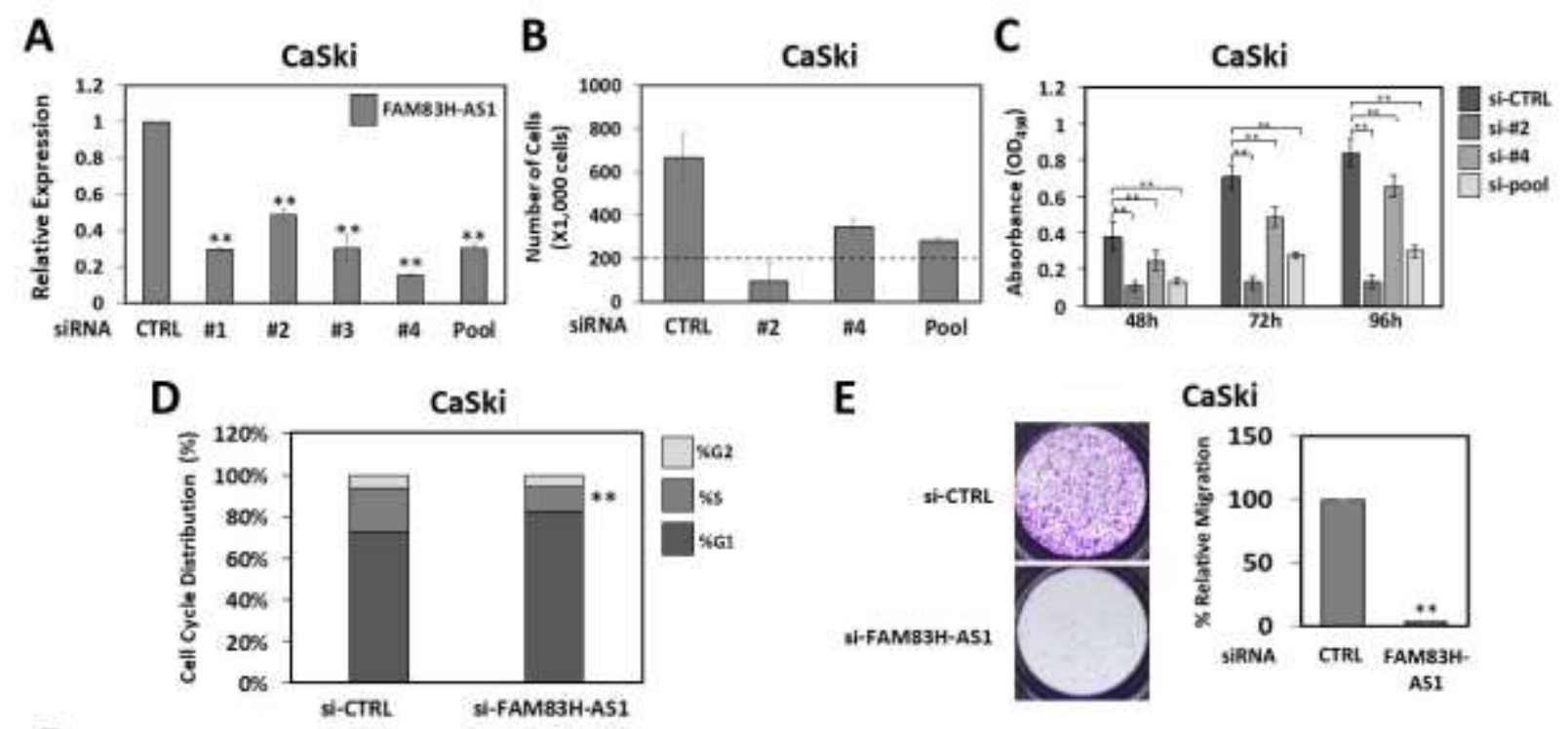

F

Early Apoptosis

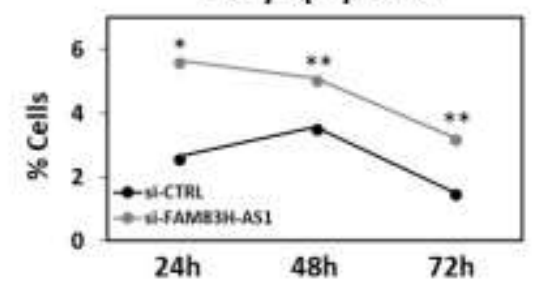

Late Apoptosis

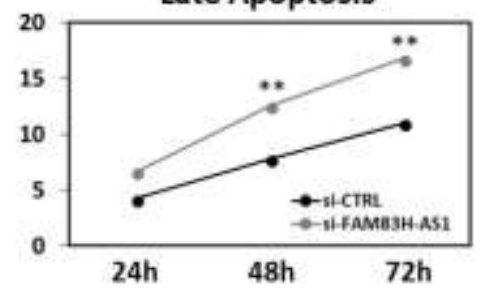

Necrosis

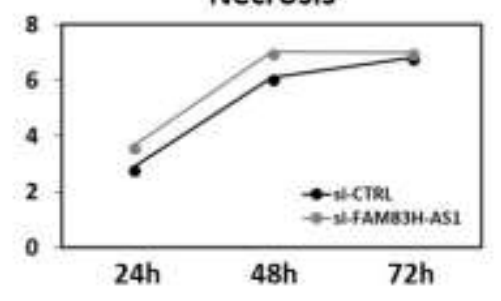


Figure 6:

A

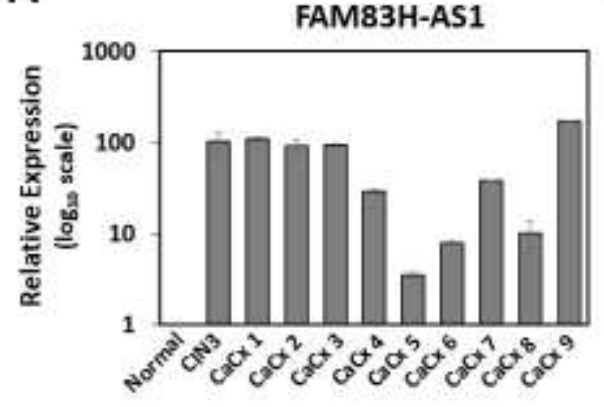

B

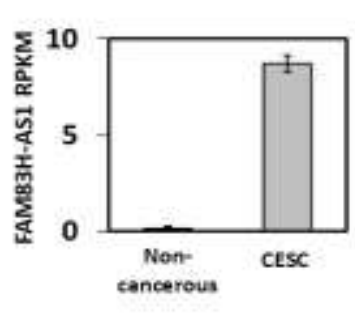

C

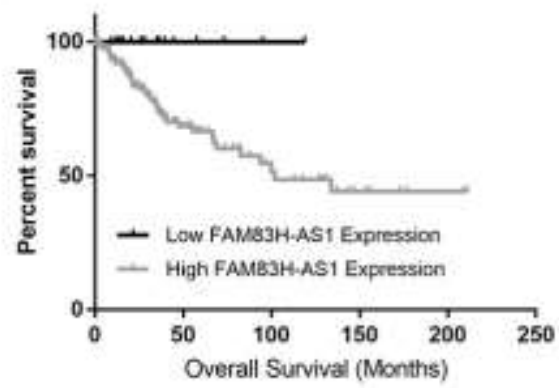


$\underline{\text { XI. Supplementary Figures }}$

\section{Supplementary Figure S1}

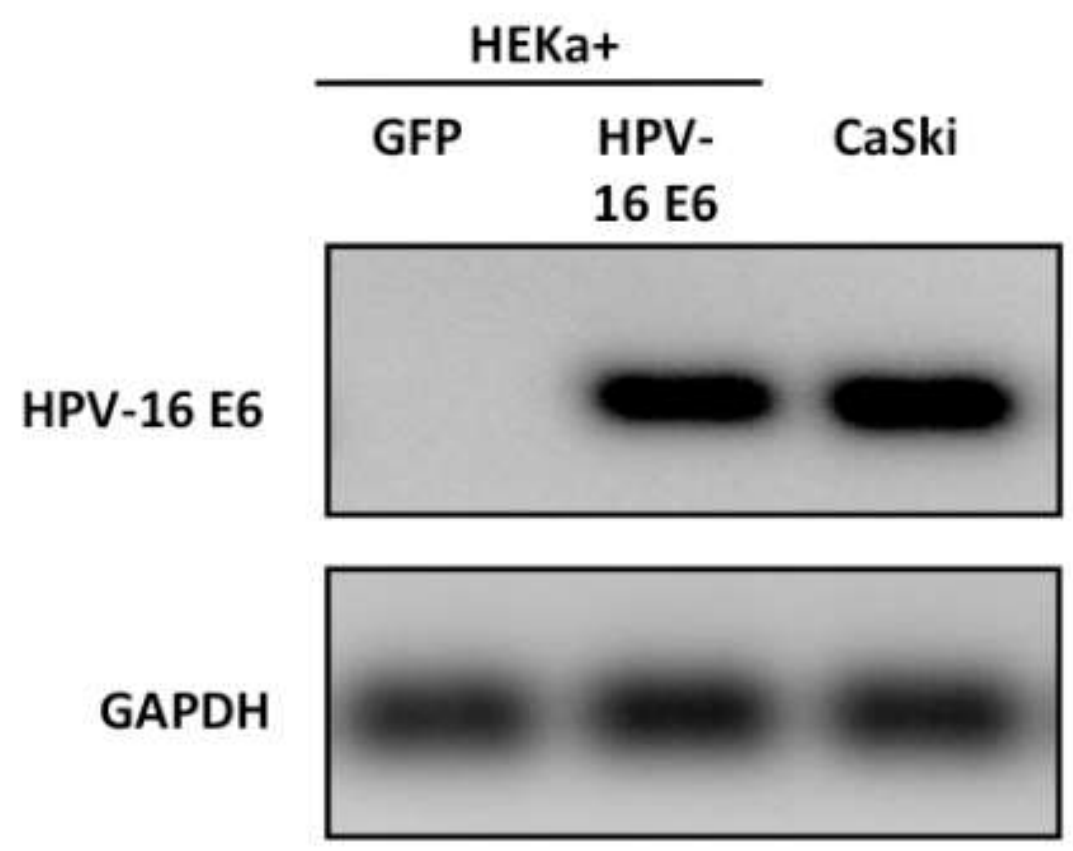

Figure S1: Validation of stable HPV-16 E6 expression in primary foreskin

keratinocytes. RT-PCR was used to confirm stable HPV-16 E6 expression in primary foreskin keratinocytes (HEKa) compared to HEKa expressing GFP control and HPV-16 positive CaSki cell line. 


\section{Supplementary Figure S2}

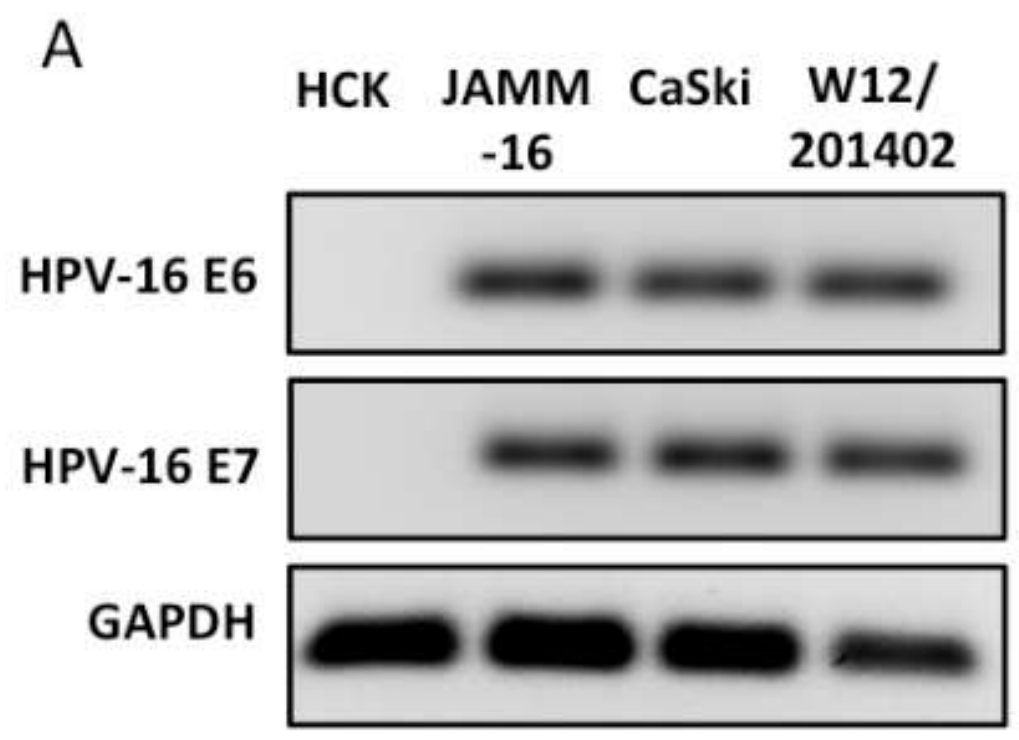

B

HCK JAMM N/A CaSki W12/ W12/ C-33A $-16$ 20863201402

p53

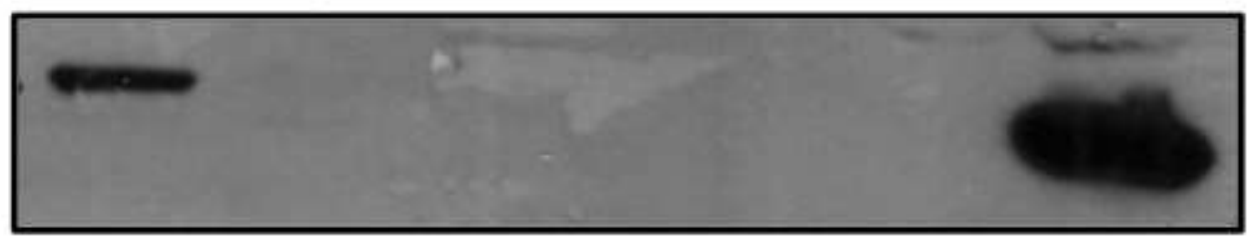

$\beta$-Actin

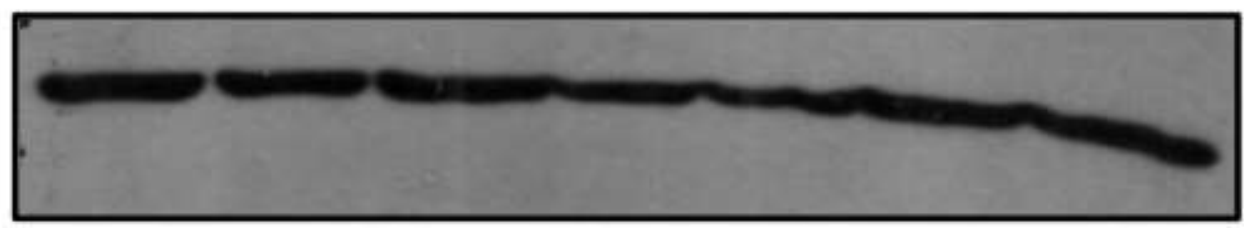

Figure S2: Validation of HPV-16 expression in primary cervical keratinocytes. RTPCR was used to confirm HPV-16 E6 and E7 expression in cervical keratinocytes transfected with entire HPV-16 genome (JAMM-16) compared to uninfected primary cervical keratinocytes (HCK) and HPV-16 positive CaSki and W12/201402 cell lines. GAPDH was used as loading control. (B) Western blot analysis was used to confirm p53 degradation in HPV-16 positive JAMM-16, CaSki, W12/20863, W12/201402 cell lines compared to HCK and HPV negative, p53-mutated C-33A cell line. Note: N/A represents a cervical cell line that was not used in this study. 


\section{Supplementary Figure S3}

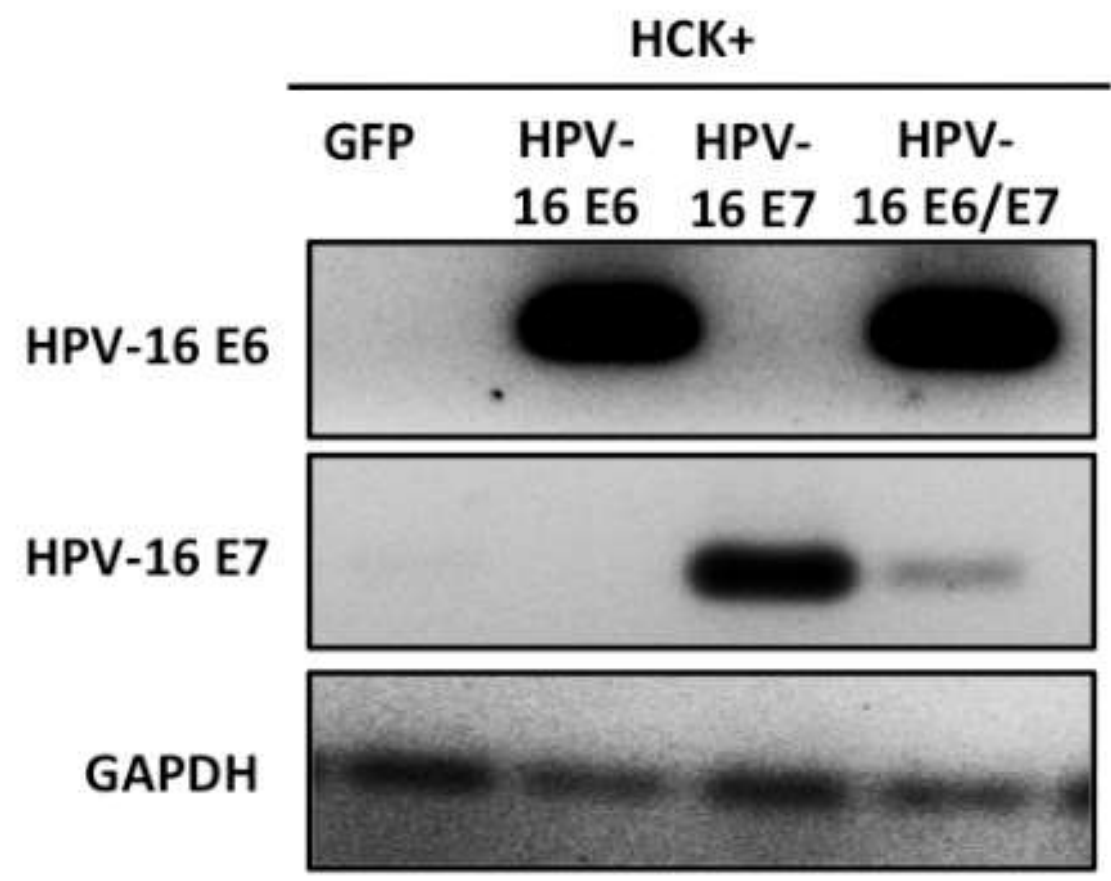

Figure S3: Validation of stable HPV-16 E6 and E7 expression in primary cervical keratinocytes. RT-PCR was used to confirm stable HPV-16 E6 and E7 expression, as desired, in primary cervical keratinocytes (HCK) compared to HCK expressing GFP control. 
Supplementary Figure S4
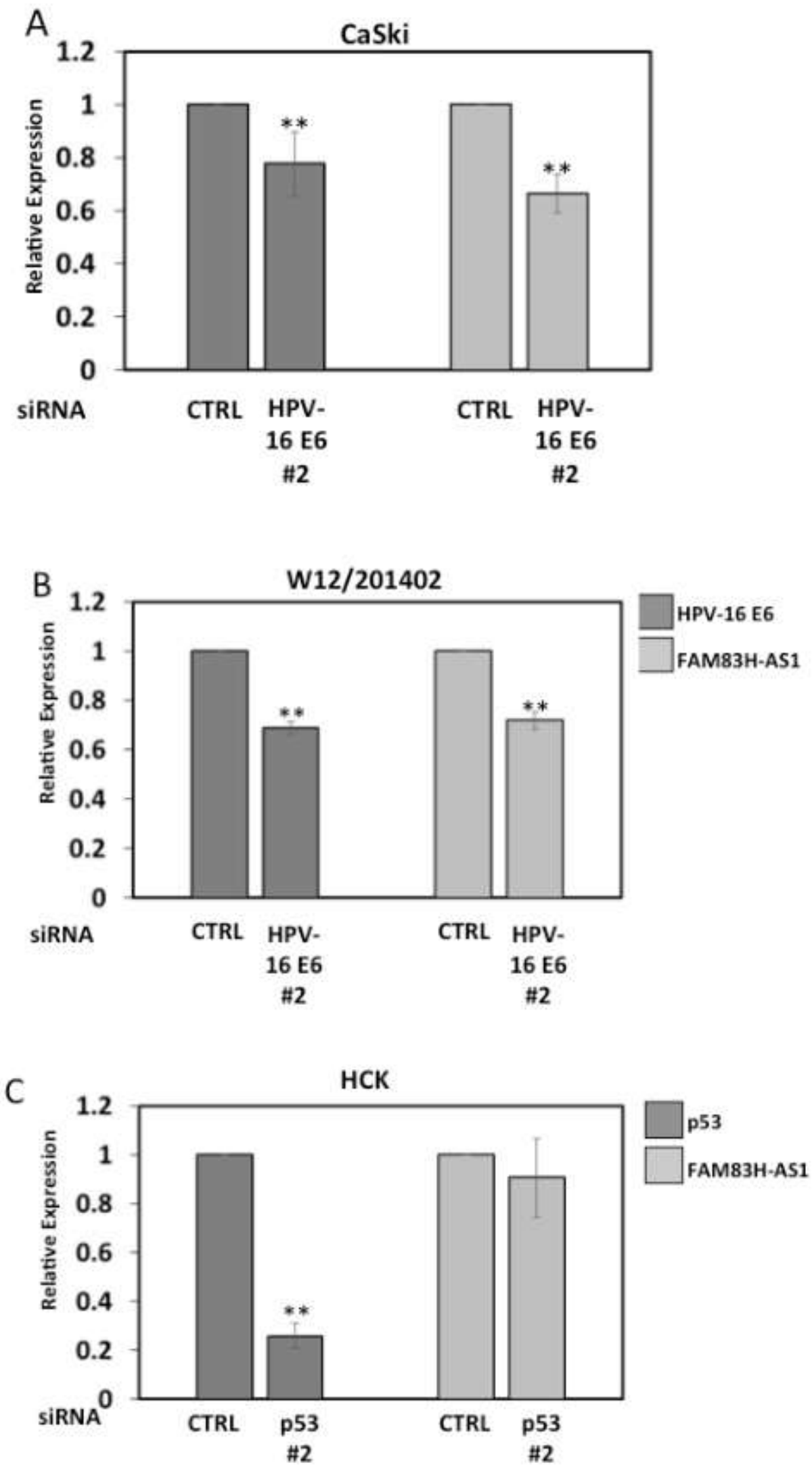

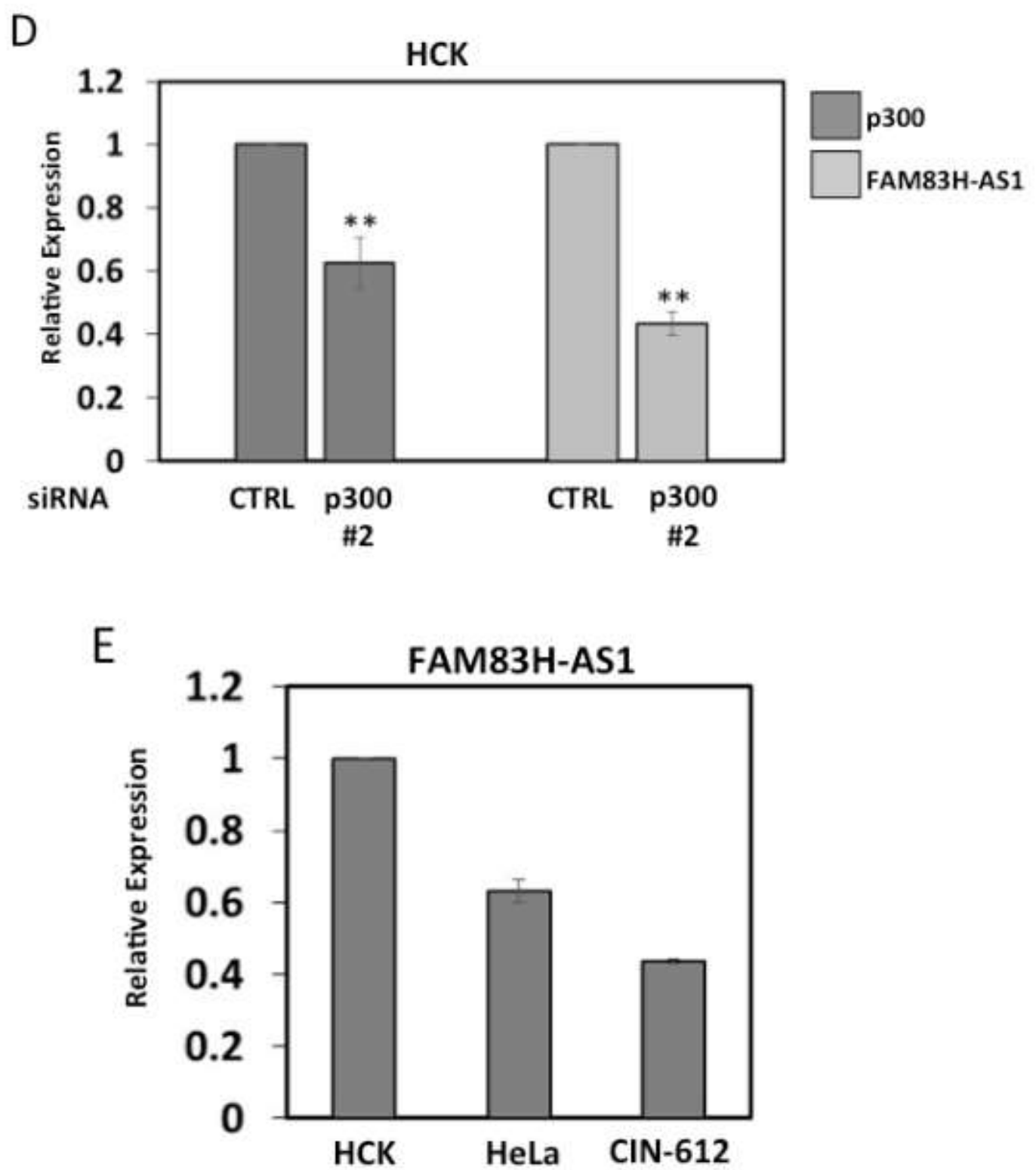

Figure S4: FAM83H-AS1 expression is regulated by HPV-16 E6 in a p53-independent, p300dependent manner. (A-B) qRT-PCR analysis of HPV-16 E6 and FAM83H-AS1 expression in HPV-16 positive Caski (A) and W12/201402 (B) cell lines transfected with a second siRNA (siRNA \#2) against HPV-16 E6 compared to siRNA control. (C) qRT-PCR analysis of p53 and FAM83H-AS1 expression in primary cervical keratinocytes (HCK) transfected with a different siRNA (siRNA \#2) against p53 compared to siRNA control. (D) p300 and FAM83H-AS1 expression in HCK transfected with a different siRNA (siRNA \#2) against p300 compared to siRNA control. (E) qRT-PCR analysis of FAM83H-AS1 expression in HPV-18 positive HeLa cells and HPV-31b positive CIN-612 cervical cell lines compared to primary cervical keratinocytes (HCK). (A-D) The graphs show the average of two independent experiments. Similar results were obtained in at least three independent experiments. GAPDH mRNA was used to normalize the qRT-PCR analyses. Two-tailed t test results are indicted as ${ }^{* *} p \leq 0.01$. CTRL, control. 


\section{Supplementary Figure S5}

A

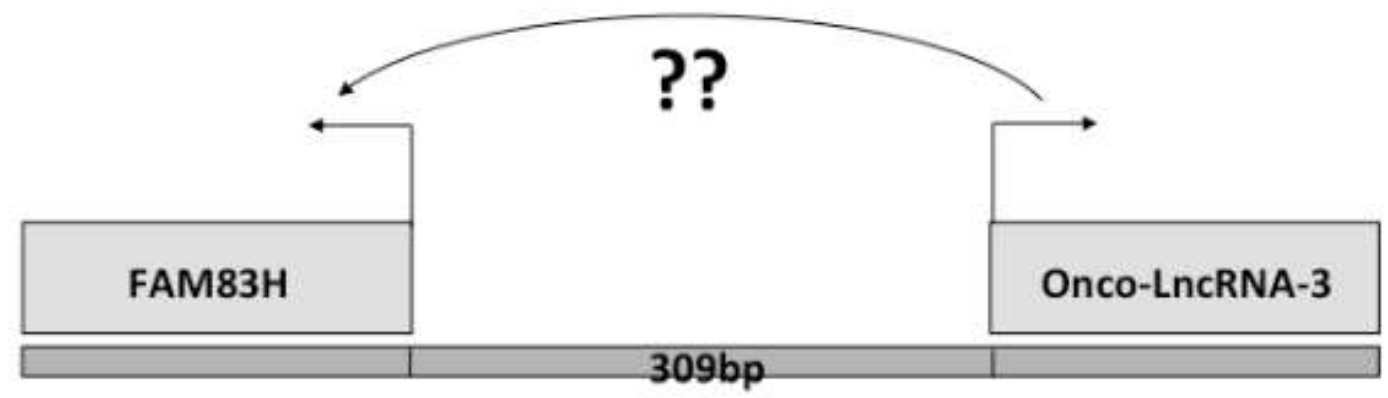

Chr8:
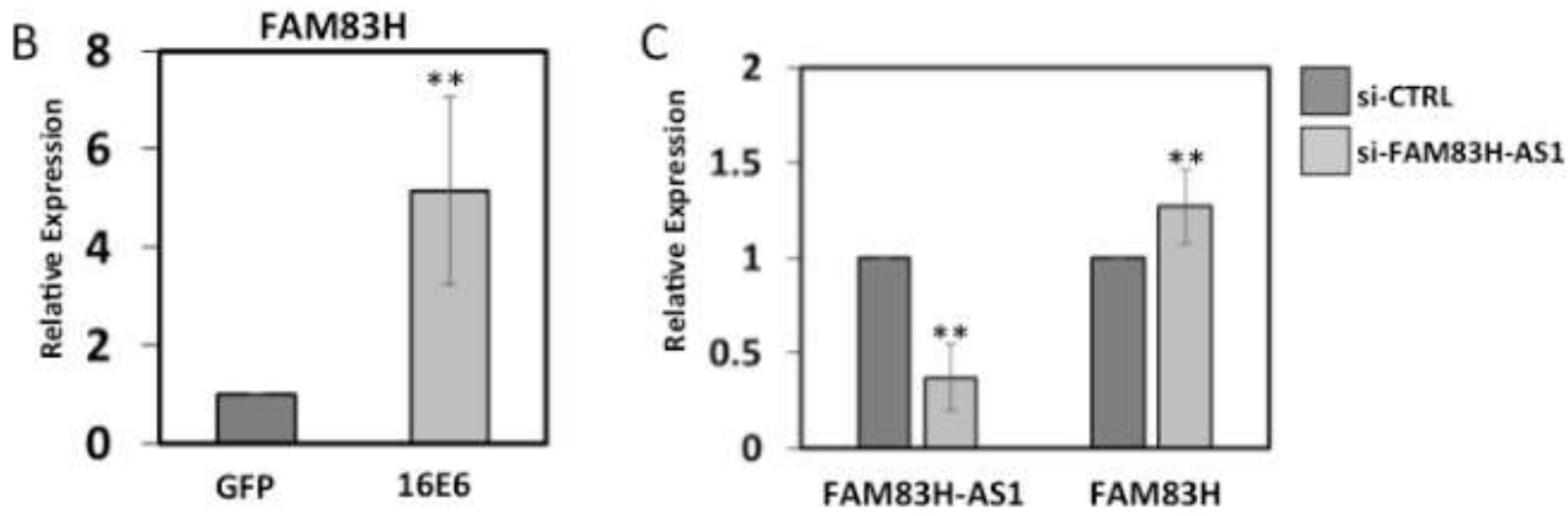

Figure S5: FAM83H expression is not regulated by FAM83H-AS1. (A) Schematic diagram depicting possible cis-regulation of protein coding gene FAM $83 \mathrm{H}$ by IncRNA FAM83H-AS1. (B) qRT-PCR analysis of FAM83H protein coding gene expression in primary cervical keratinocytes (HCK) stably expressing HPV-16 E6 compared to GFP control. (C) qRT-PCR analysis of FAM83H and FAM83H-AS1 expression in CaSki transfected with siRNA against FAM83H-AS1. The graphs shows the average of two independent experiments. Similar results were obtained in at least three independent experiments. GAPDH mRNA was used to normalize the qRT-PCR analyses. Two-tailed $t$ test results are indicted as ${ }^{* *} p \leq 0.01$. CTRL, control. 
Supplementary Figure S6

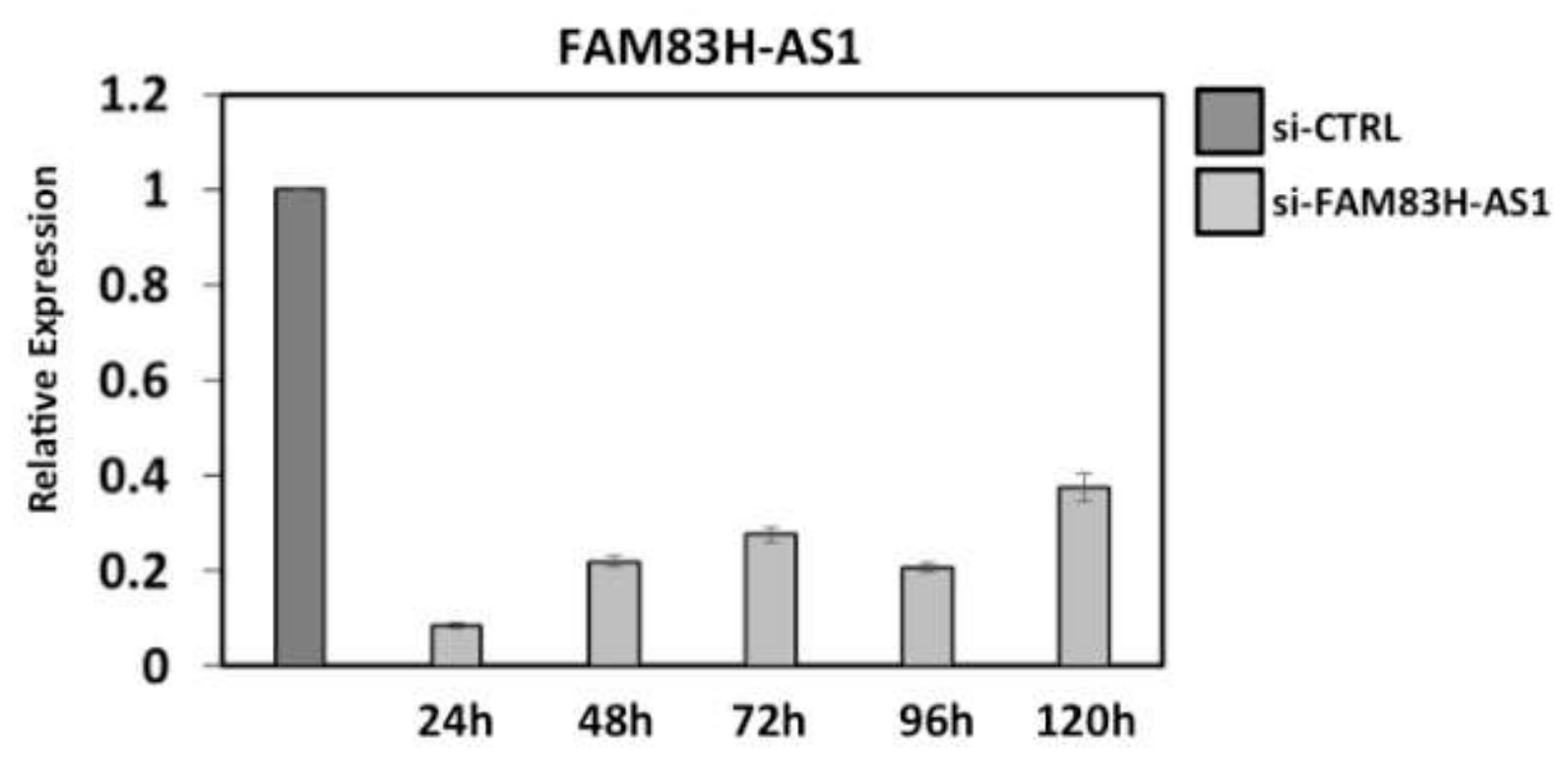

Figure S6: FAM83H-AS1 expression at 24, 48, 72, 96, and 120 hours post-transfection with FAM83H-AS1 siRNA SMARTpool by qRT-PCR analysis. 


\section{Supplementary Figure S7}

A

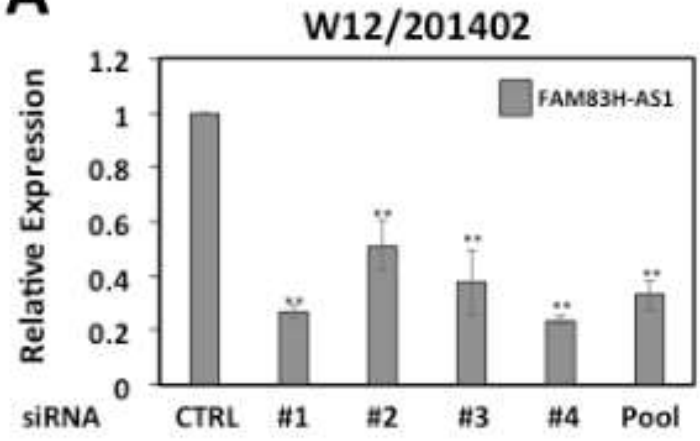

C

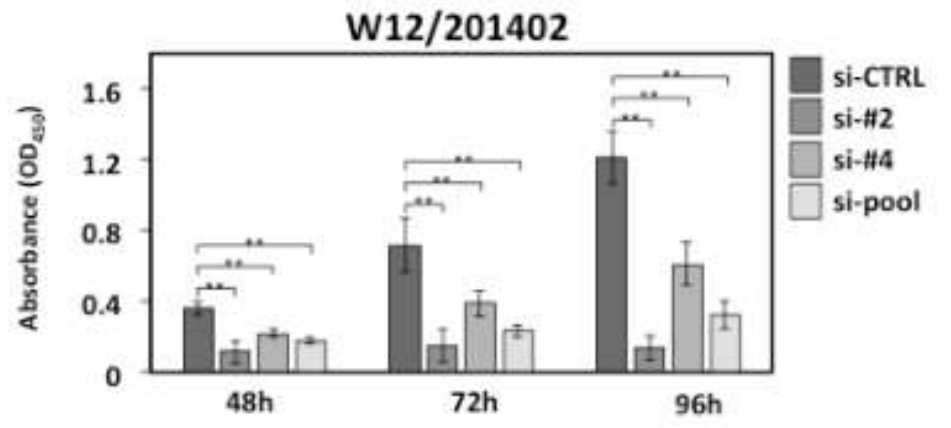

B

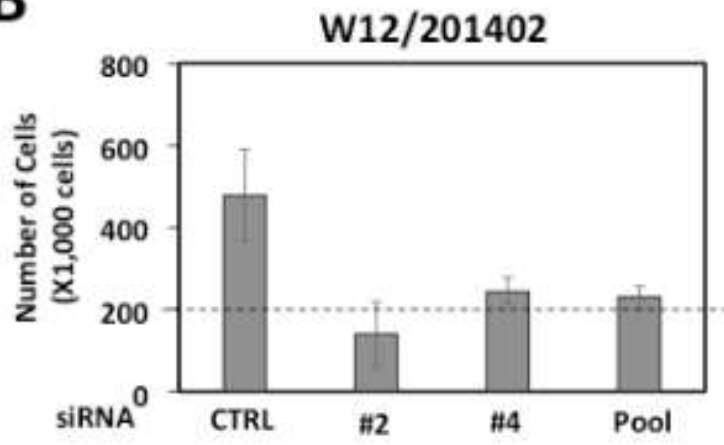

D

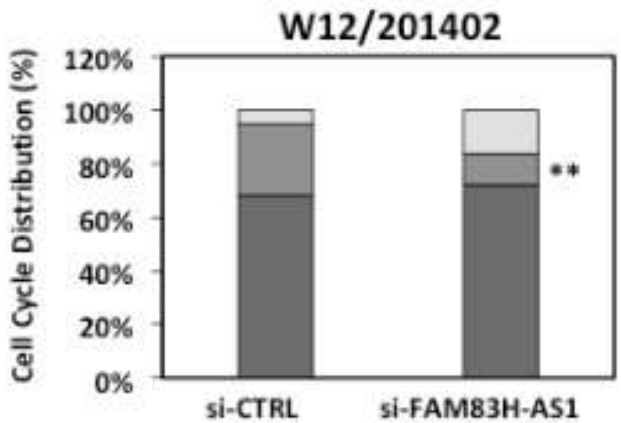

E

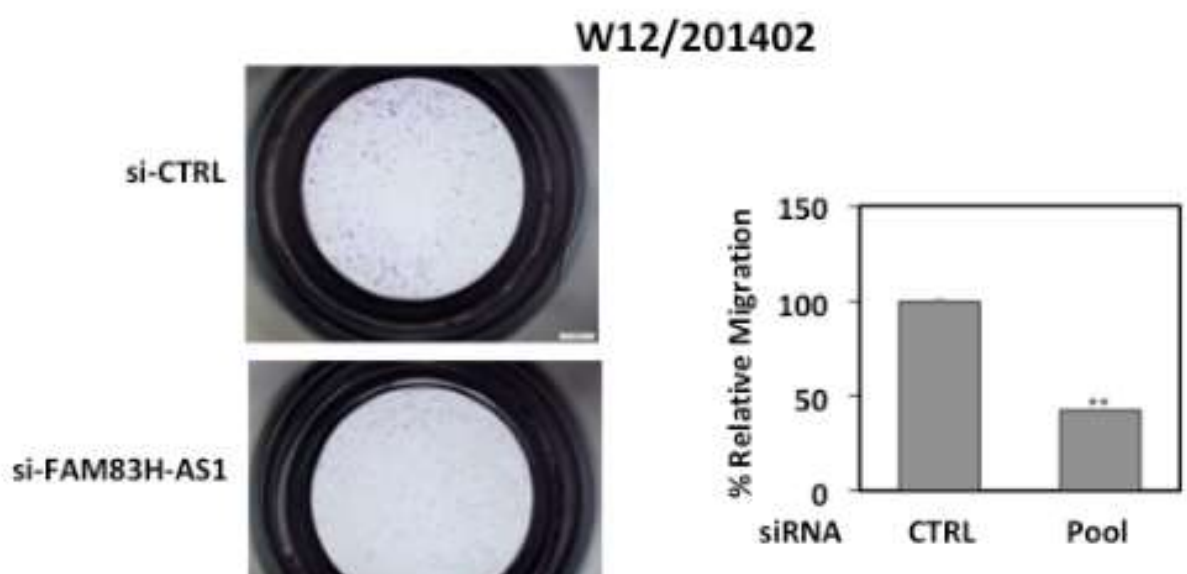

Feeders Alone 
F

\section{W12/201402}
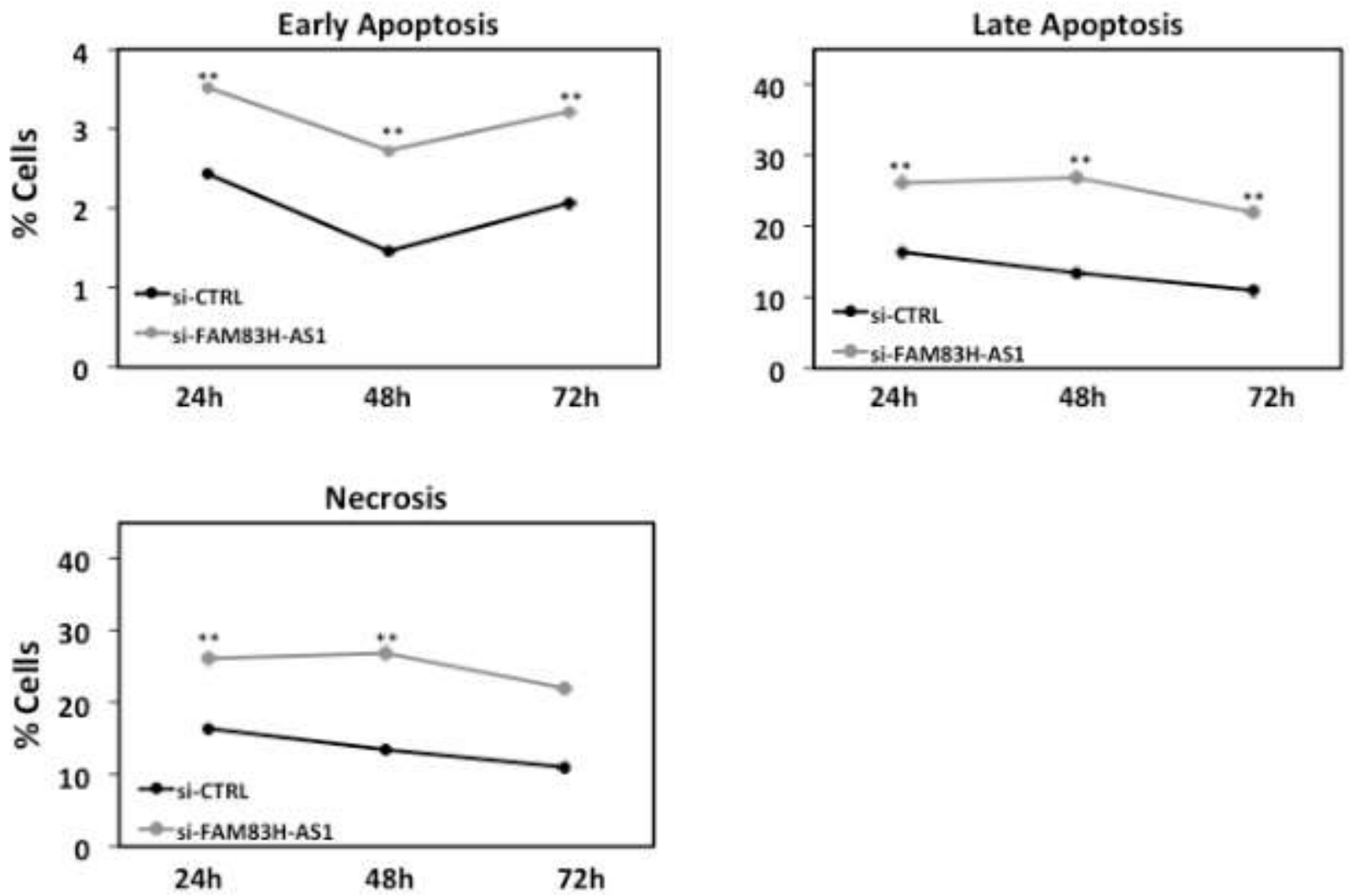


\section{2}
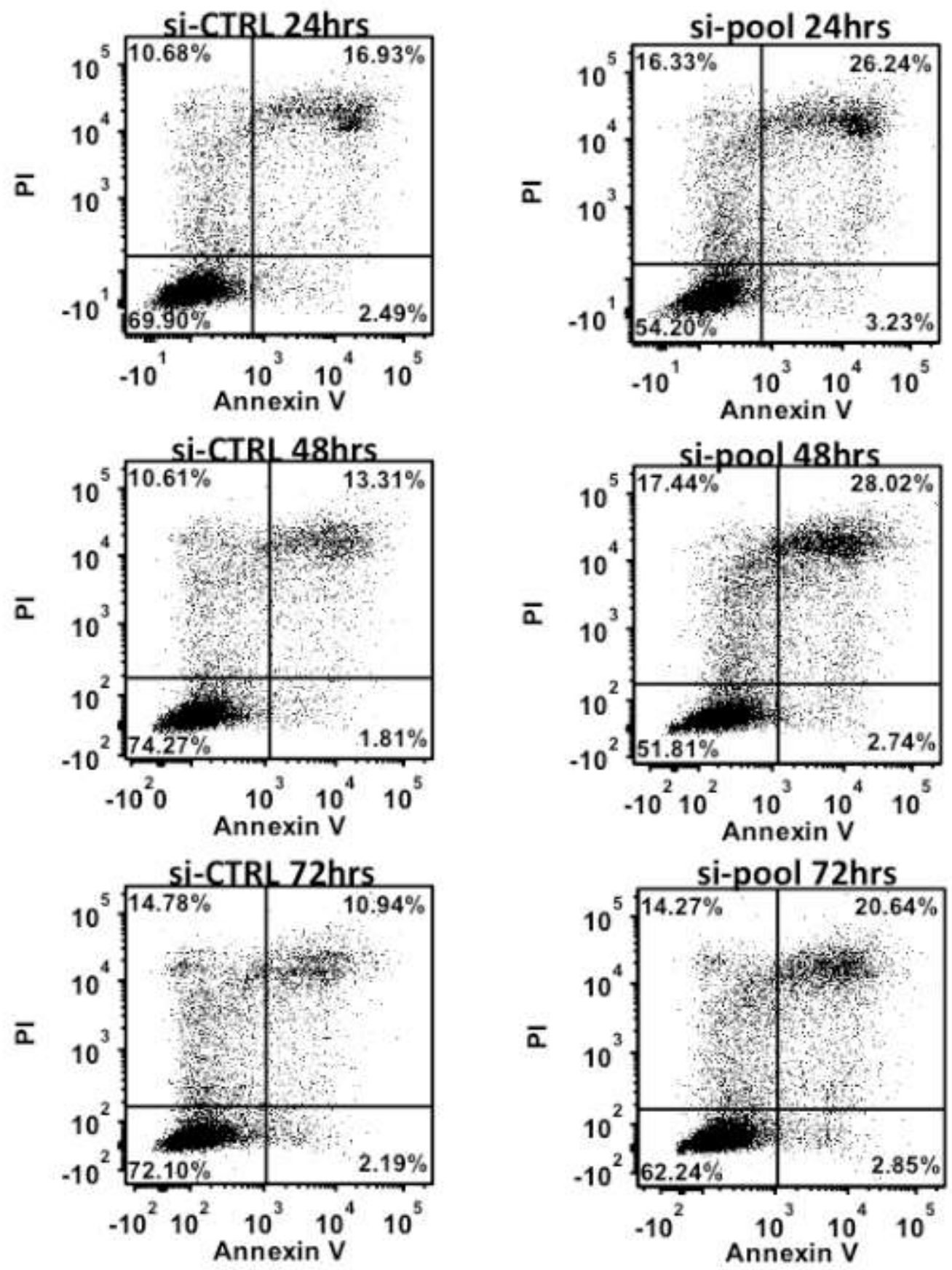


\section{CaSki}
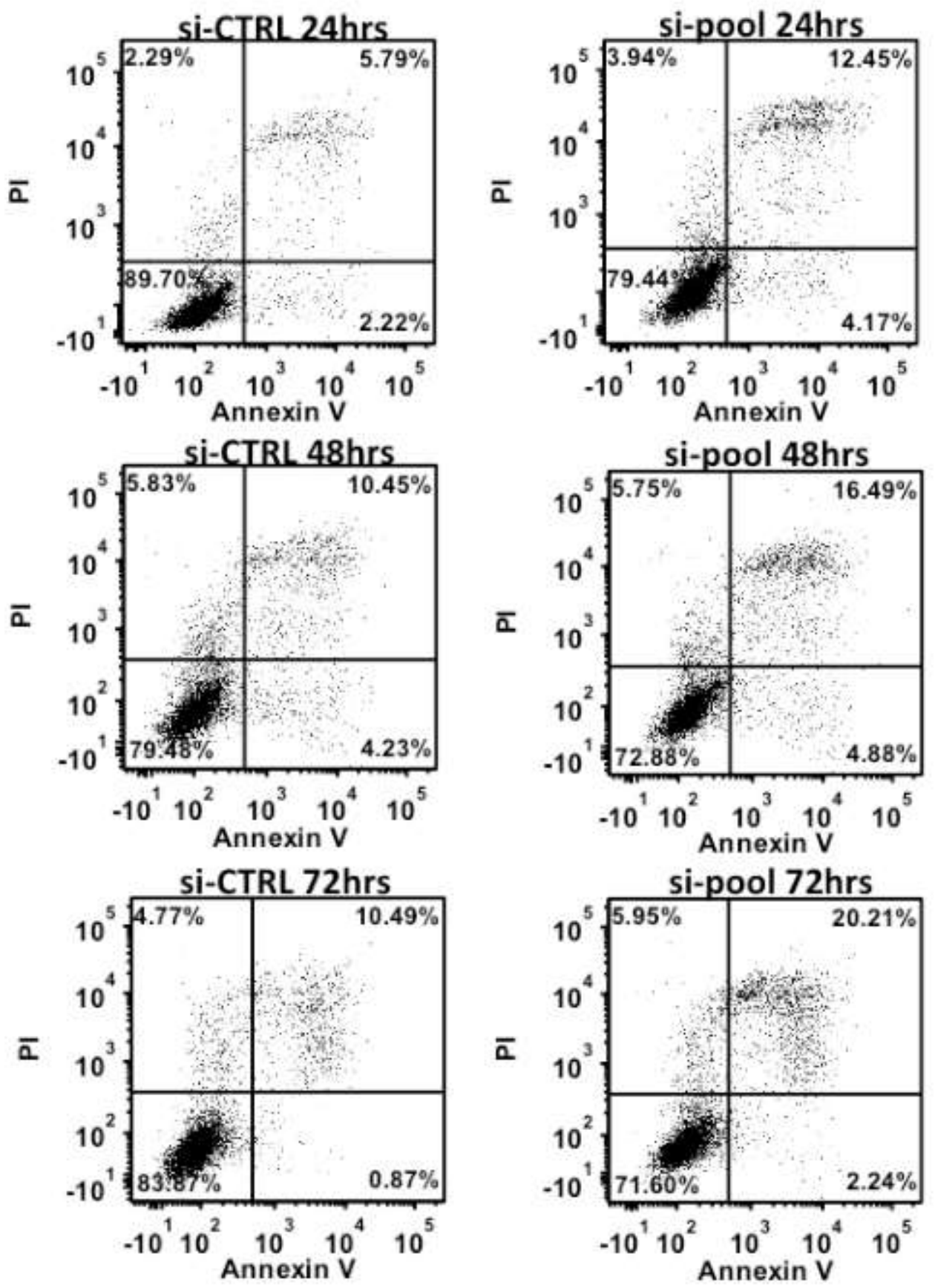
Figure S7: FAM83H-AS1 knockdown altered cell proliferation, migration, and apoptosis in W12/201402 cells. (A) Knockdown efficiency of individual and SMARTpool siRNA against FAM83H-AS1 in HPV-16 positive W12/201402, measured by qRT-PCR analysis. Because of variations in the expression of GAPDH after the knockdown of FAM83H-AS1, we used GUSB mRNA to normalize the qRT-PCR analyses. The graph shows average of two individual experiments. (B) W12/201402 cells were transfected with individual siRNAs against FAM83H-AS1, siRNA SMARTpool against FAM83H-AS1, or siRNA control for 48 hours. Cells were then replated in equal numbers (200,000 cells/well, represented by dashed line in graph) and cultured another 48 hours prior to re-counting attached cells. The graph shows the average of two individual experiments. (C) W12/201402 cells were transfected with individual siRNAs against FAM83H-AS1, siRNA SMARTpool against FAM83HAS1, or siRNA control for 48 hours then plated in equal numbers. Transfected cells were analyzed for cellular proliferation assessment by CCK- 8 assay at 48, 72, and 96 hours post-plating. The graph shows the average of two individual experiments. (DG) W12/201402 cells were transfected with siRNA SMARTpool against FAM83H-AS1 or siRNA control for 24 hours then plated in equal numbers for experiments. (D) Transfected cells were analyzed for cell cycle alterations by FACS analysis. W12/201402 cells with knockdown of FAM83H-AS1 exhibit less cells in S-phase of cell cycle compared to control cells. The graph shows the average of two individual experiments. (E) Transwell migration of transfected cells was analyzed 48 hours post-plating in upper chamber with chemoattractant in lower chamber. The graph shows the average of two individual experiments. (F-H) Transfected W12/201402 or CaSki cells were collected at 1,2 , and 3 days post-plating, stained with Annexin V/PI, and analyzed by flow cytometry to show alterations in apoptosis compared to siRNA control. (F) Quantitative analysis of Annexin V/PI flow cytometry data in W12/201402 cells. The graph shows the average of two individual experiments. (G) Dot plot analysis of Annexin V/PI flow cytometry data in W12/201402 cells. (H) Dot plot analysis of Annexin V/PI flow cytometry data in CaSki cells. Representative images shown for siRNA FAM83H-AS1 and siRNA control at each time point. Twotailed $t$ test results are indicted as ${ }^{*} p \leq 0.05$ and ${ }^{* *} p \leq 0.01$. 


\section{$\underline{\text { XII. Supplementary Tables }}$}

Table S1: Host IncRNAs altered after expression of HPV-16 E6 in primary foreskin keratinocytes.

Table shows details for each host IncRNA that was altered 1.5-fold or greater in primary foreskin keratinocytes (HEKa) expressing HPV-16 E6 compared to uninfected HEKa. Triplicates for each sample were sent for high-throughput RNA sequencing analysis.

\begin{tabular}{|c|c|c|c|c|c|c|c|c|c|c|c|c|}
\hline Gene ID & $\begin{array}{l}\text { Fold } \\
\text { Change } \\
\text { ([HPV } \\
16 E 6 \text { ] vs } \\
\text { [Control]) }\end{array}$ & Gene Symbol & $\begin{array}{l}\text { Chro } \\
\text { moso } \\
\text { me }\end{array}$ & Start & End & Strand & $\begin{array}{l}\text { RPKM } \\
\text { GFP } \\
\text { Control } \\
-1\end{array}$ & $\begin{array}{l}\text { RPKM } \\
\text { GFP } \\
\text { Control } \\
-2\end{array}$ & $\begin{array}{l}\text { RPKM } \\
\text { GFP } \\
\text { Control } \\
-3\end{array}$ & $\begin{array}{l}\text { RPKM } \\
\text { HPV- } \\
16 \text { E6- } \\
1\end{array}$ & $\begin{array}{l}\text { RPKM } \\
\text { HPV- } \\
16 \text { E6- } \\
2\end{array}$ & $\begin{array}{l}\text { RPKM } \\
\text { HPV- } \\
16 \text { E6- } \\
3\end{array}$ \\
\hline ENSG00000130600 & 4.99 & $\mathrm{H} 19$ & chr11 & 2016406 & 2022700 & - & $\begin{array}{c}2.12904 \\
93\end{array}$ & $\begin{array}{c}2.1426 \\
256\end{array}$ & $\begin{array}{c}5.6363 \\
993\end{array}$ & $\begin{array}{l}19.22 \\
9809 \\
\end{array}$ & $\begin{array}{l}10.78 \\
3182 \\
\end{array}$ & $\begin{array}{l}15.41 \\
4708 \\
\end{array}$ \\
\hline ENSG00000254236 & 3.54 & KB-1639H6.2 & chr8 & 104032415 & 104033656 & - & 0 & 0 & $\begin{array}{c}0.2873 \\
624\end{array}$ & $\begin{array}{c}3.250 \\
996 \\
\end{array}$ & $\begin{array}{c}3.968 \\
86 \\
\end{array}$ & $\begin{array}{l}3.449 \\
8966 \\
\end{array}$ \\
\hline ENSG00000244528 & 3.31 & AC134873.1 & chr2 & 243064438 & 243064620 & + & $\begin{array}{c}1.27679 \\
34 \\
\end{array}$ & $\begin{array}{c}2.8750 \\
565\end{array}$ & $\begin{array}{c}2.7582 \\
934\end{array}$ & $\begin{array}{c}6.186 \\
408 \\
\end{array}$ & $\begin{array}{c}6.602 \\
485 \\
\end{array}$ & $\begin{array}{c}9.019 \\
768 \\
\end{array}$ \\
\hline ENSG00000273413 & 3.07 & $\begin{array}{c}\text { RP11- } \\
96 C 23.15\end{array}$ & chr10 & 88729994 & 88730583 & - & $\begin{array}{c}8.39354 \\
6 \\
\end{array}$ & 0 & 0 & $\begin{array}{c}4.790 \\
1816 \\
\end{array}$ & $\begin{array}{l}7.013 \\
6943 \\
\end{array}$ & $\begin{array}{l}7.246 \\
6874 \\
\end{array}$ \\
\hline ENSG00000228626 & 3.06 & $\begin{array}{c}\text { RP11- } \\
\text { 495P10.9 }\end{array}$ & chr1 & 147760107 & 147761057 & - & $\begin{array}{c}2.02880 \\
84 \\
\end{array}$ & $\begin{array}{c}0.8839 \\
2633\end{array}$ & $\begin{array}{c}2.2444 \\
494\end{array}$ & $\begin{array}{l}6.981 \\
7734 \\
\end{array}$ & $\begin{array}{l}5.038 \\
5094 \\
\end{array}$ & $\begin{array}{l}3.696 \\
5914 \\
\end{array}$ \\
\hline ENSG00000230937 & 2.94 & MIR205HG & chr1 & 209602165 & 209606183 & + & $\begin{array}{c}61.5482 \\
64 \\
\end{array}$ & $\begin{array}{c}49.323 \\
284\end{array}$ & $\begin{array}{c}62.656 \\
292\end{array}$ & $\begin{array}{c}218.4 \\
987 \\
\end{array}$ & $\begin{array}{l}181.1 \\
5729 \\
\end{array}$ & $\begin{array}{l}121.9 \\
17755 \\
\end{array}$ \\
\hline ENSG00000254860 & 2.73 & TMEM9B-AS1 & chr11 & 8986222 & 8999074 & + & $\begin{array}{c}0.80623 \\
74 \\
\end{array}$ & $\begin{array}{c}1.7021 \\
289\end{array}$ & $\begin{array}{c}0.6363 \\
604\end{array}$ & $\begin{array}{c}4.052 \\
271 \\
\end{array}$ & $\begin{array}{l}3.137 \\
7754 \\
\end{array}$ & $\begin{array}{c}2.730 \\
444 \\
\end{array}$ \\
\hline ENSG00000249641 & 2.69 & HOXC-AS5 & chr12 & 54329112 & 54333427 & - & $\begin{array}{c}0.95938 \\
82 \\
\end{array}$ & $\begin{array}{c}1.8041 \\
867\end{array}$ & $\begin{array}{c}0.9305 \\
784\end{array}$ & $\begin{array}{c}3.716 \\
362 \\
\end{array}$ & $\begin{array}{l}3.758 \\
8704 \\
\end{array}$ & $\begin{array}{l}2.516 \\
7224 \\
\end{array}$ \\
\hline ENSG00000270168 & 2.56 & $\begin{array}{c}\text { LA16c- } \\
380 \mathrm{H} 5.4\end{array}$ & chr16 & 3051301 & 3052017 & + & $\begin{array}{c}6.47689 \\
87 \\
\end{array}$ & $\begin{array}{c}0.8551 \\
222\end{array}$ & $\begin{array}{c}4.0893 \\
016\end{array}$ & $\begin{array}{c}9.194 \\
51 \\
\end{array}$ & $\begin{array}{l}7.053 \\
4296 \\
\end{array}$ & $\begin{array}{l}6.857 \\
7585 \\
\end{array}$ \\
\hline ENSG00000235314 & 2.53 & LINC00957 & chr7 & 44078770 & 44081905 & + & $\begin{array}{c}0.35596 \\
678 \\
\end{array}$ & $\begin{array}{c}0.7332 \\
8674\end{array}$ & $\begin{array}{c}0.7004 \\
4947\end{array}$ & $\begin{array}{l}1.938 \\
0348 \\
\end{array}$ & $\begin{array}{c}2.741 \\
936 \\
\end{array}$ & $\begin{array}{l}3.045 \\
2104 \\
\end{array}$ \\
\hline ENSG00000230409 & 2.50 & TCEA1P2 & chr3 & 37317087 & 37318089 & + & $\begin{array}{c}7.46950 \\
8 \\
\end{array}$ & $\begin{array}{c}9.5921 \\
29\end{array}$ & $\begin{array}{c}10.218 \\
497\end{array}$ & $\begin{array}{l}22.00 \\
8438 \\
\end{array}$ & $\begin{array}{c}24.91 \\
3902 \\
\end{array}$ & $\begin{array}{l}20.84 \\
6043 \\
\end{array}$ \\
\hline ENSG00000233223 & 2.45 & AC113189.5 & chr17 & 7485282 & 7487390 & - & $\begin{array}{c}1.45960 \\
63 \\
\end{array}$ & $\begin{array}{c}2.9885 \\
232\end{array}$ & $\begin{array}{c}2.0333 \\
843\end{array}$ & $\begin{array}{c}3.720 \\
071 \\
\end{array}$ & $\begin{array}{l}5.512 \\
9123 \\
\end{array}$ & $\begin{array}{l}6.323 \\
3705 \\
\end{array}$ \\
\hline ENSG00000254192 & 2.36 & CTC-558O2.2 & chr5 & 168081518 & 168094766 & + & $\begin{array}{c}1.23549 \\
65 \\
\end{array}$ & $\begin{array}{c}0.9035 \\
5885\end{array}$ & $\begin{array}{c}1.6458 \\
715\end{array}$ & $\begin{array}{c}3.684 \\
764 \\
\end{array}$ & $\begin{array}{l}2.919 \\
8492 \\
\end{array}$ & $\begin{array}{l}2.477 \\
2859 \\
\end{array}$ \\
\hline ENSG00000251580 & 2.35 & $\begin{array}{c}\text { RP11- } \\
539 L 10.3 \\
\end{array}$ & chr4 & 6672452 & 6675557 & - & 0.83309 & $\begin{array}{c}1.3336 \\
157 \\
\end{array}$ & $\begin{array}{c}1.0557 \\
044 \\
\end{array}$ & $\begin{array}{c}2.159 \\
164 \\
\end{array}$ & $\begin{array}{l}2.004 \\
1137 \\
\end{array}$ & $\begin{array}{c}4.219 \\
327 \\
\end{array}$ \\
\hline ENSG00000220804 & 2.29 & AC093642.5 & chr2 & 243030784 & 243102304 & + & $\begin{array}{c}1.02285 \\
7 \\
\end{array}$ & $\begin{array}{c}1.6583 \\
912 \\
\end{array}$ & $\begin{array}{c}2.8178 \\
344 \\
\end{array}$ & $\begin{array}{l}4.230 \\
8583 \\
\end{array}$ & $\begin{array}{l}2.589 \\
8807 \\
\end{array}$ & $\begin{array}{l}5.207 \\
1075 \\
\end{array}$ \\
\hline ENSG00000201428 & 2.28 & RN7SKP71 & chr12 & 112704881 & 112705198 & + & $\begin{array}{c}0.05682 \\
099\end{array}$ & $\begin{array}{c}0.2640 \\
6604\end{array}$ & $\begin{array}{c}0.3352 \\
5583\end{array}$ & $\begin{array}{c}0.033 \\
64112 \\
6 \\
\end{array}$ & $\begin{array}{l}0.464 \\
84584\end{array}$ & $\begin{array}{c}11.79 \\
032\end{array}$ \\
\hline ENSG00000238107 & 2.27 & $\begin{array}{c}\text { RP11- } \\
495 P 10.5 \\
\end{array}$ & chr1 & 147767287 & 147769663 & + & $\begin{array}{c}1.19644 \\
03 \\
\end{array}$ & $\begin{array}{c}0.5992 \\
599 \\
\end{array}$ & $\begin{array}{c}0.7781 \\
85 \\
\end{array}$ & $\begin{array}{c}3.452 \\
5478 \\
\end{array}$ & $\begin{array}{l}1.896 \\
4818 \\
\end{array}$ & $\begin{array}{l}2.127 \\
1327 \\
\end{array}$ \\
\hline ENSG00000228463 & 2.25 & AP006222.2 & chr1 & 227615 & 267253 & - & $\begin{array}{c}0.92179 \\
984 \\
\end{array}$ & $\begin{array}{c}1.8147 \\
042\end{array}$ & $\begin{array}{c}2.4698 \\
875\end{array}$ & $\begin{array}{c}3.840 \\
908 \\
\end{array}$ & $\begin{array}{l}3.520 \\
3686 \\
\end{array}$ & $\begin{array}{l}3.766 \\
3238 \\
\end{array}$ \\
\hline ENSG00000263934 & 2.22 & SNORD3A & chr17 & 19091329 & 19092027 & + & $\begin{array}{c}56.5595 \\
66 \\
\end{array}$ & $\begin{array}{c}96.541 \\
93 \\
\end{array}$ & $\begin{array}{c}87.400 \\
215 \\
\end{array}$ & $\begin{array}{r}115.8 \\
12065 \\
\end{array}$ & $\begin{array}{l}101.6 \\
3637 \\
\end{array}$ & $\begin{array}{l}442.5 \\
1657 \\
\end{array}$ \\
\hline ENSG00000267984 & 2.21 & $\begin{array}{c}\text { CTD- } \\
\text { 2616J11.9 }\end{array}$ & chr19 & 51848423 & 51856547 & + & $\begin{array}{c}0.03900 \\
8502 \\
\end{array}$ & 0 & 0 & $\begin{array}{l}0.093 \\
65486 \\
\end{array}$ & 0 & $\begin{array}{c}10.77 \\
701 \\
\end{array}$ \\
\hline ENSG00000200488 & 2.18 & RN7SKP203 & chr2 & 76672205 & 76672536 & - & $\begin{array}{c}0.39458 \\
07 \\
\end{array}$ & $\begin{array}{c}2.4766 \\
135 \\
\end{array}$ & $\begin{array}{c}0.8919 \\
96\end{array}$ & $\begin{array}{c}1.181 \\
4926 \\
\end{array}$ & $\begin{array}{l}0.604 \\
2596 \\
\end{array}$ & $\begin{array}{c}21.76 \\
185 \\
\end{array}$ \\
\hline ENSG00000255717 & 2.13 & SNHG1 & chr11 & 62619460 & 62623386 & - & $\begin{array}{c}7.09001 \\
87 \\
\end{array}$ & $\begin{array}{c}3.8827 \\
178 \\
\end{array}$ & $\begin{array}{c}5.6702 \\
17 \\
\end{array}$ & $\begin{array}{l}13.07 \\
7036 \\
\end{array}$ & $\begin{array}{l}11.90 \\
0302 \\
\end{array}$ & $\begin{array}{c}9.669 \\
952 \\
\end{array}$ \\
\hline ENSG00000259001 & 2.12 & RPPH1 & chr14 & 20811207 & 20811844 & - & $\begin{array}{c}15.0019 \\
23 \\
\end{array}$ & $\begin{array}{c}52.592 \\
74\end{array}$ & $\begin{array}{c}31.222 \\
376\end{array}$ & $\begin{array}{l}27.47 \\
0306 \\
\end{array}$ & $\begin{array}{c}36.77 \\
239 \\
\end{array}$ & $\begin{array}{l}231.4 \\
2776 \\
\end{array}$ \\
\hline ENSG00000234741 & 2.10 & GAS5 & chr1 & 173833038 & 173838020 & - & 75.5498 & 34.682 & 63.601 & 141.4 & 116.2 & 93.72 \\
\hline
\end{tabular}




\begin{tabular}{|c|c|c|c|c|c|c|c|c|c|c|c|c|}
\hline & & & & & & & 6 & 1 & 818 & 7461 & 6347 & 997 \\
\hline ENSG00000215039 & 2.08 & CD27-AS1 & chr12 & 6548167 & 6560733 & - & $\begin{array}{c}0.66829 \\
19 \\
\end{array}$ & $\begin{array}{c}0.8538 \\
55 \\
\end{array}$ & $\begin{array}{c}0.8496 \\
1987 \\
\end{array}$ & $\begin{array}{c}3.074 \\
375\end{array}$ & $\begin{array}{c}1.768 \\
162\end{array}$ & $\begin{array}{l}1.665 \\
7759 \\
\end{array}$ \\
\hline ENSG00000259970 & 2.06 & AC099668.5 & chr3 & 49721913 & 49722416 & - & $\begin{array}{c}0.57804 \\
507\end{array}$ & $\begin{array}{c}1.3075 \\
932\end{array}$ & $\begin{array}{c}0.8779 \\
387\end{array}$ & $\begin{array}{c}2.843 \\
681\end{array}$ & $\begin{array}{l}2.354 \\
5742\end{array}$ & $\begin{array}{l}1.696 \\
4713 \\
\end{array}$ \\
\hline ENSG00000254578 & 2.04 & $\begin{array}{c}\text { CTD- } \\
\text { 2517M22.16 } \\
\end{array}$ & chr8 & 145689200 & 145690484 & + & $\begin{array}{c}2.56431 \\
48 \\
\end{array}$ & $\begin{array}{c}1.6997 \\
473 \\
\end{array}$ & $\begin{array}{l}2.1004 \\
875 \\
\end{array}$ & $\begin{array}{c}4.821 \\
358 \\
\end{array}$ & $\begin{array}{c}4.543 \\
132 \\
\end{array}$ & $\begin{array}{l}3.554 \\
0457 \\
\end{array}$ \\
\hline ENSG00000202198 & 2.04 & RN7SK & chr6 & 52860418 & 52860748 & + & $\begin{array}{c}77.7898 \\
2\end{array}$ & $\begin{array}{c}202.92 \\
421\end{array}$ & $\begin{array}{c}175.02 \\
835\end{array}$ & $\begin{array}{l}174.8 \\
5051\end{array}$ & $\begin{array}{l}183.0 \\
6963\end{array}$ & $\begin{array}{c}733.0 \\
992\end{array}$ \\
\hline ENSG00000255831 & 2.04 & AL139385.1 & chr13 & 111291555 & 111292340 & + & 0 & $\begin{array}{c}2.5623 \\
991\end{array}$ & 0 & 0 & $\begin{array}{l}5.166 \\
7576\end{array}$ & $\begin{array}{c}4.200 \\
331\end{array}$ \\
\hline ENSG00000271992 & 2.02 & RP11-42015.3 & chr1 & 70910754 & 70911219 & + & $\begin{array}{c}0.34897 \\
357\end{array}$ & $\begin{array}{c}0.0900 \\
998\end{array}$ & $\begin{array}{c}0.8007 \\
2904\end{array}$ & $\begin{array}{l}2.295 \\
6822\end{array}$ & $\begin{array}{l}1.722 \\
0101\end{array}$ & $\begin{array}{l}2.083 \\
4737\end{array}$ \\
\hline ENSG00000227195 & 2.01 & MIR663A & chr20 & 26167556 & 26232162 & - & $\begin{array}{c}0.89503 \\
71 \\
\end{array}$ & $\begin{array}{c}1.0255 \\
735 \\
\end{array}$ & $\begin{array}{c}1.4251 \\
992 \\
\end{array}$ & $\begin{array}{l}1.545 \\
9784 \\
\end{array}$ & $\begin{array}{c}1.247 \\
556 \\
\end{array}$ & $\begin{array}{l}6.125 \\
4115 \\
\end{array}$ \\
\hline ENSG00000239002 & 1.98 & SCARNA10 & chr12 & 6619388 & 6619717 & + & $\begin{array}{c}0.54754 \\
776\end{array}$ & $\begin{array}{c}1.9084 \\
775 \\
\end{array}$ & $\begin{array}{c}1.3999 \\
47 \\
\end{array}$ & $\begin{array}{l}1.167 \\
0413 \\
\end{array}$ & $\begin{array}{l}2.879 \\
6296 \\
\end{array}$ & $\begin{array}{l}6.134 \\
6283 \\
\end{array}$ \\
\hline ENSG00000263917 & 1.97 & RP11-5316.2 & chr18 & 29598792 & 29691742 & + & $\begin{array}{c}1.69259 \\
02\end{array}$ & $\begin{array}{c}0.7714 \\
8676\end{array}$ & $\begin{array}{c}1.5012 \\
345\end{array}$ & $\begin{array}{c}1.865 \\
241\end{array}$ & $\begin{array}{l}3.775 \\
4347 \\
\end{array}$ & $\begin{array}{c}2.767 \\
804\end{array}$ \\
\hline ENSG00000266929 & 1.96 & $\begin{array}{c}\text { RP11- } \\
\text { 400F19.8 } \\
\end{array}$ & chr17 & 40688528 & 40714080 & + & $\begin{array}{c}2.64025 \\
28 \\
\end{array}$ & $\begin{array}{c}2.2248 \\
166 \\
\end{array}$ & $\begin{array}{c}2.0022 \\
075 \\
\end{array}$ & $\begin{array}{l}4.118 \\
1316 \\
\end{array}$ & $\begin{array}{l}4.623 \\
1112 \\
\end{array}$ & $\begin{array}{c}4.685 \\
91 \\
\end{array}$ \\
\hline ENSG00000223891 & 1.96 & OSER1-AS1 & chr20 & 42839600 & 42854667 & + & $\begin{array}{c}0.42706 \\
716 \\
\end{array}$ & $\begin{array}{c}1.3540 \\
938 \\
\end{array}$ & $\begin{array}{c}1.7394 \\
654 \\
\end{array}$ & $\begin{array}{l}2.074 \\
9772 \\
\end{array}$ & $\begin{array}{l}3.062 \\
2957 \\
\end{array}$ & $\begin{array}{c}2.806 \\
612 \\
\end{array}$ \\
\hline ENSG00000244879 & 1.96 & GABPB1-AS1 & chr15 & 50647525 & 50659636 & + & $\begin{array}{c}1.49664 \\
08\end{array}$ & $\begin{array}{c}1.0193 \\
752\end{array}$ & $\begin{array}{c}1.1708 \\
221\end{array}$ & $\begin{array}{l}2.835 \\
0017\end{array}$ & $\begin{array}{c}2.036 \\
269\end{array}$ & $\begin{array}{l}2.337 \\
8665\end{array}$ \\
\hline ENSG00000225976 & 1.96 & $\begin{array}{c}\text { RP11- } \\
192 N 10.2 \\
\end{array}$ & chr10 & 29986832 & 29987157 & + & $\begin{array}{c}4.10156 \\
9 \\
\end{array}$ & $\begin{array}{c}1.4167 \\
225 \\
\end{array}$ & $\begin{array}{c}3.0522 \\
678 \\
\end{array}$ & $\begin{array}{l}4.856 \\
7057 \\
\end{array}$ & $\begin{array}{l}5.441 \\
2627 \\
\end{array}$ & $\begin{array}{c}5.069 \\
306 \\
\end{array}$ \\
\hline ENSG00000259827 & 1.95 & $\begin{array}{c}\text { RP11- } \\
343 \mathrm{H} 19.2 \\
\end{array}$ & chr16 & 56716382 & 56721719 & + & $\begin{array}{c}3.48532 \\
18 \\
\end{array}$ & $\begin{array}{c}2.4112 \\
535 \\
\end{array}$ & $\begin{array}{c}2.5835 \\
383 \\
\end{array}$ & $\begin{array}{c}3.920 \\
711 \\
\end{array}$ & $\begin{array}{l}3.862 \\
1109 \\
\end{array}$ & $\begin{array}{l}10.60 \\
9867 \\
\end{array}$ \\
\hline ENSG00000235499 & 1.95 & AC073046.25 & chr2 & 74212259 & 74213470 & + & $\begin{array}{c}4.49410 \\
3 \\
\end{array}$ & $\begin{array}{c}3.1759 \\
024 \\
\end{array}$ & $\begin{array}{c}2.5058 \\
224 \\
\end{array}$ & $\begin{array}{l}6.528 \\
4495 \\
\end{array}$ & $\begin{array}{c}5.577 \\
129 \\
\end{array}$ & $\begin{array}{l}7.256 \\
5813 \\
\end{array}$ \\
\hline ENSG00000258377 & 1.94 & RP11-649E7.5 & chr14 & 50087533 & 50090198 & - & 0 & $\begin{array}{c}3.7360 \\
387 \\
\end{array}$ & $\begin{array}{c}4.1599 \\
88 \\
\end{array}$ & $\begin{array}{c}5.070 \\
155\end{array}$ & $\begin{array}{c}4.762 \\
3515 \\
\end{array}$ & $\begin{array}{c}4.735 \\
923 \\
\end{array}$ \\
\hline ENSG00000233825 & 1.94 & $\begin{array}{c}\text { RP11- } \\
\text { 135A24.4 } \\
\end{array}$ & chr10 & 32635427 & 32636107 & + & $\begin{array}{c}2.00523 \\
7 \\
\end{array}$ & $\begin{array}{c}0.9287 \\
9003 \\
\end{array}$ & $\begin{array}{c}1.8029 \\
468 \\
\end{array}$ & $\begin{array}{l}2.122 \\
1492 \\
\end{array}$ & $\begin{array}{c}3.477 \\
26 \\
\end{array}$ & $\begin{array}{l}3.551 \\
1832 \\
\end{array}$ \\
\hline ENSG00000269926 & 1.93 & $\begin{array}{c}\text { RP11- } \\
442 \mathrm{H} 21.2 \\
\end{array}$ & chr10 & 74034673 & 74035738 & - & $\begin{array}{c}37.1678 \\
16 \\
\end{array}$ & $\begin{array}{c}17.135 \\
89 \\
\end{array}$ & $\begin{array}{c}27.302 \\
435 \\
\end{array}$ & $\begin{array}{l}56.37 \\
6045 \\
\end{array}$ & $\begin{array}{l}53.58 \\
8455 \\
\end{array}$ & $\begin{array}{c}41.68 \\
311 \\
\end{array}$ \\
\hline ENSG00000261824 & 1.91 & LINC00662 & chr19 & 28175488 & 28284848 & - & $\begin{array}{c}1.77218 \\
08 \\
\end{array}$ & $\begin{array}{c}0.9460 \\
5726 \\
\end{array}$ & $\begin{array}{c}1.4761 \\
61 \\
\end{array}$ & $\begin{array}{l}3.744 \\
9877 \\
\end{array}$ & $\begin{array}{l}2.442 \\
1446 \\
\end{array}$ & $\begin{array}{l}1.996 \\
9538 \\
\end{array}$ \\
\hline ENSG00000267317 & 1.90 & CTB-25B13.12 & chr19 & 1457664 & 1462764 & - & $\begin{array}{c}0.77052 \\
76 \\
\end{array}$ & $\begin{array}{c}2.7577 \\
708 \\
\end{array}$ & $\begin{array}{c}2.0533 \\
23 \\
\end{array}$ & $\begin{array}{l}3.240 \\
5212 \\
\end{array}$ & $\begin{array}{l}3.709 \\
7907 \\
\end{array}$ & $\begin{array}{c}3.223 \\
024 \\
\end{array}$ \\
\hline ENSG00000179859 & 1.89 & AC025335.1 & chr17 & 7816642 & 7819271 & - & $\begin{array}{c}2.48756 \\
43 \\
\end{array}$ & $\begin{array}{c}3.6358 \\
418 \\
\end{array}$ & $\begin{array}{c}4.2272 \\
625 \\
\end{array}$ & $\begin{array}{c}7.583 \\
128 \\
\end{array}$ & $\begin{array}{c}6.336 \\
298 \\
\end{array}$ & $\begin{array}{l}5.399 \\
1914 \\
\end{array}$ \\
\hline ENSG00000257653 & 1.87 & RP11-579D7.2 & chr12 & 49159977 & 49161106 & + & $\begin{array}{c}0.70771 \\
77 \\
\end{array}$ & $\begin{array}{c}1.0421 \\
093 \\
\end{array}$ & $\begin{array}{c}1.3791 \\
414 \\
\end{array}$ & $\begin{array}{l}2.705 \\
9877 \\
\end{array}$ & $\begin{array}{l}1.943 \\
5432 \\
\end{array}$ & $\begin{array}{l}1.797 \\
2413 \\
\end{array}$ \\
\hline ENSG00000238271 & 1.87 & IFNWP19 & chr9 & 21455483 & 21456048 & + & $\begin{array}{c}6.09118 \\
27 \\
\end{array}$ & $\begin{array}{c}4.1468 \\
25 \\
\end{array}$ & $\begin{array}{c}6.1276 \\
43 \\
\end{array}$ & $\begin{array}{l}10.78 \\
6538 \\
\end{array}$ & $\begin{array}{c}8.897 \\
233 \\
\end{array}$ & $\begin{array}{l}10.57 \\
8037 \\
\end{array}$ \\
\hline ENSG00000224032 & 1.87 & EPB41L4A-AS1 & chr5 & 111496223 & 111499973 & + & $\begin{array}{c}7.39583 \\
83 \\
\end{array}$ & $\begin{array}{c}3.9629 \\
142 \\
\end{array}$ & $\begin{array}{c}7.1221 \\
61 \\
\end{array}$ & $\begin{array}{c}9.977 \\
508\end{array}$ & $\begin{array}{l}12.22 \\
6742 \\
\end{array}$ & $\begin{array}{l}11.20 \\
5975 \\
\end{array}$ \\
\hline ENSG00000257270 & 1.87 & $\begin{array}{c}\text { RP11- } \\
\text { 521B24.5 }\end{array}$ & chr14 & 105934130 & 105936954 & - & $\begin{array}{c}6.40190 \\
6 \\
\end{array}$ & $\begin{array}{c}3.2108 \\
22 \\
\end{array}$ & $\begin{array}{c}3.5815 \\
47 \\
\end{array}$ & $\begin{array}{c}8.843 \\
35 \\
\end{array}$ & $\begin{array}{l}7.365 \\
5524 \\
\end{array}$ & $\begin{array}{l}7.390 \\
7046 \\
\end{array}$ \\
\hline ENSG00000257084 & 1.86 & U47924.27 & chr12 & 7072409 & 7073610 & + & 31.198 & $\begin{array}{c}36.405 \\
44 \\
\end{array}$ & $\begin{array}{c}20.322 \\
99 \\
\end{array}$ & $\begin{array}{l}53.07 \\
6824 \\
\end{array}$ & $\begin{array}{l}61.99 \\
1566 \\
\end{array}$ & $\begin{array}{c}44.91 \\
036 \\
\end{array}$ \\
\hline ENSG00000225377 & 1.85 & RP5-1103G7.4 & chr20 & 300957 & 328868 & - & $\begin{array}{c}2.56064 \\
6 \\
\end{array}$ & $\begin{array}{c}3.4069 \\
042 \\
\end{array}$ & $\begin{array}{c}5.1782 \\
656 \\
\end{array}$ & $\begin{array}{c}6.993 \\
363 \\
\end{array}$ & $\begin{array}{l}5.695 \\
6306 \\
\end{array}$ & $\begin{array}{c}7.200 \\
583 \\
\end{array}$ \\
\hline ENSG00000239470 & 1.84 & RP11-16F15.2 & chr11 & 9681946 & 9682503 & + & $\begin{array}{c}6.97289 \\
23 \\
\end{array}$ & $\begin{array}{c}3.0191 \\
91 \\
\end{array}$ & $\begin{array}{c}4.1343 \\
21 \\
\end{array}$ & $\begin{array}{l}10.61 \\
4801 \\
\end{array}$ & $\begin{array}{c}8.628 \\
567 \\
\end{array}$ & $\begin{array}{l}5.930 \\
6784 \\
\end{array}$ \\
\hline ENSG00000198496 & 1.84 & NBR2 & chr17 & 41277627 & 41305688 & + & $\begin{array}{c}0.83190 \\
143 \\
\end{array}$ & $\begin{array}{c}1.0633 \\
509 \\
\end{array}$ & $\begin{array}{c}1.4072 \\
425 \\
\end{array}$ & $\begin{array}{l}2.247 \\
7791 \\
\end{array}$ & $\begin{array}{l}2.288 \\
2934 \\
\end{array}$ & $\begin{array}{l}1.808 \\
5096 \\
\end{array}$ \\
\hline ENSG00000203499 & 1.84 & FAM83H-AS1 & chr8 & 144816310 & 144828507 & + & $\begin{array}{c}9.59697 \\
8 \\
\end{array}$ & $\begin{array}{c}3.3299 \\
105 \\
\end{array}$ & $\begin{array}{c}4.7020 \\
435 \\
\end{array}$ & $\begin{array}{l}12.95 \\
5384 \\
\end{array}$ & $\begin{array}{l}9.471 \\
8895 \\
\end{array}$ & $\begin{array}{c}7.570 \\
58 \\
\end{array}$ \\
\hline ENSG00000177410 & 1.83 & ZFAS1 & chr20 & 47894715 & 47905797 & + & $\begin{array}{c}211.102 \\
16\end{array}$ & $\begin{array}{c}139.73 \\
38\end{array}$ & $\begin{array}{c}252.70 \\
905\end{array}$ & $\begin{array}{l}418.7 \\
3697\end{array}$ & $\begin{array}{l}367.0 \\
7626\end{array}$ & $\begin{array}{l}297.5 \\
4745\end{array}$ \\
\hline
\end{tabular}




\begin{tabular}{|c|c|c|c|c|c|c|c|c|c|c|c|c|}
\hline ENSG00000224891 & 1.83 & AC007899.3 & chr2 & 37375673 & 37376447 & + & $\begin{array}{c}4.01025 \\
72\end{array}$ & $\begin{array}{c}2.9299 \\
183\end{array}$ & $\begin{array}{c}3.7727 \\
127\end{array}$ & $\begin{array}{c}6.872 \\
386\end{array}$ & $\begin{array}{c}6.110 \\
306\end{array}$ & $\begin{array}{c}6.431 \\
074\end{array}$ \\
\hline ENSG00000214293 & 1.82 & RSBN1L-AS1 & chr7 & 77286977 & 77325582 & - & $\begin{array}{c}1.88850 \\
9\end{array}$ & $\begin{array}{c}1.6980 \\
832\end{array}$ & $\begin{array}{c}1.8871 \\
909\end{array}$ & $\begin{array}{l}3.833 \\
9822\end{array}$ & $\begin{array}{l}3.262 \\
9604\end{array}$ & $\begin{array}{l}2.897 \\
0351\end{array}$ \\
\hline ENSG00000250902 & 1.81 & $\begin{array}{c}\text { RP11- } \\
301 \mathrm{H} 24.4\end{array}$ & chr4 & 146435767 & 146438270 & - & $\begin{array}{c}6.98976 \\
66\end{array}$ & $\begin{array}{c}.0939 \\
543 \\
\end{array}$ & $\begin{array}{c}5.3921 \\
43 \\
\end{array}$ & $\begin{array}{l}10.33 \\
7118 \\
\end{array}$ & $\begin{array}{c}8.141 \\
412 \\
\end{array}$ & $\begin{array}{c}8.172 \\
936 \\
\end{array}$ \\
\hline ENSG00000259375 & 1.81 & $\begin{array}{c}\text { RP11- } \\
815 J 21.2\end{array}$ & chr15 & 86122277 & 86123409 & - & $\begin{array}{c}1.83370 \\
24\end{array}$ & $\begin{array}{c}1.1494 \\
807\end{array}$ & $\begin{array}{c}1.1902 \\
504\end{array}$ & $\begin{array}{l}1.597 \\
2154\end{array}$ & $\begin{array}{l}2.884 \\
9528\end{array}$ & $\begin{array}{c}3.210 \\
236\end{array}$ \\
\hline ENSG00000012171 & 1.80 & SEMA3B & chr3 & 50304990 & 50314977 & + & $\begin{array}{c}0.94935 \\
43 \\
\end{array}$ & $\begin{array}{c}0.3429 \\
1044 \\
\end{array}$ & $\begin{array}{c}1.0639 \\
977 \\
\end{array}$ & $\begin{array}{l}2.247 \\
6633 \\
\end{array}$ & $\begin{array}{l}1.790 \\
5937 \\
\end{array}$ & $\begin{array}{l}1.540 \\
2615 \\
\end{array}$ \\
\hline ENSG00000273344 & 1.79 & PAXIP1-AS1 & chr7 & 154795158 & 154797413 & + & $\begin{array}{c}1.07325 \\
18\end{array}$ & $\begin{array}{c}2.6334 \\
62\end{array}$ & $\begin{array}{c}2.4573 \\
538\end{array}$ & $\begin{array}{l}3.006 \\
4073\end{array}$ & $\begin{array}{l}3.060 \\
8828\end{array}$ & $\begin{array}{c}4.358 \\
569\end{array}$ \\
\hline ENSG00000260552 & 1.79 & RP11-49I11.1 & chr18 & 33759959 & 33767411 & - & $\begin{array}{c}1.30548 \\
33\end{array}$ & $\begin{array}{c}2.1372 \\
445\end{array}$ & $\begin{array}{c}4.7266 \\
11\end{array}$ & $\begin{array}{l}3.969 \\
9843\end{array}$ & $\begin{array}{l}6.068 \\
1834\end{array}$ & $\begin{array}{l}3.154 \\
5815\end{array}$ \\
\hline ENSG00000243004 & 1.79 & AC005062.2 & chr7 & 19958604 & 20180076 & - & $\begin{array}{c}4.87558 \\
13 \\
\end{array}$ & $\begin{array}{c}7.2985 \\
44 \\
\end{array}$ & $\begin{array}{c}3.9218 \\
314 \\
\end{array}$ & $\begin{array}{l}6.931 \\
1867 \\
\end{array}$ & $\begin{array}{l}14.13 \\
0893 \\
\end{array}$ & $\begin{array}{c}8.209 \\
532 \\
\end{array}$ \\
\hline ENSG00000196756 & 1.78 & SNHG17 & chr20 & 37049235 & 37063996 & - & $\begin{array}{c}2.54244 \\
57 \\
\end{array}$ & $\begin{array}{c}1.8823 \\
376\end{array}$ & $\begin{array}{c}4.6329 \\
513 \\
\end{array}$ & $\begin{array}{l}4.370 \\
9865 \\
\end{array}$ & $\begin{array}{l}5.745 \\
9593 \\
\end{array}$ & $\begin{array}{l}5.005 \\
6214 \\
\end{array}$ \\
\hline ENSG00000272906 & 1.78 & $\begin{array}{c}\text { RP11- } \\
\text { 533E19.7 }\end{array}$ & chr1 & 179850742 & 179851730 & - & $\begin{array}{c}0.29894 \\
31 \\
\end{array}$ & $\begin{array}{c}1.2788 \\
76 \\
\end{array}$ & $\begin{array}{c}1.0368 \\
878 \\
\end{array}$ & $\begin{array}{l}1.577 \\
8978 \\
\end{array}$ & $\begin{array}{c}1.703 \\
776 \\
\end{array}$ & $\begin{array}{l}2.794 \\
0536 \\
\end{array}$ \\
\hline ENSG00000261771 & 1.78 & $\begin{array}{c}\text { DYX1C1- } \\
\text { CCPG1 }\end{array}$ & chr15 & 55647446 & 55790558 & - & $\begin{array}{c}2.09689 \\
38 \\
\end{array}$ & $\begin{array}{c}2.6955 \\
016 \\
\end{array}$ & $\begin{array}{c}2.8982 \\
72 \\
\end{array}$ & $\begin{array}{c}5.290 \\
535\end{array}$ & $\begin{array}{c}4.608 \\
052 \\
\end{array}$ & $\begin{array}{l}3.804 \\
3263 \\
\end{array}$ \\
\hline ENSG00000204054 & 1.78 & LINC00963 & chr9 & 132245730 & 132275947 & + & $\begin{array}{c}1.01223 \\
13 \\
\end{array}$ & $\begin{array}{c}0.8181 \\
0194 \\
\end{array}$ & $\begin{array}{c}0.7509 \\
8056 \\
\end{array}$ & $\begin{array}{l}2.690 \\
7895 \\
\end{array}$ & $\begin{array}{l}1.890 \\
5445 \\
\end{array}$ & $\begin{array}{l}1.125 \\
3008 \\
\end{array}$ \\
\hline ENSG00000269893 & 1.78 & SNHG8 & chr4 & 119199864 & 119200978 & + & $\begin{array}{c}95.6830 \\
75\end{array}$ & $\begin{array}{c}85.640 \\
51\end{array}$ & $\begin{array}{c}110.78 \\
4874\end{array}$ & $\begin{array}{l}193.3 \\
1099 \\
\end{array}$ & $\begin{array}{l}208.9 \\
9217\end{array}$ & $\begin{array}{l}126.6 \\
07704\end{array}$ \\
\hline ENSG00000269900 & 1.78 & RMRP & chr9 & 35657748 & 35658015 & - & $\begin{array}{c}61.7190 \\
86\end{array}$ & $\begin{array}{c}117.54 \\
486\end{array}$ & $\begin{array}{c}105.27 \\
629\end{array}$ & $\begin{array}{c}99.84 \\
465\end{array}$ & $\begin{array}{l}121.5 \\
6224\end{array}$ & $\begin{array}{l}352.0 \\
1825\end{array}$ \\
\hline ENSG00000265735 & 1.76 & RN7SL5P & chr9 & 9442060 & 9442347 & + & $\begin{array}{c}89.3426 \\
7 \\
\end{array}$ & $\begin{array}{c}171.01 \\
642 \\
\end{array}$ & $\begin{array}{c}175.36 \\
769 \\
\end{array}$ & $\begin{array}{c}146.4 \\
969 \\
\end{array}$ & $\begin{array}{l}160.5 \\
5365 \\
\end{array}$ & $\begin{array}{c}623.5 \\
067 \\
\end{array}$ \\
\hline ENSG00000229413 & 1.75 & AC018638.1 & chr7 & 128293740 & 128293989 & - & $\begin{array}{c}2.08112 \\
76 \\
\end{array}$ & $\begin{array}{c}0.8533 \\
8426 \\
\end{array}$ & $\begin{array}{c}0.6139 \\
542 \\
\end{array}$ & $\begin{array}{c}2.203 \\
358 \\
\end{array}$ & $\begin{array}{l}1.459 \\
3241 \\
\end{array}$ & $\begin{array}{c}3.445 \\
703 \\
\end{array}$ \\
\hline ENSG00000250299 & 1.74 & MRPS31P4 & chr13 & 53191693 & 53211581 & + & $\begin{array}{c}0.99767 \\
58 \\
\end{array}$ & $\begin{array}{c}0.6966 \\
341 \\
\end{array}$ & $\begin{array}{c}0.8349 \\
367 \\
\end{array}$ & $\begin{array}{l}2.199 \\
7466 \\
\end{array}$ & $\begin{array}{l}1.885 \\
6709 \\
\end{array}$ & $\begin{array}{l}1.279 \\
5835 \\
\end{array}$ \\
\hline ENSG00000226419 & 1.74 & RP11-31F15.1 & chr1 & 113499037 & 113542118 & + & $\begin{array}{c}3.05203 \\
82 \\
\end{array}$ & $\begin{array}{c}2.3166 \\
275\end{array}$ & $\begin{array}{c}2.4479 \\
396 \\
\end{array}$ & $\begin{array}{c}5.745 \\
393 \\
\end{array}$ & $\begin{array}{l}4.417 \\
4857 \\
\end{array}$ & $\begin{array}{c}3.588 \\
944 \\
\end{array}$ \\
\hline ENSG00000245060 & 1.74 & LINC00847 & chr5 & 180257957 & 180262726 & + & $\begin{array}{c}2.51798 \\
3 \\
\end{array}$ & $\begin{array}{c}1.9512 \\
683 \\
\end{array}$ & $\begin{array}{c}1.9726 \\
743 \\
\end{array}$ & $\begin{array}{l}3.667 \\
7196 \\
\end{array}$ & $\begin{array}{l}3.663 \\
7437 \\
\end{array}$ & $\begin{array}{c}3.780 \\
457 \\
\end{array}$ \\
\hline ENSG00000110811 & 1.73 & LEPREL2 & chr12 & 6937572 & 6949018 & + & $\begin{array}{c}1.43181 \\
74\end{array}$ & $\begin{array}{c}1.6433 \\
642\end{array}$ & $\begin{array}{c}1.4975 \\
213\end{array}$ & $\begin{array}{c}3.065 \\
626\end{array}$ & $\begin{array}{l}2.159 \\
1876\end{array}$ & $\begin{array}{c}2.762 \\
544\end{array}$ \\
\hline ENSG00000262160 & 1.73 & RP11-96D1.11 & chr16 & 68259872 & 68263048 & - & $\begin{array}{c}1.01642 \\
31\end{array}$ & $\begin{array}{c}1.7172 \\
104\end{array}$ & $\begin{array}{c}1.3368 \\
715 \\
\end{array}$ & $\begin{array}{l}2.896 \\
4238\end{array}$ & $\begin{array}{l}2.114 \\
6343\end{array}$ & $\begin{array}{l}1.976 \\
8672 \\
\end{array}$ \\
\hline ENSG00000250251 & 1.73 & PKD1P6 & chr16 & 15219099 & 15248421 & - & $\begin{array}{c}4.10675 \\
76 \\
\end{array}$ & $\begin{array}{c}2.8266 \\
087 \\
\end{array}$ & $\begin{array}{c}4.4332 \\
04 \\
\end{array}$ & $\begin{array}{c}4.759 \\
571 \\
\end{array}$ & $\begin{array}{c}5.149 \\
509 \\
\end{array}$ & $\begin{array}{l}10.82 \\
3436 \\
\end{array}$ \\
\hline ENSG00000203512 & 1.72 & AL353662.2 & chr9 & 34195642 & 34195883 & + & $\begin{array}{c}1.73161 \\
97 \\
\end{array}$ & $\begin{array}{c}0.8091 \\
1475 \\
\end{array}$ & $\begin{array}{c}1.1475 \\
528 \\
\end{array}$ & $\begin{array}{l}3.164 \\
7897 \\
\end{array}$ & $\begin{array}{l}1.525 \\
1368 \\
\end{array}$ & $\begin{array}{l}2.108 \\
7782 \\
\end{array}$ \\
\hline ENSG00000227615 & 1.72 & RP11-864N7.2 & chr11 & 74456761 & 74457159 & - & $\begin{array}{c}6.18152 \\
57 \\
\end{array}$ & $\begin{array}{c}1.2890 \\
593 \\
\end{array}$ & $\begin{array}{c}4.9579 \\
773 \\
\end{array}$ & $\begin{array}{c}6.729 \\
743 \\
\end{array}$ & $\begin{array}{l}6.448 \\
0925 \\
\end{array}$ & $\begin{array}{l}4.633 \\
6823 \\
\end{array}$ \\
\hline ENSG00000257086 & 1.72 & $\begin{array}{c}\text { RP11- } \\
\text { 783K16.13 } \\
\end{array}$ & chr11 & 64014411 & 64016966 & - & $\begin{array}{c}1.58257 \\
52 \\
\end{array}$ & $\begin{array}{c}1.1539 \\
651 \\
\end{array}$ & $\begin{array}{c}1.8851 \\
668 \\
\end{array}$ & $\begin{array}{l}3.347 \\
7898 \\
\end{array}$ & $\begin{array}{c}2.183 \\
432 \\
\end{array}$ & $\begin{array}{l}2.384 \\
6471 \\
\end{array}$ \\
\hline ENSG00000230795 & 1.72 & HLA-K & chr6 & 29894236 & 29897009 & + & $\begin{array}{c}0.61804 \\
247 \\
\end{array}$ & $\begin{array}{c}1.1913 \\
046 \\
\end{array}$ & $\begin{array}{c}1.5108 \\
755 \\
\end{array}$ & $\begin{array}{l}1.330 \\
7873 \\
\end{array}$ & $\begin{array}{l}2.285 \\
3572 \\
\end{array}$ & $\begin{array}{l}2.992 \\
5754 \\
\end{array}$ \\
\hline ENSG00000180672 & 1.71 & AC007362.1 & chr2 & 206642540 & 206644433 & + & $\begin{array}{c}1.37003 \\
06 \\
\end{array}$ & $\begin{array}{c}0.6789 \\
9704 \\
\end{array}$ & $\begin{array}{c}1.1776 \\
617 \\
\end{array}$ & $\begin{array}{l}3.312 \\
5985 \\
\end{array}$ & $\begin{array}{l}1.914 \\
6636 \\
\end{array}$ & $\begin{array}{c}1.281 \\
505 \\
\end{array}$ \\
\hline ENSG00000254682 & 1.71 & $\begin{array}{c}\text { RP11- } \\
\text { 660L16.2 }\end{array}$ & chr11 & 71159720 & 71163203 & + & $\begin{array}{c}1.14260 \\
32 \\
\end{array}$ & $\begin{array}{c}0.7100 \\
659 \\
\end{array}$ & $\begin{array}{c}0.4964 \\
7444 \\
\end{array}$ & $\begin{array}{l}1.667 \\
6105 \\
\end{array}$ & $\begin{array}{l}1.925 \\
3993\end{array}$ & $\begin{array}{l}1.792 \\
3354 \\
\end{array}$ \\
\hline ENSG00000267023 & 1.71 & LRRC37A16P & chr17 & 66121918 & 66148609 & - & $\begin{array}{c}1.45405 \\
14 \\
\end{array}$ & $\begin{array}{c}1.6921 \\
17 \\
\end{array}$ & $\begin{array}{c}1.4601 \\
423\end{array}$ & $\begin{array}{c}2.523 \\
746\end{array}$ & $\begin{array}{l}2.944 \\
7954 \\
\end{array}$ & $\begin{array}{c}2.423 \\
118 \\
\end{array}$ \\
\hline ENSG00000227081 & 1.71 & $\begin{array}{c}\text { RP11- } \\
\text { 543P15.1 } \\
\end{array}$ & chr12 & 3320775 & 3321096 & - & $\begin{array}{c}7.24820 \\
5 \\
\end{array}$ & $\begin{array}{c}1.5755 \\
806 \\
\end{array}$ & $\begin{array}{c}5.4170 \\
194 \\
\end{array}$ & $\begin{array}{c}7.375 \\
556 \\
\end{array}$ & $\begin{array}{c}8.514 \\
681 \\
\end{array}$ & $\begin{array}{l}4.918 \\
4346 \\
\end{array}$ \\
\hline ENSG00000223745 & 1.71 & RP4-717I23.3 & chr1 & 93727743 & 93811582 & - & $\begin{array}{c}0.95840 \\
377\end{array}$ & $\begin{array}{c}1.4749 \\
708\end{array}$ & $\begin{array}{c}1.4199 \\
601\end{array}$ & $\begin{array}{l}2.833 \\
7555\end{array}$ & $\begin{array}{l}1.944 \\
4739\end{array}$ & $\begin{array}{l}1.893 \\
2604\end{array}$ \\
\hline ENSG00000226752 & 1.70 & PSMD5-AS1 & chr9 & 123587106 & 123616651 & + & 0.90399 & 0.6311 & 1.0164 & 2.319 & 1.464 & 1.481 \\
\hline
\end{tabular}




\begin{tabular}{|c|c|c|c|c|c|c|c|c|c|c|c|c|}
\hline & & & & & & & 02 & 066 & 516 & 7796 & 0397 & 2256 \\
\hline ENSG00000227632 & 1.70 & AC018804.6 & chr2 & 130959885 & 130970201 & + & $\begin{array}{c}1.50575 \\
63\end{array}$ & $\begin{array}{c}2.2844 \\
598 \\
\end{array}$ & $\begin{array}{c}2.4223 \\
318 \\
\end{array}$ & $\begin{array}{l}2.553 \\
2963 \\
\end{array}$ & $\begin{array}{c}4.621 \\
544\end{array}$ & $\begin{array}{l}3.451 \\
2596 \\
\end{array}$ \\
\hline ENSG00000228327 & 1.69 & $\begin{array}{c}\text { RP11- } \\
\text { 206L10.2 } \\
\end{array}$ & chr1 & 700237 & 714006 & - & $\begin{array}{c}3.12584 \\
38 \\
\end{array}$ & $\begin{array}{c}1.0653 \\
372 \\
\end{array}$ & $\begin{array}{c}3.5199 \\
823 \\
\end{array}$ & $\begin{array}{l}3.532 \\
1116 \\
\end{array}$ & $\begin{array}{l}3.393 \\
6765 \\
\end{array}$ & $\begin{array}{l}4.746 \\
0814 \\
\end{array}$ \\
\hline ENSG00000228638 & 1.69 & FCF1P2 & chr3 & 48332283 & 48332865 & - & $\begin{array}{c}0.71284 \\
515\end{array}$ & $\begin{array}{c}0.7201 \\
8015 \\
\end{array}$ & $\begin{array}{c}0.2133 \\
4462 \\
\end{array}$ & $\begin{array}{l}2.789 \\
1552 \\
\end{array}$ & $\begin{array}{l}1.738 \\
6442 \\
\end{array}$ & $\begin{array}{l}0.814 \\
9583 \\
\end{array}$ \\
\hline ENSG00000258186 & 1.69 & SLC7A5P2 & chr16 & 21531151 & 21531686 & - & 8.26003 & $\begin{array}{c}6.4758 \\
79 \\
\end{array}$ & $\begin{array}{c}14.699 \\
861 \\
\end{array}$ & $\begin{array}{l}13.65 \\
3822 \\
\end{array}$ & $\begin{array}{l}13.00 \\
4153 \\
\end{array}$ & $\begin{array}{l}21.40 \\
1606 \\
\end{array}$ \\
\hline ENSG00000225648 & 1.68 & SBDSP1 & chr7 & 72300004 & 72307909 & + & $\begin{array}{c}10.7959 \\
62\end{array}$ & $\begin{array}{c}11.587 \\
327\end{array}$ & $\begin{array}{c}13.133 \\
697\end{array}$ & $\begin{array}{l}19.68 \\
8213\end{array}$ & $\begin{array}{c}19.81 \\
655\end{array}$ & $\begin{array}{l}19.96 \\
0356 \\
\end{array}$ \\
\hline ENSG00000272711 & 1.68 & $\begin{array}{c}\text { RP11- } \\
\text { 259N19.1 } \\
\end{array}$ & chr2 & 75059782 & 75061114 & - & $\begin{array}{c}1.45040 \\
58 \\
\end{array}$ & $\begin{array}{c}1.1969 \\
146 \\
\end{array}$ & $\begin{array}{c}1.6662 \\
19 \\
\end{array}$ & $\begin{array}{l}3.884 \\
3012 \\
\end{array}$ & $\begin{array}{l}2.328 \\
7628 \\
\end{array}$ & $\begin{array}{l}1.503 \\
2032 \\
\end{array}$ \\
\hline ENSG00000256462 & 1.67 & RP11-116G8.5 & chr10 & 5566939 & 5567705 & - & $\begin{array}{c}3.94108 \\
49 \\
\end{array}$ & 0 & 0 & $\begin{array}{l}3.097 \\
3866 \\
\end{array}$ & $\begin{array}{c}5.978 \\
923 \\
\end{array}$ & 0 \\
\hline ENSG00000236439 & 1.67 & RP11-175B9.3 & chr1 & 202440937 & 202441258 & - & $\begin{array}{c}6.70856 \\
76 \\
\end{array}$ & $\begin{array}{c}3.0561 \\
595 \\
\end{array}$ & $\begin{array}{c}4.4550 \\
18 \\
\end{array}$ & $\begin{array}{l}7.306 \\
8976 \\
\end{array}$ & $\begin{array}{c}8.068 \\
728 \\
\end{array}$ & $\begin{array}{l}7.226 \\
4347 \\
\end{array}$ \\
\hline ENSG00000233016 & 1.67 & SNHG7 & chr9 & 139615818 & 139622636 & - & $\begin{array}{c}3.80191 \\
66\end{array}$ & $\begin{array}{c}2.5988 \\
54 \\
\end{array}$ & $\begin{array}{c}3.9963 \\
634\end{array}$ & $\begin{array}{c}4.894 \\
757 \\
\end{array}$ & $\begin{array}{l}6.888 \\
8636 \\
\end{array}$ & $\begin{array}{c}5.446 \\
209 \\
\end{array}$ \\
\hline ENSG00000234975 & 1.67 & FTH1P2 & chr1 & 228823162 & 228823574 & + & $\begin{array}{c}2.36462 \\
16 \\
\end{array}$ & $\begin{array}{c}8.7332 \\
3 \\
\end{array}$ & $\begin{array}{c}5.5768 \\
6 \\
\end{array}$ & $\begin{array}{c}7.877 \\
958 \\
\end{array}$ & $\begin{array}{c}8.395 \\
835 \\
\end{array}$ & $\begin{array}{c}8.092 \\
968 \\
\end{array}$ \\
\hline ENSG00000228998 & 1.66 & RP11-697E2.7 & chr15 & 90818266 & 90820841 & + & $\begin{array}{c}1.66241 \\
1\end{array}$ & $\begin{array}{c}1.0105 \\
448\end{array}$ & $\begin{array}{c}1.4692 \\
17\end{array}$ & $\begin{array}{l}2.458 \\
5187\end{array}$ & $\begin{array}{c}2.098 \\
612\end{array}$ & $\begin{array}{l}2.181 \\
2184 \\
\end{array}$ \\
\hline ENSG00000251022 & 1.66 & THAP9-AS1 & chr4 & 83814162 & 83822113 & - & $\begin{array}{c}2.55584 \\
17\end{array}$ & $\begin{array}{c}2.0884 \\
433\end{array}$ & $\begin{array}{c}4.3881 \\
38\end{array}$ & $\begin{array}{c}5.688 \\
368\end{array}$ & $\begin{array}{c}4.508 \\
133\end{array}$ & $\begin{array}{c}4.144 \\
217\end{array}$ \\
\hline ENSG00000256633 & 1.64 & RP11-169D4.2 & chr11 & 72295616 & 72299023 & + & $\begin{array}{c}0.36736 \\
822 \\
\end{array}$ & $\begin{array}{c}0.2219 \\
0487 \\
\end{array}$ & $\begin{array}{c}0.2809 \\
7868 \\
\end{array}$ & $\begin{array}{l}0.585 \\
01613 \\
\end{array}$ & $\begin{array}{c}1.582 \\
57 \\
\end{array}$ & $\begin{array}{c}2.773 \\
093 \\
\end{array}$ \\
\hline ENSG00000266754 & 1.64 & RN7SL524P & chr10 & 105734277 & 105734572 & - & $\begin{array}{c}0.67657 \\
28 \\
\end{array}$ & $\begin{array}{c}0.7092 \\
3144 \\
\end{array}$ & $\begin{array}{c}1.0905 \\
253\end{array}$ & $\begin{array}{l}2.662 \\
4227 \\
\end{array}$ & $\begin{array}{l}0.618 \\
2988 \\
\end{array}$ & $\begin{array}{l}1.791 \\
2405 \\
\end{array}$ \\
\hline ENSG00000260269 & 1.63 & $\begin{array}{c}\text { CTD- } \\
\text { 2323K18.1 } \\
\end{array}$ & chr15 & 75819491 & 75893546 & - & $\begin{array}{c}1.65046 \\
27 \\
\end{array}$ & $\begin{array}{c}0.9741 \\
242 \\
\end{array}$ & $\begin{array}{c}0.6995 \\
654 \\
\end{array}$ & $\begin{array}{c}3.534 \\
106 \\
\end{array}$ & $\begin{array}{c}1.676 \\
849 \\
\end{array}$ & $\begin{array}{l}1.202 \\
5416 \\
\end{array}$ \\
\hline ENSG00000260088 & 1.62 & RP11-92G12.3 & chr1 & 200638635 & 200663378 & + & $\begin{array}{c}1.84138 \\
01\end{array}$ & $\begin{array}{c}1.6593 \\
088 \\
\end{array}$ & $\begin{array}{c}3.4227 \\
7 \\
\end{array}$ & $\begin{array}{l}4.249 \\
6977 \\
\end{array}$ & $\begin{array}{c}2.985 \\
226 \\
\end{array}$ & $\begin{array}{l}3.536 \\
2139 \\
\end{array}$ \\
\hline ENSG00000267519 & 1.62 & MIR24-2 & chr19 & 13945330 & 13947173 & - & $\begin{array}{c}4.72304 \\
44 \\
\end{array}$ & $\begin{array}{c}4.5993 \\
896 \\
\end{array}$ & $\begin{array}{c}8.6144 \\
75 \\
\end{array}$ & $\begin{array}{c}9.827 \\
661 \\
\end{array}$ & $\begin{array}{l}7.844 \\
5435 \\
\end{array}$ & $\begin{array}{l}10.23 \\
9091 \\
\end{array}$ \\
\hline ENSG00000254681 & 1.61 & PKD1P5 & chr16 & 18458147 & 18495797 & - & $\begin{array}{c}1.31439 \\
34 \\
\end{array}$ & $\begin{array}{c}1.0079 \\
812 \\
\end{array}$ & $\begin{array}{c}1.4375 \\
087 \\
\end{array}$ & $\begin{array}{l}1.722 \\
2397 \\
\end{array}$ & $\begin{array}{l}2.123 \\
2183 \\
\end{array}$ & $\begin{array}{c}2.183 \\
325 \\
\end{array}$ \\
\hline ENSG00000197989 & 1.61 & SNHG12 & chr1 & 28905050 & 28909495 & - & $\begin{array}{c}3.87161 \\
02 \\
\end{array}$ & $\begin{array}{c}2.7489 \\
998 \\
\end{array}$ & $\begin{array}{c}4.3954 \\
32 \\
\end{array}$ & $\begin{array}{c}5.069 \\
179 \\
\end{array}$ & $\begin{array}{c}5.840 \\
756 \\
\end{array}$ & $\begin{array}{l}6.601 \\
1386 \\
\end{array}$ \\
\hline ENSG00000247315 & 1.61 & AL034548.1 & chr20 & 278214 & 280961 & + & $\begin{array}{c}3.02354 \\
24 \\
\end{array}$ & $\begin{array}{c}2.0685 \\
76 \\
\end{array}$ & $\begin{array}{c}2.8194 \\
673 \\
\end{array}$ & $\begin{array}{c}4.320 \\
936 \\
\end{array}$ & $\begin{array}{l}4.353 \\
7407 \\
\end{array}$ & $\begin{array}{l}3.915 \\
0994 \\
\end{array}$ \\
\hline ENSG00000251260 & 1.61 & WDFY3-AS1 & chr4 & 85724411 & 85731544 & + & $\begin{array}{c}1.00389 \\
2 \\
\end{array}$ & $\begin{array}{c}1.4533 \\
712 \\
\end{array}$ & $\begin{array}{c}2.2153 \\
144 \\
\end{array}$ & $\begin{array}{l}3.214 \\
5927 \\
\end{array}$ & $\begin{array}{l}1.323 \\
8585 \\
\end{array}$ & $\begin{array}{c}3.147 \\
409 \\
\end{array}$ \\
\hline ENSG00000250182 & 1.60 & EEF1A1P13 & chr5 & 14652047 & 14653438 & - & 9.46529 & $\begin{array}{c}6.2075 \\
24 \\
\end{array}$ & $\begin{array}{c}6.9742 \\
58 \\
\end{array}$ & $\begin{array}{l}12.66 \\
7569 \\
\end{array}$ & $\begin{array}{r}11.56 \\
31275 \\
\end{array}$ & $\begin{array}{l}11.53 \\
3177 \\
\end{array}$ \\
\hline ENSG00000251143 & 1.60 & RP11-849H4.4 & chr11 & 71725337 & 71731956 & + & $\begin{array}{c}3.97319 \\
3 \\
\end{array}$ & $\begin{array}{c}6.8831 \\
76 \\
\end{array}$ & $\begin{array}{c}5.2716 \\
713\end{array}$ & $\begin{array}{c}9.131 \\
103 \\
\end{array}$ & $\begin{array}{c}7.891 \\
925 \\
\end{array}$ & $\begin{array}{c}8.129 \\
692 \\
\end{array}$ \\
\hline ENSG00000272888 & 1.59 & AC013394.2 & chr15 & 93425937 & 93441975 & + & $\begin{array}{c}0.84985 \\
04 \\
\end{array}$ & $\begin{array}{c}1.4388 \\
658 \\
\end{array}$ & $\begin{array}{c}1.8384 \\
619 \\
\end{array}$ & $\begin{array}{l}.750 \\
0532 \\
\end{array}$ & $\begin{array}{l}2.498 \\
7977 \\
\end{array}$ & $\begin{array}{l}1.538 \\
8129 \\
\end{array}$ \\
\hline ENSG00000173295 & 1.58 & FAM86B3P & chr8 & 8086117 & 8102387 & + & $\begin{array}{c}0.99347 \\
425 \\
\end{array}$ & $\begin{array}{c}0.8375 \\
4015 \\
\end{array}$ & $\begin{array}{c}1.0657 \\
19 \\
\end{array}$ & $\begin{array}{l}1.652 \\
3628 \\
\end{array}$ & $\begin{array}{l}1.481 \\
0578 \\
\end{array}$ & $\begin{array}{l}1.727 \\
7136 \\
\end{array}$ \\
\hline ENSG00000271581 & 1.58 & $\begin{array}{c}\text { XXbac- } \\
\text { BPG248L24.12 } \\
\end{array}$ & chr6 & 31324424 & 31325414 & + & $\begin{array}{c}10.9642 \\
39 \\
\end{array}$ & $\begin{array}{c}42.648 \\
693 \\
\end{array}$ & $\begin{array}{c}30.244 \\
503 \\
\end{array}$ & $\begin{array}{c}26.87 \\
288 \\
\end{array}$ & $\begin{array}{c}45.12 \\
537 \\
\end{array}$ & $\begin{array}{l}46.04 \\
9137 \\
\end{array}$ \\
\hline ENSG00000234694 & 1.58 & RP1-92014.3 & chr1 & 43820355 & 43824329 & - & $\begin{array}{c}1.44880 \\
64\end{array}$ & $\begin{array}{c}2.5090 \\
265 \\
\end{array}$ & $\begin{array}{c}1.8279 \\
302\end{array}$ & $\begin{array}{l}3.333 \\
9834 \\
\end{array}$ & $\begin{array}{l}4.057 \\
3297\end{array}$ & $\begin{array}{l}1.937 \\
6101 \\
\end{array}$ \\
\hline ENSG00000231970 & 1.58 & $\begin{array}{c}\text { RP11- } \\
452 \mathrm{~K} 12.7 \\
\end{array}$ & chr10 & 99160872 & 99179281 & + & $\begin{array}{c}4.40716 \\
4 \\
\end{array}$ & $\begin{array}{c}1.8063 \\
688 \\
\end{array}$ & $\begin{array}{c}1.6439 \\
916 \\
\end{array}$ & $\begin{array}{l}2.576 \\
6385 \\
\end{array}$ & $\begin{array}{c}4.841 \\
898 \\
\end{array}$ & $\begin{array}{l}4.122 \\
6187 \\
\end{array}$ \\
\hline ENSG00000258824 & 1.58 & $\begin{array}{c}\text { CTD- } \\
2555016.2\end{array}$ & chr14 & 64889653 & 64915275 & - & $\begin{array}{c}0.54871 \\
875\end{array}$ & $\begin{array}{c}0.3577 \\
88\end{array}$ & $\begin{array}{c}0.8857 \\
678\end{array}$ & $\begin{array}{l}2.461 \\
6747\end{array}$ & $\begin{array}{l}1.143 \\
732\end{array}$ & $\begin{array}{l}1.395 \\
1521\end{array}$ \\
\hline ENSG00000224886 & 1.58 & BEND3P3 & chr10 & 81444250 & 81444739 & - & $\begin{array}{c}0.14750 \\
265 \\
\end{array}$ & $\begin{array}{c}1.2424 \\
577 \\
\end{array}$ & $\begin{array}{c}0.5076 \\
731 \\
\end{array}$ & $\begin{array}{l}1.309 \\
9444 \\
\end{array}$ & $\begin{array}{l}0.603 \\
35094 \\
\end{array}$ & $\begin{array}{l}3.709 \\
9042 \\
\end{array}$ \\
\hline ENSG00000259623 & 1.57 & RP11-156E6.1 & chr17 & 40004770 & 40007699 & - & $\begin{array}{c}1.19021 \\
55 \\
\end{array}$ & $\begin{array}{c}0.7809 \\
776 \\
\end{array}$ & $\begin{array}{c}1.3159 \\
648 \\
\end{array}$ & $\begin{array}{l}2.417 \\
0635 \\
\end{array}$ & $\begin{array}{l}1.441 \\
4529 \\
\end{array}$ & $\begin{array}{l}1.755 \\
5282 \\
\end{array}$ \\
\hline
\end{tabular}




\begin{tabular}{|c|c|c|c|c|c|c|c|c|c|c|c|c|}
\hline ENSG00000214184 & 1.57 & AC012487.2 & chr2 & 109123971 & 109150652 & - & $\begin{array}{c}1.13318 \\
16 \\
\end{array}$ & $\begin{array}{c}1.5004 \\
897 \\
\end{array}$ & $\begin{array}{c}1.6879 \\
808 \\
\end{array}$ & $\begin{array}{l}2.188 \\
2014 \\
\end{array}$ & $\begin{array}{l}1.909 \\
4998 \\
\end{array}$ & $\begin{array}{c}2.645 \\
182 \\
\end{array}$ \\
\hline ENSG00000231864 & 1.57 & $\begin{array}{c}\text { RP11- } \\
\text { 229P13.23 }\end{array}$ & chr9 & 139957987 & 139959033 & + & $\begin{array}{c}2.14776 \\
64\end{array}$ & $\begin{array}{c}0.5192 \\
181\end{array}$ & $\begin{array}{c}1.6303 \\
065\end{array}$ & $\begin{array}{l}3.468 \\
3156\end{array}$ & $\begin{array}{l}2.224 \\
2556\end{array}$ & $\begin{array}{l}1.746 \\
7672\end{array}$ \\
\hline ENSG00000259948 & 1.56 & $\begin{array}{c}\text { RP11- } \\
326 A 19.5\end{array}$ & chr15 & 89744322 & 89744999 & - & 0 & 0 & 0 & 0 & $\begin{array}{l}2.250 \\
7296 \\
\end{array}$ & $\begin{array}{l}1.690 \\
6314 \\
\end{array}$ \\
\hline ENSG00000203875 & 1.56 & SNHG5 & chr6 & 86370710 & 86388451 & - & $\begin{array}{c}102.081 \\
635\end{array}$ & $\begin{array}{c}49.028 \\
21\end{array}$ & $\begin{array}{c}72.932 \\
594\end{array}$ & $\begin{array}{l}136.5 \\
7454\end{array}$ & $\begin{array}{l}115.0 \\
8786\end{array}$ & $\begin{array}{c}87.77 \\
891\end{array}$ \\
\hline ENSG00000262944 & 1.56 & RP11-473I1.9 & chr16 & 9198705 & 9200618 & + & $\begin{array}{c}4.37061 \\
83\end{array}$ & $\begin{array}{c}3.5058 \\
978\end{array}$ & $\begin{array}{c}5.2434 \\
583\end{array}$ & $\begin{array}{c}9.294 \\
138\end{array}$ & $\begin{array}{c}6.550 \\
2\end{array}$ & $\begin{array}{l}4.981 \\
1077\end{array}$ \\
\hline ENSG00000223361 & 1.56 & FTH1P10 & chr5 & 17353804 & 17354733 & - & $\begin{array}{c}1.12084 \\
73\end{array}$ & $\begin{array}{c}1.9096 \\
965\end{array}$ & $\begin{array}{c}3.1168 \\
904\end{array}$ & $\begin{array}{l}3.494 \\
8602\end{array}$ & $\begin{array}{l}3.568 \\
4345\end{array}$ & $\begin{array}{l}2.014 \\
9157\end{array}$ \\
\hline ENSG00000234797 & 1.55 & RPS3AP6 & chr15 & 60060543 & 60061347 & + & $\begin{array}{c}8.03755 \\
8\end{array}$ & $\begin{array}{c}4.6485 \\
057\end{array}$ & $\begin{array}{c}6.0571 \\
284\end{array}$ & $\begin{array}{c}10.87 \\
507\end{array}$ & $\begin{array}{c}9.480 \\
915\end{array}$ & $\begin{array}{c}8.230 \\
892\end{array}$ \\
\hline ENSG00000186831 & 1.55 & KRT17P2 & chr17 & 18330175 & 18335162 & + & $\begin{array}{c}2.67693 \\
57 \\
\end{array}$ & $\begin{array}{c}5.9414 \\
525 \\
\end{array}$ & $\begin{array}{c}8.2313 \\
9 \\
\end{array}$ & $\begin{array}{c}8.073 \\
757 \\
\end{array}$ & $\begin{array}{l}7.960 \\
8884 \\
\end{array}$ & $\begin{array}{l}7.615 \\
3316 \\
\end{array}$ \\
\hline ENSG00000235298 & 1.55 & RP11-575L7.8 & chr9 & 86587148 & 86590692 & + & $\begin{array}{c}0.99603 \\
415\end{array}$ & $\begin{array}{c}0.1577 \\
4745\end{array}$ & $\begin{array}{c}1.2684 \\
056\end{array}$ & $\begin{array}{l}1.084 \\
6498\end{array}$ & $\begin{array}{l}1.106 \\
6335\end{array}$ & $\begin{array}{l}3.923 \\
4712\end{array}$ \\
\hline ENSG00000244398 & 1.55 & $\begin{array}{c}\text { RP11- } \\
466 \mathrm{H} 18.1 \\
\end{array}$ & chr11 & 16996240 & 16996560 & - & $\begin{array}{c}210.415 \\
99 \\
\end{array}$ & $\begin{array}{c}118.90 \\
0734 \\
\end{array}$ & $\begin{array}{c}159.80 \\
867 \\
\end{array}$ & $\begin{array}{c}323.5 \\
744 \\
\end{array}$ & $\begin{array}{l}266.5 \\
1407 \\
\end{array}$ & $\begin{array}{l}171.5 \\
4987 \\
\end{array}$ \\
\hline ENSG00000232956 & 1.54 & SNHG15 & chr7 & 45022622 & 45026560 & - & $\begin{array}{c}4.96357 \\
63 \\
\end{array}$ & $\begin{array}{c}4.2213 \\
054 \\
\end{array}$ & $\begin{array}{c}6.0738 \\
254 \\
\end{array}$ & $\begin{array}{l}7.899 \\
4136 \\
\end{array}$ & $\begin{array}{l}7.568 \\
8367 \\
\end{array}$ & $\begin{array}{c}7.776 \\
898 \\
\end{array}$ \\
\hline ENSG00000265688 & 1.54 & MAFG-AS1 & chr17 & 79885705 & 79888629 & + & $\begin{array}{c}2.63169 \\
65 \\
\end{array}$ & $\begin{array}{c}1.0524 \\
323 \\
\end{array}$ & $\begin{array}{c}1.6549 \\
61 \\
\end{array}$ & $\begin{array}{l}2.523 \\
4575 \\
\end{array}$ & $\begin{array}{l}3.253 \\
9558 \\
\end{array}$ & $\begin{array}{l}2.027 \\
5886 \\
\end{array}$ \\
\hline ENSG00000205746 & 1.54 & $\begin{array}{c}\text { RP11- } \\
1212 A 22.1\end{array}$ & chr16 & 18428257 & 18488396 & - & $\begin{array}{c}2.70093 \\
97\end{array}$ & $\begin{array}{c}1.9503 \\
261\end{array}$ & $\begin{array}{c}2.9362 \\
893\end{array}$ & $\begin{array}{c}3.497 \\
657\end{array}$ & $\begin{array}{c}3.365 \\
603\end{array}$ & $\begin{array}{l}4.758 \\
7376 \\
\end{array}$ \\
\hline ENSG00000205763 & 1.53 & RP9P & chr7 & 32956427 & 32982788 & - & $\begin{array}{c}1.58876 \\
7\end{array}$ & 2.6736 & $\begin{array}{c}.2468 \\
457\end{array}$ & $\begin{array}{c}3.486 \\
325\end{array}$ & $\begin{array}{l}3.794 \\
6262\end{array}$ & $\begin{array}{l}4.901 \\
2694 \\
\end{array}$ \\
\hline ENSG00000260107 & 1.53 & AC005606.15 & chr16 & 2047655 & 2048375 & + & $\begin{array}{c}9.51927 \\
2 \\
\end{array}$ & $\begin{array}{c}5.6106 \\
744 \\
\end{array}$ & $\begin{array}{c}7.2765 \\
503 \\
\end{array}$ & $\begin{array}{c}8.348 \\
566 \\
\end{array}$ & $\begin{array}{l}12.49 \\
4355 \\
\end{array}$ & $\begin{array}{l}13.30 \\
5827 \\
\end{array}$ \\
\hline ENSG00000232445 & 1.53 & RP11-132A1.4 & chr7 & 100951627 & 100954266 & + & $\begin{array}{c}6.63814 \\
2 \\
\end{array}$ & $\begin{array}{c}1.0719 \\
961 \\
\end{array}$ & $\begin{array}{c}3.6041 \\
183 \\
\end{array}$ & $\begin{array}{c}4.977 \\
168 \\
\end{array}$ & $\begin{array}{l}4.163 \\
5494 \\
\end{array}$ & $\begin{array}{c}4.395 \\
196 \\
\end{array}$ \\
\hline ENSG00000204194 & 1.52 & RPL12P1 & chr6 & 33367836 & 33368333 & - & $\begin{array}{c}8.81644 \\
3 \\
\end{array}$ & $\begin{array}{c}4.4001 \\
51 \\
\end{array}$ & $\begin{array}{c}10.382 \\
515 \\
\end{array}$ & $\begin{array}{c}8.702 \\
698 \\
\end{array}$ & $\begin{array}{l}12.67 \\
3401 \\
\end{array}$ & $\begin{array}{l}12.93 \\
3394 \\
\end{array}$ \\
\hline ENSG00000254473 & 1.52 & RP11-522I20.3 & chr9 & 86322509 & 86328293 & + & $\begin{array}{c}0.62335 \\
21 \\
\end{array}$ & $\begin{array}{c}0.6170 \\
2996 \\
\end{array}$ & $\begin{array}{c}0.9024 \\
317 \\
\end{array}$ & $\begin{array}{l}2.064 \\
1985 \\
\end{array}$ & $\begin{array}{l}1.439 \\
8504 \\
\end{array}$ & $\begin{array}{c}1.178 \\
33\end{array}$ \\
\hline ENSG00000261971 & 1.52 & $\begin{array}{c}\text { RP11- } \\
473 \mathrm{M} 20.7 \\
\end{array}$ & chr16 & 3101992 & 3109371 & - & $\begin{array}{c}0.38817 \\
36 \\
\end{array}$ & $\begin{array}{c}0.9558 \\
703 \\
\end{array}$ & $\begin{array}{c}1.3753 \\
506 \\
\end{array}$ & $\begin{array}{l}1.559 \\
6504 \\
\end{array}$ & $\begin{array}{l}1.193 \\
0581 \\
\end{array}$ & $\begin{array}{l}2.581 \\
4805 \\
\end{array}$ \\
\hline ENSG00000235725 & 1.51 & AC007389.3 & chr2 & 65816700 & 65867311 & - & $\begin{array}{c}0.91346 \\
866\end{array}$ & $\begin{array}{c}0.7893 \\
489\end{array}$ & $\begin{array}{c}1.0001 \\
628\end{array}$ & $\begin{array}{l}1.792 \\
3195\end{array}$ & $\begin{array}{c}1.434 \\
173\end{array}$ & $\begin{array}{l}1.351 \\
0778 \\
\end{array}$ \\
\hline ENSG00000229212 & 1.51 & RP11-561C5.4 & chr15 & 85747141 & 85778026 & - & $\begin{array}{c}1.54617 \\
49\end{array}$ & $\begin{array}{c}2.1187 \\
642\end{array}$ & $\begin{array}{c}2.2953 \\
222\end{array}$ & $\begin{array}{l}2.896 \\
4856\end{array}$ & $\begin{array}{c}2.832 \\
387\end{array}$ & $\begin{array}{l}3.181 \\
3629 \\
\end{array}$ \\
\hline ENSG00000259328 & 1.51 & $\begin{array}{c}\text { RP11- } \\
\text { 152F13.7 } \\
\end{array}$ & chr15 & 82944773 & 82974312 & + & $\begin{array}{c}1.00161 \\
61 \\
\end{array}$ & $\begin{array}{c}1.3405 \\
782 \\
\end{array}$ & $\begin{array}{c}1.1754 \\
336 \\
\end{array}$ & $\begin{array}{l}1.238 \\
5976 \\
\end{array}$ & $\begin{array}{l}1.825 \\
4333 \\
\end{array}$ & $\begin{array}{c}2.403 \\
051 \\
\end{array}$ \\
\hline ENSG00000223396 & 1.51 & RPS10P7 & chr1 & 201487831 & 201499602 & + & $\begin{array}{c}3.12924 \\
72 \\
\end{array}$ & $\begin{array}{c}1.9355 \\
738 \\
\end{array}$ & $\begin{array}{c}2.9419 \\
267 \\
\end{array}$ & $\begin{array}{l}4.337 \\
2602 \\
\end{array}$ & $\begin{array}{c}4.088 \\
839 \\
\end{array}$ & $\begin{array}{l}3.454 \\
8473 \\
\end{array}$ \\
\hline ENSG00000269032 & 1.51 & AC016629.7 & chr19 & 59097525 & 59097723 & + & $\begin{array}{c}0.32712 \\
79 \\
\end{array}$ & $\begin{array}{c}0.8532 \\
135 \\
\end{array}$ & $\begin{array}{c}0.6416 \\
285 \\
\end{array}$ & 0 & $\begin{array}{l}2.120 \\
2471 \\
\end{array}$ & $\begin{array}{l}1.611 \\
3639 \\
\end{array}$ \\
\hline ENSG00000203394 & 1.51 & RP5-930J4.4 & chr1 & 21069480 & 21070455 & + & $\begin{array}{c}4.33596 \\
37 \\
\end{array}$ & $\begin{array}{c}2.2852 \\
726 \\
\end{array}$ & $\begin{array}{c}2.7163 \\
084 \\
\end{array}$ & $\begin{array}{l}5.946 \\
6834 \\
\end{array}$ & $\begin{array}{l}4.018 \\
1866 \\
\end{array}$ & $\begin{array}{l}3.844 \\
3263 \\
\end{array}$ \\
\hline ENSG00000225151 & 1.50 & AC103965.1 & chr15 & 84867600 & 84898888 & - & $\begin{array}{c}7.19354 \\
3 \\
\end{array}$ & $\begin{array}{c}9.0674 \\
305 \\
\end{array}$ & $\begin{array}{c}8.6491 \\
41 \\
\end{array}$ & \begin{tabular}{|l|}
13.35 \\
8168 \\
\end{tabular} & $\begin{array}{l}12.31 \\
3677 \\
\end{array}$ & $\begin{array}{l}11.67 \\
2823 \\
\end{array}$ \\
\hline ENSG00000244257 & 1.50 & PKD1P1 & chr16 & 16404198 & 16428047 & + & $\begin{array}{c}2.76064 \\
6 \\
\end{array}$ & $\begin{array}{c}1.8415 \\
892 \\
\end{array}$ & $\begin{array}{c}2.8608 \\
568\end{array}$ & $\begin{array}{l}3.244 \\
7884 \\
\end{array}$ & $\begin{array}{l}3.438 \\
1177 \\
\end{array}$ & $\begin{array}{l}4.434 \\
0997 \\
\end{array}$ \\
\hline ENSG00000267580 & 1.50 & $\begin{array}{c}\text { CTD- } \\
2540 B 15.11 \\
\end{array}$ & chr19 & 33790840 & 33792074 & + & $\begin{array}{c}1.09239 \\
59 \\
\end{array}$ & $\begin{array}{c}2.0792 \\
82 \\
\end{array}$ & $\begin{array}{c}1.7315 \\
762 \\
\end{array}$ & $\begin{array}{l}2.528 \\
3904 \\
\end{array}$ & $\begin{array}{l}2.831 \\
5682 \\
\end{array}$ & $\begin{array}{l}1.866 \\
6612 \\
\end{array}$ \\
\hline ENSG00000267151 & 1.50 & MIR2117 & chr17 & 41522075 & 41528568 & + & $\begin{array}{c}1.46409 \\
5 \\
\end{array}$ & $\begin{array}{c}1.9914 \\
546\end{array}$ & $\begin{array}{c}1.7382 \\
286\end{array}$ & $\begin{array}{c}2.071 \\
921\end{array}$ & $\begin{array}{c}3.630 \\
837\end{array}$ & $\begin{array}{l}2.286 \\
1967 \\
\end{array}$ \\
\hline ENSG00000270055 & 1.50 & $\begin{array}{c}\text { CTD- } \\
\text { 3092A11.2 } \\
\end{array}$ & chr15 & 30780166 & 30782516 & + & $\begin{array}{c}1.17078 \\
79 \\
\end{array}$ & $\begin{array}{c}0.9375 \\
974 \\
\end{array}$ & $\begin{array}{c}1.4183 \\
607 \\
\end{array}$ & $\begin{array}{l}2.055 \\
2423 \\
\end{array}$ & $\begin{array}{l}1.595 \\
0989 \\
\end{array}$ & $\begin{array}{l}1.714 \\
8618 \\
\end{array}$ \\
\hline ENSG00000228989 & 1.50 & AC133528.2 & chr2 & 242629829 & 242633704 & + & $\begin{array}{c}1.82867 \\
69\end{array}$ & $\begin{array}{c}1.5681 \\
707\end{array}$ & $\begin{array}{c}0.6850 \\
529\end{array}$ & $\begin{array}{l}3.815 \\
1476\end{array}$ & $\begin{array}{c}2.544 \\
251\end{array}$ & $\begin{array}{l}0.348 \\
43844\end{array}$ \\
\hline ENSG00000262791 & 1.50 & RP11- & chr17 & 1629042 & 1641879 & - & 0.61560 & 0.1723 & 0.3134 & 1.525 & 0.680 & 2.214 \\
\hline
\end{tabular}




\begin{tabular}{|c|c|c|c|c|c|c|c|c|c|c|c|c|}
\hline & & 961A15.1 & & & & & 89 & 9359 & 515 & 6633 & 255 & 0858 \\
\hline ENSG00000248092 & 1.50 & NNT-AS1 & chr5 & 43571696 & 43603332 & - & $\begin{array}{c}2.29409 \\
2 \\
\end{array}$ & $\begin{array}{c}2.9645 \\
55 \\
\end{array}$ & $\begin{array}{c}2.8210 \\
022 \\
\end{array}$ & $\begin{array}{c}4.673 \\
5754 \\
\end{array}$ & $\begin{array}{c}3.726 \\
381\end{array}$ & $\begin{array}{l}3.718 \\
8199 \\
\end{array}$ \\
\hline ENSG00000270232 & -1.51 & RP11-475J5.7 & chr5 & 99381632 & 99382078 & - & $\begin{array}{c}14.4310 \\
055\end{array}$ & $\begin{array}{c}8.3597 \\
29\end{array}$ & $\begin{array}{c}7.1948 \\
752 \\
\end{array}$ & $\begin{array}{c}7.179 \\
785\end{array}$ & $\begin{array}{l}5.739 \\
9325\end{array}$ & $\begin{array}{c}6.123 \\
285 \\
\end{array}$ \\
\hline ENSG00000255125 & -1.51 & $\begin{array}{c}\text { RP11- } \\
685 \mathrm{M} 7.5 \\
\end{array}$ & chr11 & 10804860 & 10823172 & + & $\begin{array}{c}2.00767 \\
5 \\
\end{array}$ & $\begin{array}{c}1.9517 \\
535 \\
\end{array}$ & $\begin{array}{c}4.4939 \\
42 \\
\end{array}$ & $\begin{array}{l}1.263 \\
7898 \\
\end{array}$ & $\begin{array}{l}0.861 \\
9299 \\
\end{array}$ & $\begin{array}{l}4.028 \\
4314 \\
\end{array}$ \\
\hline ENSG00000265415 & -1.52 & CTD-2510F5.4 & chr17 & 57280038 & 57281190 & - & $\begin{array}{c}2.19017 \\
36 \\
\end{array}$ & $\begin{array}{c}1.3541 \\
911 \\
\end{array}$ & $\begin{array}{c}1.1740 \\
087 \\
\end{array}$ & $\begin{array}{l}0.709 \\
3079 \\
\end{array}$ & $\begin{array}{l}0.546 \\
59224 \\
\end{array}$ & $\begin{array}{l}0.912 \\
0858 \\
\end{array}$ \\
\hline ENSG00000232358 & -1.52 & $\begin{array}{c}\text { RP5- } \\
955 \mathrm{M} 13.4 \\
\end{array}$ & chr20 & 49615907 & 49626556 & + & $\begin{array}{c}10.9595 \\
83 \\
\end{array}$ & $\begin{array}{c}13.578 \\
551 \\
\end{array}$ & $\begin{array}{c}15.932 \\
661 \\
\end{array}$ & $\begin{array}{l}5.993 \\
6986 \\
\end{array}$ & $\begin{array}{l}10.71 \\
9287 \\
\end{array}$ & $\begin{array}{l}10.58 \\
0886 \\
\end{array}$ \\
\hline ENSG00000231298 & -1.52 & LINC00704 & chr10 & 4692377 & 4720346 & - & $\begin{array}{c}3.50088 \\
14\end{array}$ & $\begin{array}{c}0.8419 \\
1686\end{array}$ & $\begin{array}{c}0.5089 \\
952\end{array}$ & $\begin{array}{c}0.122 \\
57986 \\
5 \\
\end{array}$ & 0 & $\begin{array}{l}0.204 \\
42243\end{array}$ \\
\hline ENSG00000269834 & -1.52 & $\begin{array}{c}\text { CTD- } \\
3018017.3 \\
\end{array}$ & chr19 & 52892095 & 52901019 & - & $\begin{array}{c}0.42566 \\
574 \\
\end{array}$ & $\begin{array}{c}3.0581 \\
512 \\
\end{array}$ & $\begin{array}{c}1.1532 \\
478 \\
\end{array}$ & $\begin{array}{l}0.938 \\
63595 \\
\end{array}$ & $\begin{array}{l}0.609 \\
15244 \\
\end{array}$ & $\begin{array}{l}0.861 \\
5535 \\
\end{array}$ \\
\hline ENSG00000228649 & -1.53 & AC005682.5 & chr7 & 22893797 & 22901021 & + & $\begin{array}{c}2.84680 \\
3 \\
\end{array}$ & $\begin{array}{c}1.1739 \\
081 \\
\end{array}$ & $\begin{array}{c}1.4650 \\
004 \\
\end{array}$ & $\begin{array}{l}1.375 \\
8489\end{array}$ & $\begin{array}{l}0.801 \\
59485 \\
\end{array}$ & $\begin{array}{l}0.919 \\
04426 \\
\end{array}$ \\
\hline ENSG00000250234 & -1.53 & $\begin{array}{c}\text { CTD- } \\
2024 \mathrm{P} 10.1 \\
\end{array}$ & chr5 & 34656517 & 34657355 & - & $\begin{array}{c}2.84991 \\
8 \\
\end{array}$ & $\begin{array}{c}3.6838 \\
043 \\
\end{array}$ & $\begin{array}{c}2.7612 \\
894 \\
\end{array}$ & $\begin{array}{l}1.368 \\
9165 \\
\end{array}$ & $\begin{array}{l}1.759 \\
3302 \\
\end{array}$ & $\begin{array}{l}3.366 \\
3306 \\
\end{array}$ \\
\hline ENSG00000260549 & -1.53 & MT1L & chr16 & 56651388 & 56652730 & + & $\begin{array}{c}32.0605 \\
55 \\
\end{array}$ & $\begin{array}{c}7.0488 \\
276 \\
\end{array}$ & $\begin{array}{c}13.661 \\
485 \\
\end{array}$ & $\begin{array}{c}8.433 \\
363 \\
\end{array}$ & $\begin{array}{c}8.580 \\
506 \\
\end{array}$ & $\begin{array}{l}11.88 \\
6822 \\
\end{array}$ \\
\hline ENSG00000269983 & -1.53 & $\begin{array}{c}\text { RP11- } \\
497 \mathrm{H} 16.9 \\
\end{array}$ & chr5 & 69745463 & 69746180 & + & $\begin{array}{c}2.89407 \\
18 \\
\end{array}$ & $\begin{array}{c}2.6753 \\
237 \\
\end{array}$ & $\begin{array}{c}1.4972 \\
115 \\
\end{array}$ & $\begin{array}{l}1.057 \\
8682 \\
\end{array}$ & $\begin{array}{c}2.014 \\
671 \\
\end{array}$ & $\begin{array}{c}1.510 \\
466 \\
\end{array}$ \\
\hline ENSG00000232656 & -1.54 & IDI2-AS1 & chr10 & 1068606 & 1090138 & + & 8.97962 & $\begin{array}{c}2.5590 \\
303 \\
\end{array}$ & $\begin{array}{c}4.5844 \\
545 \\
\end{array}$ & $\begin{array}{l}4.037 \\
7965 \\
\end{array}$ & $\begin{array}{l}2.612 \\
7467 \\
\end{array}$ & $\begin{array}{l}2.731 \\
6613 \\
\end{array}$ \\
\hline ENSG00000273132 & -1.54 & $\begin{array}{c}\text { RP11- } \\
\text { 350J20.12 } \\
\end{array}$ & chr6 & 150173598 & 150174328 & + & $\begin{array}{c}4.93389 \\
4 \\
\end{array}$ & $\begin{array}{c}3.8961 \\
434 \\
\end{array}$ & $\begin{array}{c}1.3101 \\
063 \\
\end{array}$ & $\begin{array}{l}0.214 \\
81651 \\
\end{array}$ & $\begin{array}{l}2.748 \\
0006 \\
\end{array}$ & $\begin{array}{l}2.503 \\
8617 \\
\end{array}$ \\
\hline ENSG00000262413 & -1.54 & RP11-498C9.3 & chr17 & 79825597 & 79826428 & + & $\begin{array}{c}131.380 \\
23 \\
\end{array}$ & $\begin{array}{c}83.971 \\
855 \\
\end{array}$ & $\begin{array}{c}65.720 \\
39 \\
\end{array}$ & $\begin{array}{l}57.11 \\
3407 \\
\end{array}$ & $\begin{array}{c}68.61 \\
178 \\
\end{array}$ & $\begin{array}{c}50.39 \\
401 \\
\end{array}$ \\
\hline ENSG00000233144 & -1.55 & RP11-537A6.9 & chr10 & 75141191 & 75143254 & + & $\begin{array}{c}1.73480 \\
49 \\
\end{array}$ & $\begin{array}{c}1.7642 \\
145 \\
\end{array}$ & $\begin{array}{c}2.0247 \\
85 \\
\end{array}$ & $\begin{array}{l}0.579 \\
00864 \\
\end{array}$ & $\begin{array}{l}1.376 \\
7893 \\
\end{array}$ & $\begin{array}{l}1.206 \\
9995 \\
\end{array}$ \\
\hline ENSG00000226963 & -1.55 & AC078883.4 & chr2 & 173292502 & 173293331 & - & 3.65149 & $\begin{array}{c}3.3694 \\
608 \\
\end{array}$ & $\begin{array}{c}3.6925 \\
917 \\
\end{array}$ & $\begin{array}{l}2.460 \\
7828 \\
\end{array}$ & $\begin{array}{l}1.833 \\
3695 \\
\end{array}$ & $\begin{array}{l}2.698 \\
5145 \\
\end{array}$ \\
\hline ENSG00000234327 & -1.55 & AC012146.7 & chr17 & 5014763 & 5017674 & + & $\begin{array}{c}1.95390 \\
83 \\
\end{array}$ & $\begin{array}{c}2.4199 \\
314 \\
\end{array}$ & $\begin{array}{c}1.5286 \\
9 \\
\end{array}$ & $\begin{array}{l}0.984 \\
7564 \\
\end{array}$ & $\begin{array}{c}1.408 \\
148 \\
\end{array}$ & $\begin{array}{c}1.369 \\
486 \\
\end{array}$ \\
\hline ENSG00000259153 & -1.56 & RP6-65G23.3 & chr14 & 71276922 & 71282120 & + & $\begin{array}{c}1.99365 \\
48 \\
\end{array}$ & $\begin{array}{c}2.7267 \\
768 \\
\end{array}$ & $\begin{array}{c}1.2656 \\
376 \\
\end{array}$ & $\begin{array}{c}1.045 \\
882 \\
\end{array}$ & $\begin{array}{l}0.530 \\
8814 \\
\end{array}$ & $\begin{array}{l}1.731 \\
0688 \\
\end{array}$ \\
\hline ENSG00000258791 & -1.58 & LINC00520 & chr14 & 56247864 & 56263406 & - & $\begin{array}{c}0.64874 \\
68 \\
\end{array}$ & $\begin{array}{c}2.9121 \\
616 \\
\end{array}$ & $\begin{array}{c}2.3695 \\
24 \\
\end{array}$ & $\begin{array}{c}0.322 \\
98782 \\
\end{array}$ & $\begin{array}{l}1.232 \\
0567 \\
\end{array}$ & $\begin{array}{l}1.418 \\
7404 \\
\end{array}$ \\
\hline ENSG00000267776 & -1.58 & AC006116.24 & chr19 & 56888073 & 56888653 & - & $\begin{array}{c}2.15047 \\
86 \\
\end{array}$ & $\begin{array}{c}3.7224 \\
712 \\
\end{array}$ & $\begin{array}{c}2.8212 \\
99 \\
\end{array}$ & $\begin{array}{l}1.832 \\
4789 \\
\end{array}$ & $\begin{array}{l}1.608 \\
6241 \\
\end{array}$ & $\begin{array}{l}1.933 \\
2796 \\
\end{array}$ \\
\hline ENSG00000257181 & -1.58 & RP11-61102.5 & chr12 & 69235068 & 69237017 & - & $\begin{array}{c}5.82705 \\
8 \\
\end{array}$ & $\begin{array}{c}7.4925 \\
43 \\
\end{array}$ & $\begin{array}{c}6.0171 \\
41 \\
\end{array}$ & $\begin{array}{c}4.169 \\
096 \\
\end{array}$ & $\begin{array}{l}3.725 \\
6134 \\
\end{array}$ & $\begin{array}{l}4.259 \\
7303 \\
\end{array}$ \\
\hline ENSG00000267774 & -1.58 & RP11-2N1.2 & chr18 & 57363691 & 57364644 & + & 4.9585 & $\begin{array}{c}2.7897 \\
93 \\
\end{array}$ & $\begin{array}{c}5.2442 \\
584 \\
\end{array}$ & $\begin{array}{c}1.467 \\
508 \\
\end{array}$ & $\begin{array}{l}3.439 \\
1992 \\
\end{array}$ & $\begin{array}{l}3.611 \\
8386 \\
\end{array}$ \\
\hline ENSG00000241749 & -1.59 & RPSAP52 & chr12 & 66151800 & 66220754 & - & $\begin{array}{c}3.20631 \\
31 \\
\end{array}$ & $\begin{array}{c}1.0826 \\
937 \\
\end{array}$ & $\begin{array}{c}1.4755 \\
359 \\
\end{array}$ & $\begin{array}{l}0.093 \\
51292 \\
\end{array}$ & $\begin{array}{l}0.572 \\
23415 \\
\end{array}$ & $\begin{array}{l}1.282 \\
0605 \\
\end{array}$ \\
\hline ENSG00000268854 & -1.59 & $\begin{array}{c}\text { CTD- } \\
2545 \mathrm{M} 3.2 \\
\end{array}$ & chr19 & 50983376 & 50986608 & - & $\begin{array}{c}.72710 \\
04 \\
\end{array}$ & $\begin{array}{c}2.3693 \\
736 \\
\end{array}$ & $\begin{array}{c}1.3470 \\
087 \\
\end{array}$ & $\begin{array}{c}3.467 \\
783 \\
\end{array}$ & $\begin{array}{l}1.066 \\
7965 \\
\end{array}$ & $\begin{array}{l}1.013 \\
8669 \\
\end{array}$ \\
\hline ENSG00000224818 & -1.59 & RP11-134G8.8 & chr1 & 201433511 & 201434274 & - & $\begin{array}{c}3.14244 \\
77 \\
\end{array}$ & $\begin{array}{c}7.4775 \\
295 \\
\end{array}$ & $\begin{array}{c}3.8924 \\
434 \\
\end{array}$ & $\begin{array}{l}2.826 \\
5297 \\
\end{array}$ & $\begin{array}{c}2.930 \\
994 \\
\end{array}$ & $\begin{array}{l}2.728 \\
9903 \\
\end{array}$ \\
\hline ENSG00000232815 & -1.59 & LINC00537 & chr9 & 68413482 & 68414196 & - & $\begin{array}{c}3.58854 \\
34 \\
\end{array}$ & $\begin{array}{c}3.3276 \\
02 \\
\end{array}$ & $\begin{array}{c}2.7501 \\
92 \\
\end{array}$ & $\begin{array}{l}2.034 \\
8413 \\
\end{array}$ & $\begin{array}{c}2.185 \\
565 \\
\end{array}$ & $\begin{array}{l}1.820 \\
1643 \\
\end{array}$ \\
\hline ENSG00000241494 & -1.61 & RP11-796G6.1 & chr14 & 102144280 & 102144717 & + & $\begin{array}{c}3.58287 \\
45 \\
\end{array}$ & $\begin{array}{c}1.8213 \\
323 \\
\end{array}$ & $\begin{array}{c}2.3123 \\
465 \\
\end{array}$ & $\begin{array}{l}1.848 \\
1115 \\
\end{array}$ & $\begin{array}{l}1.181 \\
2179 \\
\end{array}$ & $\begin{array}{l}1.666 \\
4281 \\
\end{array}$ \\
\hline ENSG00000258017 & -1.62 & $\begin{array}{c}\text { RP11- } \\
386 \text { G11.10 } \\
\end{array}$ & chr12 & 49521565 & 49541652 & + & $\begin{array}{c}27.8705 \\
02 \\
\end{array}$ & $\begin{array}{c}18.402 \\
964 \\
\end{array}$ & $\begin{array}{c}17.180 \\
283 \\
\end{array}$ & $\begin{array}{l}12.05 \\
1238 \\
\end{array}$ & $\begin{array}{l}12.65 \\
8115 \\
\end{array}$ & $\begin{array}{l}13.56 \\
6694 \\
\end{array}$ \\
\hline ENSG00000034063 & -1.62 & UHRF1 & chr19 & 4903092 & 4962165 & + & $\begin{array}{c}6.74429 \\
46 \\
\end{array}$ & $\begin{array}{c}1.2855 \\
517 \\
\end{array}$ & $\begin{array}{c}2.7702 \\
923 \\
\end{array}$ & $\begin{array}{l}2.075 \\
5224 \\
\end{array}$ & $\begin{array}{l}1.767 \\
3001 \\
\end{array}$ & $\begin{array}{l}1.536 \\
0543 \\
\end{array}$ \\
\hline ENSG00000254258 & -1.62 & RP11-398H6.1 & chr8 & 140472305 & 140475259 & - & $\begin{array}{c}4.54776 \\
14\end{array}$ & $\begin{array}{c}7.7839 \\
007\end{array}$ & $\begin{array}{c}6.3626 \\
404\end{array}$ & $\begin{array}{l}3.925 \\
3066\end{array}$ & $\begin{array}{l}3.584 \\
0328\end{array}$ & $\begin{array}{c}3.740 \\
049\end{array}$ \\
\hline
\end{tabular}




\begin{tabular}{|c|c|c|c|c|c|c|c|c|c|c|c|c|}
\hline ENSG00000213412 & -1.64 & HNRNPA1P33 & chr10 & 47133338 & 47133898 & - & $\begin{array}{c}14.4520 \\
4\end{array}$ & $\begin{array}{c}8.3262 \\
005\end{array}$ & $\begin{array}{c}6.1332 \\
52\end{array}$ & $\begin{array}{c}8.466 \\
77\end{array}$ & $\begin{array}{c}4.197 \\
107\end{array}$ & $\begin{array}{c}4.750 \\
102\end{array}$ \\
\hline ENSG00000126005 & -1.64 & MMP24-AS1 & chr20 & 33804265 & 33865934 & - & $\begin{array}{c}9.15239 \\
3\end{array}$ & $\begin{array}{c}7.7007 \\
318\end{array}$ & $\begin{array}{c}6.1065 \\
493\end{array}$ & $\begin{array}{c}5.459 \\
143\end{array}$ & $\begin{array}{l}5.335 \\
1436\end{array}$ & $\begin{array}{l}3.377 \\
0957\end{array}$ \\
\hline ENSG00000172965 & -1.65 & MIR4435-1HG & chr2 & 111953927 & 112252677 & - & $\begin{array}{c}3.55057 \\
67 \\
\end{array}$ & $\begin{array}{c}3.3823 \\
805 \\
\end{array}$ & $\begin{array}{c}3.6901 \\
011 \\
\end{array}$ & $\begin{array}{l}1.780 \\
8807 \\
\end{array}$ & $\begin{array}{l}2.279 \\
2993\end{array}$ & $\begin{array}{l}2.452 \\
1575 \\
\end{array}$ \\
\hline ENSG00000253161 & -1.65 & $\begin{array}{c}\text { RP11- } \\
150012.1\end{array}$ & chr8 & 37278859 & 37411701 & - & $\begin{array}{c}11.0290 \\
21\end{array}$ & $\begin{array}{c}12.441 \\
466\end{array}$ & $\begin{array}{c}9.9816 \\
79\end{array}$ & $\begin{array}{l}6.311 \\
8887\end{array}$ & $\begin{array}{c}8.075 \\
069\end{array}$ & $\begin{array}{l}6.012 \\
6433\end{array}$ \\
\hline ENSG00000261068 & -1.65 & RP11-7K24.3 & chr6 & 42059976 & 42061997 & - & $\begin{array}{c}1.63533 \\
17 \\
\end{array}$ & $\begin{array}{c}8.6070 \\
25 \\
\end{array}$ & $\begin{array}{c}3.6644 \\
359 \\
\end{array}$ & $\begin{array}{l}1.894 \\
0854 \\
\end{array}$ & $\begin{array}{l}2.506 \\
5024 \\
\end{array}$ & $\begin{array}{l}2.411 \\
0541 \\
\end{array}$ \\
\hline ENSG00000187653 & -1.66 & TMSB4XP8 & chr4 & 91759652 & 91760263 & - & $\begin{array}{c}8.07511 \\
6\end{array}$ & $\begin{array}{c}19.661 \\
818\end{array}$ & $\begin{array}{c}14.503 \\
13\end{array}$ & $\begin{array}{c}6.456 \\
596\end{array}$ & $\begin{array}{l}7.568 \\
5825\end{array}$ & $\begin{array}{l}10.31 \\
9572\end{array}$ \\
\hline ENSG00000226005 & -1.66 & $\begin{array}{c}\text { RP11- } \\
464 C 19.3\end{array}$ & chr10 & 3976711 & 3978005 & + & $\begin{array}{c}1.07008 \\
05\end{array}$ & $\begin{array}{c}1.8116 \\
003\end{array}$ & $\begin{array}{c}2.9764 \\
59\end{array}$ & $\begin{array}{l}0.289 \\
62108\end{array}$ & $\begin{array}{l}0.678 \\
89744\end{array}$ & $\begin{array}{l}1.258 \\
3201\end{array}$ \\
\hline ENSG00000250318 & -1.67 & CTA-963H5.5 & chr22 & 31049864 & 31050801 & - & $\begin{array}{c}1.83993 \\
59 \\
\end{array}$ & $\begin{array}{c}2.2982 \\
81 \\
\end{array}$ & $\begin{array}{c}1.8083 \\
909 \\
\end{array}$ & $\begin{array}{l}0.734 \\
8674 \\
\end{array}$ & $\begin{array}{l}0.672 \\
6246 \\
\end{array}$ & $\begin{array}{l}1.651 \\
6936 \\
\end{array}$ \\
\hline ENSG00000240225 & -1.67 & ZNF542 & chr19 & 56879468 & 56891197 & + & $\begin{array}{c}2.40770 \\
34 \\
\end{array}$ & $\begin{array}{c}2.6245 \\
768 \\
\end{array}$ & $\begin{array}{c}2.5352 \\
726\end{array}$ & $\begin{array}{l}1.434 \\
9543 \\
\end{array}$ & $\begin{array}{l}1.558 \\
2225 \\
\end{array}$ & $\begin{array}{l}1.541 \\
5956 \\
\end{array}$ \\
\hline ENSG00000240875 & -1.68 & LINC00886 & chr3 & 156465135 & 156534851 & - & $\begin{array}{c}8.04174 \\
8 \\
\end{array}$ & $\begin{array}{c}2.8910 \\
108 \\
\end{array}$ & $\begin{array}{c}2.4221 \\
4 \\
\end{array}$ & $\begin{array}{l}2.591 \\
8982 \\
\end{array}$ & $\begin{array}{l}2.259 \\
8019 \\
\end{array}$ & $\begin{array}{c}2.029 \\
531 \\
\end{array}$ \\
\hline ENSG00000256663 & -1.72 & $\begin{array}{c}\text { RP11- } \\
424 C 20.2\end{array}$ & chr12 & 20704524 & 20705946 & + & $\begin{array}{c}4.26013 \\
66 \\
\end{array}$ & $\begin{array}{c}0.7228 \\
8775 \\
\end{array}$ & $\begin{array}{c}1.1924 \\
789 \\
\end{array}$ & $\begin{array}{l}0.947 \\
2472 \\
\end{array}$ & $\begin{array}{l}0.934 \\
9184 \\
\end{array}$ & $\begin{array}{l}0.820 \\
1998 \\
\end{array}$ \\
\hline ENSG00000241990 & -1.72 & RP6-109B7.3 & chr22 & 46449585 & 46453090 & + & $\begin{array}{c}7.39844 \\
27 \\
\end{array}$ & $\begin{array}{c}3.9867 \\
473 \\
\end{array}$ & $\begin{array}{c}4.8413 \\
033 \\
\end{array}$ & $\begin{array}{c}3.091 \\
525 \\
\end{array}$ & $\begin{array}{l}3.469 \\
6312 \\
\end{array}$ & $\begin{array}{l}2.616 \\
5955 \\
\end{array}$ \\
\hline ENSG00000245112 & -1.74 & SMARCA5-AS1 & chr4 & 144434625 & 144435788 & - & $\begin{array}{c}0.00956 \\
0357\end{array}$ & $\begin{array}{c}5.2469 \\
225\end{array}$ & 0 & 0 & $\begin{array}{l}0.159 \\
80777\end{array}$ & 0 \\
\hline ENSG00000259330 & -1.74 & LINC00984 & chr15 & 40617417 & 40618916 & + & 4.89751 & $\begin{array}{c}3.6402 \\
433\end{array}$ & $\begin{array}{c}3.2306 \\
47\end{array}$ & $\begin{array}{l}2.593 \\
4253\end{array}$ & $\begin{array}{c}2.204 \\
161\end{array}$ & $\begin{array}{l}1.912 \\
7241\end{array}$ \\
\hline ENSG00000235385 & -1.75 & GS1-600G8.5 & chrX & 13284167 & 13321571 & - & $\begin{array}{c}4.73486 \\
38 \\
\end{array}$ & $\begin{array}{c}22.813 \\
477 \\
\end{array}$ & $\begin{array}{c}19.080 \\
942 \\
\end{array}$ & $\begin{array}{l}3.339 \\
2224 \\
\end{array}$ & $\begin{array}{r}14.33 \\
58755 \\
\end{array}$ & $\begin{array}{c}8.066 \\
61 \\
\end{array}$ \\
\hline ENSG00000261065 & -1.75 & RP11-74C13.4 & chr1 & 204100190 & 204101094 & + & $\begin{array}{c}1.99908 \\
89 \\
\end{array}$ & $\begin{array}{c}2.6730 \\
783 \\
\end{array}$ & $\begin{array}{c}0.4170 \\
3206 \\
\end{array}$ & $\begin{array}{l}0.414 \\
32646 \\
\end{array}$ & $\begin{array}{l}0.868 \\
4934 \\
\end{array}$ & 0 \\
\hline ENSG00000253618 & -1.76 & GRPEL2-AS1 & chr5 & 148727679 & 148737205 & - & $\begin{array}{c}1.68886 \\
54 \\
\end{array}$ & $\begin{array}{c}3.2317 \\
665 \\
\end{array}$ & 0 & $\begin{array}{l}0.215 \\
5406 \\
\end{array}$ & $\begin{array}{l}0.803 \\
7886 \\
\end{array}$ & 0 \\
\hline ENSG00000258232 & -1.77 & $\begin{array}{c}\text { RP11- } \\
161 \mathrm{H} 23.5\end{array}$ & chr12 & 49658939 & 49667089 & - & $\begin{array}{c}33.3097 \\
57 \\
\end{array}$ & $\begin{array}{c}28.819 \\
185 \\
\end{array}$ & $\begin{array}{c}28.912 \\
59 \\
\end{array}$ & $\begin{array}{l}14.44 \\
1423 \\
\end{array}$ & $\begin{array}{l}19.74 \\
7879 \\
\end{array}$ & $\begin{array}{l}17.62 \\
5807\end{array}$ \\
\hline ENSG00000272734 & -1.79 & ADIRF-AS1 & chr10 & 88725102 & 88731068 & - & $\begin{array}{c}5.43516 \\
25 \\
\end{array}$ & $\begin{array}{c}12.950 \\
257 \\
\end{array}$ & $\begin{array}{c}11.457 \\
844 \\
\end{array}$ & $\begin{array}{l}4.700 \\
653 \\
\end{array}$ & $\begin{array}{c}5.038 \\
381 \\
\end{array}$ & $\begin{array}{l}5.980 \\
5155 \\
\end{array}$ \\
\hline ENSG00000234546 & -1.81 & RP3-510D11.2 & chr1 & 9242263 & 9252148 & + & $\begin{array}{c}11.2780 \\
56\end{array}$ & $\begin{array}{c}4.5766 \\
697\end{array}$ & $\begin{array}{c}5.7239 \\
7 \\
\end{array}$ & $\begin{array}{l}2.883 \\
4605\end{array}$ & $\begin{array}{c}5.230 \\
489\end{array}$ & $\begin{array}{l}3.284 \\
4615 \\
\end{array}$ \\
\hline ENSG00000258908 & -1.82 & $\begin{array}{c}\text { RP11- } \\
203 M 5.8\end{array}$ & chr14 & 20942948 & 20945248 & - & $\begin{array}{c}7.68502 \\
6\end{array}$ & $\begin{array}{c}3.8135 \\
834\end{array}$ & $\begin{array}{c}4.4676 \\
06\end{array}$ & $\begin{array}{l}2.660 \\
3262\end{array}$ & $\begin{array}{l}2.985 \\
3904\end{array}$ & $\begin{array}{l}2.748 \\
8363 \\
\end{array}$ \\
\hline ENSG00000272159 & -1.83 & $\begin{array}{c}\text { RP11- } \\
\text { 350N15.6 } \\
\end{array}$ & chr8 & 38265566 & 38266260 & - & $\begin{array}{c}7.30503 \\
03 \\
\end{array}$ & $\begin{array}{c}2.4662 \\
013 \\
\end{array}$ & $\begin{array}{c}2.9248 \\
87 \\
\end{array}$ & $\begin{array}{l}1.585 \\
6465 \\
\end{array}$ & $\begin{array}{l}2.061 \\
9986 \\
\end{array}$ & $\begin{array}{l}2.634 \\
9046 \\
\end{array}$ \\
\hline ENSG00000226721 & -1.84 & EEF1DP2 & chr9 & 95599108 & 95599950 & - & 0 & $\begin{array}{c}6.2755 \\
63 \\
\end{array}$ & 0 & 0 & 0 & 0 \\
\hline ENSG00000204556 & -1.86 & CTD-2514C3.1 & chr20 & 25999468 & 26001320 & + & $\begin{array}{c}3.08402 \\
75 \\
\end{array}$ & $\begin{array}{c}2.3788 \\
395 \\
\end{array}$ & $\begin{array}{c}1.0570 \\
529 \\
\end{array}$ & $\begin{array}{l}1.181 \\
9186 \\
\end{array}$ & $\begin{array}{l}0.448 \\
66753 \\
\end{array}$ & $\begin{array}{l}1.024 \\
0932 \\
\end{array}$ \\
\hline ENSG00000197358 & -1.86 & BNIP3P1 & chr14 & 28733596 & 28735180 & + & $\begin{array}{c}4.02136 \\
66 \\
\end{array}$ & $\begin{array}{c}6.1853 \\
94 \\
\end{array}$ & $\begin{array}{c}4.5065 \\
994 \\
\end{array}$ & $\begin{array}{l}2.335 \\
3097 \\
\end{array}$ & $\begin{array}{l}2.544 \\
7326 \\
\end{array}$ & $\begin{array}{l}2.919 \\
4083 \\
\end{array}$ \\
\hline ENSG00000235237 & -1.88 & RP1-151B14.6 & chr22 & 37562797 & 37578890 & + & $\begin{array}{c}5.69870 \\
1 \\
\end{array}$ & $\begin{array}{c}5.5882 \\
27 \\
\end{array}$ & $\begin{array}{c}3.9706 \\
326\end{array}$ & $\begin{array}{l}2.796 \\
2503 \\
\end{array}$ & $\begin{array}{l}2.296 \\
2286 \\
\end{array}$ & $\begin{array}{l}2.966 \\
6011 \\
\end{array}$ \\
\hline ENSG00000237499 & -1.89 & RP11-356I2.4 & chr6 & 138144810 & 138189370 & - & $\begin{array}{c}2.07288 \\
89 \\
\end{array}$ & $\begin{array}{c}2.8674 \\
767 \\
\end{array}$ & $\begin{array}{c}2.2776 \\
296 \\
\end{array}$ & $\begin{array}{l}0.910 \\
7068 \\
\end{array}$ & $\begin{array}{c}1.268 \\
385\end{array}$ & $\begin{array}{c}1.577 \\
585\end{array}$ \\
\hline ENSG00000235847 & -1.89 & LDHAP7 & chr2 & 85004383 & 85005347 & - & $\begin{array}{c}2.74156 \\
7 \\
\end{array}$ & $\begin{array}{c}2.0703 \\
194 \\
\end{array}$ & $\begin{array}{c}1.2152 \\
584 \\
\end{array}$ & $\begin{array}{l}0.508 \\
1032 \\
\end{array}$ & $\begin{array}{l}1.017 \\
5685 \\
\end{array}$ & $\begin{array}{l}0.249 \\
74425 \\
\end{array}$ \\
\hline ENSG00000273038 & -1.90 & $\begin{array}{c}\text { RP11- } \\
479 G 22.8\end{array}$ & chr10 & 33176189 & 33178239 & - & $\begin{array}{c}2.26414 \\
06\end{array}$ & $\begin{array}{c}2.7482 \\
634 \\
\end{array}$ & $\begin{array}{c}1.5507 \\
42 \\
\end{array}$ & $\begin{array}{l}0.526 \\
8092 \\
\end{array}$ & $\begin{array}{l}1.410 \\
5644\end{array}$ & $\begin{array}{l}0.987 \\
04404 \\
\end{array}$ \\
\hline ENSG00000231991 & -1.91 & ANXA2P2 & chr9 & 33624223 & 33625532 & + & $\begin{array}{c}134.588 \\
53 \\
\end{array}$ & $\begin{array}{c}110.10 \\
068 \\
\end{array}$ & $\begin{array}{c}128.57 \\
472 \\
\end{array}$ & $\begin{array}{c}52.56 \\
764 \\
\end{array}$ & $\begin{array}{l}66.60 \\
7285 \\
\end{array}$ & $\begin{array}{l}77.69 \\
0636 \\
\end{array}$ \\
\hline ENSG00000256929 & -1.91 & AC067852.1 & chr17 & 40673736 & 40674065 & + & 3.25574 & $\begin{array}{c}8.0733 \\
17\end{array}$ & $\begin{array}{c}4.7597 \\
175\end{array}$ & $\begin{array}{l}3.042 \\
4023\end{array}$ & $\begin{array}{l}3.687 \\
5687\end{array}$ & $\begin{array}{l}1.590 \\
6979\end{array}$ \\
\hline ENSG00000255959 & -1.92 & RP11- & chr11 & 60603469 & 60610438 & - & 5.55156 & 2.3799 & 2.5062 & 1.125 & 1.753 & 2.367 \\
\hline
\end{tabular}




\begin{tabular}{|c|c|c|c|c|c|c|c|c|c|c|c|c|}
\hline & & $804 A 23.2$ & & & & & 56 & 477 & 263 & 2497 & 554 & 571 \\
\hline ENSG00000251292 & -1.93 & $\begin{array}{c}\text { RP11- } \\
380 P 13.2\end{array}$ & chr4 & 23724885 & 23735202 & - & $\begin{array}{c}38.6029 \\
8 \\
\end{array}$ & $\begin{array}{c}68.663 \\
376 \\
\end{array}$ & $\begin{array}{c}43.137 \\
657 \\
\end{array}$ & $\begin{array}{l}25.19 \\
0517 \\
\end{array}$ & $\begin{array}{l}34.78 \\
7884 \\
\end{array}$ & $\begin{array}{l}18.08 \\
7368 \\
\end{array}$ \\
\hline ENSG00000262410 & -1.96 & $\begin{array}{c}\text { RP11- } \\
388 C 12.8\end{array}$ & chr17 & 80702944 & 80703585 & + & $\begin{array}{c}2.04051 \\
07\end{array}$ & $\begin{array}{c}3.6950 \\
738\end{array}$ & $\begin{array}{c}4.6220 \\
4 \\
\end{array}$ & $\begin{array}{l}2.332 \\
8707\end{array}$ & $\begin{array}{l}0.493 \\
39446\end{array}$ & $\begin{array}{l}1.994 \\
9535 \\
\end{array}$ \\
\hline ENSG00000266088 & -1.97 & RP5-1028K7.2 & chr17 & 38673278 & 38683254 & + & $\begin{array}{c}4.31365 \\
35 \\
\end{array}$ & $\begin{array}{c}1.3317 \\
922 \\
\end{array}$ & $\begin{array}{c}1.3348 \\
67 \\
\end{array}$ & $\begin{array}{l}0.232 \\
17432 \\
\end{array}$ & $\begin{array}{l}0.229 \\
15247 \\
\end{array}$ & $\begin{array}{l}0.586 \\
2707 \\
\end{array}$ \\
\hline ENSG00000255234 & -1.98 & $\begin{array}{c}\text { RP11- } \\
\text { 727A23.10 }\end{array}$ & chr11 & 82997171 & 83134559 & + & $\begin{array}{c}74.2644 \\
35\end{array}$ & $\begin{array}{c}131.67 \\
998\end{array}$ & $\begin{array}{c}93.626 \\
11 \\
\end{array}$ & $\begin{array}{l}40.38 \\
3236\end{array}$ & $\begin{array}{l}64.51 \\
9875\end{array}$ & $\begin{array}{c}45.11 \\
061\end{array}$ \\
\hline ENSG00000231826 & -1.98 & AC016735.2 & chr2 & 43254992 & 43266686 & - & 16.923 & $\begin{array}{c}4.0733 \\
547\end{array}$ & $\begin{array}{c}5.2401 \\
91\end{array}$ & $\begin{array}{c}4.893 \\
341\end{array}$ & $\begin{array}{c}3.788 \\
711\end{array}$ & $\begin{array}{c}2.493 \\
266 \\
\end{array}$ \\
\hline ENSG00000235823 & -2.00 & LINC00263 & chr10 & 102133372 & 102143125 & + & $\begin{array}{c}2.90408 \\
5 \\
\end{array}$ & $\begin{array}{c}3.2670 \\
798 \\
\end{array}$ & $\begin{array}{c}2.4657 \\
273 \\
\end{array}$ & $\begin{array}{c}1.211 \\
951 \\
\end{array}$ & $\begin{array}{l}1.841 \\
8641 \\
\end{array}$ & $\begin{array}{l}1.311 \\
8396 \\
\end{array}$ \\
\hline ENSG00000265992 & -2.00 & ESRG & chr3 & 54666149 & 54673884 & - & $\begin{array}{c}233.581 \\
45 \\
\end{array}$ & $\begin{array}{c}439.66 \\
51 \\
\end{array}$ & $\begin{array}{c}314.18 \\
79 \\
\end{array}$ & $\begin{array}{l}138.6 \\
1157 \\
\end{array}$ & $\begin{array}{l}202.8 \\
8977 \\
\end{array}$ & $\begin{array}{l}142.8 \\
2372 \\
\end{array}$ \\
\hline ENSG00000260834 & -2.02 & RP11-25619.2 & chr16 & 65224876 & 65268817 & - & $\begin{array}{c}56.8772 \\
7 \\
\end{array}$ & $\begin{array}{c}96.663 \\
605 \\
\end{array}$ & $\begin{array}{c}70.333 \\
88 \\
\end{array}$ & $\begin{array}{l}29.44 \\
8397 \\
\end{array}$ & $\begin{array}{c}46.84 \\
315 \\
\end{array}$ & $\begin{array}{l}33.75 \\
8797 \\
\end{array}$ \\
\hline ENSG00000256940 & -2.06 & $\begin{array}{c}\text { RP11- } \\
783 K 16.5 \\
\end{array}$ & chr11 & 64013436 & 64015689 & + & $\begin{array}{c}13.2824 \\
1 \\
\end{array}$ & $\begin{array}{c}9.2834 \\
835 \\
\end{array}$ & $\begin{array}{c}8.4336 \\
85 \\
\end{array}$ & $\begin{array}{c}6.031 \\
071 \\
\end{array}$ & $\begin{array}{l}4.409 \\
4415 \\
\end{array}$ & $\begin{array}{l}4.459 \\
4874 \\
\end{array}$ \\
\hline ENSG00000273387 & -2.07 & RP3-412A9.16 & chr22 & 31478142 & 31479551 & - & $\begin{array}{c}3.50702 \\
29 \\
\end{array}$ & $\begin{array}{c}1.8962 \\
02 \\
\end{array}$ & $\begin{array}{c}1.4603 \\
726 \\
\end{array}$ & $\begin{array}{l}1.100 \\
5665 \\
\end{array}$ & $\begin{array}{l}0.395 \\
10265 \\
\end{array}$ & $\begin{array}{l}0.518 \\
1117 \\
\end{array}$ \\
\hline ENSG00000255021 & -2.08 & RP11-53616.2 & chr3 & 14313873 & 14345345 & - & $\begin{array}{c}14.2297 \\
64\end{array}$ & $\begin{array}{c}26.088 \\
594\end{array}$ & $\begin{array}{c}15.773 \\
34\end{array}$ & $\begin{array}{l}7.262 \\
8064\end{array}$ & $\begin{array}{l}12.21 \\
7939\end{array}$ & $\begin{array}{l}7.326 \\
7016 \\
\end{array}$ \\
\hline ENSG00000100181 & -2.08 & TPTEP1 & chr22 & 17082777 & 17179632 & + & $\begin{array}{c}20.2850 \\
84\end{array}$ & $\begin{array}{c}40.960 \\
05\end{array}$ & $\begin{array}{c}27.098 \\
47\end{array}$ & $\begin{array}{l}11.95 \\
8863\end{array}$ & $\begin{array}{l}17.23 \\
5292\end{array}$ & $\begin{array}{c}12.10 \\
646\end{array}$ \\
\hline ENSG00000236673 & -2.09 & RP11-69|8.2 & chr6 & 132223103 & 132241705 & + & $\begin{array}{c}8.28539 \\
9 \\
\end{array}$ & $\begin{array}{c}16.666 \\
319 \\
\end{array}$ & $\begin{array}{c}10.295 \\
293 \\
\end{array}$ & $\begin{array}{c}4.810 \\
559 \\
\end{array}$ & $\begin{array}{l}6.592 \\
9637 \\
\end{array}$ & $\begin{array}{c}4.929 \\
272 \\
\end{array}$ \\
\hline ENSG00000226792 & -2.09 & LINC00371 & chr13 & 51656984 & 51746524 & - & $\begin{array}{c}9.05261 \\
9 \\
\end{array}$ & $\begin{array}{c}16.953 \\
245 \\
\end{array}$ & $\begin{array}{c}10.620 \\
668 \\
\end{array}$ & $\begin{array}{l}5.284 \\
7075 \\
\end{array}$ & $\begin{array}{l}6.886 \\
0707 \\
\end{array}$ & $\begin{array}{l}4.875 \\
6647 \\
\end{array}$ \\
\hline ENSG00000236824 & -2.12 & BCYRN1 & chr2 & 47558199 & 47571656 & + & $\begin{array}{c}12.5237 \\
26 \\
\end{array}$ & $\begin{array}{c}24.176 \\
477 \\
\end{array}$ & $\begin{array}{c}18.561 \\
161 \\
\end{array}$ & $\begin{array}{l}5.118 \\
2065 \\
\end{array}$ & $\begin{array}{l}7.327 \\
3034 \\
\end{array}$ & $\begin{array}{c}15.67 \\
111 \\
\end{array}$ \\
\hline ENSG00000224081 & -2.12 & LINC01057 & chr1 & 95104017 & 95285837 & - & $\begin{array}{c}59.7651 \\
8 \\
\end{array}$ & $\begin{array}{c}112.70 \\
979 \\
\end{array}$ & $\begin{array}{c}71.806 \\
335 \\
\end{array}$ & $\begin{array}{c}32.73 \\
587\end{array}$ & $\begin{array}{l}50.64 \\
0713 \\
\end{array}$ & $\begin{array}{l}30.50 \\
0008 \\
\end{array}$ \\
\hline ENSG00000225383 & -2.15 & SFTA1P & chr10 & 10826400 & 10836943 & - & $\begin{array}{c}33.8832 \\
36 \\
\end{array}$ & $\begin{array}{c}11.625 \\
587 \\
\end{array}$ & $\begin{array}{c}16.437 \\
605 \\
\end{array}$ & $\begin{array}{c}5.999 \\
579 \\
\end{array}$ & $\begin{array}{l}6.570 \\
4207 \\
\end{array}$ & $\begin{array}{l}16.63 \\
6505 \\
\end{array}$ \\
\hline ENSG00000219445 & -2.15 & RP11-3B12.3 & chr7 & 124869633 & 124904345 & - & $\begin{array}{c}5.30390 \\
45 \\
\end{array}$ & $\begin{array}{c}10.288 \\
663 \\
\end{array}$ & $\begin{array}{c}7.6753 \\
13 \\
\end{array}$ & $\begin{array}{c}3.506 \\
465 \\
\end{array}$ & $\begin{array}{l}4.159 \\
0476 \\
\end{array}$ & $\begin{array}{l}2.902 \\
5939 \\
\end{array}$ \\
\hline ENSG00000233218 & -2.25 & SNX18P16 & chr1 & 142688379 & 142688925 & - & $\begin{array}{c}1.71771 \\
82 \\
\end{array}$ & $\begin{array}{c}2.6481 \\
433\end{array}$ & $\begin{array}{c}3.3620 \\
58 \\
\end{array}$ & $\begin{array}{l}0.430 \\
26203 \\
\end{array}$ & $\begin{array}{l}1.351 \\
1974 \\
\end{array}$ & $\begin{array}{l}0.339 \\
88443 \\
\end{array}$ \\
\hline ENSG00000233461 & -2.25 & $\begin{array}{c}\text { RP11- } \\
295 G 20.2 \\
\end{array}$ & chr1 & 231658134 & 231664302 & - & $\begin{array}{c}4.34471 \\
65 \\
\end{array}$ & $\begin{array}{c}5.2603 \\
03 \\
\end{array}$ & $\begin{array}{c}4.8380 \\
613 \\
\end{array}$ & $\begin{array}{l}0.901 \\
0945 \\
\end{array}$ & $\begin{array}{l}2.709 \\
4634 \\
\end{array}$ & $\begin{array}{l}3.601 \\
3868 \\
\end{array}$ \\
\hline ENSG00000269495 & -2.34 & CTB-147C22.8 & chr19 & 51453441 & 51466905 & + & $\begin{array}{c}4.91188 \\
86 \\
\end{array}$ & $\begin{array}{c}14.511 \\
923 \\
\end{array}$ & $\begin{array}{c}12.695 \\
248 \\
\end{array}$ & $\begin{array}{l}1.724 \\
4989 \\
\end{array}$ & $\begin{array}{l}4.951 \\
8948 \\
\end{array}$ & $\begin{array}{c}8.315 \\
971 \\
\end{array}$ \\
\hline ENSG00000257219 & -2.41 & RP11-54A9.1 & chr12 & 76653619 & 76698911 & + & $\begin{array}{c}3.86657 \\
3\end{array}$ & $\begin{array}{c}2.7792 \\
904\end{array}$ & $\begin{array}{c}2.3597 \\
717\end{array}$ & $\begin{array}{l}0.302 \\
85415\end{array}$ & $\begin{array}{l}0.949 \\
48655\end{array}$ & $\begin{array}{l}1.806 \\
0194\end{array}$ \\
\hline ENSG00000230439 & -2.41 & RP11-488P3.1 & chr1 & 94218480 & 94241000 & - & $\begin{array}{c}16.7174 \\
1 \\
\end{array}$ & $\begin{array}{c}2.3367 \\
712 \\
\end{array}$ & $\begin{array}{c}4.8807 \\
71 \\
\end{array}$ & $\begin{array}{l}2.343 \\
1618 \\
\end{array}$ & $\begin{array}{l}2.641 \\
2401 \\
\end{array}$ & $\begin{array}{c}2.188 \\
129 \\
\end{array}$ \\
\hline ENSG00000214110 & -2.44 & LDHAP4 & chr9 & 14921335 & 14922332 & - & $\begin{array}{c}6.77137 \\
7 \\
\end{array}$ & $\begin{array}{c}6.3298 \\
79 \\
\end{array}$ & $\begin{array}{c}5.6765 \\
62 \\
\end{array}$ & $\begin{array}{l}2.142 \\
0772 \\
\end{array}$ & $\begin{array}{l}2.575 \\
1235 \\
\end{array}$ & $\begin{array}{c}3.043 \\
416 \\
\end{array}$ \\
\hline ENSG00000267279 & -2.45 & $\begin{array}{c}\text { RP11- } \\
879 F 14.2 \\
\end{array}$ & chr18 & 59252979 & 59274149 & - & $\begin{array}{c}6.17703 \\
3 \\
\end{array}$ & $\begin{array}{c}1.2901 \\
783 \\
\end{array}$ & $\begin{array}{c}1.8376 \\
158 \\
\end{array}$ & $\begin{array}{l}0.892 \\
23325 \\
\end{array}$ & $\begin{array}{l}0.914 \\
65175 \\
\end{array}$ & $\begin{array}{l}0.947 \\
58815 \\
\end{array}$ \\
\hline ENSG00000254740 & -2.63 & RP11-334E6.3 & chr11 & 119243416 & 119252323 & + & $\begin{array}{c}1.65935 \\
97 \\
\end{array}$ & $\begin{array}{c}5.2611 \\
43 \\
\end{array}$ & $\begin{array}{c}5.3788 \\
548 \\
\end{array}$ & $\begin{array}{l}1.041 \\
0688 \\
\end{array}$ & $\begin{array}{l}1.639 \\
0346 \\
\end{array}$ & $\begin{array}{l}1.514 \\
5406 \\
\end{array}$ \\
\hline ENSG00000268621 & -2.68 & AC006262.5 & chr19 & 46692423 & 46706340 & - & $\begin{array}{c}2.93583 \\
7 \\
\end{array}$ & $\begin{array}{c}5.8567 \\
724 \\
\end{array}$ & $\begin{array}{c}3.7140 \\
47 \\
\end{array}$ & $\begin{array}{l}1.001 \\
9716 \\
\end{array}$ & $\begin{array}{l}2.637 \\
1474 \\
\end{array}$ & $\begin{array}{l}1.250 \\
5352 \\
\end{array}$ \\
\hline ENSG00000271573 & -2.69 & $\begin{array}{c}\text { RP11- } \\
96 \mathrm{C} 23.12 \\
\end{array}$ & chr10 & 88786941 & 88787234 & + & $\begin{array}{c}1.96670 \\
2 \\
\end{array}$ & $\begin{array}{c}9.9967 \\
87 \\
\end{array}$ & $\begin{array}{c}7.0107 \\
24 \\
\end{array}$ & $\begin{array}{l}0.436 \\
6481 \\
\end{array}$ & $\begin{array}{c}2.154 \\
825 \\
\end{array}$ & $\begin{array}{c}3.302 \\
377 \\
\end{array}$ \\
\hline ENSG00000257042 & -2.69 & $\begin{array}{c}\text { RP11- } \\
993 B 23.3\end{array}$ & chr12 & 28111450 & 28122746 & + & $\begin{array}{c}25.1891 \\
9\end{array}$ & $\begin{array}{c}26.207 \\
75\end{array}$ & $\begin{array}{c}21.461 \\
538\end{array}$ & $\begin{array}{l}4.820 \\
6534\end{array}$ & $\begin{array}{c}9.942 \\
112\end{array}$ & $\begin{array}{c}15.11 \\
63\end{array}$ \\
\hline ENSG00000255198 & -2.71 & SNHG9 & chr16 & 2014960 & 2015510 & + & $\begin{array}{c}5.08295 \\
3\end{array}$ & $\begin{array}{c}5.4102 \\
4\end{array}$ & $\begin{array}{c}2.8378 \\
108\end{array}$ & $\begin{array}{l}1.320 \\
2466 \\
\end{array}$ & $\begin{array}{l}1.149 \\
7614\end{array}$ & $\begin{array}{l}2.586 \\
8635 \\
\end{array}$ \\
\hline ENSG00000261780 & -2.74 & $\begin{array}{c}\text { CTD- } \\
\text { 2354A18.1 }\end{array}$ & chr18 & 70992176 & 71017113 & + & $\begin{array}{c}7.45266 \\
5 \\
\end{array}$ & $\begin{array}{c}13.354 \\
17 \\
\end{array}$ & $\begin{array}{c}8.3805 \\
11 \\
\end{array}$ & $\begin{array}{l}2.986 \\
0015 \\
\end{array}$ & $\begin{array}{l}4.862 \\
3896 \\
\end{array}$ & $\begin{array}{l}2.805 \\
7775 \\
\end{array}$ \\
\hline
\end{tabular}




\begin{tabular}{|c|c|c|c|c|c|c|c|c|c|c|c|c|}
\hline ENSG00000258177 & -2.75 & RP11-394J1.2 & chr12 & 96616575 & 96617751 & - & $\begin{array}{c}11.3985 \\
49\end{array}$ & $\begin{array}{c}7.2211 \\
123\end{array}$ & $\begin{array}{c}5.6029 \\
59\end{array}$ & $\begin{array}{l}1.443 \\
8808\end{array}$ & $\begin{array}{l}3.685 \\
8037\end{array}$ & $\begin{array}{l}4.163 \\
6434\end{array}$ \\
\hline ENSG00000255729 & -2.78 & AC005618.1 & chr5 & 140699661 & 140700339 & - & $\begin{array}{c}3.83701 \\
63\end{array}$ & $\begin{array}{c}7.4735 \\
03\end{array}$ & $\begin{array}{c}7.0763 \\
206\end{array}$ & $\begin{array}{l}0.756 \\
8286 \\
\end{array}$ & $\begin{array}{c}4.717 \\
6375\end{array}$ & $\begin{array}{l}1.997 \\
0802 \\
\end{array}$ \\
\hline ENSG00000238266 & -2.79 & LINC00707 & chr10 & 6821560 & 6884868 & + & $\begin{array}{c}3.58438 \\
56\end{array}$ & $\begin{array}{c}4.5337 \\
21\end{array}$ & $\begin{array}{c}3.8257 \\
384\end{array}$ & $\begin{array}{l}1.909 \\
5888\end{array}$ & $\begin{array}{c}1.506 \\
412\end{array}$ & $\begin{array}{l}0.787 \\
2698 \\
\end{array}$ \\
\hline ENSG00000239492 & -2.98 & FAM25HP & chr10 & 47740330 & 47747200 & + & $\begin{array}{c}15.1145 \\
64\end{array}$ & $\begin{array}{c}31.412 \\
348\end{array}$ & $\begin{array}{c}26.541 \\
6\end{array}$ & $\begin{array}{c}5.972 \\
542\end{array}$ & $\begin{array}{c}9.727 \\
91\end{array}$ & $\begin{array}{c}8.178 \\
021\end{array}$ \\
\hline ENSG00000253746 & -3.17 & $\begin{array}{c}\text { RP11- } \\
527 N 22.2 \\
\end{array}$ & chr8 & 37262957 & 37264242 & - & $\begin{array}{c}3.75136 \\
5 \\
\end{array}$ & $\begin{array}{c}4.0315 \\
757 \\
\end{array}$ & $\begin{array}{c}2.6437 \\
647 \\
\end{array}$ & $\begin{array}{c}0.703 \\
32074 \\
\end{array}$ & $\begin{array}{l}0.949 \\
91237 \\
\end{array}$ & $\begin{array}{l}1.250 \\
8819 \\
\end{array}$ \\
\hline ENSG00000256288 & -3.22 & $\begin{array}{c}\text { RP11- } \\
\text { 277P12.10 } \\
\end{array}$ & chr12 & 10485460 & 10490891 & - & $\begin{array}{c}6.18079 \\
3 \\
\end{array}$ & $\begin{array}{c}7.4155 \\
67 \\
\end{array}$ & $\begin{array}{c}4.6269 \\
43 \\
\end{array}$ & $\begin{array}{l}0.939 \\
5672 \\
\end{array}$ & $\begin{array}{l}3.611 \\
7387 \\
\end{array}$ & $\begin{array}{c}1.750 \\
629 \\
\end{array}$ \\
\hline ENSG00000242147 & -4.40 & $\begin{array}{c}\text { RP13- } \\
463 N 16.6\end{array}$ & chr10 & 5636954 & 5638081 & - & $\begin{array}{c}18.6134 \\
9\end{array}$ & $\begin{array}{c}1.4156 \\
137\end{array}$ & $\begin{array}{c}3.2376 \\
003\end{array}$ & $\begin{array}{l}0.521 \\
8478\end{array}$ & $\begin{array}{c}0.030 \\
29739 \\
3 \\
\end{array}$ & $\begin{array}{l}0.474 \\
20192\end{array}$ \\
\hline ENSG00000229647 & -4.79 & AC007879.7 & chr2 & 208104374 & 208110611 & + & $\begin{array}{c}31.3377 \\
27\end{array}$ & $\begin{array}{c}7.7880 \\
12\end{array}$ & $\begin{array}{c}10.499 \\
597\end{array}$ & $\begin{array}{l}3.835 \\
9287\end{array}$ & $\begin{array}{c}3.087 \\
454\end{array}$ & $\begin{array}{c}1.968 \\
523\end{array}$ \\
\hline
\end{tabular}

\section{Table S2: Comparison of FAM83H-AS1 RPKM (Reads Per Kilobase Million) with other previously} published functional LncRNAs

\begin{tabular}{|l|l|r|r|l|}
\hline LncRNA & ENSEMBL ID & Avg. RPKM GFP & \multicolumn{1}{|c|}{ Avg. RPKM 16E6 } & Known function \\
\hline FAM83H-AS1 & ENSG00000203499 & 5.876310667 & 9.9992845 & \\
\hline XIST & ENSG00000229807 & 8.137879 & 12.21193233 & miRNA sponge in cervical cancer (Zhu et al 2018) \\
\hline HOTAIR & ENSG00000228630 & 1.817852513 & 1.5785181 & miRNA sponge in cervical cancer (Liu et al 2018) \\
\hline NEAT1 & ENSG00000245532 & 34.23374033 & 27.70111733 & miRNA sponge in cervical cancer (Han et al 2018) \\
\hline TINCR & ENSG00000223573 & 16.26229083 & 16.07113433 & miRNA sponge in gastric cancer (Chen et al 2017) and breast cancer (Liu et al 2018) \\
\hline H19 & ENSG00000130600 & 3.3026914 & 15.14256633 & miRNA sponge in cervical cancer (Ou et al 2018) \\
\hline
\end{tabular}


Table S3: Primers used in this study for qRT-PCR.

\begin{tabular}{|c|c|}
\hline Primers & Sequence (5'-3') \\
\hline FAM83H-AS1 FWD & TCCCAATAAACAGGGCAGAC \\
\hline FAM83H-AS1 REV & CAAGATCACCACACCCCTCT \\
\hline Gapdh FWD & ССАСТССТССАССТTTGAC \\
\hline Gapdh REV & ACCCTGTTGCTGTAGCCA \\
\hline UBC FWD & GATTTGGGTCGCAGTTCTTG \\
\hline UBC REV & CCTTATCTTGGATCTTTGCCTTG \\
\hline Beta-Actin FWD & AGCACAGAGCCTCGCCTTT \\
\hline Beta-Actin REV & CCACGATGGAGGGGAAGAC \\
\hline U6 FWD & GTGCTCGCTTCGGCAGCACATAT \\
\hline U6 REV & AAAAATATGGAACGCTTCACGAA \\
\hline C. Elegans ama-1 FWD & GGAGCTCGAGTGGATCTTCG \\
\hline C. Elegans ama-1 REV & GCGCAGAGAGTATCCTGGAC \\
\hline HPV-16E6 FWD & AATGTTTCAGGACCCACAGG \\
\hline HPV-16E6 REV & ACTGTTGCTTGCAGTACACACA \\
\hline HPV-16E7 FWD & ACAAGCAGAACCGGACAGAG \\
\hline HPV-16E7 REV & GCCCATTAACAGGTCTTCCA \\
\hline p53 FWD & TTTGGGTCTTTGAACCCTTG \\
\hline p53 REV & CCACAACAAAACACCAGTGC \\
\hline p300 FWD & AGCGGCCTAAACTCTCATCTC \\
\hline p300 REV & CACCATTGGTTAGTCCCAATTC \\
\hline FAM83H FWD & CGACAAGTGCCGTGTCAACC \\
\hline FAM83H REV & ACTTCCCAGTGCGGCAGTAG \\
\hline H19 FWD & AGTGGACTTGGTGACGCTGTAT \\
\hline H19 REV & СTCCTGAGAGCTCATTCACTCC \\
\hline MIR205HG FWD & TCATTAAAGAGAGAAATCAACTATTCA \\
\hline MIR205HG REV & TAAAGCACCCAGTTCAGCAG \\
\hline HOXC-AS5 FWD & CTCCTTGGAGCAGTACACCTG \\
\hline HOXC-AS5 REV & GTCCATTTGCTCTCCACTTGA \\
\hline GAS5 FWD & CTTCTGGGCTCAAGTGATCCT \\
\hline GAS5 REV & TTGTGCCATGAGACTCCATCAG \\
\hline LINC00963 FWD & AACTGCCTTTGGAAGCAAGTAG \\
\hline LINC00963 REV & ACTGCTCTAGTCCAGGAGTTCG \\
\hline SNHG15 FWD & GCTGAGGTGACGGTCTCAAA \\
\hline SNHG15 REV & GCCTCCCAGTTTCATGGACA \\
\hline MAFG-AS1 FWD & AGGACTCGGGAGGAAGATAAAC \\
\hline MAFG-AS1 REV & AACACCTTCAGCTCTCTGCTG \\
\hline RP6-65G23.3 FWD & GAGATAGGAGGCCCATAATGTTTC \\
\hline RP6-65G23.3 REV & CTGCAACCAGGTGGAAGTCA \\
\hline GS1-600G8.5 FWD & ACTGTCCGGTTCACTGTGGG \\
\hline
\end{tabular}




\begin{tabular}{|l|l|} 
GS1-600G8.5 REV & CGGTGCCACTGGGTCATTTT \\
\hline RP3-510D11.2 FWD & CCAGACCGACGGGACAGCG \\
RP3-510D11.2 REV & GCTTCCCTGTCCTCCTCCTA \\
\hline RP11-479G22.8 FWD & TGGGAAACTAAAACCATTTAAGC \\
RP11-479G22.8 REV & GCTCAGAACATGGTTTTCTCTG \\
\hline LINC01057 FWD & GTGAATTCCTTGGAAGATGAGG \\
LINC01057 REV & TCATTCACTCAACCACTGAACC \\
\hline SFTA1P FWD & CAGCATTCCAGGTGGGCTTT \\
SFTA1P REV & CCTTGTTTGGCTTACTCGTGC \\
\hline RP13-463N16.6 FWD & TTGGAAATCACTCCTTCCACTT \\
RP13-463N16.6 REV & CAGAGACAATTCAACTCCCACA \\
\hline AC007879.7 FWD & ACAGGGAGCCAGGACACC \\
AC007879.7 REV & GGAACCAGCACCAGGAACC \\
\hline
\end{tabular}




\section{$\underline{\text { XIII. Supplementary Methods }}$}

\section{A. Cell Culture}

Primary human foreskin keratinocytes (HEKa) were cultured as described by supplier (EpiLife ${ }^{\circledR}$ Medium supplemented with Human Keratinocyte Growth Supplement (HKGS)). Primary human cervical keratinocytes (HCK) and J2-3T3 mouse fibroblast feeders were cultured as described by Alison McBride's laboratory (NIH, Bethesda, MD) ${ }^{1}$. HCK were maintained in F-media [3:1 F12:DMEM with 5\% Fetal Bovine Serum (FBS), 0.4ug/mL Hydrocortisone, 5ug/mL Insulin, 8.4ng/mL Cholera Toxin, 10ng/mL Epidermal Growth Factor, 24ug/mL Adenine, 10U/mL Penicillin, 10ug/mL Streptomycin, 2mM L-Glutamine, Amphotericin B] containing 10uM Y-27632 ROCK Inhibitor (Tocris). HCK cells were cocultured with J2-3T3 feeder cells rendered mitotically inactive in 8ug/mL Mitomycin C for 3 hours; these growth-arrested feeder cells were replenished every 3-4 days. J2-3T3 stock cells were cultured in Feeder media [Dulbecco's Modified Eagles Medium (DMEM), high glucose, 10\% Newborn Calf Serum, 2mM L-Glutamine, 10U/mL Penicillin, 10ug/mL Streptomycin, Amphotericin B]. J2-3T3 feeder cells are sensitive to trypsin; HCK, J2-3T3 stock, and growth-arrested J2-3T3 cells were centrifuged to remove the trypsin when passaged. HPV-16 positive CaSki cells were cultured in Roswell Park Memorial Institute (RPMI)-1640 medium containing 10\% FBS, Streptomycin-Penicillin, HEPES Buffer, Amphotericin B. HPV-16 positive W12/20863 and W12/201402 and HPV-31b positive cervical (CIN-612) were cocultured in E-media (1:1 DMEM:Ham's Nutrient Mix F12 medium supplemented with 5\% FBS, 10mM HEPES Buffer, Penicillin-Streptomycin, Amphotericin B, 0.02 $\mu$ M Triiodothyronine, $0.4 \mu \mathrm{g} / \mathrm{mL}$ Hydrocortisone, $0.1 \mu \mathrm{g} / \mathrm{mL}$ Cholera Toxin, $5 \mu \mathrm{g} / \mathrm{mL}$ Transferrin, $180 \mu \mathrm{M}$ Adenine, $5 \mu \mathrm{g} / \mathrm{mL}$ Insulin ) with murine 3T3 fibroblast cells (3T3M) that were rendered mitotically inactive with $4 \mathrm{ug} / \mathrm{mL}$ Mitomycin $\mathrm{C}$ for at least 2 hours. HPV-negative cervical C33A cells, HPV-18 positive cervical (HeLa), HPV-16 positive HNSCC cell lines UMSCC-47 and -104, HPV negative HNSCC cell line UMSCC-1, HEK293T, and 3T3M cells were all cultured in DMEM supplemented with 10\% FBS, Penicillin-Streptomycin, HEPES Buffer, and Amphotericin B. To passage stock cells, 0.25\% trypsin was utilized. Growth-arrested J2-3T3 and 3T3M fibroblasts were removed with Versene solution and gently pipetting, prior to pelleting and when otherwise desired. Cells were cultured at $37^{\circ} \mathrm{C}$ in a humidified $5 \% \mathrm{CO} 2$ cell culture incubator.

\section{B. Generation of stable cell lines}

Plasmids MSCV-N-GFP (Plasmid \#37855), MSCV-N-16E6 (Plasmid \#37875), and MSCV-N16 E7 (Plasmid \#37881) were purchased from Addgene. For retroviral production, these plasmids were 
co-transfected with packaging (pCL-ECO) and envelope (pVSV-GF) plasmids into HEK293T cells using calcium phosphate co-precipitation. 12-16 hours later, HEK293T media was replaced with fresh target cell media. After 48-hour incubation, retrovirus-containing target cell media was harvested, filtered $(0.45 \mu \mathrm{m})$, and mixed with polybrene $(4 \mu \mathrm{g} / \mathrm{mL}$ final concentration) to increase infection efficiency. 24 hours before infection, co-cultured J2-3T3 fibroblasts were removed with Versene solution and gentle pipetting from HCK cells. HEKa and HCK cells were infected with recombinant retroviruses for 8 hours, virus was removed and replaced with fresh target cell media. Growth arrested J2-3T3 were added to infected HCK and replenished every 3 days. Cells were allowed to recover for 24 hours prior to 72 -hour

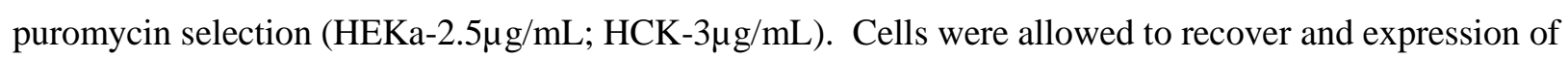
exogenous HPV-16 E6 and E7, as desired, was confirmed by RT-PCR (Fig. S1, Fig. S3).

\section{Generation of HPV-16 positive cervical JAMM-16 cell line}

According to Buck et al. protocol described previously ${ }^{2}$, the HPV-16 insert was cut out of pBR322 HPV-16 plasmid by performing a restriction digestion of two separate $25 \mathrm{ug}$ plasmid reactions in $225 \mathrm{uL}$ each with the restriction enzyme BamHI (NEB) according to manufacturer's recommended conditions $\left(37^{\circ} \mathrm{C}\right.$ for 2 hours). Digested samples were then PCR purified by QIAquick ${ }^{\circ}$ PCR Purification Kit (Qiagen) resuspended in 200uL Buffer TE. Purified samples were then ligated under dilute conditions (9mL total volume) by adding $1 \mathrm{X}$ Ligase Buffer and $6 \mathrm{uL}$ of high concentration $(2 \mathrm{~m} \mathrm{U} / \mathrm{mL}) \mathrm{T} 4 \mathrm{DNA}$ Ligase (NEB) and incubated at $16^{\circ} \mathrm{C}$ overnight. $<25 \mathrm{ug}$ in $9 \mathrm{~mL}$ ligation reactions were conducted to avoid concatemer formation. The ligated samples were then treated with $4.5 \mathrm{~mL}$ of $7.5 \mathrm{M}$ Ammonium acetate and mixed. $35 \mathrm{~mL}$ of $95 \%$ ethanol was added to each, tubes were mixed, and incubated at $4{ }^{\circ} \mathrm{C}$ overnight. The next day, the samples were brought back to room temperature and centrifuged at $\sim 5,000 \mathrm{x} g$ at room temperature for 1 hour. Pellets were washed with 70\% ethanol, spun briefly, and washed again with 70\% ethanol. The pellets were spun one last time to remove any residual ethanol, then air dried for several minutes. Pellets were resuspended in 100uL Buffer TE each, pellets were combined, and Nanodrop was used to calculate concentration retrieved. The entire product was run in $1 \%$ agarose gel for 3 hours. Desired band [expect to see supercoiled and relaxed circular bacterial backbone $(\sim 1.8$ and $\sim 3.2 \mathrm{~kb})$ and supercoiled and relaxed circular (nicked) HPV genome $(\sim 6.2$ and $>16 \mathrm{~kb})]$ was cut out and gel extracted with QIAquick® Gel Extraction Kit (Qiagen). Before transfection of the HPV-16 genomes, growtharrested J2-3T3 fibroblasts were removed by Versene solution and pipetting from co-cultured primary normal cervical keratinocytes (HCK). HCK were plated in a 6-well plate on day prior to transfecting according to manufacturer's protocol for Lipofectamine LTX (Invitrogen). On day of transfection, HCK were at about $70 \%$ confluency. Media was replenished on each well. For each well, 2.8ug of re-ligated 
HPV-16 insert was diluted in 150uL Opti-MEM I Reduced Serum Media with 2.5uL PLUS Reagent, 15uL Lipofectamine LTX was diluted in Opti-MEM I Reduced Serum Media. Diluted DNA and diluted Lipofectamine LTX/Plus complex were combined and incubated for 5 minutes at room temperature prior to adding to well. The transfected cells were passaged at least 10-15 times so HPV-16 immortalized cells could overgrowth the untransfected HCK cells prior to experimentation.

\section{RNA Extraction}

Total RNA of cultured cell lines and human tissues was purified with TRIzol reagent (Invitrogen) and treated with Turbo DNA-free DNase (20 minutes at $37^{\circ} \mathrm{C}$, Ambion) according to manufacturers' protocols. RNA concentration was determined with a Nanodrop 2000 Spectrophotometer (Thermo Scientific).

\section{E. cDNA synthesis, qualitative or quantitative RT-PCR}

RNA was reverse transcribed to complementary DNA (cDNA) under standard iScript cDNA synthesis kit (Bio-Rad) instructions in $\mathrm{T} 100^{\mathrm{TM}}$ Thermal Cycler (Bio-Rad). Exogenous spike of C. Elegans (70ng) was added for normalization of results if GAPDH mRNA levels were altered during processing

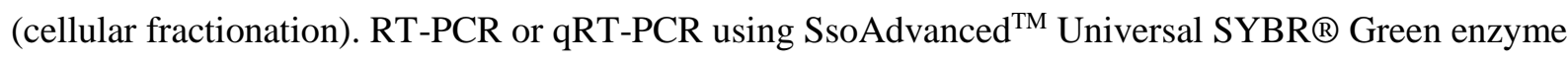
was performed according to manufacturer's protocol (Bio-Rad) in CFX Connect ${ }^{\mathrm{TM}}$ Real-Time System (Bio-Rad). The oligonucleotide primer sequences can be found in Supplementary Table S3. GAPDH was primarily used as housekeeping gene, however Ubiquitin C (UBC) or Glucuronidase Beta (GUSB) were used if GAPDH mRNA levels were altered with significant apoptosis (siRNA FAM83H-AS1 vs. siRNACTRL) and ama-1 was used for cellular fractionation studies. To validate cell fractionation efficiency, U6 snRNA was used as nuclear control and mature mRNA beta-actin as cytoplasmic control. Qualitative RT-PCR product was resolved by agarose gel electrophoresis to visualize or quantitative RT-PCR (qRT$\mathrm{PCR}$ ) relative expression was calculated using the double delta $\mathrm{CT}$ method (relative expression $=2-\Delta \mathrm{CT}$; where $\Delta \mathrm{CT}=\mathrm{CT}$ (Target RNA) - CT (mRNA endogenous GAPDH/UBC control or $C$. Elegans mRNA Ama-1 was exogenous spike control). Fold changes were calculated relative to control siRNA or the mean value of normal samples.

\section{F. Western Blot analysis}


Cell lysates were boiled in sample buffer, separated by SDS-PAGE, and transferred onto Immobilon-Fl polyvinylidene difluoride membranes. After blocking for 1 hour with 5\% milk-TBST buffer (5\% non-fat dry milk, 25mM Tris- $\mathrm{HCl} \mathrm{pH} 8.0,125 \mathrm{mM} \mathrm{NaCl}, 0.5 \%$ Tween-20), the membranes were incubated with primary antibodies against human p53 (Cell Signaling, \#9282) or Actin (C-11) (Santa Cruz, sc-1615) overnight at $4^{\circ} \mathrm{C}$. The membranes were then washed with TBST, incubated with appropriate horseradish peroxidase (HRP)-conjugated secondary antibodies [anti-goat IgG (Santa Cruz, sc-2020); anti-mouse IgG (Thermo Scientific)] for 1 hour at room temperature, washed with TBST, and the proteins were detected on the membrane using Pierce SuperSignal West Pico or Femto Maximum Sensitivity Substrate (Thermo Scientific) chemiluminescence.

\section{G. Transient transfection}

The following siRNAs were used to knock down FAM83H-AS1: Lincode Human FAM83H-AS1 siRNA - Set of 4 (Dharmacon, RU-188909-00-0002), Lincode Human FAM83H-AS1 siRNA

SMARTpool (Dharmacon, R-188909-00-000), Lincode Non-targeting siRNA \#1 (Dharmacon, D-00132001-05). Two different siRNA against HPV-16E6: 5'-GAGGUAUAUGACUUUGCUUTT-3' (Dharmacon) and 5'-UCCAUAUGCUGUAUGUGAUTT-3' (siRNA 209, Dharmacon), two different siRNA against p53 (NEB, \#2011S; Thermo Scientific Dharmacon®, J-003329-16), two different ONTARGETplus Human siRNA against p300 (Dharmacon, J-003486-11-0002; Dharmacon, J-003486-12-0002), and CTLsiRNA (Ambion, AM16104) were used.

HCK, CaSki, and W12/201402 cells were transiently transfected using standard Lipofectamine ${ }^{\circledR}$ RNAiMAX protocol (siRNA FAM83H-AS1 and siRNA HPV-16E6 \#1).W12/201402 and CaSki cells were transfected with reverse transfection protocol (siRNA HPV-16 E6 \#2 to improve transfection efficiency. For standard transfection, cells were plated at appropriate confluency to be about $70 \%$ confluent for transfection 24 hours post-plating. Growth-arrested J2-3T3 fibroblasts were co-cultured with HCK, and on day of transfection removed with Versene solution and gently pipetting . On day of CaSki, W12/201402, and HCK standard transfection, media was replenished on each well. For each well, OptiMEM I Reduced Serum Media was used to dilute Lipofectamine RNAiMAX (9uL per well of 6-well), as well as siRNA (40pmol per well of 6-well). Diluted RNAiMAX was combined with diluted siRNA, incubated 5 minutes at room temperature and added to each well. Cells were incubated 48 hours in media containing siRNA prior to harvesting, unless otherwise stated.

\section{H. Cellular fractionation}


Growth arrested 3T3M cells were removed by Versene solution and pipetting the day prior to fractionation for the W12/201402. Cellular fractionation protocol was modified from previous publication $^{3}$. On ice, CaSki ( 70\% confluent) were washed two times with cold PBS, scraped, pelleted, and resuspended in RSB Buffer (10-1.25mM Tris, $\mathrm{pH} 7.4 ; 10-1.25 \mathrm{mM} \mathrm{NaCl} ; 3-0.38 \mathrm{mM} \mathrm{MgCl} 2)$, incubated on ice for 5 minutes, centrifuged $\left(1500 \mathrm{rpm}\right.$ for 4 minutes at $\left.4^{\circ} \mathrm{C}\right)$. RSB Buffer was removed and swollen pellets were resuspended in RSB-G40 Buffer [2.5-0.63mM Tris, $\mathrm{pH} 7.4 ; 2.5-0.63 \mathrm{mM} \mathrm{NaCl}$; 0.75-0.19mM MgCl2; 2.5-0.63\% glycerol; 0.125-0.03\% NP-40; 0.125-0.03mM DTT; 100U/mL RNasin Plus RNase Inhibitor (Promega)] in $\geq 4$ times volume of pellet. Cells were disrupted with Dounce homogenizer; homogenate was centrifuged (4000 rpm, 4 minutes) to pellet nuclear fraction. The cytoplasmic fraction (supernatant) was collected. The pelleted nuclear fraction was resuspended in RSBG40 Buffer, $3.3 \%$ of sodium deoxycholate and $6.6 \%$ of Tween 20 were added to the volume of RSB-G40 (10\% of final volume was sodium deoxycholate and $10 \%$ was Tween 20 ), samples were tapped to mix, incubated on ice for 5 minutes, and pelleted by centrifugation (7000rpm, 3 minutes). The supernatant was collected and combined with first cytoplasmic fraction, and nuclear pellet was washed two times with RSB-G40 Buffer, and then resuspended in RSB-G40 Buffer. Fractionated cells were Turbo DNase Itreated, according to manufacturer's instructions (NEB), prior to RNA extraction to remove bound DNA and improve recovery of RNA. Total cell (second pellet), cytoplasmic, and nuclear RNA was extracted using TRIzol Reagent (Life Technologies) per manufacturer's instructions (Glycoblue was added during isopropanol precipitation step due to small amounts of RNA recovered from nuclear fraction) and treated with Turbo DNAfree DNase (Ambion) for 20 minutes at $37^{\circ} \mathrm{C}$. qRT-PCR analysis was performed using cDNA generated using equal concentration of RNA with exogenous C. elegans RNA (70ng) added for normalization.

\section{Cell proliferation assay}

Cell proliferation was monitored using Cell Counting Kit - 8 (CCK-8) (Sigma-Aldrich). 24 hours (CaSki) or 48 hours (201402) after transfection with FAM83H-AS1 siRNA, CaSki cells were detached with $0.05 \%$ trypsin and W12/201402 cells were detached with $0.25 \%$ trypsin. After cell detachment, trypsin was inactivated with trypsin neutralizer (CaSki) or E-media containing serum (201402), pelleted, resuspended in appropriate media containing serum, and plated at desired concentration (3E3 cells per well and of four separate 96-well plates; technical triplicates). In equal density per well, 3T3M fibroblasts treated with mitomycin C were added to the W12/201402 cells and fibroblast alone control wells were plated. At desired time (48, 72, or 96h post-plating), CCK-8 dye (100uL/well) was added to each well, 
incubated for 2 hours (CaSki) or 3 hours (201402). Then, absorbance was read with a spectrophotometer (BioTek Synergy H1 Hybrid Reader) $\left(\mathrm{OD}_{450}\right)$ according to manufacturers' protocols. Absorbances of feeders alone were subtracted from W12/201402 co-cultured with feeders. Biological replicate was conducted.

\section{J. Flow-cytometric analysis of cell cycle.}

3T3M fibroblasts were stained with CellTrace ${ }^{\mathrm{TM}}$ Far Red Cell Proliferation Kit (Invitrogen) the day before plating transfected 201402 cells. 24 hours (CaSki) or 48 hours (201402) after transfection with FAM83H-AS1 siRNA, cells were detached with $0.05 \%$ trypsin (CaSki) or $0.25 \%$ trypsin (201402). After cell detachment, trypsin was inactivated with trypsin neutralizer (CaSki) or media containing serum (201402), and cells were plated at desired concentration (4E6 for CaSki and 2.3E6 for 201402) in 100mm dishes in media containing fetal bovine serum (FBS). Mitomycin C treated, far red stained 3T3M cells were added to the 201402 cells. Cells were allowed to attach (about 5 hours) then were washed 3 times with 1X D-PBS to remove residual FBS. Cells were then serum-starved for 24 hours. After incubation, cells were trypsinized with $0.25 \%$ trypsin, suspended in serum-free media (to avoid stimulation by serum), washed with 1X PBS, pelleted, and resuspended in PBS $(200 \mu \mathrm{L})$. Harvested cells were then fixed by adding $70 \%$ cold ethanol $(1.8 \mathrm{~mL})$ while vortexing the cells and stored at $4{ }^{\circ} \mathrm{C}$ until processing. Cells in ethanol were pelleted, washed with PBS, centrifuged, and resuspended in room temperature $0.2 \%$ Tween 20 in PBS (300-1,000 $\mathrm{LL}$, depending on pellet size). 100uL of each sample was placed in a U-bottom 96well plate ( 3 wells/technical triplicates) and incubated 15 minutes at $37^{\circ} \mathrm{C}$. PBS (100uL) was added to each well, cells were pelleted, resuspended in $10 \mathrm{uL}$ of RNase A-PBS (180ug/mL stock), tapped gently to mix, and incubated at room temperature for 15 minutes. After incubation, 20uL of PI-PBS (final concentration of $50 \mu \mathrm{g} / \mathrm{mL}$ ) was added to each well, pipetted to mix, and incubated for 15 minutes at room temperature. After incubation, the volume was brought up to $300 \mathrm{uL}$ and analyzed in the dark by flow cytometry (Fortessa S10). Gates were placed around 201402 population. Biological replicate was conducted.

\section{$\underline{\text { K. Transwell migration assay }}$}

24 hours (CaSki) or 48 hours (201402) after transfection with FAM83H-AS1 siRNA, cells were detached with $0.05 \%$ trypsin. After cell detachment, trypsin was inactivated with trypsin neutralizer, pelleted, resuspended in serum-free media, and cells were plated [1E5 (CaSki) or 0.75E5 (201402), $200 \mathrm{uL}$ well, technical duplicates] in upper chambers of 24 -well transwell ( $8 \mathrm{~mm}$ pore size) with $800 \mathrm{uL}$ 
serum-free media in lower chamber. In equal density per well, 3T3M fibroblasts treated with mitomycin $\mathrm{C}$ were added to the W12/201402 cells and fibroblast alone control wells were plated. Cells were then allowed to attach for $\sim 3$ hours prior to adding fetal bovine serum (final concentration of $20 \%$ for CaSki and 5\% for W12/201402) to lower chamber. After 24 hours, media in upper chamber was replenished with appropriate fresh serum free media and lower chamber with appropriate fresh media containing with FBS chemoattractant. 48 hours after cells were plated, lower chamber/underside of transwell and upper chamber of transwell were washed with $25 \%$ D-PBS. D-PBS was removed and cells from the top of the upper chamber transwell membrane (non-migrated cells) were wiped away using a cotton swab. The lower chamber/underside of the transwell and upper chamber of transwell were washed again with $25 \%$ D-PBS and then the underside of the transwell (migrated cells) was fixed with $4 \%$ formaldehyde in $25 \%$ D-PBS in the lower chamber for 5 minutes at room temperature. The formaldehyde solution was removed and the lower chamber/underside of transwell and upper chamber of transwell were washed with 25\% DPBS. The cells on the underside of the transwell were then stained using $0.5 \%$ crystal violet in $20 \%$ ethanol in water. The stain was added to the lower chamber, submerging the transwell for 20 minutes at room temperature. The stain was removed and the lower chamber/underside of the transwell and upper chamber of transwell washed 3 times with $25 \%$ D-PBS. The top of the upper chamber transwell membrane was then cleaned with a cotton swab. The transwells were allowed to dry overnight and then the underside of the transwell imaged with an Olympus MVX10 microscope. Migration was quantitated with ImageJ. Feeders alone were subtracted from W12/201402 co-cultured with feeders. Biological replicate was conducted.

\section{Flow-cytometric analysis of apoptosis}

3T3M fibroblasts were stained with CellTrace ${ }^{\mathrm{TM}}$ Far Red Cell Proliferation Kit (Invitrogen) the day before plating transfected 201402 cells. 24 hours (CaSki) or 48 hours (201402) after transfection with FAM83H-AS1 siRNA, cells were detached with $0.05 \%$ trypsin (CaSki) or $0.25 \%$ trypsin (201402). After cell detachment, trypsin was inactivated with trypsin neutralizer (CaSki) or media containing serum (201402), pelleted, resuspended in appropriate media containing serum, and cells were plated in 100mm dishes at desired concentration (1.75E6 for CaSki and 1.1E6 for 201402). At 24, 48, and 72h later postplating, attached and floating cells were collected. On day of harvest, media containing dead cells was collected, PBS was added to the plates, collected, and added to the media. Attached cells were trypsinized with $0.25 \%$ tryspin, cells were pelleted ( 2 to $5 \mathrm{E} 6$ ) at $4{ }^{\circ} \mathrm{C}$, resuspended in PBS, and $100 \mathrm{uL}$ of each sample was aliquotted into 3 wells of a 96 well plate (technical triplicates). Cells were pelleted, PBS was removed, Annexin V Binding Buffer (100uL; diluted with autoclaved milliQ water) was added to each 
tube, Annexin V-FITC (5uL) was added to each tube, and incubated in the dark at room temperature for 10 minutes. After incubation, cold Binding buffer $(200 \mathrm{uL})$ was added to each tube, cells were pelleted, washed with cold Binding buffer two times, incubated on ice for 5 minutes in propidium iodine in Binding buffer ( $2 \mathrm{ug} / \mathrm{mL}$ final concentration, $300 \mathrm{uL}$ ), and analyzed by flow cytometry (Fortessa S10) immediately. Gates were placed around 201402 population. Biological replicate was conducted.

\section{Human cervical tissue specimen}

10 human specimens were obtained from patients under the tissue collection protocol [Prognostic Marker (IRB0406147)] at the University of Pittsburgh. Patient samples were categorized as cervical intraepithelial neoplasia stage 3 (CIN3), invasive cervical cancer $(\mathrm{CaCx})$, or non-cancerous.

\section{N. TCGA Analysis}

The Cancer Genome Atlas (TCGA) (https://cancergenome.nih.gov/) contains patient survival and RNA sequencing data from 196 cervical squamous cell carcinoma and endocervical adenocarcinoma (CESC) human patient samples. TCGA-CESC reads per kilobase million (RPKM) data was extracted, and average RPKM for all patients combined was calculated. TCGA also contains RPKM data from 3 individual non-cancerous cervical samples that were averaged together to compare to CESC RPKM values. The Atlas of non-coding RNA in Cancer (TANRIC) (MD Anderson Cancer Center) ${ }^{4}$ that utilizes TCGA-CESC dataset to characterize the expression profiles of long non-coding RNAs (lncRNAs) was used to extract FAM83H-AS1 expression in each of the TCGA-CESC patient samples. Classification and Regression Trees (CART) analysis was used to statistically define high/low lncRNA expression groups. The cut-off z score value was 3 to discern between high and low expression groups. Survival curves were estimated by the Kaplan-Meier method. The log-rank test was used to estimate statistical differences between survival curves. GraphPad software was used to make the survival plots.

\section{O. Statistics}

Student's t-test was utilized to determine mean values differences between groups examined and significance was determined at $\mathrm{p} \leq 0.05(*)$ and $\mathrm{p} \leq 0.01(* *)$. Scale bars represent Standard Deviations (SD).

\section{$\underline{\text { XIV. Supplemental References }}$}


1 Liu, X. et al. ROCK inhibitor and feeder cells induce the conditional reprogramming of epithelial cells. The American journal of pathology 180, 599-607, doi:10.1016/j.ajpath.2011.10.036 (2012).

2 Schowalter, R. M., Pastrana, D. V. \& Buck, C. B. Glycosaminoglycans and sialylated glycans sequentially facilitate Merkel cell polyomavirus infectious entry. PLoS pathogens 7, e1002161, doi:10.1371/journal.ppat.1002161 (2011).

3 Martinez, I. et al. An Exportin-1-dependent microRNA biogenesis pathway during human cell quiescence. Proceedings of the National Academy of Sciences of the United States of America 114, E4961-e4970, doi:10.1073/pnas.1618732114 (2017).

4 Li, J. et al. TANRIC: An Interactive Open Platform to Explore the Function of lncRNAs in Cancer. Cancer research 75, 3728-3737, doi:10.1158/0008-5472.can-15-0273 (2015). 


\title{
Chapter 3
}

\section{An Exportin-1-dependent microRNA biogenesis pathway during human cell quiescence}

\author{
Ivan Martinez, ${ }^{\mathrm{a}, 1,2}$ Karen E. Hayes, ${ }^{\mathrm{a}, 1}$ Jamie A. Barr, ${ }^{\mathrm{a}}$ Abby D. Harold, ${ }^{\mathrm{a}}$ Mingyi Xie, ${ }^{\mathrm{b}}$ Syed I. A. \\ Bukhari, ${ }^{\mathrm{c}}$ Shobha Vasudevan, ${ }^{\mathrm{c}}$ Joan A. Steitz, ${ }^{\mathrm{d}, \mathrm{e}, \mathrm{f}, 2}$ and Daniel DiMaio ${ }^{\mathrm{d}, \mathrm{f}, \mathrm{g}, \mathrm{h}}$
}

${ }^{a}$ Department of Microbiology, West Virginia University Cancer Institute, West Virginia University, Morgantown, WV, 26506; 'bepartment of Biochemistry and Molecular Biology, University of Florida Health Cancer Center, University of Florida, Gainesville, FL, 32610; ${ }^{\circ}$ Cancer Center, Massachusetts General Hospital, Harvard Medical School, Boston, MA, 02114; ${ }^{\mathrm{d} D e p a r t m e n t ~ o f ~ M o l e c u l a r ~ B i o p h y s i c s ~}$ and Biochemistry, Yale University, New Haven, CT, 06536; ${ }^{\mathrm{e} H o w a r d ~ H u g h e s ~ M e d i c a l ~ I n s t i t u t e, ~ Y a l e ~}$ University, New Haven, CT, 06536; ${ }^{\text {Y}}$ Yale Cancer Center, New Haven, CT, 06520; ${ }^{\mathrm{g}}$ Department of Genetics, Yale School of Medicine, New Haven, CT, 06510; hepartment of Therapeutic Radiology, Yale School of Medicine, New Haven, CT, 06510

Key Words: XPO5, XPO1, (TMG)-cap, pri-miRNA, quiescence

${ }^{1}$ Authors contributed equally to this work.

${ }^{2}$ Correspondence: Ivan Martinez (ivmartinez@hsc.wvu.edu) and Joan A. Steitz (joan.steitz@yale.edu)

Published in: Proceedings of the National Academy of Sciences in the United States of America Proc Natl Acad Sci U S A. 2017 Jun 20;114(25):E4961-E4970. PMID: 28584122 


\section{Contribution to the Project}

I assisted in numerous ways to progress the research presented in Chapter 3 to publication. I primarily conducted experiments that advanced the content in Figures 3-6. While obtaining data for these figures, I cultured cells (including human foreskin fibroblasts (HFFs) and human embryonic kidney (HEK293T)), and, on multiple occasions, I assisted with plating, inducing cellular quiescence (by serum starvation or confluency), transfecting, transducing, and pelleting cells, as needed (in experiments not described below). Additionally, I contributed to the development of several experiments stated below.

I conducted preliminary experiments in HFFs to optimize transfection conditions for future experiments. I determined optimal amounts of plasmid (GFP) and Lipofectamine for proliferating and quiescent cells (data not shown).

I conducted a preliminary experiment to determine how long microRNAs (miRNAs) could be expressed after knockdown of Exportin-1 (XPO1) followed by induction of quiescence for future experiments. To do this, I cultured and transfected HFF cells with Lipofectamine and siRNA against XPO1. Once cells were $100 \%$ confluent, I pelleted the cells at Day 0, 3, 6, 9, 12 for protein and RNA expression analysis. I conducted RNA extraction, cDNA synthesis, and qRT-PCR analysis to confirm knockdown of XPO1 and differential expression of other select genes (data not shown).

We analyzed downstream effects of individual knockdown of Exportin-5 (XPO5) (Fig. 3A, SI Appendix, Figs. S6A) and XPO1 (Figs. 3B-C, SI Appendix, Figs. S6B and S7A-E) in proliferating and quiescent HFFs using multiple siRNAs against XPO5 and XPO1, respectively. The Western Blot analysis I conducted were used in the manuscript for siRNA against XPO5 (Fig. 3A (right panel), SI Appendix Fig. S6A (middle panel)) and XPO1 (Fig. 3B (right panel), SI Appendix, Figs. S6B (middle panel) and S7D) as well as the samples I conducted RNA extraction, cDNA synthesis, and qRT-PCR analysis on to show RNA changes with individual siRNA against XPO5 (SI Appendix, Fig. S6A (left panel)) and individual siRNA against XPO1 (SI Appendix, Fig. S6B (left panel), S7A-C). Collaborators then used these samples for downstream experiments (Fig. 3C, SI Appendix, Figs. S6A-B (right panels), SI Appendix, Fig. S7E).

We exogenously expressed XPO1 in HFFs, immunoprecipitated XPO1, and discovered primary microRNAs (pri-miRNAs) that are bound to XPO1 (Fig. 3D, SI Appendix, Figs. S7F, S7G). To do this, we initially optimized transfection conditions (plasmid concentration and Lipofectamine volume) of an expression vector (not retroviral) (p3XFlag-XPO1). A collaborator transfected the cells. I then pelleted and 
conducted RNA extraction, cDNA synthesis, and qRT-PCR analysis as well as protein extraction and Western Blot analysis to confirm XPO1 expression (data not shown). We were having difficulty exogenously expressing XPO1 with this construct, so I transformed and maxi-prepped to extract fresh plasmid. I performed a restriction digestion and ran the samples in an agarose gel to confirm the correct predicted size insert was digested (data not shown). To quickly determine if fresh plasmid solved the issues, I transfected the p3XFlag-XPO1 plasmid into HEK293T cells with Lipofectamine and performed Western Blot analysis (data not shown). A collaborator attempted to transfect the XPO1 expression constructs by nucleofection, and I performed Western Blot analysis to detect XPO1 protein knockdown (data not shown). Then, a collaborator transfected proliferating and serum-starved HFFs with p3X-Flag XPO1 and attempted multiple immunoprecipitations (IP) with an antibody against Flag. For this IP, I aided by crosslinking and conducting downstream RNA extraction, cDNA synthesis, and qRT-PCR analysis as well as protein extraction and Western Blot analysis (data not shown). Due to technical issues, a collaborator cloned XPO1 into a retroviral vector (pBABE-Flag-XPO1). I transfected HEK293T with a calcium phosphate protocol to produce a retrovirus that was transduced into HFFs to successfully exogenously expressed XPO1 in HFFs. A collaborator serum-starved cells, then I crosslinked, immunoprecipitated with an antibody against Flag, performed Western Blot analysis (SI Appendix, Fig. S7F (left panel)) and initiated RNA extraction used for the figure in the manuscript to identify pri-miRNAs bound to XPO1 (Fig. 3D, SI Appendix, Fig. S7F (right panel), Fig. S7G). I performed replicates of this experiment. It was desired for potential future experiments to stably select a pool population and single cell clones of HFFs expressing XPO1. Unfortunately, after I transfected HEK293T (pBABE-Flag-XPO1), transduced HFFs, selected with antibiotics, and cultured the "stable" pool and single cell clones, they did not maintain exogenous expression of XPO1 after multiple passages (data not shown).

We knocked down TGS1 in proliferating and quiescent HFFs using siRNA against TGS1 (Fig. 4, SI Appendix, Figs. S8 and S9). Prior to my assistance, my collaborators were observing only moderate knockdown of TGS1 at the RNA level with siRNA against TGS1 in HFFs. To aid in improving TGS1 knockdown, we performed optimization experiments. I varied the amount of siRNA against TGS1 (while a collaborator varied the amount of Lipofectamine concentration) transfected into proliferating and quiescent cells to determine the optimal conditions to observe TGS1 knockdown without toxicity to the cells. I conducted RNA extraction, cDNA synthesis, and qRT-PCR analysis as well as protein extraction and Western Blot analysis on all of our samples to optimize TGS1 knockdown efficiency in HFFs (data not shown). After optimization, I significantly aided in conducting multiple protein extraction and Western Blot analysis experiments as well as multiple RNA extraction, cDNA synthesis, and qRT-PCR analysis 
using pellets provided by a collaborator who performed the upstream portion of experiments (Fig. 4, SI Appendix Figs. S8 and S9).

After we obtained results presented in Figure 4, we pursued the potential of Snurportin-1 in regulating localization of the TMG-capped pri-miRNAs induced during quiescence (data not shown). (Snurportin-1 is involved with recognizing a $\left(\mathrm{m}^{2,2,7} \mathrm{G}\right.$, TMG)-cap on certain small nucleolar RNAs (snRNAs) to import them to the nucleus from the cytoplasm(1)). I performed preliminary experiments to explore the regulation of Snurportin-1 in quiescent versus proliferating cells. To do this, I conducted Western Blot analysis of HFFs cells that were quiescent for $0,1,2,3$ days to analyze for protein expression changes in Snurportin-1 (data not shown). Additionally, I conducted Western Blot analysis on HFF cell pellets with siRNA against Snurportin-1 provided by a collaborator so I could detect knockdown of Snurportin-1 protein (data not shown).

We knocked down Drosha and XPO1 individually as well as together in proliferating and quiescent HFFs for downstream experiments (Fig. 5, SI Appendix, Figs. S10 and S12). I conducted Western Blot analysis to confirm Drosha and/or XPO1 knockdown (SI Appendix, Fig. S12 (top panel)) prior to a collaborator performing downstream experiments with these samples (Fig. 4, SI Appendix, Figs. S12 (bottom panel), S10).

After we identified an increased expression of a short isoform of Drosha in quiescent cells (Fig. 6A), we used a pool of three siRNAs to target Drosha mRNA in proliferating and quiescent cells and determined that these siRNAs were knocking down both isoforms of Drosha (SI Appendix, Fig. S13A). To do this experiment, a collaborator performed upstream knockdown experiments, and I processed the provided pellets through protein extraction and Western Blot analysis. The data I obtained was used in the manuscript (SI Appendix, Fig. S13A).

We performed cellular fractionation of quiescent and proliferating HFFs to determine localization of Drosha (Fig. 6B). While a collaborator was optimizing cellular fractionation conditions for proliferating and serum-starved HFFs, I processed multiple rounds of pellets for protein extraction and Western Blot analysis. Additionally, I obtained the data that was used in the manuscript (Fig. 6B).

Moreover, I proofread the manuscript prior to the initial submission and re-submission after revisions as well as proofread the comments to the reviewers upon re-submission. 
In summary, my experimental and writing contribution to this specific manuscript were substantial. The participation in this project helped me learn several molecular techniques as well as the process of submission and publication of its findings.

1. Huber J, Cronshagen U, Kadokura M, Marshallsay C, Wada T, Sekine M, Luhrmann R. 1998. Snurportin1, an m3G-cap-specific nuclear import receptor with a novel domain structure. Embo j 17:4114-4126. 


\section{Abstract}

The reversible state of proliferative arrest known as cellular quiescence plays an important role in tissue homeostasis and stem cell biology. By analyzing the expression of microRNAs (miRNAs) and miRNA processing factors during quiescence in primary human fibroblasts, we identified a group of miRNAs that are induced during quiescence despite markedly reduced expression of Exportin-5, a protein required for canonical miRNA biogenesis. The biogenesis of these quiescence-induced miRNAs is independent of Exportin-5 and depends instead on Exportin-1. Moreover, these quiescence-induced primary (pri)-miRNAs are modified with a 2,2,7-trimethylguanosine (TMG)-cap, which is known to bind Exportin-1, and knock-down of Exportin-1 or trimethylguanosine synthase 1, responsible for (TMG)capping, inhibits their biogenesis. Surprisingly, in quiescent cells Exportin-1-dependent pri-miR-34a is present in the cytoplasm together with a small isoform of Drosha, implying the existence of a different miRNA processing pathway in these cells. Our findings suggest that during quiescence, the canonical miRNA biogenesis pathway is down-regulated, and specific miRNAs are generated by an alternative pathway to regulate genes involved in cellular growth arrest.

\section{Significance}

Quiescence is a growth-arrested cellular state where genes involved in this process are finely regulated by several factors including miRNAs. During miRNA biogenesis, Exportin-5 transports miRNA precursors from the nucleus to the cytoplasm. In this study, we demonstrated the existence of an alternative miRNA biogenesis pathway in quiescent primary human cells. This pathway involves the repression of Exportin-5 expression by autophagy and miRNAs, and the (TMG)-cap modification of specific primiRNAs, which signals their export to the cytoplasm by Exportin-1. We further showed that these primiRNAs are rapidly processed in the cytoplasm by a small isoform of Drosha. Collectively, these results reveal a new mechanism of miRNA biogenesis that will expand our understanding of miRNA regulation in normal or disease-related cells.

\section{Introduction}

Most metazoan cells enter a reversible cell cycle arrest known as cellular quiescence when they are exposed to anti-mitogenic signals or an environment unfavorable for proliferation $(2,3)$. In mammalian cells, quiescence (also known as $\mathrm{G}_{0}$ arrest) is characterized by reduced DNA replication, altered metabolism, increased autophagy, and increased expression of cyclin-dependent kinase inhibitors such as 
$\mathrm{p} 27^{\mathrm{Kipl}}(4,5)$. In vitro, quiescence can be induced in primary cells by serum starvation, contact inhibition, and loss of adhesion to a substrate (6). Quiescence is involved in important cellular processes including the balance between differentiation and self-renewal in different types of stem cells, and dysregulation of quiescence could favor carcinogenesis. However, the molecular mechanisms that regulate quiescence are poorly understood (7).

MicroRNAs (miRNAs) are small non-coding RNAs $~ 22$-nucleotides (nt) long that regulate the expression of protein-coding genes by base-pairing with the 3' untranslated region (3'UTR) of messenger RNAs (mRNAs), repressing translation and/or inducing degradation of the target mRNA $(8,9)$. In canonical miRNA biogenesis in mammalian cells, miRNAs are transcribed by RNA polymerase II to produce 7methylguanosine-capped $\left(\mathrm{m}^{7} \mathrm{G}\right)$ primary miRNAs (pri-miRNAs) containing one or more bulged hairpin structures that are recognized by the nuclear microprocessor, which consists of the ribonuclease (RNase) III enzyme Drosha and the double-stranded RNA (dsRNA) binding protein DiGeorge syndrome critical region gene 8 (DGCR8) (10-13). Specific cleavage of the pri-miRNA by the nuclear microprocessor generates an 70-nt stem-loop structure known as the precursor miRNA (pre-miRNA), which is recognized and transported to the cytoplasm by Exportin-5 (XPO5) $(4,11,12,14)$. Subsequently, pre-miRNA is cleaved by the cytoplasmic RNase III enzyme Dicer (15-18), generating a double-stranded mature miRNA. Finally, the guide miRNA strand is loaded into the RNA-induced silencing complex (RISC) containing one of four Argonaute proteins and GW182 protein at its core (19-21).

Recent studies have identified alternative pathways of microRNA biogenesis in different cell and animal models: pre-miRNA/introns miRNAs (miRtrons), which are Drosha and DGCR8 independent (22); Drosha and DGCR8-independent viral miRNAs that use the Integrator complex to produce viral premiRNAs (23); miRNAs independent of DGCR8, Exportin-5, Dicer and Argonaute 2 (AGO2) known as Simtrons (24), which are exported from the nucleus by an unknown mechanism; Dicer-independent miRNAs, such as miR-451, which are generated by the catalytic activity of AGO2 $(25,26)$; and cytoplasmic re-localization of Drosha by RNA viruses, with no changes in endogenous miRNA expression but robust synthesis of viral miRNAs (27). Recently, we reported a novel class of $\left(\mathrm{m}^{7} \mathrm{G}\right)$-capped pre-miRNAs in human cells whose 5' end corresponds to the transcription initiation site (28); these pre-miRNAs are exported from the nucleus by Exportin-1 rather than Exportin-5.

Exportin-1 recognizes certain RNAs including viral mRNAs, cellular mRNAs, and small nuclear RNAs (snRNAs) and exports them from the nucleus to the cytoplasm (29). For example, precursors of snRNAs involved in mRNA processing such as $\mathrm{U} 1, \mathrm{U} 2, \mathrm{U} 4$, and $\mathrm{U} 5$ have a $\left(\mathrm{m}^{7} \mathrm{G}\right)$-cap, which is recognized 
by cap-binding complex (CBC) and the phosphorylated adaptor for RNA export (PHAX) in the nucleus to enable their export to the cytoplasm (30) by Exportin-1. These snRNAs are then recognized by Sm proteins in the cytoplasm and trimethylguanosine synthase 1 (TGS1) is recruited to hypermethylate the $\left(\mathrm{m}^{7} \mathrm{G}\right)$-cap into a $\left(\mathrm{m}^{2,2,7} \mathrm{G}, \mathrm{TMG}\right)$-cap. This modification is recognized by Snuportin-1 in association with Importin- $\beta$ and other factors to import the snRNAs back into the nucleus $(31,32)$. Interestingly, Exportin-1 also has high affinity for the (TMG)-capped small nucleolar RNA (snoRNA) U3 in the nucleus and transports it from Cajal bodies to the nucleoli (33). Another study showed that TGS1 enhances Rev-dependent HIV-1 RNA expression by (TMG)-capping viral mRNAs in the nucleus, thereby increasing recognition by Exportin-1 for transport to the cytoplasm (34).

miRNAs play an important role in regulating cellular quiescence. For example, it has been shown that over-expression of miR-221 and miR-222, both of which target the 3'UTRs of p27 and p57 mRNA, induce S-phase entry in quiescent cells (35). Another study reported up-regulation of let-7 and miR-125 and down-regulation of the miR-29 family in quiescent fibroblasts (36). Furthermore, over-expression of miR-29 increased cell cycle re-entry in quiescent cells, whereas over-expression of let-7 and miR-125 delayed cell cycle re-entry (36). Modulation of miR-29 can also influence senescence, an irreversible growth arrested state, by affecting B-myb expression (37). We also showed that during quiescence some miRNAs up-regulate translation of their target mRNAs (38). Finally, Exportin-5 protein expression is reduced during quiescence and promptly induced during cell cycle entry, causing a global increase in miRNA expression (39).

Here, we report the existence of an alternative miRNA biogenesis pathway in primary human foreskin fibroblasts (HFFs) during quiescence. We confirmed that the level of Exportin-5 is substantially reduced during quiescence and that certain miRNAs are induced during quiescence despite this paucity of Exportin-5. We also demonstrate that the biogenesis of pri-miRNAs corresponding to these miRNAs is dependent not on Exportin-5, but rather Exportin-1. Furthermore, the Exportin-1-dependent pri-miRNAs have a trimethylguanosine $\left(\mathrm{m}^{2,2,7} \mathrm{G}\right.$, TMG)-cap and are found in the cytoplasm during quiescence, as is a smaller isoform of Drosha. Together, our findings suggest that in quiescent cells, an alternative miRNA biogenesis pathway selectively processes and transports a specific set of miRNAs, which could be essential for reversible $\mathrm{G}_{0}$ arrest.

\section{$\underline{\text { V. Results }}$}



quiescence

To examine global miRNA expression during quiescence, miRNA microarrays that interrogated 1105 mature human miRNAs were used to compare proliferating HFFs and quiescent HFFs after 12, 48, and $72 \mathrm{~h}$ of serum starvation. We identified 88 up-regulated and 94 down-regulated miRNAs that changed 1.5-fold or more for at least two time-points of serum starvation (Fig. 1A and SI Appendix, Tables S1, S2). As assessed by qRT-PCR, mature miR-26a-2, miR-34a, miR-126, miR-199b, miR-638, miR-1228*, and miR-3188 were induced two- to four-fold by serum starvation, and miR-17, miR-18a, miR-29b, miR-155, and miR-423-3p were repressed two- to three-fold, confirming the microarray data (Fig. 1B). There was a similar pattern of miRNA expression (with the exception of miR-26a-2 and miR-126) when quiescence was induced by confluency (SI Appendix, Fig. S1A). Northern blot analysis confirmed induction of representative miRNAs in quiescence induced by serum starvation or confluency (SI Appendix, Fig. S2). By using specific primers to amplify the pri-miRNAs of these differentially expressed miRNAs (SI Appendix, Table S3), we found that the mature miRNAs induced during quiescence are also induced at the pri-miRNA level (Fig. $1 C$ and SI Appendix, Fig. S1B). In contrast, rather than being repressed, the levels of the pri-miRNAs corresponding to the repressed mature miRNAs miR-17, miR-18a, miR-29b, miR-155, and miR-423-3p were induced during quiescence (Fig. $1 C$ and SI Appendix, Fig. S1B), suggesting that repression of these miRNAs is due to post-transcriptional regulation, presumably during their processing through the microRNA biogenesis pathway.

\section{B. Exportin-5 expression is down-regulated by autophagy and miR-34a during quiescence.}

To explore the mechanism of miRNA synthesis in quiescent cells, the abundance of proteins involved in miRNA biogenesis was examined in quiescent HFF. Confirming the previous findings of Iwasaki and collaborators (39), Exportin-5 protein levels were dramatically reduced ( 30-fold) during quiescence induced by serum starvation (Fig. 2A, and SI Appendix, Fig. S3A) or contact inhibition (SI Appendix, Fig. S3B), as assessed by immunoblotting and immunofluorescence. Exportin-5 protein was also reduced in serum-starved HeLa (human cervical carcinoma) and C127 (murine mammary tumor) cell lines (SI Appendix, Fig. S3C). The level of Exportin-5 mRNA did not change during quiescence (SI Appendix, Fig. S3 $D$ and $E$ ), indicating that Exportin-5 is regulated at the post-transcriptional level. The level of Exportin-1, a protein involved in protein and RNA export (29), did not change during quiescence (Fig. 2A, and SI Appendix, Fig. S3B). The quiescence marker p27 was induced, as expected (40). 
To determine the basis for the reduction in Exportin-5 expression during quiescence, we blocked two major pathways of protein degradation, the proteasome and the autophagy pathways. Treatment of quiescent cells with the proteasome inhibitor, MG132, did not restore Exportin-5 expression, whereas the protein MDM2, which is known to be degraded by the proteasome, is maintained upon MG132 treatment, as expected (Fig. 2B, and SI Appendix, Fig. S4A). In fact, the level of Exportin-5 was further reduced in response to MG132 treatment, suggesting that a down-regulation pathway is induced by this drug (Fig. $2 B$ ). Treatment with 3-methyladenine (3-MA), an autophagy inhibitor, reduced the level of Exportin-5 in proliferating cells (Fig. 2C). Strikingly, treatment of quiescent HFFs with 3-MA restored the levels of Exportin-5 and of p62, a protein specifically degraded by autophagy (Fig. $2 C$ and SI Appendix, Fig. S4B). These data suggest that the autophagy pathway is responsible for accelerated degradation of Exportin-5 during quiescence.

We also tested whether Exportin-5 levels are modulated by miRNAs. The 3'UTR of the Exportin5 mRNA contains a conserved predicted binding site for miR-34a (Fig. 2D and SI Appendix, Fig. S5), which as shown above is induced during quiescence (Fig. $1 B$ and SI Appendix, Fig. S1A). To determine if miR34a affects expression of Exportin-5, we transfected synthetic miR-34a into proliferating HFF cells and examined the expression of Exportin-5 after $48 \mathrm{~h}$. As shown in Figure 2E, transfected miR-34a reduced the amount of endogenous Exportin-5 protein but not Exportin-5 mRNA, suggesting translational repression of Exportin-5 by miR-34a. In contrast, there was no change in expression of Exportin-1, which does not contain a miR-34a binding site in its 3'UTR. To determine if there is a direct functional interaction between miR-34a and Exportin-5 mRNA, we cloned the complete 3'UTR of the Exportin-5 gene (1,537 nt) into a luciferase reporter vector and transfected quiescent HFF cells with the empty reporter vector, the reporter vector containing the Exportin-5 3'UTR, or a reporter vector containing the Exportin-5 3'UTR with three point mutations in the miR-34a seed sequence binding site (Fig. $2 D$ ). As shown in Figure $2 F$, luciferase activity from the vector containing the wild-type Exportin-5 3'UTR was reduced about two-fold during quiescence compared to the vector lacking the 3'UTR, and there was partial recovery of luciferase activity in response to the mutant 3'UTR. These results suggest that regulation of Exportin-5 expression in quiescent cells is mediated in part by miR-34a. We also co-transfected proliferating HFFs with the Exportin-5 3'UTR luciferase reporter vectors and synthetic miR-34a or scrambled control miRNA. miR34a but not the control miRNA caused a reduction in luciferase activity from the wild-type Exportin-5 3'UTR reporter but not from the miR-34a binding site mutant or empty vector (Fig. 2G). Together these results demonstrate a functional interaction between Exportin-5 3'UTR and miR-34a. We conclude that Exportin-5 expression is regulated at two different levels during quiescence, at the protein level by autophagy and at the mRNA level by miR-34a. 


\section{Expression of quiescence-induced miRNAs is not dependent on Exportin-5.}

The increase in the levels of specific mature miRNAs in quiescent cells expressing little Exportin5 raised the possibility that these quiescence-induced miRNAs are generated by an Exportin-5-independent pathway. To investigate this possibility, we transiently transfected proliferating HFFs with an siRNA against Exportin-5 and $48 \mathrm{~h}$ later measured miRNA levels. Exportin-5 knock-down was confirmed by Western blot and qRT-PCR (Fig. 3A). As expected, Exportin-5 knock-down in proliferating cells lowered levels of mature miR-17, miR-18a, miR-29b, miR-155, and miR-423-3p, which are repressed during quiescence (Fig. 3A, dark gray bars). In contrast, levels of their corresponding primary miRNAs did not decline (Fig. 3A, light gray bars), consistent with Exportin-5 being required for maturation of these miRNAs, as it is for all canonically processed miRNAs. Strikingly, however, Exportin-5 knock-down did not repress the levels of several mature miRNAs and pri-miRNAs that are induced during quiescence, including miR-26a, miR-34a, miR-126, miR-199b, miR-638, and miR-3188 (Fig. 3A). Similar results were obtained with a second Exportin-5 siRNA in an independent experiment (SI Appendix, Fig. S6A). Although, we cannot exclude other possibilities such as a higher affinity for Exportin-5 by some quiescence-induced miRNAs (which can thus tolerate lower Exportin-5 levels), or differences in the stability of the mature miRNA (41), our findings suggest that the biogenesis of these miRNAs can proceed via an Exportin-5independent mechanism, even in proliferating cells.

\section{Exportin-1 is required for processing of quiescence-induced miRNAs.}

The results in the preceding section imply that an export factor other than Exportin-5 is required for the synthesis of mature miRNAs during quiescence. Previous studies showed that Exportin-1, an essential transport factor for certain proteins and RNAs, supports pri-miRNA processing in C. elegans and Drosophila, as well as shuttling of mature miRNA from the cytoplasm to the nucleus $(29,42,43)$. Recently, we showed that Exportin-1 is involved in the nuclear export of a group of mammalian $\left(\mathrm{m}^{7} \mathrm{G}\right)$-capped premiRNAs in proliferating cells (28).

To test whether Exportin-1 is involved in the biogenesis of the miRNAs induced in quiescent cells, we transiently transfected proliferating HFFs with an siRNA targeting Exportin-1 and confirmed siRNAmediated reduction of Exportin-1 but not Exportin-5 expression (Fig. 3B). Strikingly, Exportin-1 knock-

down in proliferating cells inhibited the expression of several of the mature miRNAs that are induced during quiescence, namely miR-26a, miR-34a, miR-126, miR-199b, miR-638, miR-1228*, and miR-3188 (Fig. 
$3 B$, dark gray bars). Notably, the levels of the pri-miRNAs corresponding to these miRNAs did not decline (Fig. 3B, light gray bars); in fact, pri-miR-34a increased five- to nine-fold in the absence of Exportin-1. The levels of Exportin-5-dependent miRNAs miR-17, miR-18a, miR-29b, miR-155, and miR-423-3p were also reduced in proliferating cells by Exportin-1 knock-down (Fig. 3B, dark gray bars), but the corresponding primary miRNAs of these mature miRNAs were also decreased, suggesting a reduction of transcription or changes in pri-miRNA turnover in the absence of Exportin-1. Mature miR-155 and the Simtron miR-1228* but not their corresponding pri-miRNAs were reduced by both Exportin- 5 and Exportin-1 knock-down (Fig. $3 A$ and $B$, dark gray bars), suggesting that both exportins are involved in the biogenesis of these miRNAs. Similar results were obtained with a second Exportin-1 siRNA experiment (SI Appendix, Fig. S6B).

To test the contribution of Exportin-1 to miRNA levels during quiescence, we knocked down Exportin-1 in serum-starved cells and measured the levels of mature miRNAs. Exportin-1 knock-down in quiescent cells did not affect the level of Exportin-5 or p27 mRNA or other proteins involved in miRNA biogenesis such as DGCR8, Drosha, Exportin-5, Dicer, and AGO2 (SI Appendix, Fig. S7A-D). Nevertheless, Exportin-1 knock-down prevented or attenuated the increase of the quiescence-induced miRNAs: miR-34a, miR-638, miR-3188, and miR-1288* (Fig. 3C). The Exportin-1-dependence of miR34a expression in quiescent cells was confirmed by Northern blot (Fig. $3 C$ ). Because the expression of the other quiescence-induced miRNAs was difficult to detect by Northern blotting, we focused on miR-34a in subsequent experiments. Interestingly, Exportin-1 knock-down did not change the percentage of HFFs entering quiescence after $24 \mathrm{~h}$ of serum starvation but caused a two-fold decrease in the percentage of cells in $\mathrm{S}$ phase $24 \mathrm{~h}$ after the addition of serum, suggesting that Exportin-1-mediated miRNA biogenesis may be required for normal exit from quiescence (SI Appendix, Fig. S7E).

We used protein-RNA crosslinking followed by Exportin-1 immunoprecipitation to determine if there is a physical interaction between Exportin-1 and pri-miR-34. For this experiment we stably expressed FLAG-tagged Exportin-1 and immunoprecipitated cell extracts with an antibody recognizing the FLAG epitope (SI Appendix, Fig. S7F). The control snoRNA U3, which is normally transported by Exportin-1 (33), was co-immunoprecipitated as expected (Fig. 3D and SI Appendix, Fig. S7G). Strikingly, the Exportin-1-dependent pri-miR-34a but not the Exportin-5-dependent pri-miR-423-3 was coimmunoprecipitated from quiescent HFFs by anti-Flag-Exportin-1 (Fig. 3D, and SI Appendix, Fig. S7G). Together, these results suggest that Exportin-1 is required for the export and/or processing of miRNAs induced during quiescence and that this activity involves a physical association between these pri-miRNAs and Exportin-1. 


\section{E. Exportin-1-dependent pri-miRNAs have a (TMG)-cap during quiescence.}

Previous reports showed that Exportin-1 can bind to (TMG)-capped RNAs such as snoRNA U3 and viral Rev-dependent HIV-1 RNAs $(33,34)$. Therefore, we investigated whether pri-miRNAs of the Exportin-1-dependent miRNAs contain a (TMG)-cap. We used an antibody against (TMG)-caps (which does not cross-react with the $\left(\mathrm{m}^{7} \mathrm{G}\right)$-cap (44)) and non-immune rabbit serum to perform immunoprecipitation from extracts of proliferating or quiescent HFFs. Specific immunoprecipitated primiRNAs were detected by RT-PCR amplification followed by gel electrophoresis. As shown in Figure $4 A$, top panel, the control (TMG)-capped U3 snoRNA was amplified following anti-(TMG)-cap pull-down from both proliferating and quiescent HFF, as expected. Strikingly, pri-miR-34a and pri-miR-3188, two quiescence-induced miRNAs, were also detected in the anti-(TMG)-cap immunoprecipitate from quiescent and to a lower extent from proliferating HFFs. qRT-PCR data from a replicate experiment confirmed that pri-miR-34a and pri-miR-3188 were immunoprecipitated by the anti-(TMG)-cap antibody during proliferation and quiescence (SI Appendix, Fig. S8 and SI Appendix, Table S4). In contrast, pri-miR-29b and pri-miR-423, which are repressed during quiescence, were not detectable in the anti-(TMG)-cap pulldowns. These results indicate that some quiescence-induced pri-miRNAs are specifically modified with a (TMG)-cap.

TGS1, the enzyme that catalyzes 5'-cap hypermethylation, has two active isoforms, a short isoform in the nucleus and a full-length isoform in the cytoplasm (45). We found that the short form of TGS1 is induced during quiescence, suggesting it may be responsible for cap hypermethylation of Exportin-1dependent pri-miRNAs (SI Appendix, Fig. S9A). Indeed, the ability of the anti-TMG antibody to immunoprecipitate pri-miR-34a and pri-miR-3188 (as well as the control U3 RNA) was dramatically diminished by siRNA-mediated knock-down of TGS1 in proliferating and quiescent HFFs (Fig. 4A, compare siRNA CTRL anti-TMG IP lanes against siRNA TGS1 anti-TMG IP lanes, and SI Appendix, Fig. S8 and S9 $B$ and $C$ ), arguing that this enzyme is required for cap hypermethylation of these miRNAs.

To further test the model that TMG capping is required for miRNA biogenesis in quiescent cells, we examined levels of mature miRNAs after knock-down of TGS1 in quiescent HFFs. As shown in Figure $4 B$, TGS1 knock-down inhibited the induction of the quiescence-induced miR-34a and miR-3188 but did not affect the abundance of the quiescence-repressed miR-423-3p and miR-29b. The knock-down of TGS1 did not affect the expression of DGCR8, Drosha, or Exportin-5, and actually increased the expression of Dicer and AGO2 (SI Appendix, Fig. S9D). To investigate whether the (TMG)-cap modification in quiescence-induced pri-miRNAs is important for the interaction with Exportin-1, we used protein-RNA 
crosslinking followed by immunoprecipitation of FLAG-Exportin-1 in quiescent HFFs that had been treated with control scrambled siRNA or siRNA against TGS1. snoRNA U3, which contains a (TMG)-cap, showed a reduced level of co-immunoprecipitation from cells treated with siRNA against TGS1, as expected (Fig. 4C). Notably, Exportin-1-dependent pri-miR-34a was also co-immunoprecipitated to a lesser extent in cells after TGS1 knock-down (Fig. 4C), whereas the Exportin-5-dependent pri-miR-423 was not coimmunoprecipitated in the presence or absence of TGS-1, reaffirming the hypothesis that this pri-miRNA does not contain a modified cap.

Taken together, these findings indicate that the pri-miRNAs of quiescence-induced miRNAs are modified with a specific hypermethylation in their 5'-cap by TGS1, a modification important for Exportin1 recognition (33).

\section{F. Pri-miR-34a is present in the cytoplasm of quiescent cells together with a small isoform of Drosha.}

To examine whether quiescence-induced pri-miRNAs are exported to the cytoplasm as a consequence of (TMG)-capping and interaction with Exportin-1, we prepared nuclear and cytoplasmic RNA from proliferating and quiescent HFFs pretreated with control siRNA or a pool of siRNAs against Drosha (in order to minimize possible rapid processing of these pri-miRNAs (SI Appendix, Fig. S10). As expected, Exportin-1-dependent pri-miR-34a and Exportin-5-dependent pri-miR-29b and pri-miR-423 were detected in the nuclear fraction of both proliferating and quiescent HFFs (Fig. 5A). These nuclear primRNAs accumulated in response to Drosha knock-down, supporting previous studies that showed their Drosha dependency $(46,47)$. Strikingly, we also detected pri-miR-34a in the cytoplasmic fraction of proliferating and quiescent HFFs following Drosha knock-down, whereas pri-miR-29b and pri-miR-423 were not detectable in this cellular fraction (Fig. $5 A$ and $B$ ). These data suggest that pri-miR-34a and possibly other quiescence-induced pri-miRNAs are transported into the cytoplasm where they are processed by an uncharacterized cytoplasmic form of Drosha. We also observed a reduction of all the pri-miRNAs in the nuclear fraction of the quiescent cells (Fig. 5A and SI Appendix, Fig. S11).

To determine if the presence of cytoplasmic pri-miR-34a required Exportin-1, we simultaneously knocked down Exportin-1 as well as Drosha in quiescent HFFs and determined the level of cytoplasmic pri-miRNAs compared to cells treated with control siRNA or Droshan siRNA alone (SI Appendix, Fig. $\mathrm{S} 12$ ). As shown in Figure $5 C$ and $D$, knock-down of Exportin-1 expression in quiescent HFFs greatly attenuated the increased amount of cytoplasmic pri-miR-34a in the Drosha knockdown cells and caused the 
accumulation of this pri-miRNA in the nuclear fraction, suggesting that Exportin-1 is involved in the transport of pri-miR34a from the nucleus to the cytoplasm.

To further explore the processing of pri-miRNAs in quiescent HFFs, we analyzed Drosha protein during quiescence. Western blotting showed the presence of full-length Drosha ( 160 kDa) in proliferating and quiescent cells (Fig. 6A). In addition, quiescent cells displayed induction of a smaller Drosha band $(\sim 130 \mathrm{kDa})$ (Fig. 6A). A pool of three siRNAs that target Drosha mRNA in different regions caused a reduction in both bands, suggesting that the smaller band is a distinct splicing isoform or cleavage product of Drosha (SI Appendix, Fig. S13 A and B). An earlier publication also detected a smaller form of Drosha $(\sim 145 \mathrm{kDa})$ in HEK-293 cells, which was observed in a different protein complex than full-length Drosha, suggesting that it associates with different binding partners (12).

To investigate the localization of these two different forms of Drosha, we examined nuclear and cytoplasmic fractions from proliferating and quiescent HFFs. The short form of Drosha was found specifically in the cytoplasmic fraction of quiescent HFFs (Fig. 6B). In addition, immunofluorescent staining of Drosha in proliferating and quiescent HFFs revealed a cytoplasmic signal during quiescence (Fig. $6 C$ and D), which was reduced by siRNA against Drosha (SI Appendix, Fig. S13E). Because DGCR8 is essential for Drosha activity, we analyzed the cytoplasmic fraction of proliferating and quiescent HFFs for DGCR8. We were unable to detect DGCR8 in the cytoplasmic fractions by direct Western blotting (Fig. $6 B$ ) or by blotting after immunoprecipitation (SI Appendix, Fig. S14A), suggesting that Drosha may interact with a different dsRNA binding protein in the cytoplasm, as was previously suggested (27). It is noteworthy that two recent publications demonstrated that alternative splicing of Drosha could affect its subcellular localization, consistent with our finding that a specific Drosha isoform exists in the cytoplasm of quiescent cells $(48,49)$. Furthermore, siRNA against DGCR8 in quiescent HFFs did not affect the biogenesis of the quiescence-induced miR-34a but altered the processing of the quiescence-repressed miR-423-3p (SI Appendix, Fig. S14B), supporting the existence of functional cytoplasmic Drosha without DGCR8.

To characterize the short form of Drosha in quiescent cells, we amplified the Drosha open reading frame mRNA by tiling RT-PCR (SI Appendix, Fig. S15). Cloning and sequencing identified two exonskipping events in the same transcript, occurring in the 5' half Drosha mRNA, during quiescence. These events disrupt the proline-rich (P-rich) region as well as the nuclear localization signal in the arginine and serine-rich (RS-rich) portion of Drosha (Fig. 6E), but leave intact the previously described minimal active fragment of the protein (18). To indirectly measure the activity of the short isoform of Drosha, we measured the levels of pri-miR-34a and pri-miR-423 after treating cells with siRNAs against both Drosha isoforms 
(siRNA Drosha both) or an siRNA that specifically targets the exon 6 region that is present only in the fulllength Drosha but not in the short isoform (siRNA Drosha full-length) (SI Appendix, Fig. S13 C and D). siRNA against both forms of Drosha caused a marked increase in the level of pri-miR-34a and pri-miR423 in quiescent cells, confirming that Drosha contributes to the processing of these miRNAs (Fig. 6F). Notably, siRNA specifically targeting full-length Drosha was much less effective in inducing pri-miR-34a or miR34a in quiescent cells than was siRNA in reducing both Drosha isoforms, implying that the short cytoplasmic form of Drosha was competent to process pri-miR-34a to mature miR-34a. In contrast, the short isoform of Drosha does not appear to be involved in processing pri-miR-423 because specific knockdown of the full-length form was sufficient to cause pri-miR-423 accumulation. Similarly, siRNA against full-length Drosha allows partial induction of mature miR-34a but not miR-423 in quiescent cells (Fig. 6G), supporting the hypothesis that the short isoform of Drosha is able to process pri-miR-34a but not pri-miR423 during quiescence.

\section{$\underline{\text { VI. Discussion }}$}

When cells reversibly exit the cell cycle and enter quiescence, they remain in a non-proliferative state until they receive appropriate mitogenic signals, but must avoid irreversible states such as apoptosis, differentiation, and senescence. Because miRNAs regulate many cellular pathways, changes in miRNA biogenesis are likely to play an important role in controlling these important cellular processes. This study revealed a number of unique features of miRNA biogenesis in proliferating and quiescent cells.

\section{A. Exportin-5 levels are regulated during cellular quiescence by autophagy and miR-34a.}

We confirmed the findings of Iwasaki and collaborators (39) that the amount of Exportin-5 protein decreases during quiescence (Fig. $2 A$ and SI Appendix, Fig. S3). We showed that at least two posttranscriptional mechanisms contribute to the reduction of Exportin-5 expression during quiescence. Because a specific inhibitor of autophagy prevented the loss of Exportin-5, a major regulator of Exportin5 levels during quiescence appears to be protein degradation by autophagy. In fact, autophagy is induced during quiescence to protect cells from apoptosis mediated by the proteasome (50), and serum starvation or treatment with an mTOR inhibitor activates autophagy, resulting in the degradation of Dicer and AGO2 (51). Together with our findings, these results indicate that several proteins involved in miRNA biogenesis

are regulated by autophagy during quiescence to avoid induction of apoptosis. In addition, our studies suggest that miR-34a binds to an evolutionarily conserved binding site in the Exportin-5 mRNA 3'UTR and represses Exportin-5 expression (Fig. 2D-G). miR-34a-mediated repression of Exportin-5 appears to 
act in conjunction with autophagy-mediated degradation to ensure low levels of Exportin-5 throughout quiescence. Consistent with this idea, miR-34a is induced relatively late after the onset of quiescence, when Exportin-5 levels are already low (Fig. $2 A$ and SI Appendix, Fig. S3). Taken together, our data suggest that Exportin-1 contributes to Exportin-5 down-regulation during quiescence by supporting the synthesis of miR-34a, which binds to Exportin-5 mRNA and reinforces the block of Exportin-5 expression. An interesting topic for future studies will be to determine if the regulation of Exportin-5 is related exclusively to the induction of quiescence or rather to the absence of specific growth factors such as IGF-1, which have been shown to be important in the regulation of autophagy in HFFs (52).

\section{B. Exportin-1-dependent microRNA biogenesis occurs during cellular quiescence.}

Our major finding is the discovery of an alternative miRNA biogenesis pathway involving Exportin-1 (also known as CRM1) during quiescence. Exportin-1, the major factor responsible for the export of proteins from the nucleus, is also involved in the export of certain RNAs such as ribosomal RNAs, small nuclear RNAs, and mRNAs encoding some proteins involved in cell cycle regulation $(29,53)$. Previous studies in C. elegans and Drosophila showed that Exportin-1 is involved in pri-miRNA processing and biogenesis (42). Our findings demonstrate that Exportin-1 is also involved in the biogenesis of specific miRNAs in proliferating mammalian cells and that this activity is enhanced during quiescence. First, there is no change in Exportin-1 levels during quiescence (Fig. 2A and SI Appendix, Fig. S3B). Second, Exportin1 knock-down reduced the levels of specific quiescence-induced mature miRNAs in proliferating and quiescent HFFs (Fig. $3 B$ and $C$ ), although the effect was not as dramatic as the reduction of quiescencerepressed miRNAs by Exportin-5 knock-down (Fig. 3A), Third, the Exportin-1-dependent pri-miR-34a but not the Exportin-5-dependent pri-miR-423 is found in a stable complex with Exportin-1 (Fig. 3D). Our data further demonstrate that some miRNAs, including miR-26a, miR-34a, miR-126, miR-199, miR-638, and miR-3188, can use an Exportin-5-independent biogenesis pathway in proliferating cells. We also identified miRNAs subject to complex regulation during quiescence. For example, siRNA-mediated knock-down of either Exportin-5 or Exportin-1 in proliferating HFFs reduced the expression of mature miR-1228* but did not affect levels of pri-miR-1228 (Fig. $3 A$ and B, and SI Appendix, Fig. S6 $A$ and B). Thus, both Exportin5 and Exportin-1 are involved in the biogenesis of miR-1228*, which is considered a Simtron, a class of miRNAs that are produced in the absence of splicing and are independent of DGCR8, Dicer, Exportin-5, and Argonaute (24). Kim and collaborators (48) demonstrated that Exportin-5 knockout in the colorectal cancer cell line HCT116 only modestly affects the processing of several miRNAs, consistent with the existence of Exportin-5-independent miRNA maturation in these cells. 


\section{Quiescence-induced primary miRNAs contain a (TMG)-cap and bind Exportin-1.}

We discovered that the primary miRNAs of Exportin-1-dependent quiescence-induced miRNAs contain a (TMG)-cap, which may be a signal for recognition by Exportin-1, whereas this cap structure is absent from the Exportin-5-dependent pri-miRNAs (Fig. 4A, SI Appendix, Fig. S8, and SI Appendix, Table S4). We also found that a short nuclear isoform of TGS1 is up-regulated during quiescence (SI Appendix, Fig. S9A). Importantly, knocking down expression of TGS1 in HFFs reduced (TMG)-capping of quiescence-induced pri-miRNAs, diminished induction of the corresponding mature miRNAs during quiescence, and reduced the interaction of pri-miR-34a with Exportin-1 (Fig. 4). Because most RNAs need to be exported to the cytoplasm to be modified with a (TMG)-cap, we have not ruled out the possibility that some pri-miRNAs could be exported by Exportin-1 with a $\left(\mathrm{m}^{7} \mathrm{G}\right)$-cap and then undergo cap hypermethylation in the cytoplasm. We recently described microprocessor-independent human and mouse pre-miRNAs that are specifically $\left(\mathrm{m}^{7} \mathrm{G}\right)$-capped and transported by the PHAX-Exportin-1 pathway and cleaved by Dicer prior to loading into the RISC complex (28). The miRNAs described here are processed differently because TMG caps are added at the pri-miRNA level, indicating that human cells contain at least two alternative Exportin-1-dependent pathways of miRNA biogenesis.

\section{Discovery of primary miRNAs and a smaller Drosha isoform in the cytoplasm of quiescent cells.}

Another important discovery is finding pri-miR-34a in the cytoplasm. We detected cytoplasmic pri-miR-34a in proliferating and quiescent HFF only after knocking down Drosha, suggesting that this primiRNA is rapidly processed once it leaves the nucleus (Fig. $5 A$ and $B$ ). We were unable to consistently detect other quiescence-induced pri-miRNAs in the cytoplasm, presumably because they are less abundant than pri-miR-34a. Drosha-mediated rapid cleavage of these cytoplasmic (TMG)-capped pri-miRNAs may prevent their recognition by Snuportin-1, which could potentially return the pri-miRNAs to the nucleus (31, 32). Moreover, we observed a reduction in the amount of pri-miR-34a in the cytoplasmic fraction of cells knocked down for both Exportin-1 and Drosha (Fig. 5C). This observation, together with the discovery of an interaction between pri-miR-34a and Exportin-1 (Fig. 3D), suggests that Exportin-1 plays a role in transporting pri-miR-34a from the nucleus to the cytoplasm. Further studies are necessary to elucidate the mechanism involved in the transport of quiescence-induced pri-miRNAs to the cytoplasm, their (TMG)capping, and their cytoplasmic processing.

We also discovered the preferential expression of a smaller, alternatively spliced cytoplasmic isoform of Drosha during quiescence (Fig. 6A-E). Recent studies demonstrated the existence of splicing 
isoforms of Drosha found in the cytoplasm in several cell lines $(48,49)$. These Drosha isoforms contain deletions in the nuclear localization region (RS-rich) similar to the small isoform of Drosha found in quiescent HFFs. These data support the hypothesis that splicing of specific genes, such as Drosha, by the spliceosome machinery is important in the regulation of cell cycle progression (54). Importantly, our data expand the concept that a specific Drosha isoform can be found in the cytoplasm of normal human cells in a particular stress condition such as serum starvation. Another study showed that certain RNA viruses trigger Exportin-1-dependent translocation of Drosha to the cytoplasm, providing a potential antiviral mechanism that acts by cleaving viral genomic RNA (55). Consistent with this model, Exportin-1 knockdown reduced the amount of cytoplasmic pri-miR-34a (Fig. 5C), and a short cytoplasmic isoform of Drosha appeared to be involved in pri-miR-34a processing during quiescence (Fig. $6 F$ and $G$ ). Our discovery of pri-miRNAs and a small isoform of Drosha in the cytoplasm suggest the existence of a new miRNA biogenesis pathway during quiescence.

\section{$\underline{E}$. The contribution of quiescence-induced miRNAs to cellular growth arrest.}

Do the quiescence-induced miRNAs play a role in growth arrest? These miRNAs may be essential for the induction of quiescence, thereby necessitating the use of an alternative miRNA biogenesis pathway when the canonical pathway is repressed. For example, the p53-regulated miR-34a regulates cellular pathways such as cell cycle, differentiation, and apoptosis $(56,57)$; it is a master regulator of tumor suppression in different human cancers and is being evaluated as a miRNA therapeutic (58). Similarly, miR-638 up-regulation in vascular smooth muscle cells was recently reported to inhibit cell proliferation and migration by targeting the orphan nuclear receptor 1 (NOR1) and consequently affecting the expression of cyclin D1 mRNA (59). In addition, miR-1228* over-expression suppresses xenograft tumor formation by negatively regulating NF-KB activity (60). Finally, miR-3188 was found to reduce cell cycle transition and proliferation in nasopharyngeal carcinoma by targeting mTOR mRNA and regulating PI3K/AKT signaling pathway through FOXO1 (61). In addition, it is possible that impaired processing of specific miRNAs in certain growth conditions may contribute to embryonic lethality of TGS1 knock-out mice (62).

The results of the cell cycle analysis in SI Appendix, Fig. S7E show that Exportin-1 regulated miRNAs are not required for entry into senescence. However, the reduced number of cells in S phase $24 \mathrm{~h}$ after serum addition suggests that Exportin-1 is required for proper exit from quiescence. We speculate that the Exportin-1-dependent, quiescence-induced miRNAs are important not to maintain growth arrest but rather to poise cells so that they can efficiently reenter the cell cycle when favorable growth conditions are restored. 
Not only do miRNAs affect cell growth, but also cell growth state can affect miRNAs. We reported previously that growth arrest can convert some miRNAs from repressors into activators of translation (38), and we show here that quiescence inhibits canonical miRNA biogenesis but stimulates an alternative miRNA biogenesis pathway. Thus, the cellular growth state may globally affect miRNA synthesis and function, which in turn may profoundly influence cellular gene expression and phenotype.

In summary, we have shown that a set of miRNAs is processed independently of the canonical miRNA pathway in quiescent cells via (TMG)-cap modification of their pri-miRNA, interaction with Exportin-1 and cytoplasmic processing by a novel isoform of Drosha (SI Appendix, Fig. S16). This pathway is constitutively active in proliferating cells, but its activity is enhanced during quiescence, presumably to regulate key processes involved in cellular growth arrest. Conversely, in quiescent cells the canonical miRNA pathway is turned off, likely contributing to the quiescent state. Future investigation will be necessary to identify additional components of this miRNA Exportin-1-dependent pathway and its impact on cell physiology.

\section{$\underline{\text { VII. Materials and Methods }}$}

Detailed experimental protocols are described in the Supporting Information Appendix (p.175).

\section{A. Cell culture and reagents}

Normal primary human foreskin fibroblasts, HeLa/E6-5K, 293T, and C127 cells were cultured in DMEM-10 at $37^{\circ} \mathrm{C}$ in the presence of $5 \% \mathrm{CO}_{2}$. Further details are in the SI Appendix.

\section{B. Quiescence induced by serum starvation and confluency}

HFFs, HeLa, and C127 cells were rinsed with DPBS, detached with $0.25 \%$ trypsin-EDTA and plated in serum-free DMEM at low density. For quiescence induced by confluency, we plated HFFs ( $2 \mathrm{X}$ $10^{6}$ cells/100 mm plate) in DMEM-10. Further details are in the SI Appendix.

\section{Immunoprecipitation of tri-methylated capped RNA}

HFFs were incubated for $72 \mathrm{~h}$ in serum-free DMEM to induce quiescence. RNA was extracted using Trizol Reagent and $10 \mu \mathrm{g}$ of RNA was diluted in NET-2 buffer, pre-cleared and incubated with 
Protein G Sepharose 4 Fast Flow beads loaded with $15 \mu \mathrm{L}$ normal rabbit serum or $15 \mu \mathrm{L}$ rabbit m3G-cap antibody. Beads were rinsed 5 times with NET-2 buffer and resuspended in G-50 buffer. RNA was extracted from the beads by phenol-chloroform-isoamyl alcohol extraction. Further details are in the SI Appendix.

\section{References}

1. Pardee AB (1974) A restriction point for control of normal animal cell proliferation. Proceedings of the National Academy of Sciences of the United States of America 71(4):1286-1290.

2. O'Farrell PH (2011) Quiescence: early evolutionary origins and universality do not imply uniformity. Philosophical transactions of the Royal Society of London. Series B, Biological sciences 366(1584):3498-3507.

3. Coller HA (2011) Cell biology. The essence of quiescence. Science 334(6059):1074-1075.

4. Valcourt JR, et al. (2012) Staying alive: metabolic adaptations to quiescence. Cell cycle 11(9):1680-1696.

5. Coller HA, Sang L, \& Roberts JM (2006) A new description of cellular quiescence. PLoS biology 4(3):e83.

6. Cheung TH \& Rando TA (2013) Molecular regulation of stem cell quiescence. Nature reviews. Molecular cell biology 14(6):329-340.

7. Bohnsack MT, Czaplinski K, \& Gorlich D (2004) Exportin 5 is a RanGTP-dependent dsRNAbinding protein that mediates nuclear export of pre-miRNAs. Rna 10(2):185-191.

8. Krol J, Loedige I, \& Filipowicz W (2010) The widespread regulation of microRNA biogenesis, function and decay. Nature reviews. Genetics 11(9):597-610.

9. Lee Y, Han J, Yeom KH, Jin H, \& Kim VN (2006) Drosha in primary microRNA processing. Cold Spring Harbor symposia on quantitative biology 71:51-57.

10. Lee Y, et al. (2003) The nuclear RNase III Drosha initiates microRNA processing. Nature 425(6956):415-419.

11. Gregory RI, et al. (2004) The Microprocessor complex mediates the genesis of microRNAs. Nature 432(7014):235-240.

12. Denli AM, Tops BB, Plasterk RH, Ketting RF, \& Hannon GJ (2004) Processing of primary microRNAs by the Microprocessor complex. Nature 432(7014):231-235.

13. Yi R, Qin Y, Macara IG, \& Cullen BR (2003) Exportin-5 mediates the nuclear export of premicroRNAs and short hairpin RNAs. Genes \& development 17(24):3011-3016.

14. Hutvagner G, et al. (2001) A cellular function for the RNA-interference enzyme Dicer in the maturation of the let-7 small temporal RNA. Science 293(5531):834-838.

15. Grishok A, et al. (2001) Genes and mechanisms related to RNA interference regulate expression of the small temporal RNAs that control C. elegans developmental timing. Cell 106(1):23-34.

16. Knight SW \& Bass BL (2001) A role for the RNase III enzyme DCR-1 in RNA interference and germ line development in Caenorhabditis elegans. Science 293(5538):2269-2271.

17. Han J, et al. (2004) The Drosha-DGCR8 complex in primary microRNA processing. Genes \& development 18(24):3016-3027.

18. Lian SL, et al. (2009) The C-terminal half of human Ago2 binds to multiple GW-rich regions of GW182 and requires GW182 to mediate silencing. Rna 15(5):804-813.

19. Hammond SM, Boettcher S, Caudy AA, Kobayashi R, \& Hannon GJ (2001) Argonaute2, a link between genetic and biochemical analyses of RNAi. Science 293(5532):1146-1150.

20. Mourelatos Z, et al. (2002) miRNPs: a novel class of ribonucleoproteins containing numerous microRNAs. Genes \& development 16(6):720-728.

21. Ruby JG, Jan $\mathrm{CH}$, \& Bartel DP (2007) Intronic microRNA precursors that bypass Drosha processing. Nature 448(7149):83-86. 
22. Cazalla D, Xie M, \& Steitz JA (2011) A primate herpesvirus uses the integrator complex to generate viral microRNAs. Molecular cell 43(6):982-992.

23. Havens MA, Reich AA, Duelli DM, \& Hastings ML (2012) Biogenesis of mammalian microRNAs by a non-canonical processing pathway. Nucleic acids research 40(10):4626-4640.

24. Cifuentes D, et al. (2010) A novel miRNA processing pathway independent of Dicer requires Argonaute2 catalytic activity. Science 328(5986):1694-1698.

25. Cheloufi S, Dos Santos CO, Chong MM, \& Hannon GJ (2010) A dicer-independent miRNA biogenesis pathway that requires Ago catalysis. Nature 465(7298):584-589.

26. Shapiro JS, Langlois RA, Pham AM, \& Tenoever BR (2012) Evidence for a cytoplasmic microprocessor of pri-miRNAs. Rna 18(7):1338-1346.

27. Xie M, et al. (2013) Mammalian 5'-capped microRNA precursors that generate a single microRNA. Cell 155(7):1568-1580.

28. Hutten S \& Kehlenbach RH (2007) CRM1-mediated nuclear export: to the pore and beyond. Trends in cell biology 17(4):193-201.

29. Ohno M, Segref A, Bachi A, Wilm M, \& Mattaj IW (2000) PHAX, a mediator of U snRNA nuclear export whose activity is regulated by phosphorylation. Cell 101(2):187-198.

30. Palacios I, Hetzer M, Adam SA, \& Mattaj IW (1997) Nuclear import of U snRNPs requires importin beta. The EMBO journal 16(22):6783-6792.

31. Kiss T (2004) Biogenesis of small nuclear RNPs. Journal of cell science 117(Pt 25):5949-5951.

32. Boulon S, et al. (2004) PHAX and CRM1 are required sequentially to transport U3 snoRNA to nucleoli. Molecular cell 16(5):777-787.

33. Yedavalli VS \& Jeang KT (2010) Trimethylguanosine capping selectively promotes expression of Rev-dependent HIV-1 RNAs. Proceedings of the National Academy of Sciences of the United States of America 107(33):14787-14792.

34. Medina R, et al. (2008) MicroRNAs 221 and 222 bypass quiescence and compromise cell survival. Cancer research 68(8):2773-2780.

35. Suh EJ, et al. (2012) A microRNA network regulates proliferative timing and extracellular matrix synthesis during cellular quiescence in fibroblasts. Genome biology 13(12):R121.

36. Martinez I, Cazalla D, Almstead LL, Steitz JA, \& DiMaio D (2011) miR-29 and miR-30 regulate B-Myb expression during cellular senescence. Proceedings of the National Academy of Sciences of the United States of America 108(2):522-527.

37. Vasudevan S, Tong Y, \& Steitz JA (2007) Switching from repression to activation: microRNAs can up-regulate translation. Science 318(5858):1931-1934.

38. Iwasaki YW, et al. (2013) Global microRNA elevation by inducible Exportin 5 regulates cell cycle entry. Rna 19(4):490-497.

39. Link S, Grund SE, \& Diederichs S (2016) Alternative splicing affects the subcellular localization of Drosha. Nucleic acids research 44(11):5330-5343.

40. Rissland OS, Hong SJ, \& Bartel DP (2011) MicroRNA destabilization enables dynamic regulation of the miR-16 family in response to cell-cycle changes. Molecular cell 43(6):993-1004.

41. Bussing I, Yang JS, Lai EC, \& Grosshans H (2010) The nuclear export receptor XPO-1 supports primary miRNA processing in C. elegans and Drosophila. The EMBO journal 29(11):1830-1839.

42. Castanotto D, Lingeman R, Riggs AD, \& Rossi JJ (2009) CRM1 mediates nuclear-cytoplasmic shuttling of mature microRNAs. Proceedings of the National Academy of Sciences of the United States of America 106(51):21655-21659.

43. Luhrmann R, et al. (1982) Isolation and characterization of rabbit anti-m3 2,2,7G antibodies. Nucleic acids research 10(22):7103-7113.

44. Girard C, et al. (2008) Characterization of a short isoform of human Tgs1 hypermethylase associating with small nucleolar ribonucleoprotein core proteins and produced by limited proteolytic processing. The Journal of biological chemistry 283(4):2060-2069.

45. Chong MM, et al. (2010) Canonical and alternate functions of the microRNA biogenesis machinery. Genes \& development 24(17):1951-1960. 
46. Kawai S \& Amano A (2012) BRCA1 regulates microRNA biogenesis via the DROSHA microprocessor complex. The Journal of cell biology 197(2):201-208.

47. Kim YK, Kim B, \& Kim VN (2016) Re-evaluation of the roles of DROSHA, Export in 5, and DICER in microRNA biogenesis. Proceedings of the National Academy of Sciences of the United States of America 113(13):E1881-1889.

48. Ha M \& Kim VN (2014) Regulation of microRNA biogenesis. Nature reviews. Molecular cell biology 15(8):509-524.

49. Legesse-Miller A, et al. (2012) Quiescent fibroblasts are protected from proteasome inhibitionmediated toxicity. Molecular biology of the cell 23(18):3566-3581.

50. Gibbings D, et al. (2012) Selective autophagy degrades DICER and AGO2 and regulates miRNA activity. Nature cell biology 14(12):1314-1321.

51. Bitto A, et al. (2010) Long-term IGF-I exposure decreases autophagy and cell viability. PloS one 5(9):e12592.

52. Verheggen C \& Bertrand E (2012) CRM1 plays a nuclear role in transporting snoRNPs to nucleoli in higher eukaryotes. Nucleus 3(2):132-137.

53. Karamysheva Z, Diaz-Martinez LA, Warrington R, \& Yu H (2015) Graded requirement for the spliceosome in cell cycle progression. Cell cycle 14(12):1873-1883.

54. Shapiro JS, et al. (2014) Drosha as an interferon-independent antiviral factor. Proceedings of the National Academy of Sciences of the United States of America 111(19):7108-7113.

55. He L, et al. (2007) A microRNA component of the p53 tumour suppressor network. Nature 447(7148):1130-1134.

56. Chen F \& Hu SJ (2012) Effect of microRNA-34a in cell cycle, differentiation, and apoptosis: a review. Journal of biochemical and molecular toxicology 26(2):79-86.

57. Bader AG (2012) miR-34 - a microRNA replacement therapy is headed to the clinic. Frontiers in genetics 3:120.

58. Li P, et al. (2013) MicroRNA-638 is highly expressed in human vascular smooth muscle cells and inhibits PDGF-BB-induced cell proliferation and migration through targeting orphan nuclear receptor NOR1. Cardiovascular research 99(1):185-193.

59. Jia L, et al. (2013) Restoration of miR-1228* expression suppresses epithelial-mesenchymal transition in gastric cancer. PloS one 8(3):e58637.

60. Lund E, Guttinger S, Calado A, Dahlberg JE, \& Kutay U (2004) Nuclear export of microRNA precursors. Science 303(5654):95-98.

61. Jia Y, et al. (2012) Early embryonic lethality of mice with disrupted transcription cofactor PIMT/NCOA6IP/Tgs1 gene. Mechanisms of development 129(9-12):193-207. 


\section{$\underline{\text { IX. Figure Legends }}$}

Figure 1. Differential expression of mature miRNAs and their corresponding pri-miRNAs during quiescence. $(A)$ Heat map of mature miRNA microarray data comparing proliferating HFFs to HFFs serumstarved (SS) for 24,48 , or $72 \mathrm{~h}$. Only differentially expressed miRNAs during quiescence are shown. $(B)$ qRT-PCR validation of representative differentially expressed mature miRNAs during quiescence. $(C)$ qRT-PCR quantitation of pri-miRNAs corresponding to differentially expressed mature miRNAs (using primers that bind upstream and downstream of the miRNA stem loop). Red bars represent the miRNAs upregulated and the green bars represent the miRNAs down-regulated during quiescence by miRNA microarray analysis. Numbers refer to days of serum starvation. Black bars represent miRNAs in proliferating (P) cells. RNU43 and GAPDH mRNA were used to normalize the qRT-PCR analyses, which are shown relative to expression in proliferating cells. Similar results were obtained in three independent experiments.

Figure 2. Regulation of Exportin-5 during quiescence by autophagy and miR-34a. (A) Western blot showing reduction of Exportin-5 but not Exportin-1 levels during the induction of quiescence by serum starvation. p27 was used as a marker of quiescence induction, and $\beta$-actin was used as a loading control. $(B)$ Western blot showing Exportin-5 and MDM2 in proliferating and quiescent HFFs treated with $10 \mu \mathrm{M}$ of the proteasome inhibitor, MG132. Mock indicates cells proliferating in the presence of serum; cells were serum-starved for 3, 6, or $12 \mathrm{~h}$ as indicated to induce quiescence. $(C)$ Western blot of Exportin-5 and p62 in proliferating and quiescent HFFs treated with $10 \mu \mathrm{M}$ of the autophagy inhibitor, 3-MA. Similar results were obtained in three independent experiments. $(D)$ The sequence of hsa-miR-34a and its putative wildtype (WT) or mutant (mt) binding site in the Exportin-5 3'UTR are shown. Vertical lines indicate predicted base pairing between hsa-miR-34a and the Exportin-5 3' UTR, and the dots indicate potential G-U base pairs. Point mutations in the Exportin-5 3'UTR are shown with asterisks. Numbers represent the nucleotide sequence of Exportin-5 3'UTR numbered from the beginning of the untranslated region. $(E)$ miR-34a regulates the expression of Exportin-5. Left panels, Western blot of Exportin-5 and Exportin-1 $48 \mathrm{~h}$ after transfection of exogenous miR-34a. Right panel, qRT-PCR analysis of Exportin-5 mRNA $48 \mathrm{~h}$ after transfection of miR-34a. $(F)$ Activity of the Exportin-5 3'UTR is regulated by cell growth state. Luciferase activity from the empty reporter vector (Control), the vector containing the wild-type Exportin-5 3'UTR (WT) or the Exportin-5 3'UTR containing a mutant miR-34a binding site (mt). Assays were conducted in proliferating $(\mathrm{P})$ or quiescent $(\mathrm{Q}) \mathrm{HFFs}$ as described in the experimental procedures. $(G)$ miR-34a regulates the Exportin-5 3'UTR. Luciferase activity from the reporter vectors described in panel $(F)$ was measured in proliferating HFFs transfected with a scrambled miRNA control (miR-CTRL) or miR-34a. In panels $(F)$ and $(G)$ firefly luciferase activity was measured and normalized to Renilla luciferase activity expressed from the same plasmid (Relative luciferase units, RLUs). Data were obtained in triplicate, and the graph shows the average of two individual experiments. Two-tailed $t$-test results are indicated by $*$ for $\mathrm{P}<0.05$ and $* *$ for $\mathrm{P}<0.01$.

Figure 3. Biogenesis of quiescence-induced miRNAs requires Exportin-1 but not Exportin-5. (A) qRTPCR analysis showing relative expression of primary miRNAs (light gray bars) and their corresponding mature miRNAs (dark gray bars) in proliferating HFFs $48 \mathrm{~h}$ after transfection with an siRNA scrambled control (dashed line) or an siRNA targeting Exportin-5 (Dharmacon) (dark and light gray bars). Right panel shows Western blot analysis of Exportin-1 and Exportin-5 after siRNA transfection. (B) qRT-PCR analysis showing relative levels of primary miRNAs (light gray bars) and their corresponding mature miRNAs (dark gray bars) in proliferating HFFs $48 \mathrm{~h}$ after transfection with an siRNA scrambled control (dashed line) or an siRNA targeting Exportin-1 (ID s14937, Ambion) (dark and light gray bars). Two-tailed $t$-test results are indicated by $*$ for $\mathrm{P}<0.05$ and $* *$ for $\mathrm{P}<0.01$. Western blot analysis of Exportin- 1 and Exportin-5 is also shown after siRNA transfection. $(C)$ qRT-PCR analysis of quiescence-induced miRNAs and miR-34a Northern blot $48 \mathrm{~h}$ after transfection of an siRNA scrambled control (black bars) or an siRNA targeting Exportin-1 (ID s14937, Ambion) (gray bars) into proliferating HFFs in the presence of serum (P) or into 
HFFs serum-starved (SS) for 24 or $72 \mathrm{~h}(24,48$, or $72 \mathrm{~h}$ for miR-34a Northern blot). Transfection of quiescent cells was done $24 \mathrm{~h}$ prior to serum starvation. $(D)$ Exportin-1 physically associates with pri-miR34a in quiescent cells. qRT-PCR were used to detect U3 snoRNA, pri-miR-34a, or pri-miR-423 in control Flag-Vector (Flag, black bars) or Flag-Exportin-1 (Flag-XPO1, gray bars) immunoprecipitates from quiescent HFFs (expression data in Fig. S7E). Exogenous spike of $C$. elegans RNA was used for qRT-PCR normalization by amplifying Ama-1 mRNA. Similar results were obtained in two independent experiments.

Figure 4. Quiescence-induced primary miRNAs are (TMG)-capped by TGS1. (A) Quiescence-induced primiRNAs contain a TMG cap. Extracts were prepared from proliferating or serum starved quiescent HFFs transfected with control siRNA or siRNA targeting TGS1 (ID s41313, Ambion). siRNAs were transfected $24 \mathrm{~h}$ prior to serum starvation. RNA was harvested $48 \mathrm{~h}$ after transfection of siRNAs in proliferating HFFs or $72 \mathrm{~h}$ after removal of serum. Extracts were then immunoprecipitated with control antibody or antibody recognizing the TMG cap, and immunoprecipitated U3 snoRNA and pri-miRNAs were amplified by RTPCR and visualized after gel electrophoresis. Labels: input (I), supernatant (S), immunoprecipitate (IP), control anti-rabbit serum (C), and anti-TMG antibody (T). (B) TGS1 knock-down inhibits expression of quiescence-induced miRNAs. HFFs were transfected with an siRNA scrambled control (black bars) or an siRNA targeting TGS1 (sc-45875, Santa Cruz) (gray bars). $24 \mathrm{~h}$ later, cells were serum starved (SS) for 24 or $72 \mathrm{~h}$, or maintained in serum $(\mathrm{P})$. RNA was then prepared and quantified by qRT-PCR. $(C)$ TGS1 knockdown affects the binding of pri-miR-34a to Exportin-1 in quiescent cells. qRT-PCR was used to detect U3 snoRNA, pri-miR-34a, or pri-miR-423 in immunoprecipitate samples of control Flag-Vector or FlagExportin-1 in quiescent HFF cells. Cells were transfected with siRNA control (black bars) or siRNA against TGS1 (gray bars). Two-tailed t-test results are indicated by $* *$ for $\mathrm{P}<0.01$. Similar results were obtained in two independent experiments.

Figure 5. Detection of cytoplasmic pri-miR-34a is dependent on Exportin-1 expression. (A) Detection of pri-miR-34a in the cytoplasmic fraction of Drosha knock-down HFFs. Proliferating (P) and quiescent (Q) HFFs were transfected with control siRNA or siRNA targeting Drosha (sc-44080, Santa Cruz) and after 48 $\mathrm{h}$ subjected to cytoplasmic and nuclear RNA fractionation. RT-PCR was used to detect pri-miR-34a, primiR-29b, and pri-miR-423. U6 snRNA is a nuclear control and mitochondrial (mt) tRNA-val is a cytoplasmic marker. $(B)$ qRT-PCR of cytoplasmic fractions as in Figure 5A was used to quantify the levels of pri-miR-34a. Two-tailed $t$-test results are indicated by $* *$ for $\mathrm{P}<0.01$. ( $C$ and $D$ ) Exportin-1 knockdown affects the distribution of pri-miR-34a in quiescent HFFs. Cells were transfected with an siRNA scrambled control, an siRNA targeting Drosha (sc-44080, Santa Cruz), or siRNAs targeting Drosha (sc44080, Santa Cruz) and Exportin-1 (ID s14937, Ambion). 24 h later, cells were serum starved for $72 \mathrm{~h}$. RNA was then prepared from the cytoplasmic (C) or nuclear (D) fraction for the quantification of pri-miR$34 \mathrm{a}$ and pri-miR-423 by qRT-PCR. Normalization was done using C. elegans total RNA as an exogenous spike for the amplification of the worm-specific ama-1 gene. Similar results were obtained in two independent experiments.

Figure 6. Detection of a small cytoplasmic isoform of Drosha during quiescence. (A) Western blot showing reduction of full-length Drosha and appearance of a more rapidly migrating form of Drosha (Drosha ${ }_{\text {short }}$ ) during the induction of quiescence by serum starvation. (B) A small form of Drosha (Drosha short $_{\text {) }}$ is found in the cytoplasmic fraction of quiescent cells. Western blot analysis of Drosha and DGCR8 in total, nuclear, and cytoplasmic fractions in proliferating (P) and quiescent (Q) HFFs. 10\% of the total loading was used for the nuclear fraction in the Drosha Western blot. Histone deacetylase 1 (HDAC1) was used as nuclear marker and $\beta$-tubulin as cytoplasmic marker. $(C)$ Cytoplasmic localization of Drosha in quiescent HFF cells. Immunofluorescence of Drosha (green) in proliferating and quiescent HFFs. Nuclear fluorescence (DAPI, blue) and cytoskeleton immunofluorescence (actin, red) were used to visualize the localization of Drosha. (D) Quantification of cytoplasmic Drosha immunofluorescence. Proliferating and quiescent HFF cells were incubated with digitonin (to selectively permeabilize only the plasma membrane) to diminish masking of cytoplasmic Drosha staining with nuclear Drosha signal. Confocal microscopy was used to 
visualize the cells, and fluorescent images were used to obtain relative pixel area quantification using Image $\mathrm{J}$ software. Two-tailed $t$-test results are indicated by $*$ for $\mathrm{P}<0.05$. Similar results were obtained in three independent experiments. $(E)$ Drosha mRNA exon skipping during quiescence. Illustration and sequencing data show two alternative splicing events (red lines) between exon 3 and 4 and between exon 5 and 7 in Drosha mRNA and the affected regions in Drosha protein. Numbers represent the nucleotide sequence of Drosha mRNA. P-rich indicates the proline-rich region, RS-rich indicates the arginine and serine-rich region, RIIIDa and RIIIDb indicate RNase III domains a and b, respectively, and dsRBD indicates doublestranded RNA-binding domain. $(F)$ The short isoform of Drosha is responsible for processing of pri-miR34a. Quantitative RT-PCR of pri-miR-34a and pri-miR-423 was conducted in quiescent HFFs transfected with control siRNA or siRNA against the full-length Drosha (IDs26491, Ambion) or both Drosha isoforms (both) (sc-44080, Santa Cruz). Reduced accumulation of pri-miR-34a in cells treated with siRNA targeting full-length Drosha compared to siRNA against both Drosha isoforms (both) suggests the short Drosha isoform can process pri-miR-34a. $(G)$ qRT-PCR of mature miR-34a and miR-423-3p was conducted from the same experiments shown in Figure $6 F$. Two-tailed $t$-test results are indicated by $* *$ for $\mathrm{P}<0.01$. Similar results were obtained in two independent experiments. 


\section{Figures}

Figure 1

A

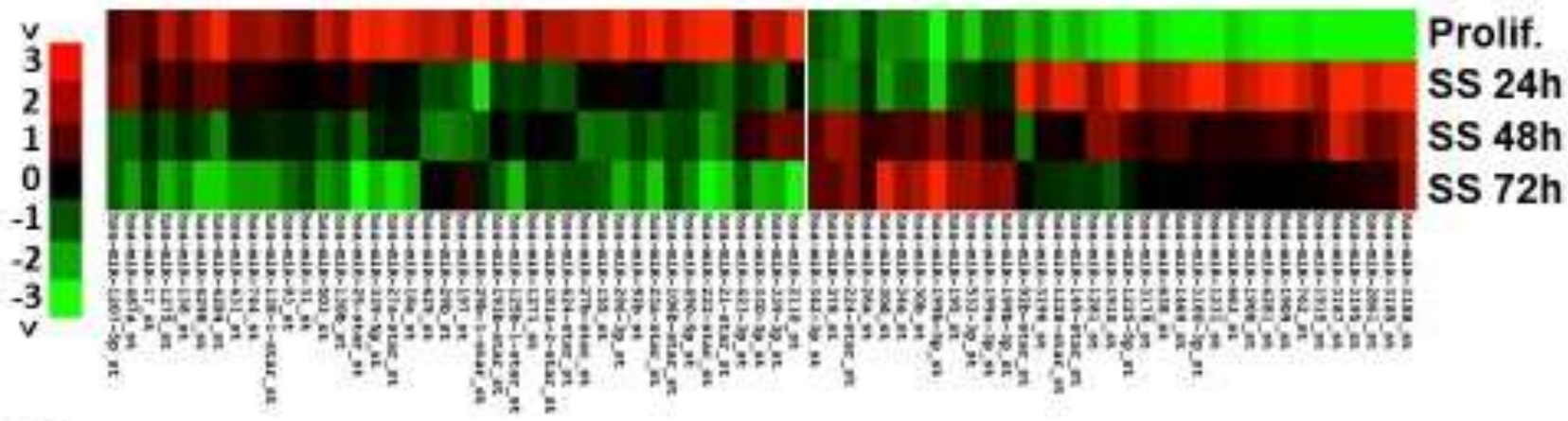

B

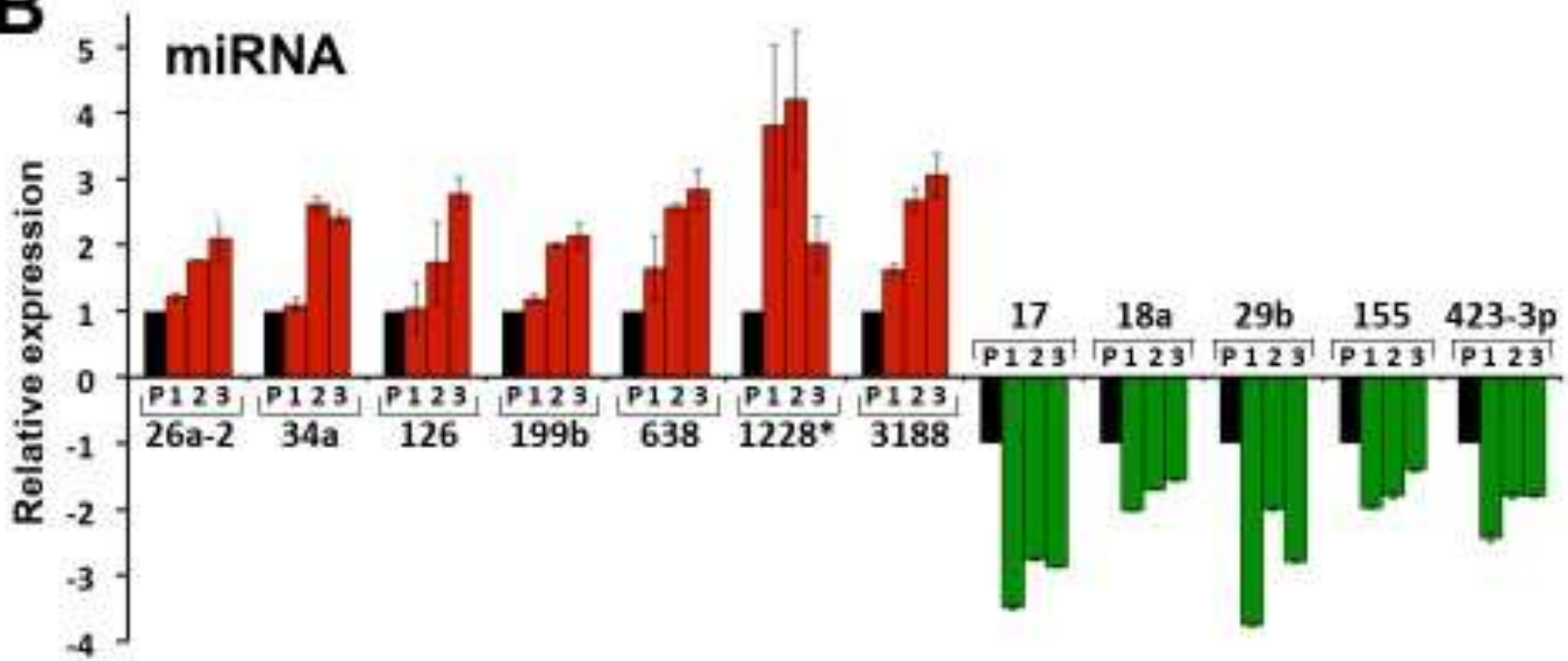

c

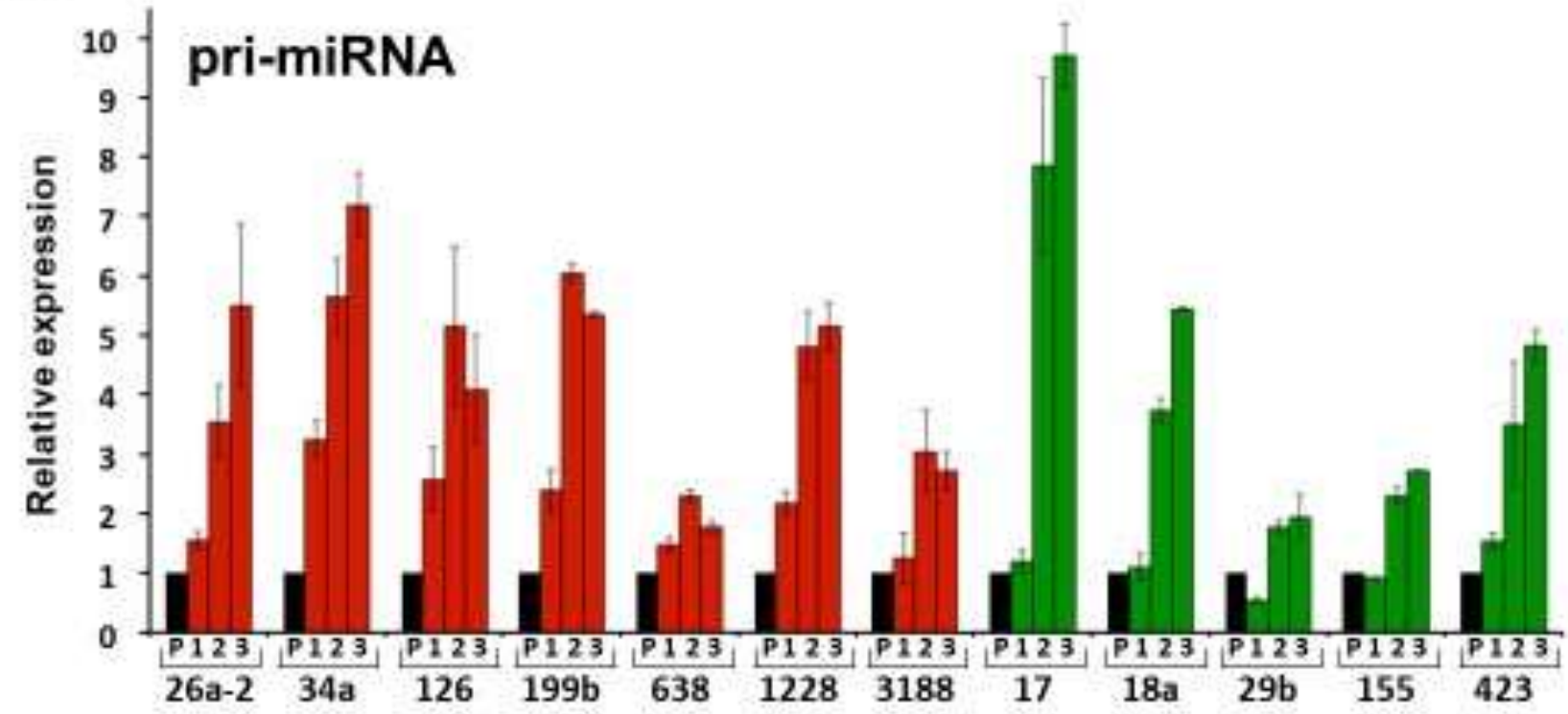


Figure 2
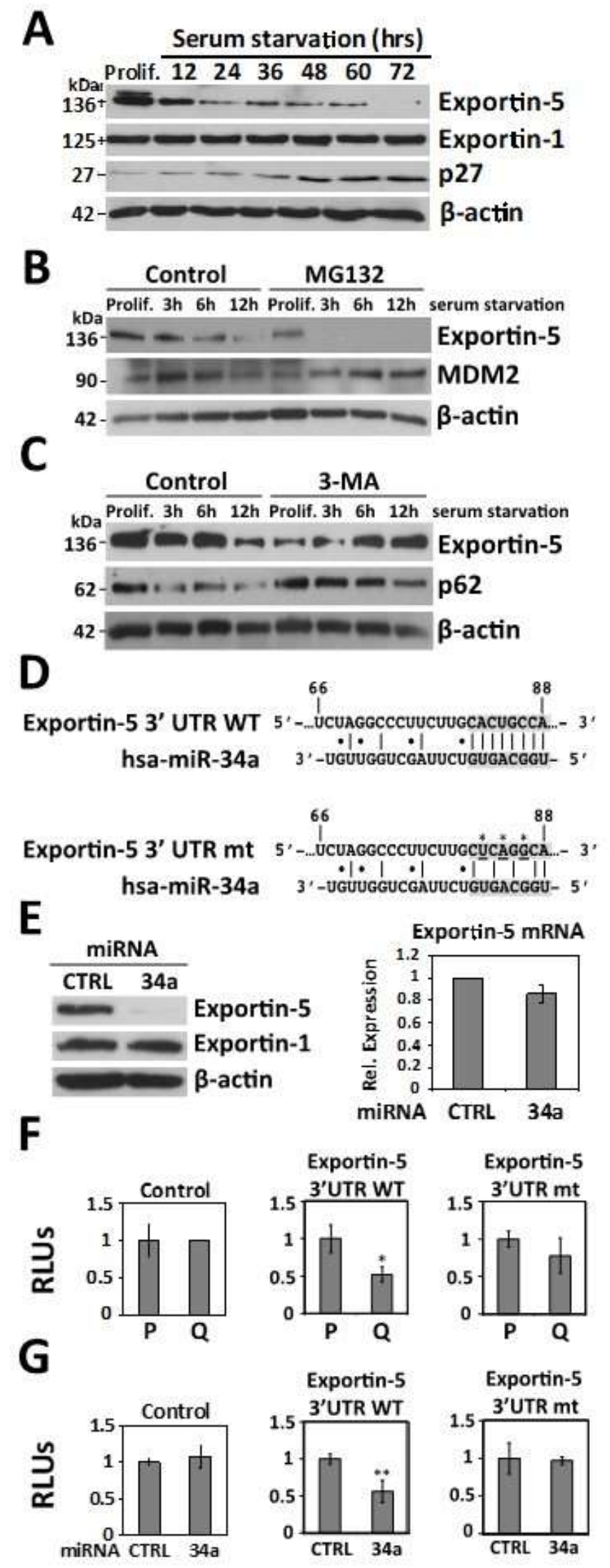
Figure 3

A
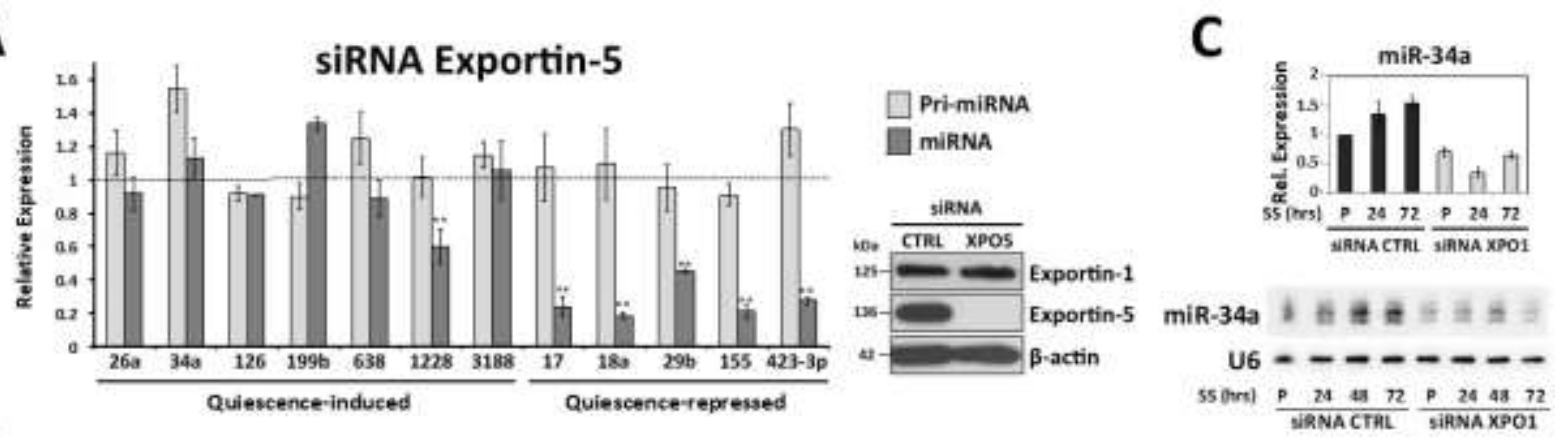

B

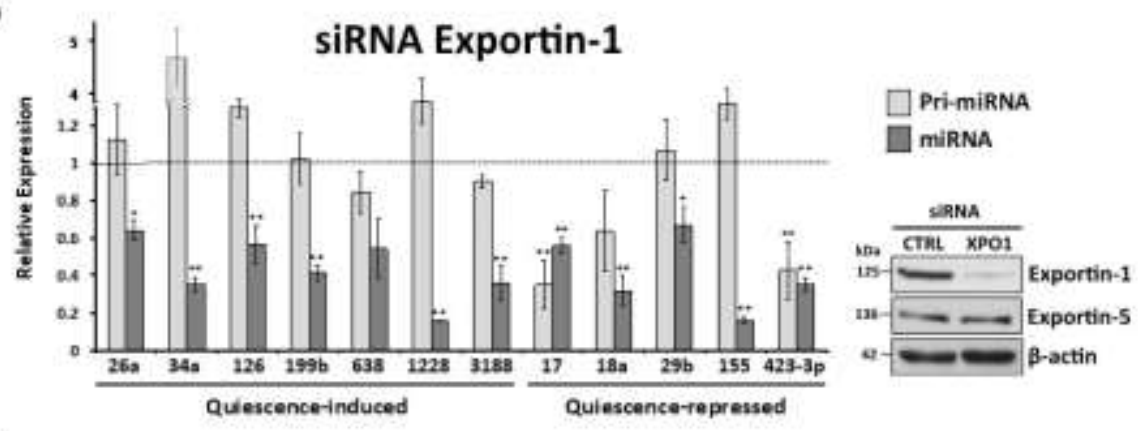

D
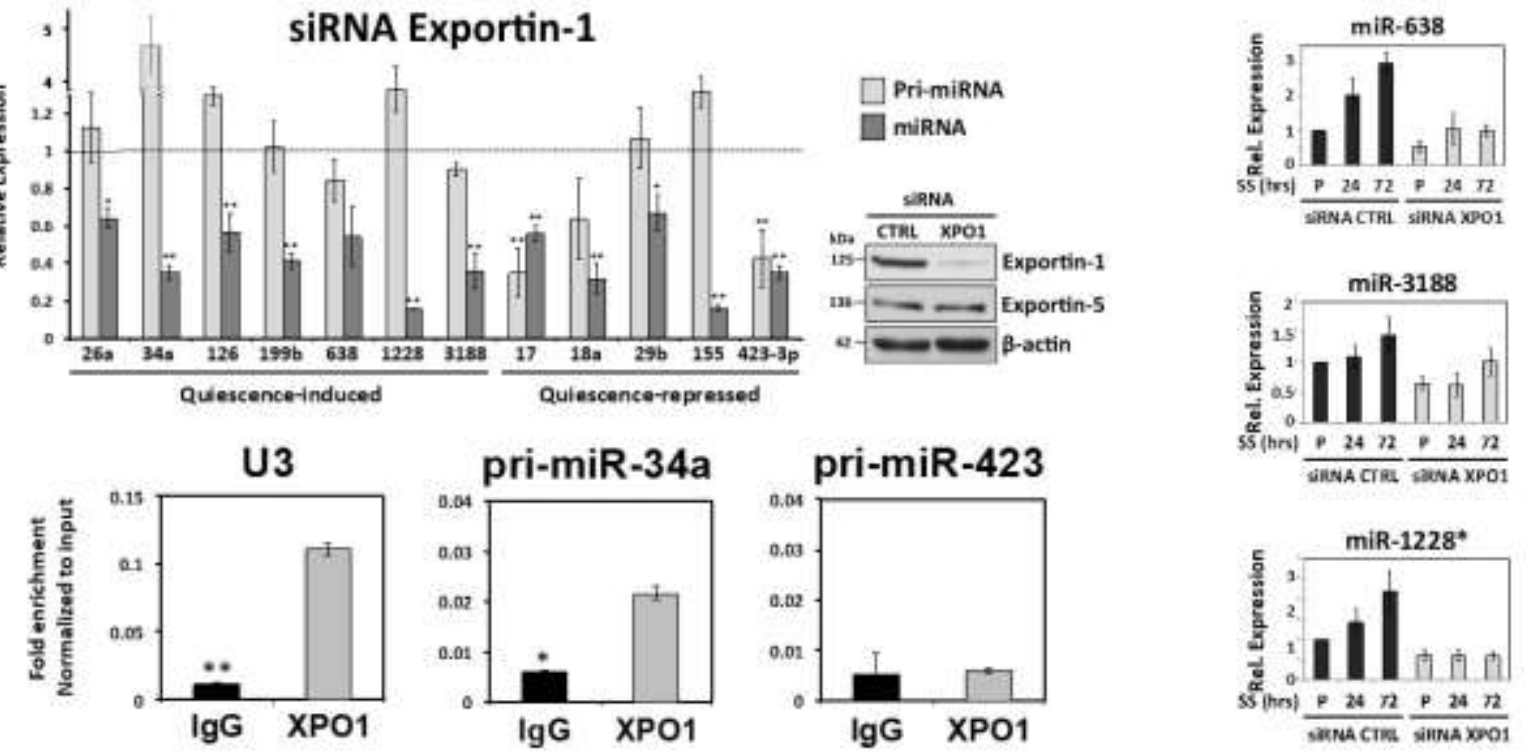
Figure 4

A

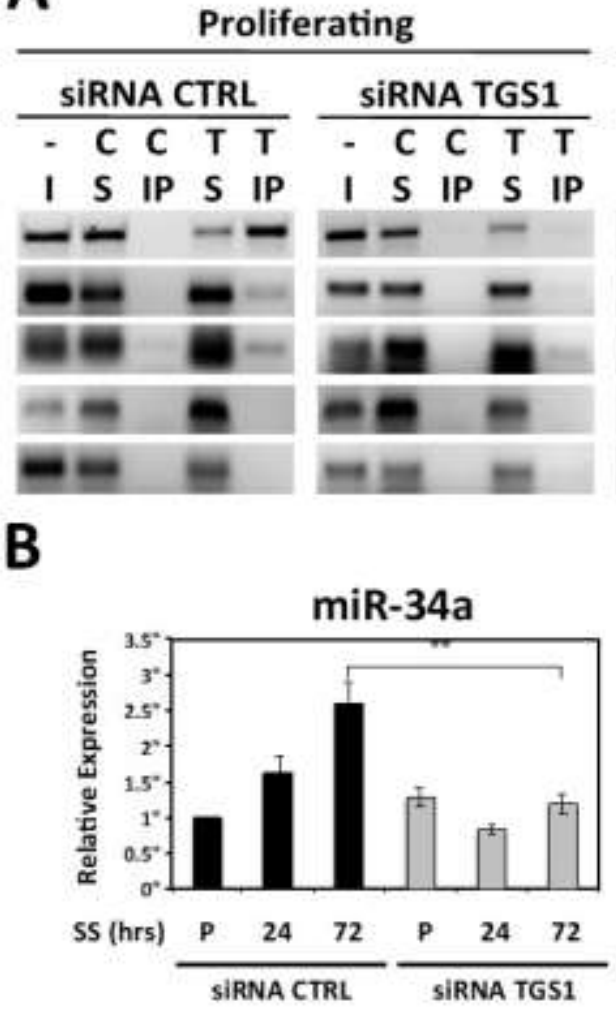

Quiescent

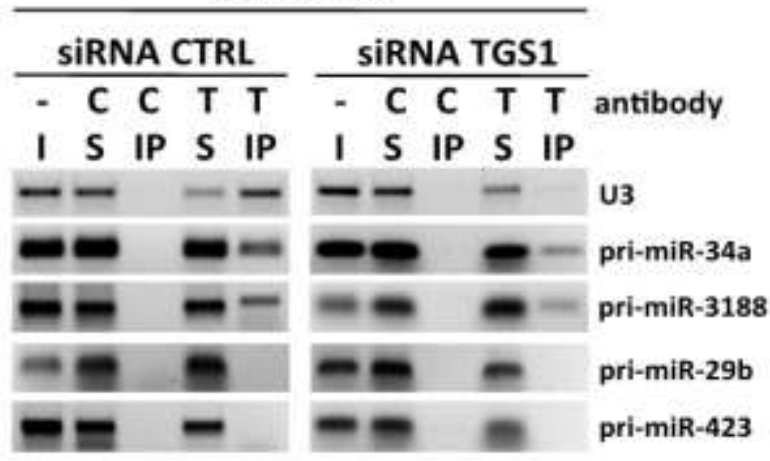


Figure 5

A

\begin{tabular}{|c|c|c|c|c|c|c|c|c|}
\hline \multicolumn{4}{|c|}{ Cytoplasmic } & \multicolumn{4}{|c|}{ Nuclear } & \\
\hline \multicolumn{2}{|c|}{ siRNA CTRL } & \multicolumn{2}{|c|}{ siRna Drosha } & \multicolumn{2}{|c|}{ siRnA CTRL } & \multicolumn{2}{|c|}{ siRna Drosha } & \\
\hline \multirow[t]{5}{*}{$\mathbf{P}$} & $\mathbf{Q}$ & $\mathbf{P}$ & $\mathbf{Q}$ & $\mathbf{P}$ & $\mathbf{Q}$ & $\mathbf{P}$ & $\mathbf{Q}$ & \\
\hline & & etsin & T5 & ames & $=$ & & & pri-miR-34a \\
\hline & & & * & & - & & & pri-miR-29b \\
\hline & & & & & - & & & pri-miR-423 \\
\hline & & & & & $m$ & $=$ & -5 & $\begin{array}{l}\text { U6 } \\
\text { (nuclear marker) }\end{array}$ \\
\hline & & & & & & & & $\begin{array}{l}\text { mt tRNAval } \\
\text { (cytoplasmic marker) }\end{array}$ \\
\hline
\end{tabular}

B

Cytoplasmic pri-miR-34a

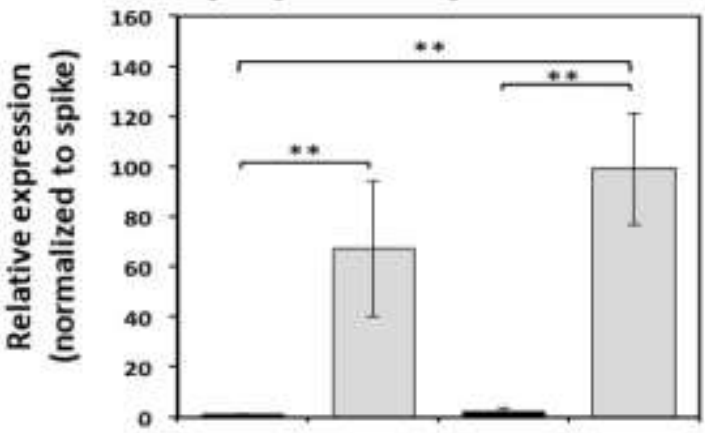

siRNA Ctrl Drosha Ctrl Drosha

$\overline{\text { Proliferating }}$ Quiescent
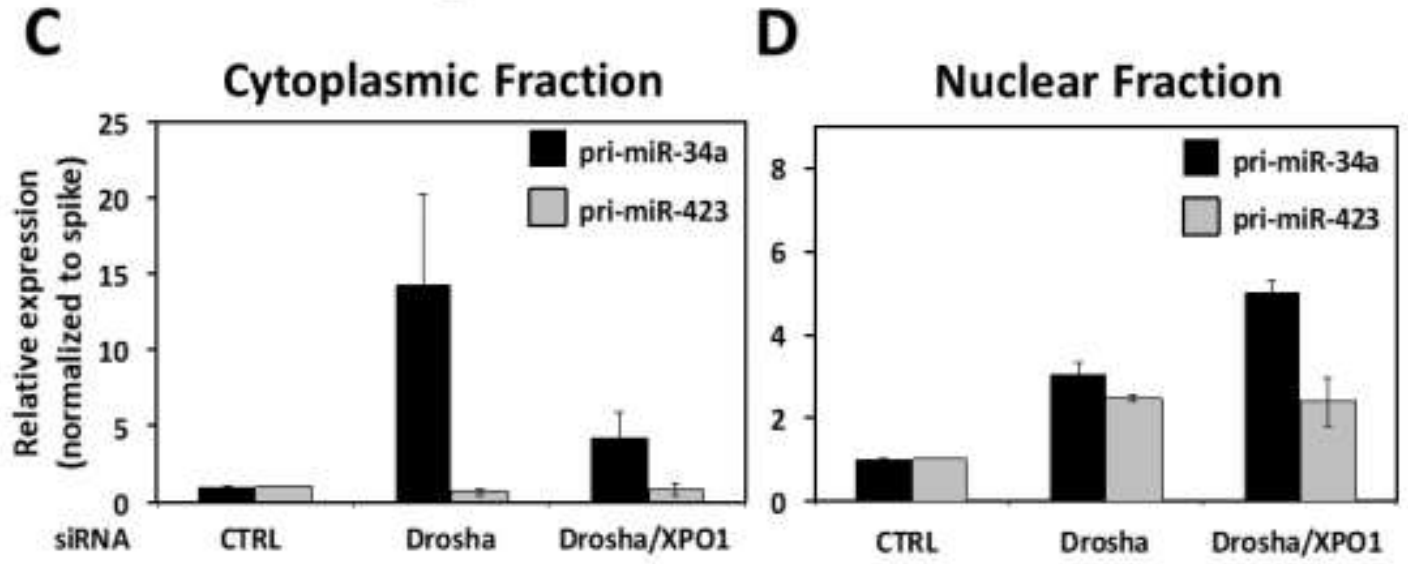
Figure 6

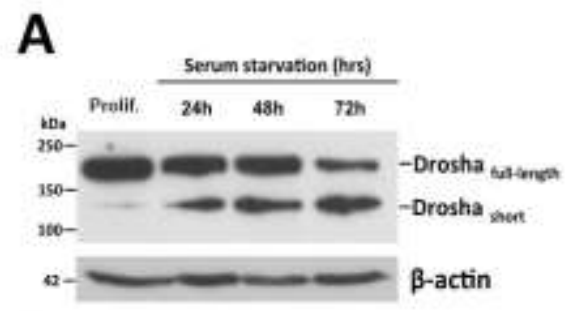

E

B
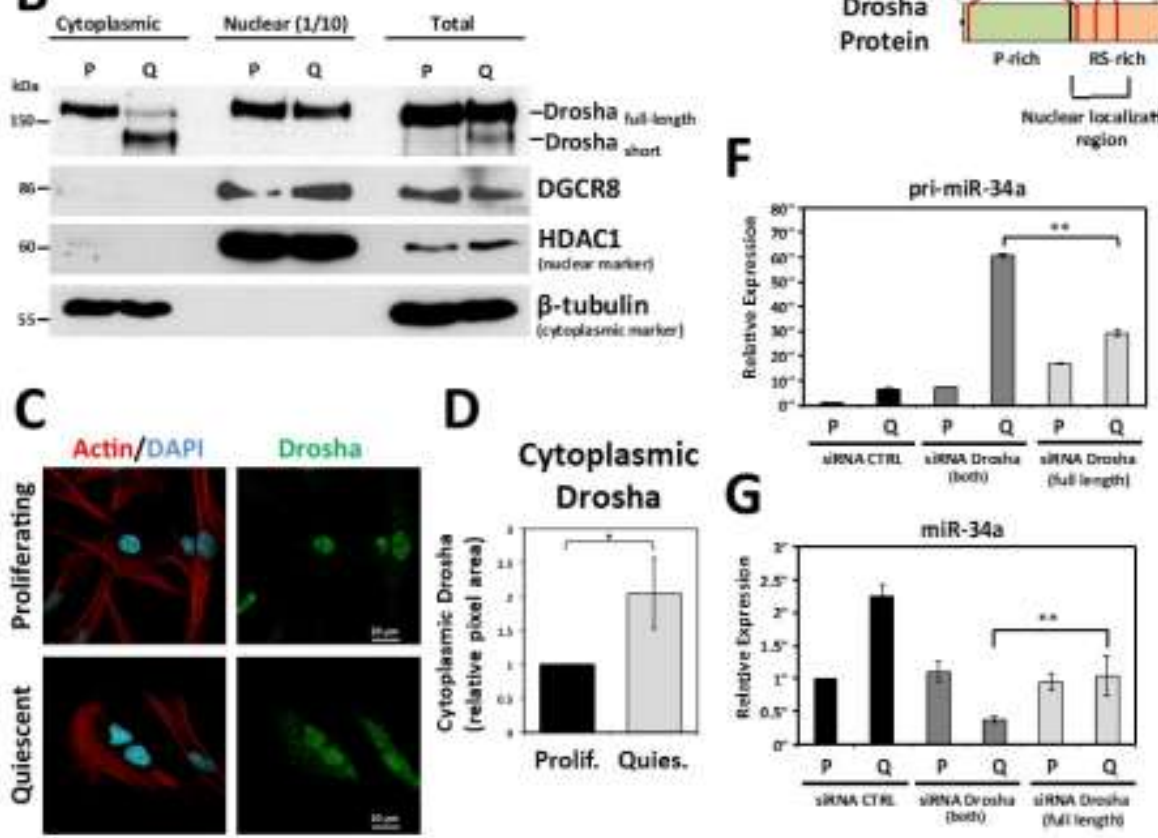

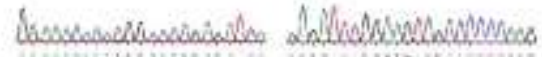
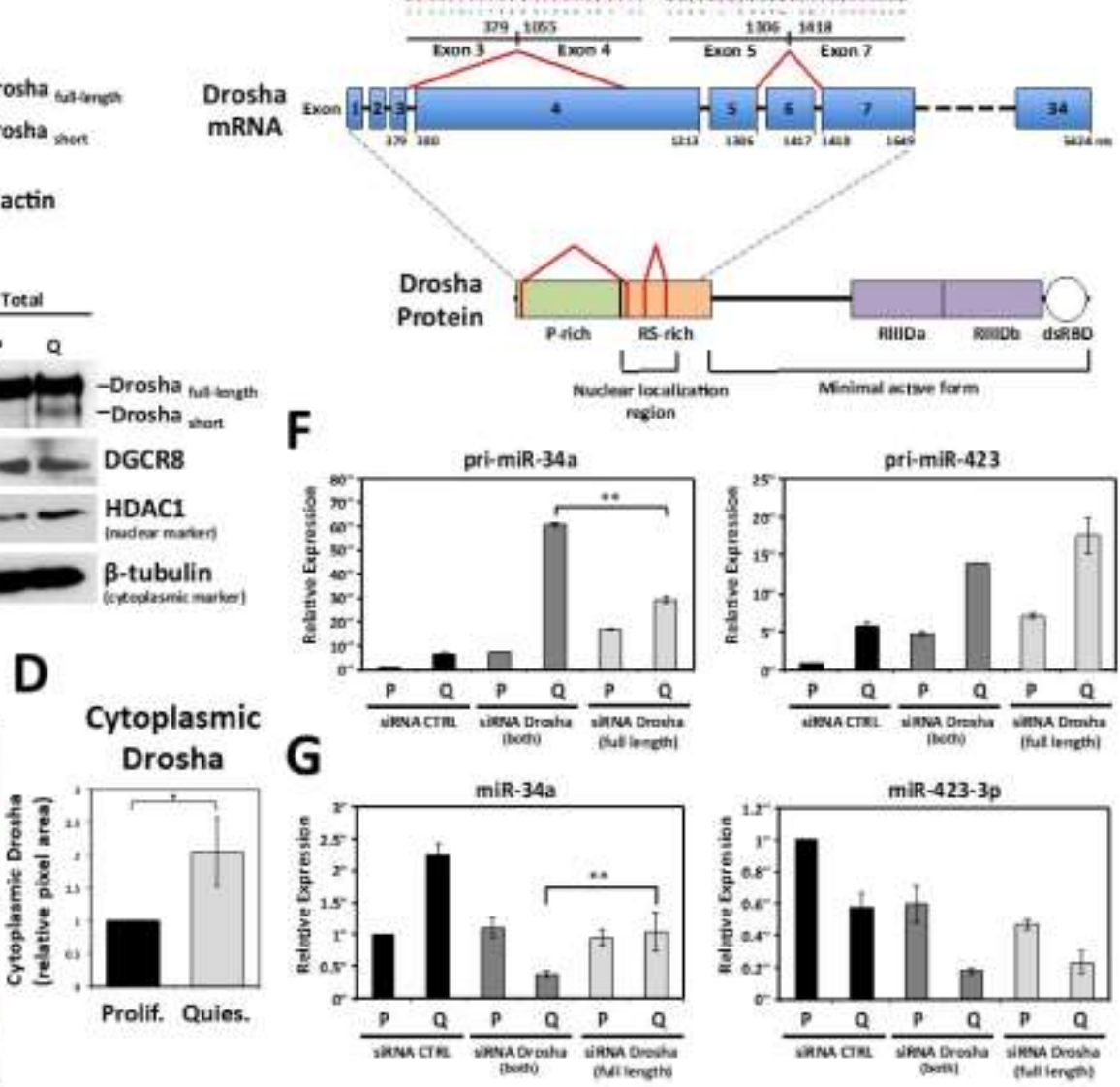


\section{$\underline{\text { XI. Supplementary Tables }}$}

\section{Table S1}

\begin{tabular}{|c|c|c|c|}
\hline miRNA Name & $\begin{array}{l}\text { 12hrs SS vs } \\
\text { mock }\end{array}$ & $\begin{array}{l}48 \mathrm{hrs} \text { SS vs } \\
\text { mock }\end{array}$ & $\begin{array}{l}\text { 72hrs SS vs } \\
\text { mock }\end{array}$ \\
\hline hsa-let-7g-star_st & 1.15 & 1.53 & 2.23 \\
\hline hsa-miR-101_st & 1.68 & 1.79 & 1.94 \\
\hline hsa-miR-1208_st & 2.10 & 1.55 & 1.46 \\
\hline hsa-miR-1225-5p_st & 3.21 & 2.13 & 1.70 \\
\hline hsa-miR-1228-star_st & 4.48 & 2.10 & 1.67 \\
\hline hsa-miR-1231_st & 5.31 & 2.61 & 2.47 \\
\hline hsa-miR-1246_st & 3.00 & 3.68 & 4.87 \\
\hline hsa-miR-126_st & 1.18 & 1.79 & 3.16 \\
\hline hsa-miR-1281_st & 2.04 & 2.05 & 1.37 \\
\hline hsa-miR-133b_st & 1.71 & 1.55 & 1.87 \\
\hline hsa-miR-1469_st & 5.39 & 3.37 & 2.54 \\
\hline hsa-miR-149-star_st & 3.90 & 1.78 & 1.46 \\
\hline hsa-miR-150-star_st & 2.62 & 1.90 & 1.53 \\
\hline hsa-miR-154_st & 1.39 & 1.71 & 2.74 \\
\hline hsa-miR-1825_st & 1.82 & 1.69 & 1.25 \\
\hline hsa-miR-187-star_st & 2.92 & 3.97 & 1.76 \\
\hline hsa-miR-1908_st & 4.59 & 2.49 & 2.21 \\
\hline hsa-miR-1909_st & 5.49 & 2.72 & 2.32 \\
\hline hsa-miR-1909-star_st & 1.92 & 1.92 & 1.50 \\
\hline hsa-miR-1910_st & 2.73 & 2.36 & 1.39 \\
\hline hsa-miR-1915_st & 4.59 & 3.64 & 2.58 \\
\hline hsa-miR-194_st & 2.04 & 2.05 & 3.50 \\
\hline hsa-miR-195_st & 1.15 & 1.50 & 1.83 \\
\hline hsa-miR-199a-3p_st & 1.34 & 1.66 & 2.33 \\
\hline hsa-miR-199b-3p_st & 1.35 & 1.78 & 2.54 \\
\hline hsa-miR-199b-5p_st & 1.21 & 2.42 & 4.31 \\
\hline hsa-miR-224-star_st & 1.05 & 1.74 & 1.71 \\
\hline hsa-miR-2277_st & 3.10 & 1.95 & 1.34 \\
\hline hsa-miR-26a_st & 1.09 & 1.55 & 1.57 \\
\hline hsa-miR-26b_st & 1.86 & 1.38 & 2.18 \\
\hline hsa-miR-2861_st & 7.14 & 4.60 & 3.70 \\
\hline hsa-miR-2909_st & 1.63 & 1.67 & 1.32 \\
\hline hsa-miR-301a_st & 1.78 & 1.74 & 1.66 \\
\hline hsa-miR-302a_st & 1.52 & 1.56 & 1.53 \\
\hline hsa-miR-30b_st & 1.13 & 1.64 & 2.13 \\
\hline
\end{tabular}




\begin{tabular}{|c|c|c|c|}
\hline hsa-miR-30d_st & -1.05 & 1.54 & 2.34 \\
\hline hsa-miR-30e-star_st & 1.77 & 1.50 & 2.00 \\
\hline hsa-miR-3144-5p_st & 2.09 & 1.68 & 1.39 \\
\hline hsa-miR-3154_st & 1.65 & 1.32 & 1.51 \\
\hline hsa-miR-3168_st & 1.55 & 1.66 & 1.78 \\
\hline hsa-miR-3178_st & 3.79 & 2.50 & 2.12 \\
\hline hsa-miR-3180-3p_st & 4.44 & 2.24 & 2.13 \\
\hline hsa-miR-3181_st & 4.08 & 3.60 & 3.01 \\
\hline hsa-miR-3185_st & 7.28 & 4.50 & 3.59 \\
\hline hsa-miR-3187_st & 5.49 & 4.27 & 2.83 \\
\hline hsa-miR-3188_st & 8.77 & 5.21 & 4.93 \\
\hline hsa-miR-3194_st & 1.12 & 2.15 & 1.59 \\
\hline hsa-miR-3195_st & 6.20 & 4.79 & 3.22 \\
\hline hsa-miR-3196_st & 3.29 & 1.87 & 1.48 \\
\hline hsa-miR-342-3p_st & -1.05 & 1.55 & 1.61 \\
\hline hsa-miR-346_st & 3.20 & 2.69 & 2.15 \\
\hline hsa-miR-34a_st & 1.12 & 1.95 & 2.39 \\
\hline hsa-miR-34a-star_st & 1.01 & 1.55 & 2.44 \\
\hline hsa-miR-378_st & -1.04 & 1.82 & 1.58 \\
\hline hsa-miR-422a_st & 1.81 & 2.87 & 3.07 \\
\hline hsa-miR-4259_st & 1.64 & 1.49 & 1.61 \\
\hline hsa-miR-4271_st & 2.69 & 1.98 & 1.56 \\
\hline hsa-miR-4281_st & 5.29 & 2.70 & 2.32 \\
\hline hsa-miR-4292_st & 2.18 & 2.06 & 1.90 \\
\hline hsa-miR-4320_st & 1.58 & 1.67 & 1.59 \\
\hline hsa-miR-4321_st & 2.18 & 1.90 & 1.31 \\
\hline hsa-miR-4322_st & 3.43 & 2.57 & 2.47 \\
\hline hsa-miR-455-5p_st & 2.41 & 2.43 & 3.06 \\
\hline hsa-miR-498_st & 2.39 & 1.71 & 1.20 \\
\hline hsa-miR-514_st & 1.30 & 1.56 & 1.50 \\
\hline hsa-miR-523_st & -1.19 & 1.68 & 1.55 \\
\hline hsa-miR-532-3p_st & 1.33 & 1.81 & 1.99 \\
\hline hsa-miR-557_st & 1.89 & 1.22 & 1.54 \\
\hline hsa-miR-564_st & 2.44 & 2.30 & 1.79 \\
\hline hsa-miR-566_st & 1.70 & 1.25 & 1.55 \\
\hline hsa-miR-572_st & 3.60 & 2.18 & 1.21 \\
\hline hsa-miR-575_st & 1.57 & 1.66 & 1.53 \\
\hline hsa-miR-596_st & 2.92 & 1.80 & 1.08 \\
\hline hsa-miR-602_st & 4.50 & 1.90 & 2.12 \\
\hline hsa-miR-638_st & 4.67 & 3.06 & 2.30 \\
\hline hsa-miR-654-5p_st & 1.69 & 2.12 & 1.33 \\
\hline
\end{tabular}




\begin{tabular}{|l|r|r|r|}
\hline hsa-miR-663_st & 3.62 & 2.22 & 1.95 \\
\hline hsa-miR-664-star_st & 1.29 & 1.85 & 1.87 \\
\hline hsa-miR-675_st & 1.59 & 1.52 & 1.67 \\
\hline hsa-miR-675-star_st & 1.76 & 1.56 & 1.71 \\
\hline hsa-miR-762_st & 4.73 & 2.52 & 2.17 \\
\hline hsa-miR-768-3p_st & 1.11 & 1.67 & 2.78 \\
\hline hsa-miR-885-3p_st & 3.26 & 2.56 & 2.34 \\
\hline hsa-miR-885-5p_st & 2.61 & 2.17 & 1.81 \\
\hline hsa-miR-888-star_st & 1.61 & 1.25 & 1.52 \\
\hline hsa-miR-92b-star_st & 3.45 & 1.19 & 1.61 \\
\hline hsa-miR-943_st & 3.41 & 1.69 & 2.13 \\
\hline hsa-miR-99a-star_st & 1.41 & 1.85 & 1.59 \\
\hline
\end{tabular}

Table S1. Mature microRNAs up-regulated during the induction of quiescence in HFFs. Table show microRNAs that were up-regulated 1.5-fold or greater in at least two-time points of serum starvation (red values) in comparison to proliferating HFF cells. GeneChip® miRNA 2.0 Array (Affymetrix, Santa Clara, CA) were used for this analysis. 
Table S2

\begin{tabular}{|c|c|c|c|}
\hline miRNA Name & $\begin{array}{l}\text { 12hrs SS vs } \\
\text { mock }\end{array}$ & $\begin{array}{l}\text { 48hrs SS vs } \\
\text { mock }\end{array}$ & $\begin{array}{l}\text { 72hrs SS vs } \\
\text { mock }\end{array}$ \\
\hline hsa-let-7a-2-star_st & -1.92 & -1.39 & -1.58 \\
\hline hsa-miR-106b-star_st & -1.71 & -1.84 & -1.84 \\
\hline hsa-miR-1184_st & -1.17 & -2.08 & -1.62 \\
\hline hsa-miR-1207-5p_st & 1.08 & -1.59 & -1.59 \\
\hline hsa-miR-1244_st & -1.50 & -1.51 & 1.30 \\
\hline hsa-miR-1247_st & -1.95 & -1.80 & -1.17 \\
\hline hsa-miR-1249_st & -1.58 & -1.49 & -1.74 \\
\hline hsa-miR-125b-1-star_st & -2.50 & -2.33 & -3.34 \\
\hline hsa-miR-1271_st & -1.54 & -1.35 & -1.59 \\
\hline hsa-miR-1275_st & -1.30 & -1.95 & -2.44 \\
\hline hsa-miR-1285_st & -1.20 & -1.89 & -2.51 \\
\hline hsa-miR-1292_st & 1.35 & -1.59 & -2.87 \\
\hline hsa-miR-1296_st & -2.18 & -1.73 & -2.20 \\
\hline hsa-miR-1308_st & -1.94 & -2.64 & -3.48 \\
\hline hsa-miR-130b_st & -1.49 & -1.76 & -2.24 \\
\hline hsa-miR-138_st & -1.33 & -1.78 & -2.34 \\
\hline hsa-miR-138-1-star_st & -1.46 & -1.72 & -2.31 \\
\hline hsa-miR-139-3p_st & -2.04 & -3.03 & -2.42 \\
\hline hsa-miR-1470_st & -7.07 & -7.07 & -7.07 \\
\hline hsa-miR-1471_st & -4.02 & -1.98 & -1.25 \\
\hline hsa-miR-1538_st & -2.36 & -2.61 & -1.67 \\
\hline hsa-miR-155_st & -1.79 & -2.51 & -2.42 \\
\hline hsa-miR-155-star_st & -1.86 & -1.76 & -1.63 \\
\hline hsa-miR-17_st & -1.20 & -1.50 & -1.69 \\
\hline hsa-miR-181a-2-star_st & -1.78 & -1.46 & -1.77 \\
\hline hsa-miR-184_st & -1.89 & -2.01 & -1.62 \\
\hline hsa-miR-185-star_st & -1.84 & -1.42 & -1.51 \\
\hline hsa-miR-188-5p_st & -1.85 & -1.58 & -1.28 \\
\hline hsa-miR-18a_st & -1.87 & -1.90 & -2.81 \\
\hline hsa-miR-18a-star_st & -1.81 & -2.26 & -1.57 \\
\hline hsa-miR-18b_st & -1.25 & -1.77 & -1.50 \\
\hline hsa-miR-190_st & -1.28 & -1.70 & -1.57 \\
\hline hsa-miR-1914_st & -4.22 & -3.43 & -1.18 \\
\hline hsa-miR-1915-star_st & -1.63 & -2.08 & -1.55 \\
\hline hsa-miR-193b-star_st & -1.54 & -1.34 & -1.66 \\
\hline hsa-miR-197_st & -1.72 & -1.79 & -1.25 \\
\hline hsa-miR-1972_st & -1.74 & -1.93 & -1.95 \\
\hline
\end{tabular}




\begin{tabular}{|c|c|c|c|}
\hline hsa-miR-21-star_st & -2.91 & -2.96 & -3.50 \\
\hline hsa-miR-2110_st & -1.72 & -1.37 & -3.06 \\
\hline hsa-miR-22-star_st & -1.46 & -2.50 & -1.67 \\
\hline hsa-miR-222-star_st & -3.11 & -3.56 & -4.22 \\
\hline hsa-miR-23a-star_st & -2.05 & -2.55 & -3.15 \\
\hline hsa-miR-25-star_st & -1.97 & -2.74 & -3.91 \\
\hline hsa-miR-27a-star_st & -1.81 & -1.84 & -2.85 \\
\hline hsa-miR-27b-star_st & -1.48 & -1.81 & -1.67 \\
\hline hsa-miR-296-3p_st & -1.66 & -2.11 & -2.38 \\
\hline hsa-miR-298_st & -1.74 & -1.23 & -1.50 \\
\hline hsa-miR-29b_st & -1.69 & -1.89 & -1.52 \\
\hline hsa-miR-29b-1-star_st & -4.04 & -2.91 & -2.55 \\
\hline hsa-miR-31_st & -1.42 & -1.60 & -1.89 \\
\hline hsa-miR-3130-5p_st & -2.00 & -1.14 & -2.56 \\
\hline hsa-miR-3131_st & -2.08 & -1.83 & -1.84 \\
\hline hsa-miR-3136_st & -1.91 & -2.01 & -2.19 \\
\hline hsa-miR-3158_st & -1.60 & -1.81 & -1.39 \\
\hline hsa-miR-3170_st & -1.70 & 1.08 & -1.93 \\
\hline hsa-miR-3183_st & -2.05 & -2.32 & -1.32 \\
\hline hsa-miR-3198_st & -1.18 & -1.56 & -1.93 \\
\hline hsa-miR-323-5p_st & -2.64 & -1.93 & 1.25 \\
\hline hsa-miR-330-3p_st & -1.68 & -1.31 & -2.09 \\
\hline hsa-miR-339-3p_st & -1.75 & -1.12 & -1.91 \\
\hline hsa-miR-339-5p_st & -1.79 & -1.87 & -2.54 \\
\hline hsa-miR-33b-star_st & -1.25 & -1.61 & -2.71 \\
\hline hsa-miR-376a_st & -1.08 & -2.53 & -1.78 \\
\hline hsa-miR-378b_st & -1.35 & -1.51 & -1.60 \\
\hline hsa-miR-423-3p_st & -1.51 & -1.18 & -1.70 \\
\hline hsa-miR-424-star_st & -2.07 & -1.75 & -2.03 \\
\hline hsa-miR-4284_st & -1.48 & -2.51 & -2.91 \\
\hline hsa-miR-4298_st & -1.31 & -2.18 & -3.29 \\
\hline hsa-miR-431_st & -1.31 & -1.51 & -2.04 \\
\hline hsa-miR-4315_st & -1.17 & -1.53 & -1.63 \\
\hline hsa-miR-487a_st & 1.06 & -1.56 & -1.75 \\
\hline hsa-miR-490-3p_st & -3.55 & -1.88 & -1.68 \\
\hline hsa-miR-490-5p_st & -1.92 & -2.03 & -2.34 \\
\hline hsa-miR-503_st & -1.72 & -2.19 & -2.71 \\
\hline hsa-miR-508-5p_st & -2.34 & -1.49 & -1.68 \\
\hline hsa-miR-519c-3p_st & -1.14 & -1.59 & -1.55 \\
\hline hsa-miR-548a-3p_st & -2.24 & -1.20 & -1.68 \\
\hline hsa-miR-550_st & -1.58 & -2.01 & -1.86 \\
\hline
\end{tabular}




\begin{tabular}{|l|c|c|c|}
\hline hsa-miR-550-star_st & -1.77 & -1.85 & -1.58 \\
\hline hsa-miR-608_st & -5.90 & -2.98 & -4.15 \\
\hline hsa-miR-629_st & -1.65 & -1.81 & -1.44 \\
\hline hsa-miR-629-star_st & -2.25 & -2.62 & -2.05 \\
\hline hsa-miR-639_st & -1.52 & -1.47 & -1.65 \\
\hline hsa-miR-658_st & -2.66 & -1.88 & -1.06 \\
\hline hsa-miR-671-3p_st & -2.03 & -2.37 & -3.83 \\
\hline hsa-miR-720_st & -1.05 & -1.83 & -1.56 \\
\hline hsa-miR-744_st & -1.34 & -1.59 & -2.22 \\
\hline hsa-miR-758_st & -1.73 & -1.29 & -1.50 \\
\hline hsa-miR-770-5p_st & -1.51 & -2.69 & -2.08 \\
\hline hsa-miR-886-3p_st & -2.39 & -6.22 & -5.80 \\
\hline hsa-miR-886-5p_st & -1.84 & -2.32 & -3.08 \\
\hline hsa-miR-92a-1-star_st & -1.71 & -1.73 & -1.60 \\
\hline hsa-miR-92b_st & -1.68 & -1.90 & -2.14 \\
\hline hsa-miR-93_st & -1.50 & -1.69 & -2.18 \\
\hline
\end{tabular}

Table S2. Mature microRNAs down-regulated during the induction of quiescence in HFFs. Table shows microRNAs that were down-regulated as defined in the legend to Table S1, shown in green. 


\section{Table S3}

\begin{tabular}{|c|c|}
\hline primers & sequence $\left(5^{\prime}-3^{\prime}\right)$ \\
\hline $\begin{array}{ll}\text { pri-miR-26a-2 } & \text { FWD } \\
\text { pri-miR-26a-2 } & \text { REV }\end{array}$ & $\begin{array}{l}\text { ACCTAATCATGACCTGGACAGAC } \\
\text { AAGAAGCGCACACACCATGT }\end{array}$ \\
\hline $\begin{array}{ll}\text { pri-miR-34a } & \text { FWD } \\
\text { pri-miR-34a } & \text { REV }\end{array}$ & $\begin{array}{l}\text { CTGGGGAGAGGCAGGACA } \\
\text { GGCATCTCTCGCTTCATCTT }\end{array}$ \\
\hline $\begin{array}{ll}\text { pri-miR-126 } & \text { FWD } \\
\text { pri-miR-126 } & \text { REV }\end{array}$ & $\begin{array}{l}\text { AGCGCAGCATTCTGGAAGAC } \\
\text { AGGGAGGTCAAGGCTGAGGT }\end{array}$ \\
\hline $\begin{array}{ll}\text { pri-miR-199b } & \text { FWD } \\
\text { pri-miR-199b } & \text { REV } \\
\end{array}$ & $\begin{array}{l}\text { GGGGTTCTCGGATCTCCA } \\
\text { GTCGGTCCAGCTCTCCAGT }\end{array}$ \\
\hline $\begin{array}{ll}\text { pri-miR-638 } & \text { FWD } \\
\text { pri-miR-638 } & \text { REV }\end{array}$ & $\begin{array}{l}\text { AGCTCGGTGCTGGAGAACTT } \\
\text { GAAAGCTGGGACCAGATGGA }\end{array}$ \\
\hline $\begin{array}{ll}\text { pri-miR-1228 } & \text { FWD } \\
\text { pri-miR-1228 } & \text { REV }\end{array}$ & $\begin{array}{l}\text { GGGGCAGGTGTGTGGTG } \\
\text { GGCGAGGCAGGTGTGAG }\end{array}$ \\
\hline $\begin{array}{ll}\text { pri-miR-3188 } & \text { FWD } \\
\text { pri-miR-3188 } & \text { REV } \\
\end{array}$ & $\begin{array}{l}\text { CCTTCTGGAGAGGCTTTGTG } \\
\text { GAGGGAGGGAAGGAAGGAG }\end{array}$ \\
\hline $\begin{array}{ll}\text { pri-miR-17 } & \text { FWD } \\
\text { pri-miR-17 } & \text { REV }\end{array}$ & $\begin{array}{l}\text { AGAGAACATCACCTTGTAAAACTGAA } \\
\text { CCTGCACTTTAAAGCCCAACT }\end{array}$ \\
\hline $\begin{array}{ll}\text { pri-miR-18a } & \text { FWD } \\
\text { pri-miR-18a } & \text { REV } \\
\end{array}$ & $\begin{array}{l}\text { CCTGCTGATGTTGAGTGCTTT } \\
\text { ACACCTATATACTTGCTTGGCTTG }\end{array}$ \\
\hline $\begin{array}{ll}\text { pri-miR-29b } & \text { FWD } \\
\text { pri-miR-29b } & \text { REV } \\
\end{array}$ & $\begin{array}{l}\text { CCTTTCTAGGTTGTCTTGGGTTT } \\
\text { TACTTCAGAGCTGTCCCATTCA }\end{array}$ \\
\hline $\begin{array}{ll}\text { pri-miR-155 } & \text { FWD } \\
\text { pri-miR-155 } & \text { REV }\end{array}$ & $\begin{array}{l}\text { TTTATGCCTCATCCTCTGAGTG } \\
\text { CAATTTGTTCCATGTGAATGCT }\end{array}$ \\
\hline $\begin{array}{ll}\text { pri-miR-423 } & \text { FWD } \\
\text { pri-miR-423 } & \text { REV } \\
\end{array}$ & $\begin{array}{l}\text { CGAAGTTTGAGGGAGAAACTTG } \\
\text { TCTAAGGAAGCCAGGAACTGTC }\end{array}$ \\
\hline $\begin{array}{ll}\text { U3 } & \text { FWD } \\
\text { U3 } & \text { REV }\end{array}$ & $\begin{array}{l}\text { CGAAAACCACGAGGAAGAGA } \\
\text { CACTCCCCAATACGGAGAGA }\end{array}$ \\
\hline $\begin{array}{ll}\text { U6 } & \text { FWD } \\
\text { U6 } & \text { REV }\end{array}$ & $\begin{array}{l}\text { ATCTTGTGGAAAGGACGAAACA } \\
\text { AAATATGGAACGCTTCACGAAT }\end{array}$ \\
\hline $\begin{array}{lll}\text { mt } & \text { tRNAval } & \text { FWD } \\
\text { mt } & \text { tRNAval } & \text { REV } \\
\end{array}$ & $\begin{array}{l}\text { CAGAGTGTAGCTTAACACAAAGCA } \\
\text { CAAGTTAAGTTGAAATCTCCTAAGTG }\end{array}$ \\
\hline $\begin{array}{ll}\text { Exportin-5 } & \text { FWD } \\
\text { Exportin-5 } & \text { REV }\end{array}$ & $\begin{array}{l}\text { AGTGGAAATCCAATCTTCCGTA } \\
\text { АTTTTGGCTAGCATTTCTGGTG }\end{array}$ \\
\hline $\begin{array}{ll}\text { Exportin-1 } & \text { FWD } \\
\text { Exportin-1 } & \text { REV } \\
\end{array}$ & $\begin{array}{l}\text { AAAATACGTTGTTGGCCTCATT } \\
\text { GGGCCATTCTTGTTTCAGTATC }\end{array}$ \\
\hline $\begin{array}{ll}\text { TGS1 } & \text { FWD } \\
\text { TGS1 } & \text { REV } \\
\end{array}$ & $\begin{array}{l}\text { GGATTCAAGTATGGCTCAGGAC } \\
\text { TGTCTGCGCATGTCTAGGTACT }\end{array}$ \\
\hline $\begin{array}{ll}\text { p27 } & \text { FWD } \\
\text { p27 } & \text { REV }\end{array}$ & $\begin{array}{l}\text { GGGGCTCGTCTTTTCGGGGTGTTT } \\
\text { GAGCGGGAGGGCGGAGAGGAG }\end{array}$ \\
\hline $\begin{array}{ll}\text { GAPDH } & \text { FWD } \\
\text { GAPDH } & \text { REV } \\
\end{array}$ & $\begin{array}{l}\text { CCACTCCTCCACCTTTGAC } \\
\text { АCCCTGTTGCTGTAGCCA }\end{array}$ \\
\hline $\begin{array}{lll}\text { C. elegans ama-1 FWD } \\
\text { C. elegans ama-1 REV }\end{array}$ & $\begin{array}{l}\text { GGAGCTCGAGTGGATCTTCG } \\
\text { GCGCAGAGAGTATCCTGGAC }\end{array}$ \\
\hline 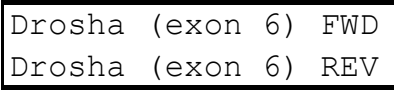 & $\begin{array}{l}\text { ACAACCGAAGATCACCATCTCT } \\
\text { CATTCTTGTCAGGCATGGTCT }\end{array}$ \\
\hline $\begin{array}{ll}\text { Drosha } & \text { FWD } \\
\text { Drosha } & \text { REV }\end{array}$ & $\begin{array}{l}\text { GAATGCAAAGGCATGATTGTTA } \\
\text { AAAGTGGACGATAATCGGAAAA }\end{array}$ \\
\hline
\end{tabular}

Table S3. Primers used in this study for qRT-PCR. 
Table S4

\begin{tabular}{|c|c|c|c|c|c|c|c|c|}
\cline { 2 - 9 } \multicolumn{1}{c|}{} & $\begin{array}{c}\text { Proliferating } \\
\text { + siRNA } \\
\text { CTRL }\end{array}$ & $\begin{array}{c}\text { Proliferating } \\
+ \text { siRNA } \\
\text { CTRL }\end{array}$ & $\begin{array}{c}\text { Proliferating } \\
+ \text { siRNA } \\
\text { TGS1 }\end{array}$ & $\begin{array}{c}\text { Proliferating } \\
\text { + siRNA } \\
\text { TGS1 }\end{array}$ & $\begin{array}{c}\text { Quiescent } \\
\text { + siRNA } \\
\text { CTRL }\end{array}$ & $\begin{array}{c}\text { Quiescent } \\
+ \text { siRNA } \\
\text { CTRL }\end{array}$ & $\begin{array}{c}\text { Quiescent } \\
\text { + siRNA } \\
\text { TGS1 }\end{array}$ & $\begin{array}{c}\text { Quiescent } \\
\text { + siRNA } \\
\text { TGS1 }\end{array}$ \\
\cline { 2 - 9 } & IP CTRL & IP TMG & IP CTRL & IP TMG & IP CTRL & IP TMG & IP CTRL & IP TMG \\
\hline $\begin{array}{c}\text { pri- } \\
\text { miR- } \\
\mathbf{3 4 a}\end{array}$ & 2.35 & 39.84 & 6.22 & 15.56 & 1.62 & 147.25 & 0.09 & 35.08 \\
\hline $\begin{array}{c}\text { pri- } \\
\text { miR- } \\
\mathbf{3 1 8 8}\end{array}$ & 11.87 & 62.32 & 1.84 & 29.57 & 3.46 & 235.98 & 1.48 & 53.29 \\
\hline $\begin{array}{c}\text { pri- } \\
\text { miR- } \\
\mathbf{4 2 3}\end{array}$ & 2.86 & 2.97 & 1.00 & 4.02 & 0.58 & 2.68 & 0.38 & 4.09 \\
\hline
\end{tabular}

Table S4. Quiescence-induced pri-miRNAs contain a TMG cap. Amounts of pri-miR-34a, pri-miR3188 and pri-miR-423 immunoprecipitated with anti-rabbit serum (IP CTRL) or antibody recognizing TMG-cap RNA (IP TMG) in proliferating or quiescent HFFs transfected with control siRNA or siRNA targeting TSG1. Relative expression was obtained by comparing input RNA and normalizing against a spike-in of non-endogenous RNA using qRT-PCR. Values in the table were multiplied by 1000 for clarity. 


\section{Supplementary Figures}

\section{Figure S1}
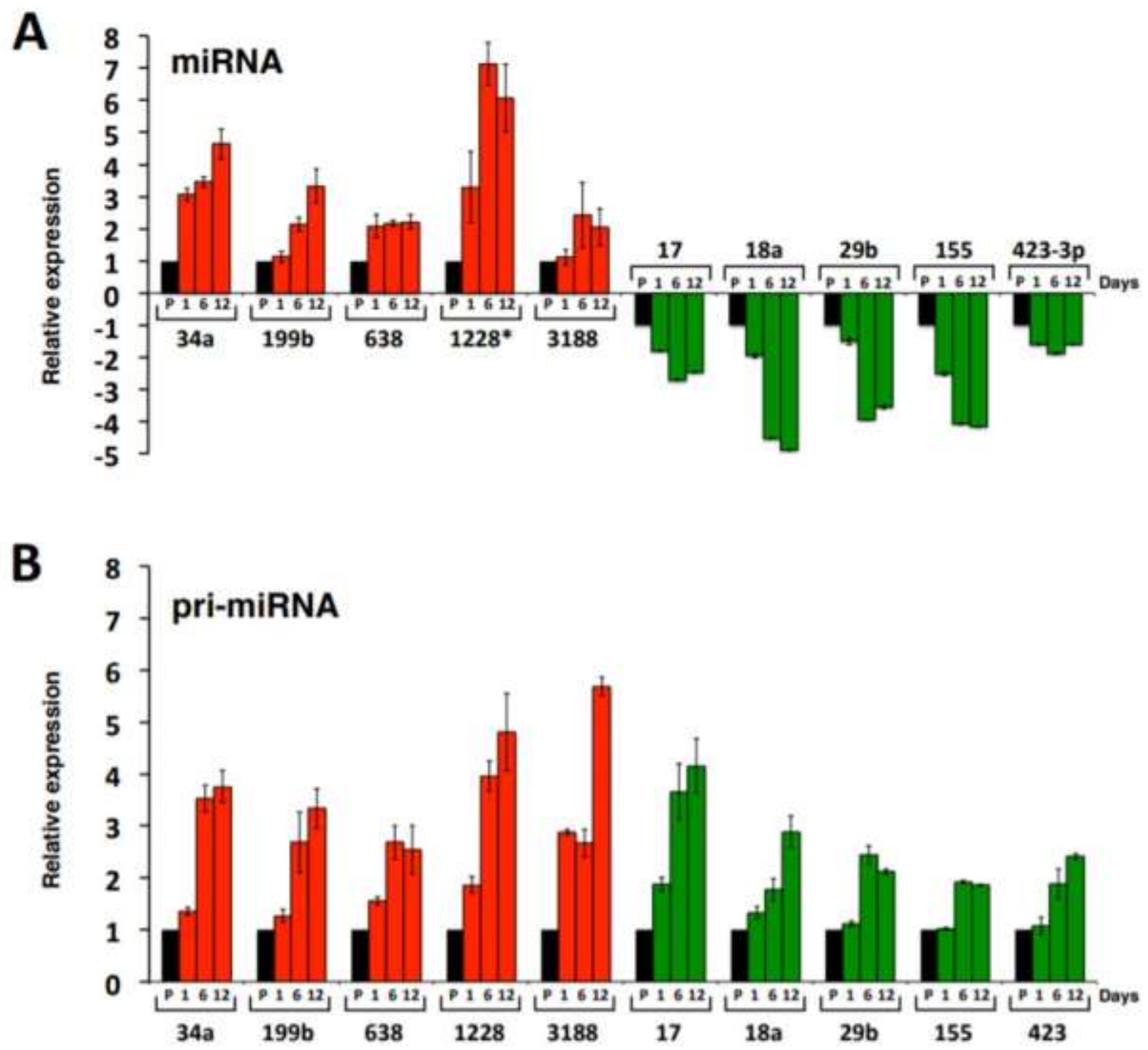

Figure S1. Differential expression of mature miRNAs and their corresponding primary miRNAs during the induction of quiescence by cell confluency. (A) qRT-PCR analysis of mature miRNAs. RNA was extracted 1, 6 and 12 days after reaching complete confluency and compared to RNA isolated from proliferating HFFs (P). (B) qRT-PCR analysis of primary miRNAs by using primers that bind upstream and downstream of the miRNA stem loop. In both A and B, red bars represent the miRNAs up-regulated during serum starvation by miRNA microarray analysis. Green bars represent mature miRNAs down-regulated during serum starvation by miRNA microarray analysis. Black bars represent miRNAs in proliferating (P) cells. Results were normalized to RNU43 and GAPDH mRNA expression and expressed relative to proliferating cells. Similar results were obtained in two independent experiments. 


\section{Figure S2}
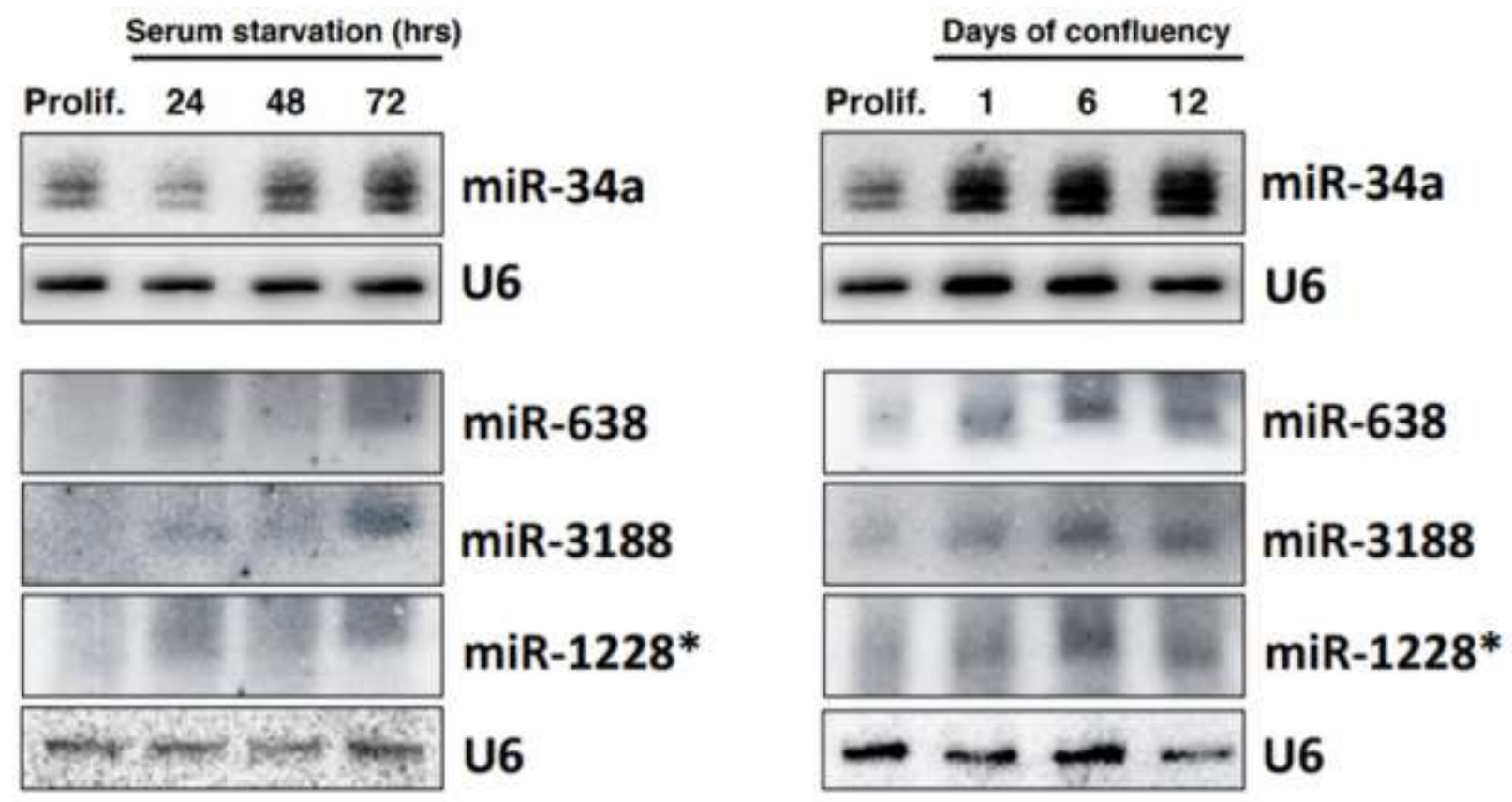

Figure S2. Northern blot analysis validating several miRNAs up-regulated during the induction of quiescence by serum starvation or cell confluency. U6 was used as loading control. 


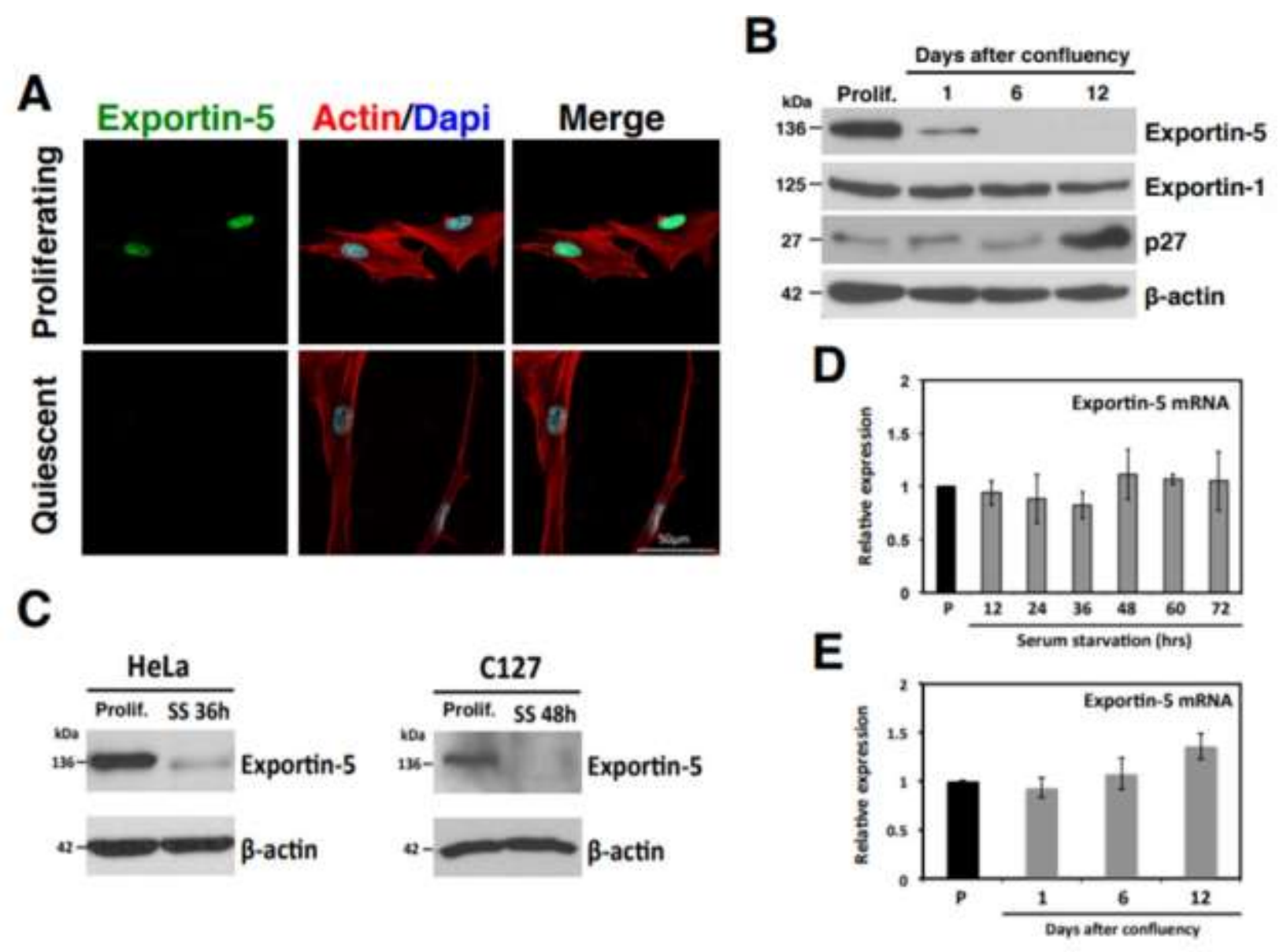

Figure S3. Exportin-5 expression is reduced post-transcriptionally during quiescence. (A) Immunofluorescence of Exportin-5 (green) in proliferating and quiescent HFFs by serum starvation. Nuclear fluorescence (DAPI, blue) and cytoskeleton immunofluorescence (actin, red) were used to visualize the nuclear localization of Exportin-5. (B) Western blot analysis showing reduction of Exportin5 but not Exportin-1 levels after induction of quiescence by confluency. (C) Exportin-5 protein expression is markedly reduced during quiescence by serum starvation in cervical carcinoma (HeLa) and murine mammary tumor (C127) cell lines. $\beta$-actin was used as a loading control. (D and E) qRT-PCR analysis of Exportin-5 mRNA, showing no changes in levels during serum starvation or confluency compared to proliferating cells (P). Exportin-5 mRNA levels were normalized to GAPDH mRNA. Results show the average of three independent experiments. 


\section{Figure S4}
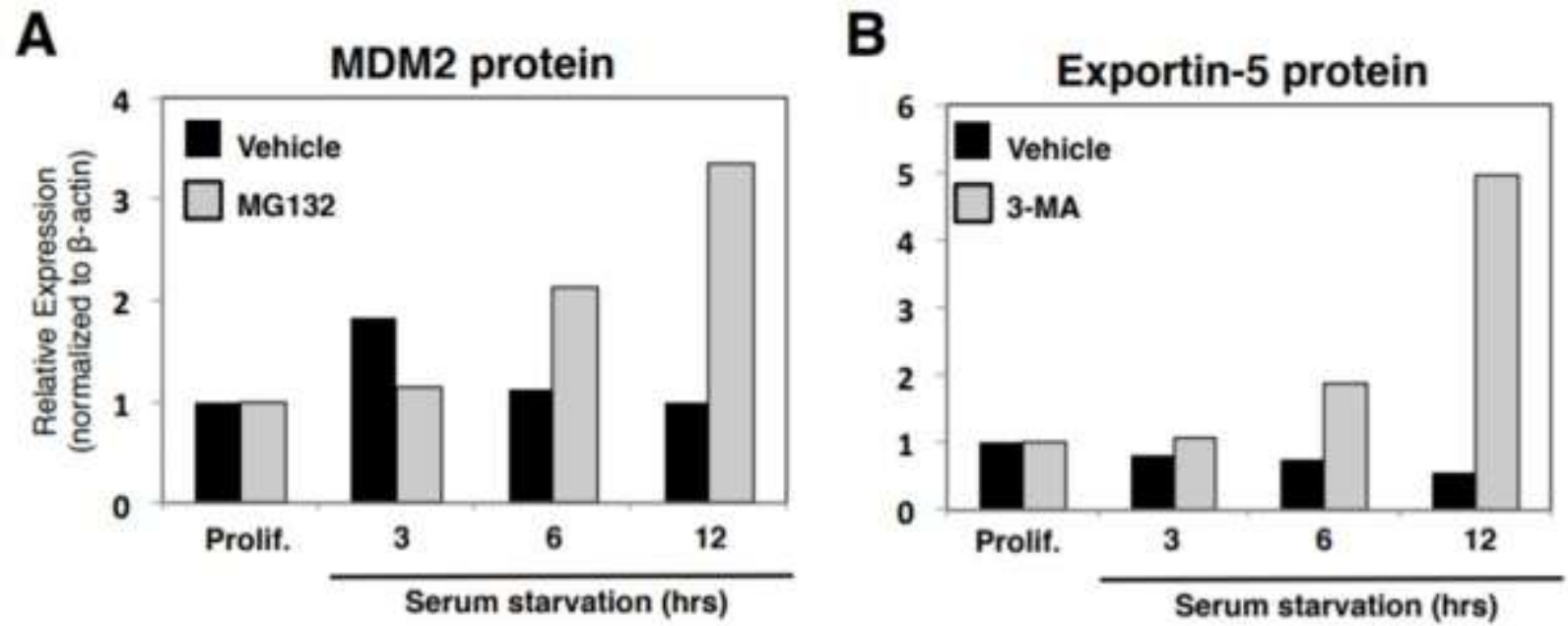

Figure S4. Accumulation of MDM2 and Exportin-5 caused by the inhibition of the proteasome or the autophagy pathways, respectively, during the induction of quiescence. (A) Western blot quantification of MDM2 protein in cells treated with vehicle (DMSO) or proteasome inhibitor MG132. (B) Exportin-5 protein in cells treated with vehicle (water) or autophagy inhibitor 3MA was measured by western blotting and quantified by using Image J software 1.48 (http://imagej.nih.gov/ij/download.html). Results were normalized to $\beta$-actin levels and compared to proliferating HFFs. Experiment was performed in triplicate with similar results. The figure shows a representative experiment. 


\section{Figure S5}

H. sapiens (Human)

P. troglodytes (Chimpanzee)

M. mulatta (Rhesus)

o. garnetti (Bushbaby)

T. belangeri (Treeshrew)

M. musculus (Mouse)

R. norvegicus (Rat)

C. porcellus (Guinea pig)

o. cuniculus (Rabbit)

S. araneus (Shrew)

E. europaeus (Hedgehog)

C. familiaris (Dog)

F. catus (Cat)

E. caballus (Horse)

B. Taurus (Cow)

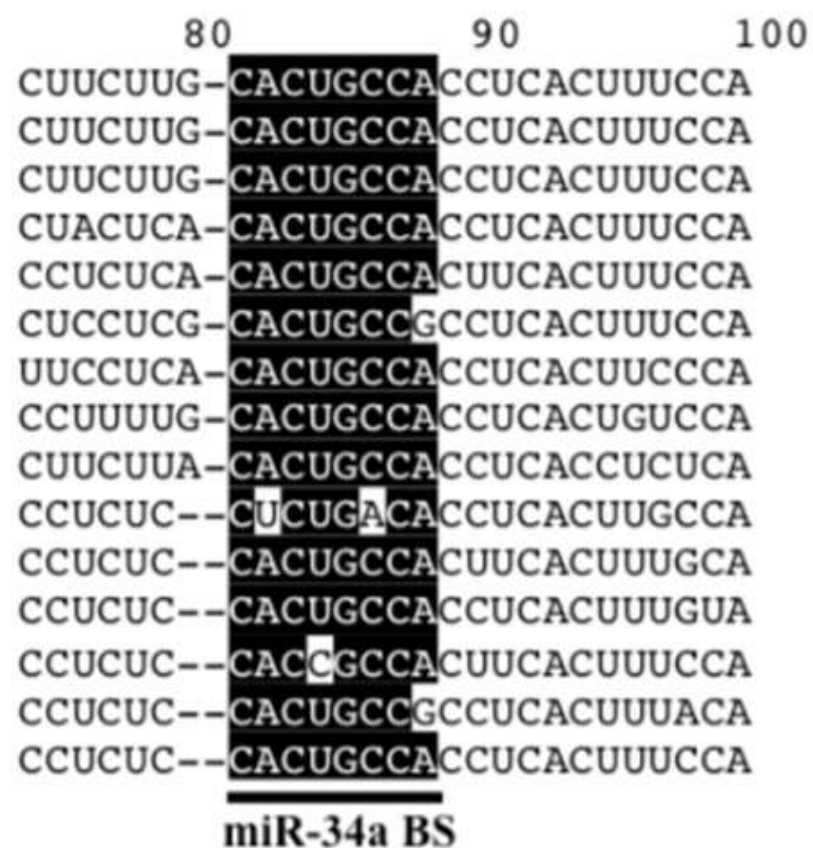

miR-34a BS

Figure S5. The Exportin-5 3'UTR contains a conserved miR-34 binding site. Comparison of the Exportin53 'UTR (nucleotide 74 to 100) in different organisms, showing a conserved binding site for the miR-34 family (nucleotides in black box). Analysis was obtained using TargetScanHuman 6.2 software (www.targetscan.org). 

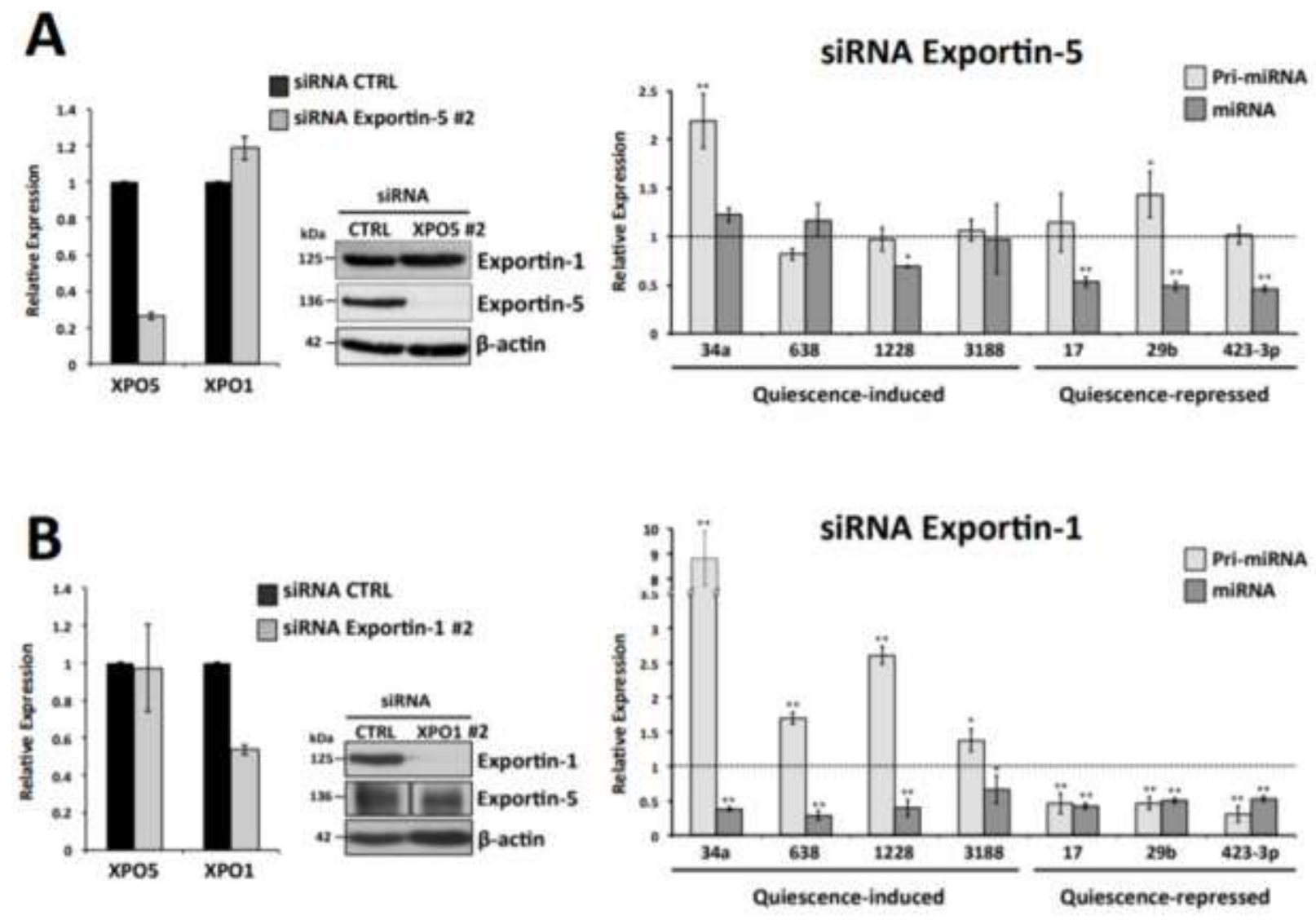

Figure S6. Biogenesis of quiescence-induced miRNAs requires Exportin-1 but not Exportin-5. (A) qRTPCR analysis showing relative expression of quiescence-induced and quiescence-repressed primary miRNAs (light gray bars) and their corresponding mature miRNAs (dark gray bars) in proliferating HFFs $48 \mathrm{hrs}$ after transfection with an siRNA scrambled control (dashed line) or an siRNA targeting Exportin-5 (siRNA XPO5 \#2) (IDs33191, Ambion) (dark and light gray bars). Western blot and qRT-PCR analysis of Exportin-1 and Exportin-5 using XPO5-depleted or control HFF lysates confirms specific knock-down of Exportin-5. (B) qRT-PCR analysis showing relative levels of the RNAs tested in panel (A) in proliferating HFFs $48 \mathrm{hrs}$ after transfection with an siRNA scrambled control (dashed line) or an siRNA targeting Exportin-1 (siRNA XPO1 \#2) (ID118206, Ambion) (dark and light gray bars). Western blot and qRT-PCR analysis of Exportin-1 and Exportin-5 using XPO1-depleted or control HFF lysates confirm specific knockdown of Exportin-1. Two-tailed t-test results are indicated by $*$ for $\mathrm{P}<0.05$ and $* *$ for $\mathrm{P}<0.01$. 
Figure S7
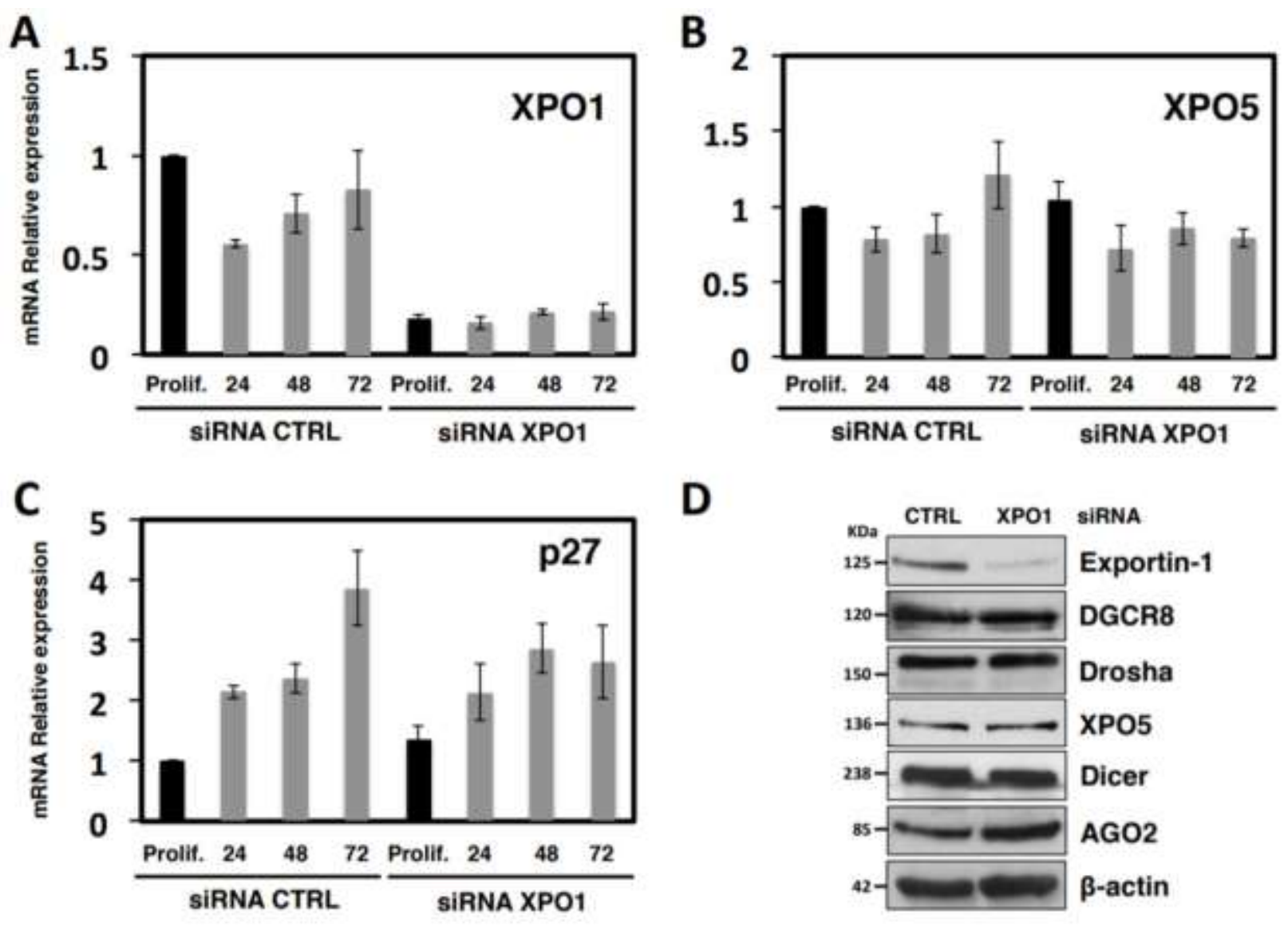

토
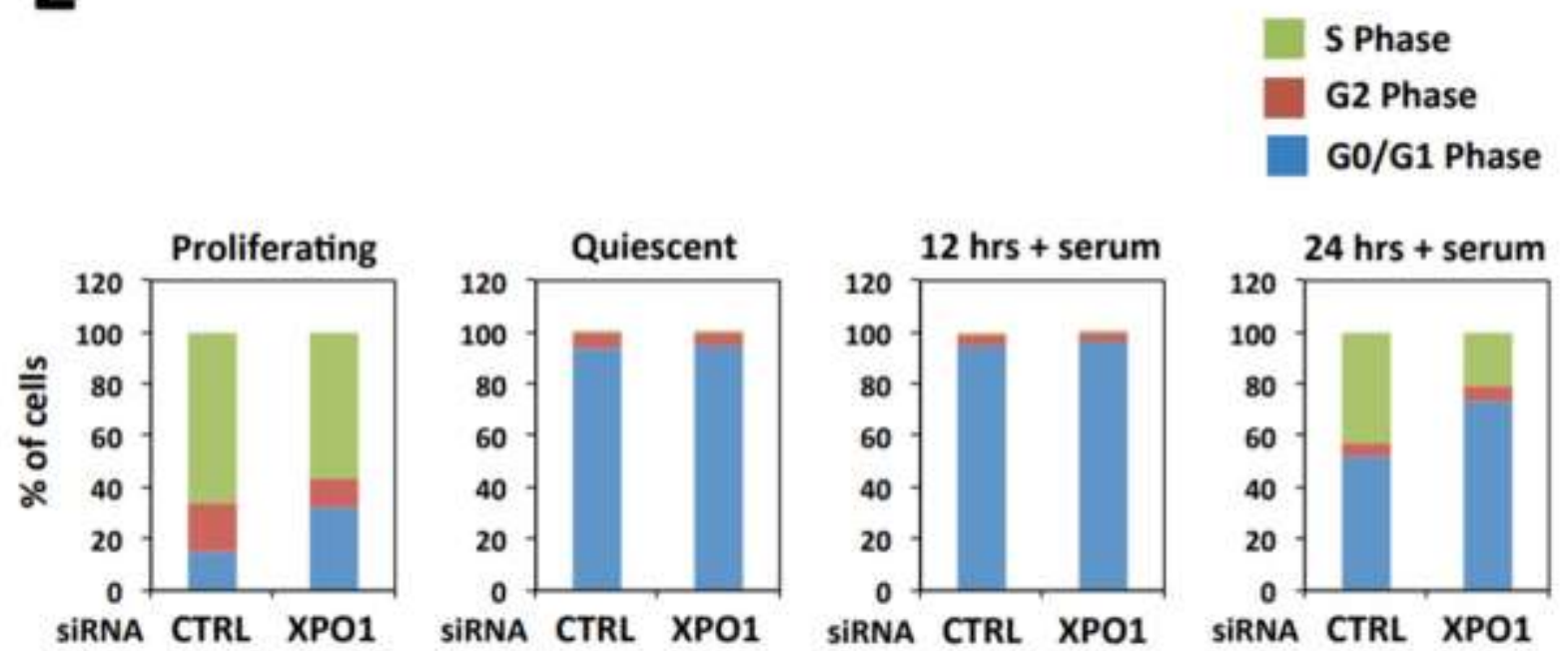

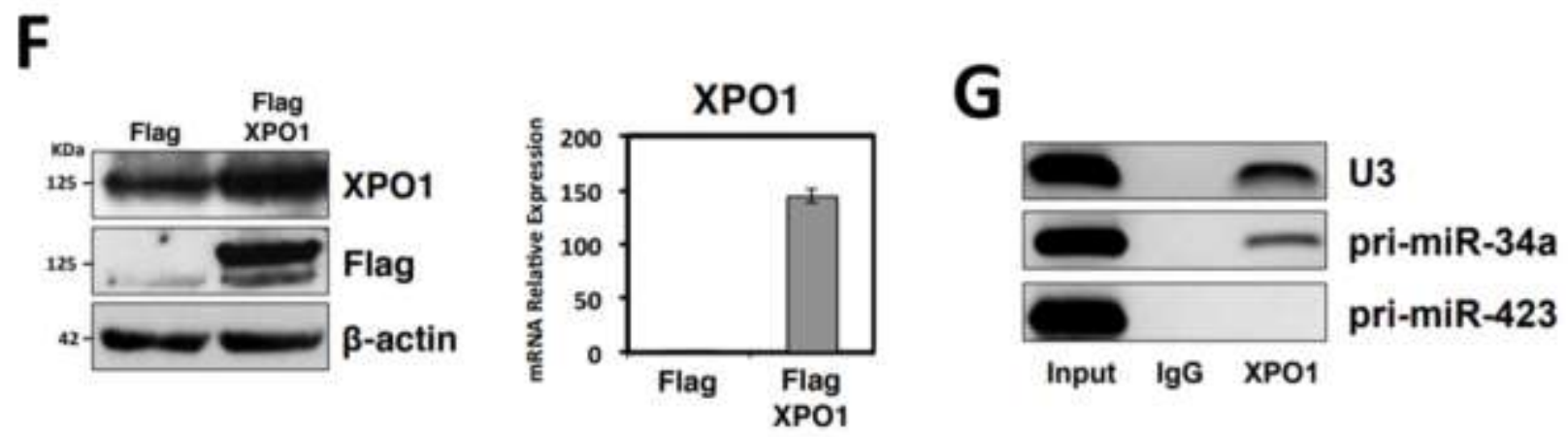

Figure S7. Exportin-1 knock-down during serum starvation does not alter the expression of Exportin-5, p27 or key proteins involved in miRNA biogenesis. HFFs were transfected with a siRNA scrambled control (CTRL) or a siRNA targeting Exportin-1 (XPO1) (ID s14937, Ambion) 24 hrs before the induction of quiescence by serum starvation. Expression of Exportin-1 (A), Exportin-5 (XPO5) (B), or p27 (C) mRNA in proliferating cells (Prolif) or at the indicated hours after serum starvation was determined by qRT-PCR and normalized to GAPDH mRNA. (D) Western blot showing repression of Exportin-1 but not other proteins involved in miRNA biogenesis in proliferating HFF cells transfected with an siRNA against Exportin-1 (XPO1). Similar results were obtained in two independent experiments. (E) Exportin-1 knockdown do not change the entrance of HFFs to quiescence. Cell cycle assays in proliferating, quiescent (24 hours after serum starvation), and synchronized (12 and 24 hours after the addition of serum) HFFs with siRNA scrambled control (CTRL) or a siRNA targeting Exportin-1 (XPO1). Similar results were obtained in two independent experiments. (F) Overexpression of Flag-Exportin-1. Western blot and qRT-PCR of HFF cells expressing Flag-tagged empty vector or Flag-tagged Exportin-1 (G) Exportin-1 physically associates with pri-miR-34a in quiescent cells. RT-PCR was used to detect U3 snoRNA, pri-miR-34a, or pri-miR-423 in control Flag-Vector (IgG) or Flag-Exportin-1 (XPO1) immunoprecipitates from quiescent HFFs. Similar results were obtained in two independent experiments. 


\section{Figure S8}
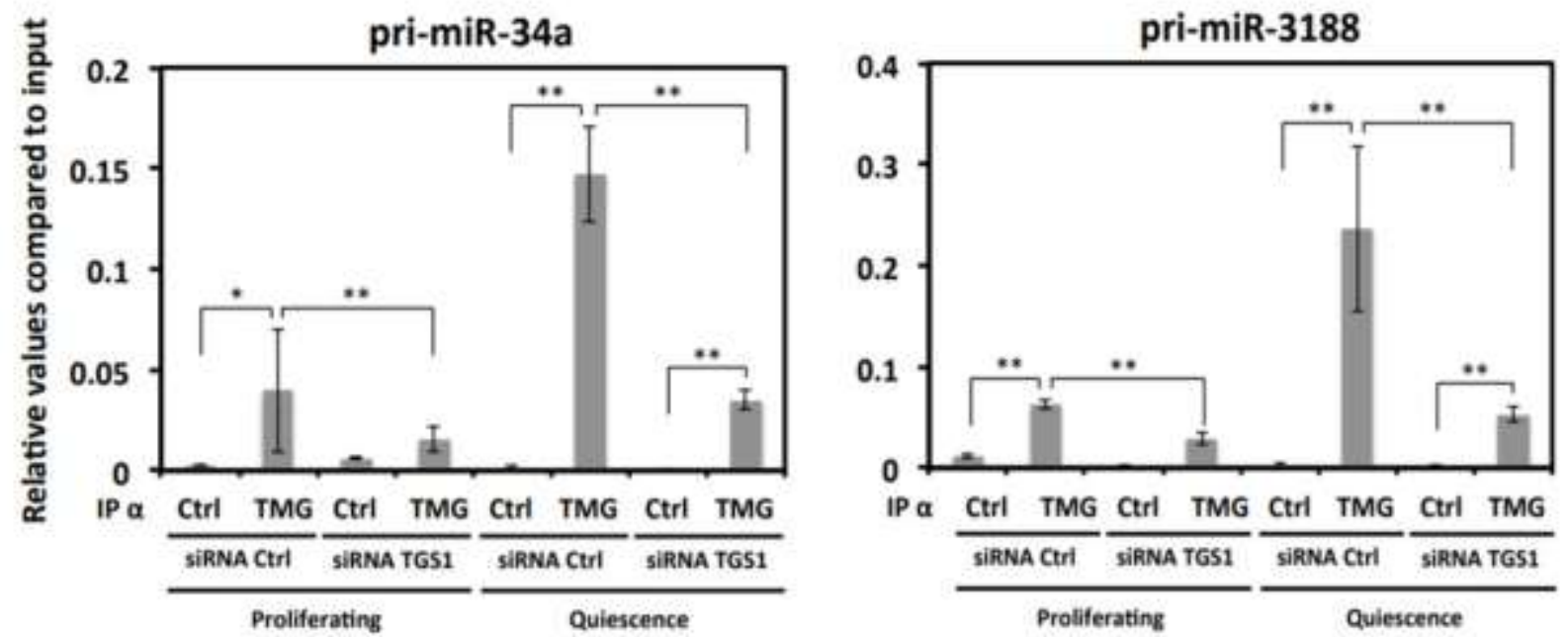

Figure S8. Quiescence-induced primary miRNAs are trimethylguanosine (TMG)-capped by TGS1. qRTPCR of anti-TMG pull-downs as in Figure 4A to quantify levels of miR-34a and miR-3188. Relative expression was compared to input RNA and normalized against a spike-in of non-endogenous RNA. Twotailed t-test results are indicated by $*$ for $\mathrm{P}<0.05$ and $* *$ for $\mathrm{P}<0.01$. 
Figure S9
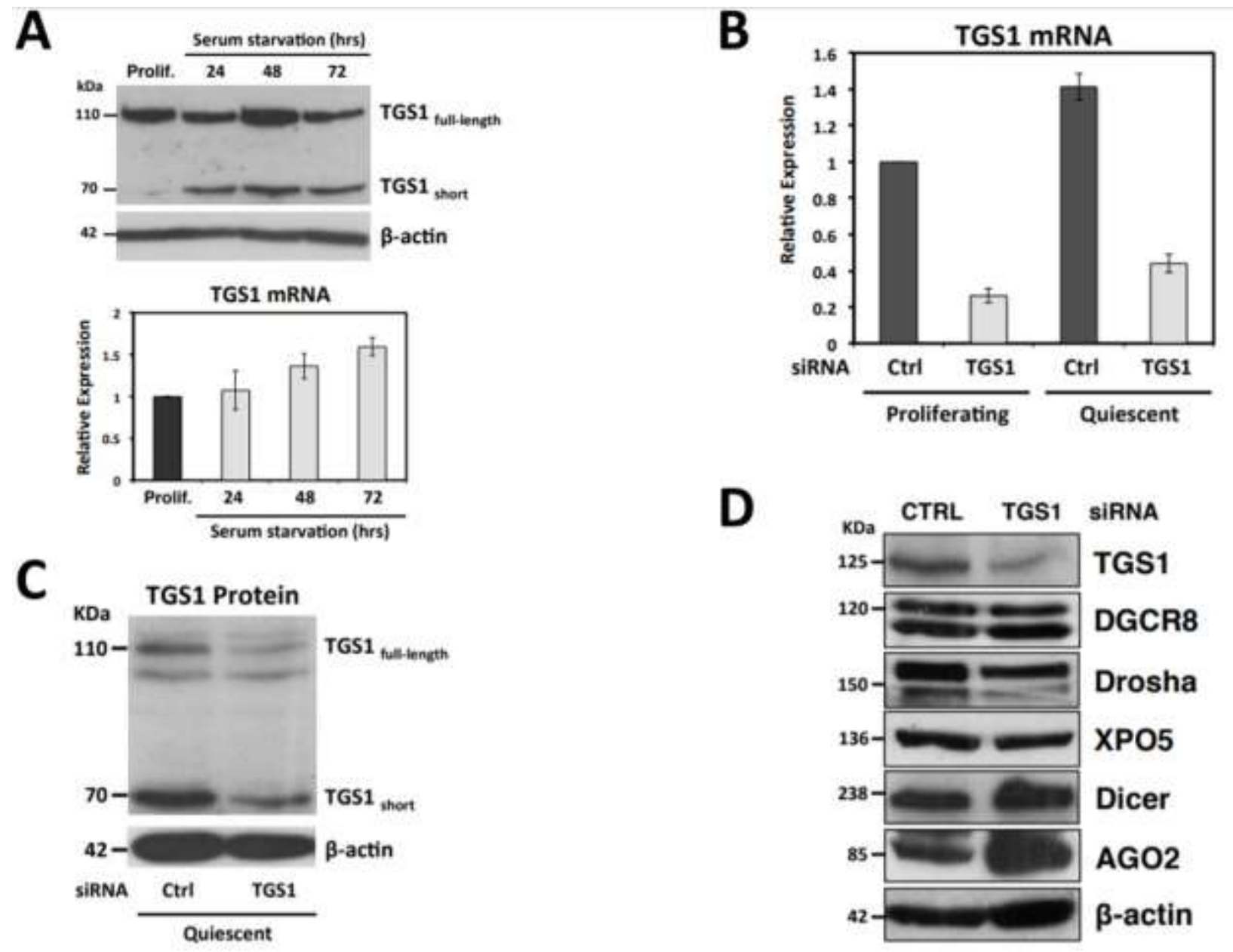

Figure S9. Analysis of TGS1 protein knock-down in proliferating and quiescent HFFs. (A) TGS1 expression increases during quiescence. Western blot (top panel) showing increased expression of the short isoform of TGS1 protein and qRT-PCR analysis (bottom panel) showing increased expression of total TGS1 mRNA in HFFs after serum starvation. (B) qRT-PCR analysis showing relative expression of TGS1 mRNA in proliferating and quiescent HFFs transfected with an siRNA scrambled control (dark grey bars) or an siRNA targeting TGS1 mRNA (Ambion) (light grey bars). (C) Western blot showing partial knockdown of TGS1 by using a siRNA against TGS1 (Ambion) in quiescent HFFs. (D) Western blot showing repression of full-length TGS1 but not other proteins involved in miRNA biogenesis in proliferating HFF cells transfected with a siRNA pool against TGS1 (Santa Cruz). Similar results were obtained in two independent experiments. 
Figure S10

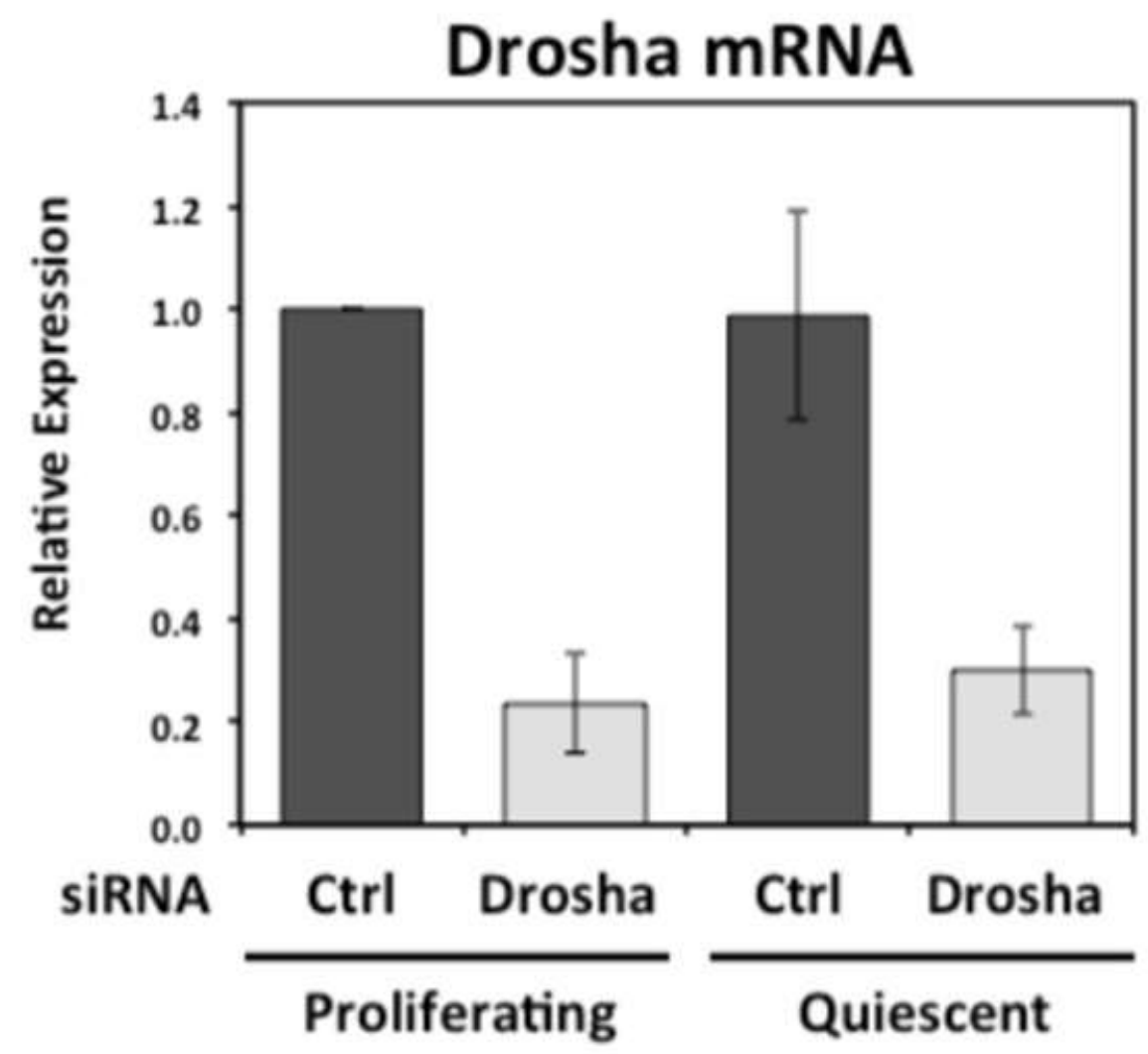

Figure S10. Analysis of Drosha knock-down in proliferating and quiescent HFFs. qRT-PCR analysis showing relative expression of Drosha mRNA in proliferating and quiescent HFFs transfected with siRNA scrambled control (dark grey bars) or siRNA pool targeting Drosha (Santa Cruz) (light grey bars). Cells were made quiescent by serum starvation for $48 \mathrm{hrs}$. Similar results were obtained in three independent experiments. The figure shows a representative experiment. 
Figure S11

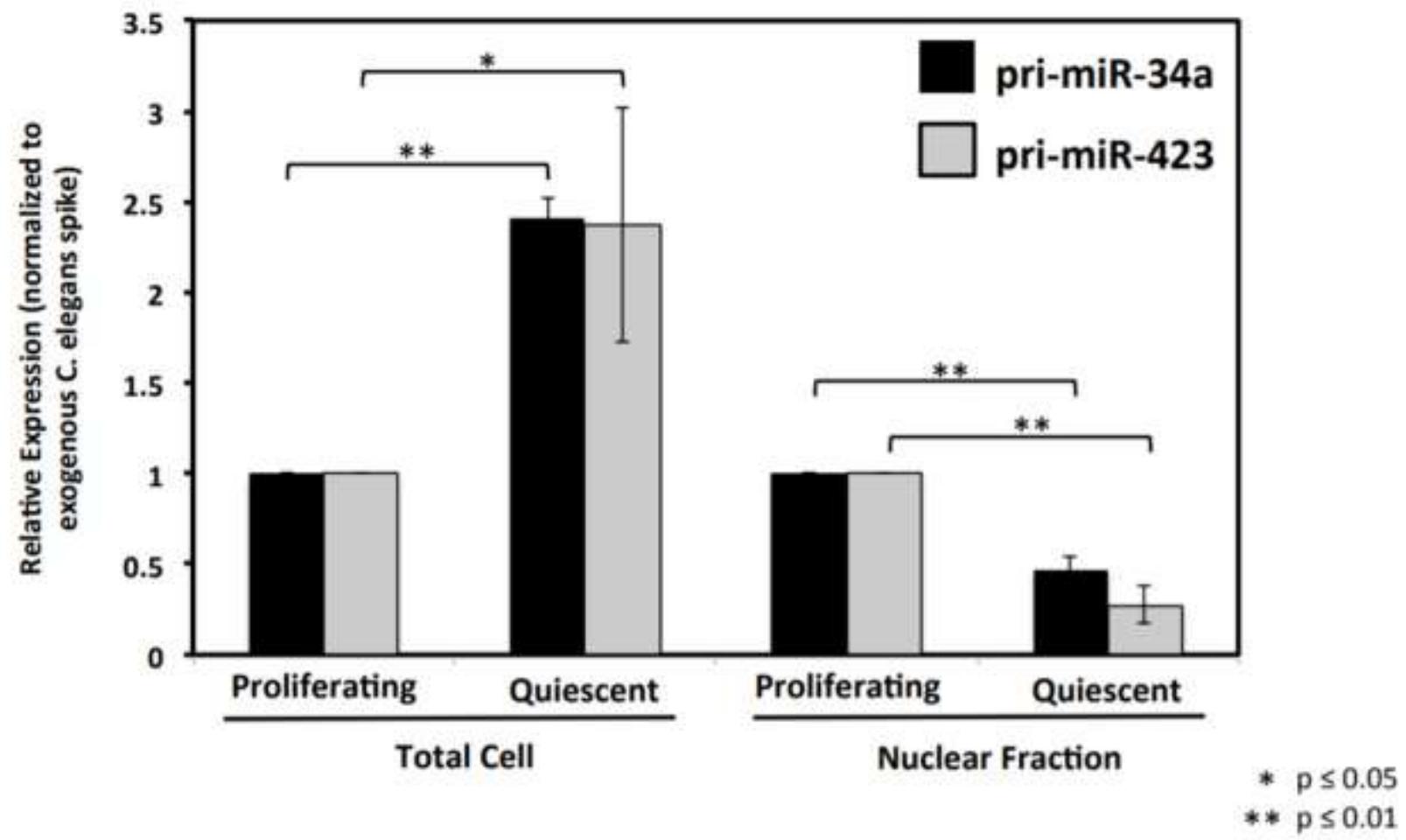

Figure S11. Comparison of pri-miRNAs present in total cell versus nuclear fraction. qRT-PCR analysis shows a significant reduction of pri-miR-34a and pri-miR-423 in nuclear fractions in comparison to total cell in quiescent HFF cells. Results show the average of three independent experiments. Two-tailed t-test results are indicated by $*$ for $\mathrm{P}<0.05$ and $* *$ for $\mathrm{P}<0.01$. 
Figure S12
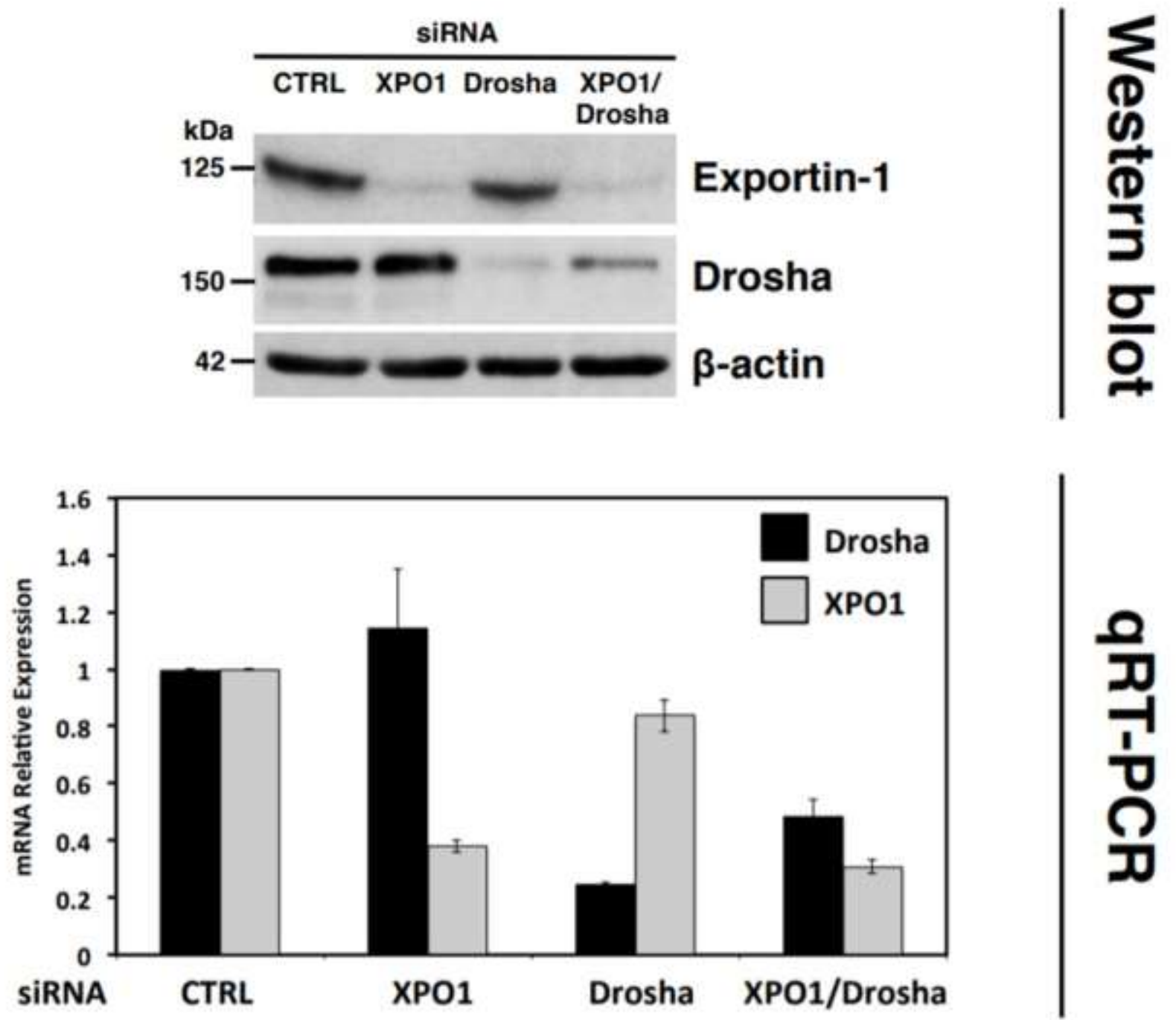

Figure S12. Verification of double knock-down of Drosha and XPO1. Knock-down of Drosha (Santa Cruz) and/or Exportin-1 (ID s14937, Ambion) was performed by using siRNAs against these proteins in proliferating HFFs and confirmed by Western blot analysis and qRT-PCR. siRNA control was added to XPO1 only and Drosha only siRNA transfections to equalize total siRNA amounts. Similar results were obtained in two independent experiments. 
Figure S13
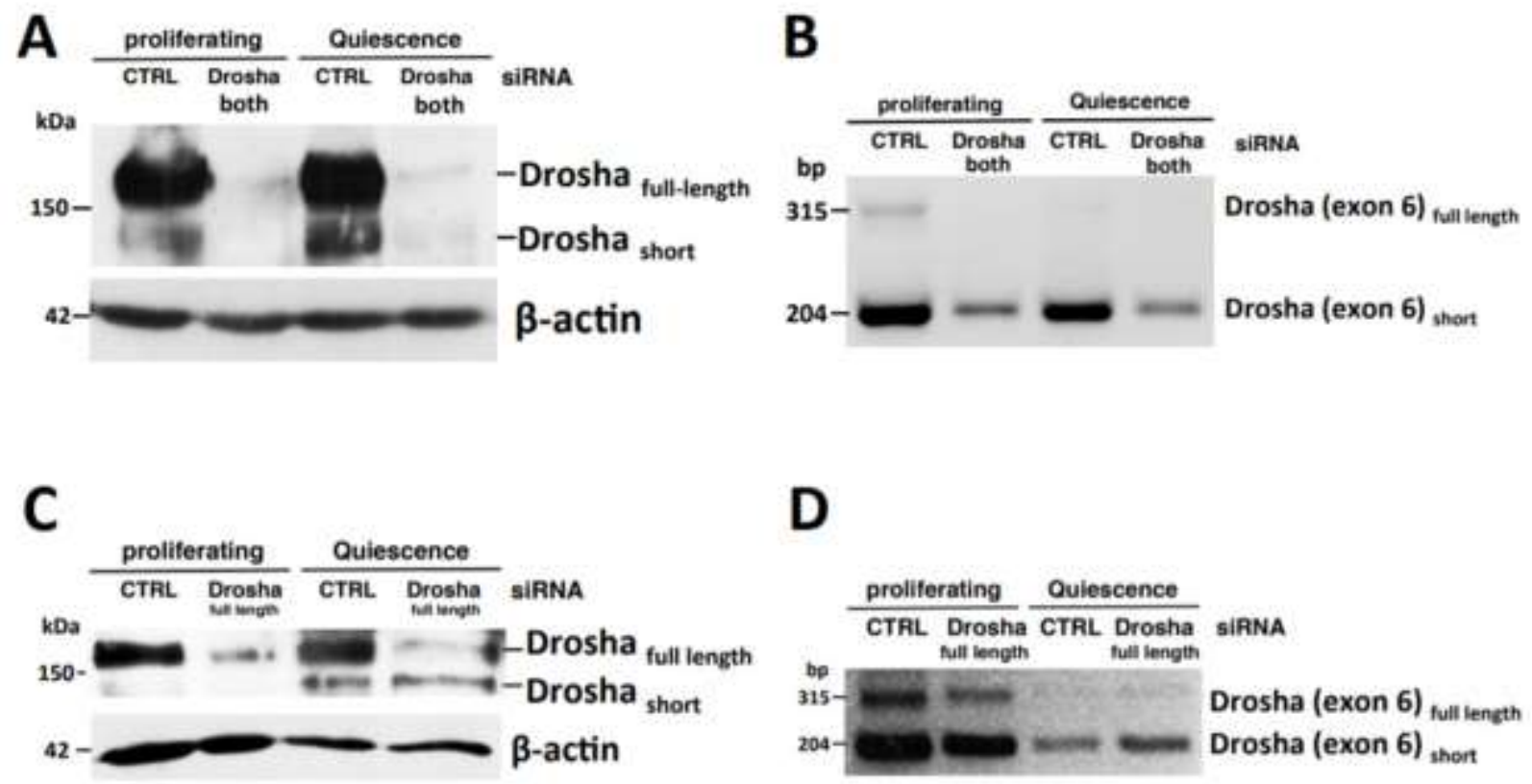

D
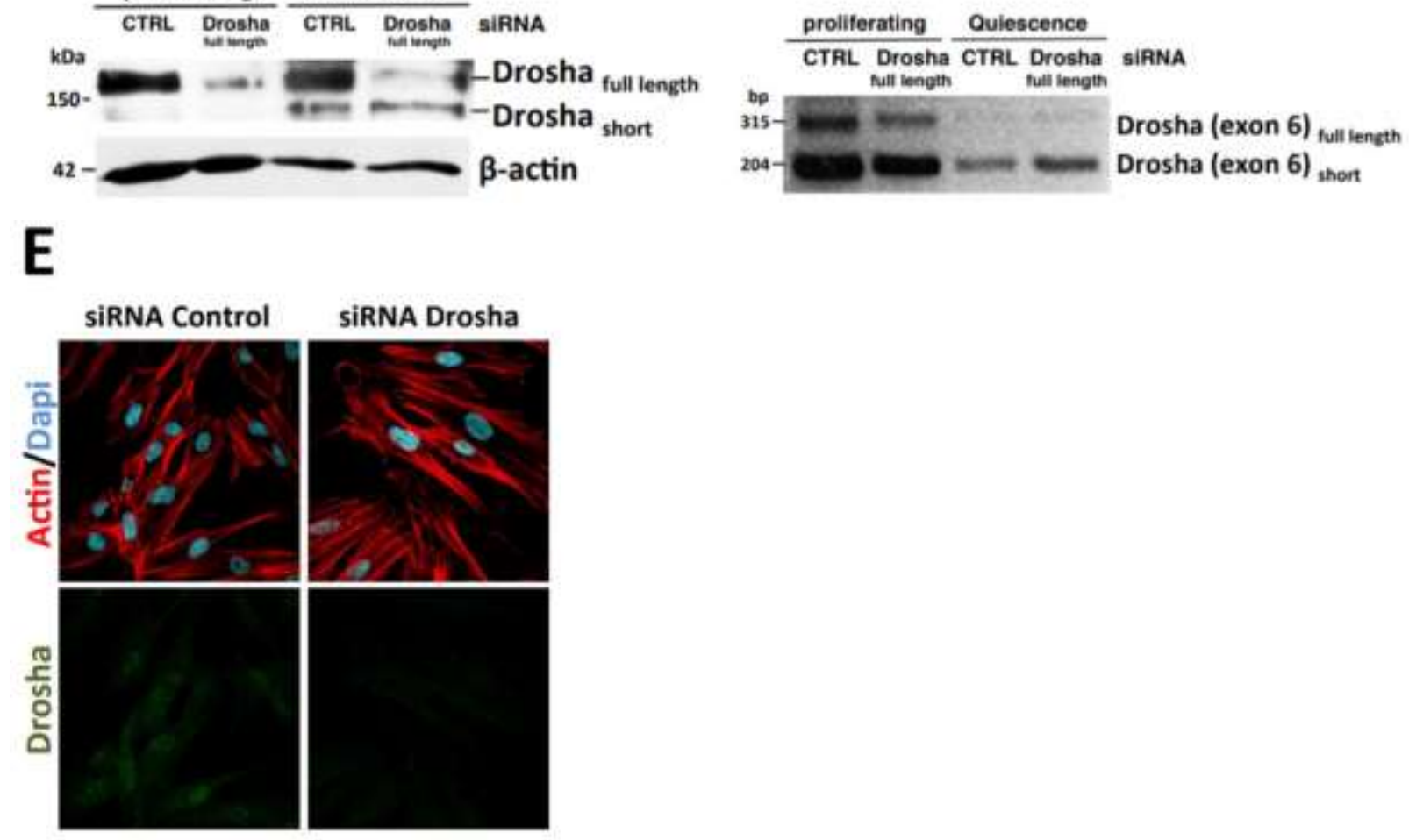

Figure S13. Transient knock-down of Drosha isoforms. (A) Western blot analysis of Drosha in proliferating and quiescent HFF cells $72 \mathrm{hrs}$ after knock-down of both Drosha isoforms by using a pool of siRNAs against different regions of Drosha mRNA (Drosha pool, Santa Cruz). (B) qRT-PCR of the exon 6 region of Drosha mRNA (204bp for the short Drosha and 315bp for the full length Drosha) after knock-down of both isoforms of Drosha. (C) Western blot analysis of Drosha in proliferating and quiescent HFF cells 72 hrs after selective knock-down of full length Drosha isoform only by using a siRNAs targeting exon 4 of Drosha mRNA (Drosha full length) that is absent (alternative splicing) from the short isoform of Drosha. (D) qRT-PCR of the exon 6 region of Drosha, as detailed in (B), in proliferating and quiescent HFF cells 72 hrs after specific knock-down of Drosha full length. (E) To verify specificity of Drosha antibody for immunofluorescence, confocal microscopy images were obtained using the same settings. HFF treated with siRNA Drosha (Drosha pool, Santa Cruz) demonstrate a loss of Drosha staining (pseudo-color green). Nuclear (DAPI, blue) and cytoskeleton (actin, red) immunofluorescent staining were used to visualize the cellular localization of Drosha. 


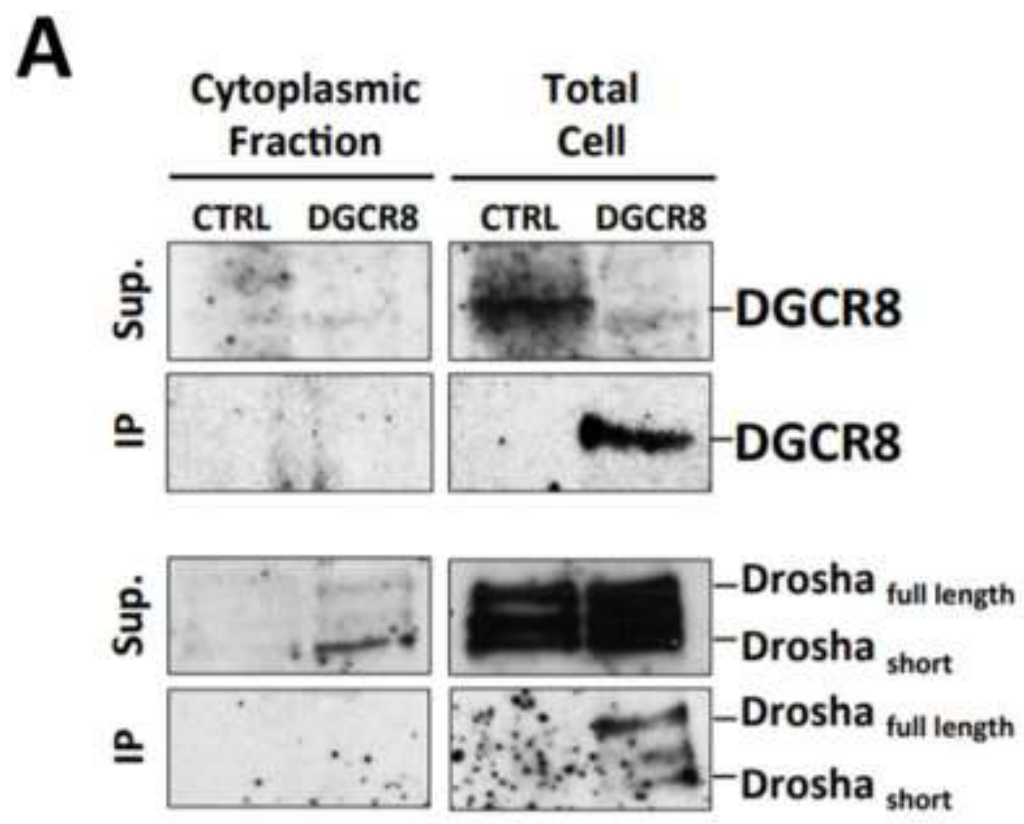

B
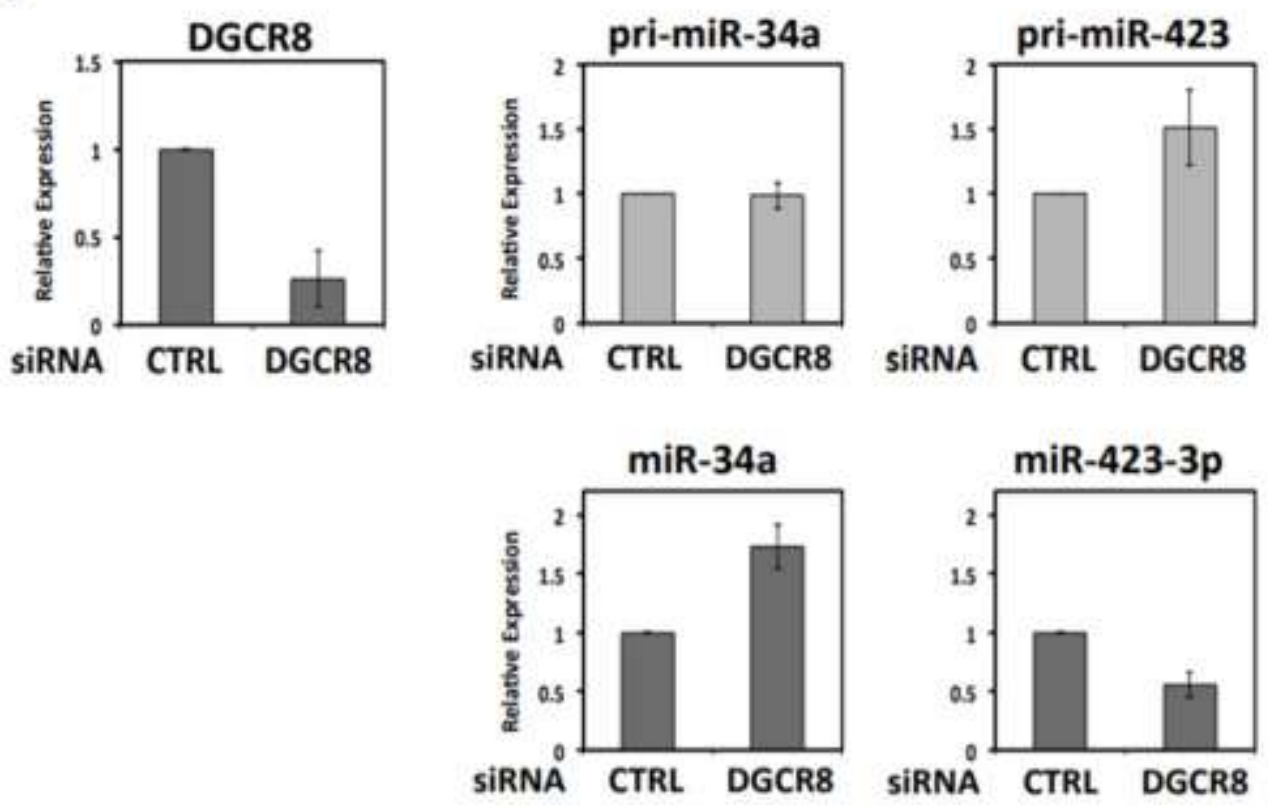

Figure S14. DGCR8 is not present in the cytoplasm of quiescent HFFs and the processing of quiescentinduced miR-34a is not affect by DGCR8 knock-down (A) DGCR8 is not interacting with the cytoplasmic isoform of Drosha. Immunoprecipitation (IP) of DGCR8 in quiescent HFFs shows absence of this protein in the cytoplasmic fraction and no pull-down of the small isoform of Drosha. DGCR8 polyclonal antibody (Proteintech) was used for the IP and rabbit IgG as a negative control (CTRL). Supernatant (Sup.) is also shown as an IP control. Similar results were obtained in two independent experiments. (B) miR-34a processing is not affected by DGCR8 knock-down in quiescent HFFs. Knock-down of DGCR8 was performed by using siRNAs against these proteins in quiescent HFFs and confirmed by qRT-PCR. siRNA scramble was used as a control (CTRL). Similar results were obtained in two independent experiments. 


\section{Figure S15}
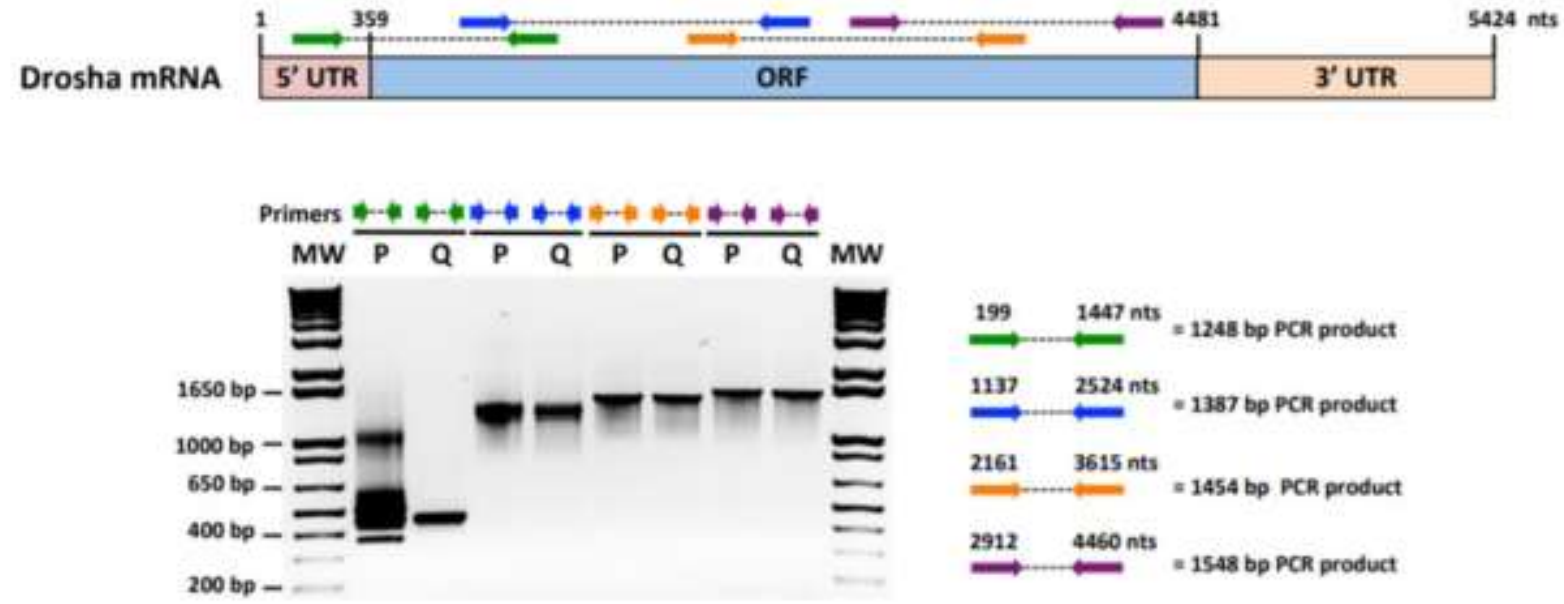

Figure S15. Detection of a short isoform of Drosha in quiescent HFF cells. Tiling RT-PCR of The Drosha mRNA open reading frame (ORF). Colored arrows represent different pairs of primers at different positions in Drosha mRNA with the predicted sizes of the wild-type amplification products shown. $P$ indicates proliferating HFF cells, Q indicates quiescent HFF cells, and MW indicates molecular weight ladder. Similar results were obtained in three independent experiments. 

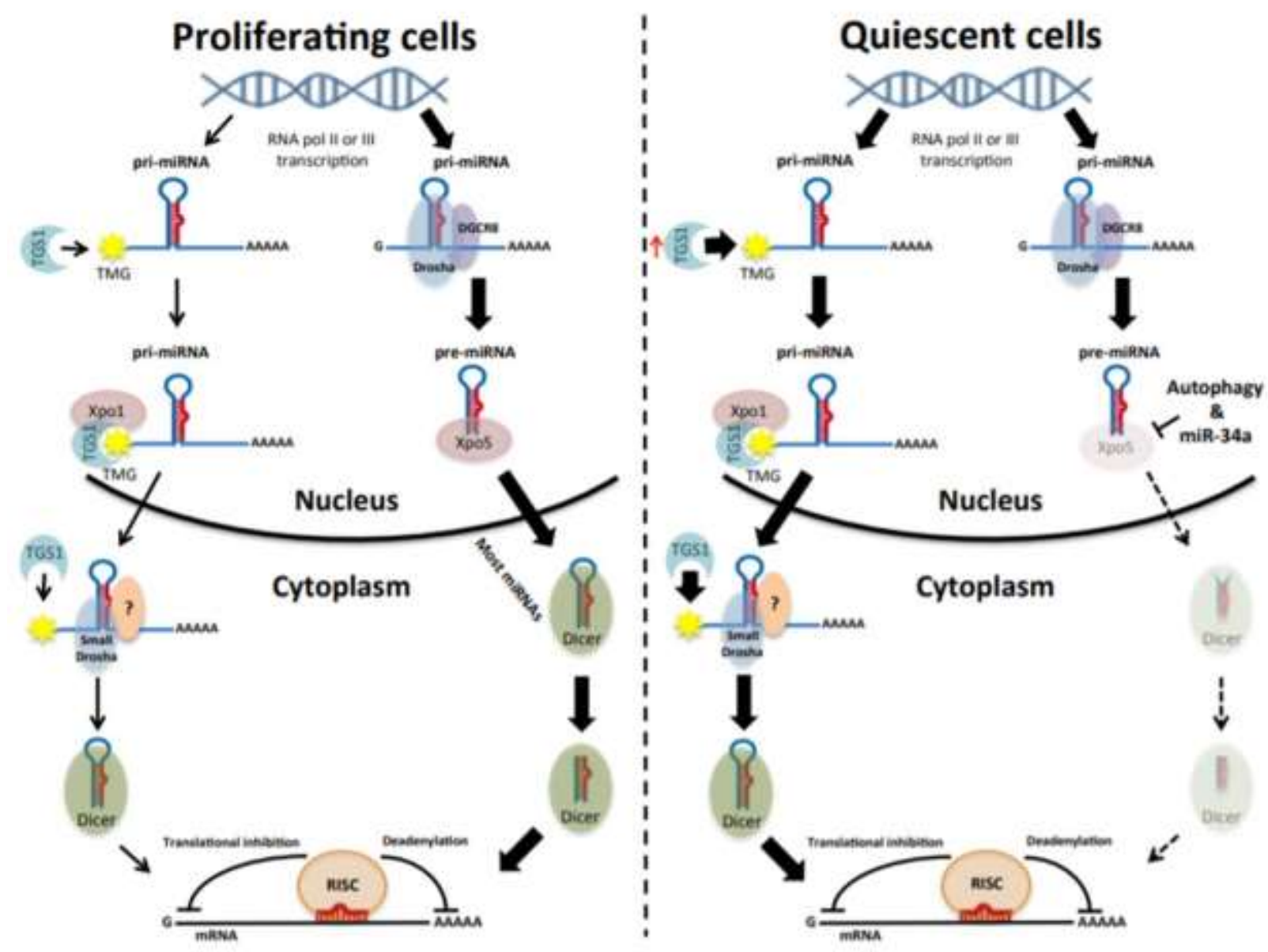

Figure S16. Model showing an alternative miRNA biogenesis pathway of (TMG)-capped pri-miRNAs. In proliferating cells, most miRNAs are processed through the canonical Exportin-5-dependent miRNA biogenesis pathway, but the $\left(\mathrm{m}^{7} \mathrm{G}\right)$-cap of a small group of pri-miRNAs is hypermethylated by TGS1 to form a (2,2,7-TMG)-cap, allowing recognition by Exportin-1. In quiescent cells, markedly reduced expression of Exportin-5, due to autophagy and miR-34a-mediated repression, limits the processing of most miRNAs, but increased expression of TGS1 supports the production of (TMG)-capped pri-miRNAs, which can be exported by Exportin-1 to the cytoplasm. A short form of Drosha in the cytoplasm (possibly exported from the nucleus by Exportin-1) processes the (TMG)-capped pri-miRNAs. 


\section{$\underline{\text { XII. Acknowledgements }}$}

We thank Demian Cazalla, Brian Adams, Kasandra Riley, Antonio Giraldez, Anne Edwards, Sara Marlatt, and Jan Zulkeski at Yale University for advice and assistance. We also thank Dr. Arjumand Ghazi of the University of Pittsburgh, and Dr. Frank Slack of Harvard University, for their support with reagents. Microarray processing was carried out in the Yale Center for Genome Analysis (YCGA). Imaging experiments and image analysis were performed in the West Virginia University (WVU) Microscope Imaging Facility, supported by the WVU Cancer Institute and NIH grants P20 RR016440, P30 GM103488, and P20 GM103434. I.M., J.B., and K.H. were supported in part by a WVU Foundation Fund (2V882). K.H. was supported in part by funding from the Ladies Auxillary to the VFW of the United States (CK 003229). S.I.A.B. and S.V. were supported by Massachusetts General Hospital startup funds. M.X. is supported by NIH grant R00 CA190886. J.A.S. is an Investigator of the Howard Hughes Medical Institute. This work was supported by Program Project Grant CA016038 from the National Cancer Institute (D.D. and J.A.S.).

\section{Supplementary Materials and Methods}

\section{A. Cell culture and reagents}

Normal primary human foreskin fibroblasts (HFFs, obtained from the Yale Skin Disease Research Center), HeLa/E6-5K, 293T, and C127 cells were cultured in DMEM (Sigma-Aldrich, St. Louis, MO) supplemented with $10 \%$ fetal bovine serum (FBS), L-glutamine, $10 \mathrm{mM}$ Hepes pH 7.3, penicillinstreptomycin, and amphotericin B (DMEM-10) at $37^{\circ} \mathrm{C}$ in the presence of $5 \% \mathrm{CO}_{2}$.

\section{B. Quiescence induced by serum starvation and confluency}

HFFs, HeLa, and C127 cells were rinsed with Dulbecco's phosphate buffered saline (DPBS, Life Technologies, Grand Island, NY), detached using minimal amounts of $0.25 \%$ trypsin-EDTA (Life Technologies) and plated in serum-free DMEM supplemented with MEM non-essential amino acids at low density (approx. $1 \times 10^{6}$ cells $/ 150 \mathrm{~cm}^{2}$ flask). After various times at $37^{\circ} \mathrm{C}$, cells were collected by scraping, pelleted by centrifugation, and rinsed twice with phosphate buffered saline (PBS, Sigma). For proliferating samples ( 0 h), HFFs, HeLa, and C127 cells were plated in DMEM supplemented with 10\% FBS and MEM non-essential amino acids at a density of approximately $1.5 \times 10^{6}$ cells $/ 100 \mathrm{~mm}$ plate, cultured for $24-48 \mathrm{~h}$, scraped, and pelleted. For quiescence induced by confluency, we plated HFFs ( 2 X $10^{6}$ cells $/ 100 \mathrm{~mm}$ plate) 
in DMEM-10. After cells reached 100\% confluency (day 0), DMEM-10 was replaced every second day. Cells were scraped and pelleted by centrifugation at days 1, 6, and 12. Proliferating HFFs (Mock, day 0) were plated at $1 \times 10^{6}$ cells $/ 100 \mathrm{~mm}$ plate in DMEM-10 and harvested at approximately $80 \%$ confluency.

\section{Immunoprecipitation of tri-methylated capped RNA}

Proliferating HFFs ( 2 X $10^{6}$ cells/100 mm plate) were transiently transfected with 500 pmol siRNA TGS1 (ID S14937, Ambion) or siRNA negative control \#1 (Ambion) using Lipofectamine RNAiMAX (Invitrogen) and incubated at $37^{\circ} \mathrm{C}$ for $24 \mathrm{~h}$ in antibiotic-free DMEM-10. Cells were then trypsinized, plated and incubated for $72 \mathrm{~h}$ in serum-free, antibiotic-free DMEM supplemented with MEM non-essential amino acids to induce quiescence or in the same medium supplemented with $10 \%$ FBS for $24 \mathrm{~h}$ to maintain proliferation. RNA was extracted from pelleted cells using Trizol Reagent (Life Technologies) per manufacturer's instructions and treated with Turbo DNAfree DNase (Ambion) for $20 \mathrm{~m}$ at $37^{\circ} \mathrm{C}$. RNA concentration was determined with a Nanodrop 2000 Spectrophotometer (Thermo Scientific, Wilmington, DE). $1 \mu \mathrm{L}$ of RNasin Plus RNase inhibitor (Promega, Madison, WI) was added to $10 \mu \mathrm{g}$ of RNA and incubated for $3-5 \mathrm{~m}$ at $85^{\circ} \mathrm{C}$; an additional $1 \mu \mathrm{L}$ of RNase inhibitor was added after heating. RNA diluted in NET-2 buffer (150 mM NaCl; $50 \mathrm{mM}$ Tris, $\mathrm{pH} 7.4$; and 0.05\% NP-40) was incubated for $2 \mathrm{~h}$ at $4^{\circ} \mathrm{C}$ with $40 \mu \mathrm{L} / 10 \mu \mathrm{g}$ RNA of Protein G Sepharose 4 Fast Flow beads (GE Healthcare, Buckinghamshire, UK). The beads were then removed by centrifugation. Pre-cleared RNA was rotated overnight at $4^{\circ} \mathrm{C}$ with $40 \mu \mathrm{L}$ Protein G Sepharose 4 Fast Flow beads loaded with $15 \mu \mathrm{L}$ normal rabbit serum (control, EMD-Millipore, Billerica, MA) or $15 \mu \mathrm{L}$ rabbit m3G-cap antibody (Synaptic Systems, Goettingen, Germany). Beads were rinsed 5 times with NET-2 buffer and resuspended in G-50 buffer (20 mM Tris, pH 7.5; $300 \mathrm{mM} \mathrm{NaOAc}$; 2 mM EDTA; and $0.25 \%$ SDS). RNA was extracted from the beads by phenol-chloroform-isoamyl alcohol extraction and resuspended in $20 \mu \mathrm{L}$ of nuclease-free water. $1 \mu \mathrm{L}$ of GlycoBlue Coprecipitant (Ambion) was added to aid in visualization of the RNA pellet. Immunoprecipitated tri-methylated capped RNA was analyzed by RT-PCR and qRT-PCR for pri-miRNA. Relative expression was normalized using Solaris RNA spike kit (Thermo Scientific, Rockford, IL).

\section{Microarray analysis}

For global miRNA expression, we used the GeneChip® miRNA 2.0 Array (Affymetrix, Santa Clara, CA, USA) that assesses 1,105 mature human miRNAs. Microarray processing was carried out in the Yale Center for Genome Analysis (YCGA). Briefly, $1 \mu \mathrm{g}$ of total RNA was labeled using the Flashtag RNA labeling kit (Genisphere, Hatfield, PA, USA) according to the manufacturer's instructions. Each sample 
was hybridized to the array at $48^{\circ} \mathrm{C}$ and $60 \mathrm{rpm}$ for 16 hours then washed and stained on Fluidics Station 450 (Fluidics script FS450_0003) and finally scanned on a GeneChip® Scanner 3000 7G (Affymetrix, Santa Clara, CA, USA). Feature intensities were extracted by using miRNA 2.0 array library files and analyzed with the miRNA QC Tool software. Array data were deposited into ArrayExpress: E-MTAB2760.

\section{E. Quantitative RT-PCR}

Total RNA from HFFs was extracted using Trizol Reagent (Life Technologies) per manufacture instructions and treated with Turbo DNAfree DNase (Ambion) for 20 minutes at $37^{\circ} \mathrm{C}$. RNA concentrations were determined with a Nanodrop 2000 Spectrophotometer (Thermo Scientific, Wilmington, DE). For mature miRNAs, $1 \mu \mathrm{g}$ of total RNA was reverse transcribed using the TaqMan ${ }^{\circledR}$ miRNA Reverse Transcription Kit (Life Technologies) according to the manufacturer's instructions. For qRT-PCR of individual mature microRNAs, we used specific TaqMan® miRNA Assays (Life Technologies) analyzed on a Real-Time thermocycler iQ5 (Bio-Rad, Hercules, CA) according to the manufacturer's protocol. To measure pre-miRNAs, pri-miRNAs and mRNA levels, total RNA was converted to cDNA using the iScript cDNA synthesis kit (Bio-Rad), followed by RT-PCR or qRT-PCR using the iQ SYBR green supermix (BioRad). Relative expression was calculated using the double delta CT method (relative expression $=2-\Delta \mathrm{CT}$; where $\Delta \mathrm{CT}=\mathrm{CT}$ (Target RNA) - CT (endogenous control RNA)), where the endogenous control for miRNAs was the small nucleolar RNA RNU43 and for pre-miRNAs, pri-miRNAs and mRNA was GAPDH. For qRT-PCR normalization by exogenous spike, we used C. elegans total RNA for the amplification of the worm-specific ama-1 gene. Primer sequences are shown in supplementary Table 4.

\section{F. Western blot analysis}

Proliferating or quiescent HFFs were lysed in NP-40 lysis buffer (50 mM Tris-HCl pH 8.0, 150 $\mathrm{mM} \mathrm{NaCl}, 1$ mM EDTA, $1 \%$ NP-40, 0.1\% SDS, 0.5 mM sodium metavanadate, Protease Inhibitor Cocktail (Sigma-Aldrich) for 12 minutes at $4^{\circ} \mathrm{C}$ and clarified by centrifugation at $4{ }^{\circ} \mathrm{C}(5 \mathrm{~min} ., 13,000 \mathrm{rpm})$. The concentration of extracted proteins was determined by using the Pierce BCA Protein Assay (Thermo Scientific, Rockford, IL). Cell lysates (15-30 $\mu \mathrm{g}$ of total cell protein) were separated by SDS-PAGE, transferred to Immobilon-Fl membranes (Millipore, Bedford, MA) and blocked with 5\% milk-TBST buffer (5\% nonfat dried milk, $25 \mathrm{mM}$ Tris- $\mathrm{HCl} \mathrm{pH} 8.0,125 \mathrm{mM} \mathrm{NaCl}$ and $0.5 \%$ Tween-20) for $1 \mathrm{~h}$ at room temperature. Membranes were incubated overnight at $4^{\circ} \mathrm{C}$ with primary antibodies, washed with TBST and incubated with appropriate horseradish peroxidase (HPR)-conjugated secondary antibodies in 5\% milk- 
TBST buffer for $1 \mathrm{~h}$ at room temperature [1:10.000; Pierce goat anti-mouse or Pierce donkey anti-rabbit (Thermo Scientific), anti-Rat IgG HRP-linked antibody (Cell Signaling), or donkey anti-goat (Santa Cruz Biotechnologies, Dallas, TX)]. After rinsing with TBST, proteins were detected using the Pierce SuperSignal West Pico Chemiluminescent Substrate or Pierce SuperSignal West Femto Maximum Sensitivity Substrate (Thermo Scientific, Rockford, IL). The following primary antibodies were used: goat anti-Actin (C-11, 1:5000, Santa Cruz Biotechnologies), rabbit anti-MDM2 (1:250, Santa Cruz Biotechnologies), rabbit anti-p27 (C-19, 1:1000, Santa Cruz Biotechnologies), rabbit anti-p62/SQSTM1 (NBP1-49954, 1:1000, Novus Biologicals, Littleton, CO), rabbit anti-TGS1 (1:1000, Proteintech), mouse anti-Exportin-1 (C-1, 1:1000, Santa Cruz Biotechnologies), mouse anti-Exportin-5 (1:1000, Abnova, Walnut, CA), goat anti-Exportin-5 (E-16, 1:1000, Santa Cruz Biotechnologies), rabbit anti-Drosha (1:1000 ab12286, Abcam, Cambridge, MA), rabbit anti-Drosha (1:1000 D28B1, Cell Signaling Technology, MA), rabbit anti-DGCR8 (1:1000 10996-1-AP, Proteintech), rabbit anti- $\beta$-Tubulin (1:1000 9F3, Cell Signaling, Danvers, MA), rat anti-AGO2 $(1 \mu \mathrm{g} / \mathrm{mL}$ clone 11A9, Sigma-Aldrich), rabbit anti-Dicer (1:1000, Cell Signaling), and rabbit anti-HDAC1 (1:1000 H52, SantaCruz Biotechnologies).

\section{G. Immunofluorescence and signal intensity quantitation}

HFFs were plated on poly-L-lysine-coated glass coverslips at $5 \times 10^{4}$ cells/coverslip in serum-free DMEM supplemented with MEM non-essential amino acids (quiescent) and at $2.5 \times 10^{4}$ cells/coverslip in DMEM supplemented with 10\% FBS and MEM non-essential amino acids (proliferating). Cells were rinsed with PBS and fixed with 10\% buffered formalin phosphate (Fisher Scientific, Fair Lawn, NJ) for 15 minutes at room temperature $24 \mathrm{~h}, 48 \mathrm{~h}$, and $72 \mathrm{~h}$ after plating; proliferating cells were fixed at $24 \mathrm{~h}$. Cells were permeabilized for 10 minutes at room temperature with $0.4 \%$ Triton X-100 (Ameresco, Solon, OH) in PBS and incubated with mouse anti-Exportin-5 (1:500) or rhodamine-conjugated phalloidin (F-actin, 1:500; Molecular Probes, Eugene, OR) in 5\% bovine serum albumin (BSA) in PBS for $1 \mathrm{~h}$ at room temperature. Immunostained cells were then incubated for one $\mathrm{h}$ at room temperature with Alexa Fluor 488-conjugated goat anti-mouse secondary antibodies (1:2000; Molecular Probes) in 5\% BSA in PBS. Cells were mounted on glass slides with ProLong Gold Anti-fade Reagent with Dapi (Life Technologies, Eugene, OR) to visualize nuclei. Zeiss LSM510 confocal microscope using AIM software (Carl Zeiss MicroImaging, Thornwood, NY, USA) was utilized to acquire fluorescent images. For immunofluorescence quantification, HFF cells were plated and cultured on poly-L-lysine coated coverslips (Neuvitro Corp., El Monte, CA) as described above. To diminish masking of cytoplasmic signal with nuclear staining, HFF cells were incubated in $3 \mu \mathrm{g} / \mathrm{mL}$ of Digitonin (Promega, Madison, WI) in Buffer B (20 mM Hepes, $110 \mathrm{mM}$ Potassium Acetate, $5 \mathrm{mM}$ Sodium Acetate, $2 \mathrm{mM}$ magnesium Acetate, $1 \mathrm{mM}$ 
EGTA and $2 \mathrm{mM}$ DTT) for $3 \mathrm{~min}$ to selectively permeabilize only the plasma membrane (http://www.nature.com/protocolexchange/protocols/1994\#/figures). After fixation with 3.7\% formaldehyde in PBS, HFF cells were blocked with 5\% BSA in PBS for $1 \mathrm{~h}$ at room temperature and immunolabeled as described above with rabbit anti-Drosha (D28131; 1:500; Cell Signaling) and Rhodamine-Phalloidin and mounted on glass slides with ProLong Gold Anti-fade Reagent with Dapi (Life Technologies). Confocal microscopy was used to visualize cells; fluorescent images were converted to TIF Files and localization of Drosha in the cytoplasm was quantitated utilizing Image $\mathbf{J}$ (http://www.macbiophotonics.ca/imagej/). In short, the area (outline) of the entire cell and nucleus were determined using F-actin and Dapi images, respectively, by the "Analyze/Analyze Particle" command after the "Threshold" was set for the images. "Nuclear" Drosha and "Total Cell" Drosha were measured by total pixel area from threshold Drosha image contained in the "outline" of the cell and nucleus (identified regions of interests, ROIs, obtained from F-actin and Dapi images). Quantification of "cytoplasmic" Drosha was generated by the subtraction of "Nuclear" Drosha due to partial permeabilization of the nuclear fraction with digitonin from "Total Cell” Drosha. Results were normalized to proliferating HFF cells; experiments were performed in triplicate.

\section{$\underline{H . \text { Inhibition of proteasome activity and autophagy }}$}

HFFs plated the day before at $1.5 \times 10^{6}$ cells $/ 100 \mathrm{~mm}$ plate were pre-treated with $10 \mu \mathrm{M}$ MG132 (2-Leu-Leu-H Aldehyde, Peptide International, Louisville, KY) or vehicle (DMSO) for $2 \mathrm{~h}$ at $37^{\circ} \mathrm{C}$. Treated cells were trypsinized and plated at $1 \times 10^{6}$ cells/T150 flask in serum-free DMEM supplemented with MEM non-essential amino acids containing $10 \mu \mathrm{M}$ MG132 to induce quiescence. Cells were harvested by scraping at 3,6, and $12 \mathrm{~h}$ and pelleted. Alternatively, MG132-treated HFF cells were scraped and pelleted after pretreatment for $0 \mathrm{~h}$ time point (mock). Pelleted cells were lysed and analyzed by Western blotting. For the 3-MA treatment, HFFs were plated at $1.5 \times 10^{6}$ cells/100 mm plate in DMEM-10 and incubated overnight. Cells pre-treated with $10 \mathrm{mM}$ 3-methyladenine, 3-MA (Sigma-Aldrich) or vehicle (water) for $2 \mathrm{~h}$ were induced to quiescence by trypsinizing and plating at $0.9 \times 10^{6}$ cells/T150 flask and serum starved in the presence of $10 \mathrm{mM} 3 \mathrm{MA}$. Cells were scraped at 3,6, and $12 \mathrm{~h}$ and pelleted to collect. Additionally, Mock $(0 \mathrm{~h})$ was generated by pelleting 3MA-treated proliferating HFFs. Harvested cells were lysed with NP40 lysis buffer and analyzed by Western blotting.

\section{Transient transfection of siRNAs and mimicRNAs}


$75 \mu \mathrm{L}$ of Lipofectamine RNAiMAX (Invitrogen, Grand Island, NY) was added to 500 pmol (total) of siRNAs or mimicRNAs diluted in $2.5 \mathrm{~mL}$ of Opti-MEM I Reduced Serum Media (Gibco). To form complexes, Lipofectamine RNAiMAX/RNA solution was incubated for 20 minutes at room temperature then spread in a $100 \mathrm{~mm}$ plate. $2.0 \times 10^{6} \mathrm{HFFs}$ were added dropwise to the $100 \mathrm{~mm}$ plate containing complexes and incubated for $48 \mathrm{~h}$. siRNAs and mimicRNAs were preMir Negative Control \#1 (Ambion, Grand Island, NY), Silencer Select Negative Control \#1 (Ambion), Silencer Select Validated siRNA Exportin-1 (ID s14937, Ambion), Silencer Select Validated Exportin-1 (ID118206, Ambion), Silencer Select Predesigned siRNA TGS1 (ID s41313, Ambion), siGenome siRNA Exportin-5 (Thermo Scientific, Dharmacon), Silencer Select Validated Exportin-5 (IDs33191, Ambion), Silencer Select Validated RNASEN (Drosha long, IDs26491, Ambion), PIMT (TGS1) siRNA (280 pmol, sc-45875, Santa Cruz), and hsa-miR-34a (ID PM11030, Ambion). For cellular fractionation to determine localization of pri-miRNA in the cytoplasm; 280 pmol of a pool of 3 target-specific 19-25 nt siRNAs against Drosha (sc-44080, Santa Cruz) or siRNA Control was utilized as detailed above to knockdown Drosha in HFF cells.

\section{J. Transient transfection of plasmids}

$87 \mu \mathrm{L}$ of Lipofectamine 2000 (Invitrogen) was diluted in $1.2 \mathrm{~mL}$ of Opti-MEM I Reduced Serum Media (Gibco) and incubated at room temperature for 5 minutes. Separately, $11 \mu \mathrm{g}$ of plasmid was diluted in 1.2 $\mathrm{mL}$ of Opti-MEM I Reduced Serum Media. The Lipofectamine solution and the DNA/OptiMEM solution were mixed and incubated for 20 minutes at room temperature. The Lipofectamine/DNA complexes were added drop-wise to HFFs plated the previous day at a density of $1.5 \times 10^{6}$ cells $/ 100 \mathrm{~mm}$ plate in antibioticfree DMEM-10. Antibiotic-free DMEM 10 medium was changed after 4-6 h, and HFFs were incubated overnight at $37^{\circ} \mathrm{C}$.

\section{K. Retroviral infection of plasmids}

293 cells were transiently transfected with the retroviral plasmid pBABE-puro-Flag-XPO1 or pBABEpuro-Flag-vector and retroviral packaging plasmids (pCL-Eco and VSV-G) using calcium phosphate transfection. Retrovirus was collected after $48 \mathrm{hrs}$, filtered and supplemented with polybrene $(4 \mu \mathrm{g} / \mathrm{mL})$ to increase infection efficiency. HFF cells were exposed to retrovirus for $8 \mathrm{hrs}$ and allowed to recover for 48 hrs before use.

\section{Luciferase assays}


Luciferase plasmids pmirGlo dual-luciferase expression vector (Promega) and derivatives containing the wild-type or mutant Exportin-5 3'UTR (three point mutations in the binding sequence of the miRNA seed region) were transiently transfected into HFFs by using Lipofectamine 2000 as described above. After incubation for $24 \mathrm{~h}$, transfected cells were trypsinized and plated at a density of $5.0 \times 10^{4}$ cells/well in 6well plates in antibiotic-free, serum-free DMEM medium supplemented with MEM non-essential amino acids (for quiescent cells) and $1.0 \times 10^{5}$ cells/well in antibiotic-free, DMEM supplemented with $10 \%$ FBS and MEM non-essential amino acids (for proliferating cells) and incubated for $48 \mathrm{~h}$. Luciferase activity was analyzed by using the Dual-Luciferase Reporter Assay System (Promega) per manufacturer's protocol. In short, cells were lysed with Passive Lysis Buffer $(250 \mu \mathrm{L}$ for quiescent cells and $500 \mu \mathrm{L}$ for proliferating cells) by rapid shaking for 30 minutes at room temperature and aliquoted $(20 \mu \mathrm{L}$ for proliferating cells, 80 $\mu \mathrm{L}$ for quiescent cells) into 96 well Costar white plate (Corning Incorporated, Corning, NY). Luminescence was measured by using a Modulus II Microplate Luminometer with dual injector system (Turner BioSystems, Sunnyvale, CA). All transfections and luciferase assays were done in triplicate. For microRNA and anti-miR transfections, we transiently transfected $7 \times 10^{4}$ cells/well in a 24 well plate with 17 pmol pmiR Negative Control \#1 (Ambion) or hsa-miR-34a (PM11030, Ambion) diluted in $87 \mu \mathrm{L}$ OptiMEM I Reduced Serum Medium mixed with Lipofectamine RNAiMAX (Life Technologies) as described above. Transfected cells were incubated overnight and then transiently transfected with pmirGlo dual-luciferase expression vector, Exportin-5 3'UTR WT, and Exportin-5 3'UTR MT (0.5 $\mu \mathrm{g} /$ well) using Lipofectamine $2000(1 \mu \mathrm{L} /$ well $)$ as described above. Cells were then incubated $48 \mathrm{~h}$ in antibiotic-free DMEM supplemented with 10\% FBS and non-essential amino acids and luciferase assays were performed. Firefly luciferase activity was measured, normalized to Renilla luciferase activity and converted to Relative luciferase units (RLUs). Data average ratios were obtained in each experiment from three replicates; the graph shows the average of two individual experiments with a standard deviation shown as error bars. Twotailed t-test results are indicated by * for $\mathrm{P}<0.05$ and $* *$ for $\mathrm{P}<0.01$.

\section{Cellular Fractionation}

Proliferating and quiescent HFF cells were pelleted and immediately processed for cell fractionation to extract either protein or RNA (protocol adapted from Wang, Y. et. al. 2006) (63). Pelleted cells were resuspended in RSB buffer (10 mM Tris, pH 7.5; $0.3 \mathrm{mM}$ sodium chloride; $0.1 \mathrm{mM}$ magnesium chloride), incubated on ice for $5 \mathrm{~min}$, and centrifuged at $1500 \mathrm{rpm}$ for $4 \mathrm{~min}$ to re-pellet. Swollen pellets were resuspended in a volume of RSBG40 buffer (10 mM Tris, $\mathrm{pH} 7.5 ; 0.3 \mathrm{mM}$ sodium chloride; $0.1 \mathrm{mM}$ magnesium chloride; $10 \%$ glycerol; 0.5\% NP-40; $100 \mathrm{U} / \mathrm{mL}$ RNasin Plus RNase inhibitor (Promega, Madison, WI) and 0.5 mM DTT) at least 4 times the volume of the pellet. Cells were disrupted using an 
autoclaved Dounce homogenizer; homogenate was centrifuged at $4000 \mathrm{rpm}$ for $4 \mathrm{~min}$ to pellet the nuclear fraction. The cytoplasmic fraction (supernatant) was collected. The pelleted nuclear fraction was resuspended in RSB-G40; $3.3 \%$ of sodium deoxycholate and $6.6 \%$ of Tween 20 was added to $1 / 10^{\text {th }}$ of the volume of RSB-G40. Nuclei were re-pelleted by centrifugation at $7000 \mathrm{rpm}$ for three min after five min incubation on ice. The supernatant was collected and added to the cytoplasmic fraction, which was then subjected to Western blot protein analysis, or RNA was extracted using Trizol Reagent (Life Technologies) per manufacturer's instructions and treated with Turbo DNAfree DNase (Ambion) for 20 minutes at $37^{\circ} \mathrm{C}$. RNA concentrations were obtained using a Nanodrop 2000 Spectrophotometer (Thermo Scientific, Wilmington, DE). The nuclear pellet was washed with RSB-G40 and resuspended in NP-40 lysis buffer for protein analysis by Western blot or Trizol for RNA extraction and RT-PCR analysis.

\section{N. Immunoprecipitation of Exportin-1}

Immunoprecipitation protocol was adapted from Niranjanakumari S et al. 2002; Vasudevan S and Steitz JA. 2007; and Vasudevan S et al. 2007 (38, 64, 65). Quiescence was induced in HFF cells expressing pBABE-puro-Flag-XPO1 or pBABE-puro-Flag-vector pFlag by serum-starvation (48 hrs). Quiescent HFFs were crosslinked with $1 \%$ formaldehyde in PBS for $10 \mathrm{~min}$ at RT on a shaker. Glycine was added to a final concentration of $0.125 \mathrm{M}$ to quench the reaction for $5 \mathrm{~min}$ at RT, shaking occasionally. HFF cells were washed with PBS, scraped and pelleted by centrifugation. Cell pellets lysed with RIPA buffer (50mM tris, pH 7.5, 1\% NP-40, 0.5\% sodium deoxycholate, 0.05\% SDS, 1mM EDTA, 150mM NaCl), and sonicated. Pre-washed anti-Flag M2 Magnetic Bead (20 $\mu \mathrm{L}$, Sigma-Aldrich) were incubated with 275-300 $\mu \mathrm{g}$ of lysates, RNasin Plus RNase Inhibitor (Promega), and $100 \mu \mathrm{g} / \mathrm{mL}$ tRNA (Sigma-Aldrich) while rotating for approximately $4 \mathrm{hrs}$ at $4^{\circ} \mathrm{C}$. Beads were washed 6 times with RIPA buffer. RNA/beads were un-crosslinked; briefly, beads were resuspended in 6M urea, 50mM Tris, $\mathrm{pH} 6.8$, and 3mM DTT and incubated for $45 \mathrm{~min}$ at $60^{\circ} \mathrm{C}$. Proteinase $\mathrm{K}$ buffer $(50 \mathrm{mM}$ Tris-HCL, $\mathrm{pH} 8.0,10 \mathrm{mM} \mathrm{CaCl} 2)$ was added to dilute urea and 75 $\mu \mathrm{g} / \mathrm{mL}$ Proteinase K (Fisher Scientific) was added to digest protein. Samples were incubated for $1 \mathrm{hr}$ at $45^{\circ}$ $\mathrm{C}$, pelleted and supernatant was collected. RNA was extracted with TRIzol Reagent (Invitrogen) per manufacturer's instructions; GlycoBlue Coprecipitant (Life Technologies) was added.

\section{$\underline{\text { XIV. Supplemental References }}$}

1. Huber J, Cronshagen U, Kadokura M, Marshallsay C, Wada T, Sekine M, Luhrmann R. 1998. Snurportin1, an m3G-cap-specific nuclear import receptor with a novel domain structure. Embo j 17:4114-4126.

2. Pardee AB. 1974. A restriction point for control of normal animal cell proliferation. Proc Natl Acad Sci U S A 71:1286-1290. 
3. O'Farrell PH. 2011. Quiescence: early evolutionary origins and universality do not imply uniformity. Philos Trans R Soc Lond B Biol Sci 366:3498-3507.

4. Coller HA. 2011. Cell biology. The essence of quiescence. Science 334:1074-1075.

5. Valcourt JR, Lemons JM, Haley EM, Kojima M, Demuren OO, Coller HA. 2012. Staying alive: metabolic adaptations to quiescence. Cell Cycle 11:1680-1696.

6. Coller HA, Sang L, Roberts JM. 2006. A new description of cellular quiescence. PLoS Biol 4:e83.

7. Cheung TH, Rando TA. 2013. Molecular regulation of stem cell quiescence. Nat Rev Mol Cell Biol 14:329-340.

8. Bohnsack MT, Czaplinski K, Gorlich D. 2004. Exportin 5 is a RanGTP-dependent dsRNAbinding protein that mediates nuclear export of pre-miRNAs. Rna 10:185-191.

9. Krol J, Loedige I, Filipowicz W. 2010. The widespread regulation of microRNA biogenesis, function and decay. Nat Rev Genet 11:597-610.

10. James MA, Lee JH, Klingelhutz AJ. 2006. HPV16-E6 associated hTERT promoter acetylation is E6AP dependent, increased in later passage cells and enhanced by loss of p300. Int J Cancer 119:1878-1885.

11. Lee Y, Ahn C, Han J, Choi H, Kim J, Yim J, Lee J, Provost P, Radmark O, Kim S, Kim VN. 2003. The nuclear RNase III Drosha initiates microRNA processing. Nature 425:415-419.

12. Gregory RI, Yan KP, Amuthan G, Chendrimada T, Doratotaj B, Cooch N, Shiekhattar R. 2004. The Microprocessor complex mediates the genesis of microRNAs. Nature 432:235-240.

13. Denli AM, Tops BB, Plasterk RH, Ketting RF, Hannon GJ. 2004. Processing of primary microRNAs by the Microprocessor complex. Nature 432:231-235.

14. Yi R, Qin Y, Macara IG, Cullen BR. 2003. Exportin-5 mediates the nuclear export of premicroRNAs and short hairpin RNAs. Genes Dev 17:3011-3016.

15. Hutvagner G, McLachlan J, Pasquinelli AE, Balint E, Tuschl T, Zamore PD. 2001. A cellular function for the RNA-interference enzyme Dicer in the maturation of the let-7 small temporal RNA. Science 293:834-838.

16. Grishok A, Pasquinelli AE, Conte D, Li N, Parrish S, Ha I, Baillie DL, Fire A, Ruvkun G, Mello CC. 2001. Genes and mechanisms related to RNA interference regulate expression of the small temporal RNAs that control C. elegans developmental timing. Cell 106:23-34.

17. Knight SW, Bass BL. 2001. A role for the RNase III enzyme DCR-1 in RNA interference and germ line development in Caenorhabditis elegans. Science 293:2269-2271.

18. Han J, Lee Y, Yeom KH, Kim YK, Jin H, Kim VN. 2004. The Drosha-DGCR8 complex in primary microRNA processing. Genes Dev 18:3016-3027.

19. Lian SL, Li S, Abadal GX, Pauley BA, Fritzler MJ, Chan EK. 2009. The C-terminal half of human Ago2 binds to multiple GW-rich regions of GW182 and requires GW182 to mediate silencing. RNA 15:804-813.

20. Hammond SM, Boettcher S, Caudy AA, Kobayashi R, Hannon GJ. 2001. Argonaute2, a link between genetic and biochemical analyses of RNAi. Science 293:1146-1150.

21. Mourelatos Z, Dostie J, Paushkin S, Sharma A, Charroux B, Abel L, Rappsilber J, Mann M, Dreyfuss G. 2002. miRNPs: a novel class of ribonucleoproteins containing numerous microRNAs. Genes Dev 16:720-728.

22. Ruby JG, Jan CH, Bartel DP. 2007. Intronic microRNA precursors that bypass Drosha processing. Nature 448:83-86.

23. Cazalla D, Xie M, Steitz JA. 2011. A primate herpesvirus uses the integrator complex to generate viral microRNAs. Mol Cell 43:982-992.

24. Havens MA, Reich AA, Duelli DM, Hastings ML. 2012. Biogenesis of mammalian microRNAs by a non-canonical processing pathway. Nucleic Acids Res 40:4626-4640.

25. Cifuentes D, Xue H, Taylor DW, Patnode H, Mishima Y, Cheloufi S, Ma E, Mane S, Hannon GJ, Lawson ND, Wolfe SA, Giraldez AJ. 2010. A novel miRNA processing pathway independent of Dicer requires Argonaute2 catalytic activity. Science 328:1694-1698. 
26. Cheloufi S, Dos Santos CO, Chong MM, Hannon GJ. 2010. A dicer-independent miRNA biogenesis pathway that requires Ago catalysis. Nature 465:584-589.

27. Shapiro JS, Langlois RA, Pham AM, Tenoever BR. 2012. Evidence for a cytoplasmic microprocessor of pri-miRNAs. Rna 18:1338-1346.

28. Xie M, Li M, Vilborg A, Lee N, Shu MD, Yartseva V, Sestan N, Steitz JA. 2013. Mammalian 5'-capped microRNA precursors that generate a single microRNA. Cell 155:1568-1580.

29. Hutten S, Kehlenbach RH. 2007. CRM1-mediated nuclear export: to the pore and beyond. Trends Cell Biol 17:193-201.

30. Ohno M, Segref A, Bachi A, Wilm M, Mattaj IW. 2000. PHAX, a mediator of U snRNA nuclear export whose activity is regulated by phosphorylation. Cell 101:187-198.

31. Palacios I, Hetzer M, Adam SA, Mattaj IW. 1997. Nuclear import of U snRNPs requires importin beta. EMBO J 16:6783-6792.

32. Kiss T. 2004. Biogenesis of small nuclear RNPs. J Cell Sci 117:5949-5951.

33. Boulon S, Verheggen C, Jady BE, Girard C, Pescia C, Paul C, Ospina JK, Kiss T, Matera AG, Bordonne R, Bertrand E. 2004. PHAX and CRM1 are required sequentially to transport U3 snoRNA to nucleoli. Mol Cell 16:777-787.

34. Yedavalli VS, Jeang KT. 2010. Trimethylguanosine capping selectively promotes expression of Rev-dependent HIV-1 RNAs. Proc Natl Acad Sci U S A 107:14787-14792.

35. Medina R, Zaidi SK, Liu CG, Stein JL, van Wijnen AJ, Croce CM, Stein GS. 2008. MicroRNAs 221 and 222 bypass quiescence and compromise cell survival. Cancer Res 68:27732780 .

36. Suh EJ, Remillard MY, Legesse-Miller A, Johnson EL, Lemons JM, Chapman TR, Forman JJ, Kojima M, Silberman ES, Coller HA. 2012. A microRNA network regulates proliferative timing and extracellular matrix synthesis during cellular quiescence in fibroblasts. Genome Biol 13:R121.

37. Martinez I, Cazalla D, Almstead LL, Steitz JA, DiMaio D. 2011. miR-29 and miR-30 regulate B-Myb expression during cellular senescence. Proc Natl Acad Sci U S A 108:522-527.

38. Vasudevan S, Tong Y, Steitz JA. 2007. Switching from repression to activation: microRNAs can up-regulate translation. Science 318:1931-1934.

39. Iwasaki YW, Kiga K, Kayo H, Fukuda-Yuzawa Y, Weise J, Inada T, Tomita M, Ishihama Y, Fukao T. 2013. Global microRNA elevation by inducible Exportin 5 regulates cell cycle entry. RNA 19:490-497.

40. Link S, Grund SE, Diederichs S. 2016. Alternative splicing affects the subcellular localization of Drosha. Nucleic Acids Res 44:5330-5343.

41. Rissland OS, Hong SJ, Bartel DP. 2011. MicroRNA destabilization enables dynamic regulation of the miR-16 family in response to cell-cycle changes. Mol Cell 43:993-1004.

42. Bussing I, Yang JS, Lai EC, Grosshans H. 2010. The nuclear export receptor XPO-1 supports primary miRNA processing in C. elegans and Drosophila. EMBO J 29:1830-1839.

43. Castanotto D, Lingeman R, Riggs AD, Rossi JJ. 2009. CRM1 mediates nuclear-cytoplasmic shuttling of mature microRNAs. Proc Natl Acad Sci U S A 106:21655-21659.

44. Luhrmann R, Appel B, Bringmann P, Rinke J, Reuter R, Rothe S, Bald R. 1982. Isolation and characterization of rabbit anti-m3 2,2,7G antibodies. Nucleic Acids Res 10:7103-7113.

45. Girard C, Verheggen C, Neel H, Cammas A, Vagner S, Soret J, Bertrand E, Bordonne R. 2008. Characterization of a short isoform of human Tgs1 hypermethylase associating with small nucleolar ribonucleoprotein core proteins and produced by limited proteolytic processing. $\mathrm{J}$ Biol Chem 283:2060-2069.

46. Chong MM, Zhang G, Cheloufi S, Neubert TA, Hannon GJ, Littman DR. 2010. Canonical and alternate functions of the microRNA biogenesis machinery. Genes Dev 24:1951-1960.

47. Kawai S, Amano A. 2012. BRCA1 regulates microRNA biogenesis via the DROSHA microprocessor complex. J Cell Biol 197:201-208. 
48. Kim YK, Kim B, Kim VN. 2016. Re-evaluation of the roles of DROSHA, Export in 5, and DICER in microRNA biogenesis. Proc Natl Acad Sci U S A 113:E1881-1889.

49. Ha M, Kim VN. 2014. Regulation of microRNA biogenesis. Nat Rev Mol Cell Biol 15:509-524.

50. Legesse-Miller A, Raitman I, Haley EM, Liao A, Sun LL, Wang DJ, Krishnan N, Lemons JM, Suh EJ, Johnson EL, Lund BA, Coller HA. 2012. Quiescent fibroblasts are protected from proteasome inhibition-mediated toxicity. Mol Biol Cell 23:3566-3581.

51. Gibbings D, Mostowy S, Jay F, Schwab Y, Cossart P, Voinnet O. 2012. Selective autophagy degrades DICER and AGO2 and regulates miRNA activity. Nat Cell Biol 14:1314-1321.

52. Bitto A, Lerner C, Torres C, Roell M, Malaguti M, Perez V, Lorenzini A, Hrelia S, Ikeno Y, Matzko ME, McCarter R, Sell C. 2010. Long-term IGF-I exposure decreases autophagy and cell viability. PLoS One 5:e12592.

53. Verheggen C, Bertrand E. 2012. CRM1 plays a nuclear role in transporting snoRNPs to nucleoli in higher eukaryotes. Nucleus 3:132-137.

54. Karamysheva Z, Diaz-Martinez LA, Warrington R, Yu H. 2015. Graded requirement for the spliceosome in cell cycle progression. Cell Cycle 14:1873-1883.

55. Shapiro JS, Schmid S, Aguado LC, Sabin LR, Yasunaga A, Shim JV, Sachs D, Cherry S, tenOever BR. 2014. Drosha as an interferon-independent antiviral factor. Proc Natl Acad Sci U S A 111:7108-7113.

56. He L, He X, Lim LP, de Stanchina E, Xuan Z, Liang Y, Xue W, Zender L, Magnus J, Ridzon D, Jackson AL, Linsley PS, Chen C, Lowe SW, Cleary MA, Hannon GJ. 2007. A microRNA component of the p53 tumour suppressor network. Nature 447:1130-1134.

57. Chen F, Hu SJ. 2012. Effect of microRNA-34a in cell cycle, differentiation, and apoptosis: a review. J Biochem Mol Toxicol 26:79-86.

58. Bader AG. 2012. miR-34 - a microRNA replacement therapy is headed to the clinic. Front Genet 3:120.

59. Li P, Liu Y, Yi B, Wang G, You X, Zhao X, Summer R, Qin Y, Sun J. 2013. MicroRNA-638 is highly expressed in human vascular smooth muscle cells and inhibits PDGF-BB-induced cell proliferation and migration through targeting orphan nuclear receptor NOR1. Cardiovasc Res 99:185-193.

60. Jia L, Wu J, Zhang L, Chen J, Zhong D, Xu S, Xie C, Cai J. 2013. Restoration of miR-1228* expression suppresses epithelial-mesenchymal transition in gastric cancer. PLoS One 8: 58637.

61. Lund E, Guttinger S, Calado A, Dahlberg JE, Kutay U. 2004. Nuclear export of microRNA precursors. Science 303:95-98.

62. Jia Y, Viswakarma N, Crawford SE, Sarkar J, Sambasiva Rao M, Karpus WJ, Kanwar YS, Zhu YJ, Reddy JK. 2012. Early embryonic lethality of mice with disrupted transcription cofactor PIMT/NCOA6IP/Tgs1 gene. Mech Dev 129:193-207.

63. Wang Y, Zhu W, Levy DE. 2006. Nuclear and cytoplasmic mRNA quantification by SYBR green based real-time RT-PCR. Methods 39:356-362.

64. Vasudevan S, Steitz JA. 2007. AU-rich-element-mediated upregulation of translation by FXR1 and Argonaute 2. Cell 128:1105-1118.

65. Niranjanakumari S, Lasda E, Brazas R, Garcia-Blanco MA. 2002. Reversible cross-linking combined with immunoprecipitation to study RNA-protein interactions in vivo. Methods 26:182190. 


\section{Chapter 4}

\section{Overall Discussion and Future Directions}

Traditionally, biomedical research was conducted based on the central dogma of molecular biology, which is that DNA contains the genomic information, RNA is the "messenger" of this information, and proteins are the final product. In 2007, the Encyclopedia of DNA Elements (ENCODE) showed that more than $80 \%$ of the human genome is transcribed, but less than $3 \%$ of these molecules become proteins(1). These numerous newly discovered transcribed RNAs are classified as non-coding RNAs (ncRNAs) and have now evoked researchers to identify novel ncRNA regulators of cellular pathways besides the 30,000 protein-coding genes encoded by the human genome. A single ncRNA can regulate many different genes involved in many different cellular pathways, so it is critical to further study the role(s) of these individual ncRNAs in more detail.

NcRNA expressions are altered by a variety of cellular processes that are involved with the progression to cancer, such as those occurring after infection with oncogenic viruses (e.g. human papillomavirus $)(2,3)$ or specifically involved with cell cycle regulation, such as cellular growth arrest (e.g. quiescence) (Chapter 3)(4). Our group's overall goal is to continue to identify early molecular changes in ncRNAs that occur in response to cellular stresses and contribute to carcinogenesis. In the context of cancer, traditionally, ncRNA expression changes are identified by comparing established tumors to normal tissues. One of the specific goals of our group is to continue to elucidate early molecular events that cause initial dysregulation but are also critical enough to be maintained throughout carcinogenesis.

Oncogenic viruses, such as human papillomavirus (HPV), are useful models to study early events in cancer because they are single exogenous agents involved in the initial steps of carcinogenesis. HPV infection can regulate some unique cellular pathways, but also some analogous pathways other carcinogenic factors utilize. Thus, identifying early molecular events that are critical for progression to carcinogenesis in HPV-associated cancers could potentially be applicable to other cancers and would add to the marketability of these involved genes as therapeutic targets or diagnostic markers.

Additionally, it is necessary to continue to study HPV, as it continues to be linked to development of $4.5 \%$ of total cancer incidences worldwide. Certain HPV-associated cancers, such as the oropharyngeal 
subset of head and neck squamous cell carcinoma (HNSCC), are on the rise even in industrialized countries(5). In principle, HPV-associated cancer should be preventable as there are prophylactic vaccinations available to prevent infection with strains commonly associated with oncogenesis. However, more than two-thirds of the cervical cancer incidences occur in less-developed countries(6), which are limited by financial and logistical issues for access to the vaccines(7). Worldwide, as of October 2014, only a small minority (7.5\%) of 10 to 20-year-old females are estimated to have been administered at least one shot of an HPV vaccine(8), and this statistic does not guarantee that all of these patients completed the full administration of shots required for full efficacy. Additionally, since the HPV vaccinations are prophylactic, they do not protect those who have already been exposed to the virus. According to the CDC, "HPV is so common that nearly all men and women get exposed to the virus at some point in their lives" (https://www.cdc.gov/std/hpv/stats.htm). Thus, these many individuals who already have been exposed to oncogenic strains of the virus risk persistent infection contributing to carcinogenesis, and new cancer therapies need to be developed.

HPV regulates a variety of downstream genes to contribute to carcinogenesis $(9,10)$. Our group recently revealed novel coding genes (including LAT1 and NAP1L3) that are differentially expressed in HPV-positive compared to HPV-negative cervical cancer lines and normal cervical keratinocytes. Future studies will be conducted to elucidate downstream effects of their up-regulation as well as potential use as biomarkers associated with the development of cervical cancer. Different types of ncRNAs [microRNAs (miRNAs) and long non-coding RNAs (lncRNAs)] also exhibit global differential expression when comparing cervical cancer patients (expressing the full HPV genome) to normal matched cervical tissues $(2,3)$.

Notably, lncRNAs are a more recent field of interest and many have not been fully characterized. There is not a clear, set standard collection of lncRNAs that all research groups analyze; certain lncRNAs might not be analyzed in every dataset so it can be difficult to compare between different groups' studies. In the initial stages of the project presented in Chapter 2, MD Anderson's The Atlas of ncNA in $\underline{\text { Cancer }}$ (TANRIC) database (http://ibl.mdanderson.org/tanric/_design/basic/index.html) was the most userfriendly, open access resource for extracting information from large high-throughput RNA sequencing (RNA-seq) datasets showing lncRNAs expression changes involved in cancer. More recently, additional

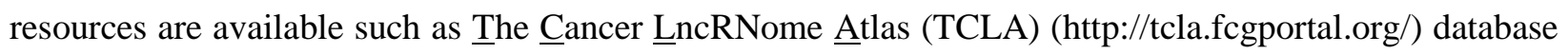
hosted by University of Pennsylvania. 
Two specific oncogenic components of the HPV genome, E6 and E7, affect two main tumor suppressors $\mathrm{p} 53$ (11) and $\mathrm{Rb}(12)$, respectively, however they have been found to regulate a plethora of genes involved in cancer progression(10,13). Since the HPV oncogenes regulate pathways commonly altered in cancer (even in HPV-negative cancers), looking at downstream pathways regulated by the HPV oncogenes can also be applicable to other types of cancers. In addition to coding gene regulation specifically by HPV oncogenes, certain studies have shown ncRNA regulation. Martinez et al. showed high-risk HPV-16 E6 regulation of a miRNA (miR-218) that regulates a coding gene (LAMB3) to potentially increase viral infection of surrounding tissue and contribute to carcinogenesis (14). Additionally, a couple previous studies showed that the HPV-16 E6/E7 oncogenes regulate the expression of lncRNA MALAT1(15) and CCEPR(16) to contribute to cervical cancer. Our group recently showed that HPV E6 expression alone causes differential expression alterations in additional host lncRNAs (Chapter 2, Figure 1A and Supplementary Table S1). Future studies elucidating the mechanism(s) of these HPV E6-regulated lncRNAs could contribute significantly to the cancer field.

Another interesting future route to study HPV E6 regulation of lncRNAs is to evaluate if any lncRNAs are directly bound to the HPV-16 E6 oncoprotein. A variety of proteins are shown to directly interact with HPV-16 E6(17), but enhancement of knowledge on ncRNA E6 binding partners could reveal alternative downstream regulators of carcinogenesis. Since HPV-16 E6 is localized to the nucleus(18), it is predicted the bound ncRNAs will be nuclear. The primary goal of this direction would be to identify host lncRNAs that are bound to E6, however, with advanced high-throughput RNA sequencing technologies it would be possible to identify novel host and viral ncRNAs bound to HPV. The Martinez lab has unpublished data showing HPV produces viral circular RNAs that alter different hallmarks of cancer (proliferation and apoptosis) (data not shown). Our lab has not yet elucidated if this is directly or indirectly by HPV-16 E6 or the presence of the full genome. Preliminary experiments were conducted to attempt to transfect a Flag-HPV-16 E6 construct into cervical cells and immunoprecipitate out by an antibody against Flag. There are well-known technical difficulties in the HPV field when trying to detect HPV-16 E6 because good quality antibodies are lacking and it has such a low molecular weight ( $21 \mathrm{kDa})$ that is difficult by Western Blot analysis.

Our lab is particularly interested in researching the host lncRNA FAM83H-AS1, which our group identified to be HPV-16 E6 regulated in cervical cells (Chapter 2, Figure 3A-C, Supplementary Figure S4A,B), and its importance in cancer initiation and progression. Most lncRNAs are tissue specific(19). FAM83H-AS1 is classified as an "onco-lncRNA", which describes the few lncRNAs that have been determined to be altered in many different cancer tissue lineages unrelated to HPV infection. This is an 
important feature because if a lncRNA is affected in multiple types of cancers, it suggests its universal importance in essential cellular pathways involved in carcinogenesis. The study of these onco-lncRNAs could increase the ability to develop potential diagnostic, prognostic, or therapeutic applications for several types of cancers(20). FAM83H-AS1 expression was shown to be up-regulated in the following cancers and high expression of FAM83H-AS1 in each of these cancers correlates with poor overall survival: breast(20, 21), colorectal(20, 22, 23), lung(20, 24), pancreatic(25), brain(26), and cervical (Chapter 2, Figure 6) (Figure 1). Therefore, further elucidating the FAM83H-AS1 in HPV-related cancers could be beneficial to a broad range of cancer patients.

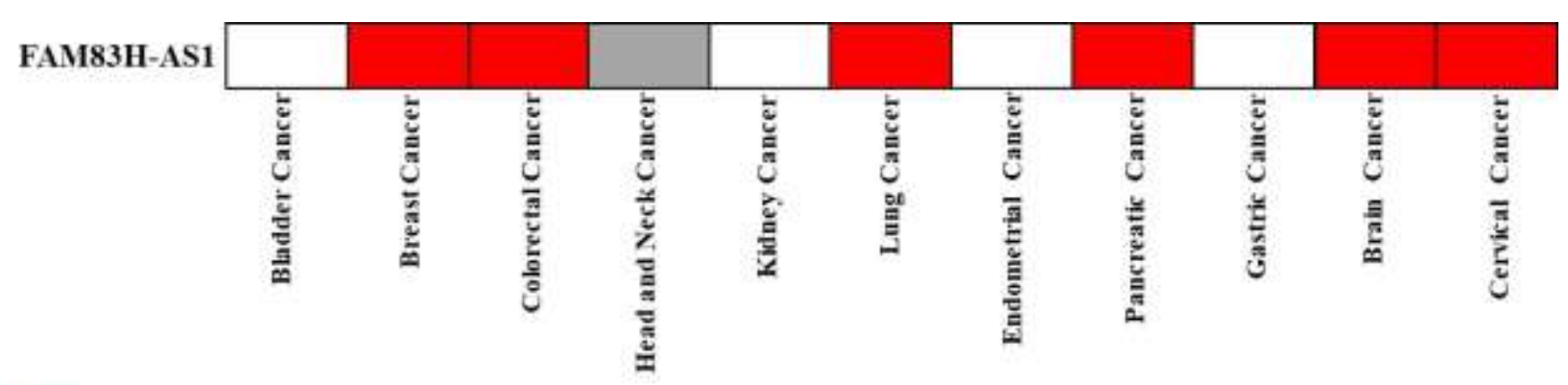

Up-regulated

Outlier

Not altered

Figure 1: Heat map of FAM83H-AS1 expression alterations in multiple cancer types.

Adapted from: Cabanski et al., RNA Biology: 2015.

Our group showed for the first time that FAM83H-AS1 expression is altered at an early stage of HPV infection in cervical cells (Chapter 2, Figure 2A), and this alteration is maintained throughout later stages of cervical carcinogenesis (Chapter 2, Figure 2B and Figure 6A,B). These results could be further strengthened in the future with access to larger sample sizes of normal, pre-malignant (cervical intraepithelial neoplasia), and cancerous human patient tissues to analyze FAM83H-AS1 expression differences. These data would contribute to the conclusion that initial up-regulation of FAM83H-AS1 is an early event similar to HPV infection itself. This increases its applicability for utilization as a biomarker to predict patients with high FAM83H-AS1 expression changes early on with HPV infection who will likely progress to cervical carcinogenesis.

Our group analyzed FAM83H-AS1 expression after persistent HPV infection by extracting data from TANRIC to show high expression of FAM83H-AS1 in cervical squamous cell carcinoma and endocervical adenocarcinoma (CESC) patients and that high expression of FAM83H-AS1 correlated with poor overall survival (Chapter 2, Figure 6B,C). HPV is detected in almost all cervical cancers. HPV-16 is 
the most prevalent strain (50\%), HPV-18 the second most common (15\%), and there are many other strains $(-31,-33,-35,-39,-45,-51,-52,-56,-58,-59$, and 68) classified as carcinogenic(27). Dividing TANRIC CESC patients into subgroups by strain could increase the impact if it is found that FAM83H-AS1 expression is altered in more than just HPV-16 positive samples. The original article that classified FAM83H-AS1 as an onco-lncRNA showed that, in their analysis, its expression in head and neck cancer is an outlier, where "outlier" is defined as a subset that had an alteration in expression(20). It is speculated that if these head and neck samples were divided into HPV-positive and HPV-negative subsets that there could have been a correlation determined. Further data mining from TANRIC of other cancer types, and segregation of samples based on HPV status, could be utilized to enhance the idea that FAM83H-AS1 is up-regulated in an HPV-dependent manner in certain cancer types.

HNSCC is a significant cancer type to study further as recently it was identified that the incidence of HPV-positive oropharyngeal cancer has surpassed cervical cancer in the United States(5). Our group showed that FAM83H-AS1 expression was also shown to be higher in HPV-positive versus HPV-negative HNSCC cell lines (Chapter 2, Figure 2C). Future access to human HPV-positive oropharyngeal HNSCC tissue samples as well as functional assays after FAM83H-AS1 knockdown in HNSCC cell lines would be beneficial to strengthen the HPV regulation of FAM83H-AS1 in HNSCC samples.

Overall, since individual knockdown of FAM83H-AS1 showed positive responses desired for cervical cancer cells (decreased proliferation and migration, and increased apoptosis) (Chapter 2, Figure 5 and Supplementary Figure S7), FAM83H-AS1 expression could potentially be targeted in either early stages of HPV infection (when the increase in expression is detected such that it could potentially prevent progression to carcinogenesis) or in later stages of cervical cancer.

Genes can be alternatively spliced under cellular stresses, which can contribute to carcinogenesis, and specifically HPV gene expression is controlled by alternative splicing to regulate its viral life cycle(28). Our group has obtained unpublished data showing alternative splicing events upon HPV infection can produce a viral circular RNA that regulates proliferation in cervical cancer cells (data not shown). Our lab plans to continue to elucidate alternatively spliced host and virus-produced ncRNAs critical for the initiation and progression to cancer.

Certain lncRNAs are predicted to have multiple isoforms according to online databases such as LNCipedia (lncipedia.org). We plan to fully sequence the specific functional isoform(s) of FAM83H-AS1 that is important in cervical cancer. Northern Blot probes, developed by in vitro transcription, will be used 
to detect the sizes of the FAM83H-AS1 isoforms. Then, TANRIC's RNA sequencing data of FAM83HAS1 could be extracted and bioinformatics analysis conducted to determine the frequency of reads at certain regions of RNA transcript. The sequences of these regions could be extracted from UCSC Genome Browser and used to design sets of primers to confirm (via RT-PCR) the presence of these certain RNA segments and map the entire functional RNA sequence. Once the functional FAM83H-AS1 isoform(s) is characterized in cervical cells, the presence and functionality of this specific isoform(s) in other cancer lineages could be determined to increase the universal potential of this lncRNA as a therapeutic target and/or diagnostic marker. Sequencing the functional isoform of FAM83H-AS1 would enable multiple future studies described in the paragraphs below.

Elucidation of upstream regulators of FAM83H-AS1 could determine ways to control and/or predict the lncRNA's expression changes. Our group showed that FAM83H-AS1 expression is regulated by HPV-16 E6 in cervical cells (Chapter 2, Figure 3A,B,C, Supplementary Figure S4A,B). This regulation of FAM83H-AS1 by HPV could be characterized further. Reporter assays, using a luciferase reporter vector containing the promoter region of FAM83H-AS1, could be transfected into HPV-positive and HPVnegative cells with or without siRNAs against HPV oncogenes E6 and/or E7. It is expected that there will be changes in luciferase activity in HPV-positive cell lines after the repression of the HPV oncogenes, suggesting a regulation of FAM83H-AS1 at the transcriptional level. If the hypothesis is correct, different luciferase constructs with specific mutations in the FAM83H-AS1 promoter could be used to identify the regulatory region in the genomic sequence.

HPV-16 E6 contributes to the degradation of the tumor suppressor p53, which alters the expression of many p53-regulated genes and affects critical cellular pathways (e.g. proliferation, DNA repair, and apoptosis $)(9,29)$ to contribute to carcinogenesis. Interestingly, FAM83H-AS1 was determined to be regulated in a p53-independent manner in cervical keratinocytes (Chapter 2, Figure 3D, Supplementary Figure S4C). Since HPV-16 E6 interacts directly with p300(17, 30, 31), and that there are three p300 binding sites in the predicted promoter region of FAM83H-AS1 (Chapter 2, Figure 3E) as determined by using the transcription factor binding site prediction software ConTra v3(32) we analyzed FAM83H-AS1 regulation by $\mathrm{p} 300$. The transcription activator p300 was identified as a regulator of FAM83H-AS1 expression in cervical keratinocytes (Chapter 2, Figure 3F, Supplementary Figure S4D). To further strengthen this, an inducible p300 knockdown construct could be stably transduced into cervical cancer cells and FAM83H-AS1 expression could be monitored by a dose-dependent progressive knockdown of p300. Reciprocally, p300 could be added to cervical keratinocytes (likely exhibiting low baseline expression of p300) and FAM83H-AS1 expression could be monitored; it would be expected that 
FAM83H-AS1 expression would increase with p300 expression. A reporter assay, using a luciferase reporter vector containing the promoter-region of FAM83H-AS1, could be used to determine direct binding between p300 and FAM83H-AS1. The expression of FAM83H-AS1 could be monitored between a luciferase reporter vector containing the wild-type promoter as well as different constructs containing mutations in each of the individual p300 binding sites. Additionally, to confirm direct binding, chromatin immunoprecipitation (ChIP) with a p300 antibody could be used to determine direct binding of p300 to the FAM83H-AS1 promoter region of the genome.

Since other groups previously showed FAM83H-AS1 expression is altered in cancers not regulated by HPV expression(20-26), HPV is not the only potential driver of increased FAM83H-AS1 expression. It is speculated that HPV-16 E6 could be regulating a downstream factor, and this downstream factor is more directly responsible for regulating FAM83H-AS1 expression. A group previously analyzed specific proteins that are bound to the E6 oncoproteins expressed by different high-risk strains of HPV. Interestingly, of the high-risk strains that were analyzed, the only high-risk strain E6 oncoprotein that bound transcription activator p300 was HPV-16(17) (Figure 2). Our group performed preliminary analysis of FAM83H-AS1 expression in an HPV-18 positive cervical carcinoma cell line (HeLa) and found that FAM83H-AS1 is

A

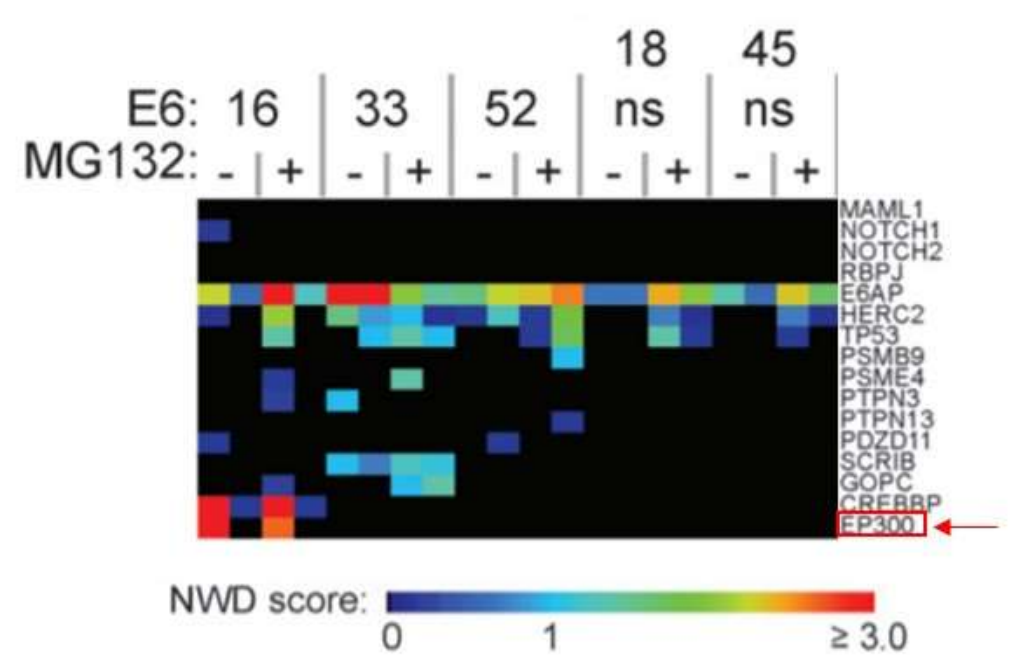

B

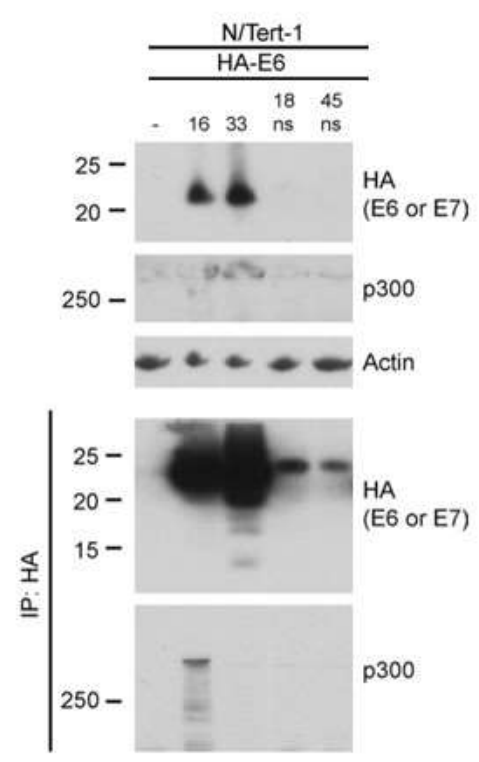

Figure 2: HPV-16 was the only high-risk strain analyzed that demonstrated E6 oncoprotein binding to p300. A) Heat map of interactions observed after immunoprecipitation of E6 oncoprotein and mass spectroscopy analysis of bound proteins. B) Western blot analysis to confirm that p300 immunoprecipitated out with HA-tagged E6 proteins. Adapted from: Cabanski et al., RNA Biology, 2015. 
expressed at similar levels to normal cervical keratinocytes (Chapter 2, Supplementary Figure S4E). Therefore, our group speculates that HPV-16 E6 regulates FAM83H-AS1 indirectly in cervical cancer, and p300 is directly regulating FAM83H-AS1 expression in HPV-16 positive cervical cancer. Furthermore, other types of cancers where FAM83H-AS1 is found overexpressed also showed overexpression of the transcription factor p300(33-37).

Many antisense lncRNAs can share the same promoter as nearby coding genes and their expressions correlate $(38,39)$. LncRNA FAM83H-AS1 is located near the coding gene FAM83H in the genome and transcribed antisense to the coding gene. Thus, it was hypothesized that they share the same promoter. FAM83H is up-regulated in many different types of cancer(40), and HPV-16 E6 up-regulates FAM83H as well as FAM83H-AS1 expression (Chapter 2, Figure 3E, Supplementary Figure S5B). Thus, it can be speculated that the lncRNA and coding gene share the same promoter. To confirm this, the sense and antisense sequences could be cloned independently in a luciferase reporter vector and their activity verified. Additionally, knocking down p300 and analyzing for changes in FAM83H expression would provide evidence that FAM83H and FAM83H-AS1 share the same promoter region.

In addition to identifying upstream regulators of FAM83H-AS1, it is critical to identify specific downstream effects of dysregulated FAM83H-AS1 expression. Previous publications showed that FAM83H-AS1 regulates CDKN1A in glioma cells(26), Notch signaling in colorectal cancer(22), and MET/EGFR signaling in lung cancer(24). These publications specifically looked at FAM83H-AS1 downstream regulation in single cancer types, thus it could be determined if FAM83H-AS1 functions similarly in other cancer types including cervical cancers.

In the ncRNA field, it is understood that the stoichiometry between a ncRNA and its targets can predict the chances of interactions and consequential downstream effects. NcRNA expression is usually quantified using techniques such as qRT-PCR analysis that show relative expression. However, it is important to determine absolute expression of RNAs in cells. To do this, in vitro transcription followed by qRT-PCR analysis can be used. We believe, however, that FAM83H-AS1 is expressed at a reasonable functional level, as its RPKM value in our RNAseq data is similar to the RPKM values of known functional lncRNAs (Chapter 2, Supplementary Table S2).

Determining the localization of a lncRNA can aid in elucidating the downstream function of the lncRNA. For example, if a lncRNA is nuclear it can be speculated that it regulates at the transcriptional level. Other groups have shown the localization of FAM83H-AS1 in the nucleus in lung and breast cancer 
cells $(24,26)$, and our group recently found FAM83H-AS1 in the nucleus of cervical cancer cells (as seen in Chapter 2 with cellular fractionation in Figure 4A,B). Fully sequencing FAM83H-AS1 would provide information necessary to design RNA fluorescent in situ hybridization (FISH) probes that could be used to detect the localization of lncRNAs and strengthen the finding that FAM83H-AS1 is localized in the nucleus. RNA FISH probes could hybridize with cervical cells as well as cervical tissue microarrays to analyze FAM83H-AS1 expression levels in human cervical samples at early stage infection (cervical intraepithelial neoplasia) through late stage carcinogenesis. Successful design of RNA FISH probes for FAM83H-AS1 would be beneficial to study other cancer types as well, as RNA FISH probes have not been used to detect FAM83H-AS1 in any cellular context to our knowledge.

Many nuclear lncRNAs can function in cis(41). Since FAM83H-AS1 is localized in the nucleus in cervical cancer cells (Chapter 2, Figure 4A,B), it was initially hypothesized that FAM83H-AS1 regulates the nearby coding gene FAM83H in cis. Knockdown of FAM83H-AS1 lncRNA does not appear to regulate FAM83H expression (Chapter 2, Supplementary Figure S5C), thus our group speculates that FAM83HAS1 does not regulate FAM83H in cis in our system. FAM83H-AS1 is likely functioning in trans in our model, but it does not eliminate the possibility that FAM83H-AS1 could function in cis in other cancer types or cellular stress situations (e.g. serum starvation and hypoxia).

Determining RNA and protein expressions that are affected by FAM83H-AS1 would potentially allow us to further narrow down the lncRNA mechanism of action. Using samples with efficient knockdown of FAM83H-AS1 in cervical cancer cells, it is proposed to conduct RNA high-throughput sequencing and mass spectroscopy to determine specific direct and/or indirect downstream effectors of FAM83H-AS1 that are contributing to the observed carcinogenic effects. A previous study analyzed differential gene expression changes with two different siRNAs against FAM83H-AS1 in a pancreatic cancer cell line, Aspc1(25). The theoretical dataset in cervical cancer could be compared to the pancreatic cancer dataset to identify similar molecular regulation by FAM83H-AS1 between cancer types. To elucidate direct binding partners, FAM83H-AS1 could be pulled out by a biotinylated RNA probe to identify its direct RNA/DNA (high-throughput RNA sequencing) and protein (mass spectroscopy) binding partners. A previous publication showed that in glioma cells, FAM83H-AS1 binds to EZH2(26), thus this would be a binding target we would look for in cervical cancer cells, as EZH2 regulates many genes at the transcriptional level by DNA methylation. Both cytoplasmic(42) and nuclear(43) lncRNAs can bind to miRNAs to act as competing endogenous RNAs. To verify potential binding interactions between FAM83H-AS1 and miRNAs to suggest a post-transcriptional regulation of miRNAs, crosslinking immunoprecipitation (CLIP) assays using Argonaute antibodies (that is the protein of the RNA induced silencing complex that miRNAs 
are loaded into) could be conducted. Once the analysis is narrowed down to critical lncRNAs, Target scan could be used to determine potential FAM83H-AS1 miRNA binding sites. To confirm direct miRNA binding to lncRNA, luciferase reporter experiments could be conducted by cloning FAM83H-AS1 regions with potential miRNA binding sites to a luciferase reporter vector. Subsequently, FAM83H-AS1 activity could be measured by using siRNAs against specific miRNAs and then mutating the potential binding sites in these constructs. Other FAM83H-AS1 binding partners are yet to be determined, thus this experiment would provide novel information.

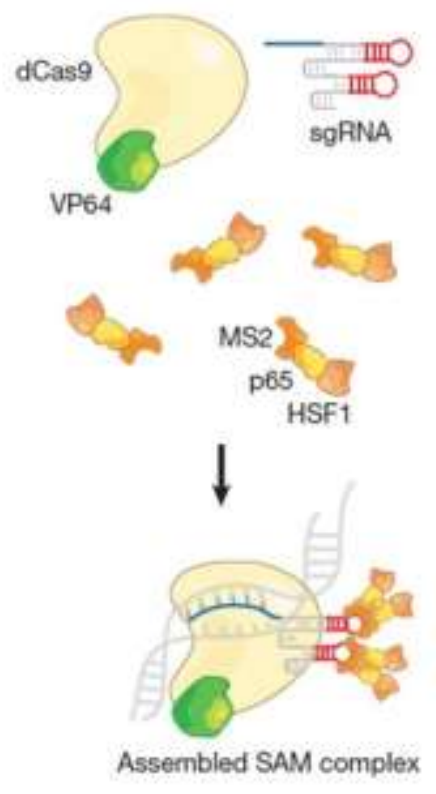

Figure 3: Schematic of the SAM complex. Complex is composed of three components: dCas9-VP64; MS2-p65HSF 1; sgRNA. Adapted from: Konermann et al., Nature, 2015.
In addition to specific genes regulated by FAM83H-AS1, we are interested in monitoring functional changes with alterations in FAM83H-AS1 expression. Knockdown of FAM83H-AS1 in cervical cancer cells resulted in decreased proliferation and migration, increased cells in S-phase, and increased apoptosis (Chapter 2, Figure 6 and Supplementary Figure S7). An ideal next step would be to express the FAM83H-AS1 in cells with low baseline FAM83H-AS1 expression and monitor for functional changes. The hypothesis for this future direction is that expression of FAM83H-AS1 would increase proliferation and migration. There was a group recently that cloned the PCR-amplified complementary DNA (cDNA) sequence that encoded FAM83HAS1 into an expression vector and transfected this into cells to monitor functional changes(26). However, to ensure expression of the entire functional lncRNA, we propose cloning the full FAM83H-AS1 sequence into a retroviral plasmid (e.g. PLPCX) or use the lentiviral CRISPR-Cas9 mutant technology known as SAM complex (Figure 3). This system requires sequentially expressing the lentiviral plasmids (Addgene) MS2-P65-HSF1_Hygro (Plasmid \#1), dCAS9-VP64_Blast (Plasmid \#2), and sgRNA(MS2)_Zeo (Plasmid \#3)(44). Briefly, Plasmid \#1 expresses the MS2 protein that binds to p65 and heat-shock factor 1 (HSF1) proteins that enhance transcription factor signals. Plasmid \#2 contains a mutant Cas9 (dCas9) that inhibits its endonuclease activity, and is bound to the transcription factor VP64. Plasmid \#3 was cloned such that it would specifically target a predicted FAM83H-AS1 promoter site. Preliminary data was obtained in the lab showing successful stable transfection of Plasmids \#1 (MS2 mRNA), \#2 (dCas9 mRNA), and \#3 (Zeocin 


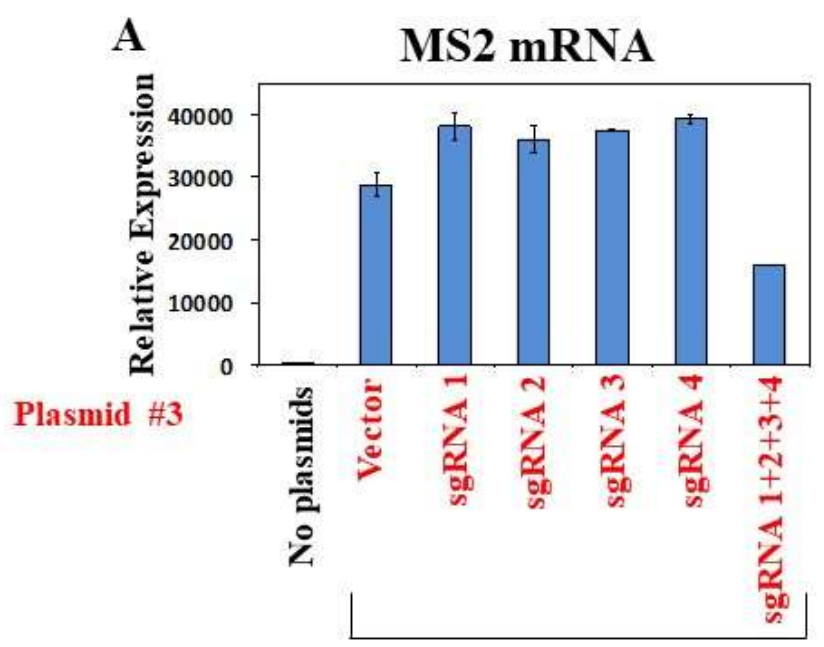

Plasmid \#1 + Plasmid \#2

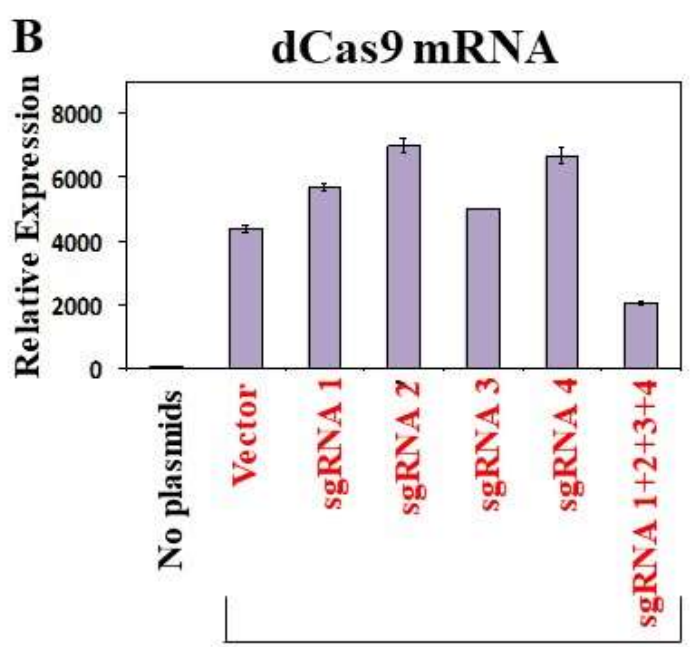

Plasmid \#1 + Plasmid \#2
C

Plasmid \#3

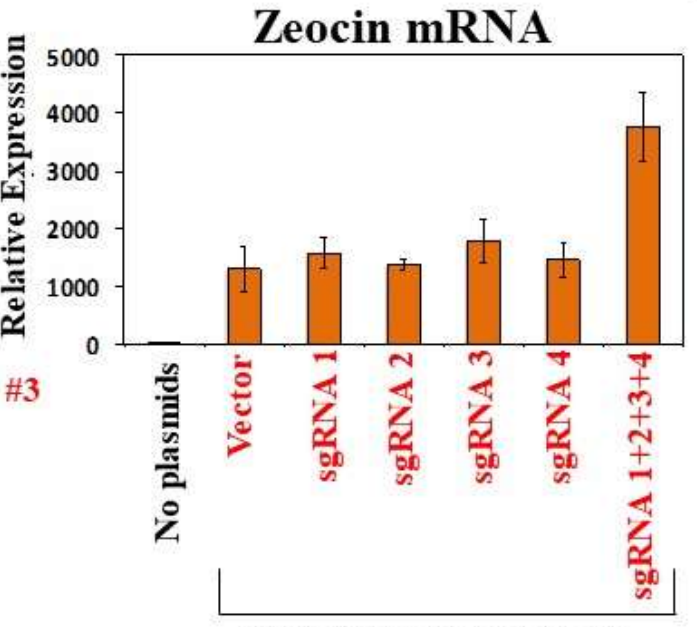

Plasmid \#1 + Plasmid \#2
D

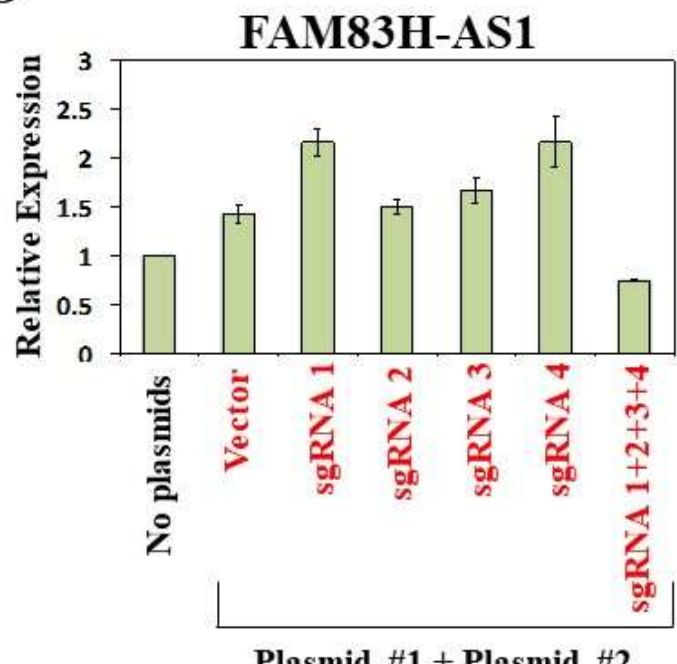

Figure 4: CRISPR Cas9 mutant technology to over-express FAM83H-AS1 in C-33A cell line. Confirmation that A) Plasmids \#1, B) Plasmid \#2, and $\mathbf{C}$ ) and Plasmid \#3 are stably expressing desired genes. D) Plasmid \#3 sgRNA designed to target FAM83H-AS1 promoter did not induce FAM83H-AS1.

mRNA) in an HPV-negative cervical carcinoma cell line (C-33A) and immortalized keratinocytes (HaCat). However, there was not significant up-regulation of FAM83H-AS1 observed (Figure 4). After further analysis, it was determined that the predicted promoter sequence inserted into Plasmid \#3 was not the region that we currently believe is the predicted promoter site (region containing p300 binding sites). This would explain the lack of increased FAM83H-AS1 expression observed in Figure 4. A future direction is to redesign sgRNAs to target the more characterized FAM83H-AS1 promoter site and over-express FAM83H- 
AS1 in cervical cells. This system would be useful to study the effects of increased FAM83H-AS1 expression in other cancers as well.

In the knockdown experiments described in Chapter 2, FAM83H-AS1 expression was transiently knocked down. It would be required to develop a system to stably knockdown FAM83H-AS1 for future in vivo experiments and long-term in vitro experiments (e.g. colony formation assays); it is important to note that the transient knockdown of FAM83H-AS1 was maintained for at least 120 hours (Chapter 2, Supplementary Figure S6). In developing a system to stably knockdown FAM83H-AS1, it would also be ideal to develop a stable population from a single cell clone population to ensure that all cells have knockdown of FAM83H-AS1 and there is a lack of non-transfected cell background.

It is clear that a significant portion of the cells had knockdown of FAM83H-AS1, as we saw significant functional changes, however the transfection efficiency was not determined. In the future, a system could be developed to express a fluorescent protein (e.g. GFP) to confirm siRNA successfully transfected into cell nuclei. This would allow for either visualization under fluorescent microscope to qualitatively determine efficiency, or flow cytometry could be potentially used to sort and collect the GFP positive population for future experiments. It is speculated that cells that had successful knockdown of FAM83H-AS1 were the ones that underwent apoptosis, therefore there would be technical difficulties if this was true; if knockdown efficiency was increased then there would not be any viable cells to conduct experiments with. One alternative strategy could be the knockdown of FAM83H-AS1 using tetracyclineinducible shRNA viral vector that could express an siRNA against FAM83H-AS1 in a dose-dependent manner. If successful knockdown of FAM83H-AS1 induced cellular death in cells with knockdown, however, it would be a beneficial therapeutic approach to induce cell death in cancer cells. It would be critical to determine the effect of FAM83H-AS1 knockdown on surrounding normal and microenvironment cells, however, before moving forward. Simply transfecting normal cervical keratinocytes with siRNA against FAM83H-AS1 would be a good start to obtain this data.

If it is confirmed that knockdown of FAM83H-AS1 kills all the cells, it would eliminate the potential in vivo experimental set-up to knockdown siRNA in cervical cancer cells prior to injecting into a human tumor to see the reduction in tumor formation. It would be ideal to develop an in vivo system to develop a mouse tumor and then reduce FAM83H-AS1 expression and observe alterations in tumor growth. Currently, patient-derived xenograft (PDX) murine models are utilized in the cancer field as they allow for direct engraftment of human tumor tissues. A group previously showed the utilization of a PDX model with cervical cancer tumor engraftment(45). The Martinez lab has collaborated with West Virginia University 
(WVU) PDX core to establish PDX cervical cancer tumors. It would be ideal to have the molecular background (FAM83H-AS1 expression) of the human tumors prior to implantation into the mouse so we could compare therapeutic effects with higher and lower FAM83H-AS1 expression in the tumor. Additionally, we could compare the therapeutic effects observed in our model to the human clinical outcomes. In the future, siRNA against FAM83H-AS1 could be injected into the mice and tumor size could be monitored. There is a collaborator at WVU with expertise in targeted drug delivery with nanoparticles (Dr. Werner Geldenhuys). Theoretically, siRNAs could be bound to nanoparticles to increase the internalization of siRNA into the nucleus. FAM83H-AS1 knockdown could be combined with current chemotherapies to improve their efficacy. If benefits are observed with FAM83H-AS1 knockdown with siRNAs, further studies could be developed on a drug to target FAM83H-AS1 that might be more clinically appealing than siRNAs.

Overall, FAM83H-AS1 regulates many of the cellular hallmarks of cancer in a variety of different cancers and should be studied further as a therapeutic target, diagnostic, and/or prognostic marker.

Another interesting phenomenon is that artificial inhibition of the HPV oncoproteins E6 and E7 in cervical cancer cells can induce cellular growth arrest by recovering the expression of the wild-type p53 and $\mathrm{Rb}$ tumor suppresor genes. More specifically, Martinez et al. showed that inhibition of HPV E7 can induce an irreversible form of growth arrest known as senescence through the Rb pathway by regulating specific miRNAs(46). Another important form of reversible growth arrest, known as quiescence, has been shown to be important in maintaining the homeostasis between differentiation and self-renewal of stem cells, and dysregulation of quiescence is related to cancer(47). Increasing knowledge on this cellular process will benefit research on aging-related diseases, wound healing treatment, organ regeneration, and cancer prevention. Our group, and others, have shown that ncRNAs (e.g. miRNAs and lncRNAs) are dysregulated in cellular quiescence (Chapter 3, Figure 1)(4).

Our group discovered that quiescent cells display an alternative miRNA biogenesis pathway in normal, primary foreskin fibroblast cells (Chapter 3), and further characterization of this pathway could reveal novel therapeutic and diagnostic markers. Currently, research on abnormal cellular growth arrest is focused on genetic alterations or mutations in cellular factors involved in the canonical pathway, and it has been demonstrated that mutations of some miRNA-processing factors have been linked to tumor formation(48). The findings in Chapter 3 show that there are alternations in the miRNA biogenesis pathway, which are not due to genetic mutations, that need to be researched further. It is hypothesized that quiescent cells disrupt the canonical miRNA biogenesis pathway to induce cellular growth arrest but continue the 
production of essential miRNAs by the alternative pathway identified in Chapter 3. Other groups have demonstrated certain cells exhibit non-canonical miRNA processing $(49,50)$, but these non-canonical pathways only affect certain miRNAs without modifying the global canonical biogenesis pathway.

Specifically, our group discovered that Exportin-5 (XPO5) expression is reduced in quiescence by autophagy and miR-34a (Chapter 3, Figure 2), and certain miRNAs are induced in quiescence despite the absence of XPO5 (Chapter 3, Figure 3). Primary microRNAs (pri-miRNAs) corresponding to the quiescence-induced miRNAs are exported by Exportin-1 (XPO1) (Chapter 3, Figure 3) potentially via a trimethylguanosine $\left(\mathrm{m}^{2,2,7} \mathrm{G}, \mathrm{TMG}\right)$-cap (Chapter 3, Figure 4).

Pri-miRNAs are canonically processed into precursor miRNAs (pre-miRNAs) in the nucleus(51), however certain pri-miRNAs (e.g. pri-miR-34a) were identified in the cytoplasm in quiescent cells (Chapter 3 , Figure 5). The components involved in processing of these pri-miRNAs are not fully characterized. It is unclear if the pri-miRNA cap modification of these quiescence-induced miRNAs occurs in the nucleus or the cytoplasm. Future studies could identify the molecular components (and their localization) necessary for TMG capping of these pri-miRNA. ( $\mathrm{m}^{7} \mathrm{G}$ )-capped nuclear RNAs [small nuclear RNAs (snRNAs)] can be exported by XPO1 in collaboration with cap-binding complex (CBC) and the phosphorylated adaptor for RNA export (PHAX)(52). In the cytoplasm, these snRNAs are recognized by Sec1/Munc18 (Sm) proteins and the cap is hypermethylated into a $\left(\mathrm{m}^{2,2,7} \mathrm{G}, \mathrm{TMG}\right)$-cap by TGS1. These snRNAs are then imported back into the nucleus by Snurportin-1 in association with other factors(53). It is hypothesized that quiescence-induced pri-miRNAs could be capped in a similar fashion. To test this hypothesis, cellular localization of $\left(\mathrm{m}^{2,2,7} \mathrm{G}, \mathrm{TMG}\right)$-capped pri-miRNAs will be determined. Briefly, quiescent cells will be fractionated, an anti-TMG cap antibody will be used to perform RNA immunoprecipitation (RIP) and recovered RNA will be sent for RNA-seq. Our group published a protocol to immunoprecipitate TMGcapped RNA that could be used (Appendix). It will be determined if Sm protein is directly binding to these pri-miRNAs in the cytoplasmic fraction by performing RIP assays and mutational experiments.

Typically, Drosha does not cleave pri-miRNAs alone, as it forms a nuclear microprocessor complex with DGCR8 in the nucleus of cells to recognize and cleave pri-miRNAs and form pre-miRNAs. DGCR8 acts as a molecular anchor necessary for the recognition of pri-miRNAs at the double-stranded RNA junction and directs Drosha to cleave and release the hairpin-shape pre-miRNAs in the nucleus(54). Our group additionally showed that there is a cytoplasmic short form of Drosha in quiescent cells that is functional and potentially involved in the processing (Chapter 3, Figure 6), thus it was speculated that DGCR8 would also be detected in the cytoplasm. Interestingly, we were unable to detect DGCR8 in the 
cytoplasm during quiescence (Chapter 3, Figure 6B). DGCR8 was knocked down by siRNA in quiescent cells and the biogenesis of quiescence-induced miR-34a was not affected (Chapter 3, Supplementary Figure 14B). It is possible, however, that DGCR8 in the cytoplasm has a cleaved form present in the cytoplasm (similar to Drosha) that was not targeted by the siRNA against DGCR8 or detected by the specific DGCR8 antibody used. If this is not the case, it is speculated that there are alternative DGCR8-like, double stranded RNA binding proteins in the cytoplasm aiding Drosha in miRNA processing. A future study to characterize the Drosha-cytoplasmic microprocessor complex during cellular quiescence would be to conduct coimmunoprecipitations using an antibody against Drosha in cytoplasmic fractions of quiescent cells, followed by mass spectroscopy. Once a DGCR8-like protein(s) is identified as an interactor with Drosha in the cytoplasm, it would need to be proven to be involved in the pri-miRNA processing. Briefly, the DGCR8like protein(s) could be knocked out by CRISPR/Cas9 technology and downstream pri-miRNA and mature miRNA levels could be monitored.

A specific group of miRNAs is induced during cellular quiescence. Individually identifying genes targeted by these individual miRNAs could identify critical regulators in cellular growth arrest. It would be interesting to further identify global regulation by these induced miRNAs during quiescence. To identify interaction between the active miRNAs and their real biological mRNAs, high-throughput sequencing of RNA isolated by crosslinking immunoprecipitation (HITS-CLIP) could be conducted in quiescent cells.

Normal human foreskin fibroblasts were used in Chapter 3 to identify and study the non-canonical miRNA biogenesis pathway in quiescent cells. To broaden the applications, future studies analyzing miRNA biogenesis of stem cells and cancer stem cells, which undergo quiescence before they differentiate or start self-renewal, could be conducted.

Overall, further elucidation of this quiescence-induced miRNA biogenesis pathway could identify specific novel target genes that are involved in cellular proliferation, apoptosis inhibition, and autophagy pathways and could be potential therapeutic targets or diagnostic markers. These findings could thus be globally impactful, as essentially every cell in the human body has the potential to undergo these critical cellular processes. 


\section{$\underline{\text { References }}$}

1. Birney E, Stamatoyannopoulos JA, Dutta A, Guigo R, Gingeras TR, Margulies EH, Weng Z, Snyder M, Dermitzakis ET, Thurman RE, Kuehn MS, Taylor CM, Neph S, Koch CM, Asthana S, Malhotra A, Adzhubei I, Greenbaum JA, Andrews RM, Flicek P, Boyle PJ, Cao H, Carter NP, Clelland GK, Davis S, Day N, Dhami P, Dillon SC, Dorschner MO, Fiegler H, Giresi PG, Goldy J, Hawrylycz M, Haydock A, Humbert R, James KD, Johnson BE, Johnson EM, Frum TT, Rosenzweig ER, Karnani N, Lee K, Lefebvre GC, Navas PA, Neri F, Parker SC, Sabo PJ, Sandstrom R, Shafer A, Vetrie D, et al. 2007. Identification and analysis of functional elements in 1\% of the human genome by the ENCODE pilot project. Nature 447:799816.

2. Wang H, Zhao Y, Chen M, Cui J. 2017. Identification of Novel Long Non-coding and Circular RNAs in Human Papillomavirus-Mediated Cervical Cancer. Front Microbiol 8:1720.

3. Huang J, Liu T, Shang C, Zhao Y, Wang W, Liang Y, Guo L, Yao S. 2018. Identification of lncRNAs by microarray analysis reveals the potential role of lncRNAs in cervical cancer pathogenesis. Oncol Lett 15:5584-5592.

4. Bierhoff H, Dammert MA, Brocks D, Dambacher S, Schotta G, Grummt I. 2014. Quiescenceinduced LncRNAs trigger H4K20 trimethylation and transcriptional silencing. Mol Cell 54:675682.

5. Berman TA, Schiller JT. 2017. Human papillomavirus in cervical cancer and oropharyngeal cancer: One cause, two diseases. Cancer 123:2219-2229.

6. de Martel C, Plummer M, Vignat J, Franceschi S. 2017. Worldwide burden of cancer attributable to HPV by site, country and HPV type. Int J Cancer 141:664-670.

7. Schiller JT, Muller M. 2015. Next generation prophylactic human papillomavirus vaccines. Lancet Oncol 16:e217-225.

8. Bruni L, Diaz M, Barrionuevo-Rosas L, Herrero R, Bray F, Bosch FX, de Sanjose S, Castellsague X. 2016. Global estimates of human papillomavirus vaccination coverage by region and income level: a pooled analysis. Lancet Glob Health 4:e453-463.

9. Munger K, Howley PM. 2002. Human papillomavirus immortalization and transformation functions. Virus Res 89:213-228.

10. Moody CA, Laimins LA. 2010. Human papillomavirus oncoproteins: pathways to transformation. Nat Rev Cancer 10:550-560.

11. Scheffner M, Werness BA, Huibregtse JM, Levine AJ, Howley PM. 1990. The E6 oncoprotein encoded by human papillomavirus types 16 and 18 promotes the degradation of p53. Cell 63:11291136.

12. Boyer SN, Wazer DE, Band V. 1996. E7 protein of human papilloma virus-16 induces degradation of retinoblastoma protein through the ubiquitin-proteasome pathway. Cancer Res 56:4620-4624.

13. Yim EK, Park JS. 2005. The role of HPV E6 and E7 oncoproteins in HPV-associated cervical carcinogenesis. Cancer Res Treat 37:319-324.

14. Martinez I, Gardiner AS, Board KF, Monzon FA, Edwards RP, Khan SA. 2008. Human papillomavirus type 16 reduces the expression of microRNA-218 in cervical carcinoma cells. Oncogene 27:2575-2582.

15. Jiang Y, Li Y, Fang S, Jiang B, Qin C, Xie P, Zhou G, Li G. 2014. The role of MALAT1 correlates with HPV in cervical cancer. Oncol Lett 7:2135-2141.

16. Sharma S, Munger K. 2018. Expression of the cervical carcinoma expressed PCNA regulatory (CCEPR) long noncoding RNA is driven by the human papillomavirus E6 protein and modulates cell proliferation independent of PCNA. Virology 518:8-13.

17. White EA, Kramer RE, Tan MJ, Hayes SD, Harper JW, Howley PM. 2012. Comprehensive analysis of host cellular interactions with human papillomavirus E6 proteins identifies new E6 binding partners and reflects viral diversity. J Virol 86:13174-13186. 
18. Masson M, Hindelang C, Sibler AP, Schwalbach G, Trave G, Weiss E. 2003. Preferential nuclear localization of the human papillomavirus type $16 \mathrm{E} 6$ oncoprotein in cervical carcinoma cells. J Gen Virol 84:2099-2104.

19. Yan X, Hu Z, Feng Y, Hu X, Yuan J, Zhao SD, Zhang Y, Yang L, Shan W, He Q, Fan L, Kandalaft LE, Tanyi JL, Li C, Yuan CX, Zhang D, Yuan H, Hua K, Lu Y, Katsaros D, Huang Q, Montone K, Fan Y, Coukos G, Boyd J, Sood AK, Rebbeck T, Mills GB, Dang CV, Zhang L. 2015. Comprehensive Genomic Characterization of Long Non-coding RNAs across Human Cancers. Cancer Cell 28:529-540.

20. Cabanski CR, White NM, Dang HX, Silva-Fisher JM, Rauck CE, Cicka D, Maher CA. 2015. Pan-cancer transcriptome analysis reveals long noncoding RNAs with conserved function. RNA Biol 12:628-642.

21. Yang F, Lv SX, Lv L, Liu YH, Dong SY, Yao ZH, Dai XX, Zhang XH, Wang OC. 2016. Identification of lncRNA FAM83H-AS1 as a novel prognostic marker in luminal subtype breast cancer. Onco Targets Ther 9:7039-7045.

22. Lu S, Dong W, Zhao P, Liu Z. 2018. IncRNA FAM83H-AS1 is associated with the prognosis of colorectal carcinoma and promotes cell proliferation by targeting the Notch signaling pathway. Oncol Lett 15:1861-1868.

23. Yang L, Xu L, Wang Q, Wang M, An G. 2016. Dysregulation of long non-coding RNA profiles in human colorectal cancer and its association with overall survival. Oncol Lett 12:4068-4074.

24. Zhang J, Feng S, Su W, Bai S, Xiao L, Wang L, Thomas DG, Lin J, Reddy RM, Carrott PW, Lynch WR, Chang AC, Beer DG, Guo YM, Chen G. 2017. Overexpression of FAM83H-AS1 indicates poor patient survival and knockdown impairs cell proliferation and invasion via MET/EGFR signaling in lung cancer. Sci Rep 7:42819.

25. Arnes L, Liu Z, Wang J, Carlo Maurer H, Sagalovskiy I, Sanchez-Martin M, Bommakanti N, Garofalo DC, Balderes DA, Sussel L, Olive KP, Rabadan R. 2018. Comprehensive characterisation of compartment-specific long non-coding RNAs associated with pancreatic ductal adenocarcinoma. Gut doi:10.1136/gutjnl-2017-314353.

26. Bi YY, Shen G, Quan Y, Jiang W, Xu F. 2018. Long noncoding RNA FAM83H-AS1 exerts an oncogenic role in glioma through epigenetically silencing CDKN1A (p21). J Cell Physiol doi:10.1002/jcp.26813.

27. Guan P, Howell-Jones R, Li N, Bruni L, de Sanjose S, Franceschi S, Clifford GM. 2012. Human papillomavirus types in 115,789 HPV-positive women: a meta-analysis from cervical infection to cancer. Int J Cancer 131:2349-2359.

28. Graham SV, Faizo AAA. 2017. Control of human papillomavirus gene expression by alternative splicing. Virus Res 231:83-95.

29. Bieging KT, Mello SS, Attardi LD. 2014. Unravelling mechanisms of p53-mediated tumour suppression. Nat Rev Cancer 14:359-370.

30. Patel D, Huang SM, Baglia LA, McCance DJ. 1999. The E6 protein of human papillomavirus type 16 binds to and inhibits co-activation by CBP and p300. Embo j 18:5061-5072.

31. Zimmermann H, Degenkolbe R, Bernard HU, O'Connor MJ. 1999. The human papillomavirus type 16 E6 oncoprotein can down-regulate p53 activity by targeting the transcriptional coactivator CBP/p300. J Virol 73:6209-6219.

32. Kreft L, Soete A, Hulpiau P, Botzki A, Saeys Y, De Bleser P. 2017. ConTra v3: a tool to identify transcription factor binding sites across species, update 2017. Nucleic Acids Res 45:W490-w494.

33. Fermento ME, Gandini NA, Salomon DG, Ferronato MJ, Vitale CA, Arevalo J, Lopez Romero A, Nunez M, Jung M, Facchinetti MM, Curino AC. 2014. Inhibition of p300 suppresses growth of breast cancer. Role of p300 subcellular localization. Exp Mol Pathol 97:411-424.

34. Ishihama K, Yamakawa M, Semba S, Takeda H, Kawata S, Kimura S, Kimura W. 2007. Expression of HDAC1 and CBP/p300 in human colorectal carcinomas. J Clin Pathol 60:12051210. 
35. Hou X, Li Y, Luo RZ, Fu JH, He JH, Zhang LJ, Yang HX. 2012. High expression of the transcriptional co-activator p300 predicts poor survival in resectable non-small cell lung cancers. Eur J Surg Oncol 38:523-530.

36. Ono H, Basson MD, Ito H. 2016. P300 inhibition enhances gemcitabine-induced apoptosis of pancreatic cancer. Oncotarget 7:51301-51310.

37. Panicker SP, Raychaudhuri B, Sharma P, Tipps R, Mazumdar T, Mal AK, Palomo JM, Vogelbaum MA, Haque SJ. 2010. p300- and Myc-mediated regulation of glioblastoma multiforme cell differentiation. Oncotarget 1:289-303.

38. Sigova AA, Mullen AC, Molinie B, Gupta S, Orlando DA, Guenther MG, Almada AE, Lin C, Sharp PA, Giallourakis CC, Young RA. 2013. Divergent transcription of long noncoding RNA/mRNA gene pairs in embryonic stem cells. Proc Natl Acad Sci U S A 110:2876-2881.

39. Khachane AN, Harrison PM. 2010. Mining mammalian transcript data for functional long noncoding RNAs. PLoS One 5:e10316.

40. Snijders AM, Lee SY, Hang B, Hao W, Bissell MJ, Mao JH. 2017. FAM83 family oncogenes are broadly involved in human cancers: an integrative multi-omics approach. Mol Oncol 11:167179.

41. Yu B, Shan G. 2016. Functions of long noncoding RNAs in the nucleus. Nucleus 7:155-166.

42. Chen G, Peng L, Zhu Z, Du C, Shen Z, Zang R, Su Y, Xia Y, Tang W. 2017. LncRNA AFAP1AS Functions as a Competing Endogenous RNA to Regulate RAP1B Expression by sponging miR181a in the HSCR. Int J Med Sci 14:1022-1030.

43. Liu P, Yang H, Zhang J, Peng X, Lu Z, Tong W, Chen J. 2017. The lncRNA MALAT1 acts as a competing endogenous RNA to regulate KRAS expression by sponging miR-217 in pancreatic ductal adenocarcinoma. Sci Rep 7:5186.

44. Konermann S, Brigham MD, Trevino AE, Joung J, Abudayyeh OO, Barcena C, Hsu PD, Habib N, Gootenberg JS, Nishimasu H, Nureki O, Zhang F. 2015. Genome-scale transcriptional activation by an engineered CRISPR-Cas9 complex. Nature 517:583-588.

45. Chaudary N, Pintilie M, Schwock J, Dhani N, Clarke B, Milosevic M, Fyles A, Hill RP. 2012. Characterization of the Tumor-Microenvironment in Patient-Derived Cervix Xenografts (OCICx). Cancers (Basel) 4:821-845.

46. Martinez I, Cazalla D, Almstead LL, Steitz JA, DiMaio D. 2011. miR-29 and miR-30 regulate B-Myb expression during cellular senescence. Proc Natl Acad Sci U S A 108:522-527.

47. Cheung TH, Rando TA. 2013. Molecular regulation of stem cell quiescence. Nat Rev Mol Cell Biol 14:329-340.

48. Melo SA, Moutinho C, Ropero S, Calin GA, Rossi S, Spizzo R, Fernandez AF, Davalos V, Villanueva A, Montoya G, Yamamoto H, Schwartz S, Jr., Esteller M. 2010. A genetic defect in exportin-5 traps precursor microRNAs in the nucleus of cancer cells. Cancer Cell 18:303-315.

49. Havens MA, Reich AA, Duelli DM, Hastings ML. 2012. Biogenesis of mammalian microRNAs by a non-canonical processing pathway. Nucleic Acids Res 40:4626-4640.

50. Shapiro JS, Langlois RA, Pham AM, Tenoever BR. 2012. Evidence for a cytoplasmic microprocessor of pri-miRNAs. Rna 18:1338-1346.

51. Lee Y, Ahn C, Han J, Choi H, Kim J, Yim J, Lee J, Provost P, Radmark O, Kim S, Kim VN. 2003. The nuclear RNase III Drosha initiates microRNA processing. Nature 425:415-419.

52. Boulon S, Verheggen C, Jady BE, Girard C, Pescia C, Paul C, Ospina JK, Kiss T, Matera AG, Bordonne R, Bertrand E. 2004. PHAX and CRM1 are required sequentially to transport U3 snoRNA to nucleoli. Mol Cell 16:777-787.

53. Kiss T. 2004. Biogenesis of small nuclear RNPs. J Cell Sci 117:5949-5951.

54. Gregory RI, Yan KP, Amuthan G, Chendrimada T, Doratotaj B, Cooch N, Shiekhattar R. 2004. The Microprocessor complex mediates the genesis of microRNAs. Nature 432:235-240. 


\title{
Appendix
}

\section{Immunoprecipitation of Tri-methylated Capped RNA}

\author{
Karen E. Hayes ${ }^{1}$, Jamie A. Barr ${ }^{1}$, Mingyi Xie ${ }^{2}$, Joan A. Steitz ${ }^{3,4,5}$ and Ivan Martinez ${ }^{1, *}$ \\ ${ }^{1}$ Department of Microbiology, Program in Cancer Cell Biology, WVU Cancer Institute, West Virginia \\ University, Morgantown, WV, USA; ${ }^{2}$ Department of Biochemistry \& Molecular Biology, U.F. Health \\ Cancer Center, University of Florida, Gainesville, FL, USA; ${ }^{3}$ Department of Molecular Biophysics \& \\ Biochemistry, Yale University, New Haven, CT, USA; ${ }^{4}$ Howard Hughes Medical Institute, Yale \\ University, New Haven, CT 06536, USA; ${ }^{5}$ Yale Cancer Center, New Haven, CT, USA \\ *For correspondence: ivmartinez@hsc.wvu.edu
}

Keywords: $\mathrm{m}^{2,2,7}$ G-cap RNA, TMG-cap RNA, Tri-methylated RNA, RNA immunoprecipitation, PrimiRNA

Published in: Bio-protocol journal

Bio Protoc. 2018 Feb 5;8(3). PMID: 29527542 


\section{$\underline{\text { I. Abstract }}$}

Cellular quiescence (also known as $\mathrm{G}_{0}$ arrest) is characterized by reduced DNA replication, increased autophagy, and increased expression of cyclin-dependent kinase p27 ${ }^{\mathrm{Kip} 1}$. Quiescence is essential for wound healing, organ regeneration, and preventing neoplasia. Previous findings indicate that microRNAs (miRNAs) play an important role in regulating cellular quiescence. Our recent publication demonstrated the existence of an alternative miRNA biogenesis pathway in primary human foreskin fibroblast (HFF) cells during quiescence. Indeed, we have identified a group of pri-miRNAs (whose mature miRNAs were found induced during quiescence) modified with a 2,2,7-trimethylguanosine (TMG)-cap by the trimethylguanosine synthase 1 (TGS1) protein and transported to the cytoplasm by the Exportin-1 (XPO1) protein. We used an antibody against (TMG)-caps (which does not cross-react with the ( $\left.\mathrm{m}^{7} \mathrm{G}\right)$-caps that most pri-miRNAs or mRNAs contain [Luhrmann et al., 1982]) to perform RNA immunoprecipitations from total RNA extracts of proliferating or quiescent HFFs. The novelty of this assay is the specific isolation of pri-miRNAs as well as other non-coding RNAs containing a TMG-cap modification.

\section{$\underline{\text { II. Background }}$}

Cellular quiescence, a type of reversible growth arrest, is an important cellular state involved in wound healing, organ regeneration, and preventing neoplasia (Coller, 2011; Valcourt et al., 2012). Small non-coding RNAs such as miRNAs have been found involved in the regulation of cellular quiescence. miRNAs are small non-coding RNAs $\sim 22$-nucleotides long that regulate the expression of protein-coding genes by base-pairing with the 3' untranslated region (3'UTR) of messenger RNAs (mRNAs) (Esteller, 2011). The canonical miRNA biogenesis pathway is based on a stepwise processing machinery (Ha and Kim, 2014; Kim et al., 2016). miRNAs are transcribed to produce a primary miRNA (pri-miRNA) with an imperfect loop structure that is recognized by the enzyme Drosha and its binding partner DGCR8 in the nucleus. Cleavage of the pri-miRNA generates a precursor miRNA (pre-miRNA) that is recognized and transported to the cytoplasm by the Exportin-5 (XPO5) protein. The pre-miRNA is cleaved by the enzyme Dicer (mature miRNA) and loaded into the RNA-induced silencing complex (RISC). On the other hand, precursors of small nuclear RNAs (snRNAs) involved in mRNA processing such as U1, U2, U4, and U5 have a $\left(\mathrm{m}^{7} \mathrm{G}\right)$-cap, which is recognized by cap-binding complex $(\mathrm{CBC})$ and the phosphorylated adaptor for RNA export (PHAX) in the nucleus to enable their export to the cytoplasm by XPO1 (Ohno et al., 2000). These snRNAs are then recognized by Sec1/Munc18 $(\mathrm{Sm})$ proteins (by binding to Sm binding site sequences) in the cytoplasm and TGS1 is recruited to hypermethylate the $\left(\mathrm{m}^{7} \mathrm{G}\right)$-cap into a $\left(\mathrm{m}^{2,2,7} \mathrm{G}, \mathrm{TMG}\right)$ cap. This modification is recognized by Snuportin- 1 in association with Importin- $\beta$ and other factors to 
import the snRNAs back into the nucleus (Palacios et al., 1997; Kiss, 2004). Interestingly, XPO1 also has high affinity for the (TMG)-capped small nucleolar RNA (snoRNA) U3 in the nucleus and transports it from Cajal bodies to the nucleoli (Boulon et al., 2004). A previous study showed that TGS1 enhances Revdependent HIV-1 RNA expression by (TMG)-capping viral mRNAs in the nucleus, thereby increasing recognition by XPO1 for transport to the cytoplasm (Yedavalli and Jeang, 2010). These findings suggest that TMG-capping of RNAs gives plasticity to different types of RNA molecules in order to regulate their processing and cellular localization. Our recent findings demonstrated the existence of a group of primiRNAs modified with a 2,2,7-trimethylguanosine (TMG)-cap by TGS1 protein and transported to the cytoplasm by XPO1 during quiescence. Previous publications have shown the ability to pull-down (TMG)cap RNAs, such as snRNAs and snoRNAs, with specific antibodies against (TMG)-cap RNAs (Luhrmann et al., 1982). Our previous publications demonstrated for the first time the pull-down of (TMG)-cap primiRNAs in human cells (Martinez et al., 2017). Understanding which RNAs could be modified with a TMG-cap will provide new important insights into RNA biogenesis in normal or disease-related conditions.

\section{Materials and Reagents}

1. $1.5 \mathrm{ml}$ microcentrifuge tubes (Fisher Scientific, catalog number: $\underline{05-408-129}$ )

2. $15 \mathrm{ml}$ conical centrifuge tubes (DNase-/RNase-free) (Corning, catalog number: $\underline{430052}$ )

3. Gilson ${ }^{\mathrm{TM}}$ EXPERT $^{\mathrm{TM}}$ University Fit pipette filter tips (Gilson, catalog numbers: F1731031, $\underline{F 1733031}, \underline{F 1735031}, \underline{F 1737031)}$

4. Gel-loading pipet tips (Fisher Scientific, catalog number: 02-707-139)

5. Large-orifice pipet tips (Fisher Scientific, catalog number: 02-707-134)

6. Sterile polystyrene disposable serological pipettes (Greiner Bio One International, catalog number: 710180)

7. Serological pipettes

$2 \mathrm{ml}$ serological pipettes (Fisher Scientific, catalog number: 13-678-11C)

$5 \mathrm{ml}$ serological pipettes (Fisher Scientific, catalog number: 13-678-11D)

$10 \mathrm{ml}$ serological pipettes (Fisher Scientific, catalog number: 13-678-11E)

$25 \mathrm{ml}$ serological pipettes (Fisher Scientific, catalog number: 13-678-11)

8. $150 \mathrm{~cm}^{2}$ vented tissue culture treated flasks (Corning, Falcon ${ }^{\circledR}$, catalog number: $\underline{355001}$ )

9. $100 \mathrm{~mm}$ TC-treated cell culture dish (Corning, Falcon ${ }^{\circledR}$, catalog number: 353033 )

10. Cell Scrapers (Fisher Scientific, catalog number: $\underline{08-100-242}$ )

11. HFF cells (obtained from the Yale Skin Disease Research Center) (Alternative source of HFF cells from ATCC: Hs27 (ATCC, catalog number: CRL-1634) 
12. DMEM (Sigma-Aldrich, catalog number: D7777)

13. Minimum essential medium (MEM) non-essential amino acids, 100x (Thermo Fisher Scientific, catalog number: $\underline{11140076}$ )

14. $0.05 \%$ Trypsin-EDTA with phenol red (Thermo Fisher Scientific, catalog number: 25300054)

15. 10x PBS (Sigma-Aldrich, catalog number: $\underline{\text { P5493) }}$

16. Nuclease-free water (not DEPC-Treated) (Thermo Fisher Scientific, catalog number: AM9937)

17. TRIzol ${ }^{\mathrm{TM}}$ Reagent (Thermo Fisher Scientific, catalog number: $\underline{15596026)}$

18. RNase AWAY ${ }^{\mathrm{TM}}$ Surface Decontaminant (Thermo Fisher Scientific, catalog number: 7002 )

19. Chloroform (Sigma-Aldrich, catalog number: $\underline{\mathrm{C} 2432}$ )

20. Ethanol, molecular biology grade (Fisher Scientific, catalog number: BP2818-500)

21. Isopropanol, molecular biology grade (Fisher Scientific, catalog number: BP26184)

22. GlycoBlue ${ }^{\mathrm{TM}}$ Coprecipitant $(15 \mathrm{mg} / \mathrm{ml})$ (Thermo Fisher Scientific, catalog number: $\underline{\text { AM9516) }}$

23. TURBO DNA-free ${ }^{\mathrm{TM}}$ Kit (Thermo Fisher Scientific, catalog number: AM1907)

24. Anti-m3G-cap, rabbit polyclonal, antiserum (Synaptic Systems)*

Note: *Synaptic Systems discontinued the production of the Anti-m3G-cap, rabbit polyclonal, antiserum. Creative Diagnostics has a rabbit anti-TMG antibody (Anti-m3G-cap polyclonal antibody, Creative Diagnostics, catalog number: DPAB29202) that would be similar to the one we previously used but the experimental conditions have to be re-evaluated.

As we mentioned above, the company (Synaptic Systems) discontinued this antibody. For that reason, we recommend to use a similar antibody from Creative Diagnostics. We agree to not mention the catalog number of the previous company to avoid confusion.

25. Protein G Sepharose ${ }^{\circledR} 4$ Fast Flow Beads (GE Healthcare, catalog number: 17061801)

26. Normal rabbit serum (control, EMD Millipore, catalog number: NS01L-1ML)

27. $\mathrm{NaCl}$ (Fisher Scientific, catalog number: $\underline{\mathrm{S} 671-3}$ )

28. NP-40 (Thermo Fisher Scientific, catalog number: 28324)

29. Tris base (Fisher Scientific, catalog number: BP152-5)

30. $\mathrm{HCl}$ (VWR, catalog number: $\underline{\mathrm{BDH} 7204-1)}$

31. RNasin ${ }^{\mathrm{TM}}$ Plus RNase inhibitor (Promega, catalog number: $\underline{\mathrm{N} 2611}$ )

32. NaOAc (Amresco, catalog number: 0602)

33. EDTA, pH 8 (Thermo Fisher Scientific, catalog number: AM9260G)

34. EDTA (Amresco, catalog number: 0105)

35. SDS (Thermo Fisher Scientific, catalog number: AM9822)

36. Phenol/Chloroform/Isoamyl Alcohol; 125:24:1 mixture, pH 4.5 (Thermo Fisher Scientific, catalog number: AM9720) 
37. Agarose LE (Denville Scientific, catalog number: $\underline{\text { CA3510-8) }}$

38. iScript ${ }^{\mathrm{TM}}$ cDNA Synthesis Kit (Bio-Rad Laboratories, catalog number: 1708891)

39. Sso Advanced ${ }^{\mathrm{TM}}$ Universal $\mathrm{SYBR}^{\circledR}$ Green Supermix (Bio-Rad Laboratories, catalog number: 1725274)

40. PARIS ${ }^{\mathrm{TM}}$ Kit (Thermo Fisher Scientific, catalog number: $\underline{\text { AM1921) }}$

41. Boric acid (Fisher Scientific, catalog number: A73-1)

42. NET-2 Buffer (see Recipes)

43. G-50 Buffer (see Recipes)

44. 1x TBE (see Recipes)

\section{Equipment}

1. Micropipettes (Gilson, model: Pipetman ${ }^{\circledR}$ L, catalog number: F167370)

2. Forma ${ }^{\mathrm{TM}}$ Steri-Cycle ${ }^{\mathrm{TM}} \mathrm{CO}_{2}$ Incubator (Thermo Scientific, model: Forma ${ }^{\mathrm{TM}}$ Steri-Cycle ${ }^{\mathrm{TM}} \mathrm{CO}_{2}$ Incubators, catalog number: 370 )

3. $-80{ }^{\circ} \mathrm{C}$ freeze

4. Sorvall ${ }^{\mathrm{TM}}$ Legend $^{\mathrm{TM}}$ Micro 21R Microcentrifuge (Thermo Fisher Scientific, model: Sorvall ${ }^{\mathrm{TM}}$ Legend $^{\mathrm{TM}}$ Micro 21R, catalog number: 75002490)

5. Eppendorf ${ }^{\mathrm{TM}}$ Thermomixer ${ }^{\mathrm{TM}} \mathrm{R}$ (Eppendorf, model: Thermomixer R, catalog number: 05-412-401)

6. Labquake ${ }^{\mathrm{TM}}$ Tube Shaker/Rotator (Thermo Fisher Scientific, catalog number: C4152110Q)

7. Sorvall ${ }^{\mathrm{TM}}$ ST 40R Centrifuge (Thermo Fisher Scientific, model: Sorvall ${ }^{\mathrm{TM}}$ ST 40R, catalog number: 75004525)

8. NanoDrop ${ }^{\mathrm{TM}} 2000$ Spectrophotometer (Thermo Fisher Scientific, model: NanoDrop ${ }^{\mathrm{TM}}$ 2000, catalog number: ND-2000)

9. $\mathrm{T} 100^{\mathrm{TM}}$ Thermal Cycler (Bio-Rad Laboratories, catalog number: $\underline{1861096}$ )

10. CFX Connect ${ }^{\mathrm{TM}}$ Real-Time PCR Detection System (Bio-Rad Laboratories, catalog number: $\underline{1855200)}$

11. UV transilluminator

\section{$\underline{\text { E. Procedure }}$}

1. Plate HFF cells in serum-free DMEM supplemented with MEM non-essential amino acids at low density (approx. 1 x $10^{6}$ cells $/ 150 \mathrm{~cm}^{2}$ flask). Incubate HFF cells in humidified atmosphere of $5 \%$ $\mathrm{CO}_{2}$ at $37^{\circ} \mathrm{C}$ for $48 \mathrm{~h}$ (Figure 1 ). 
Notes:

a. In order to obtain enough RNA for each experiment, use 4 to $6150 \mathrm{~cm}^{2}$ flasks.

b. To plate HFF cells in serum-free DMEM; wash 70-80\% confluent HFF cells (100 mm TCtreated cell culture dish) three times each with $10 \mathrm{ml}$ phosphate buffered saline, add $1 \mathrm{ml} 0.05 \%$ trypsin with phenol red to detach cells, add $10 \mathrm{ml}$ serum-free DMEM media and collect cells in $15 \mathrm{ml}$ conical tube, pellet cells by centrifuging at $300 \times \mathrm{g}$ at room temperature, and resuspend pellet in serum-free DMEM supplemented medium.

c. It is important to remove trypsin from HFF cells before re-plating since medium doesn't contain FBS to inactive the trypsin.

2. Collect HFF cells by scraping flask on ice, pellet at $300 \times \mathrm{g}$ for $5 \mathrm{~min}$ at $4{ }^{\circ} \mathrm{C}$, and wash two times each with $2 \mathrm{ml}$ of phosphate buffered saline. Cell pellets can be frozen at $-80{ }^{\circ} \mathrm{C}$ until RNA extraction (cell pellets must be kept on ice until lysed with TRIzol ${ }^{\mathrm{TM}}$ ) (Figure 1).

Notes: To pellet HFF cells, remove old serum-free DMEM from cells and add $3 \mathrm{ml}$ of fresh serumfree DMEM. Use the blade of cell scraper to collect cells in one corner of flask taking care to not splash the cells in flask. Add 1-2\% of volume of FBS or BSA to cells on ice to assist in pelleting. Extract RNA from pelleted cells using TRIzol ${ }^{\mathrm{TM}}$ Reagent per manufacturer's instructions:

Note: All steps in this protocol should be performed in an RNase free bench area by using RNase AWA $Y^{T M}$ Surface Decontaminant to spray and wipe most surface areas including micropipettes and ice bucket.

a. Resuspend cell pellets thawed on ice in $1 \mathrm{ml}$ of TRIzol ${ }^{\mathrm{TM}}$ Reagent for $5 \mathrm{~min}$ to lyse.

b. Add chloroform ( $20 \%$ of TRIzol ${ }^{\mathrm{TM}}$ Reagent volume), mix samples well, and incubate at room temperature for 2-3 $\mathrm{min}$.

c. Centrifuge samples at $12,000 \times \mathrm{g}$ for $15 \mathrm{~min}$ at $4{ }^{\circ} \mathrm{C}$ to separate red phenol-chloroform (red/lower layer), interphase, and aqueous phase (clear/upper layer).

d. Collect aqueous layer and precipitate RNA for $10 \mathrm{~min}$ at room temperature with isopropanol ( 0.5 volume of original TRIzol ${ }^{\mathrm{TM}}$ Reagent); GlycoBlue ${ }^{\mathrm{TM}}$ Coprecipitant $(15 \mu \mathrm{g})$ can be added at this time to aid RNA precipitation.

e. Pellet precipitated RNA by centrifugation $\left(12,000 \times \mathrm{g}\right.$ for $10 \mathrm{~min}$ at $\left.4{ }^{\circ} \mathrm{C}\right)$.

f. Wash RNA pellets with $75 \%$ ethanol (add an equal volume of $75 \%$ ethanol to TRIzol ${ }^{\mathrm{TM}}$ Reagent and briefly vortex) and re-pellet by centrifuging at 7,500 $\mathrm{xg}$ for $5 \mathrm{~min}$ at $4{ }^{\circ} \mathrm{C}$.

g. Air-dry RNA pellets for a few minutes and re-suspend in nuclease-free water.

Note: Do not completely dry the RNA pellet because it will be difficult to re-suspend. Incubation at $55^{\circ} \mathrm{C}$ for 10 min can help solubilize the RNA. 
3. Use DNase from TURBO DNA-free ${ }^{\mathrm{TM}}$ Kit to digest DNA contamination in RNA samples according to manufacturer's protocol:

a. Mix RNA with 10x TURBO DNase Buffer (0.1 volume) and $1 \mu l$ of TURBO DNase.

b. Incubate RNA at $37^{\circ} \mathrm{C}$ for $20 \mathrm{~min}$ and halt the reaction by adding DNase Inactivation Reagent (0.1 volume)

c. Incubate RNA samples for 5 min with intermittent mixing by flicking tube then centrifuge at $10,000 \times g$ at room temperature for $2 \mathrm{~min}$.

d. Collect RNA (supernatant) and quantitate with Nanodrop ${ }^{\mathrm{TM}} 2000$ Spectrophotometer.

Note: The average amount of total RNA obtained from each flask is 1.5-2 $\mu$ g.

4. Pre-load Protein G Sepharose ${ }^{\circledR} 4$ Fast beads with rabbit serum control or anti-m3G-cap antibody (Figure 1).

Note: Use large-orifice pipet tips or clip the tip off of a pipet tip to aid the transfer of Protein $G$ Sepharose ${ }^{\circledR}$ Fast beads.

a. Wash 4 times the Protein G Sepharose ${ }^{\circledR} 4$ Fast beads ( $40 \mu 1$ slurry/IP sample) each with $1 \mathrm{ml}$ NET-2 Buffer (see Recipes) using short $30 \mathrm{sec}$ spins at $1000 \times \mathrm{g}$ to pellet beads.

Note: Non-filtered gel-loading pipet tips can be used during 'wash' steps to help prevent accidental loss of beads.

b. Dilute washed Protein G Sepharose beads in $500 \mu \mathrm{l}$ of NET-2 Buffer and add rabbit anti-m3Gcap antibody $(15 \mu \mathrm{l} / \mathrm{IP}$ sample, approximately $150 \mu \mathrm{g})$ or rabbit serum control (15 $\mu \mathrm{l} / \mathrm{IP}$ sample, approximately $150 \mu \mathrm{g}$ ). To achieve equal loading of beads with antibodies only use one microcentrifuge tube/antibody; aliquot the beads into separate tubes after they are pre-loaded.

c. Incubate beads/antibody for $1.5 \mathrm{~h}$ at room temperature on tube rotator; make sure samples are actually mixing.

d. Remove excess antibody by washing Protein G Sepharose Beads 5 times each with $1 \mathrm{ml}$ NET2 Buffer (short $30 \mathrm{sec}$ spins at 1,000 $x \mathrm{~g}$ ); Resuspend beads in NET-2 Buffer ( $50 \mu 1 /$ sample).

e. Aliquot antibody-bound Protein G Sepharose Beads into 1.5 microcentrifuge tubes; use enough tubes to have one of each antibody (Anti-m3G-cap and control) per sample.

5. Pre-clear RNA with Protein G Sepharose Beads to reduce non-specific binding:

a. Add RNasin Plus RNase inhibitor $(1 \mu \mathrm{l})$ to RNA ( $10 \mu \mathrm{g} / 500 \mu \mathrm{l}$ diluted with NET-2 buffer $)$ and incubate for 3 to $5 \mathrm{~min}$ at $85^{\circ} \mathrm{C}$.

Note: Plunge RNA into ice immediately after heating to avoid refolding of RNA.

b. After heating, add an additional $1 \mu \mathrm{l}$ of RNasin Plus RNase inhibitor to RNA.

Note: Remember to wipe tubes with RNase AWA $Y^{T M}$ Surface Decontaminant before opening to prevent possible contamination and degradation of RNA. 
c. Rotate RNA (10 $\mu \mathrm{g} / \mathrm{sample}$ for each antibody) slowly (without vibration) with $40 \mu 1$ of NET 2washed Protein G Sepharose 4 Fast Flow beads (without antibody) for $2 \mathrm{~h}$ at $4{ }^{\circ} \mathrm{C}$.

d. Centrifuge RNA at 1,000 $x \mathrm{~g}$ for 2 min to remove pre-clearing beads.

6. Rotate pre-cleared RNA slowly (without vibration) with anti-m3G-cap or control pre-loaded Protein G Sepharose ${ }^{\circledR} 4$ Fast beads for 4 to $16 \mathrm{~h}$ at $4{ }^{\circ} \mathrm{C}$. Confirm that samples are mixing (Figure 1).

7. Collect the beads by centrifuging at $1,000 \times g$ for 2 min and remove supernatant (save $250 \mu$ for RNA extraction).

8. Wash beads 5 times with $1 \mathrm{ml}$ of NET-2 Buffer using short $30 \mathrm{sec}$ spins at 1,000 $x \mathrm{~g}$.

Note: RNasin Plus RNase inhibitor can be added to NET-2 Buffer (see Recipes) to prevent degradation of RNA.

9. Resuspend beads in $250 \mu \mathrm{l}$ of G-50 Buffer (see Recipes) to elute TMG-capped RNA (Figure 1).

10. Purify RNA by Phenol-chloroform-isoamyl alcohol (PCI) extraction (also extract RNA from TMGcapped depleted supernatant)

a. Add $250 \mu \mathrm{l}$ of Phenol-chloroform-isoamyl alcohol to RNA in G-50 Buffer.

b. Vortex RNA samples for $20 \mathrm{sec}$ (10 up and 10 angled) and centrifuge samples for $10 \mathrm{~min}$ at $12,000 \times g$ at room temperature.

c. Transfer the aqueous phase with RNA (upper layer) to a clean microcentrifuge tube containing $50 \mu \mathrm{l}$ of $3 \mathrm{M}$ sodium acetate ( $\mathrm{NaOAc}), \mathrm{pH} 5.2$.

d. Add $1 \mathrm{ml}$ of $100 \%$ ethanol (precooled to $-20{ }^{\circ} \mathrm{C}$ ) to precipitate RNA; $1 \mu$ of GlycoBlue can be added to help precipitate RNA and visualize pellets.

e. Incubate RNA at $-20{ }^{\circ} \mathrm{C}$ for $48 \mathrm{~h}$.

f. Pellet RNA by centrifuging sample at $12,000 \times \mathrm{g}$ for $10 \mathrm{~min}$ at $4{ }^{\circ} \mathrm{C}$.

g. Aspirate ethanol and wash RNA pellet with $500 \mu \mathrm{l}$ of $75 \%$ ethanol (precooled to $-20{ }^{\circ} \mathrm{C}$ ).

h. Re-pellet purified RNA by short spin for $1 \mathrm{~min}$ at $12,000 \times \mathrm{g}$ at $4{ }^{\circ} \mathrm{C}$ and aspirate $75 \%$ ethanol.

i. Dissolve the dried m3G-capped RNA pellet in nuclease-free water (20 to $30 \mu 1)$.

Note: The average amount of pull-down m3G-capped RNA obtained from each experiment (5 flasks) is $1.5-2 \mu g$.

11. RT-PCR of m3G-capped RNAs by using iScript ${ }^{\mathrm{TM}}$ cDNA Synthesis Kit and 2x SsoAdvanced ${ }^{\mathrm{TM}}$ $\mathrm{SYBR}^{\circledR}$ Green Supermix PCR according to manufacturer's protocols:

Note: Perform RT-PCR using un-processed RNA (input) and RNA extracted from supernatant to determine expression levels of RNA of interest (m3G-capped and uncapped).

a. Mix $5 \mu 1$ of m3G-capped RNA with 5x iScript Reaction Mix (0.2 volume) and $1 \mu 1$ of iScript Reverse Transcriptase. 
b. Process RNA to cDNA in a thermocycler by undergoing the following conditions: $25{ }^{\circ} \mathrm{C}$ for 5 $\min , 46{ }^{\circ} \mathrm{C}$ for $20 \mathrm{~min}, 95^{\circ} \mathrm{C}$ for $1 \mathrm{~min}$, and held at $4{ }^{\circ} \mathrm{C}$. The reaction can be stored at $4{ }^{\circ} \mathrm{C}$ for short-term storage or $-20{ }^{\circ} \mathrm{C}$ for long-term storage.

c. Mix the resulting cDNA (around $1 / 4$ of the total cDNA reaction mix) with $2 x$ SsoAdvanced ${ }^{\mathrm{TM}}$ $\mathrm{SYBR}^{\circledR}$ Green Supermix (0.5 volume) and the desired forward and reverse primers $(12.5 \mu \mathrm{M}$ stock; 0.04 volume).

d. cDNA then undergoes the following conditions to produce a PCR product: $95^{\circ} \mathrm{C}$ for $1.5 \mathrm{~min}$; 40 cycles: $95^{\circ} \mathrm{C}$ for $30 \mathrm{sec}, 60^{\circ} \mathrm{C}$ for $30 \mathrm{sec}$.

e. The PCR product is ran on a $1 \%$ agarose gel (made with 1x TBE [see Recipes] and ethidium bromide) at $100 \mathrm{~V}$ for $2 \mathrm{~h}$, and bands are imaged with a UV transilluminator.

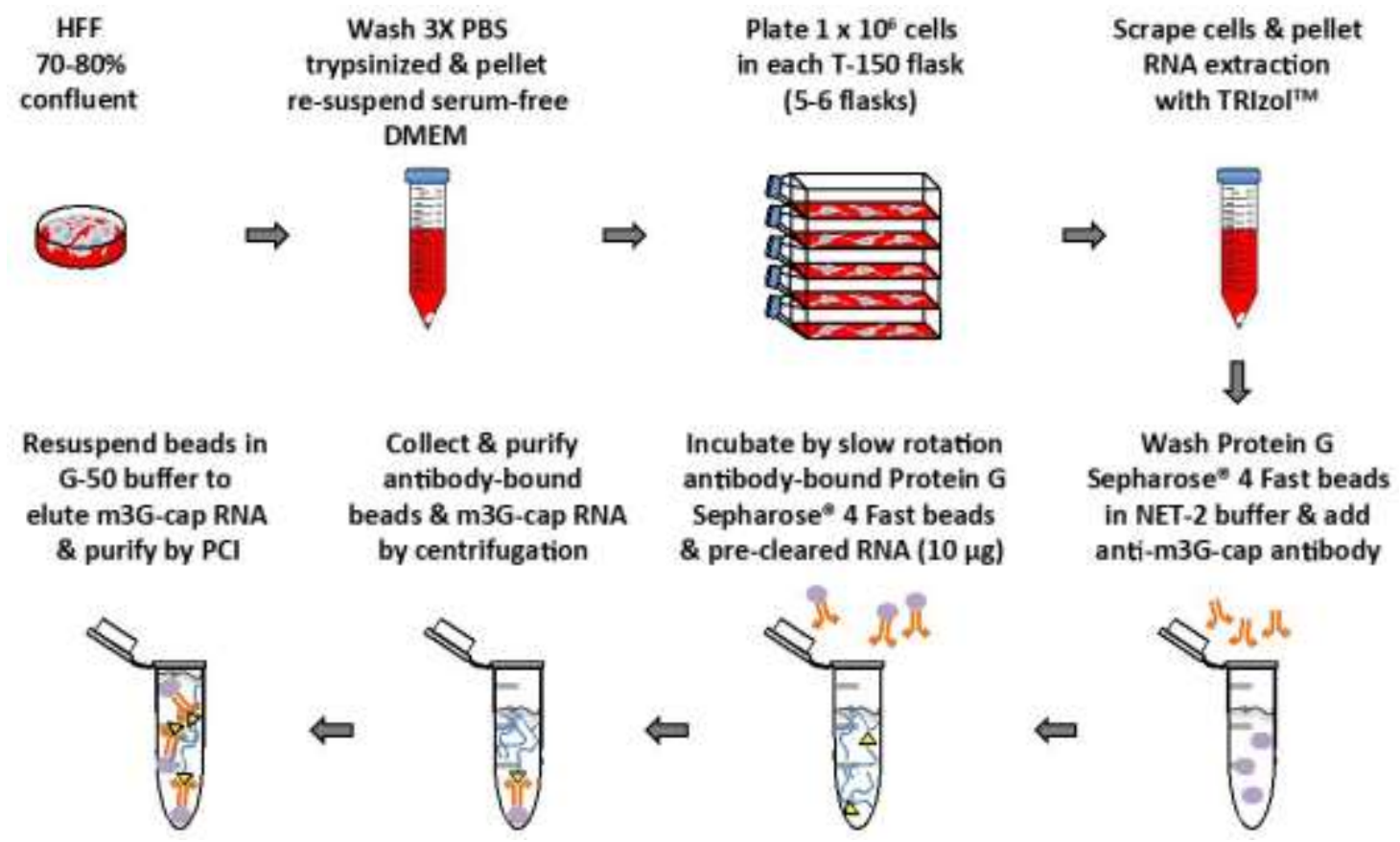

Figure 1. Main steps diagram of this protocol

\section{$\underline{\text { F. Data analysis }}$}

The recovery of (TMG)-capped RNAs was measured by RT-PCR amplification of well-known hypermethylated RNAs such as small nuclear RNA (snRNA) U7 (Figure 2) or small nucleolar RNA (snoRNA) U3 (Martinez et al., 2017). 


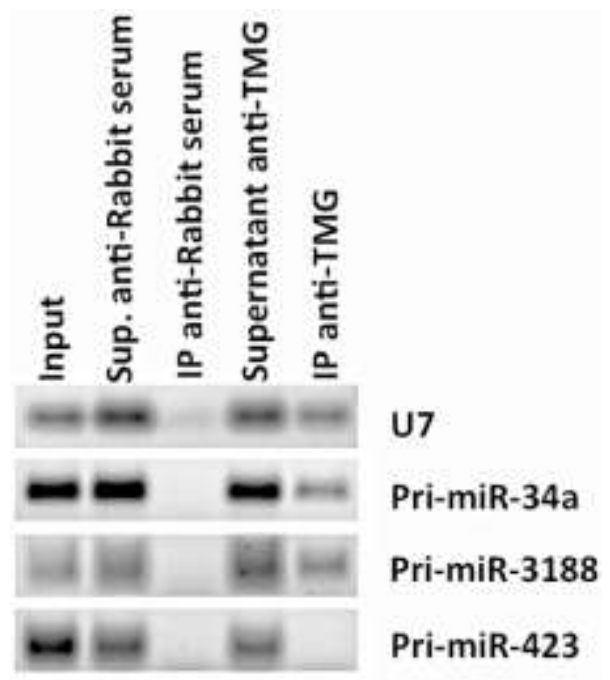

Figure 2. RNA immunoprecipitation of (TMG)-capped primary miRNAs (Pri-miRNAs) in quiescent human foreskin fibroblasts (HFFs). RT-PCR data shows the RNA immunoprecipitation of Pri-miR-34a and Pri-miR-3188 in quiescent HFFs (as well as the positive control snRNA U7), but not Pri-miR-423 using an antibody against (TMG)-capped RNAs. Total RNA was extracted using TRIzol Reagent, and $10 \mu \mathrm{g}$ of RNA was diluted in NET-2 buffer, precleared and incubated with Protein G Sepharose 4 Fast Flow beads loaded with $15 \mu 1$ of control antibody (Normal Rabbit Serum, EMD-Millipore) or with antibody recognizing the (TMG)-cap (Anti-m3G-cap, rabbit polyclonal, Synaptic Systems). Beads were rinsed five times with NET-2 buffer and were resuspended in G-50 buffer. RNA was extracted from the beads by phenolchloroform-isoamyl alcohol extraction and resuspended in $20 \mu \mathrm{l}$ of nuclease-free water. Immunoprecipitated tri-methylated capped RNA was converted to cDNA using iScript cDNA synthesis kit (Bio-Rad), followed by RT-PCR, and visualized after gel electrophoresis.

\section{G. Notes}

This protocol could be modified to determine the location of TMG-capped RNA by separation of nuclear and cytoplasmic RNA fractions from fresh cultured cells (PARIS ${ }^{\mathrm{TM}} \mathrm{Kit}$ ).

\section{$\underline{\text { H. Recipes }}$}

Note: All reagents should be made with nuclease-free water and autoclaved.

1. NET-2 Buffer

$150 \mathrm{mM} \mathrm{NaCl}$ 
$0.05 \%$ NP-40

50 mM Tris-HCl, $\mathrm{pH} 7.4$

2. G-50 buffer

$20 \mathrm{mM}$ Tris, $\mathrm{pH} 7.5$

$300 \mathrm{mM}$ NaOAc

2 mM EDTA, pH 8

$0.25 \%$ SDS

3. $1 \mathrm{x}$ TBE

$216 \mathrm{~g}$ Tris base

$110 \mathrm{~g}$ boric acid

$80 \mathrm{ml} 0.5 \mathrm{M}$ EDTA, pH 8

$1.5 \mathrm{~L}$ water

(Bring solution to $\mathrm{pH} 8.3$ )

\section{Acknowledgments}

We thank Dr. Joan Steitz's laboratory for sharing part of this protocol. I.M., J.A.B., and K.E.H. were supported in part by a WVU Foundation Fund (2V882). K.E.H. was supported in part by funding from the Ladies Auxiliary to the VFW of the United States (CK 003229). M.X. is supported by NIH grant R00 CA190886. J.A.S. is an Investigator of the Howard Hughes Medical Institute. This protocol was modified from previous work (Yu et al., 1998). We do not have any conflict of interest or competing interests.

\section{J. References}

1. Boulon, S., Verheggen, C., Jady, B. E., Girard, C., Pescia, C., Paul, C., Ospina, J. K., Kiss, T., Matera, A. G., Bordonne, R. and Bertrand, E. (2004). PHAX and CRM1 are required sequentially to transport U3 snoRNA to nucleoli. Mol Cell 16(5): 777-787.

2. Coller, H. A. (2011). Cell biology. The essence of quiescence. Science 334(6059): 1074-1075.

3. Esteller, M. (2011). Non-coding RNAs in human disease. Nat Rev Genet 12(12): 861-874.

4. Ha, M. and Kim, V. N. (2014). Regulation of microRNA biogenesis. Nat Rev Mol Cell Biol 15(8): 509-524.

5. Kim, Y. K., Kim, B. and Kim, V. N. (2016). Re-evaluation of the roles of DROSHA, Export in 5, and DICER in microRNA biogenesis. Proc Natl Acad Sci U S A 113(13): E1881-1889.

6. Kiss, T. (2004). Biogenesis of small nuclear RNPs. J Cell Sci 117(Pt 25): 5949-5951.

7. Luhrmann, R., Appel, B., Bringmann, P., Rinke, J., Reuter, R., Rothe, S. and Bald, R. (1982). Isolation and characterization of rabbit anti-m3 2,2,7G antibodies. Nucleic Acids Res 10(22): 71037113. 
8. Martinez, I., Hayes, K. E., Barr, J. A., Harold, A. D., Xie, M., Bukhari, S. I. A., Vasudevan, S., Steitz, J. A. and DiMaio, D. (2017). An Exportin-1-dependent microRNA biogenesis pathway during human cell quiescence. Proc Natl Acad Sci U S A 114(25): E4961-E4970.

9. Ohno, M., Segref, A., Bachi, A., Wilm, M. and Mattaj, I. W. (2000). PHAX, a mediator of U snRNA nuclear export whose activity is regulated by phosphorylation. Cell 101(2): 187-198.

10. Palacios, I., Hetzer, M., Adam, S. A. and Mattaj, I. W. (1997). Nuclear import of U snRNPs requires importin 3. EMBO J 16(22): 6783-6792.

11. Valcourt, J. R., Lemons, J. M., Haley, E. M., Kojima, M., Demuren, O. O. and Coller, H. A. (2012). Staying alive: metabolic adaptations to quiescence. Cell Cycle 11(9): 1680-1696.

12. Yedavalli, V. S. and Jeang, K. T. (2010). Trimethylguanosine capping selectively promotes expression of Rev-dependent HIV-1 RNAs. Proc Natl Acad Sci U S A 107(33): 14787-14792.

13. Yu, Y. T., Shu, M. D. and Steitz, J. A. (1998). Modifications of U2 snRNA are required for snRNP assembly and pre-mRNA splicing. EMBO J 17(19): 5783-5795. 


\section{Jamie Ann Barr}

jamieabarr@gmail.com

\section{Professional Summary}

I aspire to work in a position that allows me to contribute to the translation of research discoveries to the public by utilizing my knowledge in technology transfer and commercialization, biology, and engineering principles. Experiences during my academic career and positions as a technology scout have shown me that I enjoy discussing scientific research topics, working with researchers to evaluate their technologies for commercial potential, and advising on the process of technology commercialization and protection.

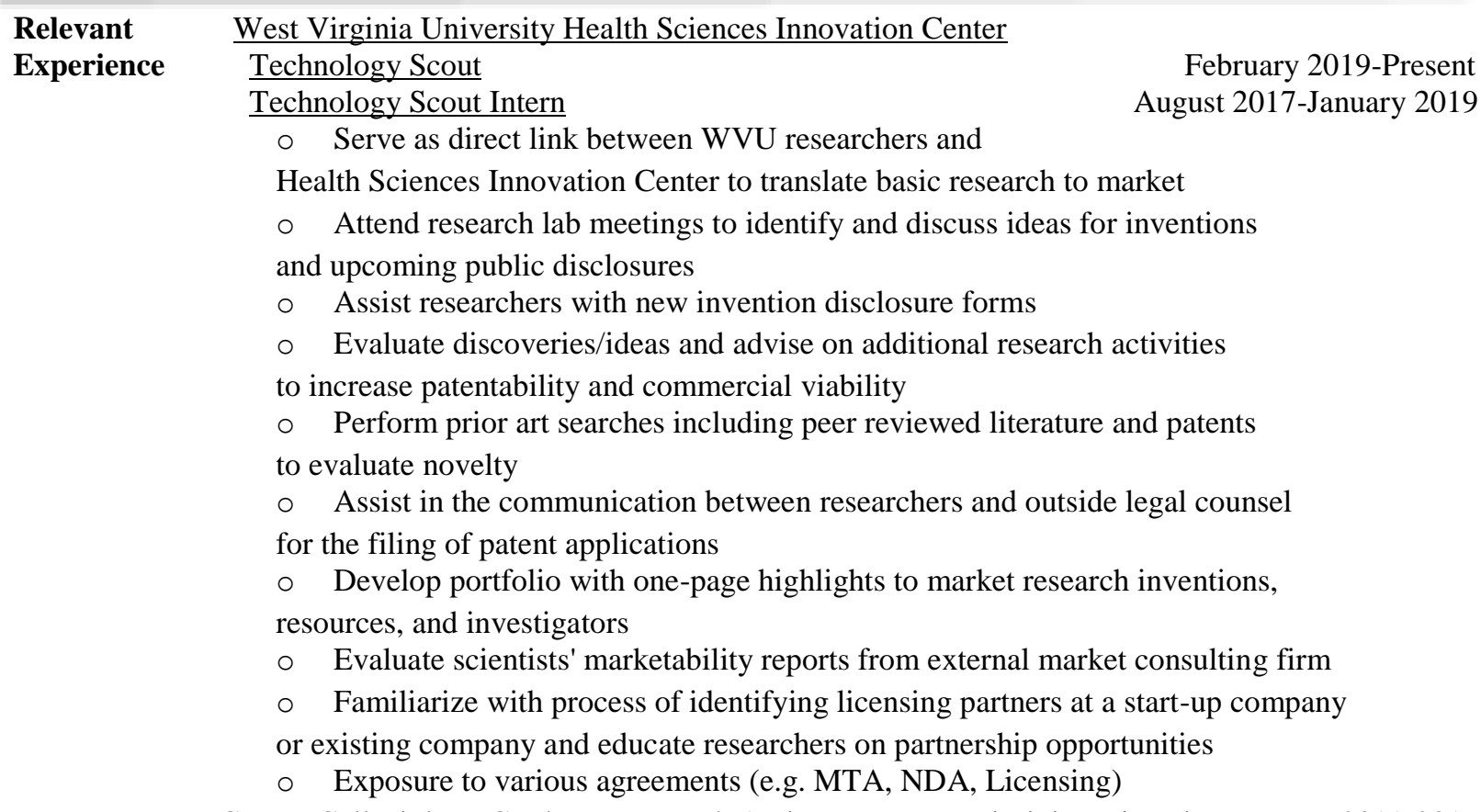

Cancer Cell Biology Graduate Research Assistant at West Virginia University

2011-2019

Principal Investigator: Ivan Martinez, Ph.D. Spring 2013-Spring 2019

- Dissertation Thesis: Regulation of long non-coding RNAs by the human papillomavirus oncoproteins

- Independently plan and conduct scientific experiments for multiple research projects

- Plan projects to train multiple research rotation students

- Perform weekly ordering for the lab based on lab budget

- Maintain Radiation Safety compliance for the lab

Principal Investigator: Yon Rojanasakul, Ph.D.

Spring 2012-Spring 2013

- Researched the role of a cardiac glycoside as an anti-cancer therapeutic agent

- Analyzed the toxicity of carbon nanotubes and their risk of inducing and promoting lung carcinogenesis

Lectured a Cellular Methods Class to students in Ph.D. program

Biomedical \& Chemical Engineering Senior Research at West Virginia University

Principal Investigator: Robin S. Hissam, Ph.D.

- Performed research on elastin-based vesicles for the transport and delivery of drugs

Pharmaceutical Sciences Summer Research Experience at West Virginia University

Principal Investigator: Peter M. Gannett, Ph.D. 
- Performed research using nanoparticles in order to improve detection and treatment of diseases

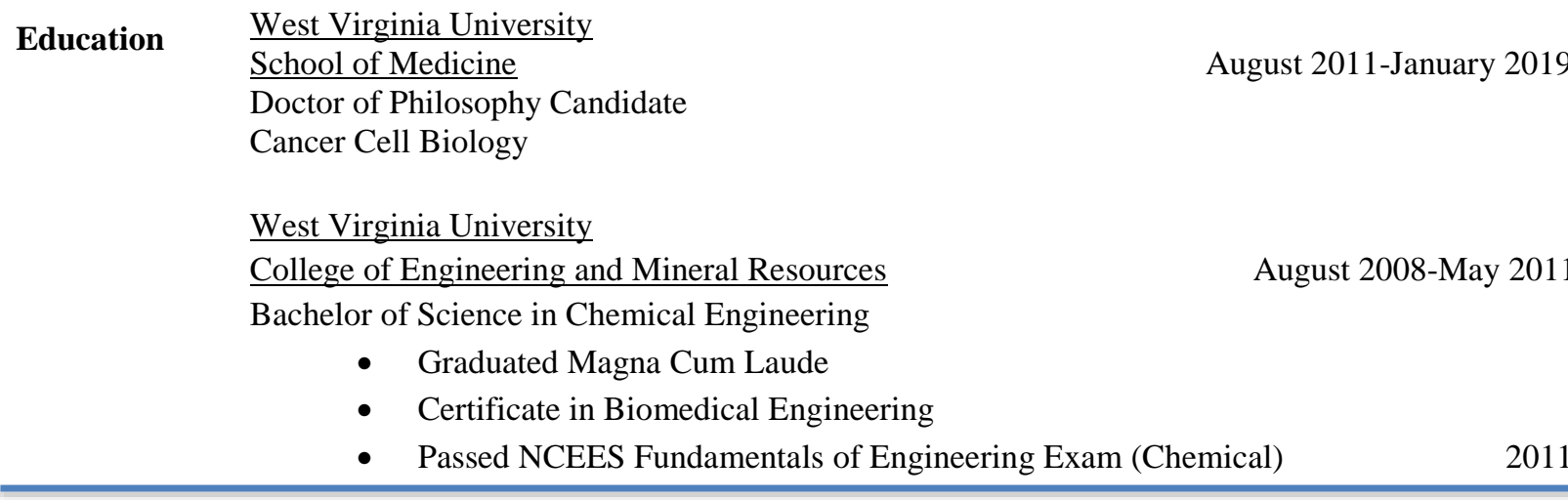
Fellowships,
Honors, and
Scholarships
Graduate Student Travel Award to visit Broad Institute in Boston, MA Principal Investigator: John Rinn, Ph.D.

- Trained on RNA Immunoprecipitation technique

Outstanding Graduate Student Award - Captain of Team Award

"Mad Scientists" - Relay for Life Team

$1^{\text {st }}$ Place Van Liere Poster Presentation Award - Basic Science

Year 4

Year 3

WVNano/NanoSAFE Graduate Fellowship Program

Dean's List

February 2015

August 2011-August 2013

Fall 2007 \& 2009, Spring 2008, 2010, \& 2011

WVNano Summer Undergraduate Research Experience (SURE) program

Kincaid Memorial Scholarship
April-July 2010

Fall 2009, Spring 2010

\section{Skills}

- Completing and compiling documents for the disclosure of new technologies

- Discussing and evaluating scientists' research ideas and guide inventors to advance their technologies

- Identifying marketable attributes to researchers' inventions

- Communication: oral presentation and written (assisted Principal Investigator on editing grant submissions from 2013-present) (writer, co-author, and editor on multiple scientific, technical writing journal submissions) (presented research at many student forums, conferences, and meetings)

- Computer proficient: Microsoft Office Excel, PowerPoint, Word; Adobe Illustrator

- Developing and optimizing detailed scientific protocols

- RNA preparation and analysis for human transcriptome microarray (Affymetrix)

- RNA-specific techniques: RNA \& library preparation for high-throughput sequencing, RNA extraction, real-time reverse transcription PCR (q-RTPCR), reverse transcription PCR (RT-PCR), PCR, RNA Immunoprecipitation, northern blotting, rapid amplification of cDNA ends (RACE), RNA fluorescence in situ hybridization (FISH) in cell lines and human tissue arrays

$\circ$ Experience with confocal microscopy

- Genetic expression manipulation: CRISPR/Cas9, antisense oligonucleotides (ASOs), siRNA, shRNA

- DNA extraction

- Cellular Fractionation 
- Standard cell culture techniques (primary and immortalized mammalian cells)

- Mammalian cell retroviral and lentiviral transfection (Lipofectamine- and calcium phosphate-based) and infection

- Cloning and transformation of constructs in bacteria

- Restriction digestion \& TOPO cloning

- In vitro cellular function assays (Transwell migration and invasion, flow cytometry for PI cell cycle and Annexin V/PI apoptosis, cell proliferation/viability)

- Protein-specific techniques: protein extraction, immunoprecipitation, western blotting

\section{Publications Manuscripts published:}

Barr, J.A., Hayes, K.E., Brownmiller, T., Harold, A.D., Jagannathan, R., Lockman, P., Martinez, I. Long non-coding RNA FAM83H-AS1 is regulated by human papillomavirus 16 E6 independently of p53 in cervical cancer cells. Sci Rep. 2019 Mar 6;9(1):3662.

Hayes, K.E., Barr, J.A., Xie, M., Steitz, J.A., Martinez, I. (2017) Immunoprecipitation of trimethylated capped RNA. Bio Protoc. 2018 Feb 5;8(3).

Martinez, I., Hayes, K.E., Barr, J.A., Harold, A., Xie, M., Bukhari, S.I.A., Vasudevan, S., Steitz, J.A., DiMaio, D. (2017) An Exportin-1-dependent microRNA Biogenesis Pathway During Human Cell Quiescence. Proc Natl Acad Sci U S A. 114(25):E4961-E4970.

Manuscripts in preparation for submission:

Khan, S.A., Barr, J.A., Brownmiller, T., Martinez, I. Global Expression Analysis and Tissuearrays Identify the Regulation of LAT1 and NAP1L3 Genes by Human Papillomavirus Oncogenes in Cervical-Cancer Cells. 2018 (Manuscript under review at Journal of Virology).

Hayes, K.E., Barr, J.A., Harold, A., Wilusz, J.E., Martinez, I. Discovery of Novel Non-Coding Circular RNAs Generated by High-Risk Human Papillomaviruses. 2018.

Hayes, K.E., Barr, J.A., Harold, A., Wilusz, J.E., Ziegelbauer, J.M., Martinez, I. HPV Oncogene E6 Regulates the Human Non-Coding Circular RNA circMYBL2 During Cervical Carcinogenesis. 2018.

Organizations Association of University Technology Managers (AUTM) American Association for the Advancement of Science (AAAS)

Cell Biology Training Program (CBPT)

American Association for Cancer Research (AACR)

Graduate Student Organization (GSO)

- Secretary

Tau Beta Pi (TBP): The Engineering Honors Society

First Year Student Mentorship Program

WVNano/NanoSAFE Graduate Fellowship Program

The American Institute of Chemical Engineers (AIChE)

- Secretary

- Assistant Treasurer

Society of Women Engineers (SWE)

Society of Biological Engineers (SBE)
December 2017-Present

August 2016-Present

Fall 2013-Spring 2019

2012-Present

2012-2019

2014-2016

2010-Present

Fall 2013-Spring 2014

August 2011-August 2013

2008-2011

Fall 2010-Spring 2011

Fall 2009-Spring 2010

2008-2011

2010-2011 